\title{
Combined Theoretical and Experimental Studies Unravel Multiple Pathways to Convergent Asymmetric Hydrogenation of Enamides.
}

Jianping Yang $\ddagger^{[\mathrm{a}]}$, Luca Massaro $\ddagger^{[\mathrm{a}]}$, Suppachai Krajangsri ${ }^{[\mathrm{a}]}$, Thishana Singh ${ }^{[\mathrm{b}]}$, $\mathrm{Hao} \mathrm{Su}{ }^{[\mathrm{c}]}$, Emanuele Silvi ${ }^{[\mathrm{a}]}$, Sudipta Ponra ${ }^{[\mathrm{a}]}$, Lars Eriksson ${ }^{[\mathrm{d}]}$, Mårten S. G. Ahlquist ${ }^{[\mathrm{c}]}$ and Pher G. Andersson* [a],[b]

[a] Department of Organic Chemistry, Stockholm University, Arrhenius Laboratory, 106 91, Stockholm, Sweden. Corresponding author: pher.andersson@su.se

[b] School of Chemistry and Physics, University of Kwazulu-Natal, Private Bag X54001, Durban, 4000, South Africa.

[c] School of Biotechnology, KTH Royal Institute of Technology, 106 91, Stockholm, Sweden.

[d] Department of Materials and Environmental Chemistry, Stockholm University, Svante Arrhenius väg 16C, 106 91, Stockholm, Sweden.

$\$$ Authors contributed equally to this work

Table of contents

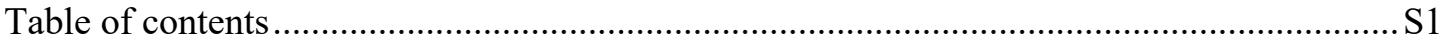

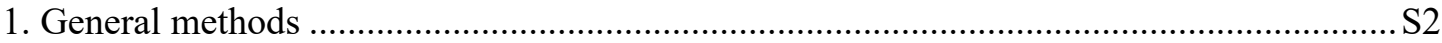

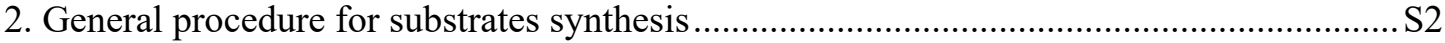

2.1. General procedure for preparation of 1,2-diarylethan-1-one .............................. S2

2.2 General procedure for preparation $\alpha$-Aryl-Alkyl ketones ...................................... S2

2.3 General procedure for preparation of aldehydes ..................................................... S2

2.4. General procedure for the preparation of the $\alpha, \beta$-enamides ( $Z$-isomer) $) . . \ldots \ldots \ldots \ldots \ldots . . . . . . . S 3$

2.5. General procedure for the preparation of the $\alpha, \beta$ - enamides $(E$-isomer) ................. S4

2.6. General procedure for the preparation of the $(Z)$-a, $\beta$-enamides from $E$ isomer....... S6

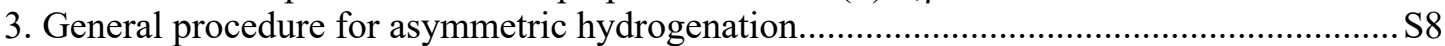

4. General procedure for the preparation of iridium complexes ..........................................S13

5. Separation method data and specific rotation of chiral compounds ..................................S15

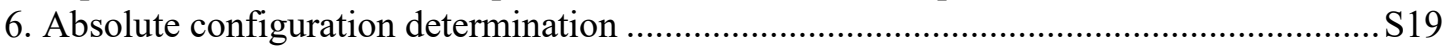

6.1 The absolute configuration of the different classes of hydrogenated products.......S19

6.2 Di-aryl enamides by single crystal X-ray diffraction .............................................. 19

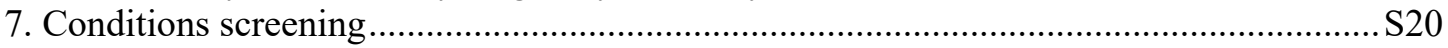

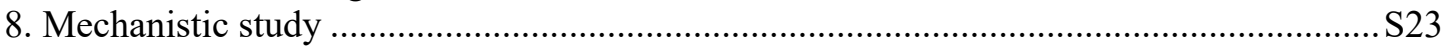

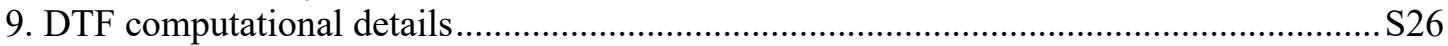

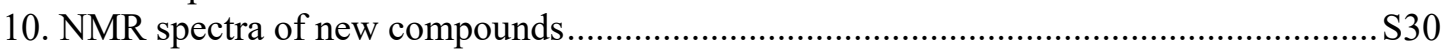

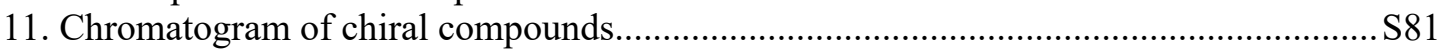

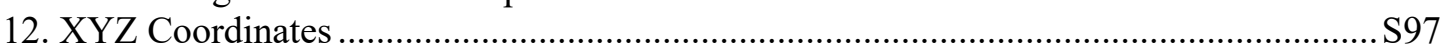

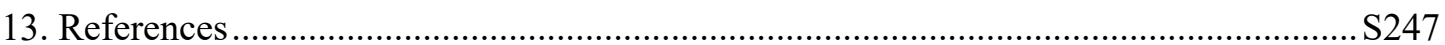




\section{General methods}

All reactions were conducted under dry and inert atmosphere using magnetic stirring. $\mathrm{CH}_{2} \mathrm{Cl}_{2}$, used in the hydrogenation, was freshly distilled from $\mathrm{CaH}_{2}$ under nitrogen. THF was freshly distilled from sodium-benzophenone under nitrogen. All reagents were used as supplied commercially without further purification. Chromatographic separations were performed on Kiesel gel $60 \mathrm{H}$ silica gel (particle size: 0.063-0.100 mm) or Brockmann I, activated. Thin layer chromatography (TLC) was performed on aluminum plates coated with Kieselgel 60 $(0.20 \mathrm{~mm}, \mathrm{UV} 254)$ and visualized under ultraviolet light $(v=254 \mathrm{~nm}) .{ }^{1} \mathrm{H}$ NMR spectra were recorded on a Bruker 400 or 500 at $400 / 500 \mathrm{MHz}$ in $\mathrm{CDCl}_{3}$ and referenced internally to the residual $\mathrm{CHCl}_{3}$ peak $(7.26 \mathrm{ppm}) .{ }^{13} \mathrm{C}$ NMR spectra were recorded at $100 \mathrm{MHz}$ in $\mathrm{CDCl}_{3}$ and referenced to the central peak of $\mathrm{CDCl}_{3}(77.16 \mathrm{ppm})$. Chemical shifts are reported in ppm $(\delta$ scale). Enantiomeric excesses were determined either using chiral HPLC, SFC or GC with a diode array detector at 220 and $254 \mathrm{~nm}$. Racemic compounds were used for comparison. HRMS data were obtained using a Bruker MicroTOF-Q II instrument operation at ambient temperature. Optical rotations were recorded on an Autopol IV polarimeter from Rudolp Research Analytical, equipped with a sodium lamp (589 nm) and a $10 \mathrm{~cm}$ cell.

\section{General procedure for substrates synthesis}

\subsection{General procedure for preparation of 1,2-diarylethan-1-one}

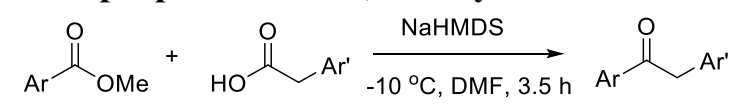

To a solution of aromatic acetic acid (10 mmol, 1.0 equiv.) and aromatic methyl ester (10 mmol, 1.0 equiv.) in DMF (30 mL) was added NaHMDS (2.0 M in THF) $(20 \mathrm{~mL}, 40 \mathrm{mmol}$, 4.0 equiv.) at $-10^{\circ} \mathrm{C}$ over $1 \mathrm{~min}$. The resulting mixture was stirred at $-10^{\circ} \mathrm{C}$ for 3.5 hours. To the resulting solution was then added saturated aqueous $\mathrm{NH}_{4} \mathrm{Cl}$ solution. The resulting mixture was extracted with EtOAc $(2 \times 100 \mathrm{ml})$. The combined organic phase was washed with brine, dried over $\mathrm{Na}_{2} \mathrm{SO}_{4}$ and concentrated in vacuo. The residue was purified by silica gel column chromatography.

\subsection{General procedure for preparation $\alpha$-Aryl-Alkyl ketones}

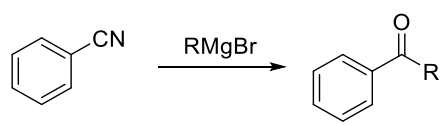

Grignard reagent ( $15 \mathrm{mmol}, 1.5$ equiv.) was slowly added to a solution of cyano (-CN) (10 mmol, 1.0 equiv.) aromatic compounds in dry THF $(30 \mathrm{~mL})$ at $0^{\circ} \mathrm{C}$. The reaction was allowed to stir at room temperature for $16 \mathrm{~h}$. The reaction was quenched by addition of a saturated aqueous $\mathrm{NH}_{4} \mathrm{Cl}$ solution, and $1 \mathrm{M} \mathrm{HCl}$ was added and the mixture was stirred for $1 \mathrm{~h}$. The mixture was extracted with $\mathrm{Et}_{2} \mathrm{O}(3 \times 100 \mathrm{ml})$. The combined organic phases were combined and washed brine and dried over $\mathrm{Na}_{2} \mathrm{SO}_{4}$. The solvent was evaporated under reduced pressure to afford the crude products which then were purified by column chromatography on silica gel.

\subsection{General procedure for preparation of aldehydes}

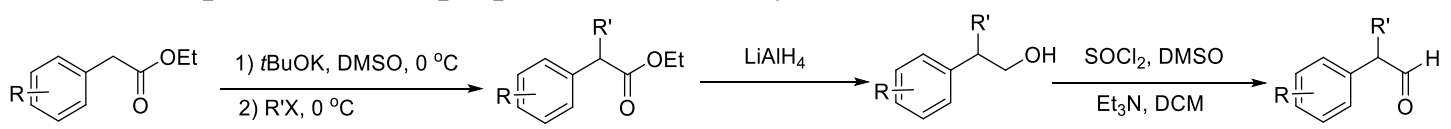

Potassium tert-butoxide ( 1 equiv.) was suspended in dry DMF $(7 \mathrm{~mL} / 10 \mathrm{mmol})$ at $0^{\circ} \mathrm{C}$ under nitrogen atmosphere and methyl phenylacetate (1 equiv.) was added in one portion, followed 
by alkyl halide (1 equiv.) after $2 \mathrm{~min}$. The reaction was allowed to warm up to room temperature and magnetic stirring was continued for $1 \mathrm{~h}$. Water $(10 \mathrm{~mL})$ was added and the solution was extracted with $\mathrm{CH}_{2} \mathrm{Cl}_{2}$ (2 times). The organic layers were washed with $10 \mathrm{~mL}$ of a saturated, aqueous solution of $\mathrm{NH}_{4} \mathrm{Cl}$ and $10 \mathrm{~mL}$ of water, and dried over $\mathrm{Na}_{2} \mathrm{SO}_{4}$. The solvent was evaporated and the crude product was purified by column chromatography.

$\mathrm{LiAlH}_{4}$ (1.2 equiv.) was suspended in $\operatorname{dry~}_{\mathrm{Et}} \mathrm{O}(3 \mathrm{~mL} / 10 \mathrm{mmol})$. Then, a solution of alkylated ester ( 1 equiv.) in $20 \mathrm{~mL}$ of $\mathrm{Et}_{2} \mathrm{O}$ was added slowly while stirring. The reaction was monitored by TLC and after complete consumption of the ester the reaction was quenched by adding water. The layers were separated and the aqueous layer was extracted with $\mathrm{CH}_{2} \mathrm{Cl}_{2}$ (2 times). The combined organic layers were dried over $\mathrm{Na}_{2} \mathrm{SO}_{4}$. The solvent was evaporated and the product was obtained as colorless oil after purification by column chromatography.

To a solution of oxalyl chloride (1.1 equiv.) in $\mathrm{CH}_{2} \mathrm{Cl}_{2}(13 \mathrm{~mL} / 10 \mathrm{mmol})$ was added a solution of DMSO (2.4 equiv.) in $\mathrm{CH}_{2} \mathrm{Cl}_{2}(3 \mathrm{~mL} / 10 \mathrm{mmol})$ at $-78{ }^{\circ} \mathrm{C}$. The solution was stirred for $10 \mathrm{~min}$ and alcohol (1 equiv.) in $\mathrm{CH}_{2} \mathrm{Cl}_{2}(20 \mathrm{~mL} / 10 \mathrm{mmol})$ was added. After $15 \mathrm{~min}, \mathrm{Et}_{3} \mathrm{~N}$ $(7.5 \mathrm{~mL} / 10 \mathrm{mmol}$ of alcohol) was added, and the solution was allowed to warm up to room temperature and stirred for $1 \mathrm{~h}$. Water was added to quench the reaction, the phases were separated, and the aqueous layer was extracted with $\mathrm{CH}_{2} \mathrm{Cl}_{2}$ (2 times). The organic phase was washed with water, dried over $\mathrm{Na}_{2} \mathrm{SO}_{4}$, and the solvent was evaporated. The corresponding aldehyde was obtained as colorless solid after silica-gel flash chromatography.

\subsection{General procedure for the preparation of the $\alpha, \beta$-enamides ( $Z$-isomer)}

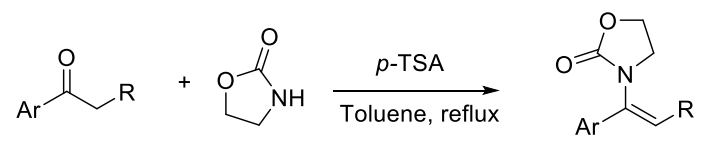

A solution of the ketone (10 mmol, 1.0 equiv.), 2-oxazolidinone (30 mmol, 2.610g, 3 equiv.), and $10 \mathrm{~mol} \%$ of $p$-toluenesulfonic acid $(1 \mathrm{mmol}, 190 \mathrm{mg})$ in toluene $(30 \mathrm{~mL}: 10 \mathrm{mmol}$ ketone) was heated to reflux and the formed water was removed by azeotropic distillation over $36 \mathrm{~h}$. After cooling the solution to room temperature, it was washed with a saturated, aqueous solution of $\mathrm{NH}_{4} \mathrm{Cl}(10 \mathrm{~mL})$ and water $(10 \mathrm{~mL})$, and the combined organic layers were dried over $\mathrm{Na}_{2} \mathrm{SO}_{4}$. After evaporation of the organic solvent and purification by silicagel flash chromatography (pentane/ethyl acetate 80:20 to 70:30), the pure Z-isomers of the $\alpha, \beta$-enamides were obtained.

Characterization for the following compounds have been previously reported: $\mathbf{1 a}-\mathbf{1} \mathbf{k},{ }^{1} \mathbf{2 a - 2} \mathbf{c},{ }^{1}$ $\mathbf{2 f - 2 h},{ }^{1} 4 \mathbf{a}^{2}, \mathbf{4} \mathbf{b}^{3}, \mathbf{4} \mathbf{c}^{1}, \mathbf{1 1} \mathrm{c}^{4,5}, \mathbf{1 1} \mathbf{d}^{6}$.

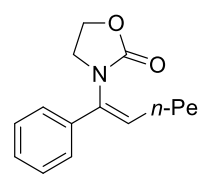

(Z)-3-(1-phenylhept-1-en-1-yl)oxazolidin-2-one(Z-2d) White solid. ${ }^{1} \mathrm{H}$ NMR (400 MHz, Chloroform- $d$ ) $\delta 7.39-7.27(\mathrm{~m}, 5 \mathrm{H}), 6.01(\mathrm{t}, J=7.2 \mathrm{~Hz}, 1 \mathrm{H}), 4.54-4.41(\mathrm{~m}, 2 \mathrm{H}), 3.70-$ $3.55(\mathrm{~m}, 2 \mathrm{H}), 2.22(\mathrm{q}, J=7.3 \mathrm{~Hz}, 2 \mathrm{H}), 1.58-1.44(\mathrm{~m}, 2 \mathrm{H}), 1.44-1.31(\mathrm{~m}, 4 \mathrm{H}), 0.91(\mathrm{t}, J=$ $7.4 \mathrm{~Hz}, 3 \mathrm{H}) .{ }^{13} \mathrm{C}$ NMR $(101 \mathrm{MHz}$, Chloroform- $d$ ) $\delta 156.6,136.0,133.8,131.0,128.8,128.3$, 126.0, 62.4, 45.8, 31.8, 28.7, 28.4, 22.6, 14.1. HRMS-ESI calcd for $\mathrm{C}_{16} \mathrm{H}_{21} \mathrm{NO}_{2}[\mathrm{M}+\mathrm{Na}]^{+}$: 282.1465 , found: 282.1463 .

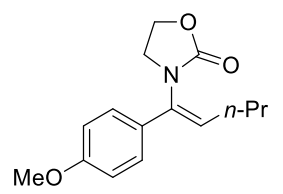


(Z)-3-(1-(4-methoxyphenyl)pent-1-en-1-yl)oxazolidin-2-one(Z-2e) Colorless oil. ${ }^{1} \mathrm{H}$ NMR $(400 \mathrm{MHz}$, Chloroform- $d$ ) $\delta 7.30-7.23(\mathrm{~m}, 2 \mathrm{H}), 6.92-6.83(\mathrm{~m}, 2 \mathrm{H}), 5.89(\mathrm{t}, J=7.2 \mathrm{~Hz}$, $1 \mathrm{H}), 4.53-4.41(\mathrm{~m}, 2 \mathrm{H}), 3.81(\mathrm{~s}, 3 \mathrm{H}), 3.68-3.60(\mathrm{~m}, 2 \mathrm{H}), 2.18$ (q, $J=7.3 \mathrm{~Hz}, 2 \mathrm{H}), 1.62-$ $1.48(\mathrm{~m}, 2 \mathrm{H}), 0.98(\mathrm{t}, J=7.4 \mathrm{~Hz}, 3 \mathrm{H}) .{ }^{13} \mathrm{C}$ NMR (101 MHz, Chloroform-d) $\delta 159.8,156.6$, 133.6, 128.9, 128.6, 127.3, 114.2, 62.4, 55.5, 45.9, 30.4, 22.4, 14.2. HRMS-ESI calcd for $\mathrm{C}_{15} \mathrm{H}_{19} \mathrm{NO}_{3}[\mathrm{M}+\mathrm{Na}]^{+}: 284.1257$, found: 284.1244 .

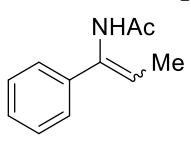

(Z/E)- $N$-(1-phenylprop-1-en-1-yl)acetamide(11a) ${ }^{7} \quad$ White $\quad$ solid. $\quad(Z)-N$-Acetyl-1phenylpropenamine (rotamers ratio 1.9:1): Major rotamer: ${ }^{1} \mathrm{H}$ NMR $\left(400 \mathrm{MHz}, \mathrm{CDCl}_{3}\right): \delta$ 7.23-7.45 (m, 5H), 6.57 (br, s, 1H), 5.97 (q, $J=6.9 \mathrm{~Hz}, 1 \mathrm{H}$ ), 2.19 (s, 3H) 1.77 (d, $J=7.0 \mathrm{~Hz}$, $3 \mathrm{H}$,). Minor rotamer: $\delta$ 7.23-7.45 (m, 5H), $6.58(\mathrm{br}, \mathrm{s}, 1 \mathrm{H}), 6.05(\mathrm{q}, J=7.0 \mathrm{~Hz}, 1 \mathrm{H}),, 1.86(\mathrm{~d}$, $J=7.0 \mathrm{~Hz}, 3 \mathrm{H}), 1.82(\mathrm{~s}, 3 \mathrm{H})$.

(Z)- $N$-Acetyl-1-phenylpropenamine (rotamers are not splitted in DMSO): ${ }^{1} \mathrm{H}$ NMR (400 MHz, DMSO- $\left.d_{6}\right) \delta 9.10(\mathrm{~s}, 1 \mathrm{H}), 7.21-7.40(\mathrm{~m}, 5 \mathrm{H}), 5.90(\mathrm{q}, J=6.8 \mathrm{~Hz}, 1 \mathrm{H}), 2.01(\mathrm{~s}, 3 \mathrm{H})$, $1.63-1.70(\mathrm{~m}, 3 \mathrm{H})$.

Isolated $(E)$ - $N$-Acetyl-1-phenylpropenamine: $\left.1 \mathrm{H} \mathrm{NMR} \mathrm{(400} \mathrm{MHz,} \mathrm{CDCl}_{3}\right): \delta 7.30-7.40(\mathrm{~m}$, $5 \mathrm{H}), 6.62(\mathrm{br}, \mathrm{s}, 1 \mathrm{H}), 6.33(\mathrm{q}, J=6.42,1 \mathrm{H}), 2.03-2.04(\mathrm{~m}, 3 \mathrm{H}), 1.83-1.86(\mathrm{~m}, 1 \mathrm{H}), 1.70(\mathrm{~d}, J$ $=7.2 \mathrm{~Hz}, 3 \mathrm{H})$. HRMS-ESI calcd for $\mathrm{C}_{11} \mathrm{H}_{13} \mathrm{NO}[\mathrm{M}+\mathrm{Na}]^{+}:$198.0889, found: 198.0895.

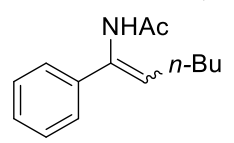

(Z/E)- $N$-(1-phenylhex-1-en-1-yl)acetamide(11b) White solid. (Z)- $N$-(1-phenylhex-1-en-1yl)acetamide (rotamers ratio 1.6:1) Major rotamer: ${ }^{1} \mathrm{H}$ NMR (400 MHz, Chloroform- $d$ ) $\delta 7.47$ $-7.12(\mathrm{~m}, 5 \mathrm{H}), 6.61(\mathrm{br}, \mathrm{s}, 1 \mathrm{H}), 5.86(\mathrm{t}, J=7.11 \mathrm{~Hz}, 1 \mathrm{H}), 2.21-2.11(\mathrm{~m}, 5 \mathrm{H}), 1.52-1.33$ $(\mathrm{m}, 4 \mathrm{H}), 0.93$ (t, $J=7.20 \mathrm{~Hz}, 3 \mathrm{H})$. Minor rotamer: ${ }^{1} \mathrm{H}$ NMR (400 MHz, Chloroform- $d$ ) $\delta 7.46$ $-7.20(\mathrm{~m}, 5 \mathrm{H}), 6.61(\mathrm{br}, \mathrm{s}, 1 \mathrm{H}), 5.94(\mathrm{t}, J=7.42 \mathrm{~Hz}, 1 \mathrm{H}), 2.28-2.21(\mathrm{~m}, 2 \mathrm{H}), 2.17(\mathrm{~s}, 3 \mathrm{H})$, $1.52-1.33(\mathrm{~m}, 4 \mathrm{H}), 0.93(\mathrm{t}, J=7.30 \mathrm{~Hz}, 3 \mathrm{H})$.

(E)- $N$-(1-phenylhex-1-en-1-yl)acetamide. ${ }^{1} \mathrm{H}$ NMR (400 MHz, Chloroform- $d$ ) $\delta 7.46-7.22$ (m, 5H), 6.52 (br, s, 1H), 6.30 (t, $J=7.79 \mathrm{~Hz}, 1 \mathrm{H}), 2.07-2.00(\mathrm{~m}, 5 \mathrm{H}), 1.35-1.17(\mathrm{~m}, 4 \mathrm{H})$, 0.83 (t, $J=7.19 \mathrm{~Hz}, 3 \mathrm{H})$. HRMS-ESI calcd for $\mathrm{C}_{14} \mathrm{H}_{19} \mathrm{NO}[\mathrm{M}+\mathrm{Na}]^{+}: 240.1359$, found: 240.1346.

\subsection{General procedure for the preparation of the $\alpha, \beta$ - enamides ( $E$-isomer)}

$$
\text { 年 }
$$

A nitrogen-flooded sealed vial containing corresponding ketones (1.1 equiv.) in dry $\mathrm{CH}_{2} \mathrm{Cl}_{2}$ was cooled down to $0^{\circ} \mathrm{C}$ before $\mathrm{TiCl}_{4}\left(1 \mathrm{M}\right.$ solution in $\mathrm{CH}_{2} \mathrm{Cl}_{2}$ or neat, 1.2 equiv.) was added dropwise. The resulting light yellow/orange solution was stirred at this temperature for $5 \mathrm{~min}$ and $0^{\circ} \mathrm{C}$ 2-oxazolidone (1 equiv.) pre-dissolved in $\mathrm{CH}_{2} \mathrm{Cl}_{2}(5 \mathrm{~mL})$ was added slowly followed by dropwise addition of $\mathrm{NEt}_{3}$ ( 5 equiv.). The color of the reaction mixture turned black upon addition of the base, a color change that did not occur when using most other bases. The reaction mixture was allowed to warm up to r.t. and was stirred over $3 \mathrm{~h}$ with regular monitoring using thin layer chromatography plates (eluent: ethyl acetate 1:2 pentane). The crude mixture was cooled to $0^{\circ} \mathrm{C}$, quenched by water (4 equiv.) and diluted with $\mathrm{CH}_{2} \mathrm{Cl}_{2}$ 
followed by portwise addition of slica. The mixture was filtered by celite. The filtrate was washed with water. The layers were separated and the organic phase was extracted with $\mathrm{CH}_{2} \mathrm{Cl}_{2}(3 \times 50 \mathrm{~mL})$. The combined organic layers were dried over $\mathrm{Na}_{2} \mathrm{SO}_{4}$ and concentrated under reduced pressure. The residue was then purified by flash chromatography on silica gel (ethyl acetate/pentane, gradient from 1:4 to 1:2) to provide clean $E$-enamides.

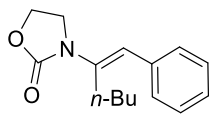

(E)-3-(1-phenylhex-1-en-2-yl)oxazolidin-2-one(E-3a) Colorless oil. ${ }^{1} \mathrm{H}$ NMR (400 MHz, Chloroform- $d$ ) $\delta 7.36-7.30(\mathrm{~m}, 2 \mathrm{H}), 7.25-7.19(\mathrm{~m}, 3 \mathrm{H}), 6.18(\mathrm{~s}, 1 \mathrm{H}), 4.44-4.35(\mathrm{~m}, 2 \mathrm{H})$, $3.94-3.83(\mathrm{~m}, 2 \mathrm{H}), 2.77-2.66(\mathrm{~m}, 2 \mathrm{H}), 1.56-1.47(\mathrm{~m}, 2 \mathrm{H}), 1.33(\mathrm{~h}, J=7.3 \mathrm{~Hz}, 2 \mathrm{H}), 0.87$ (t, $J=7.3 \mathrm{~Hz}, 3 \mathrm{H}) .{ }^{13} \mathrm{C}$ NMR (101 MHz, Chloroform- $d$ ) 155.2, 140.1, 136.4, 129.0, 128.4, 126.7, 117.3, 61.5, 46.5, 30.8, 27.4, 22.6, 14.0, HRMS-ESI calcd for $\mathrm{C}_{15} \mathrm{H}_{19} \mathrm{NO}_{2}[\mathrm{M}+\mathrm{Na}]^{+}$: 268.1313, found: 268.1308 .

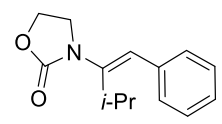

(E)-3-(3-methyl-1-phenylbut-1-en-2-yl)oxazolidin-2-one( $\boldsymbol{E}$-3b) White solid. ${ }^{1} \mathrm{H}$ NMR (400 $\mathrm{MHz}$, Chloroform- $d$ ) $\delta 7.35-7.27(\mathrm{~m}, 4 \mathrm{H}), 7.26-7.21(\mathrm{~m}, 1 \mathrm{H}), 6.34(\mathrm{~s}, 1 \mathrm{H}), 4.31-4.21(\mathrm{~m}$, 2H), $3.49-3.40(\mathrm{~m}, 2 \mathrm{H}), 3.00$ (heptd, $J=6.8,1.4 \mathrm{~Hz}, 1 \mathrm{H}), 1.19$ (d, $J=6.9 \mathrm{~Hz}, 6 \mathrm{H}) .{ }^{13} \mathrm{C}$ NMR (101 MHz, Chloroform- $d$ ) $\delta$ 156.8, 142.5, 135.6, 128.7, 128.0, 127.6, 123.0, 62.6, 46.0, 31.8, 20.8. HRMS-ESI calcd for $\mathrm{C}_{14} \mathrm{H}_{17} \mathrm{NO}_{2}[\mathrm{M}+\mathrm{Na}]^{+}: 254.1157$, found: 254.1169 .<smiles>O=C1OCCN1C(=[PH2+])c1ccccc1</smiles>

(E)-3-(1-phenylpent-1-en-2-yl)oxazolidin-2-one( $\boldsymbol{E}$-3c) Colorless oil. ${ }^{1} \mathrm{H}$ NMR (400 MHz, Chloroform- $d$ ) $\delta 7.36-7.30(\mathrm{~m}, 2 \mathrm{H}), 7.25-7.18(\mathrm{~m}, 3 \mathrm{H}), 6.19(\mathrm{~s}, 1 \mathrm{H}), 4.44-4.36(\mathrm{~m}, 2 \mathrm{H})$, $3.94-3.87(\mathrm{~m}, 2 \mathrm{H}), 2.74-2.62(\mathrm{~m}, 2 \mathrm{H}), 1.62-1.48(\mathrm{~m}, 2 \mathrm{H}), 0.92(\mathrm{t}, J=7.4 \mathrm{~Hz}, 3 \mathrm{H}) .{ }^{13} \mathrm{C}$ NMR (101 MHz, Chloroform- $d$ ) $\delta$ 155.2, 139.9, 136.4, 129.0, 128.4, 126.7, 61.5, 46.5, 29.5, 21.9, 13.9, HRMS-ESI calcd for $\mathrm{C}_{14} \mathrm{H}_{17} \mathrm{NO}_{2}[\mathrm{M}+\mathrm{Na}]^{+}: 254.1157$, found: 254.11538 .<smiles>[Li]/C(=C\c1ccccc1)N1CCOC1=O</smiles>

(E)-3-(1-cyclohexyl-2-phenylvinyl)oxazolidin-2-one( $\boldsymbol{E}$-3d) Colorless oil. ${ }^{1} \mathrm{H}$ NMR (400 $\mathrm{MHz}$, Chloroform- $d$ ) $\delta 7.35-7.26(\mathrm{~m}, 4 \mathrm{H}), 7.25-7.20(\mathrm{~m}, 1 \mathrm{H}), 6.30(\mathrm{~s}, 1 \mathrm{H}), 4.31-4.20(\mathrm{~m}$, $2 \mathrm{H}), 3.49-3.34(\mathrm{~m}, 2 \mathrm{H}), 2.61(\mathrm{tdt}, J=10.1,3.0,1.4 \mathrm{~Hz}, 1 \mathrm{H}), 2.06-1.94(\mathrm{~m}, 2 \mathrm{H}), 1.82(\mathrm{dt}, J$ $=12.4,3.0 \mathrm{~Hz}, 2 \mathrm{H}), 1.77-1.69(\mathrm{~m}, 1 \mathrm{H}), 1.45-1.09(\mathrm{~m}, 5 \mathrm{H}) \cdot{ }^{13} \mathrm{C}$ NMR $(101 \mathrm{MHz}$, Chloroform- $d$ ) $\delta 156.9,141.9,135.7,128.7,128.1,127.5,123.5,62.5,46.2,41.7,31.5,26.4$, 26.4. HRMS-ESI calcd for $\mathrm{C}_{17} \mathrm{H}_{21} \mathrm{NO}_{2}[\mathrm{M}+\mathrm{Na}]^{+}: 294.1470$, found: 294.1479 .<smiles>[13CH]c1ccccc1</smiles>

(E)-3-(3,3-dimethyl-1-phenylbut-1-en-2-yl)oxazolidin-2-one( $\boldsymbol{E}$-3e) White solid. ${ }^{1} \mathrm{H}$ NMR $(400 \mathrm{MHz}$, Chloroform- $d$ ) $\delta 7.38-7.27(\mathrm{~m}, 2 \mathrm{H}), 7.30-7.20(\mathrm{~m}, 3 \mathrm{H}), 6.64(\mathrm{~s}, 1 \mathrm{H}), 4.43-$ $3.88(\mathrm{~m}, 2 \mathrm{H}), 3.71-3.17(\mathrm{~m}, 2 \mathrm{H}), 1.27(\mathrm{~d}, J=1.0 \mathrm{~Hz}, 9 \mathrm{H}) .{ }^{13} \mathrm{C}$ NMR $(101 \mathrm{MHz}$, Chloroform- $d$ ) $\delta 158.1,145.4,135.5,128.8,128.0,127.7,126.6,62.3,47.3,38.8,29.7$, HRMS-ESI calcd for $\mathrm{C}_{15} \mathrm{H}_{19} \mathrm{NO}_{2}[\mathrm{M}+\mathrm{Na}]^{+}: 268.1313$, found: 268.1302 .

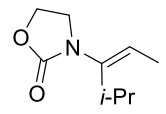


(E)-3-(4-methylpent-2-en-3-yl)oxazolidin-2-one(E-3f) Colorless oil. ${ }^{1} \mathrm{H}$ NMR (400 MHz, Chloroform- $d$ ) $\delta 5.50$ (q, $J=7.1 \mathrm{~Hz}, 1 \mathrm{H}), 4.38-4.29$ (m, 2H), $3.77-3.67$ (m, 2H), 2.96 (p, $J$ $=7.0 \mathrm{~Hz}, 1 \mathrm{H}), 1.72(\mathrm{~d}, J=7.1 \mathrm{~Hz}, 3 \mathrm{H}), 1.10(\mathrm{~d}, J=7.0 \mathrm{~Hz}, 6 \mathrm{H}) \cdot{ }^{13} \mathrm{C}$ NMR $(101 \mathrm{MHz}$, Chloroform- $d$ ) $\delta$ 158.1, 141.7, 123.6, 61.8, 49.7, 29.2, 20.8, 12.9, HRMS-ESI calcd for $\mathrm{C}_{9} \mathrm{H}_{15} \mathrm{NO}_{2}[\mathrm{M}+\mathrm{Na}]^{+}: 192.1000$, found: 192.0993 .

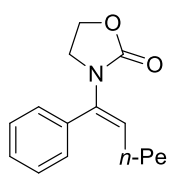

(E)-3-(1-phenylhept-1-en-1-yl)oxazolidin-2-one(E-2d) White solid. ${ }^{1} \mathrm{H}$ NMR (500 MHz, Chloroform- $d$ ) $\delta 7.40-7.35(\mathrm{~m}, 2 \mathrm{H}), 7.35-7.31(\mathrm{~m}, 1 \mathrm{H}), 7.28-7.25(\mathrm{~m}, 2 \mathrm{H}), 5.86(\mathrm{t}, J=$ $7.8 \mathrm{~Hz}, 1 \mathrm{H}), 4.36-4.23(\mathrm{~m}, 2 \mathrm{H}), 3.59-3.44(\mathrm{~m}, 2 \mathrm{H}), 2.10(\mathrm{q}, J=7.7 \mathrm{~Hz}, 2 \mathrm{H}), 1.42(\mathrm{p}, J=$ $7.3 \mathrm{~Hz}, 2 \mathrm{H}), 1.30-1.20(\mathrm{~m}, 4 \mathrm{H}), 0.86(\mathrm{t}, J=7.0 \mathrm{~Hz}, 3 \mathrm{H}) .{ }^{13} \mathrm{C}$ NMR $(126 \mathrm{MHz}$, Chloroformd) $\delta 156.6,134.8,134.4,129.0,128.5,128.3,126.0,61.6,46.2,31.5,29.7,28.1,22.6,14.1$. HRMS-ESI calcd for $\mathrm{C}_{16} \mathrm{H}_{21} \mathrm{NO}_{2}[\mathrm{M}+\mathrm{Na}]^{+}: 282.1465$, found: 282.1463 .<smiles>COc1ccc(C(C=CC(=O)O)N2CCOC2=O)cc1</smiles>

(E)-3-(1-(4-methoxyphenyl)pent-1-en-1-yl)oxazolidin-2-one( $\boldsymbol{E}$-2e) White solid. ${ }^{1} \mathrm{H}$ NMR $(400 \mathrm{MHz}$, Chloroform- $d$ ) $\delta 7.30-7.21(\mathrm{~m}, 2 \mathrm{H}), 6.90-6.82(\mathrm{~m}, 2 \mathrm{H}), 5.88(\mathrm{t}, J=7.2 \mathrm{~Hz}$, $1 \mathrm{H}), 4.52-4.40$ (m, 2H), $3.81(\mathrm{~s}, 3 \mathrm{H}), 3.68-3.58(\mathrm{~m}, 2 \mathrm{H}), 2.18$ (q, $J=7.3 \mathrm{~Hz}, 2 \mathrm{H}), 1.60-$ $1.46(\mathrm{~m}, 2 \mathrm{H}), 0.97(\mathrm{t}, J=7.4 \mathrm{~Hz}, 3 \mathrm{H}) .{ }^{13} \mathrm{C}$ NMR (101 MHz, Chloroform- $d$ ) $\delta 159.8,156.6$, 133.6, 128.9, 128.6, 127.3, 114.2, 62.4, 55.5, 45.9, 30.4, 22.3, 14.2. HRMS-ESI calcd for $\mathrm{C}_{15} \mathrm{H}_{19} \mathrm{NO}_{3}[\mathrm{M}+\mathrm{Na}]^{+}: 284.1257$, found: 284.1232 .<smiles>O=C1OCCN1C(=Cc1cccc([N+](=O)[O-])c1)c1ccccc1</smiles>

(E)-3-(1-(m-tolyl)pent-1-en-1-yl)oxazolidin-2-one( $\boldsymbol{E}-\mathbf{2 g})$ Colorless oil. ${ }^{1} \mathrm{H}$ NMR (400 MHz, Chloroform- $d$ ) $\delta 7.30-7.23(\mathrm{~m}, 1 \mathrm{H}), 7.14(\mathrm{~d}, J=7.6 \mathrm{~Hz}, 1 \mathrm{H}), 7.06(\mathrm{~d}, J=7.4 \mathrm{~Hz}, 2 \mathrm{H}), 5.84$ $(\mathrm{t}, J=7.7 \mathrm{~Hz}, 1 \mathrm{H}), 4.34-4.25(\mathrm{~m}, 2 \mathrm{H}), 3.59-3.47(\mathrm{~m}, 2 \mathrm{H}), 2.36(\mathrm{~s}, 3 \mathrm{H}), 2.08$ (q, $J=7.6 \mathrm{~Hz}$, $2 \mathrm{H}), 1.44$ (h, $J=7.3 \mathrm{~Hz}, 2 \mathrm{H}), 0.90$ (t, $J=7.4 \mathrm{~Hz}, 3 \mathrm{H}) .{ }^{13} \mathrm{C}$ NMR (101 MHz, Chloroform- $d$ ) $\delta$ $156.7,138.2,134.8,134.7,129.5,129.1,128.4,126.2,125.8,61.6,46.2,30.2,23.2$, 21.6, 13.9. HRMS-ESI calcd for $\mathrm{C}_{15} \mathrm{H}_{19} \mathrm{NO}_{2}[\mathrm{M}+\mathrm{Na}]^{+}: 268.1308$, found: 268.1314 .

\subsection{General procedure for the preparation of the (Z)-a, $\beta$-enamides from $E$ isomer}
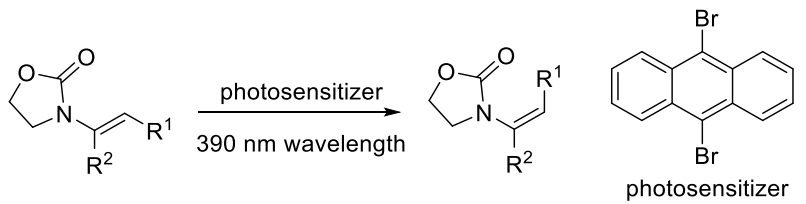

A solution of $(E)$-a, $\beta$-enamides (1.0 equiv.) and a catalytic amount $(5 \mathrm{~mol} \%)$ of photosensitizer in $\mathrm{CH}_{2} \mathrm{Cl}_{2}(2 \mathrm{~mL} / 2 \mathrm{mmol}$ enamides $)$ was irradiated using $390 \mathrm{~nm}$ sodium lamp overnight. The solvent was removed under vacuum. After purification by silica-gel flash chromatography, the pure $Z$-enamides were obtained.

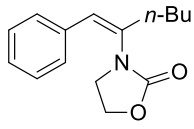


(Z)-3-(1-phenylhex-1-en-2-yl)oxazolidin-2-one(Z-3a) Colorless oil. ${ }^{1} \mathrm{H}$ NMR (400 MHz, Chloroform- $d$ ) $\delta 7.36-7.27(\mathrm{~m}, 4 \mathrm{H}), 7.26-7.21(\mathrm{~m}, 1 \mathrm{H}), 6.28(\mathrm{~s}, 1 \mathrm{H}), 4.34-4.24(\mathrm{~m}, 2 \mathrm{H})$, $3.52-3.44(\mathrm{~m}, 2 \mathrm{H}), 2.59-2.47(\mathrm{~m}, 2 \mathrm{H}), 1.55-1.32(\mathrm{~m}, 4 \mathrm{H}), 0.95(\mathrm{t}, J=7.2 \mathrm{~Hz}, 3 \mathrm{H}) .{ }^{13} \mathrm{C}$ NMR (101 MHz, Chloroform- $d$ ) $\delta$ 156.6, 137.1, 135.6, 128.7, 128.2, 127.6, 124.5, 62.7, 45.6, 33.9, 29.5, 22.5, 14.1, HRMS-ESI calcd for $\mathrm{C}_{15} \mathrm{H}_{19} \mathrm{NO}_{2}[\mathrm{M}+\mathrm{Na}]^{+}: 268.1313$, found: 268.1309.<smiles>CCCC1=C(c2ccccc2)CCN1C(=O)OC</smiles>

(Z)-3-(3-methyl-1-phenylbut-1-en-2-yl)oxazolidin-2-one(Z-3b) White solid. ${ }^{1} \mathrm{H}$ NMR (400 $\mathrm{MHz}$, Chloroform- $d$ ) $\delta 7.35-7.28(\mathrm{~m}, 2 \mathrm{H}), 7.27-7.20(\mathrm{~m}, 3 \mathrm{H}), 6.47(\mathrm{~s}, 1 \mathrm{H}), 4.41-4.31(\mathrm{~m}$, 2H), 3.85 (ddd, $J=9.2,6.7,1.6 \mathrm{~Hz}, 2 \mathrm{H}), 3.15$ (pd, $J=7.0,1.5 \mathrm{~Hz}, 1 \mathrm{H}), 1.13$ (dt, $J=7.0,1.3$ $\mathrm{Hz}, 6 \mathrm{H}) .{ }^{13} \mathrm{C}$ NMR (101 MHz, Chloroform- $d$ ) $\delta 157.8,143.7,135.7,128.9,128.4,128.4$, $127.5,61.9,49.9,30.2,21.3$. HRMS-ESI calcd for $\mathrm{C}_{14} \mathrm{H}_{17} \mathrm{NO}_{2}[\mathrm{M}+\mathrm{Na}]^{+}: 254.1157$, found: 254.1154 .<smiles>O=C1OCCN1/C([PH+])=C\c1ccccc1</smiles>

(Z)-3-(1-phenylpent-1-en-2-yl)oxazolidin-2-one(Z-3c) Colorless oil. ${ }^{1} \mathrm{H}$ NMR (400 MHz, Chloroform- $d$ ) $\delta 7.35-7.28(\mathrm{~m}, 4 \mathrm{H}), 7.25-7.20(\mathrm{~m}, 1 \mathrm{H}), 6.28(\mathrm{~s}, 1 \mathrm{H}), 4.33-4.22(\mathrm{~m}, 2 \mathrm{H})$, $3.51-3.42(\mathrm{~m}, 2 \mathrm{H}), 2.57-2.47(\mathrm{~m}, 2 \mathrm{H}), 1.63-1.49(\mathrm{~m}, 2 \mathrm{H}), 0.99(\mathrm{t}, J=7.3 \mathrm{~Hz}, 3 \mathrm{H}) .{ }^{13} \mathrm{C}$ NMR (101 MHz, Chloroform- $d$ ) $\delta$ 156.6, 136.9, 135.6, 128.7, 128.2, 127.6, 124.7, 62.7, 45.6, 36.3, 20.5, 13.8, HRMS-ESI calcd for $\mathrm{C}_{14} \mathrm{H}_{17} \mathrm{NO}_{2}[\mathrm{M}+\mathrm{Na}]^{+}: 254.1157$, found: 254.1157 .<smiles>O=C1OCCN1/C(Cl)=C/c1ccccc1</smiles>

\section{(Z)-3-(1-cyclohexyl-2-phenylvinyl)oxazolidin-2-one (Z-3d)}

${ }^{1} \mathrm{H}$ NMR (400 MHz, Chloroform- $d$ ) $\delta 7.40-7.32(\mathrm{~m}, 2 \mathrm{H}), 7.31-7.21(\mathrm{~m}, 3 \mathrm{H}), 6.49(\mathrm{~s}, 1 \mathrm{H})$, $4.44-4.35(\mathrm{~m}, 2 \mathrm{H}), 3.92-3.83(\mathrm{~m}, 2 \mathrm{H}), 2.81(\mathrm{tt}, J=12.1,3.1 \mathrm{~Hz}, 1 \mathrm{H}), 1.90-1.70(\mathrm{~m}, 4 \mathrm{H})$, $1.70-1.60(\mathrm{~m}, 1 \mathrm{H}), 1.53-1.37(\mathrm{~m}, 2 \mathrm{H}), 1.31-1.16(\mathrm{~m}, 3 \mathrm{H}) .{ }^{13} \mathrm{C}$ NMR $(101 \mathrm{MHz}$, Chloroform- $d$ ) $\delta 157.9,143.2,135.6,129.2,128.9,128.5,127.5,61.9,50.1,41.1,31.4,26.2$, 26.0. HRMS-ESI calcd for $\mathrm{C}_{17} \mathrm{H}_{21} \mathrm{NO}_{2}[\mathrm{M}+\mathrm{Na}]^{+}: 294.1465$, found: 294.1453 .<smiles></smiles>

(Z)-3-(3,3-dimethyl-1-phenylbut-1-en-2-yl)oxazolidin-2-one(Z-3e) White solid. ${ }^{1} \mathrm{H}$ NMR $(400 \mathrm{MHz}$, Chloroform- $d$ ) $\delta 7.34-7.28(\mathrm{~m}, 2 \mathrm{H}), 7.28-7.21(\mathrm{~m}, 3 \mathrm{H}), 6.68(\mathrm{~s}, 1 \mathrm{H}), 4.44-$ $4.36(\mathrm{~m}, 2 \mathrm{H}), 3.93-3.85(\mathrm{~m}, 2 \mathrm{H}), 1.08(\mathrm{~s}, 9 \mathrm{H}) .{ }^{13} \mathrm{C}$ NMR (101 MHz, Chloroform- $d$ ) $\delta 158.1$, $146.5,136.9,131.6,128.7,128.0,127.1,61.9,50.8,38.6,30.7$. HRMS-ESI calcd for $\mathrm{C}_{15} \mathrm{H}_{19} \mathrm{NO}_{2}[\mathrm{M}+\mathrm{Na}]^{+}: 268.1313$, found: 268.1324 .<smiles>C/C=C(\C(=O)OCC)P(CC)CC</smiles>

(Z)-3-(4-methylpent-2-en-3-yl)oxazolidin-2-one(Z-3f) Colorless oil. ${ }^{1} \mathrm{H}$ NMR (400 MHz, Chloroform- $d$ ) $\delta 5.48(\mathrm{qd}, J=6.8,1.3 \mathrm{~Hz}, 1 \mathrm{H}), 4.46-4.36(\mathrm{~m}, 2 \mathrm{H}), 3.75-3.66(\mathrm{~m}, 2 \mathrm{H})$, 2.49 (ttt, $J=6.8,5.4,1.5 \mathrm{~Hz}, 1 \mathrm{H}), 1.61(\mathrm{dd}, J=6.9,1.4 \mathrm{~Hz}, 3 \mathrm{H}), 1.08(\mathrm{~s}, 3 \mathrm{H}), 1.07$ (s, 3H). ${ }^{13} \mathrm{C}$ NMR (101 MHz, Chloroform- $d$ ) $\delta$ 156.5, 140.8, 120.0, 62.3, 46.2, 31.6, 20.9, 13.0. HRMS-ESI calcd for $\mathrm{C}_{9} \mathrm{H}_{15} \mathrm{NO}_{2}[\mathrm{M}+\mathrm{Na}]^{+}: 192.1000$, found: 192.0992 . 


\section{General procedure for asymmetric hydrogenation}

A vial was charged with substrate $(0.015 \mathrm{mmol})$ and Ir-complex (1 mol\%). Dry $\mathrm{CH}_{2} \mathrm{Cl}_{2}(1.5$ $\mathrm{mL}$ ) was added (so that the concentration of the substrate was $0.1 \mathrm{M}$ ) and the vial was placed in a high-pressure hydrogenation apparatus. The reactor was purged three times with Ar gas, then filled with $\mathrm{H}_{2}$. The reaction was stirred at room temperature for 16 hours before the $\mathrm{H}_{2}$ pressure was released and the solvent was removed in vacuo. The crude product was filtered through on a short plug of silica. Conversions were determined by ${ }^{1} \mathrm{H}$ NMR spectroscopy and ee values were determined by HPLC, SFC or GCMS using a chiral stationary phase.

Characterization for the following compounds have been previously reported: $\mathbf{1 2 a}^{8}, \mathbf{1 2}^{9}$, $12 \mathbf{c}^{10}, 12 \mathbf{d}^{11}, 13 \mathbf{a}^{12}$.

The characterization of the hydrogenated compounds:

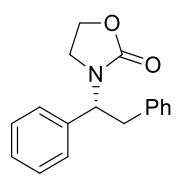

(R)-3-(1,2-diphenylethyl)oxazolidin-2-one(5a) White solid, 98\% yield. ${ }^{1} \mathrm{H}$ NMR (400 MHz, Chloroform- $d$ ) $\delta 7.44-7.34(\mathrm{~m}, 4 \mathrm{H}), 7.34-7.29(\mathrm{~m}, 2 \mathrm{H}), 7.27$ (tt, $J=3.5,2.0 \mathrm{~Hz}, 3 \mathrm{H}), 7.24$ - $7.18(\mathrm{~m}, 1 \mathrm{H}), 5.35$ (dd, $J=9.7,6.7 \mathrm{~Hz}, 1 \mathrm{H}), 4.29-3.87$ (m, 2H), 3.53 (td, $J=8.2,6.9 \mathrm{~Hz}$, 1H), $3.45-3.03(\mathrm{~m}, 3 \mathrm{H}) .{ }^{13} \mathrm{C}$ NMR (101 MHz, Chloroform- $d$ ) $\delta$ 158.0, 138.4, 137.4, 128.8 , $128.8,128.7,128.2,127.7,126.8,61.9,57.5,40.9,36.8$, HRMS-ESI calcd for $\mathrm{C}_{17} \mathrm{H}_{17} \mathrm{NO}_{2}$ $[\mathrm{M}+\mathrm{Na}]^{+}: 290.1151$, found: 290.1157.

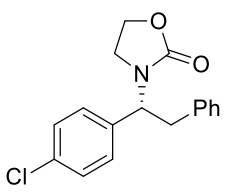

(R)-3-(1-(4-chlorophenyl)-2-phenylethyl)oxazolidin-2-one(5b) white solid, 92\% yield. ${ }^{1} \mathrm{H}$ NMR (400 MHz, Chloroform-d) $\delta 7.34$ (s, 4H), 7.32 - 7.27 (m, 2H), 7.25 - 7.19 (m, 3H), $5.31(\mathrm{dd}, J=9.4,7.1 \mathrm{~Hz}, 1 \mathrm{H}), 4.25-4.12(\mathrm{~m}, 2 \mathrm{H}), 3.52(\mathrm{td}, J=8.3,6.7 \mathrm{~Hz}, 1 \mathrm{H}), 3.39-3.11$ (m, 3H). ${ }^{13} \mathrm{C}$ NMR (101 MHz, Chloroform- $d$ ) $\delta$ 158.0, 137.1, 136.9, 134.1, 129.1, 129.1, $128.8,128.8,127.0,62.0,57.0,41.0,36.9$, HRMS-ESI calcd for $\mathrm{C}_{17} \mathrm{H}_{16} \mathrm{ClNO}_{2}[\mathrm{M}+\mathrm{Na}]^{+}$: 324.0762, found: 324.0757 .

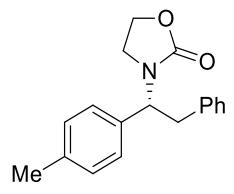

(R)-3-(2-phenyl-1-(p-tolyl)ethyl)oxazolidin-2-one(5c) White solid, 95\% yield. ${ }^{1} \mathrm{H}$ NMR (400 MHz, Chloroform- $d$ ) $\delta 7.32-7.24(\mathrm{~m}, 6 \mathrm{H}), 7.23-7.15(\mathrm{~m}, 3 \mathrm{H}), 5.32$ (dd, $J=9.6,6.7$ $\mathrm{Hz}, 1 \mathrm{H}), 4.18-4.10(\mathrm{~m}, 2 \mathrm{H}), 3.52(\mathrm{td}, J=8.2,6.9 \mathrm{~Hz}, 1 \mathrm{H}), 3.36-3.20(\mathrm{~m}, 3 \mathrm{H}), 2.35(\mathrm{~s}, 3 \mathrm{H})$. ${ }^{13} \mathrm{C}$ NMR (101 MHz, Chloroform-d) $\delta$ 158.0, 138.0, 137.6, 135.4, 129.5, 128.9, 128.7, 127.6, $126.8,61.9,57.2,40.8,36.9,21.2$, HRMS-ESI calcd for $\mathrm{C}_{18} \mathrm{H}_{19} \mathrm{NO}_{2}[\mathrm{M}+\mathrm{Na}]^{+}: 304.1308$, found: 304.1306 .<smiles>COc1ccc(CCc2ccccc2)c(N2CCOC2=O)c1</smiles> 
(R)-3-(1-(4-methoxyphenyl)-2-phenylethyl)oxazolidin-2-one(5d) white solid, 90\% yield. ${ }^{1} \mathrm{H}$ NMR (400 MHz, Chloroform- $d$ ) $\delta 7.35-7.26(\mathrm{~m}, 4 \mathrm{H}), 7.26-7.18(\mathrm{~m}, 3 \mathrm{H}), 6.93-6.83$ $(\mathrm{m}, 2 \mathrm{H}), 5.30(\mathrm{dd}, J=9.5,6.9 \mathrm{~Hz}, 1 \mathrm{H}), 4.18-4.12(\mathrm{~m}, 2 \mathrm{H}), 3.80(\mathrm{~s}, 3 \mathrm{H}), 3.51(\mathrm{td}, J=8.2$, $6.8 \mathrm{~Hz}, 1 \mathrm{H}), 3.35-3.18(\mathrm{~m}, 3 \mathrm{H}) .{ }^{13} \mathrm{C}$ NMR $(101 \mathrm{MHz}$, Chloroform- $d$ ) $\delta 159.4,158.0,137.6$, 130.5, 129.0, 128.9, 128.7, 126.8, 114.2, 61.9, 57.0, 55.4, 40.8, 37.1, HRMS-ESI calcd for $\mathrm{C}_{18} \mathrm{H}_{19} \mathrm{NO}_{3}[\mathrm{M}+\mathrm{Na}]^{+}: 320.1257$, found: 320.1259 .<smiles>O=C1OCCN1Cc1ccc(C(F)(F)F)cc1</smiles>

(R)-3-(2-phenyl-1-(4-(trifluoromethyl)phenyl)ethyl)oxazolidin-2-one(5e) White solid, 92\% yield. ${ }^{1} \mathrm{H}$ NMR (400 MHz, Chloroform- $d$ ) $\delta 7.63(\mathrm{~d}, J=8.1 \mathrm{~Hz}, 2 \mathrm{H}), 7.53(\mathrm{~d}, J=8.1 \mathrm{~Hz}, 2 \mathrm{H})$, $7.38-7.18(\mathrm{~m}, 5 \mathrm{H}), 5.38(\mathrm{dd}, J=9.3,7.1 \mathrm{~Hz}, 1 \mathrm{H}), 4.30-4.10(\mathrm{~m}, 2 \mathrm{H}), 3.56(\mathrm{td}, J=8.3,6.7$ $\mathrm{Hz}, 1 \mathrm{H}), 3.39-3.14(\mathrm{~m}, 3 \mathrm{H}) .{ }^{13} \mathrm{C}$ NMR (101 MHz, Chloroform-d) $\delta 158.0,142.5,136.8$, $130.5(\mathrm{q}, \mathrm{J}=32.7 \mathrm{~Hz}), 128.9,128.8,128.1,128.1,127.1,125.9$ (q, J = 3.8 Hz), 122.7 (q, J = $272.2 \mathrm{~Hz}), 62.0,57.2,41.1,36.8 .{ }^{19} \mathrm{~F}$ NMR $(377 \mathrm{MHz}$, Chloroform- $d$ ) $\delta$-62.7. HRMS-ESI calcd for $\mathrm{C}_{18} \mathrm{H}_{16} \mathrm{~F}_{3} \mathrm{NO}_{2}[\mathrm{M}+\mathrm{Na}]^{+}: 358.1025$, found: 358.1024 .

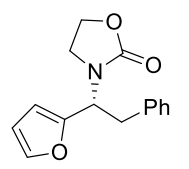

(R)-3-(1-(furan-2-yl)-2-phenylethyl)oxazolidin-2-one(5f) Colorless oil, 80\% yield. ${ }^{1} \mathrm{H}$ NMR (400 MHz, Chloroform-d) $\delta 7.26(\mathrm{dd}, J=1.8,0.8 \mathrm{~Hz}, 1 \mathrm{H}), 7.17-7.12(\mathrm{~m}, 2 \mathrm{H}), 7.10-7.03$ (m, 4H), $6.20-6.04(\mathrm{~m}, 2 \mathrm{H}), 5.23-5.13(\mathrm{~m}, 1 \mathrm{H}), 4.15-4.00(\mathrm{~m}, 2 \mathrm{H}), 3.46(\mathrm{td}, J=8.7,6.1$ $\mathrm{Hz}, 1 \mathrm{H}), 3.28-3.16(\mathrm{~m}, 2 \mathrm{H}), 3.01(\mathrm{dd}, J=14.1,8.8 \mathrm{~Hz}, 1 \mathrm{H}) .{ }^{13} \mathrm{C}$ NMR $(101 \mathrm{MHz}$, Chloroform- $d$ ) $\delta$ 158.0, 152.0, 142.5, 136.8, 128.9, 128.7, 127.0, 110.5, 108.5, 62.1, 52.0, 41.4, 36.6. HRMS-ESI calcd for $\mathrm{C}_{15} \mathrm{H}_{15} \mathrm{NO}_{3}[\mathrm{M}+\mathrm{Na}]^{+}: 280.0950$, found: 280.0967 .

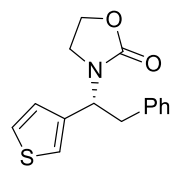

(R)-3-(2-phenyl-1-(thiophen-3-yl)ethyl)oxazolidin-2-one(5g) Colorless oil, 90\% yield. ${ }^{1} \mathrm{H}$ NMR (400 MHz, Chloroform- $d$ ) $\delta 7.32-7.28(\mathrm{~m}, 1 \mathrm{H}), 7.28-7.25(\mathrm{~m}, 2 \mathrm{H}), 7.25-7.17(\mathrm{~m}$, 4H), 7.09 (dd, $J=5.0,1.4 \mathrm{~Hz}, 1 \mathrm{H}), 5.39$ (dd, $J=9.5,6.4 \mathrm{~Hz}, 1 \mathrm{H}), 4.21-4.04$ (m, 2H), 3.46 $(\mathrm{dd}, J=8.7,5.9 \mathrm{~Hz}, 1 \mathrm{H}), 3.34(\mathrm{dd}, J=14.3,6.3 \mathrm{~Hz}, 1 \mathrm{H}), 3.25-3.13(\mathrm{~m}, 2 \mathrm{H}) .{ }^{13} \mathrm{C}$ NMR $(101$ $\mathrm{MHz}$, Chloroform- $d$ ) $\delta$ 158.0, 139.8, 137.2, 128.7, 128.7, 127.1, 126.9, 126.5, 122.6, 61.9, 53.3, 40.7, 37.7, HRMS-ESI calcd for $\mathrm{C}_{15} \mathrm{H}_{15} \mathrm{NO}_{2} \mathrm{~S}$ [M+Na] $]^{+}$296.0716, found: 296.0721 .

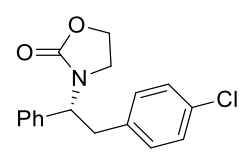

(R)-3-(2-(4-chlorophenyl)-1-phenylethyl)oxazolidin-2-one(5h) White solid, 98\% yield. ${ }^{1} \mathrm{H}$ NMR (400 MHz, Chloroform- $d$ ) $\delta 7.34(\mathrm{~s}, 4 \mathrm{H}), 7.31-7.27(\mathrm{~m}, 2 \mathrm{H}), 7.25-7.19(\mathrm{~m}, 3 \mathrm{H})$, $5.31(\mathrm{dd}, J=9.4,7.1 \mathrm{~Hz}, 1 \mathrm{H}), 4.22-4.11(\mathrm{~m}, 2 \mathrm{H}), 3.52(\mathrm{td}, J=8.3,6.7 \mathrm{~Hz}, 1 \mathrm{H}), 3.34-3.15$ $(\mathrm{m}, 3 \mathrm{H}) .{ }^{13} \mathrm{C}$ NMR (101 MHz, Chloroform- $d$ ) $\delta$ 158.0, 137.1, 136.9, 134.1, 129.1, 129.1, $128.8,128.8,127.0,62.0,57.0,41.0,36.9$, HRMS-ESI calcd for $\mathrm{C}_{17} \mathrm{H}_{16} \mathrm{ClNO}_{2}[\mathrm{M}+\mathrm{Na}]^{+}$: 324.0762, found: 324.0754 . 


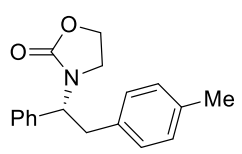

(R)-3-(1-phenyl-2-(p-tolyl)ethyl)oxazolidin-2-one(5i) White solid, 95\% yield. ${ }^{1} \mathrm{H}$ NMR (400 $\mathrm{MHz}$, Chloroform- $d$ ) $\delta 7.43-7.34(\mathrm{~m}, 4 \mathrm{H}), 7.34-7.28(\mathrm{~m}, 1 \mathrm{H}), 7.14(\mathrm{~d}, J=8.1 \mathrm{~Hz}, 2 \mathrm{H})$, 7.09 (d, $J=7.9 \mathrm{~Hz}, 2 \mathrm{H}), 5.33(\mathrm{dd}, J=9.8,6.5 \mathrm{~Hz}, 1 \mathrm{H}), 4.20-4.09(\mathrm{~m}, 2 \mathrm{H}), 3.53(\mathrm{td}, J=8.3$, $6.9 \mathrm{~Hz}, 1 \mathrm{H}), 3.37-3.18(\mathrm{~m}, 3 \mathrm{H}), 2.30$ (s, 3H). ${ }^{13} \mathrm{C}$ NMR (101 MHz, Chloroform- $d$ ) $\delta 158.1$, 138.6, 136.4, 134.3, 129.4, 128.9, 128.7, 128.2, 127.7, 61.9, 57.5, 40.8, 36.5, 21.2, HRMSESI calcd for $\mathrm{C}_{18} \mathrm{H}_{19} \mathrm{NO}_{2}[\mathrm{M}+\mathrm{Na}]^{+}$: 304.1308 , found: 304.1304 .

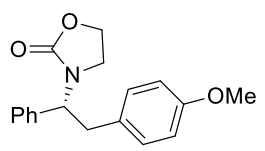

(R)-3-(2-(4-methoxyphenyl)-1-phenylethyl)oxazolidin-2-one(5j) Colorless oil, 98\% yield. ${ }^{1} \mathrm{H}$ NMR (400 MHz, Chloroform-d) $\delta 7.42-7.28(\mathrm{~m}, 5 \mathrm{H}), 7.19-7.14(\mathrm{~m}, 2 \mathrm{H}), 6.85-6.80$ $(\mathrm{m}, 2 \mathrm{H}), 5.29(\mathrm{dd}, J=9.7,6.6 \mathrm{~Hz}, 1 \mathrm{H}), 4.19-4.13(\mathrm{~m}, 2 \mathrm{H}), 3.77(\mathrm{~s}, 3 \mathrm{H}), 3.52(\mathrm{td}, J=8.2$, $7.0 \mathrm{~Hz}, 1 \mathrm{H}), 3.32-3.18(\mathrm{~m}, 3 \mathrm{H}) .{ }^{13} \mathrm{C}$ NMR $(101 \mathrm{MHz}$, Chloroform- $d$ ) $\delta$ 158.4, 158.1, 138.5, 129.8, 129.4, 128.9, 128.2, 127.7, 114.1, 61.9, 57.6, 55.3, 40.9, 36.0, HRMS-ESI calcd for $\mathrm{C}_{18} \mathrm{H}_{19} \mathrm{NO}_{3}[\mathrm{M}+\mathrm{Na}]^{+}: 320.1257$, found: 320.1242 .

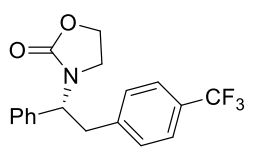

(R)-3-(1-phenyl-2-(4-(trifluoromethyl)phenyl)ethyl)oxazolidin-2-one(5k) White solid, 93\% yield. ${ }^{1} \mathrm{H}$ NMR $(400 \mathrm{MHz}$, Chloroform- $d$ ) $\delta 7.54(\mathrm{~d}, J=8.0 \mathrm{~Hz}, 2 \mathrm{H}), 7.42-7.32(\mathrm{~m}$, $7 \mathrm{H}), 5.34(\mathrm{dd}, J=8.6,7.7 \mathrm{~Hz}, 1 \mathrm{H}), 4.18(\mathrm{dd}, J=8.6,7.4 \mathrm{~Hz}, 2 \mathrm{H}), 3.57-3.45$ (m, $1 \mathrm{H}), 3.39$ $(\mathrm{d}, J=8.2 \mathrm{~Hz}, 2 \mathrm{H}), 3.24(\mathrm{q}, J=8.3 \mathrm{~Hz}, 1 \mathrm{H}) .{ }^{13} \mathrm{C}$ NMR $(101 \mathrm{MHz}$, Chloroform- $d$ ) $\delta 158.0$, $142.5,136.8,130.5$ (q, $J=32.7 \mathrm{~Hz}), 128.9,128.8,128.1,128.1,127.1,125.9$ (q, $J=3.8 \mathrm{~Hz}$ ), 122.7 (q, $J=272.2 \mathrm{~Hz}), 62.0,57.2,41.1,36.8 .{ }^{19} \mathrm{~F}$ NMR (376 MHz, Chloroform- $d$ ) $\delta-62.5$. HRMS-ESI calcd for $\mathrm{C}_{18} \mathrm{H}_{16} \mathrm{~F}_{3} \mathrm{NO}_{2}[\mathrm{M}+\mathrm{Na}]^{+}: 358.1025$, found: 358.1028 .

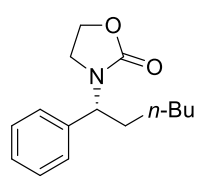

(R)-3-(1-phenylhexyl)oxazolidin-2-one(6a) Colorless oil, 99\% yield. ${ }^{1} \mathrm{H}$ NMR (400 MHz, Chloroform- $d$ ) $\delta 7.44-7.27(\mathrm{~m}, 5 \mathrm{H}), 4.99(\mathrm{dd}, J=8.6,7.2 \mathrm{~Hz}, 1 \mathrm{H}), 4.35-4.15(\mathrm{~m}, 2 \mathrm{H})$, $3.54-3.39(\mathrm{~m}, 1 \mathrm{H}), 3.25-3.11(\mathrm{~m}, 1 \mathrm{H}), 2.02-1.85(\mathrm{~m}, 2 \mathrm{H}), 1.40-1.26(\mathrm{~m}, 6 \mathrm{H}), 0.89(\mathrm{t}, J$ $=7.1 \mathrm{~Hz}, 3 \mathrm{H}) .{ }^{13} \mathrm{C}$ NMR $(101 \mathrm{MHz}$, Chloroform- $d) \delta 158.3,138.9,128.8,128.1,127.8,62.0$, $56.6,40.3,31.7,30.4,26.2,22.7,14.1$. HRMS-ESI calcd for $\mathrm{C}_{15} \mathrm{H}_{21} \mathrm{NO}_{2}[\mathrm{M}+\mathrm{Na}]^{+}: 270.1465$, found: 270.1479 .

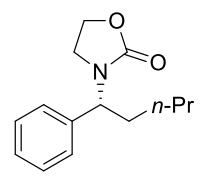

(R)-3-(1-phenylpentyl)oxazolidin-2-one(6b) Colorless oil, 92\% yield. ${ }^{1} \mathrm{H}$ NMR (400 MHz, Chloroform- $d$ ) $\delta 7.39-7.27(\mathrm{~m}, 5 \mathrm{H}), 4.98(\mathrm{dd}, J=8.5,7.3 \mathrm{~Hz}, 1 \mathrm{H}), 4.33-4.25(\mathrm{~m}, 1 \mathrm{H})$, $4.24-4.17(\mathrm{~m}, 1 \mathrm{H}), 3.51-3.43(\mathrm{~m}, 1 \mathrm{H}), 3.22-3.14(\mathrm{~m}, 1 \mathrm{H}), 2.03-1.87(\mathrm{~m}, 2 \mathrm{H}), 1.48-$ $1.28(\mathrm{~m}, 4 \mathrm{H}), 0.92(\mathrm{t}, J=7.1 \mathrm{~Hz}, 3 \mathrm{H}) .{ }^{13} \mathrm{C}$ NMR $(101 \mathrm{MHz}$, Chloroform- $d$ ) $\delta$ 158.13, 138.74, 128.64, 127.87, 127.55, 61.82, 56.36, 40.09, 29.89, 28.48, 22.36, 13.92. HRMS-ESI calcd for $\mathrm{C}_{14} \mathrm{H}_{19} \mathrm{NO}_{2}[\mathrm{M}+\mathrm{Na}]^{+}: 256.1308$, found: 256.1313 . 


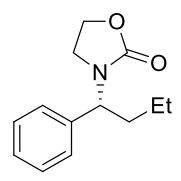

(R)-3-(1-phenylbutyl)oxazolidin-2-one(6c) Colorless oil, 93\% yield. ${ }^{1} \mathrm{H}$ NMR (400 MHz, Chloroform- $d$ ) $\delta 7.38-7.27(\mathrm{~m}, 5 \mathrm{H}), 5.00(\mathrm{~s}, 1 \mathrm{H}), 4.33-4.14(\mathrm{~m}, 2 \mathrm{H}), 3.52-3.40(\mathrm{~m}, 1 \mathrm{H})$, $3.24-3.14(\mathrm{~m}, 1 \mathrm{H}), 1.99-1.88(\mathrm{~m}, 2 \mathrm{H}), 1.46-1.32(\mathrm{~m}, 2 \mathrm{H}), 0.99(\mathrm{t}, J=7.4 \mathrm{~Hz}, 3 \mathrm{H}) .{ }^{13} \mathrm{C}$ NMR (101 MHz, Chloroform- $d$ ) $\delta 158.3,138.9,128.8,128.1,127.7,62.0,56.2,40.2,32.5$, 19.7, 13.9. HRMS-ESI calcd for $\mathrm{C}_{13} \mathrm{H}_{17} \mathrm{NO}_{2}[\mathrm{M}+\mathrm{Na}]^{+}: 242.1151$, found: 242.1165 .

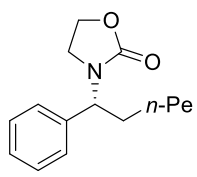

(R)-3-(1-phenylheptyl)oxazolidin-2-one(6d) Colorless oil, 93\% yield. Colorless oil, 98\% yield. ${ }^{1} \mathrm{H}$ NMR (400 MHz, Chloroform- $d$ ) $\delta 7.40-7.27$ (m, 5H), $\delta 5.03-4.92(\mathrm{~m}, 1 \mathrm{H}), 4.37$ $-4.09(\mathrm{~m}, 2 \mathrm{H}), 3.53-3.42(\mathrm{~m}, 1 \mathrm{H}), 3.29-3.13(\mathrm{~m}, 1 \mathrm{H}), 2.04-1.84(\mathrm{~m}, 2 \mathrm{H}), 1.45-1.20(\mathrm{~m}$, $8 \mathrm{H}), 0.88(\mathrm{t}, J=6.9 \mathrm{~Hz}, 3 \mathrm{H}) .{ }^{13} \mathrm{C}$ NMR $(101 \mathrm{MHz}$, Chloroform- $d$ ) $\delta 158.3,138.9,128.8$, $128.1,127.7,62.0,56.6,40.3,31.8,30.4,29.1,26.5,22.7,14.2$. HRMS-ESI calcd for $\mathrm{C}_{16} \mathrm{H}_{23} \mathrm{NO}_{2}[\mathrm{M}+\mathrm{Na}]^{+}: 284.1621$, found: 284.1641 .

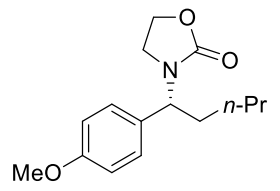

(R)-3-(1-(4-methoxyphenyl)pentyl)oxazolidin-2-one(6e) Colorless oil, 95\% yield. ${ }^{1} \mathrm{H}$ NMR $(400 \mathrm{MHz}$, Chloroform- $d) \delta 7.31-7.21(\mathrm{~m}, 2 \mathrm{H}), 6.92-6.82(\mathrm{~m}, 2 \mathrm{H}), 4.93(\mathrm{t}, J=7.9 \mathrm{~Hz}$, $1 \mathrm{H}), 4.33-4.14(\mathrm{~m}, 2 \mathrm{H}), 3.44(\mathrm{td}, J=8.8,6.1 \mathrm{~Hz}, 1 \mathrm{H}), 3.23-3.09(\mathrm{~m}, 1 \mathrm{H}), 1.98-1.84(\mathrm{~m}$, $3 \mathrm{H}), 1.47-1.23(\mathrm{~m}, 5 \mathrm{H}), 0.91$ (t, $J=7.1 \mathrm{~Hz}, 2 \mathrm{H}) .{ }^{13} \mathrm{C}$ NMR (101 MHz, Chloroform- $d$ ) $\delta$ $159.3,158.3,131.0,128.9,114.1,62.0,56.0,55.4,40.2,30.3,28.7,22.5,14.1$. HRMS-ESI calcd for $\mathrm{C}_{15} \mathrm{H}_{21} \mathrm{NO}_{3}[\mathrm{M}+\mathrm{Na}]^{+}: 286.1414$, found: 286.1389 .

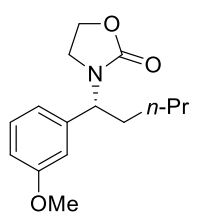

(R)-3-(1-(3-methoxyphenyl)pentyl)oxazolidin-2-one(6f) Colorless oil, 90\% yield. ${ }^{1} \mathrm{H}$ NMR $(400 \mathrm{MHz}$, Chloroform- $d$ ) $\delta 7.27(\mathrm{t}, J=7.9 \mathrm{~Hz}, 1 \mathrm{H}), 6.93-6.81(\mathrm{~m}, 3 \mathrm{H}), 4.95(\mathrm{dd}, J=8.7$, $7.1 \mathrm{~Hz}, 1 \mathrm{H}), 4.37-4.09(\mathrm{~m}, 2 \mathrm{H}), 3.50-3.42(\mathrm{~m}, 1 \mathrm{H}), 3.24-3.15(\mathrm{~m}, 1 \mathrm{H}), 2.00-1.80(\mathrm{~m}$, $2 \mathrm{H}), 1.49-1.18(\mathrm{~m}, 4 \mathrm{H}), 0.91(\mathrm{t}, J=7.0 \mathrm{~Hz}, 3 \mathrm{H}) .{ }^{13} \mathrm{C}$ NMR $(101 \mathrm{MHz}$, Chloroform- $d) \delta$ $160.0,158.3,140.5,129.8,119.9,113.8,113.2,62.0,56.5,55.4,40.3,30.1,28.6,22.6,14.1$. HRMS-ESI calcd for $\mathrm{C}_{15} \mathrm{H}_{21} \mathrm{NO}_{3}[\mathrm{M}+\mathrm{Na}]^{+}: 286.1414$, found: 286.1420 .

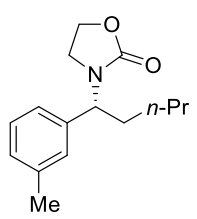

(R)-3-(1-(m-tolyl)pentyl)oxazolidin-2-one(6g) Colorless oil, 99\% yield. ${ }^{1} \mathrm{H}$ NMR (400 MHz, Chloroform-d) $\delta 7.23(\mathrm{t}, J=7.5 \mathrm{~Hz}, 1 \mathrm{H}), 7.16-7.07(\mathrm{~m}, 3 \mathrm{H}), 4.94(\mathrm{t}, J=7.9 \mathrm{~Hz}, 1 \mathrm{H}), 4.33-$ $4.23(\mathrm{~m}, 1 \mathrm{H}), 4.23-4.14(\mathrm{~m}, 1 \mathrm{H}), 3.52-3.41(\mathrm{~m}, 1 \mathrm{H}), 3.25-3.12(\mathrm{~m}, 1 \mathrm{H}), 2.34(\mathrm{~s}, 3 \mathrm{H})$, $1.99-1.86(\mathrm{~m}, 2 \mathrm{H}), 1.46-1.26(\mathrm{~m}, 4 \mathrm{H}), 0.91(\mathrm{t}, J=7.1 \mathrm{~Hz}, 3 \mathrm{H}) .{ }^{13} \mathrm{C}$ NMR $(101 \mathrm{MHz}$, Chloroform- $d$ ) $\delta 158.12,138.63,138.35,128.59,128.49,128.42,124.31,61.79,56.30,40.05$, 
29.86, 28.48, 22.35, 21.40, 13.91. HRMS-ESI calcd for $\mathrm{C}_{15} \mathrm{H}_{21} \mathrm{NO}_{2}[\mathrm{M}+\mathrm{Na}]^{+}:$270.1465, found: 270.1467 .

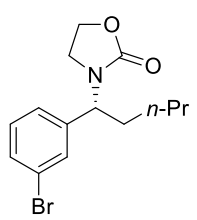

(R)-3-(1-(3-bromophenyl)pentyl)oxazolidin-2-one(6h) Colorless oil, 97\% yield. ${ }^{1} \mathrm{H}$ NMR $(400 \mathrm{MHz}$, Chloroform- $d$ ) $\delta 7.49-7.41(\mathrm{~m}, 2 \mathrm{H}), 7.30-7.19(\mathrm{~m}, 2 \mathrm{H}), 4.94(\mathrm{t}, J=7.9 \mathrm{~Hz}$, $1 \mathrm{H}), 4.35-4.18(\mathrm{~m}, 2 \mathrm{H}), 3.47$ (td, $J=8.8,6.2 \mathrm{~Hz}, 1 \mathrm{H}), 3.25-3.15(\mathrm{~m}, 1 \mathrm{H}), 1.91$ (q, $J=8.4$, $8.0 \mathrm{~Hz}, 1 \mathrm{H}), 1.46-1.24(\mathrm{~m}, 4 \mathrm{H}), 0.92(\mathrm{t}, J=7.1 \mathrm{~Hz}, 3 \mathrm{H}) .{ }^{13} \mathrm{C}$ NMR $(101 \mathrm{MHz}$, Chloroformd) $\delta 158.05,141.19,131.05,130.29,126.32,122.79,61.87,55.88,40.04,29.80,28.38,22.29$, 13.89. HRMS-ESI calcd for $\mathrm{C}_{14} \mathrm{H}_{18} \mathrm{BrNO}_{2}[\mathrm{M}+\mathrm{Na}]^{+}: 334.0413$, found: 334.0419 .

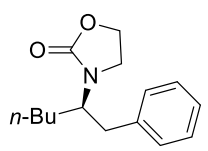

(R)-3-(1-phenylhexan-2-yl)oxazolidin-2-one(7a) Colorless oil, 99\% yield. ${ }^{1} \mathrm{H}$ NMR (400 $\mathrm{MHz}$, Chloroform- $d$ ) $\delta 7.32-7.26(\mathrm{~m}, 2 \mathrm{H}), 7.24-7.17(\mathrm{~m}, 3 \mathrm{H}), 4.19(\mathrm{dtd}, J=31.0,8.7,7.0$ $\mathrm{Hz}, 2 \mathrm{H}), 4.09-3.95(\mathrm{~m}, 1 \mathrm{H}), 3.45-3.28(\mathrm{~m}, 2 \mathrm{H}), 2.90-2.74(\mathrm{~m}, 2 \mathrm{H}), 1.62-1.50(\mathrm{~m}, 2 \mathrm{H})$, $1.41-1.20(\mathrm{~m}, 4 \mathrm{H}), 0.88(\mathrm{t}, J=7.0 \mathrm{~Hz}, 3 \mathrm{H}) .{ }^{13} \mathrm{C}$ NMR $(101 \mathrm{MHz}$, Chloroform- $d$ ) $\delta 158.2$, 138.0, 129.0, 128.6, 126.7, 62.0, 54.7, 40.7, 39.2, 31.6, 28.6, 22.5, 14.1, HRMS-ESI calcd for $\mathrm{C}_{15} \mathrm{H}_{21} \mathrm{NO}_{2}[\mathrm{M}+\mathrm{Na}]^{+}: 270.1470$, found: 270.1459 .

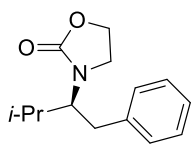

(S)-3-(3-methyl-1-phenylbutan-2-yl)oxazolidin-2-one(7b) Colorless oil, 93\% yield. ${ }^{1} \mathrm{H}$ NMR (400 MHz, Chloroform- $d$ ) $\delta 7.31-7.24(\mathrm{~m}, 2 \mathrm{H}), 7.20(\mathrm{dt}, J=5.9,1.4 \mathrm{~Hz}, 3 \mathrm{H}), 4.19-$ $4.09(\mathrm{~m}, 1 \mathrm{H}), 4.05-3.91(\mathrm{~m}, 1 \mathrm{H}), 3.80-3.67(\mathrm{~m}, 1 \mathrm{H}), 3.41-3.21(\mathrm{~m}, 2 \mathrm{H}), 3.12(\mathrm{dd}, J=$ 14.6, 4.4 Hz, 1H), 2.67 (dd, $J=14.6,11.4 \mathrm{~Hz}, 1 \mathrm{H}), 1.97-1.79(\mathrm{~m}, 1 \mathrm{H}), 1.08$ (d, $J=6.7 \mathrm{~Hz}$, $3 \mathrm{H}), 0.97(\mathrm{~d}, J=6.7 \mathrm{~Hz}, 3 \mathrm{H}) .{ }^{13} \mathrm{C}$ NMR (101 MHz, Chloroform- $d$ ) $\delta$ 158.4, 138.2, 128.6, 126.6, 61.9, 60.5, 41.5, 36.1, 31.1, 20.2, 19.9. HRMS-ESI calcd for $\mathrm{C}_{14} \mathrm{H}_{19} \mathrm{NO}_{2}[\mathrm{M}+\mathrm{Na}]^{+}$: 256.1313 , found: 256.1308 .

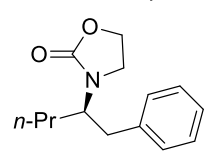

(R)-3-(1-phenylpentan-2-yl)oxazolidin-2-one(7c) Colorless oil, 92\% yield. ${ }^{1} \mathrm{H}$ NMR (400 MHz, Chloroform- $d$ ) $\delta 7.31-7.26(\mathrm{~m}, 2 \mathrm{H}), 7.23-7.18(\mathrm{~m}, 3 \mathrm{H}), 4.26-4.02(\mathrm{~m}, 3 \mathrm{H}), 3.43-$ $3.29(\mathrm{~m}, 2 \mathrm{H}), 2.87-2.76(\mathrm{~m}, 2 \mathrm{H}), 1.63-1.46(\mathrm{~m}, 2 \mathrm{H}), 1.42-1.24(\mathrm{~m}, 2 \mathrm{H}), 0.92(\mathrm{t}, J=7.3$ $\mathrm{Hz}, 3 \mathrm{H}) .{ }^{13} \mathrm{C}$ NMR (101 MHz, Chloroform- $d$ ) $\delta$ 158.2, 138.0, 129.0, 128.6, 126.7, 62.0, 54.4, 40.7, 39.2, 34.0, 19.7, 13.9, HRMS-ESI calcd for $\mathrm{C}_{14} \mathrm{H}_{19} \mathrm{NO}_{2}[\mathrm{M}+\mathrm{Na}]^{+}: 256.1313$, found: 256.1317.

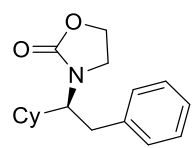

(S)-3-(1-cyclohexyl-2-phenylethyl)oxazolidin-2-one(7d) Colorless oil, 90\% yield. ${ }^{1} \mathrm{H}$ NMR (400 MHz, Chloroform- $d$ ) $\delta 7.31-7.26(\mathrm{~m}, 2 \mathrm{H}), 7.20$ (s, 3H), 4.13 (ddd, $J=9.3,8.4,6.2 \mathrm{~Hz}$, $1 \mathrm{H}), 4.03-3.95(\mathrm{~m}, 1 \mathrm{H}), 3.78$ (ddd, $J=11.4,9.4,4.5 \mathrm{~Hz}, 1 \mathrm{H}), 3.34$ (ddd, $J=9.3,8.3,6.2$ $\mathrm{Hz}, 1 \mathrm{H}), 3.29-3.20$ (m, 1H), 3.13 (dd, $J=14.6,4.5 \mathrm{~Hz}, 1 \mathrm{H}), 2.68$ (dd, $J=14.6,11.4 \mathrm{~Hz}$, 
1H), $1.96-1.88(\mathrm{~m}, 1 \mathrm{H}), 1.85-1.55(\mathrm{~m}, 5 \mathrm{H}), 1.36-1.00(\mathrm{~m}, 5 \mathrm{H}) .{ }^{13} \mathrm{C}$ NMR $(101 \mathrm{MHz}$, Chloroform- $d$ ) $\delta 158.4,138.4,128.7,128.7,126.6,61.9,59.6,42.0,40.4,35.8,30.6,30.1$, 26.4, 26.1, 26.1, HRMS-ESI calcd for $\mathrm{C}_{17} \mathrm{H}_{23} \mathrm{NO}_{2}[\mathrm{M}+\mathrm{Na}]^{+}: 296.1626$, found: 296.1615 .

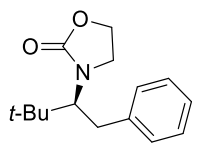

(S)-3-(3,3-dimethyl-1-phenylbutan-2-yl)oxazolidin-2-one(7e) Colorless oil, 93\% yield. ${ }^{1} \mathrm{H}$ NMR (400 MHz, Chloroform- $d$ ) $\delta 7.32-7.26(\mathrm{~m}, 2 \mathrm{H}), 7.23-7.16(\mathrm{~m}, 3 \mathrm{H}), 4.26-4.00(\mathrm{~m}$, $2 \mathrm{H}), 3.97-3.74(\mathrm{~m}, 1 \mathrm{H}), 3.70-3.52(\mathrm{~m}, 1 \mathrm{H}), 3.51-3.40(\mathrm{~m}, 1 \mathrm{H}), 3.17-3.00(\mathrm{~m}, 1 \mathrm{H}), 2.87$ $-2.60(\mathrm{~m}, 1 \mathrm{H}), 1.08(\mathrm{~s}, 9 \mathrm{H}) .{ }^{13} \mathrm{C}$ NMR $(101 \mathrm{MHz}$, Chloroform-d) $\delta 159.2,138.7,128.8$, $128.5,126.7,77.4,62.0,42.1,35.9,32.5,27.7$. HRMS-ESI calcd for $\mathrm{C}_{15} \mathrm{H}_{21} \mathrm{NO}_{2}[\mathrm{M}+\mathrm{Na}]^{+}$: 270.1465 , found: 270.1478 .<smiles>CCC(C(C)C)N1CCOC1=O</smiles>

(S)-3-(2-methylpentan-3-yl)oxazolidin-2-one(7f) Colorless oil, 95\% yield. ${ }^{1} \mathrm{H}$ NMR (400 MHz, Chloroform- $d$ ) $\delta 4.31(\mathrm{dd}, J=8.4,7.7 \mathrm{~Hz}, 2 \mathrm{H}), 3.41(\mathrm{td}, J=7.8,2.0 \mathrm{~Hz}, 2 \mathrm{H}), 3.33$ (ddd, $J=11.4,9.4,3.7 \mathrm{~Hz}, 1 \mathrm{H}), 1.81-1.60(\mathrm{~m}, 2 \mathrm{H}), 1.40-1.24(\mathrm{~m}, 1 \mathrm{H}), 0.95$ (d, $J=6.6 \mathrm{~Hz}$, $3 \mathrm{H}), 0.91-0.83(\mathrm{~m}, 6 \mathrm{H}) .{ }^{13} \mathrm{C}$ NMR (101 MHz, Chloroform- $d$ ) $\delta$ 159.1, 62.0, 61.2, 40.2, 31.0, 22.5, 20.0, 19.9, 11.0, HRMS-ESI calcd for $\mathrm{C}_{9} \mathrm{H}_{17} \mathrm{NO}_{2}[\mathrm{M}+\mathrm{Na}]^{+}:$194.1151, found: 194.1156.<smiles>CC(CN1CCOC1=O)c1ccccc1</smiles>

3-(2-phenylpropyl)oxazolidin-2-one (8a) Colorless oil, ${ }^{1} \mathrm{H}$ NMR (400 MHz, Chloroform- $d$ ) $\delta 7.35-7.27(\mathrm{~m}, 2 \mathrm{H}), 7.27-7.19(\mathrm{~m}, 3 \mathrm{H}), 4.14(\mathrm{dd}, J=8.5,7.6 \mathrm{~Hz}, 2 \mathrm{H}), 3.54(\mathrm{dd}, J=13.9$, $6.9 \mathrm{~Hz}, 1 \mathrm{H}), 3.35-3.21(\mathrm{~m}, 2 \mathrm{H}), 3.18-3.00(\mathrm{~m}, 2 \mathrm{H}), 1.28(\mathrm{~d}, J=7.0 \mathrm{~Hz}, 3 \mathrm{H}) .{ }^{13} \mathrm{C} \mathrm{NMR}$ $\left(101 \mathrm{MHz}, \mathrm{CDCl}_{3}\right) \delta 158.5,143.8,128.6,127.0,126.7,61.6,51.2,45.1,38.6,19.1$. HRMSESI calcd for $\mathrm{C}_{12} \mathrm{H}_{15} \mathrm{NO}_{2}[\mathrm{M}+\mathrm{Na}]^{+}$: 228.0995, found: 228.1000 .<smiles>CCC(CN1CCOC1=O)c1ccccc1</smiles>

3-(2-phenylbutyl)oxazolidin-2-one (8b) Colorless oil, ${ }^{1} \mathrm{H}$ NMR (400 MHz, Chloroform- $d$ ) $\delta$ $7.35-7.27(\mathrm{~m}, 2 \mathrm{H}), 7.27-7.19(\mathrm{~m}, 3 \mathrm{H}), 4.14(\mathrm{dd}, J=8.5,7.6 \mathrm{~Hz}, 2 \mathrm{H}), 3.54(\mathrm{dd}, J=13.9$, $6.9 \mathrm{~Hz}, 1 \mathrm{H}), 3.35-3.21(\mathrm{~m}, 2 \mathrm{H}), 3.18-3.00(\mathrm{~m}, 2 \mathrm{H}), 1.60-1.50(\mathrm{~m}, 2 \mathrm{H}), 0.95(\mathrm{t}, J=7.3$ $\mathrm{Hz}, 3 \mathrm{H}) .{ }^{13} \mathrm{C}$ NMR $\left(101 \mathrm{MHz}, \mathrm{CDCl}_{3}\right) \delta 158.5,143.7,128.5,127.0,126.7,62.0,54.4,40.7$, 39.2, 24.0, 21.2 HRMS-ESI calcd for $\mathrm{C}_{13} \mathrm{H}_{17} \mathrm{NO}_{2}[\mathrm{M}+\mathrm{Na}]^{+}$: 242.1157, found: 242.1162 .<smiles>CCCC(CN1CCOC1=O)c1ccccc1</smiles>

3-(2-phenylpentyl)oxazolidin-2-one (8c) Colorless oil, ${ }^{1} \mathrm{H}$ NMR $\left(400 \mathrm{MHz}, \mathrm{CDCl}_{3}\right) \delta 7.36-$ $7.28(\mathrm{~m}, 2 \mathrm{H}), 7.26-7.18(\mathrm{~m}, 3 \mathrm{H}), 4.17-4.02(\mathrm{~m}, 2 \mathrm{H}), 3.62(\mathrm{dd}, J=13.9,6.2 \mathrm{~Hz}, 1 \mathrm{H}), 3.31$ $-3.17(\mathrm{~m}, 2 \mathrm{H}), 3.07-2.97(\mathrm{~m}, 1 \mathrm{H}), 2.90(\mathrm{tt}, J=9.5,5.8 \mathrm{~Hz}, 1 \mathrm{H}), 1.70-1.50(\mathrm{~m}, 2 \mathrm{H}), 1.20$ (h, $J=7.5 \mathrm{~Hz}, 2 \mathrm{H}), 0.85$ (t, $J=7.3 \mathrm{~Hz}, 3 \mathrm{H}) .{ }^{13} \mathrm{C} \mathrm{NMR}\left(101 \mathrm{MHz}, \mathrm{CDCl}_{3}\right) \delta$ 158.5, 143.7, 128.5 127.0, 126.7, 54.4, 46.0, 40.7, 39.2, 34.0, 19.9, 13.9. HRMS-ESI calcd for $\mathrm{C}_{14} \mathrm{H}_{19} \mathrm{NO}_{2}$ $[\mathrm{M}+\mathrm{Na}]^{+}: 256.1313$, found: 256.1307 .

\section{General procedure for the preparation of iridium complexes}




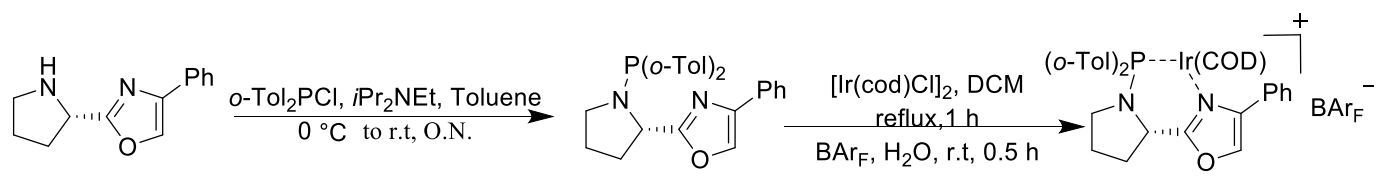

Amine $(1 \mathrm{mmol})$ was co-evaporated with dry toluene $(3 \times 20 \mathrm{~mL})$ and dissolved in dry toluene or THF $(6 \mathrm{~mL})$ under $\mathrm{N}_{2}$. Freshly distilled di-iso-propylethylamine (3 mmol) was added and the solution was cooled to $0{ }^{\circ} \mathrm{C}$ in an ice-bath. Freshly distilled (prepared) suitable phosphine chloride $(1.2 \mathrm{mmol})$ was dissolved in toluene $(2 \mathrm{~mL})$ and added dropwise to the reaction mixture and stirred at room temperature for 24 hours. After completion of the reaction, monitored by TLC, it was quenched with saturated $\mathrm{NaHCO}_{3}$ solution and the reaction mixture was extracted in $\mathrm{CH}_{2} \mathrm{Cl}_{2}(3 \times 10 \mathrm{~mL})$. The combined organic layers were dried over $\mathrm{Na}_{2} \mathrm{SO}_{4}$, filtered and concentrated under reduced pressure. The oily residual was quickly passed through a small pad of silica and used to make the Ir-complex without further characterization.

The synthesized oxazoline ligand was dissolved in $\mathrm{CH}_{2} \mathrm{Cl}_{2}(20 \mathrm{~mL})$ and $[\operatorname{Ir}(\mathrm{COD}) \mathrm{Cl}]_{2}(0.25$ mmol) was added. The atmosphere in the flask was evacuated and replenished three times with $\mathrm{N}_{2}$. The mixture was heated to reflux for 1 hour. After the solution was cooled to room temperature, distilled $\mathrm{H}_{2} \mathrm{O}(20 \mathrm{~mL})$ was added. Under vigorous stirring, $\mathrm{NaBAr}_{\mathrm{F}} \cdot \mathrm{xH}_{2} \mathrm{O}(0.6$ $\mathrm{mmol}$ ) was added to the biphasic solution in one portion. The mixture was stirred vigorously for 30 minutes and extracted with $\mathrm{CH}_{2} \mathrm{Cl}_{2}(3 \times 10 \mathrm{~mL})$. Combined organic phase was dried over $\mathrm{Na}_{2} \mathrm{SO}_{4}$. After concentration in vacuum, the residue was purified on silica gel with $\mathrm{CH}_{2} \mathrm{Cl}_{2}$ : pentane $(1: 1)$ as the eluent to afford the iridium complex as an orange solid.<smiles>c1ccc(-c2coc(C3CCCN3)n2)cc1</smiles>

White solid. ${ }^{1} \mathrm{H}$ NMR (400 MHz, Chloroform-d) $\delta 7.83(\mathrm{~s}, 1 \mathrm{H}), 7.77-7.68(\mathrm{~m}, 2 \mathrm{H}), 7.42-$ $7.33(\mathrm{~m}, 2 \mathrm{H}), 7.33-7.26(\mathrm{~m}, 1 \mathrm{H}), 4.39(\mathrm{ddd}, \mathrm{J}=7.6,6.0,1.2 \mathrm{~Hz}, 1 \mathrm{H}), 3.23-3.13(\mathrm{~m}, 1 \mathrm{H})$, $3.09-2.89(\mathrm{~m}, 1 \mathrm{H}), 2.44(\mathrm{~s}, 1 \mathrm{H}), 2.32-2.05(\mathrm{~m}, 2 \mathrm{H}), 2.05-1.69(\mathrm{~m}, 2 \mathrm{H}) .{ }^{13} \mathrm{C}$ NMR $(101$ $\mathrm{MHz}$, Chloroform- $d$ ) $\delta 166.8,140.6,133.5,131.2,128.8,128.03,125.6,55.74,47.0,31.0$, 25.5. HRMS-EI calcd for $\mathrm{C}_{13} \mathrm{H}_{14} \mathrm{NO}[\mathrm{M}+\mathrm{Na}]^{+}:$727.2425, found: 727.2445 .

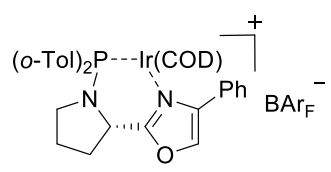

Orange solid. $[\alpha]_{\mathrm{D}}^{25.0}=+51\left(\mathrm{C}=0.10\right.$ in $\left.\mathrm{CHCl}_{3}\right) .{ }^{1} \mathrm{H}$ NMR $(400 \mathrm{MHz}$, Chloroform- $d): \delta 7.73$ (p, $J=2.2 \mathrm{~Hz}, 8 \mathrm{H}), 7.62(\mathrm{~s}, 1 \mathrm{H}), 7.52(\mathrm{~s}, 5 \mathrm{H}), 7.43(\mathrm{q}, J=8.4,7.7 \mathrm{~Hz}, 4 \mathrm{H}), 7.32(\mathrm{~d}, J=7.7$ $\mathrm{Hz}, 3 \mathrm{H}), 7.25-7.20$ (m, 1H), 7.11 (dd, $J=12.7,7.9 \mathrm{~Hz}, 2 \mathrm{H}), 6.98$ (d, $J=7.6 \mathrm{~Hz}, 2 \mathrm{H}), 6.66$ $(\mathrm{t}, J=9.2 \mathrm{~Hz}, 1 \mathrm{H}), 5.22(\mathrm{t}, J=7.1 \mathrm{~Hz}, 1 \mathrm{H}), 4.73-4.56(\mathrm{~m}, 1 \mathrm{H}), 4.20(\mathrm{~s}, 1 \mathrm{H}), 3.62-3.48(\mathrm{~m}$, $1 \mathrm{H}), 3.33$ (t, $J=6.8 \mathrm{~Hz}, 1 \mathrm{H}), 3.07$ (dd, $J=11.2,5.4 \mathrm{~Hz}, 1 \mathrm{H}), 2.86(\mathrm{~s}, 3 \mathrm{H}), 2.74(\mathrm{~s}, 3 \mathrm{H}), 2.65$ $(\mathrm{td}, J=11.9,10.0,5.4 \mathrm{~Hz}, 2 \mathrm{H}), 2.49(\mathrm{dd}, J=13.5,6.9 \mathrm{~Hz}, 1 \mathrm{H}), 2.28-1.82(\mathrm{~m}, 6 \mathrm{H}), 1.68(\mathrm{~d}$, $J=10.9 \mathrm{~Hz}, 1 \mathrm{H}), 1.41(\mathrm{td}, J=9.8,8.7,4.7 \mathrm{~Hz}, 2 \mathrm{H}) \cdot{ }^{13} \mathrm{C}$ NMR $(100 \mathrm{MHz}$, Chloroform- $d$ ) $\delta$ 165.6, 161.8 (ddd, $J=99.7,49.8,3.2 \mathrm{~Hz}$ ), 141.9, 140.9 (dd, $J=105.7,14.0 \mathrm{~Hz}$ ), 136.1, 134.9, $133.3-132.2(\mathrm{~m}), 131.8,131.6(\mathrm{~d}, J=47.2 \mathrm{~Hz}), 130.7,129.4,129.1(\mathrm{dt}, J=5.8,3.1 \mathrm{~Hz})$, $129.0-128.6(\mathrm{~m}), 128.5(\mathrm{~d}, J=3.6 \mathrm{~Hz}), 128.0,127.5(\mathrm{~d}, J=7.4 \mathrm{~Hz}), 126.9(\mathrm{~d}, J=10.1 \mathrm{~Hz})$, 126.4, 126.0 (d, $J=2.2 \mathrm{~Hz}), 123.3(\mathrm{~d}, J=2.2 \mathrm{~Hz}), 121.8(\mathrm{~d}, J=62.7 \mathrm{~Hz}), 120.6,117.6,94.5$ $(\mathrm{d}, J=10.9 \mathrm{~Hz}), 86.9$ (d, $J=14.0 \mathrm{~Hz}), 69.7,68.0,59.0(\mathrm{~d}, J=11.5 \mathrm{~Hz}), 47.8,34.3,31.0(\mathrm{~d}, J$ $=170.9 \mathrm{~Hz}), 27.9-26.6(\mathrm{~m}), 26.4,25.1(\mathrm{~d}, J=6.3 \mathrm{~Hz}), 21.3(\mathrm{~d}, J=6.7 \mathrm{~Hz}) .{ }^{31} \mathrm{P}$ NMR $(162$ 
$\mathrm{MHz}$, Chloroform- $d$ ) $\delta 51.1$ (s). HRMS-EI calcd for $\mathrm{C}_{35} \mathrm{H}_{40} \mathrm{IrN}_{2} \mathrm{OP}[\mathrm{M}]^{+}:$727.2425, found: 727.2445 .

\section{Separation method data and specific rotation of chiral compounds}

Entry Product

1<smiles>CCCCC(Cc1ccccc1)N1CCOC1=O</smiles>

2<smiles>O=C(c1ccccc1)C(Cc1ccccc1)N1CCOC1=O</smiles>

3<smiles>O=C1OCCN1C(Cc1ccccc1)C(F)I</smiles>

4<smiles>O=C1OCCN1C(Cl)Cc1ccccc1</smiles>

5<smiles>CCCC(C(C)C)N1CCOC1=O</smiles>

6<smiles>CC(C)(C)[C@H](Cc1ccccc1)N1CCOC1=O</smiles>

7<smiles>CCCCC(c1ccccc1)N1CCOC1=O</smiles>

8<smiles>CCCCC(c1ccccc1)N1CCOC1=O</smiles>

Separation method

Optical rotation ee $(\%)$

SFC, OZH column, $[\alpha]_{\mathrm{D}}^{25.0}=-18(\mathrm{C}=95$ $10 \% \mathrm{MeOH}, 2 \mathrm{ml} / \mathrm{min}, \quad 0.10$ in $\mathrm{CHCl}_{3}$ )

$\mathrm{t}_{\mathrm{R}}=7.4 \mathrm{~min}$ (major)/8.2 (minor)

SFC, IF column, 15\% $\mathrm{MeOH}, 2 \mathrm{ml} / \mathrm{min}, \mathrm{t}_{\mathrm{R}}=$ $5.8 \min$ (minor) $/ 6.1$ (major)

SFC, ASH column, $[\alpha]_{\mathrm{D}}^{25.0}=-45(\mathrm{C}=96$ $15 \% \mathrm{MeOH}, 2 \mathrm{ml} / \mathrm{min}, \quad 0.10$ in $\mathrm{CHCl}_{3}$ ) $\mathrm{t}_{\mathrm{R}}=2.8 \mathrm{~min}$ (minor)/2.9 (major)

SFC, OZH column, $[\alpha]_{\mathrm{D}}^{25.0}=-22(\mathrm{C}=96$ $15 \% \mathrm{MeOH}, 2 \mathrm{ml} / \mathrm{min}, \quad 0.10$ in $\mathrm{CHCl}_{3}$ ) $\mathrm{t}_{\mathrm{R}}=7.5 \mathrm{~min}$ (major)/8.4 (minor)

GC-MS, Hydrodex $\beta-[\alpha]_{\mathrm{D}}^{25.0}=-4 \quad(\mathrm{C}=93$ $3 \mathrm{P}, 50{ }^{\circ} \mathrm{C}$ to $175{ }^{\circ} \mathrm{C}, 1 \quad 0.10$ in $\mathrm{CHCl}_{3}$ ) degree $/ \mathrm{min}, \mathrm{t}_{\mathrm{R}}=87.6$ $\min \quad$ (minor) $/ 88.7$ (major)

SFC, IF column, $15 \% \quad[\alpha]_{\mathrm{D}}^{25.0}=-12(\mathrm{C}=99$ $\mathrm{MeOH}, 2 \mathrm{ml} / \mathrm{min}, \mathrm{t}_{\mathrm{R}}=0.10$ in $\mathrm{CHCl}_{3}$ ) $5.3 \mathrm{~min}$ (minor) $/ 5.8$ (major)

SFC, IF column, $15 \% \quad[\alpha]_{\mathrm{D}}^{25.0}=+16592$ $\mathrm{MeOH}, 2 \mathrm{ml} / \mathrm{min}, \mathrm{t}_{\mathrm{R}}=(\mathrm{C}=0.73$ in $6.0 \quad \min \quad$ (major) $/ 7.7 \quad \mathrm{CHCl}_{3}$ ) (minor)

SFC, IF column, $15 \% \quad[\alpha]_{\mathrm{D}}^{25.0}=+106.8 \quad 89$ $\mathrm{MeOH}, 2 \mathrm{ml} / \mathrm{min}, \mathrm{t}_{\mathrm{R}}=(\mathrm{C}=0.54$ in $5.5 \min$ (major) $/ 6.3 \quad \mathrm{CHCl}_{3}$ ) (minor) 
9<smiles>CCCC(c1ccccc1)N1CCOC1=O</smiles>

10<smiles>O=PC[C@@H](c1ccccc1)N1CCOC1=O</smiles>

11<smiles>COc1ccc(C(CPC(C)C)N2CCOC2=O)cc1</smiles>

12<smiles>COc1cccc(C(CC(C)C)N2CCOC2=O)c1</smiles>

13<smiles>Cc1cccc(C(CPC(C)C)N2CCOC2=O)c1</smiles>

14<smiles>CCCC(=O)N1CCOC1=O</smiles>

15<smiles>O=C1OCCN1C(Cc1ccccc1)c1ccccc1</smiles>

16<smiles>O=C1OCCN1[C@@H](Cc1ccccc1)c1ccc(Cl)cc1</smiles>

HPLC, ASH column, $[\alpha]_{\mathrm{D}}^{25.0}=+9788$

$25 \% \mathrm{iPrOH}$ in hexane, $(\mathrm{C}=0.10 \quad$ in $\left.0.5 \mathrm{ml} / \mathrm{min}, \mathrm{t}_{\mathrm{R}}=30.5 \quad \mathrm{CHCl}_{3}\right)$ $\min$ (major) $/ 40.8 \mathrm{~min}$ (minor)

SFC, IF column, $15 \% \quad[\alpha]_{\mathrm{D}}^{25.0}=+101(\mathrm{C} \quad 93$ $\mathrm{MeOH}, 2 \mathrm{ml} / \mathrm{min}, \mathrm{t}_{\mathrm{R}}==0.10$ in $\mathrm{CHCl}_{3}$ ) $6.1 \min$ (major)/7.1 (minor)

SFC, IF column, ASH $[\alpha]_{\mathrm{D}}^{25.0}=+18093$ column, $25 \% i \mathrm{PrOH}$ in $(\mathrm{C}=\quad 0.10$ in hexane, $\left.t_{R}=45.3 \min \mathrm{CDCl}_{3}\right)$ (major)/53.6 (minor)

SFC, IF column, $10 \% \quad[\alpha]_{\mathrm{D}}^{25.0}=\quad+6393$ $\mathrm{MeOH}, 2 \mathrm{ml} / \mathrm{min}, \mathrm{t}_{\mathrm{R}}=(\mathrm{C}=0.10 \quad$ in 11.0 min (major)/13.7 $\quad \mathrm{CHCl}_{3}$ ) (minor)

SFC, ASH column, $[\alpha]_{\mathrm{D}}^{25.0}=+100.795$ $15 \% \mathrm{MeOH}, 2 \mathrm{ml} / \mathrm{min}, \quad$ (C $\mathrm{t}_{\mathrm{R}}=3.2 \mathrm{~min}=0.77$ in $\left.\mathrm{CHCl}_{3}\right)$ (minor)/3.6 (major)

SFC, ASH column, $[\alpha]_{\mathrm{D}}^{25.0}=+85.691$ $15 \% \mathrm{MeOH}, 2 \mathrm{ml} / \mathrm{min}, \quad(\mathrm{C}=0.73$ in $\left.\mathrm{t}_{\mathrm{R}}=4.4 \min \mathrm{CHCl}_{3}\right)$ (minor)/5.0 (major)

SFC, IF column, $15 \% \quad[\alpha]_{\mathrm{D}}^{25.0}=\quad+8293$ $\mathrm{MeOH}, 2 \mathrm{ml} / \mathrm{min}, \mathrm{t}_{\mathrm{R}}=(\mathrm{C}=0.10 \quad$ in $12.2 \min$ (major) / $\mathrm{CHCl}_{3}$ ) 13.2 (minor)

SFC, IF column, 15\% $[\alpha]_{\mathrm{D}}^{25.0}=\quad+6797$ $\mathrm{MeOH}, 2 \mathrm{ml} / \mathrm{min}, \mathrm{t}_{\mathrm{R}}=(\mathrm{C}=\quad 0.45 \mathrm{in}$ 14.6 min (minor)/ $\left.14.9 \quad \mathrm{CHCl}_{3}\right)$ (major) 
<smiles>Cc1ccc(C(Cc2ccccc2)N2CCOC2=O)cc1</smiles>

18<smiles>COc1ccc(C(Cc2ccccc2)N2CCOC2=O)cc1</smiles>

19<smiles>O=C1OCCN1[C@@H](Cc1ccccc1)c1ccc(C(F)(F)F)cc1</smiles>

20<smiles>O=C1OCCN1C(Cc1ccccc1)c1ccco1</smiles>

21<smiles>O=C1OCCN1C(Cc1ccccc1)c1ccsc1</smiles>

22<smiles>O=C1OCCN1[C@@H](Cc1ccc(Cl)cc1)c1ccccc1</smiles>

23<smiles>Cc1ccc(CC(c2ccccc2)N2CCOC2=O)cc1</smiles>

24<smiles>COc1ccc(CC(c2ccccc2)N2CCOC2=O)cc1</smiles>

25<smiles>O=C1OCCN1[C@@H](Cc1ccc(C(F)(F)F)cc1)c1ccccc1</smiles>

SFC, OJH column, $[\alpha]_{\mathrm{D}}^{25.0}=\quad+8292$

$15 \% \mathrm{MeOH}, 2 \mathrm{ml} / \mathrm{min}, \quad(\mathrm{C}=0.10 \quad$ in

$\mathrm{t}_{\mathrm{R}}=5.3 \mathrm{~min}$ (major)/ $\mathrm{CHCl}_{3}$ )

9.3(minor)

SFC, OZH column, $[\alpha]_{\mathrm{D}}^{25.0}=+90(\mathrm{C}=94$

$10 \% \mathrm{MeOH}, 2 \mathrm{ml} / \mathrm{min}, \quad 0.10$ in $\mathrm{CHCl}_{3}$ )

$\mathrm{t}_{\mathrm{R}}=7.0 \mathrm{~min}$

(minor)/7.8 (major)

SFC, OZH column, $[\alpha]_{\mathrm{D}}^{25.0}=+68(\mathrm{C}=95$

$15 \% \mathrm{MeOH}, 2 \mathrm{ml} / \mathrm{min}, \quad 0.10$ in $\mathrm{CHCl}_{3}$ )

$\mathrm{t}_{\mathrm{R}}=4.5 \mathrm{~min}$

(minor)/5.0 (major)

SFC, ODH column, $[\alpha]_{\mathrm{D}}^{25.0}=+83(\mathrm{C}=99$ $15 \% \mathrm{MeOH}, 2 \mathrm{ml} / \mathrm{min}, \quad 0.10$ in $\mathrm{CHCl}_{3}$ )

$\mathrm{t}_{\mathrm{R}}=4.4 \mathrm{~min}$

(major)/4.6 (minor)

SFC, IF column, $15 \% \quad[\alpha]_{\mathrm{D}}^{25.0}=\quad+78 \quad 88$

$\mathrm{MeOH}, 2 \mathrm{ml} / \mathrm{min}, \mathrm{t}_{\mathrm{R}}=(\mathrm{C}=0.10 \quad$ in 16.5 min (major)/ $18.0 \quad \mathrm{CHCl}_{3}$ ) (minor)

SFC, ASH column, $[\alpha]_{\mathrm{D}}^{25.0}=\quad+79 \quad 92$

$15 \% \mathrm{MeOH}, 2 \mathrm{ml} / \mathrm{min}, \quad(\mathrm{C}=0.10 \quad$ in $\left.\mathrm{t}_{\mathrm{R}}=6.9 \min \mathrm{CHCl}_{3}\right)$

(minor)/9.3 (major)

SFC, ASH column, $[\alpha]_{\mathrm{D}}^{25.0}=+3792$ $15 \% \mathrm{MeOH}, 2 \mathrm{ml} / \mathrm{min}, \quad(\mathrm{C}=0.10 \quad$ in $\left.\mathrm{t}_{\mathrm{R}}=6.1 \min \mathrm{CHCl}_{3}\right)$

(minor)/7.2 (major)

SFC, ASH column, $[\alpha]_{\mathrm{D}}^{25.0}=+6795$

$15 \% \mathrm{MeOH}, 2 \mathrm{ml} / \mathrm{min}, \quad(\mathrm{C}=0.10 \quad$ in

$\left.\mathrm{t}_{\mathrm{R}}=6.4 \min \quad \mathrm{CHCl}_{3}\right)$

(minor)/7.5 (major)

SFC, IF column, $15 \% \quad[\alpha]_{\mathrm{D}}^{25.0}=\quad+73 \quad 96$

$\mathrm{MeOH}, 2 \mathrm{ml} / \mathrm{min}, \mathrm{t}_{\mathrm{R}}=(\mathrm{C}=0.10 \quad$ in

$6.0 \min$ (minor) $/ 6.6 \quad \mathrm{CHCl}_{3}$ )

(major) 
26<smiles>CC[C](N=C(C)C)c1ccccc1</smiles>

27<smiles>CC(CN1CCOC1=O)c1ccccc1</smiles>

28<smiles>[M]C(CN1CCOC1=O)c1ccccc1</smiles>

29<smiles>CCC(CN1CCOC1=O)c1ccccc1</smiles>

30<smiles>CCC(CN1CCOC1=O)c1ccccc1</smiles>

31<smiles>O=C1OCCN1CC(P)c1ccccc1</smiles>

32<smiles>O=C1OCCN1CC([PH2+])c1ccccc1</smiles>

SFC, ADH column, $[\alpha]_{\mathrm{D}}^{25.0}=-110.598$

$15 \% \mathrm{MeOH}, 2 \mathrm{ml} / \mathrm{min}, \quad(\mathrm{C}=0.20 \quad$ in $\left.\mathrm{t}_{\mathrm{R}}=5.7 \quad \min \mathrm{CHCl}_{3}\right)$

(minor)/6.6 (major)

SFC, ASH column, $[\alpha]_{\mathrm{D}}^{25.0}=-31(\mathrm{C}=97$

$15 \% \mathrm{MeOH}, 2 \mathrm{ml} / \mathrm{min}, \quad 0.1$ in $\mathrm{CHCl}_{3}$ )

$15 \mathrm{~min}, \mathrm{t}_{\mathrm{R}}=4.7 \mathrm{~min}$

(minor) $/ 5.2 \quad \min$

(major)

SFC, ASH column, $[\alpha]_{\mathrm{D}}^{25.0}=31(\mathrm{C}=97$

$15 \% \mathrm{MeOH}, 2 \mathrm{ml} / \mathrm{min}, \quad 0.1$ in $\mathrm{CHCl}_{3}$ )

$15 \mathrm{~min}, t_{R}=4.7 \mathrm{~min}$

(majorr) $/ 5.2 \quad \min$

(minor)

SFC, IF column, $15 \%[\alpha]_{\mathrm{D}}^{25.0}=-22(\mathrm{C}=96$

$\mathrm{MeOH}, 2 \mathrm{ml} / \mathrm{min}, \mathrm{t}_{\mathrm{R}}=0.10$ in $\mathrm{CHCl}_{3}$ )

$5.1 \mathrm{~min}$ (minor) $/ 5.5$

(major)

SFC, IF column, $15 \% \quad[\alpha]_{\mathrm{D}}^{25.0}=21(\mathrm{C}=93$

$\mathrm{MeOH}, 2 \mathrm{ml} / \mathrm{min}, \mathrm{t}_{\mathrm{R}}=0.10$ in $\mathrm{CHCl}_{3}$ )

$5.1 \min$ (minor) $/ 5.5$

(major)

SFC, IF column, $15 \% \quad[\alpha]_{\mathrm{D}}^{25.0}=-25(\mathrm{C}=96$

$\mathrm{MeOH}, 2 \mathrm{ml} / \mathrm{min}, \mathrm{t}_{\mathrm{R}}=0.10$ in $\mathrm{CHCl}_{3}$ )

$5.4 \min$ (minor) $/ 5.7$

(major)

SFC, IF column, $15 \% \quad[\alpha]_{\mathrm{D}}^{25.0}=22(\mathrm{C}=90$

$\mathrm{MeOH}, 2 \mathrm{ml} / \mathrm{min}, \mathrm{t}_{\mathrm{R}}=0.10$ in $\mathrm{CHCl}_{3}$ )

$5.4 \min$ (minor) $/ 5.7$

(major) 


\section{Absolute configuration determination}

\subsection{The absolute configuration of the different classes of hydrogenated products}<smiles>CC[C](N)c1ccccc1</smiles>

$13 a$<smiles></smiles><smiles>CCC(NCCO)c1ccccc1</smiles>

$15 a$<smiles>CC[C@H](c1ccccc1)N1CCOC1=O</smiles>
$[\alpha]_{\mathrm{D}}^{25}=-109\left(\mathrm{c} 0.10, \mathrm{CHCl}_{3}\right)$<smiles>CC(C)[C@H](N)Cc1cccc(Cc2cccc(Cc3ccccc3)c2)c1</smiles>

$14 b$

$15 b$<smiles>C[C](CNCCOC(C)C)c1ccccc1</smiles><smiles>C[C@@H](CN1CCOC1=O)c1ccccc1</smiles>

$[\alpha]_{D}^{25}=+58\left(\mathrm{c} 0.10, \mathrm{CHCl}_{3}\right)$
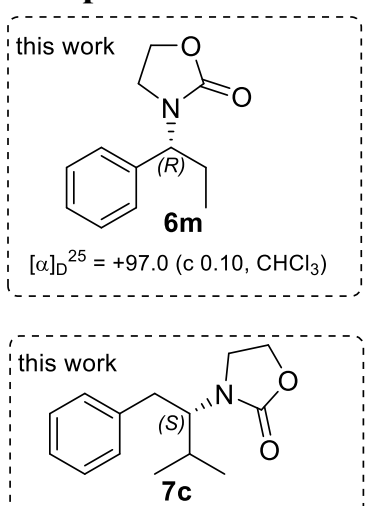

$[\alpha]_{D}^{25}=-45.0\left(\mathrm{c} 0.10, \mathrm{CHCl}_{3}\right)$

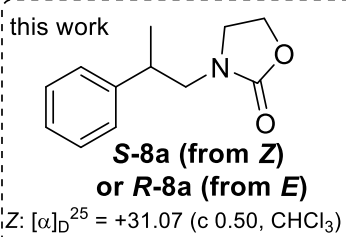

$E:[\alpha]_{D_{-}}^{25}=-31.07\left(\mathrm{c} 0.50, \mathrm{CHCl}_{3}\right) ;$

Reaction condition (1) ethyl 2-chloro-2-oxoacetate, $\mathrm{NEt}_{3}, \mathrm{CH}_{2} \mathrm{Cl}_{2}$, r.t., 2 h. (2) LAH, THF, reflux. o.n. (3) triphosgene, $\mathrm{NEt}_{3}$, rt. o.n.

The absolute configuration of the different classes of hydrogenated product was determined by transformation of known compounds 13a, 14b, 14c into compound 15a-15c and comparison with hydrogenated products $\mathbf{6 m}, \mathbf{7 c}, S-8 \mathrm{a}$ and $Z \mathbf{Z - 8 a}$. First, the animes 12a-12c were treated with ethyl 2-chloro-2-oxoacetate to furnish compounds 13a, 14b, 14c. Then a cyclization using triphosgene and $\mathrm{NEt}_{3}$ afforded compound 15a-15c, of which optical rotation were compared to hydrogenated products $\mathbf{6 m}, \mathbf{7 c}, S-8 \mathrm{a}$ and $Z \mathbf{Z - 8 a}$. The absolute configuration of the other products has been tentatively assigned by comparison of elution order from HPLC and SFC, using a chiral stationary phase and the optical rotation of products $6 \mathbf{m}, 7 \mathbf{c}, S$ 8a and $Z-8$ a.

\subsection{Di-aryl enamides by single crystal $X$-ray diffraction}

Single crystal of $5 \mathbf{a}$ was grown by dissolving in pentane diethyl ether and slow evaporation at room temperature. Detailed information for this crystal is available in CCDC 1955080.

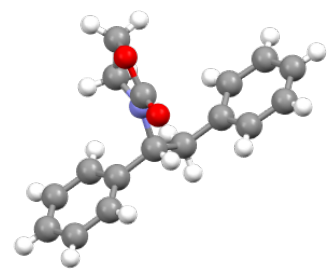

X-ray crystal structure of 5. 


\section{Conditions screening}

Table S1 The catalyst screening ${ }^{\text {a }}$

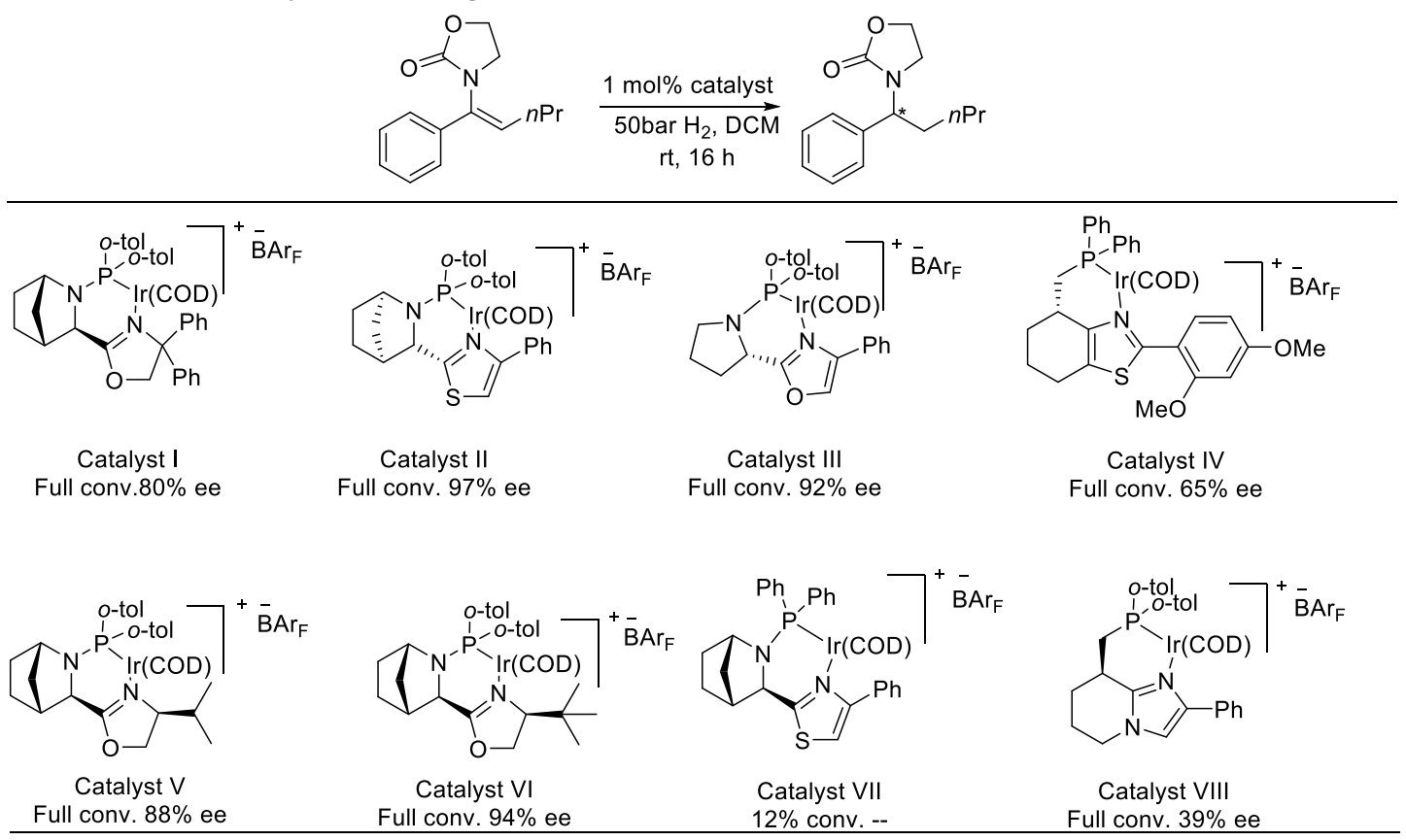

${ }^{a} 0.05 \mathrm{mmol}$ of substrates in $0.5 \mathrm{~mL}$ of DCM. Conversion was determined by ${ }^{1} \mathrm{H}$ NMR spectroscopy. Enantiomeric excess was determined by SFC analysis, using chiral stationary phases.

Table S2 The solvent screening ${ }^{\mathrm{a}}$

\begin{tabular}{cccc} 
Entry & Solvent & Conv.(\%) & ee (\%) \\
\hline 1 & DCM & Full & 97 \\
2 & Toluene & Full & 97 \\
3 & PhCF3 & Full & 97 \\
4 & Benzent, rt, 16 h & Full & 97 \\
5 & DCE & Full & 97
\end{tabular}

${ }^{\text {a }} 0.05 \mathrm{mmol}$ of substrates in $0.5 \mathrm{~mL}$ of solvent. Conversion was determined by ${ }^{1} \mathrm{H}$ NMR spectroscopy. Enantiomeric excess was determined by SFC analysis, using chiral stationary phases. 
Table S3 The condition screening of class 1
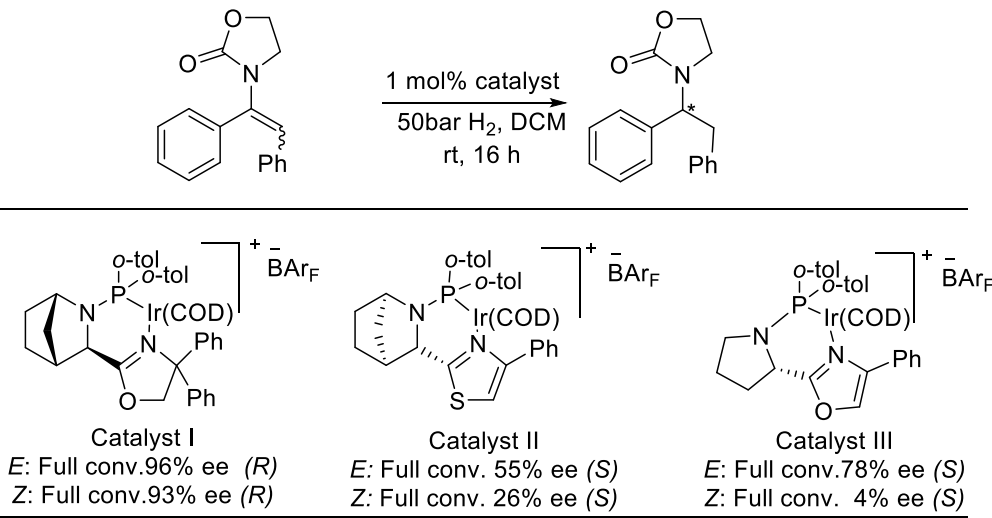

${ }^{a} 0.05 \mathrm{mmol}$ of substrates in $0.5 \mathrm{~mL}$ of DCM. Conversion was determined by ${ }^{1} \mathrm{H}$ NMR spectroscopy. Enantiomeric excess was determined by SFC analysis, using chiral stationary phase

Table S4 The additional condition screening of class $2^{\text {a }}$
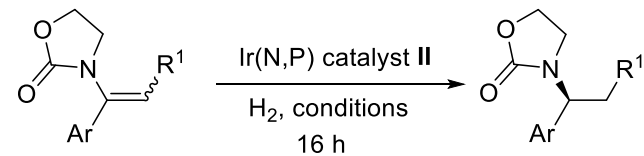

\begin{tabular}{|c|c|c|c|c|c|c|}
\hline Entry & Subs. & Solvent & Press.(bar) & Temp. $\left({ }^{\circ} \mathrm{C}\right)$ & Conv.(\%) & $\mathrm{ee}(\%)$ \\
\hline \multirow{2}{*}{1} & $(E)-2 c$ & $\mathrm{DCM}$ & 50 & r.t & 99 & $43(S)$ \\
\hline & $(Z)-2 c$ & DCM & 50 & r.t & 99 & 98(R) \\
\hline \multirow{2}{*}{2} & $(E)-2 \mathrm{c}$ & Toluene & 50 & 60 & 99 & $10(R)$ \\
\hline & $(Z)-2 c$ & Toluene & 50 & 60 & 99 & $97(\mathrm{R})$ \\
\hline \multirow{2}{*}{3} & $(E)-2 \mathrm{c}$ & Toluene & 3 & 60 & 99 & $47(\mathrm{R})$ \\
\hline & $(Z)-2 c$ & Toluene & 3 & 60 & 99 & $97(\mathrm{R})$ \\
\hline \multirow{2}{*}{4} & $(E)-2 c$ & Toluene & 1 & 60 & 76 & $60(\mathrm{R})$ \\
\hline & $(Z)-2 c$ & Toluene & 1 & 60 & 99 & $97(\mathrm{R})$ \\
\hline \multirow{2}{*}{5} & $(E)-2 c$ & DCE & 1 & 60 & 99 & 63(R) \\
\hline & $(Z)-2 c$ & DCE & 1 & 60 & 99 & $97(\mathrm{R})$ \\
\hline \multirow{2}{*}{6} & $(E)-2 \mathrm{a}$ & DCE & 1 & 60 & 99 & $80(\mathrm{R})$ \\
\hline & $(Z)-2 a$ & DCE & 1 & 60 & 99 & $97(\mathrm{R})$ \\
\hline
\end{tabular}

a $0.05 \mathrm{mmol}$ of substrates in $0.5 \mathrm{~mL}$ of solvent. Conversion was determined by ${ }^{1} \mathrm{H}$ NMR spectroscopy. Enantiomeric excess was determined by SFC analysis, using chiral stationary phase 
Table S5 The condition screening of class $3^{\mathrm{a}}$
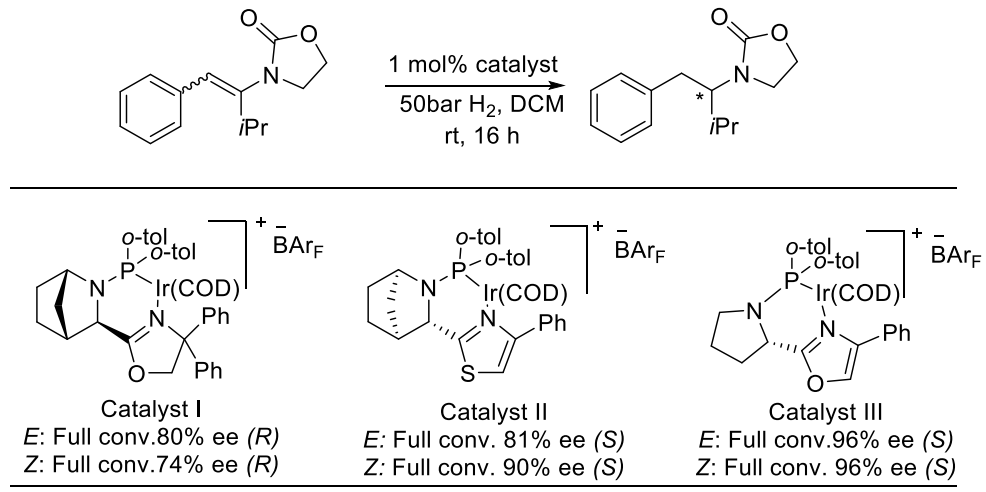

${ }^{a} 0.05 \mathrm{mmol}$ of substrates in $0.5 \mathrm{~mL}$ of DCM. Conversion was determined by ${ }^{1} \mathrm{H}$ NMR spectroscopy. Enantiomeric excess was determined by SFC analysis, using chiral stationary phase 


\section{Mechanistic study}

Table S6 Kinetic study on hydrogenation of separated geometric isomer

\begin{tabular}{|c|c|c|c|c|c|}
\hline \multirow{2}{*}{ Entry } & \multirow{2}{*}{ Time $(\min )$} & \multicolumn{2}{|c|}{ E } & \multicolumn{2}{|l|}{$z$} \\
\hline & & Z/E (ratio) & conv.to $\mathbf{5 a}$ & Z/E (ratio) & conv.to $5 \mathrm{a}$ \\
\hline 1 & 5 & 1:1.1 & $22 \%$ & $5.8: 1$ & $10 \%$ \\
\hline 2 & 15 & 1:1.1 & $31 \%$ & $5.9: 1$ & $27 \%$ \\
\hline 3 & 30 & $5: 1$ & $51 \%$ & $6: 1$ & $31 \%$ \\
\hline 4 & 60 & $6: 1$ & $69 \%$ & $6: 1$ & $40 \%$ \\
\hline
\end{tabular}

Table S7 Acid catalyzed isomerization

\begin{tabular}{|c|c|c|c|}
\hline Entry & $\mathrm{H}^{+}$source & Z/E (ratio) & Time (days) \\
\hline 1 & $\begin{array}{c}\mathrm{AcOH} \\
(100 \mathrm{~mol} \%)\end{array}$ & $2: 1$ & 14 \\
\hline 2 & $\begin{array}{c}\text { TCA } \\
\text { (100 mol\%) }\end{array}$ & $6: 1$ & 7 \\
\hline 3 & $\begin{array}{c}\text { TCA } \\
(5 \mathrm{~mol} \%)\end{array}$ & $5: 1$ & 14 \\
\hline 4 & $\begin{array}{c}\text { Ir-H } \\
(5 \mathrm{~mol} \%)\end{array}$ & $6: 1$ & 8 \\
\hline
\end{tabular}

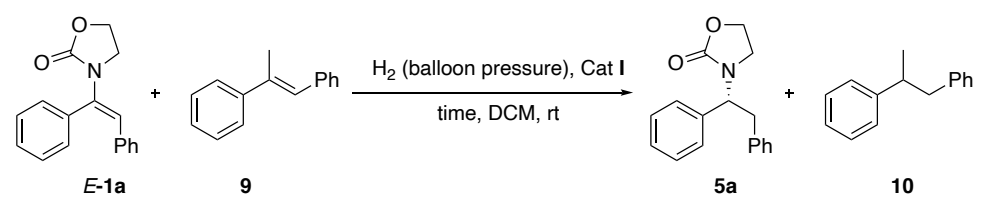

Competition (Enamide Class 1 vs Stilbene)

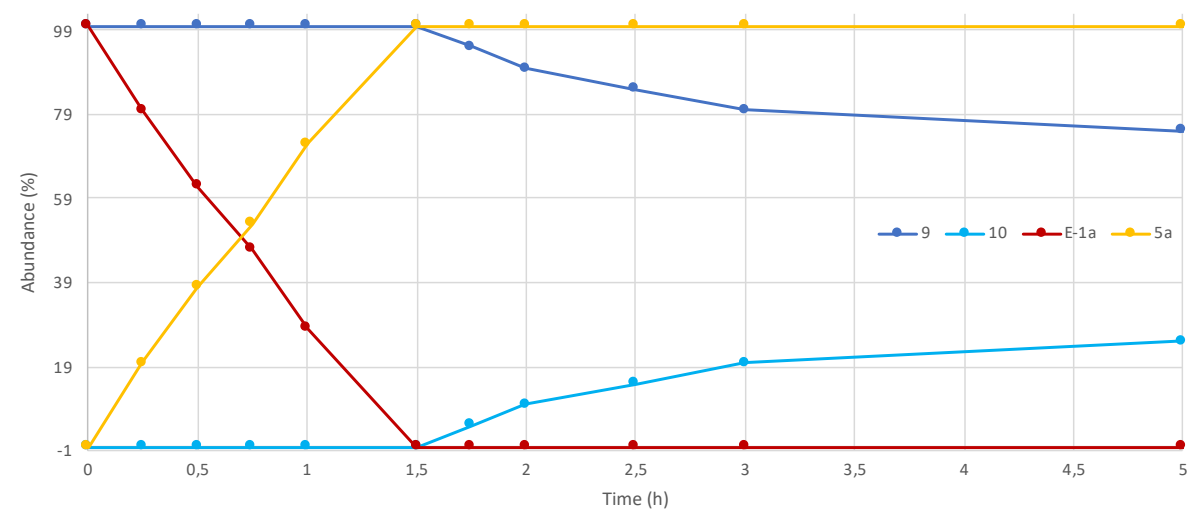

Figure S1 Competition experiments for class 1 enamide and trans methyl stilbene. 


\section{Labeling study}

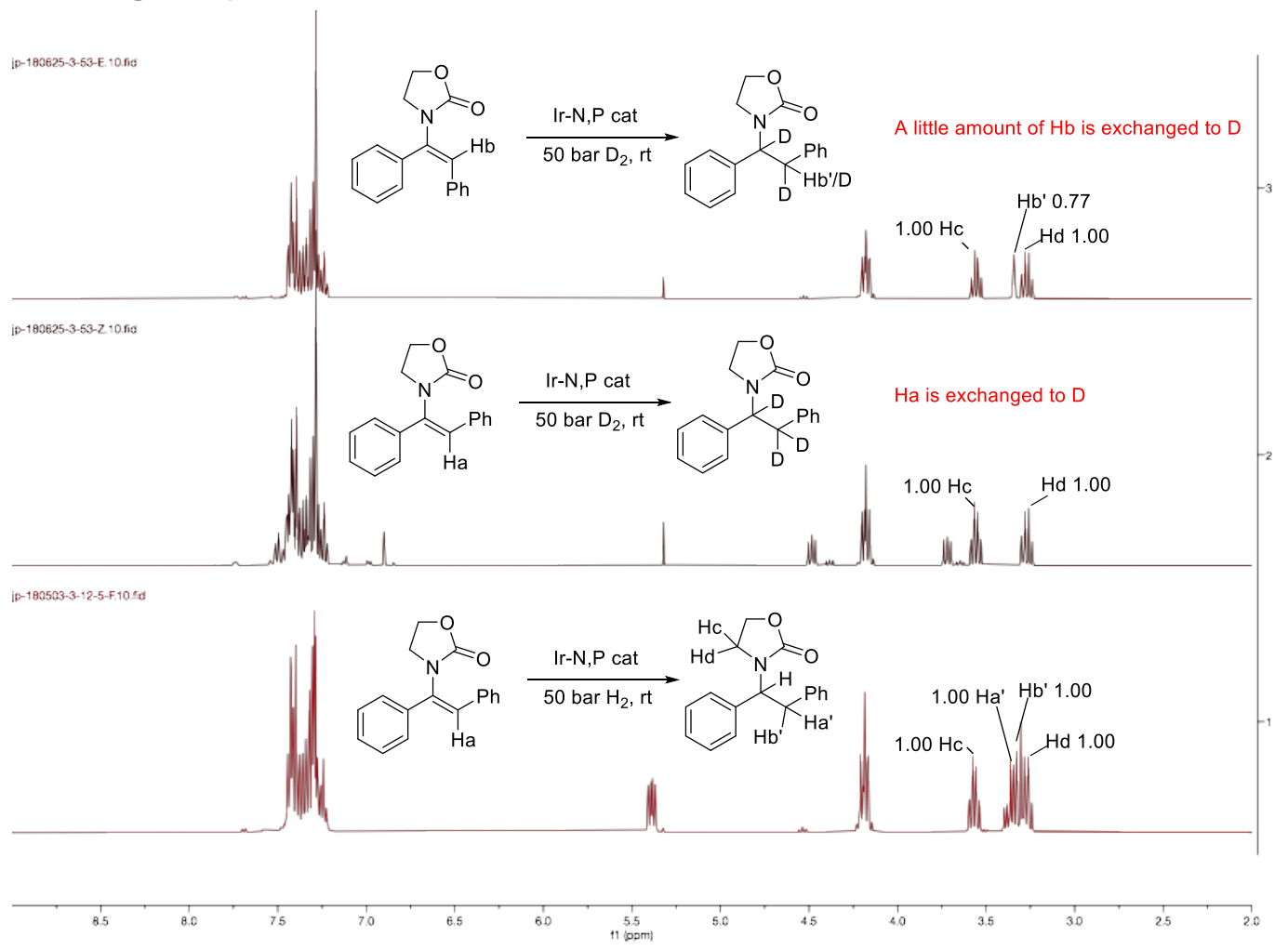

Figure S2 Deuterium study on 1a

YJ-5beta-nBu-Z-D2.21.fid

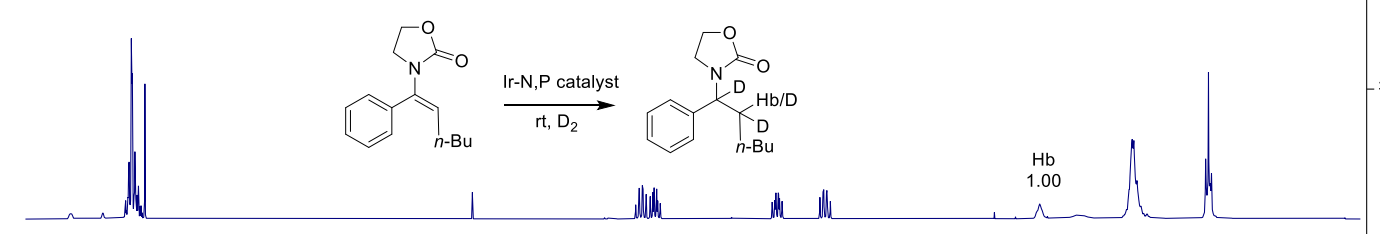

YJ-5beta-nBu-E-D2.20.fid

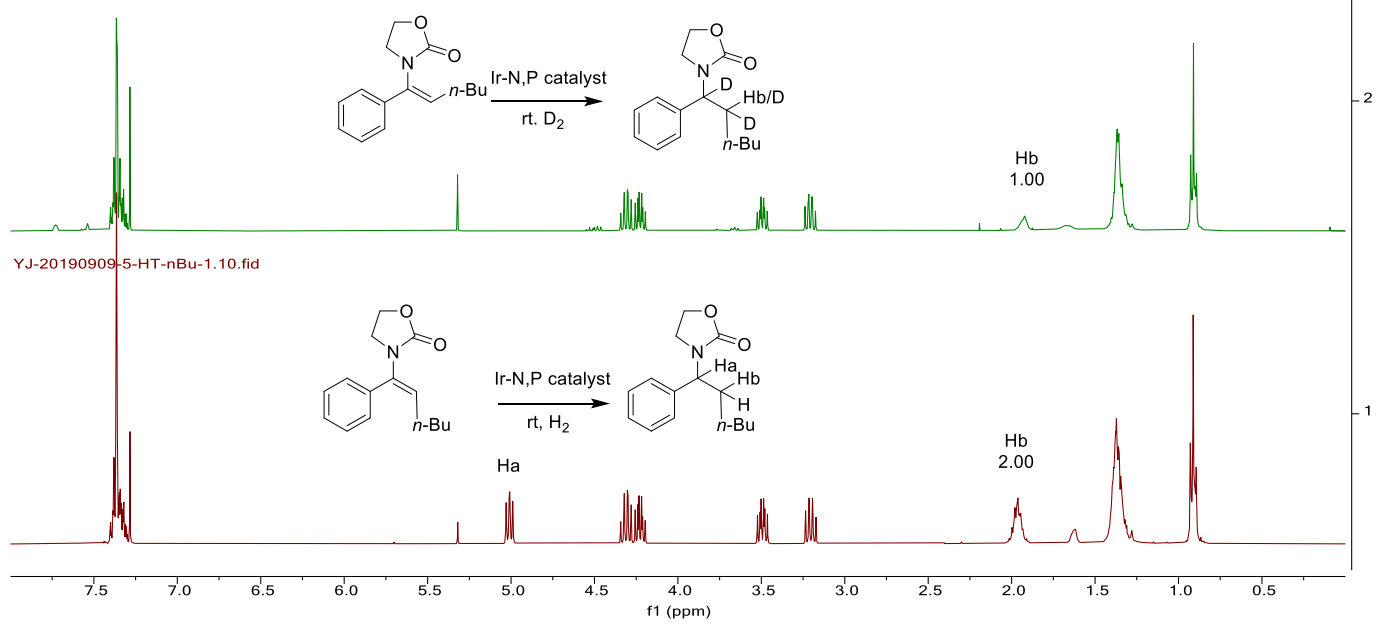

Figure S3 Deuterium study on 2a (rt, 50bar) 

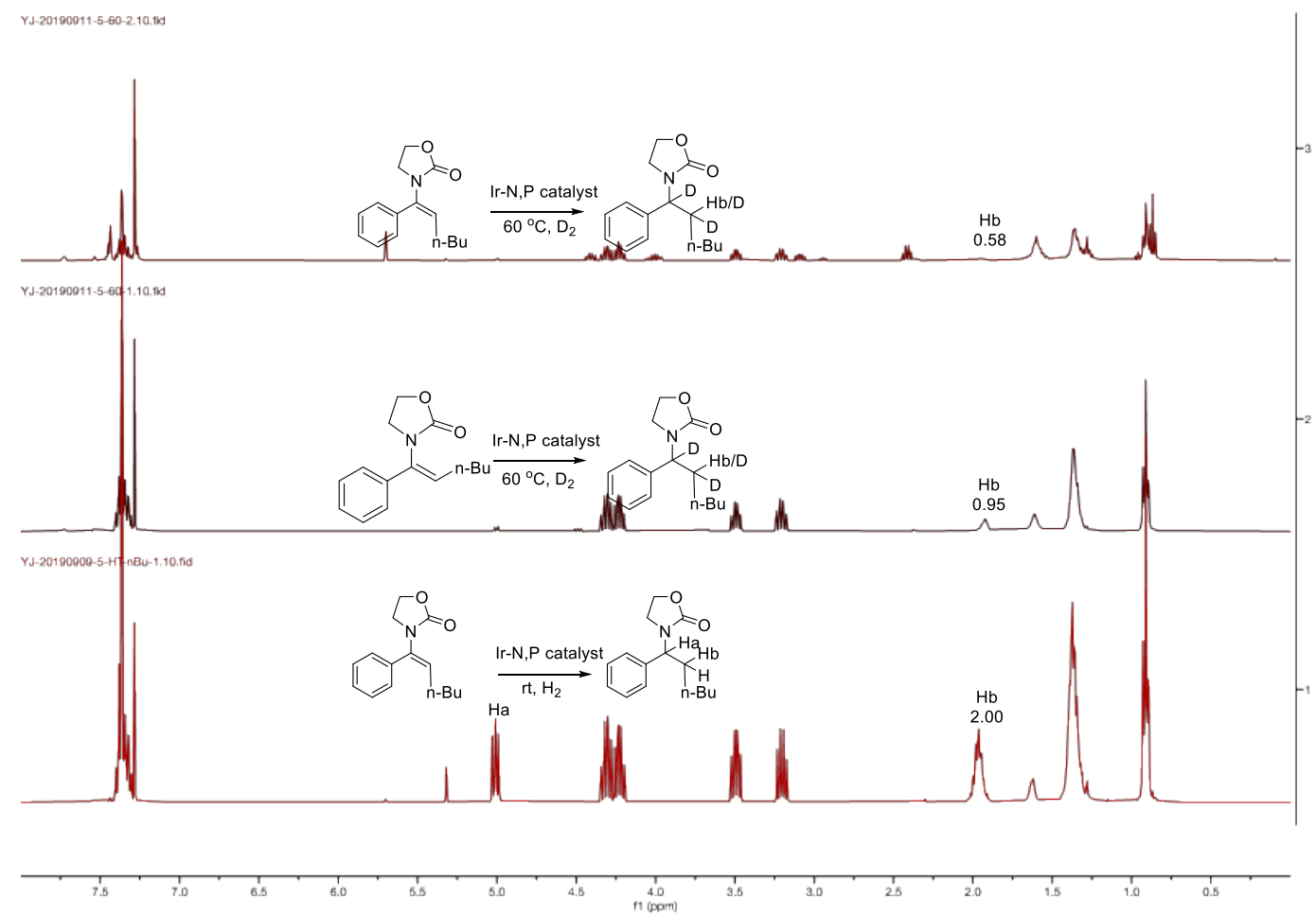

Figure S4 Deuterium study on $\mathbf{2 a}\left(60{ }^{\circ} \mathrm{C}, 1\right.$ bar $)$
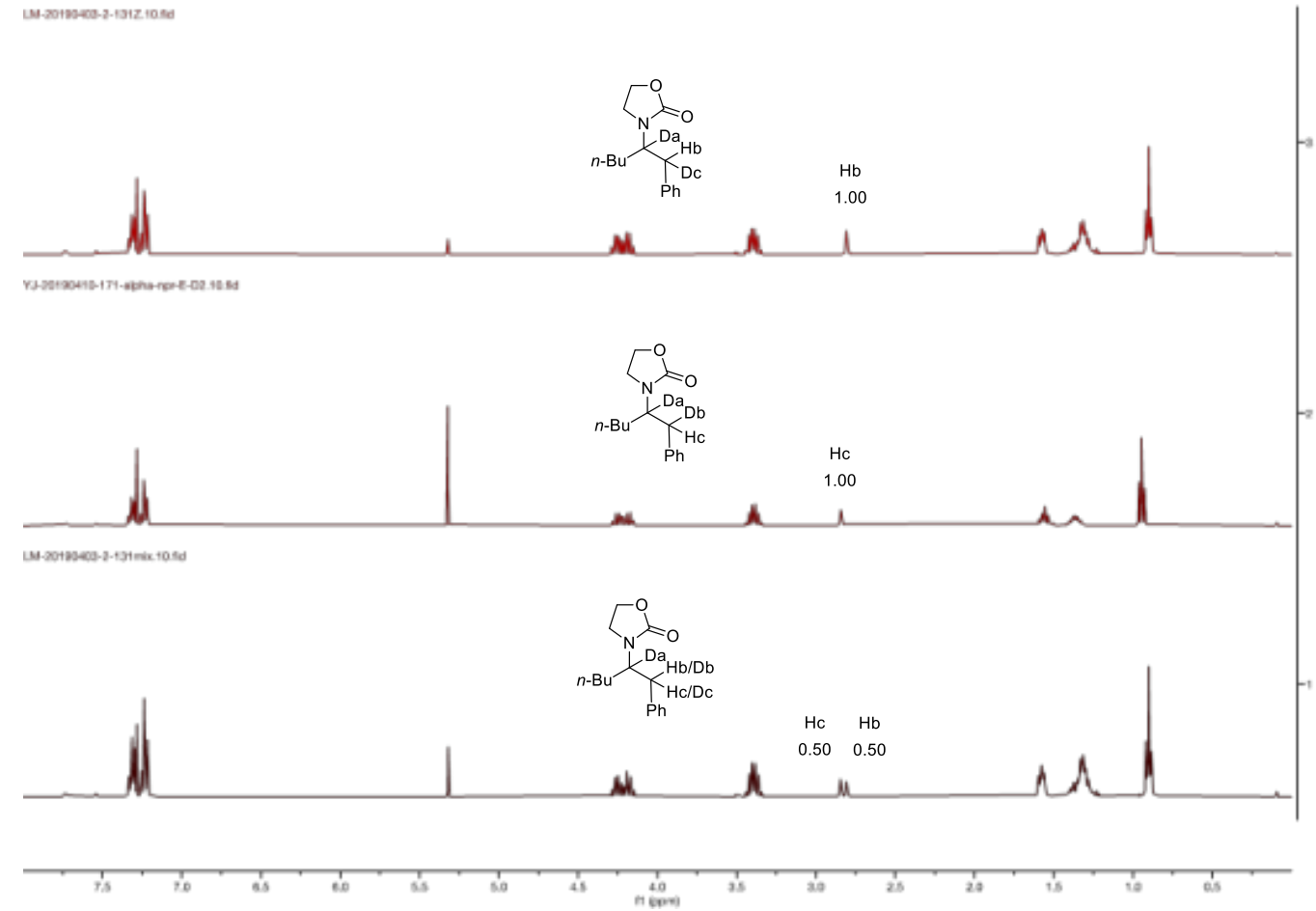

Figure S5 Deuterium study on 3a (rt) 


\section{DTF computational details}

Single point free energy with solvation (dichloromethane) were carried out with Jaguar ${ }^{13}$ (version 10.1) using the B3LYP-D $3^{14,15}$ functional in combination with the psLACVP** basis set. ${ }^{16}$ The structures were first optimized in the gas phase. The transition states were characterized by one negative vibrational frequency and $\mathrm{QRC}^{17}$ (quick reaction coordinate) calculations. The solvent energies for the optimized structures were then calculated using the Poisson-Boltzmann solver with dichloromethane as the solvent. The energy that is depicted with the XYZ coordinates (listed below) was obtained from the optimized gas phase calculation. All computations were carried out using the computational cluster resources at the National Supercomputer Centre based at Linkoping University, Sweden and at the Center for High Performance Computing, South Africa.

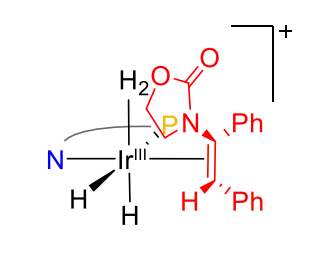

(RE=26.20) ER_H $H_{2}$ SM_Trans-N

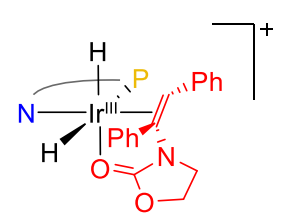

(RE=0.52) ZR_chelbot_SM_Trans-N

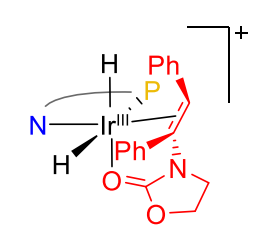

(RE=0)

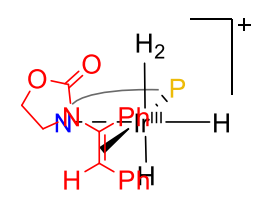

(RE=12.26)

ER_H ${ }_{2}$ SM_Trans-P

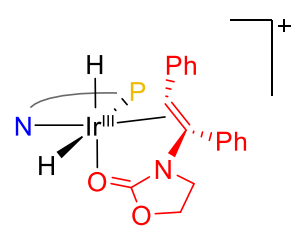

(RE=0.63)

ZS_chelbot_SM_Trans-N

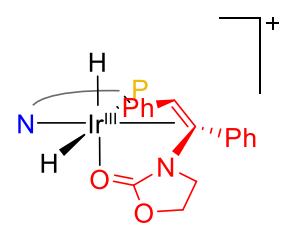

(RE=3.64)

ER_chelbot_SM_Trans-N ES_chelbot_SM_Trans-N ES_cheltop_SM_Trans-N ER_cheltop_SM_Trans-N

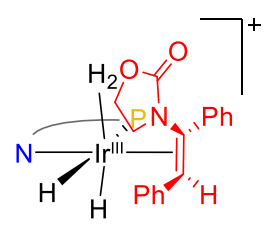

(RE=16.29)

ZR_H ${ }_{2}$ SM_Trans-N

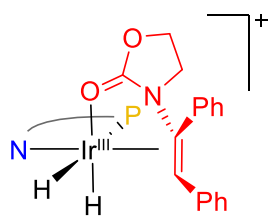

(RE=2.57)

ZS_cheltop_SM_Trans-N

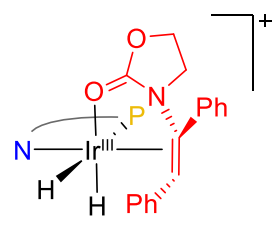

(RE=4.91)

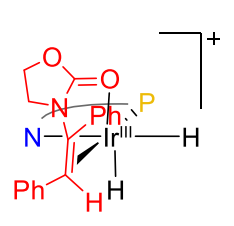

$(\mathrm{RE}=2.50)$

ER_cheltop_SM_Trans-P

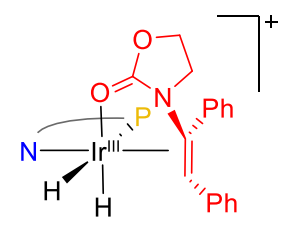

(RE=5.90)

ZR_cheltop_SM_Trans-N

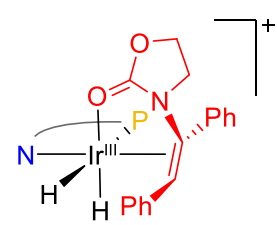

(RE=8.55)

Figure S6 The substrate-catalyst complexes explored in this calculation. The P, N ligand is abbreviated as $\mathrm{P}$ and $\mathrm{N}$; the relative energies are shown in parentheses. 


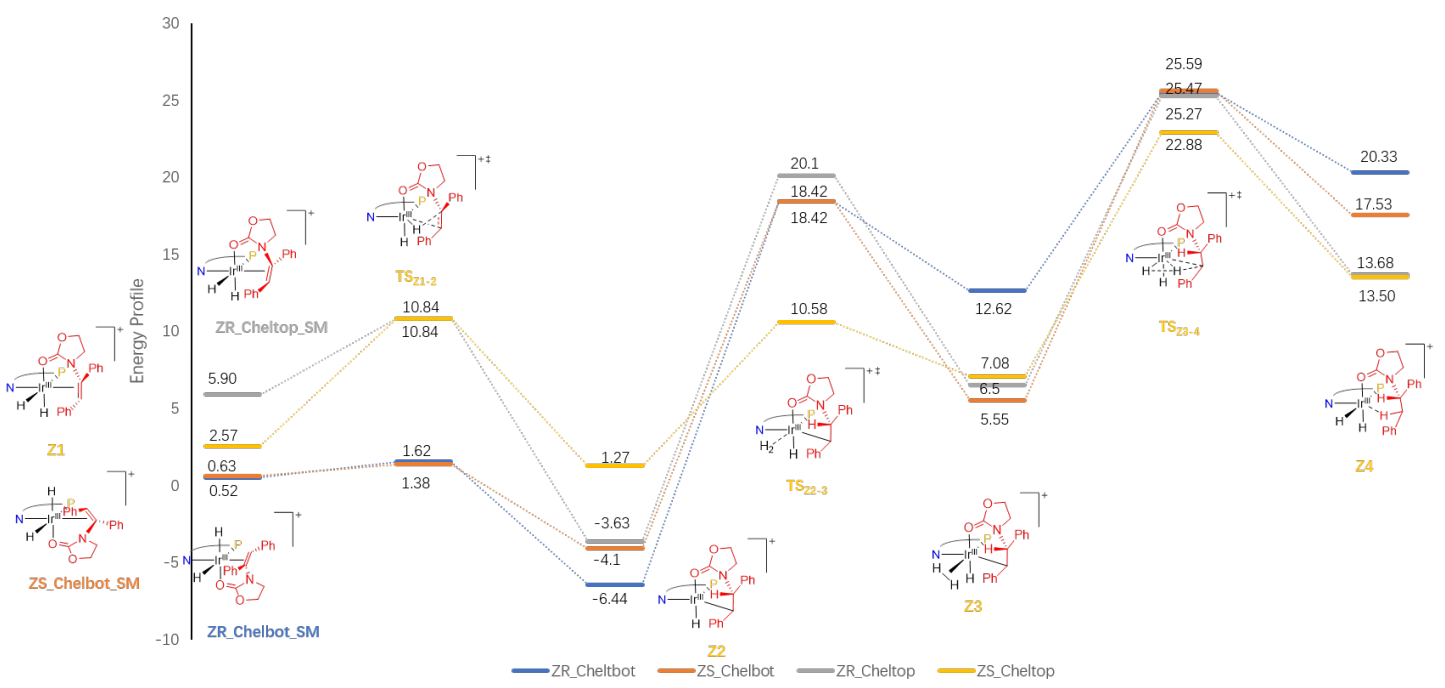

Figure S7 Free energy profile of hydrogenation for the $Z$ isomer. ZS_cheltop is the most favored pathway.

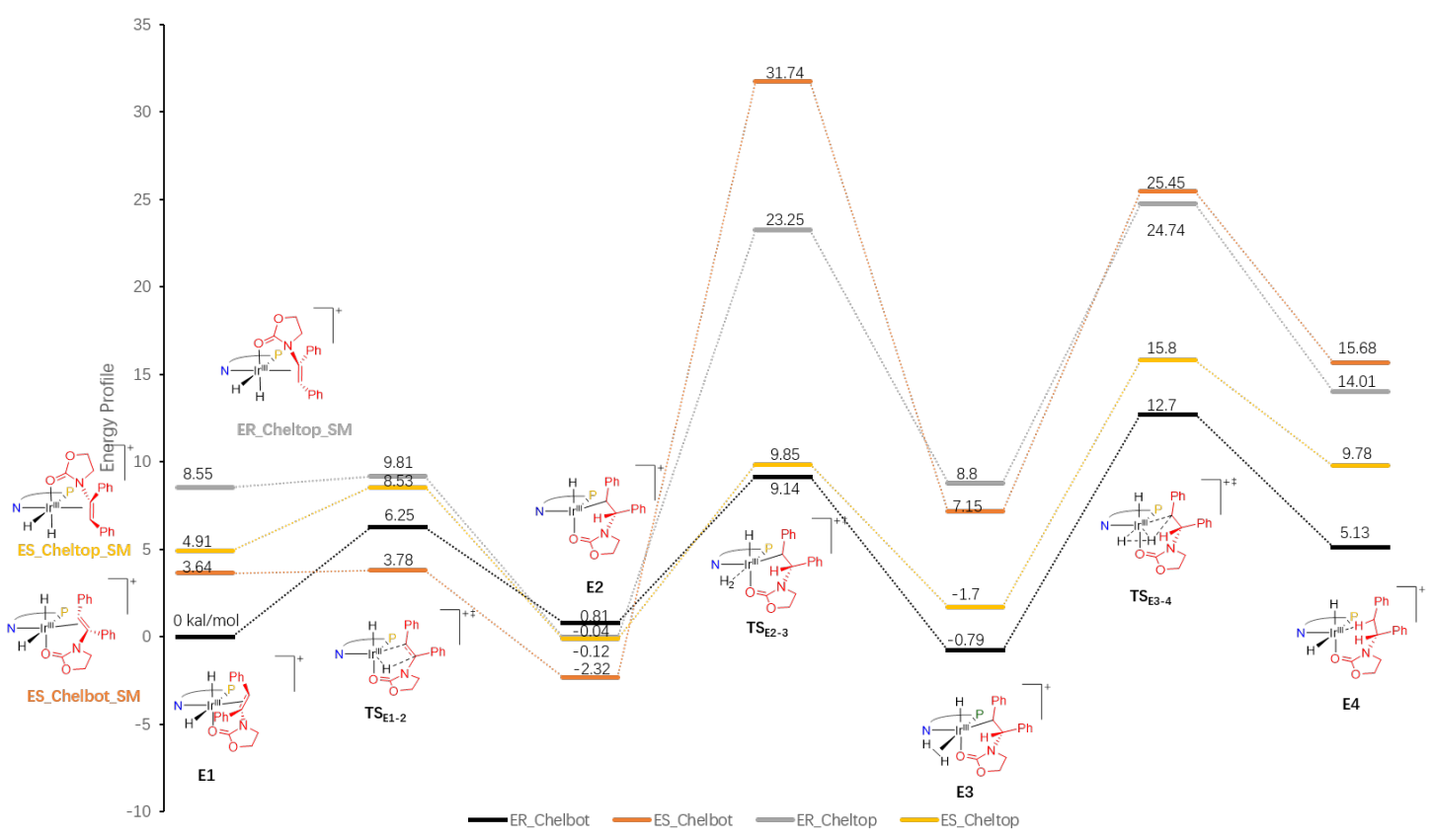

Figure S8 Free energy profile of hydrogenation for the $E$ isomer. ER_Chelbot is the most favored pathway. 


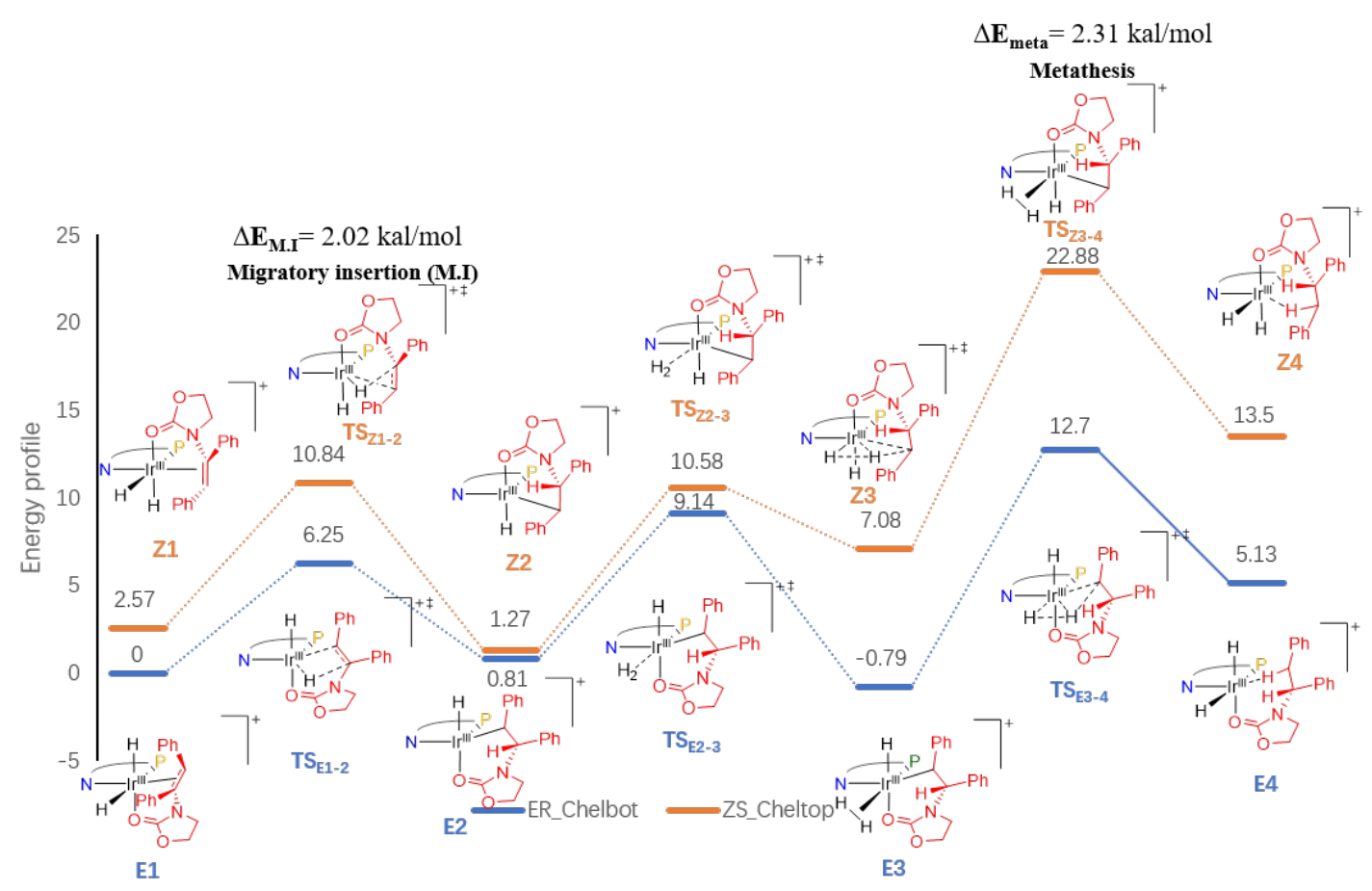

Figure S9 Energy comparison of the favored pathway for the hydrogenation of $Z$-isomer and $E$-isomer.

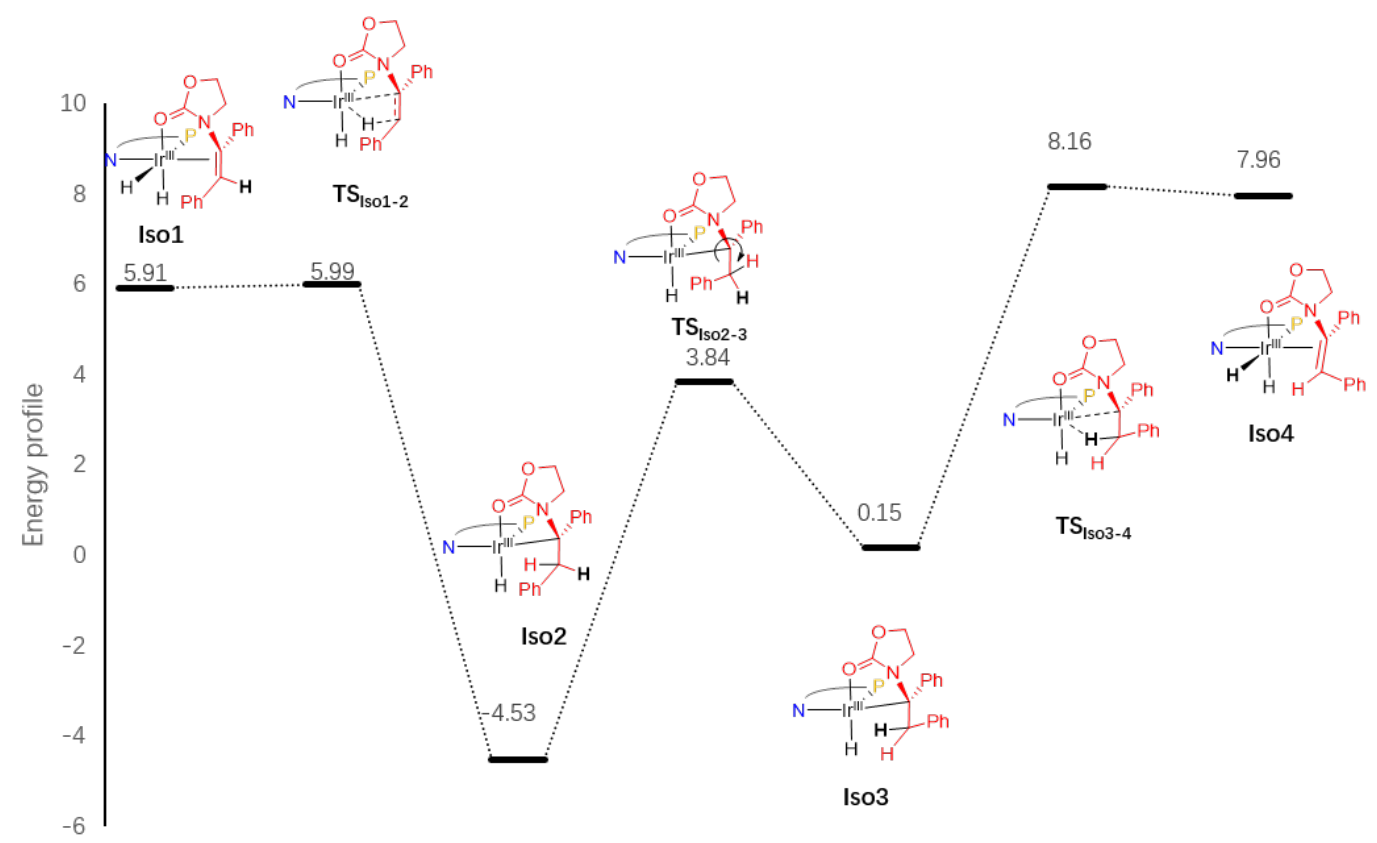

Figure S10 Free energy profile of $Z-E$ isomerization. 


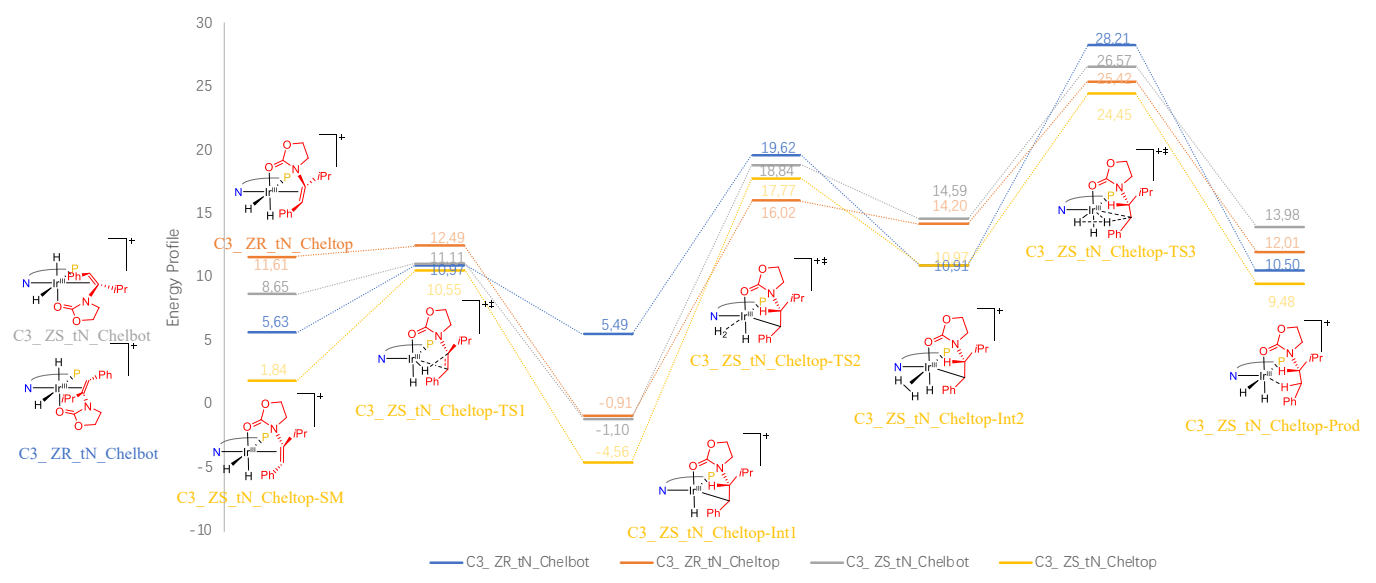

Figure S11 Free energy profile of hydrogenation for the $Z$ isomer (class 3). ZS_Cheltop is the most favored pathway.

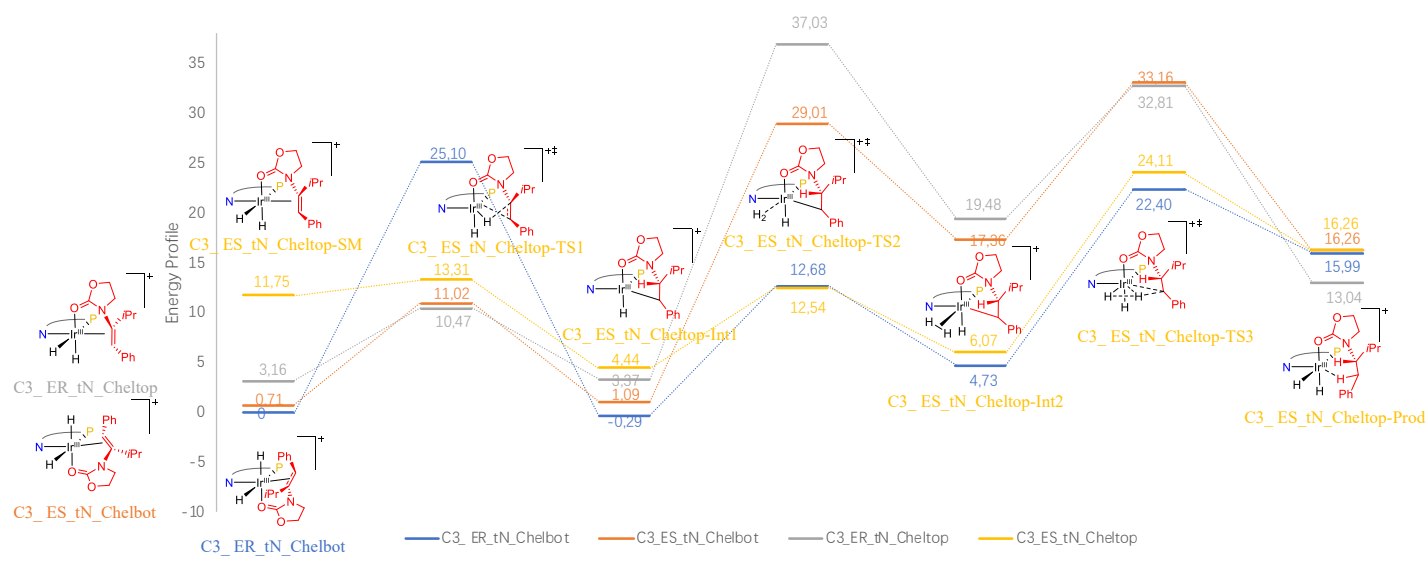

Figure S12 Free energy profile of hydrogenation for the $E$ isomer (class 3). ES_Cheltop is the most favored pathway. 
10. NMR spectra of new compounds
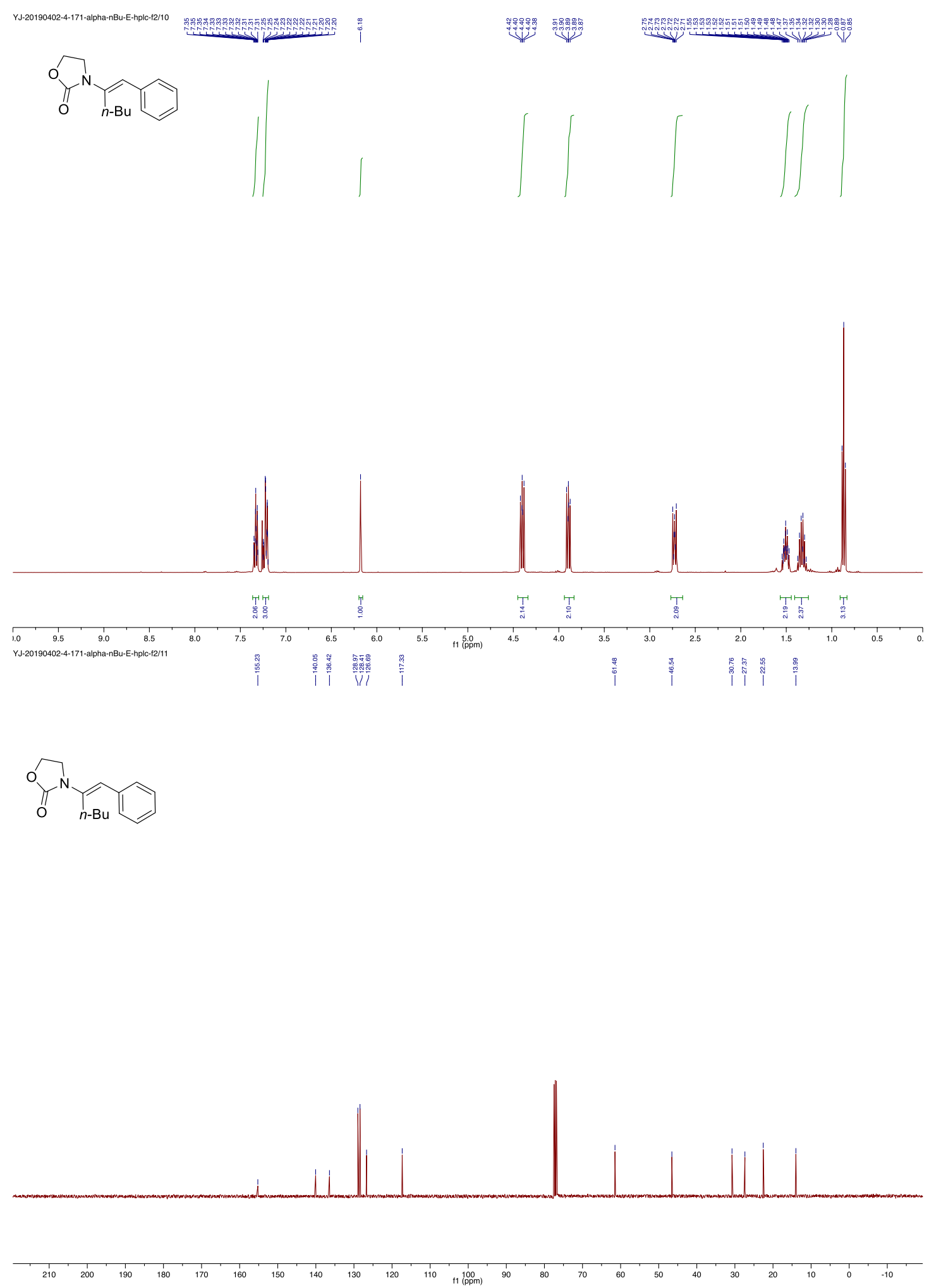
jp-190309-4-146-nbu-Z-ł2/10
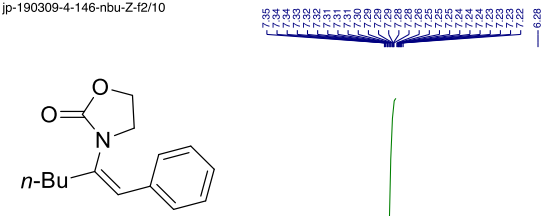

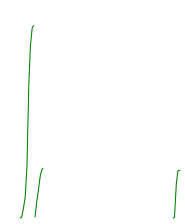

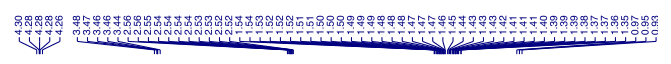

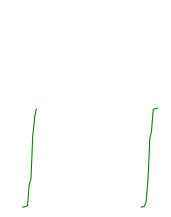

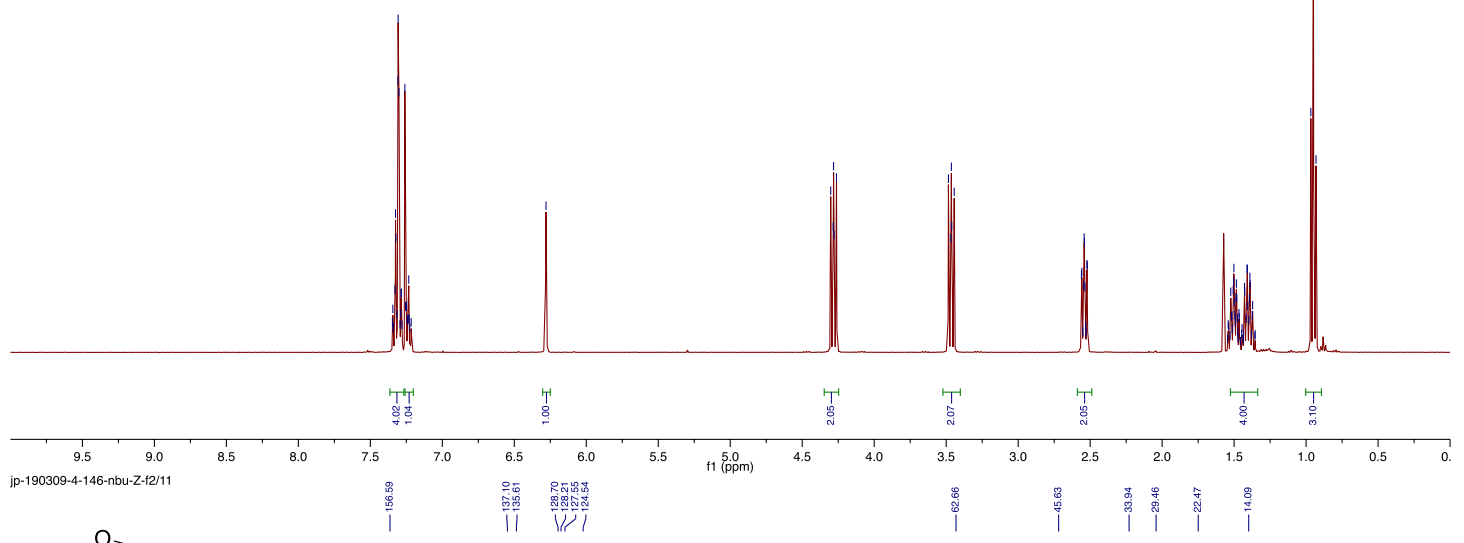
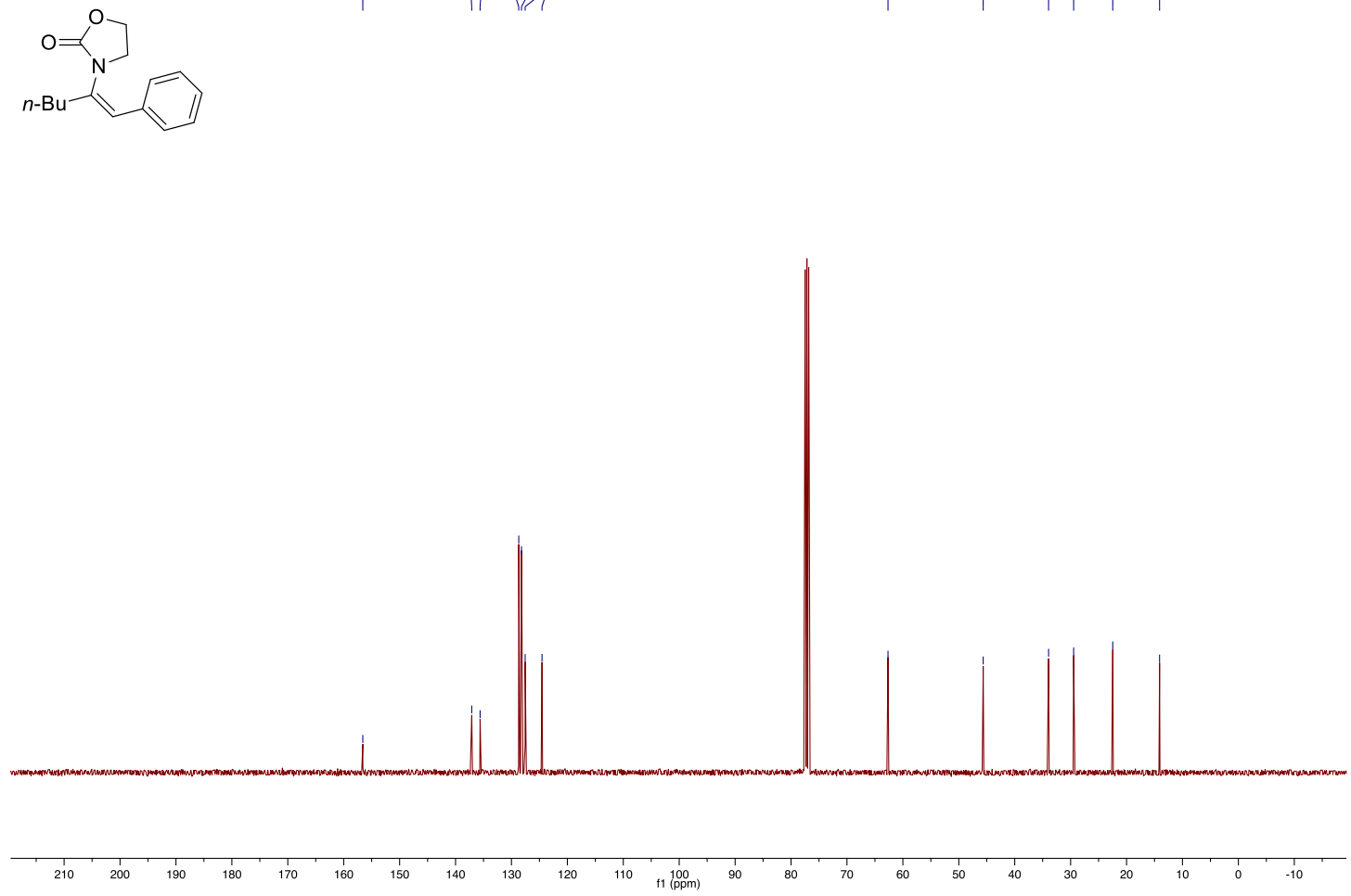

S31 

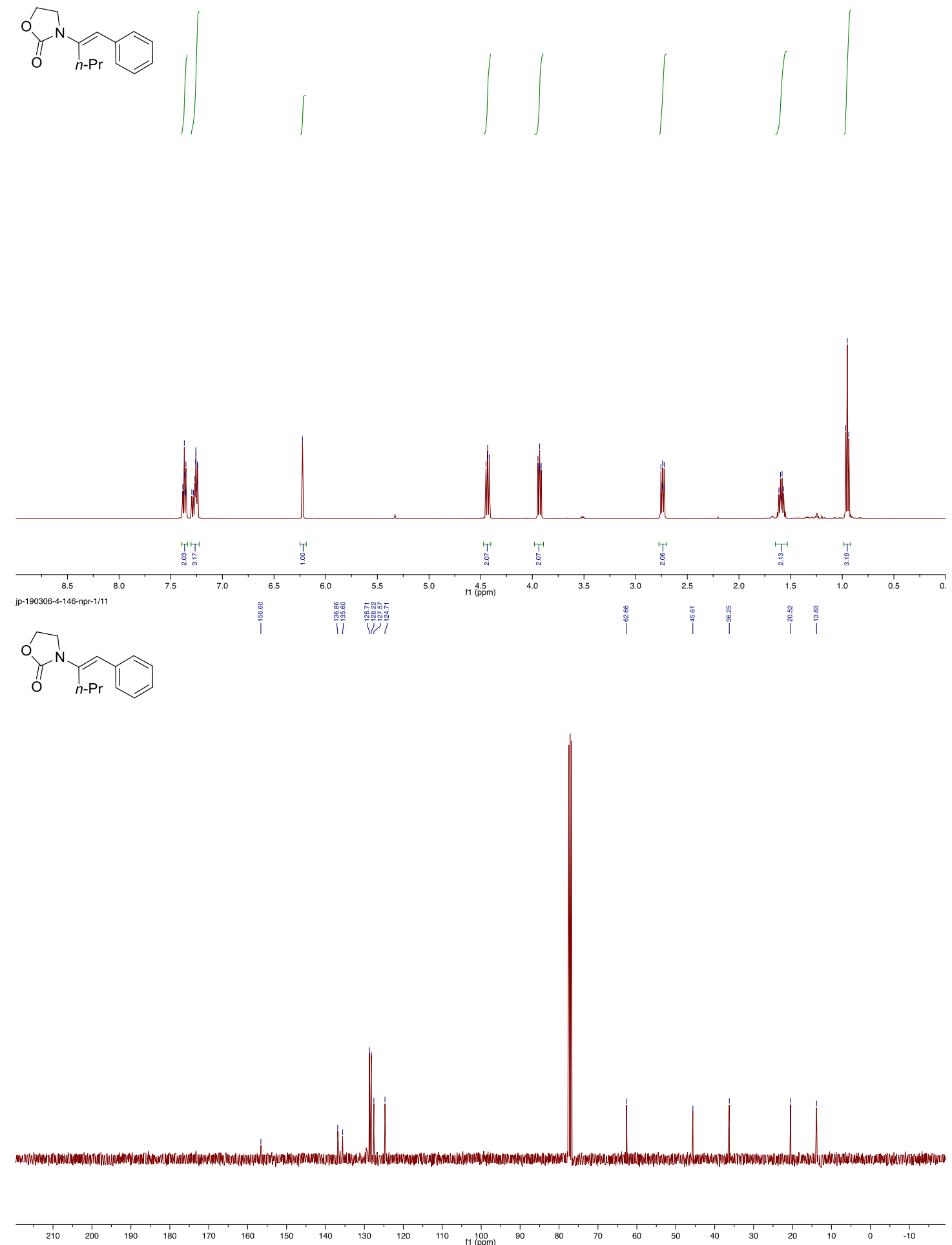

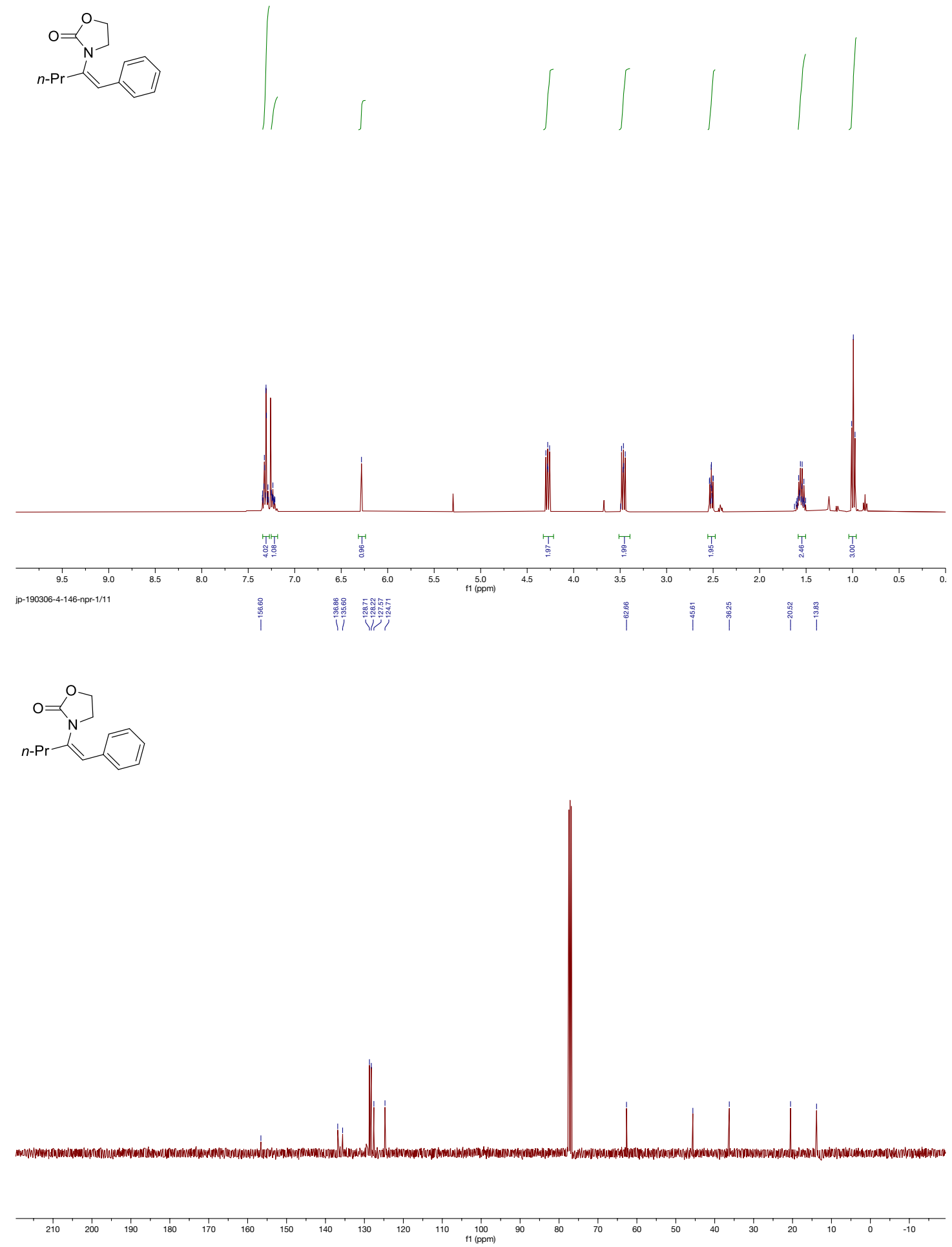
YJ-20180430-3-7-ipr-f1/10
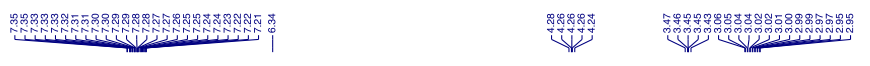

$\sqrt{2}$

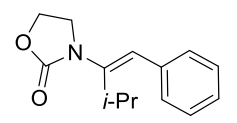

1
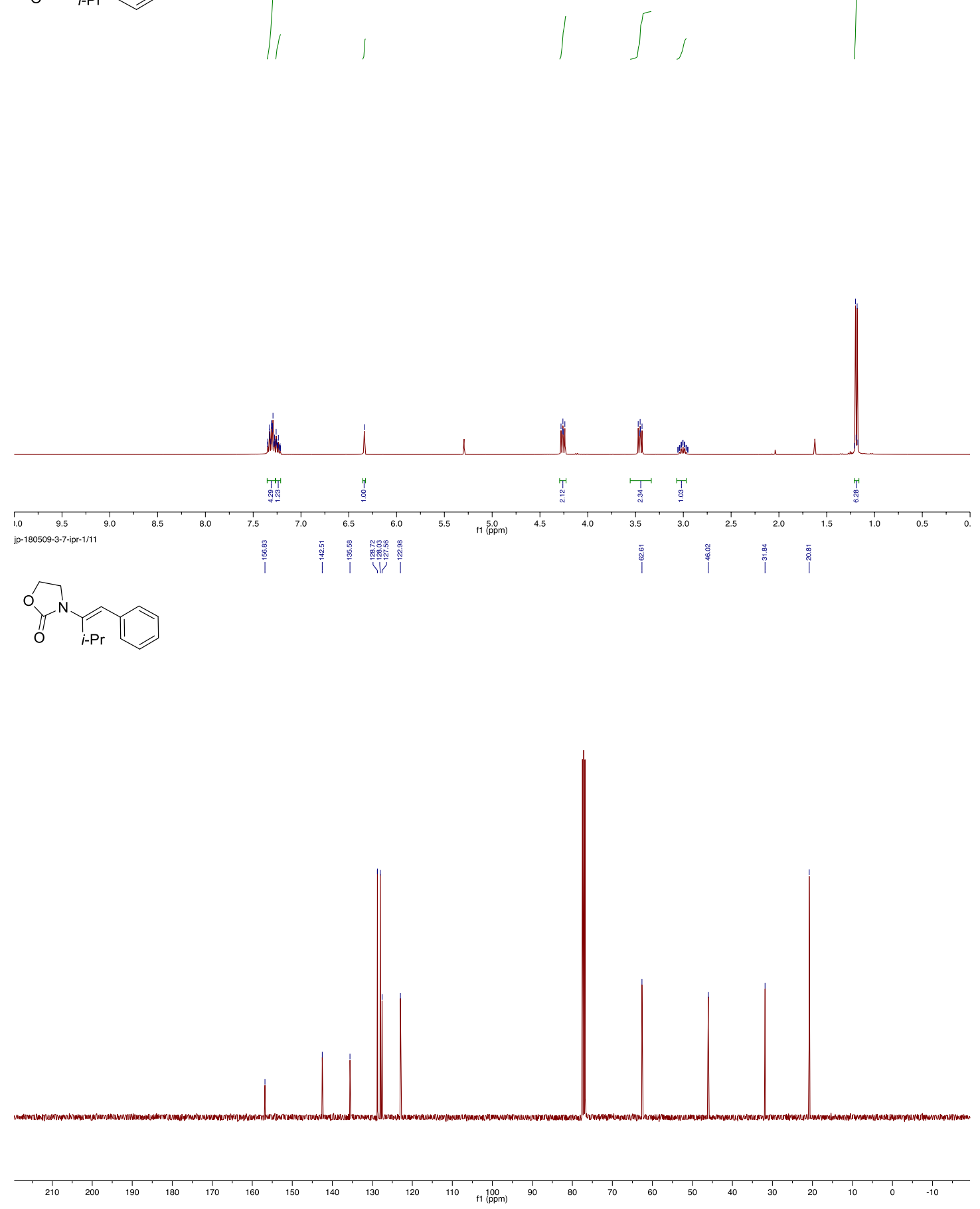

S34 


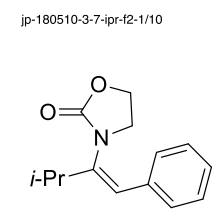

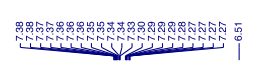

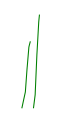

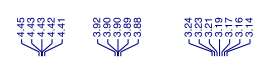

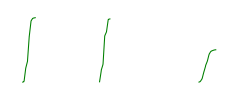

锭

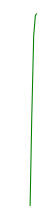

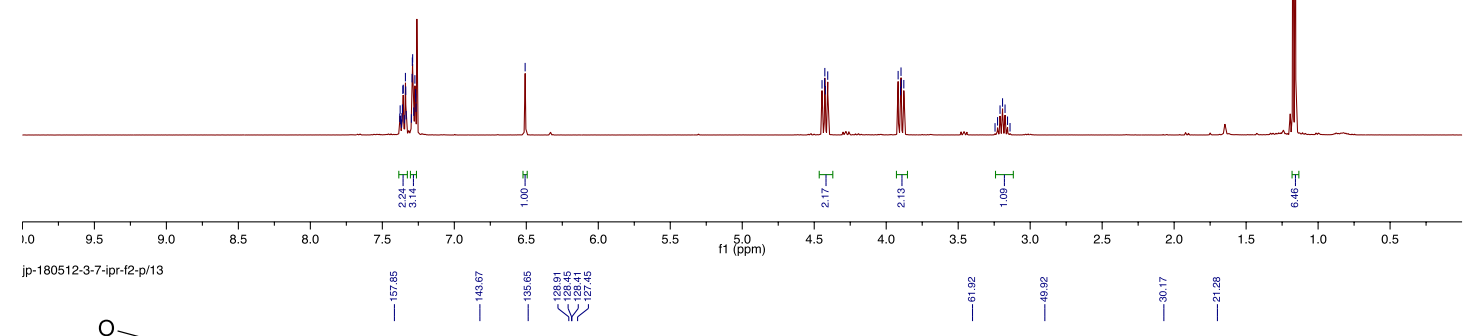
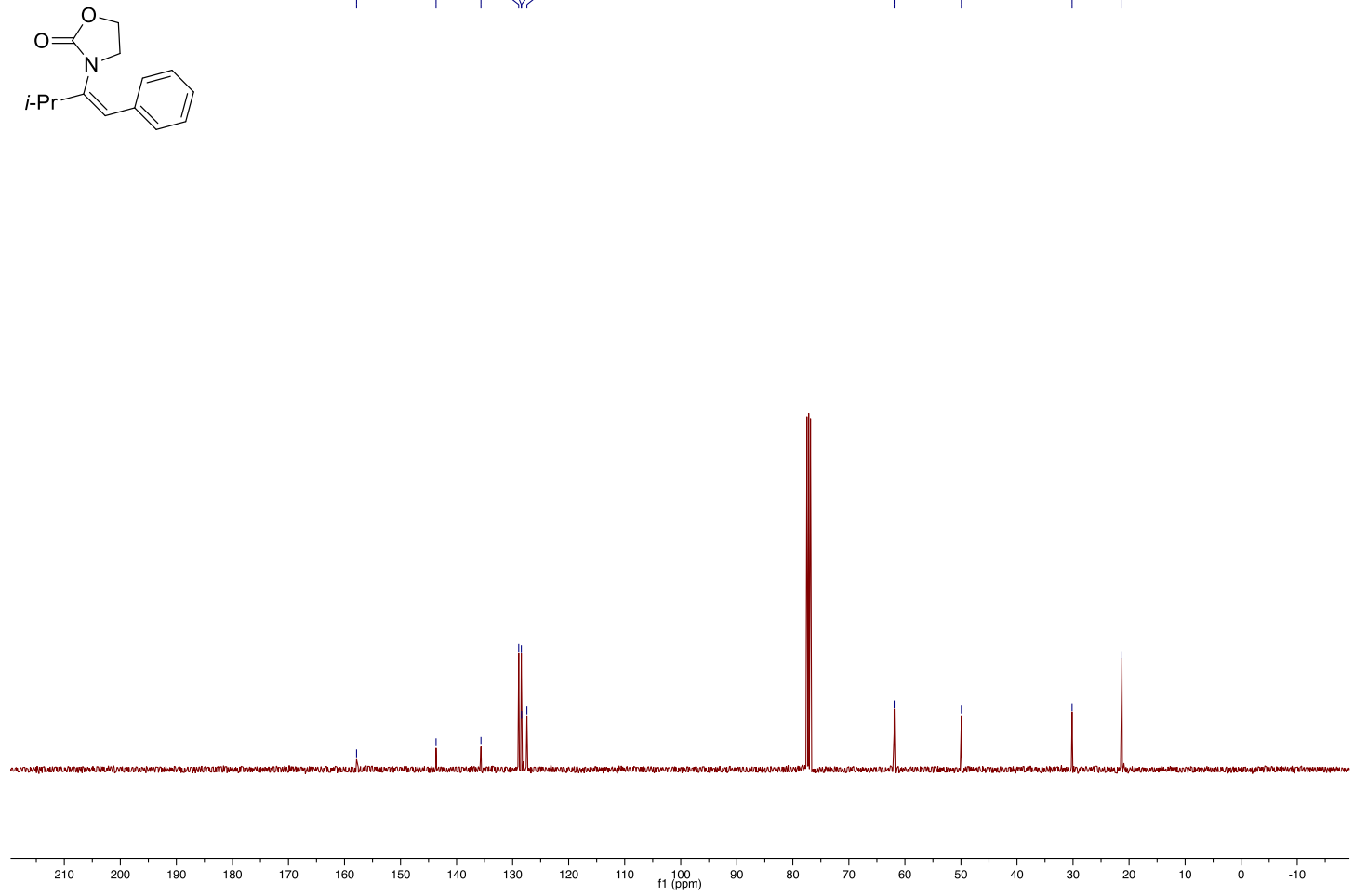

S35 
LM-20190402-2-130Zdry.10.fid

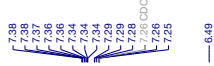

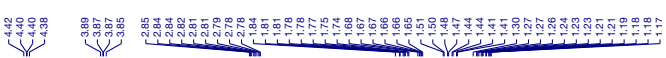

型
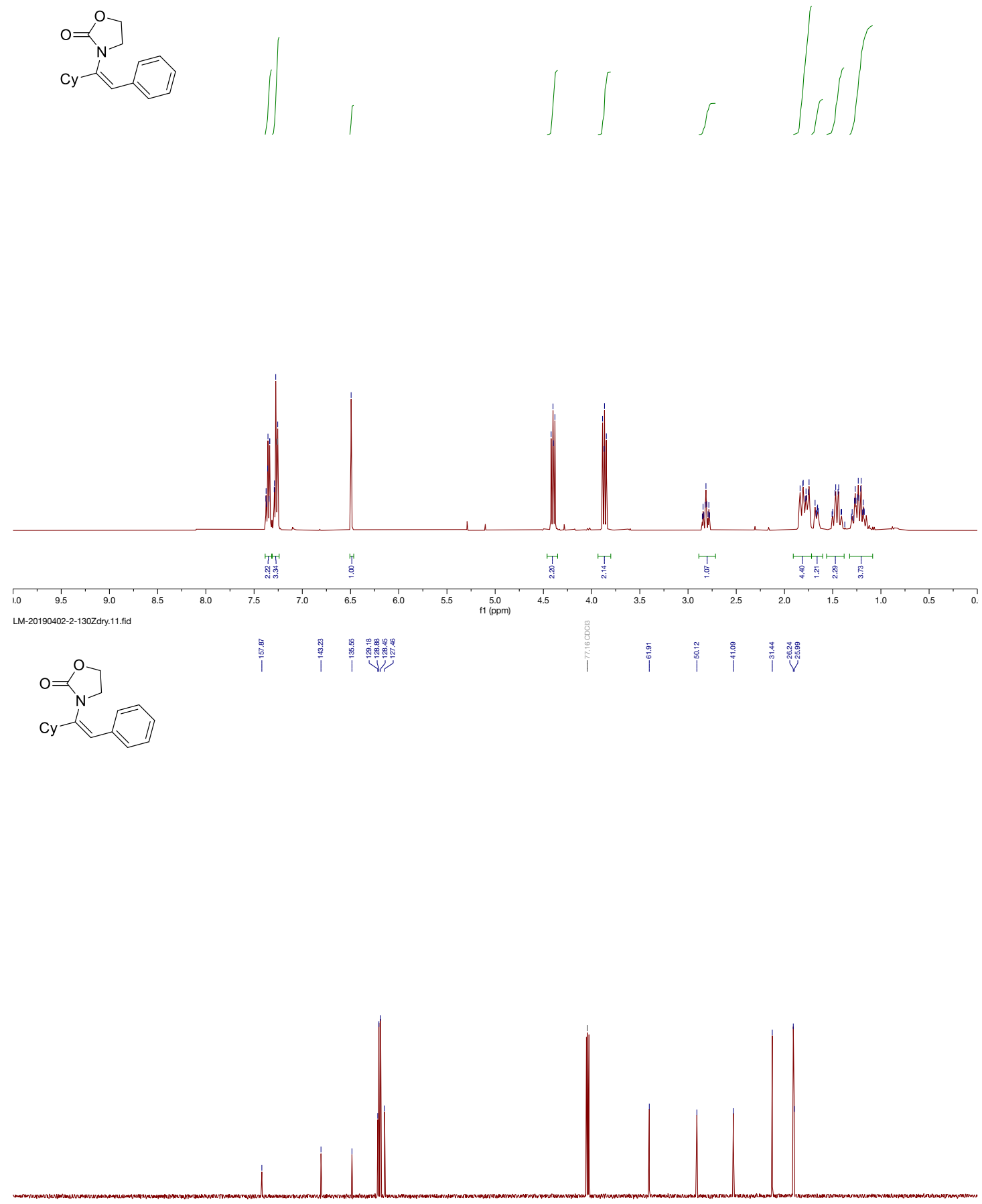

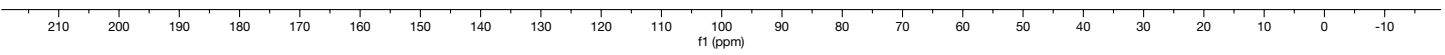


<smiles>O=C1OCCN1C(=O)c1ccccc1</smiles><smiles>C=CC</smiles>
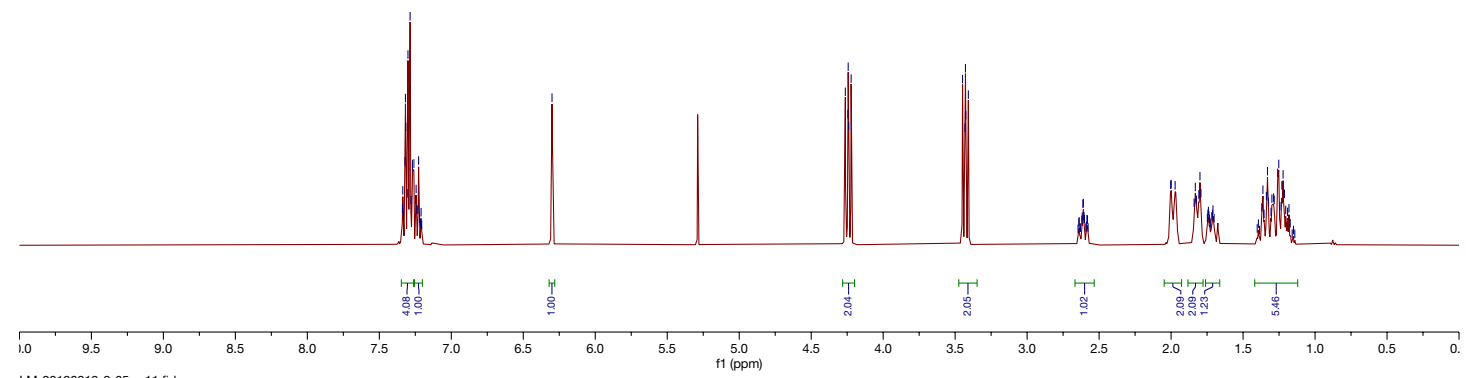
LM-20190219-2-65cy.11.fid $<C_{\text {Cy }}^{O}$

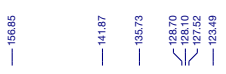
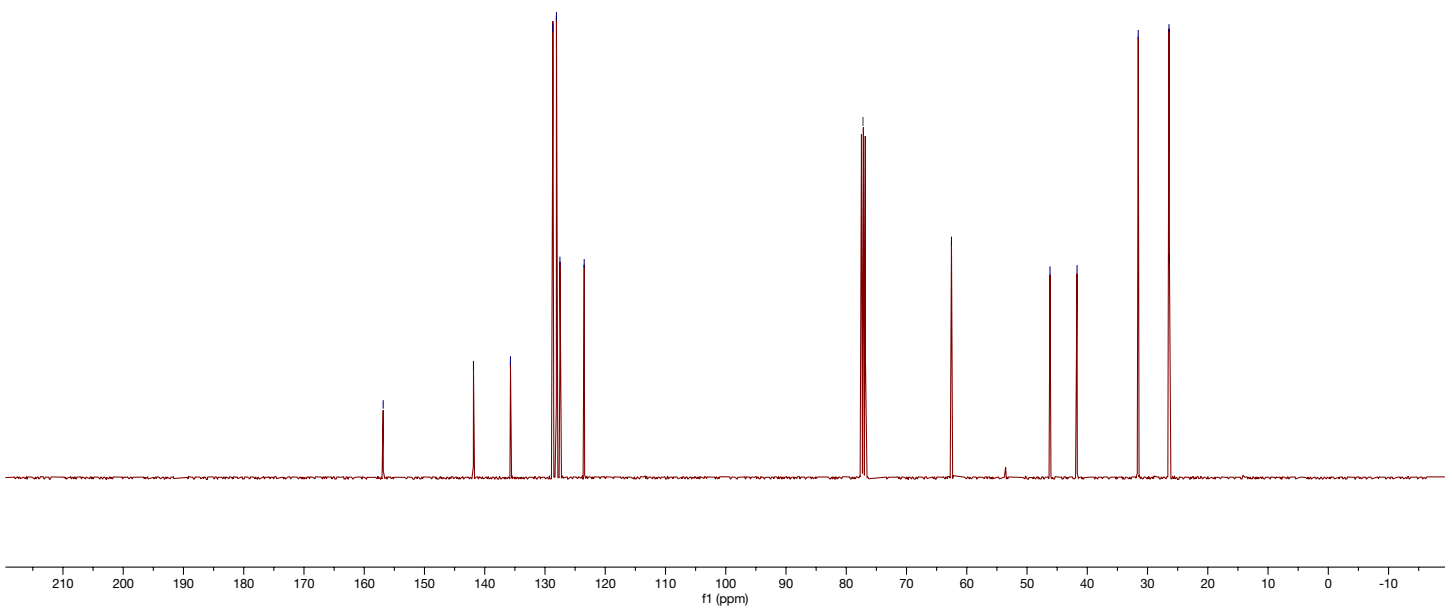
<smiles>CN1CCOC1=O</smiles>

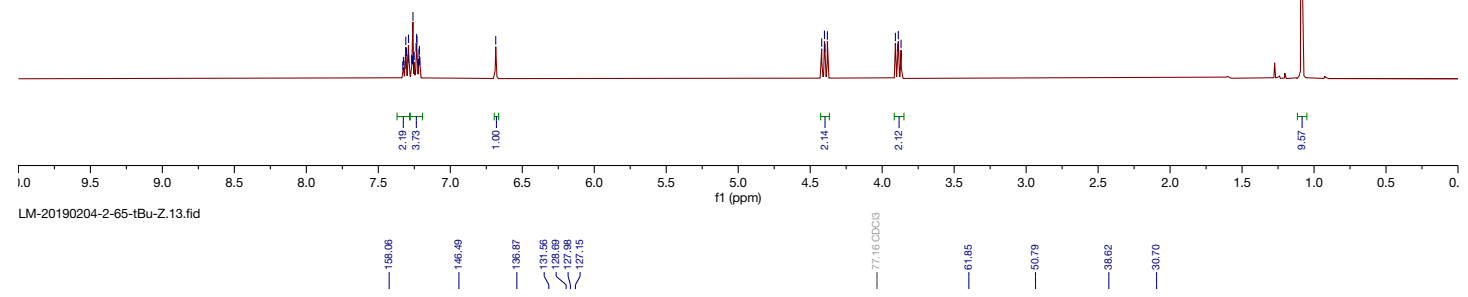

$\underset{t-\mathrm{Bu}}{\lambda_{N}^{O}} \geqslant$
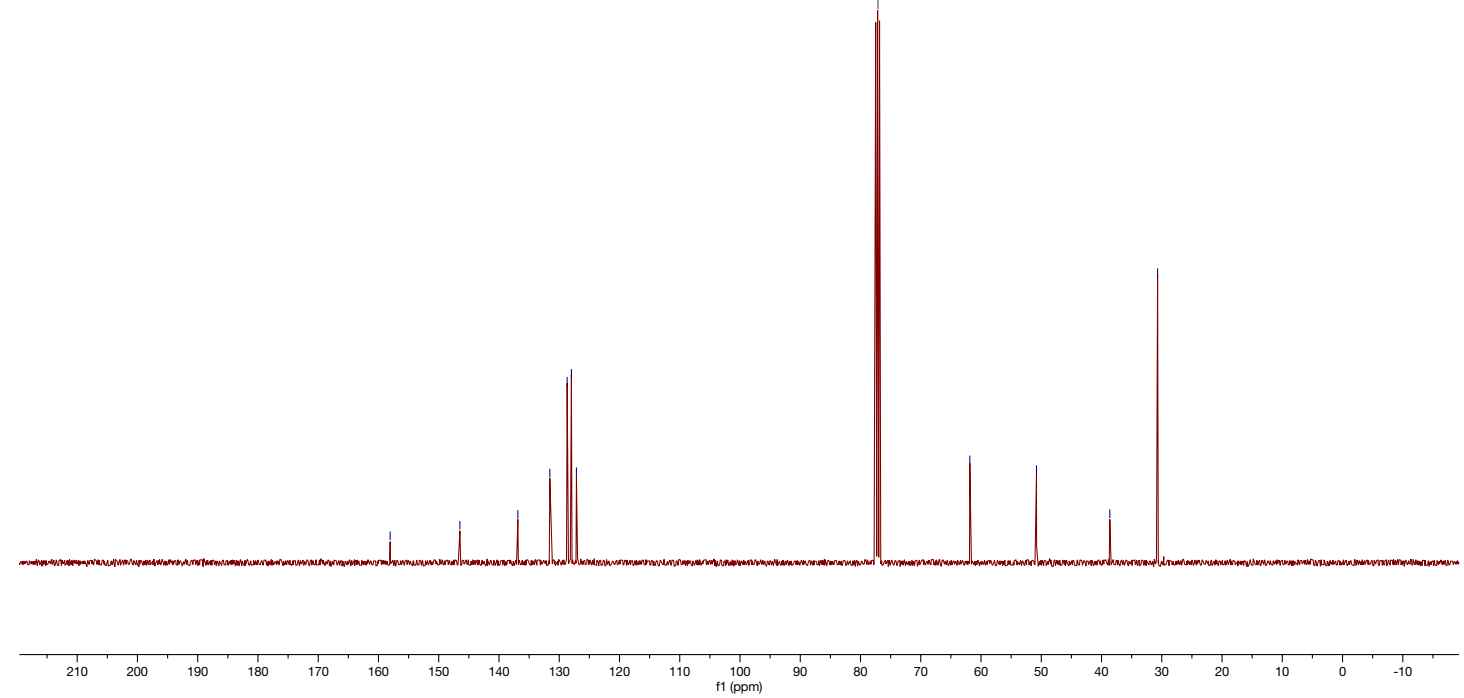


$$
L_{t-\mathrm{Bu}}^{\mathrm{O}}
$$
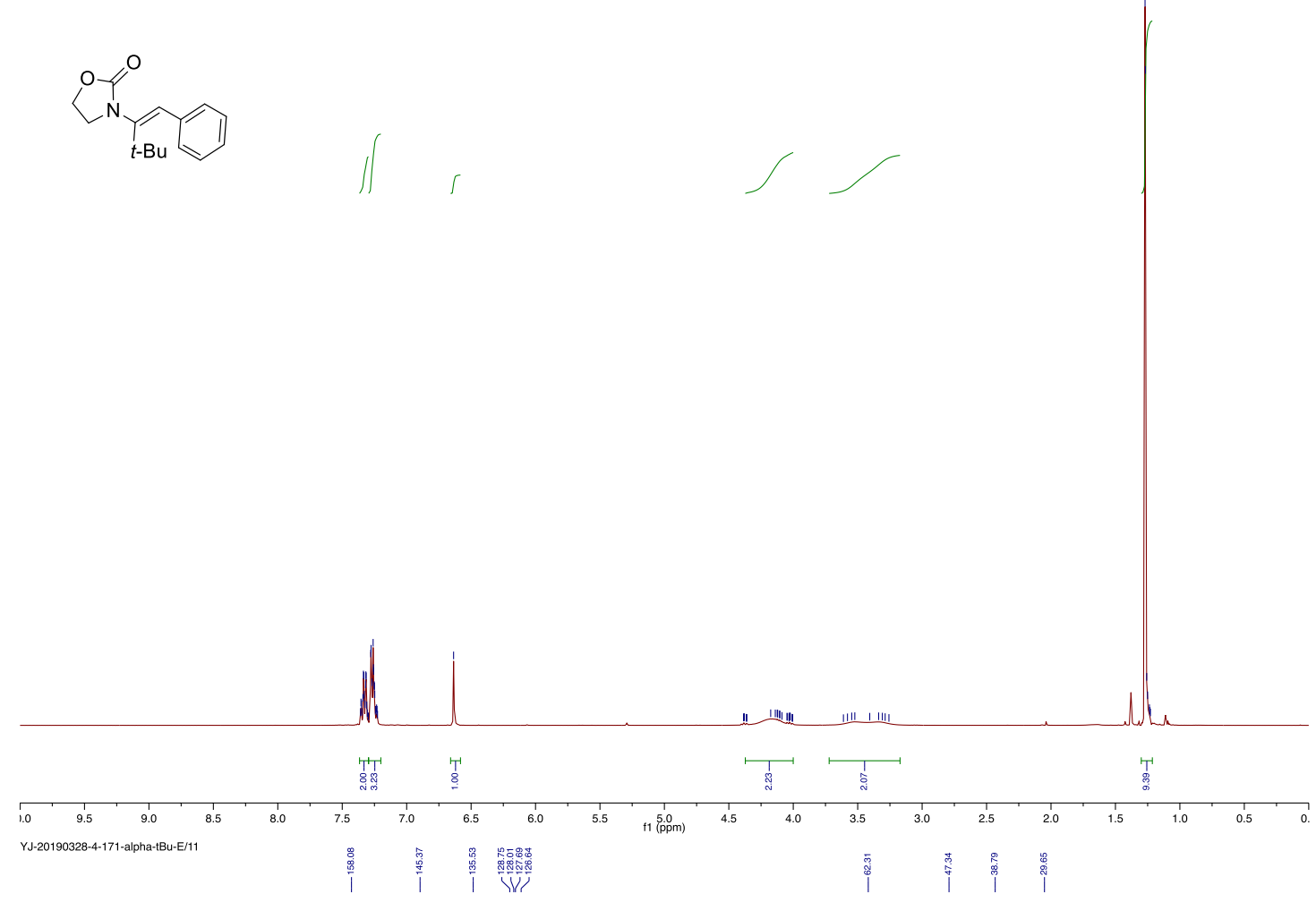<smiles>O=C1OCCN1C([18F])=Cc1ccccc1</smiles>
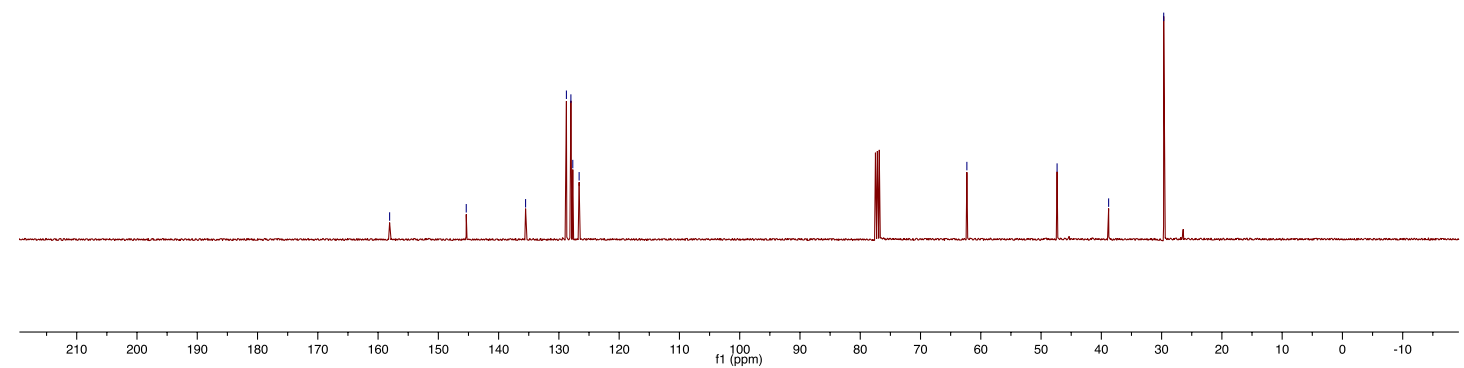

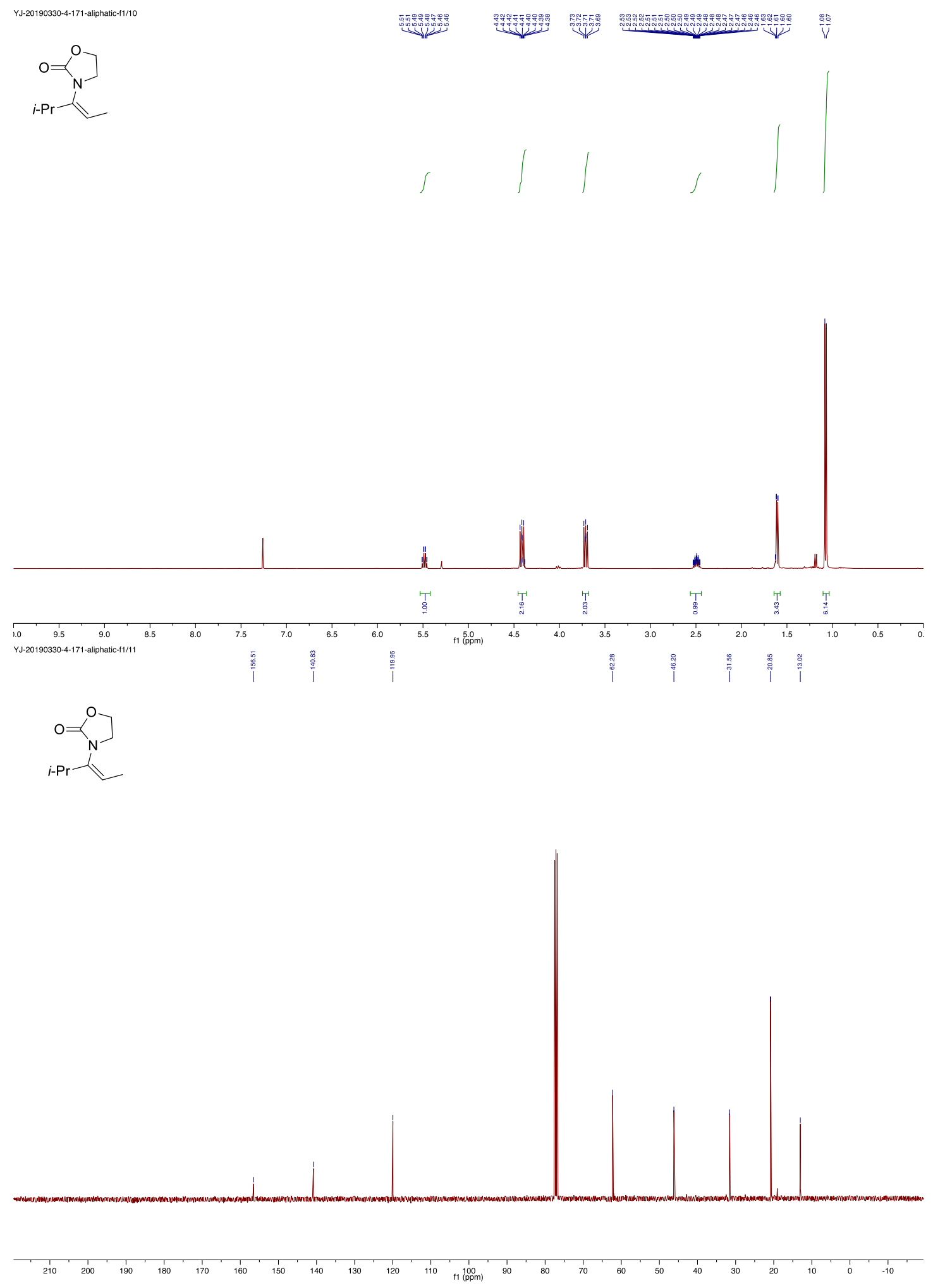

S40 

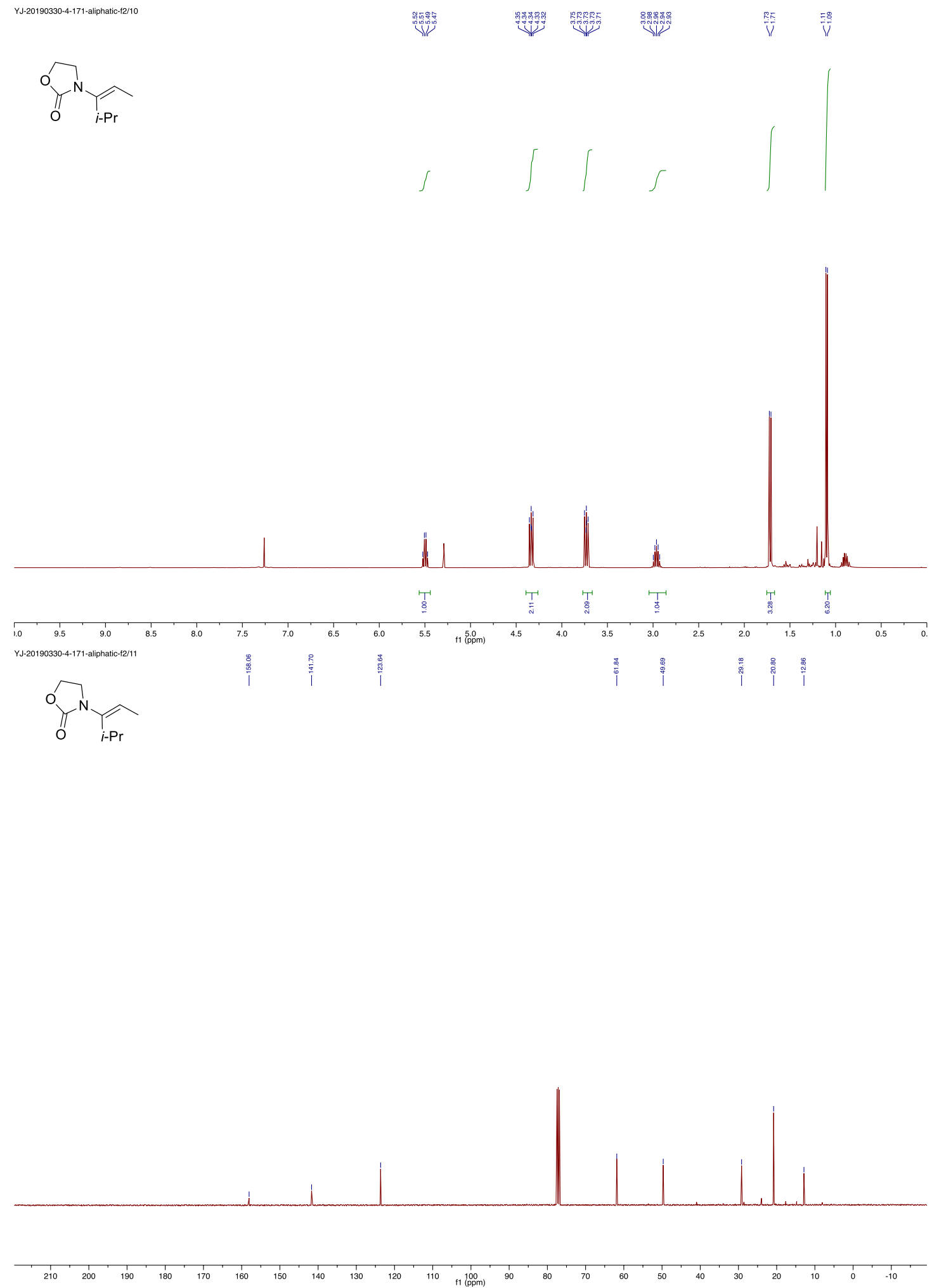
$>_{n-\mathrm{Bu}}^{2}$

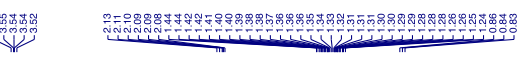
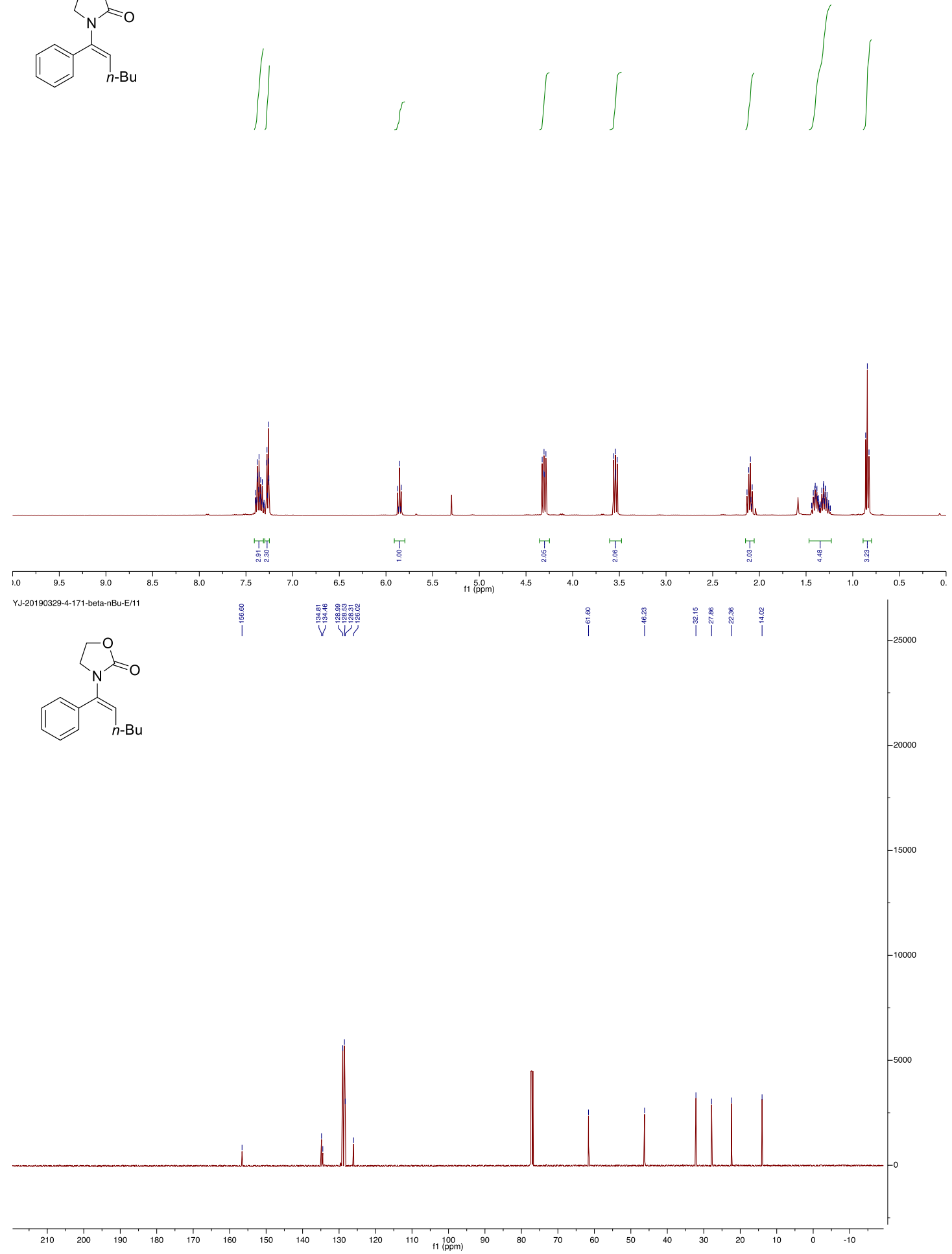

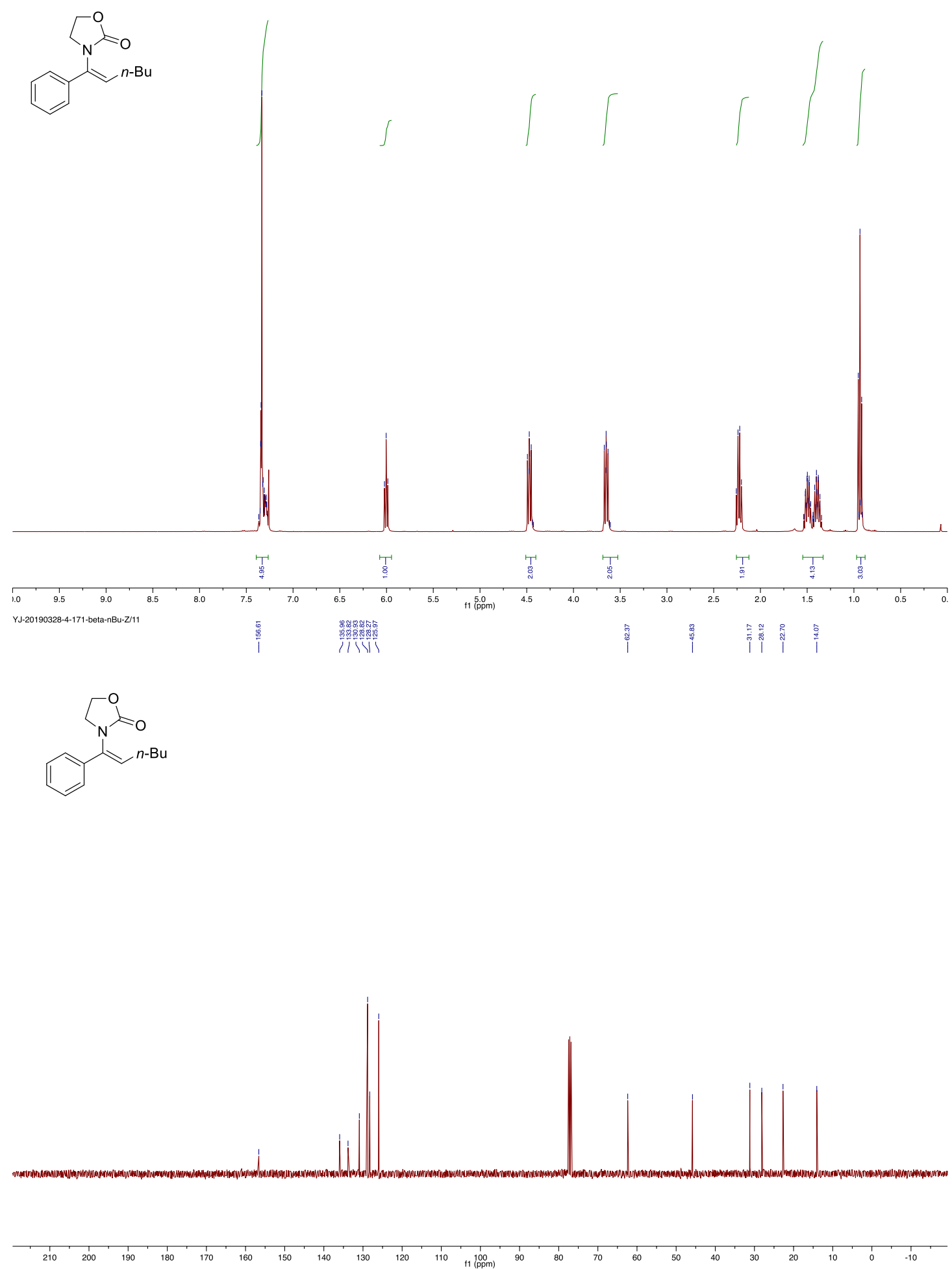
yanji-20210904-8-89-4-CDCl3-BASE.3.fid

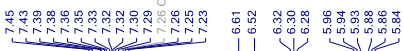

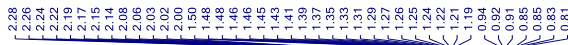

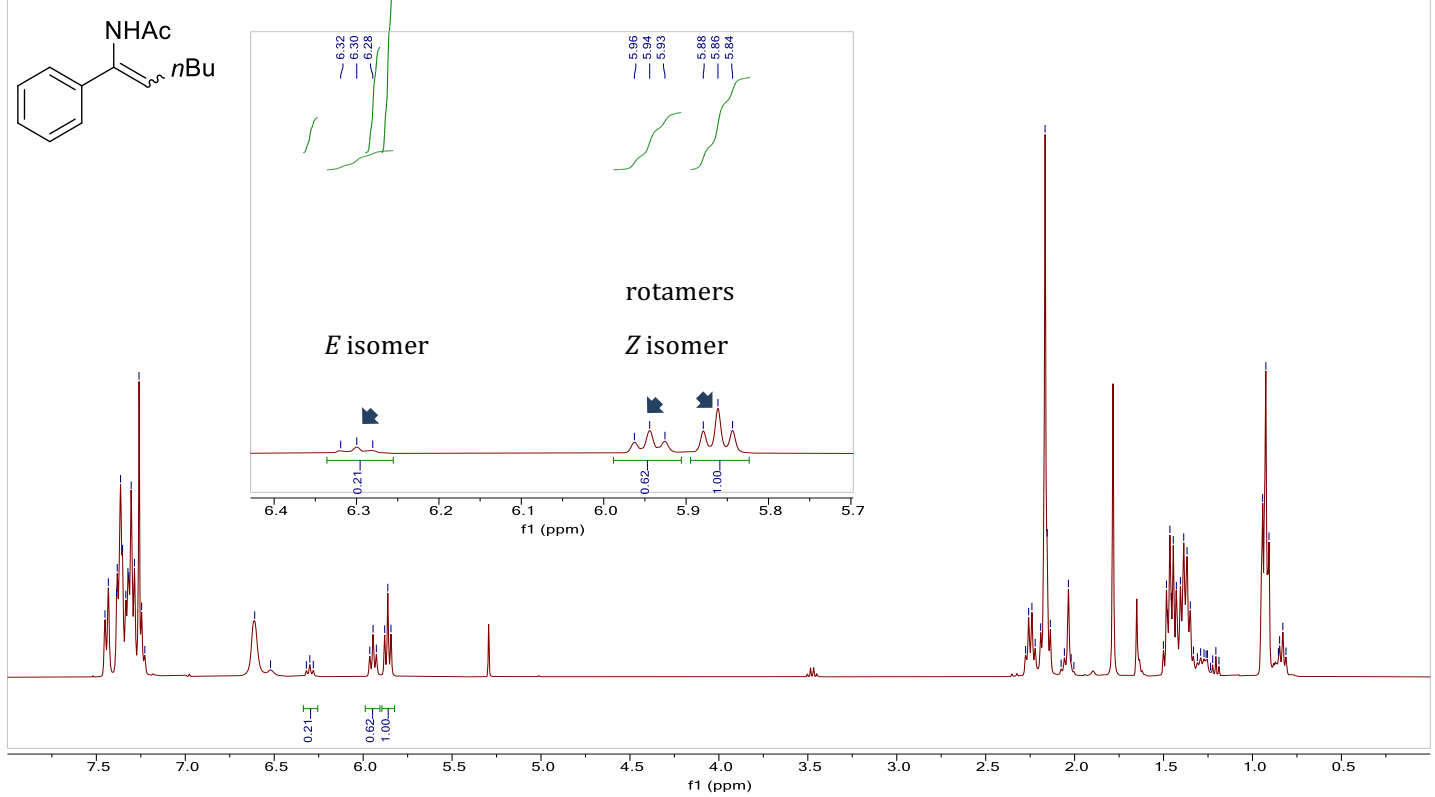


LM3-mMe.3.fic

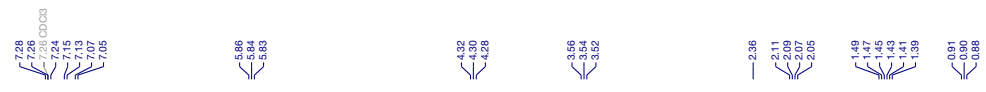<smiles>O=C1OCCN1C(=C[P+](F)(F)F)c1cccc([N+](=O)[O-])c1</smiles>

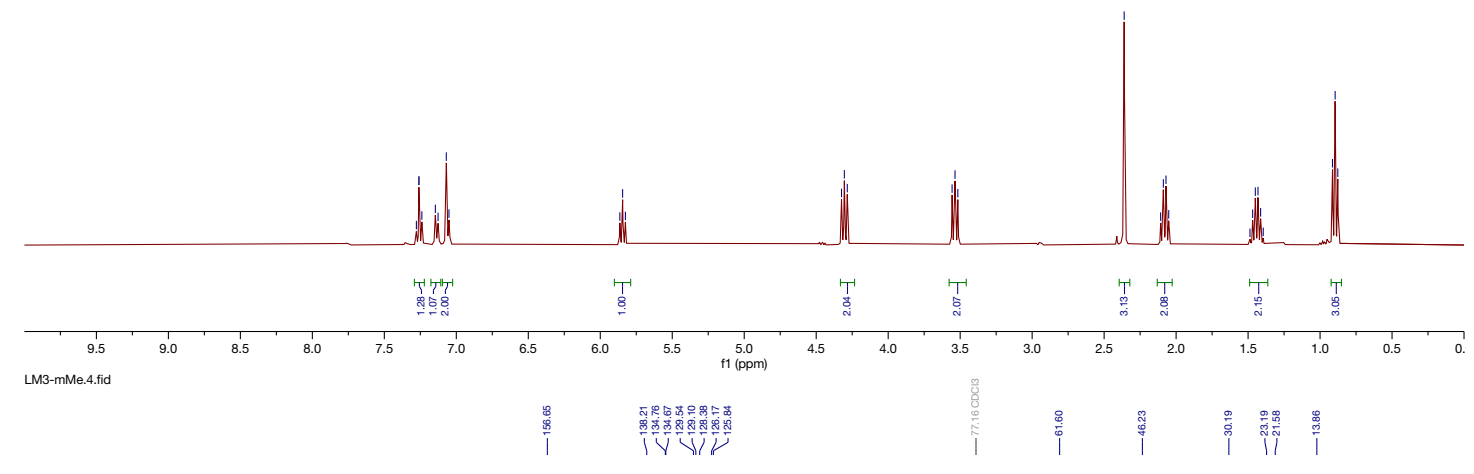<smiles>CCCC=C(c1cccc([N+](=O)[O-])c1)N1CCOC1=O</smiles>

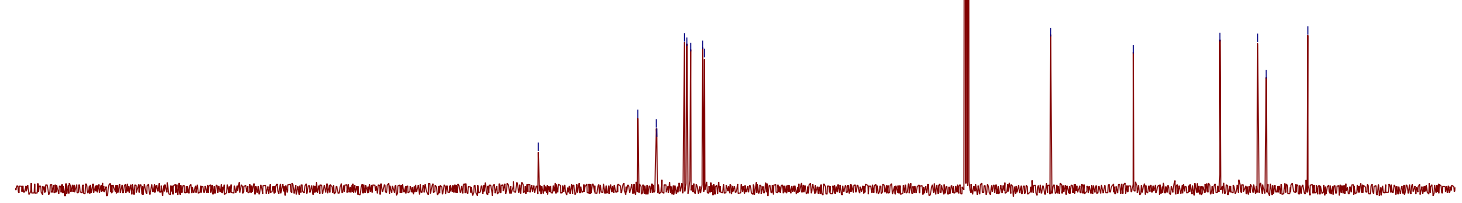

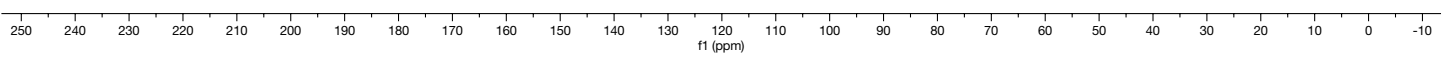



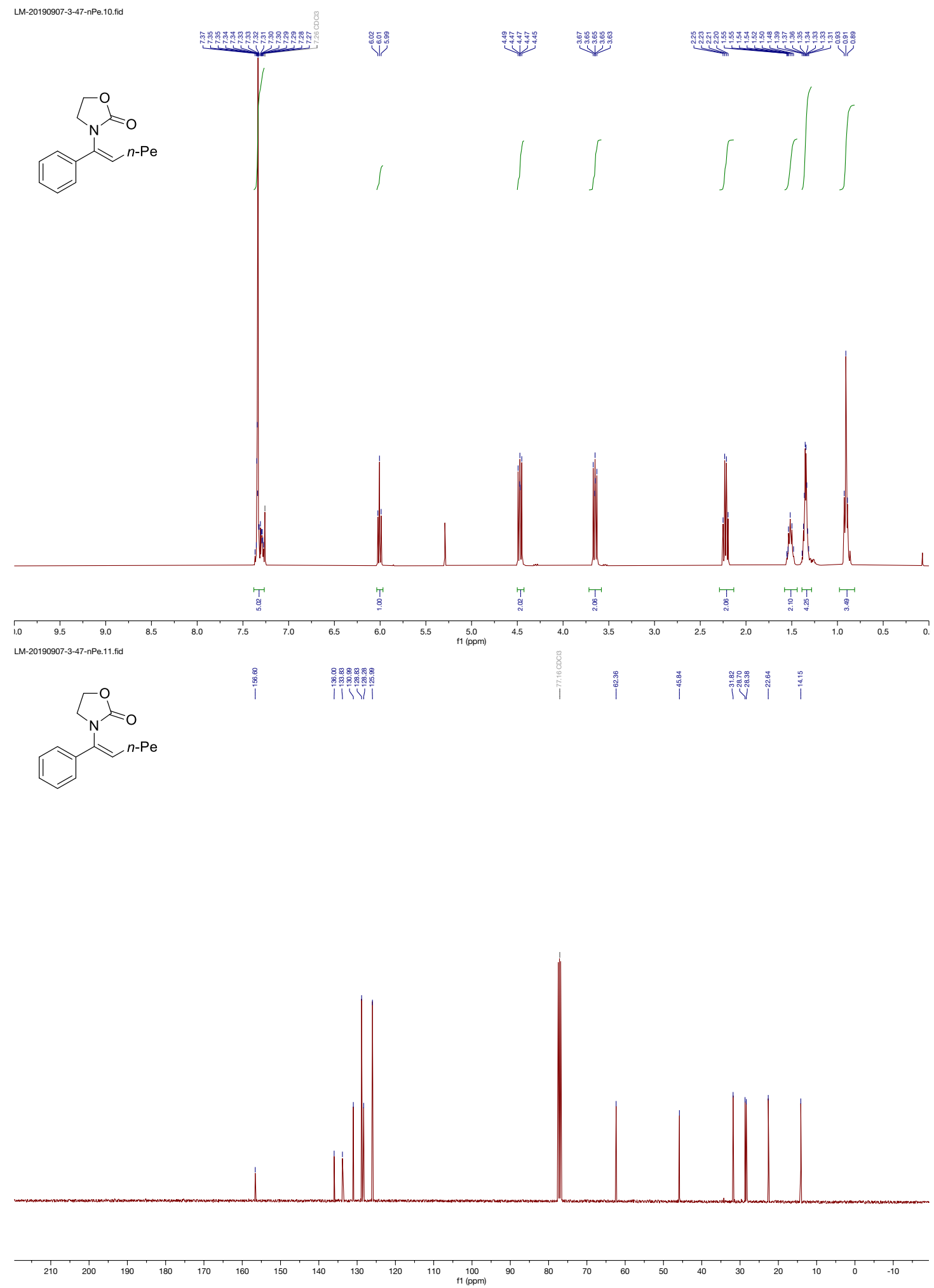


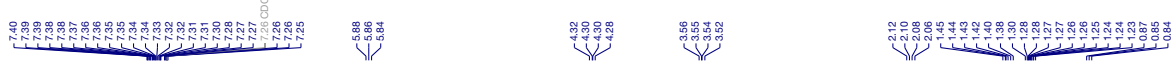<smiles>O=C1OCCN1C(=Cc1ccccc1)c1ccccc1</smiles>
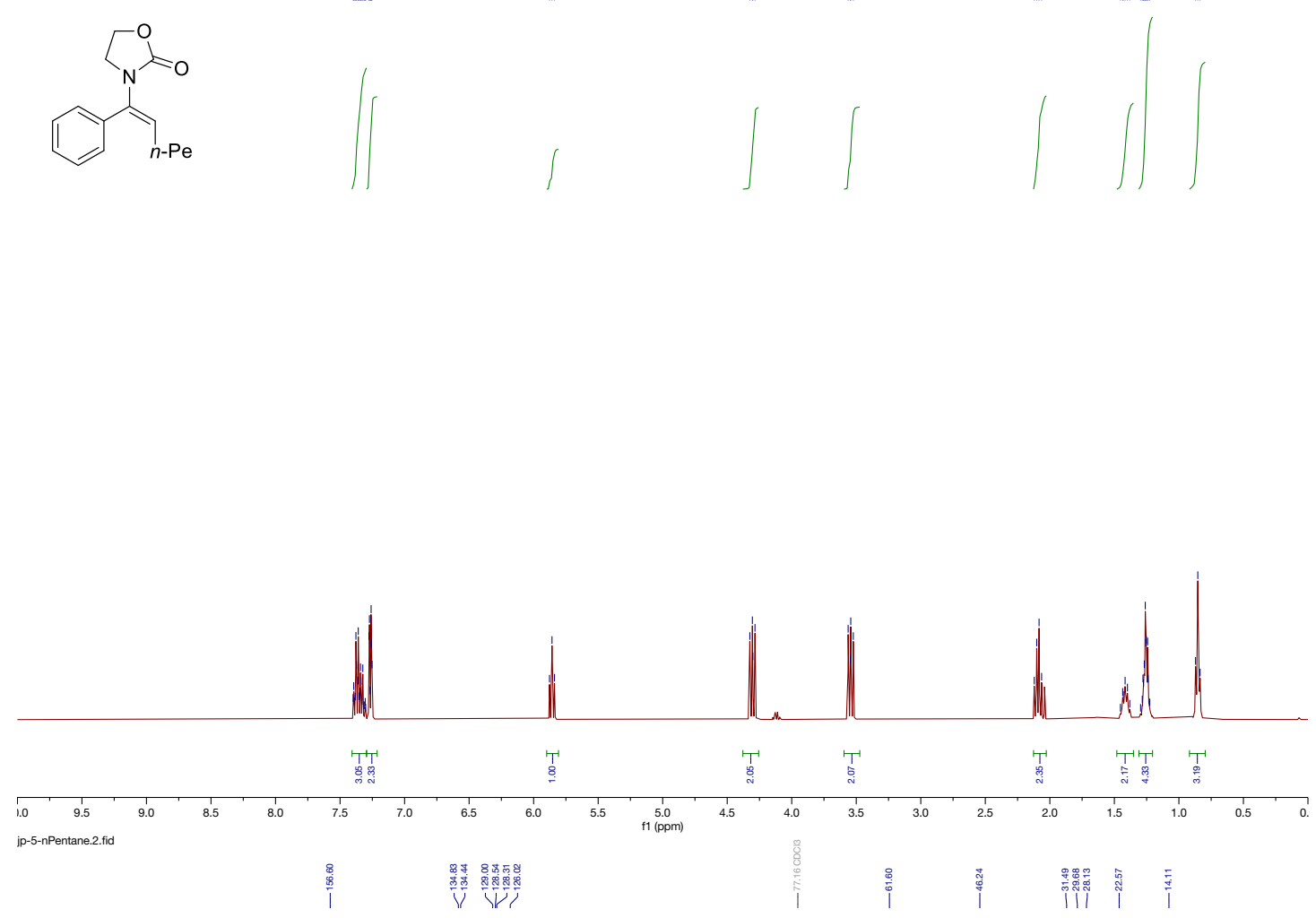

$\sum_{n-\mathrm{Pe}}^{2}$
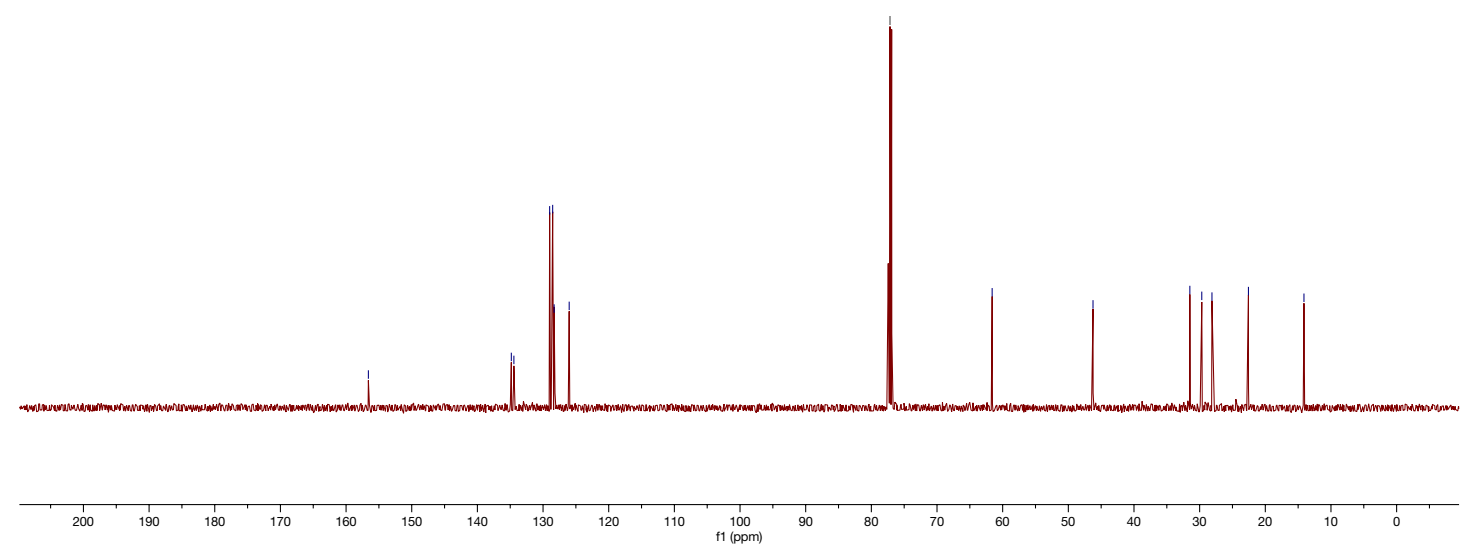

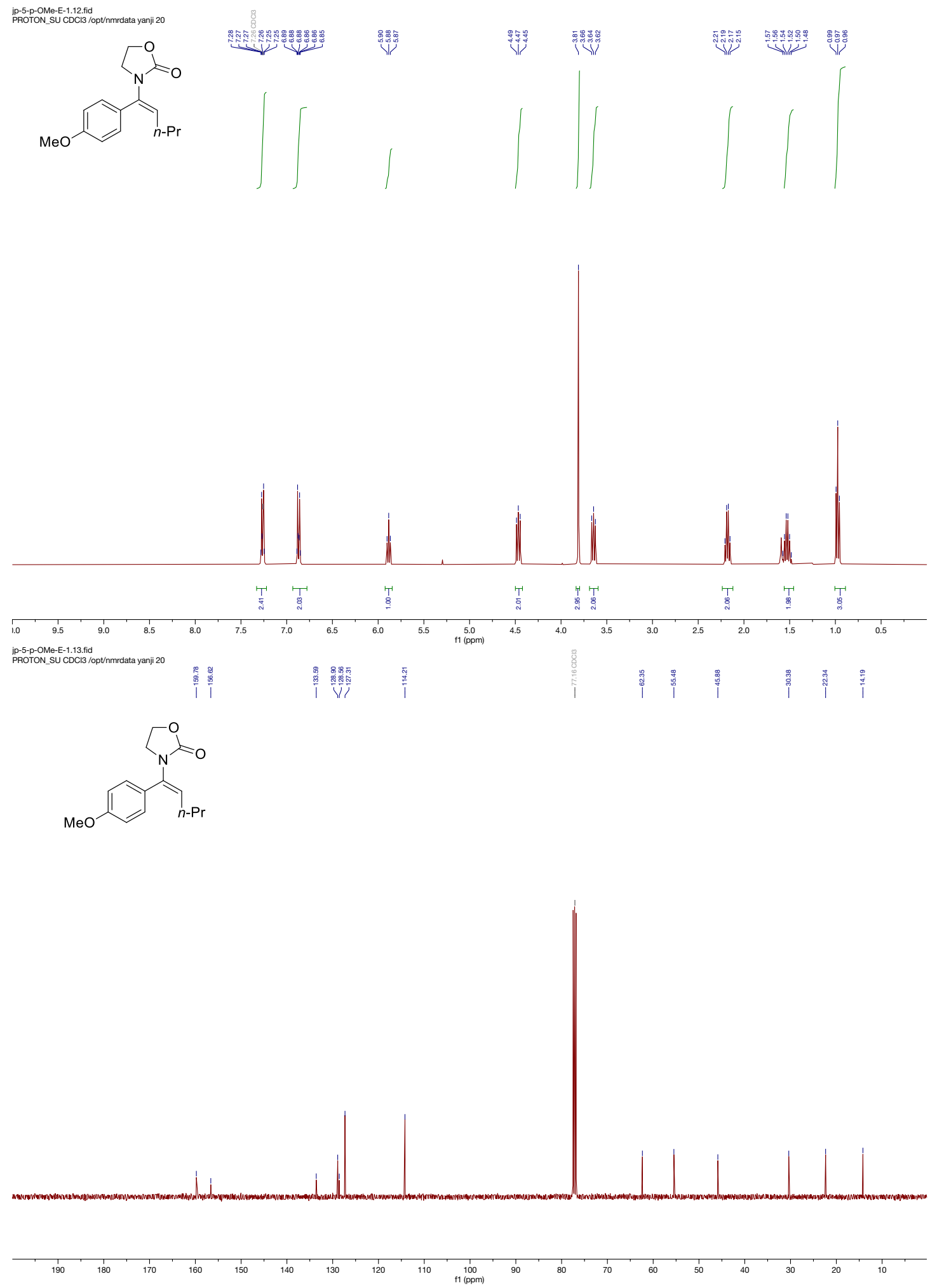

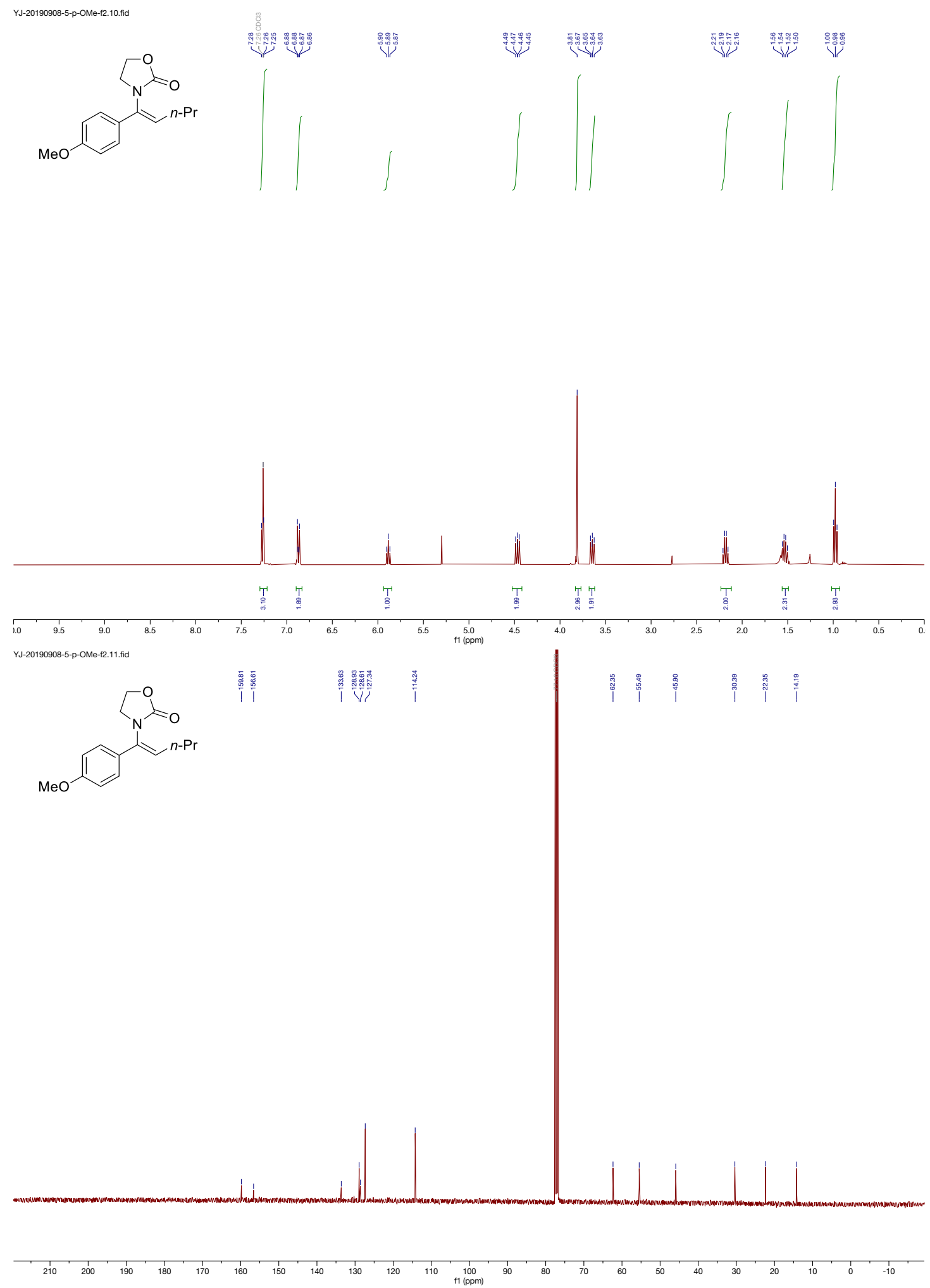


$$
\text { of reing }
$$
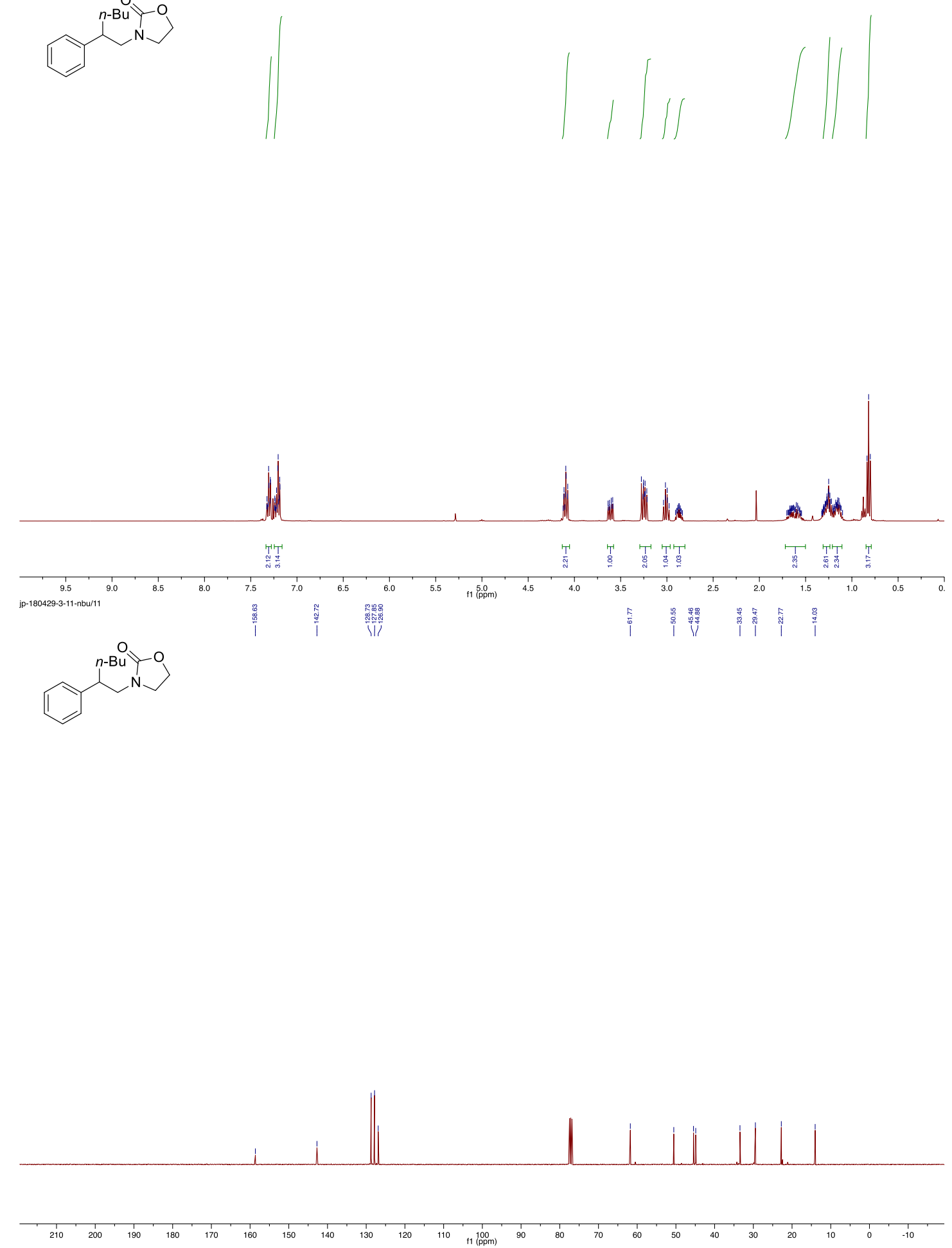

S50 
<smiles>CCCC(Cc1ccccc1)N1CCOC1=O</smiles>
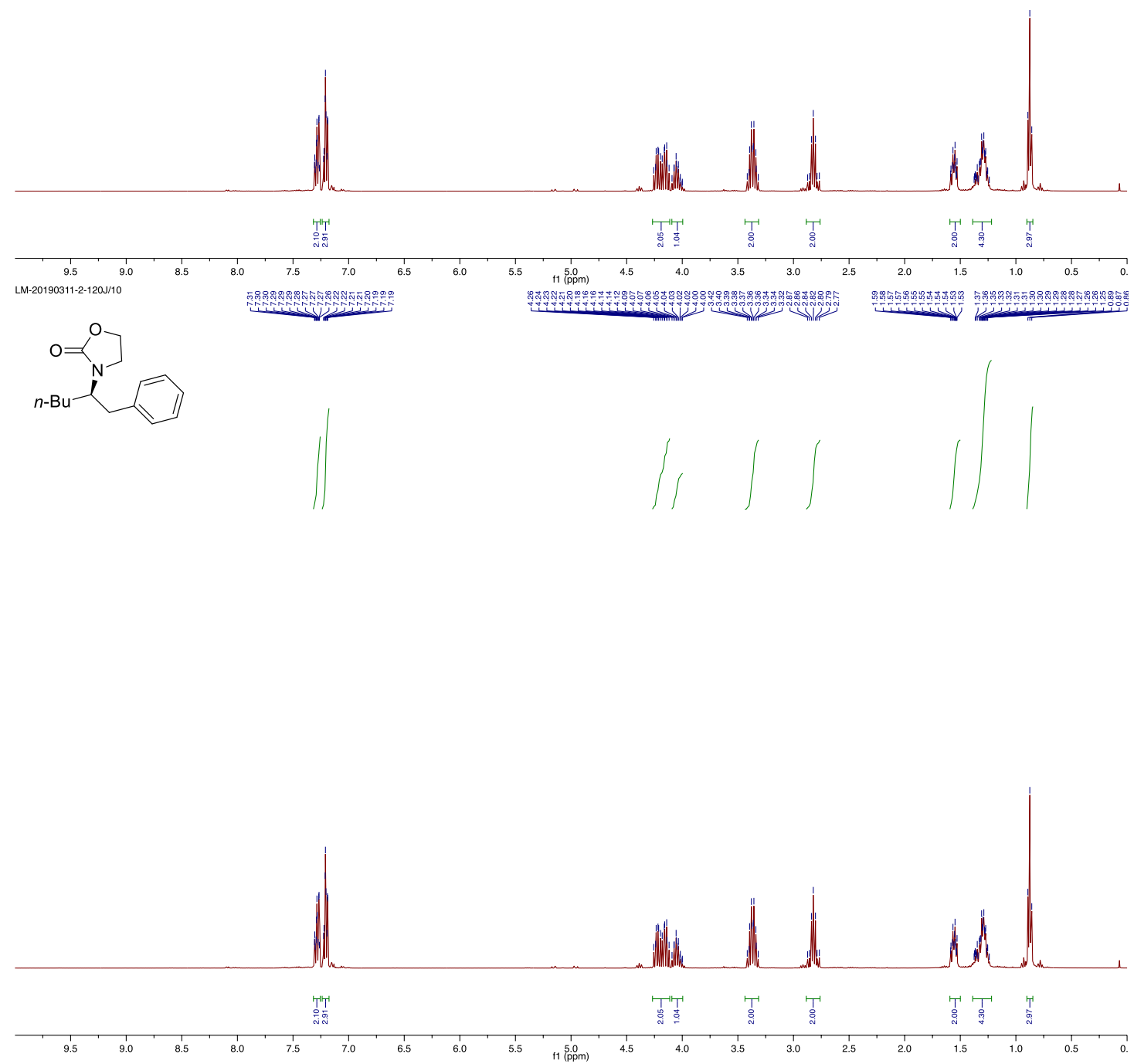

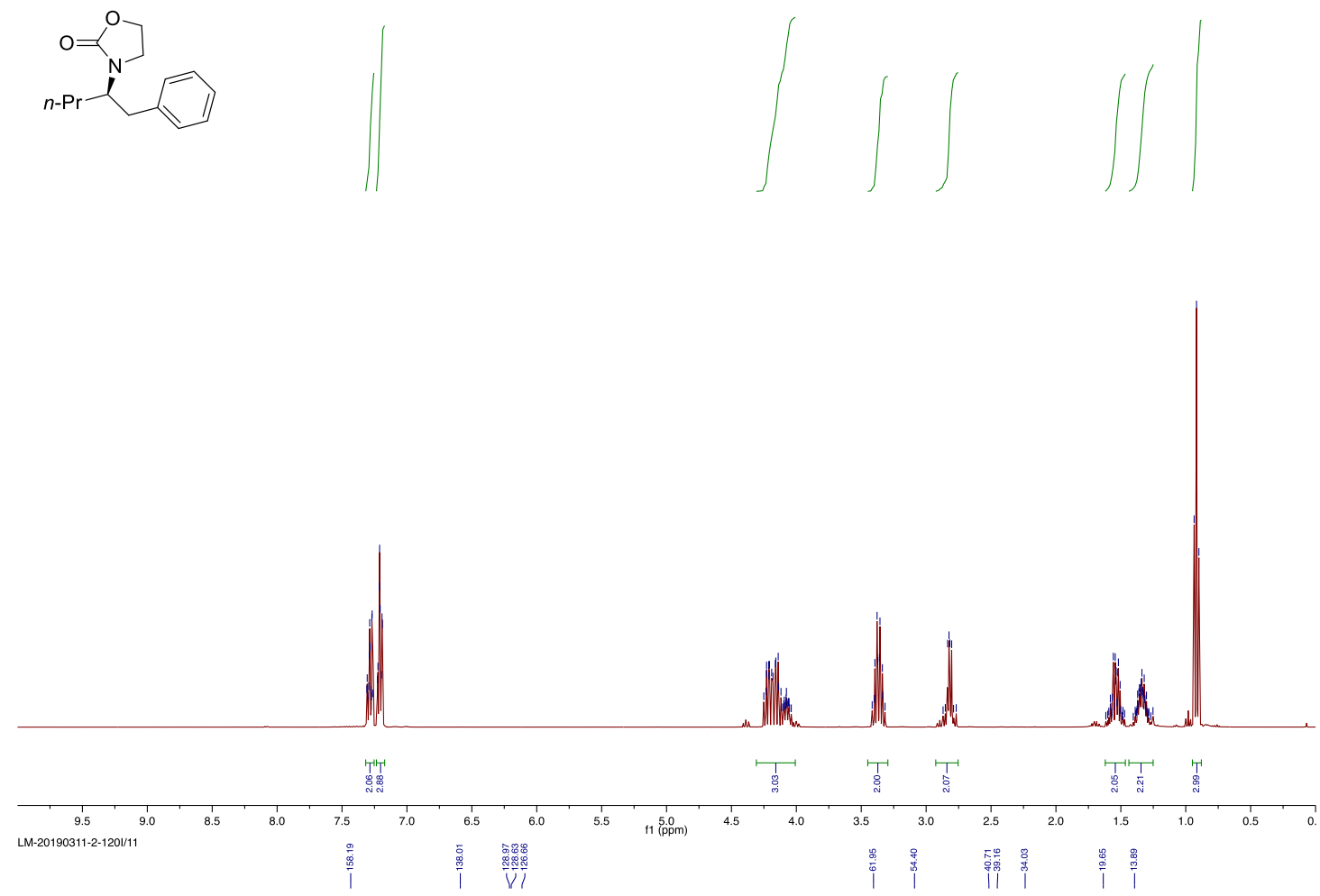

$$
=
$$
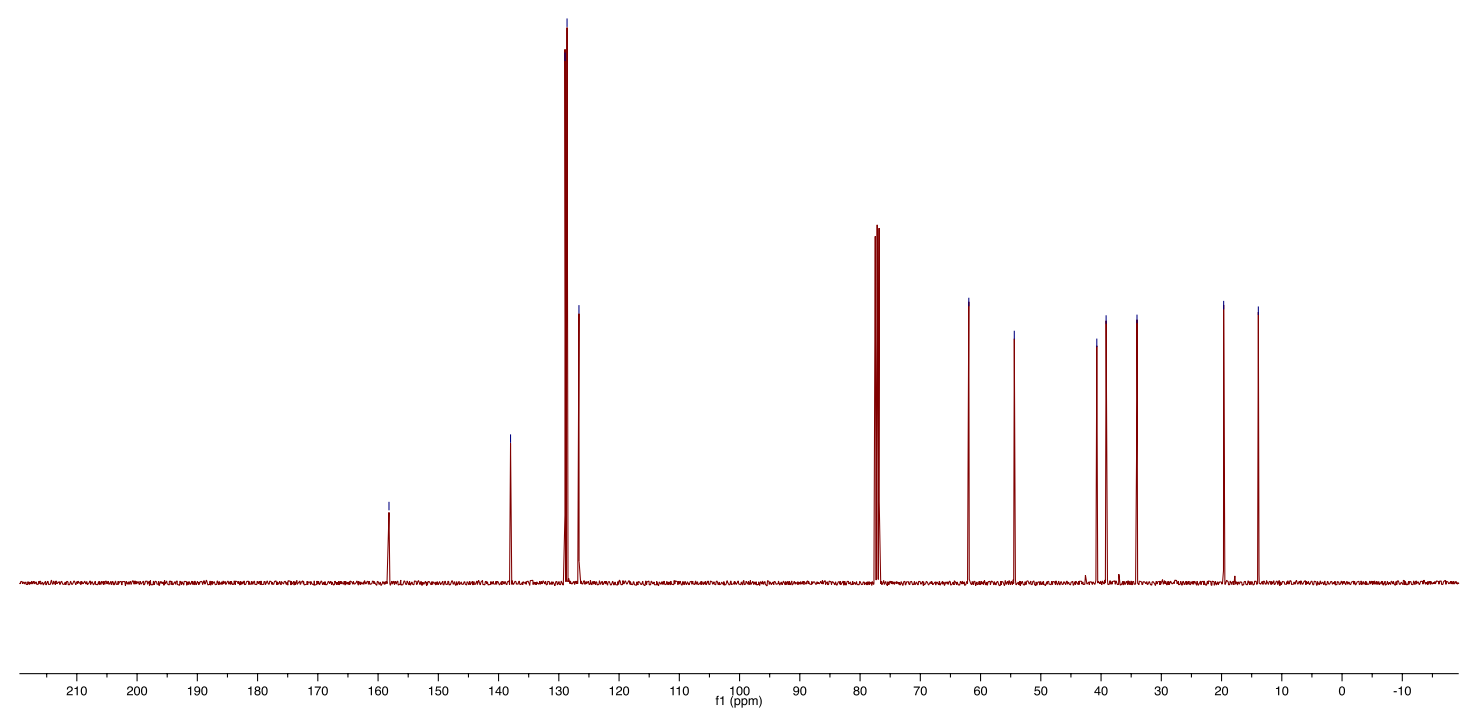
<smiles>CC(C)C(Cc1ccccc1)N1CCOC1=O</smiles>
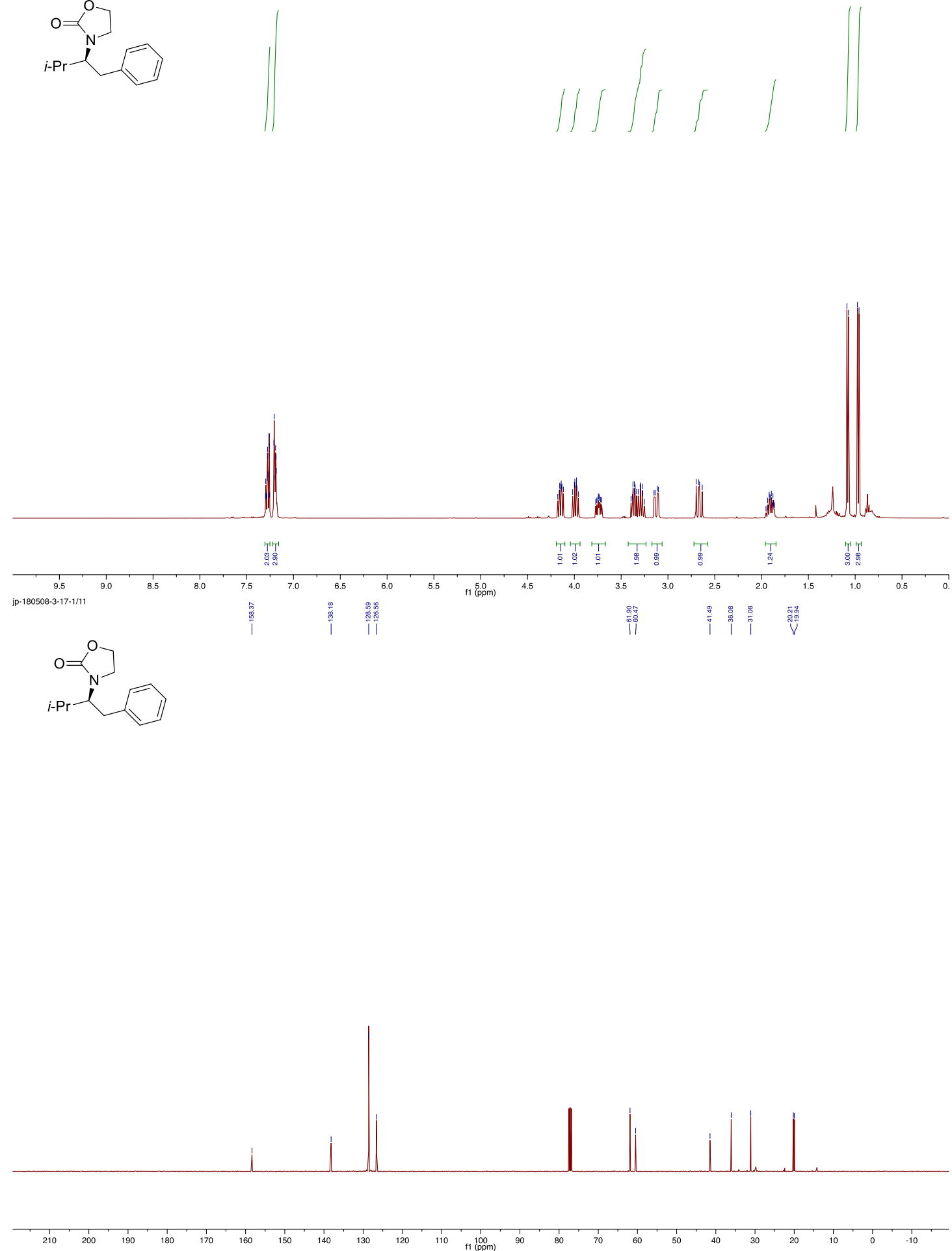


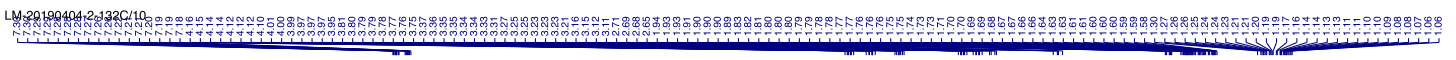
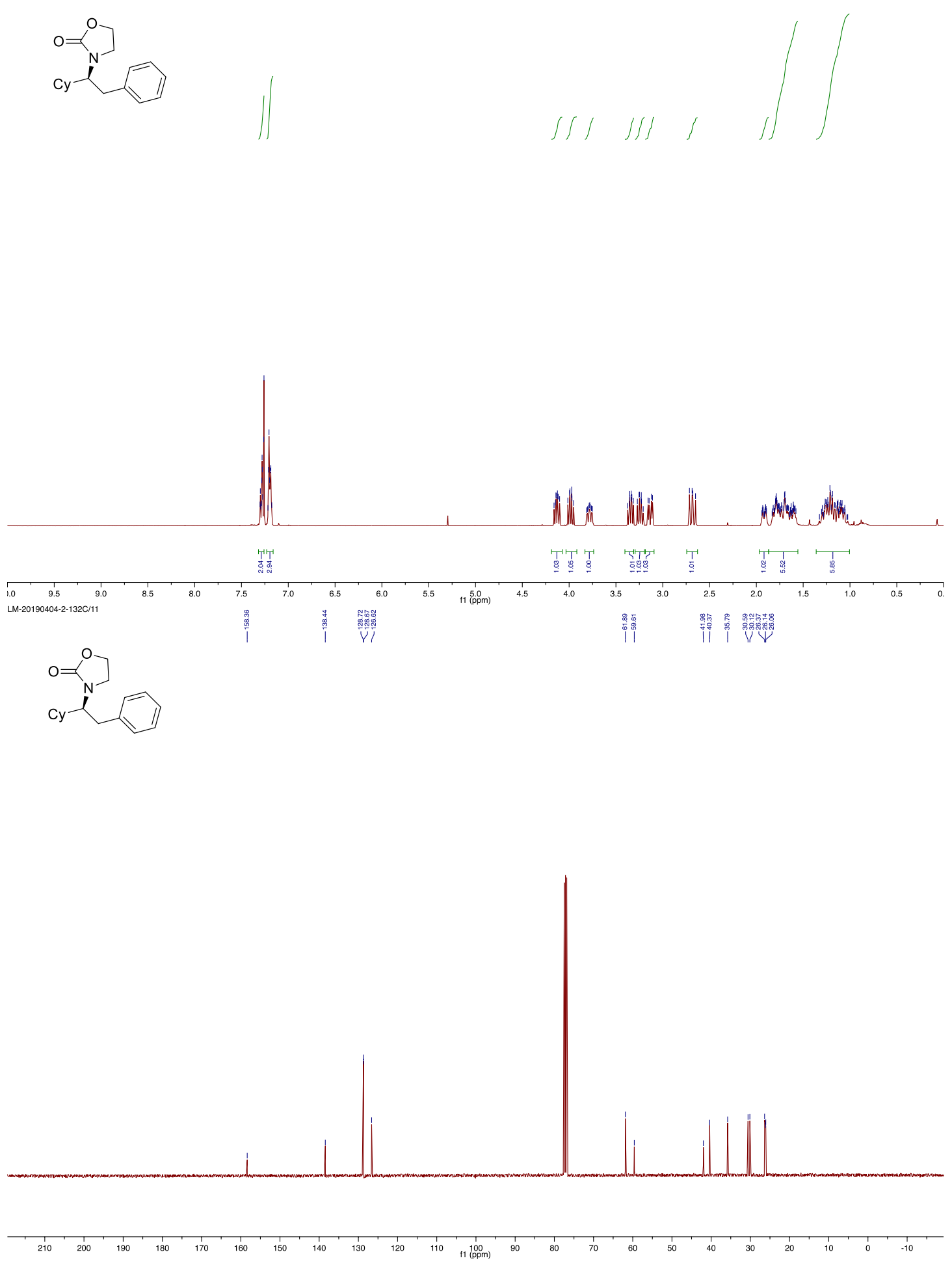

S54 


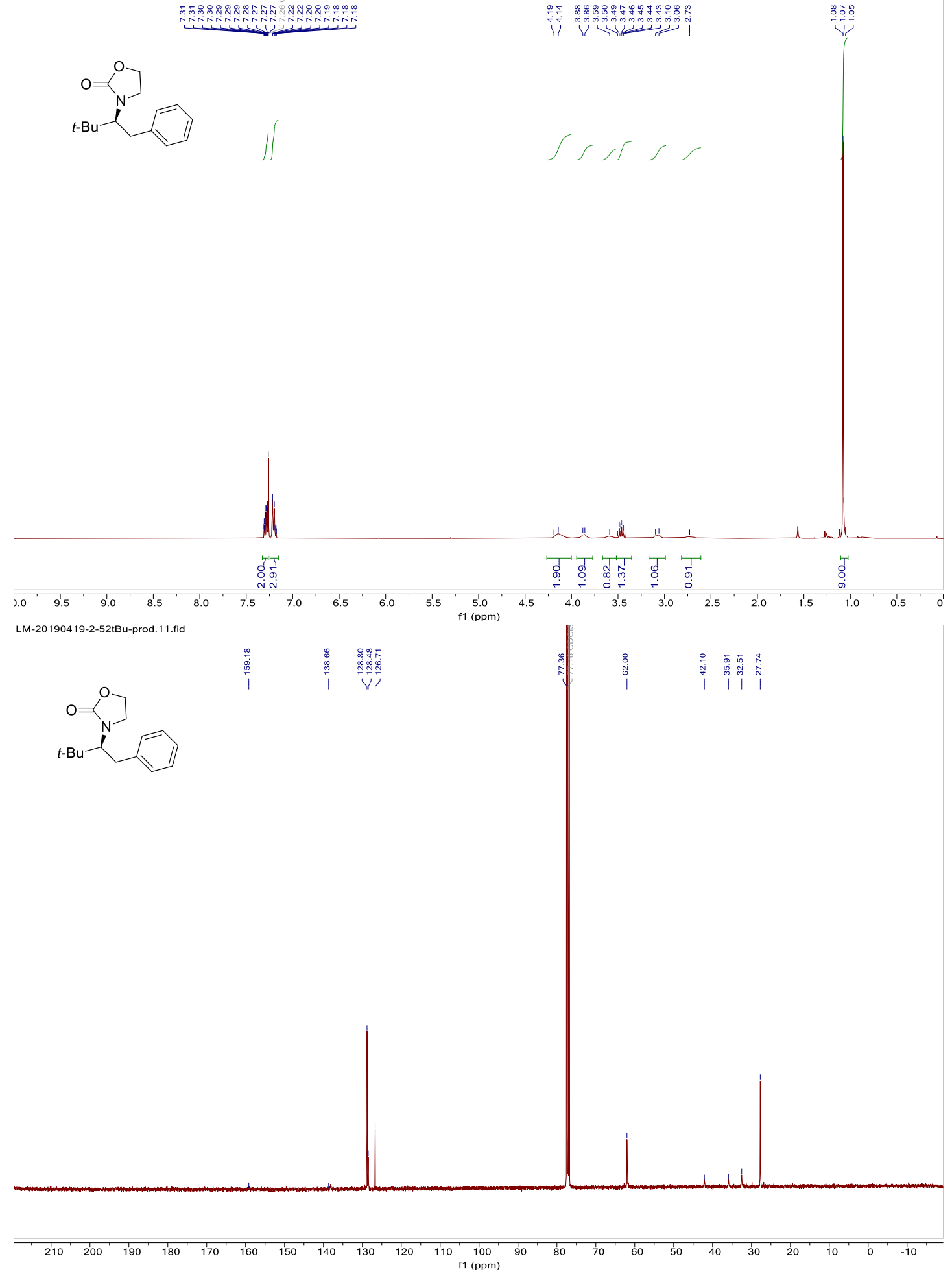


LM-20190210-2-81su/10
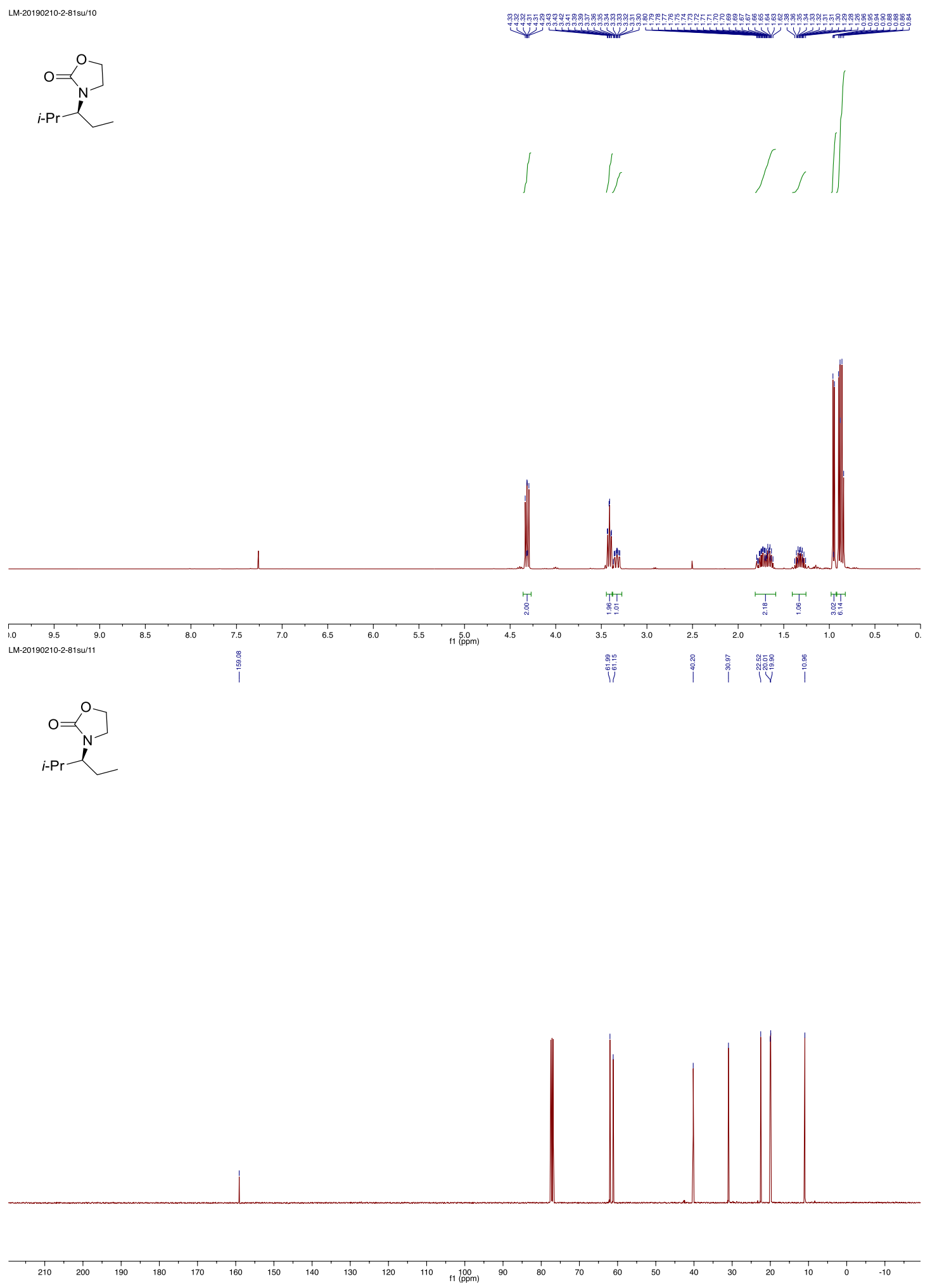

S56 
$\overbrace{}^{n-B u}$
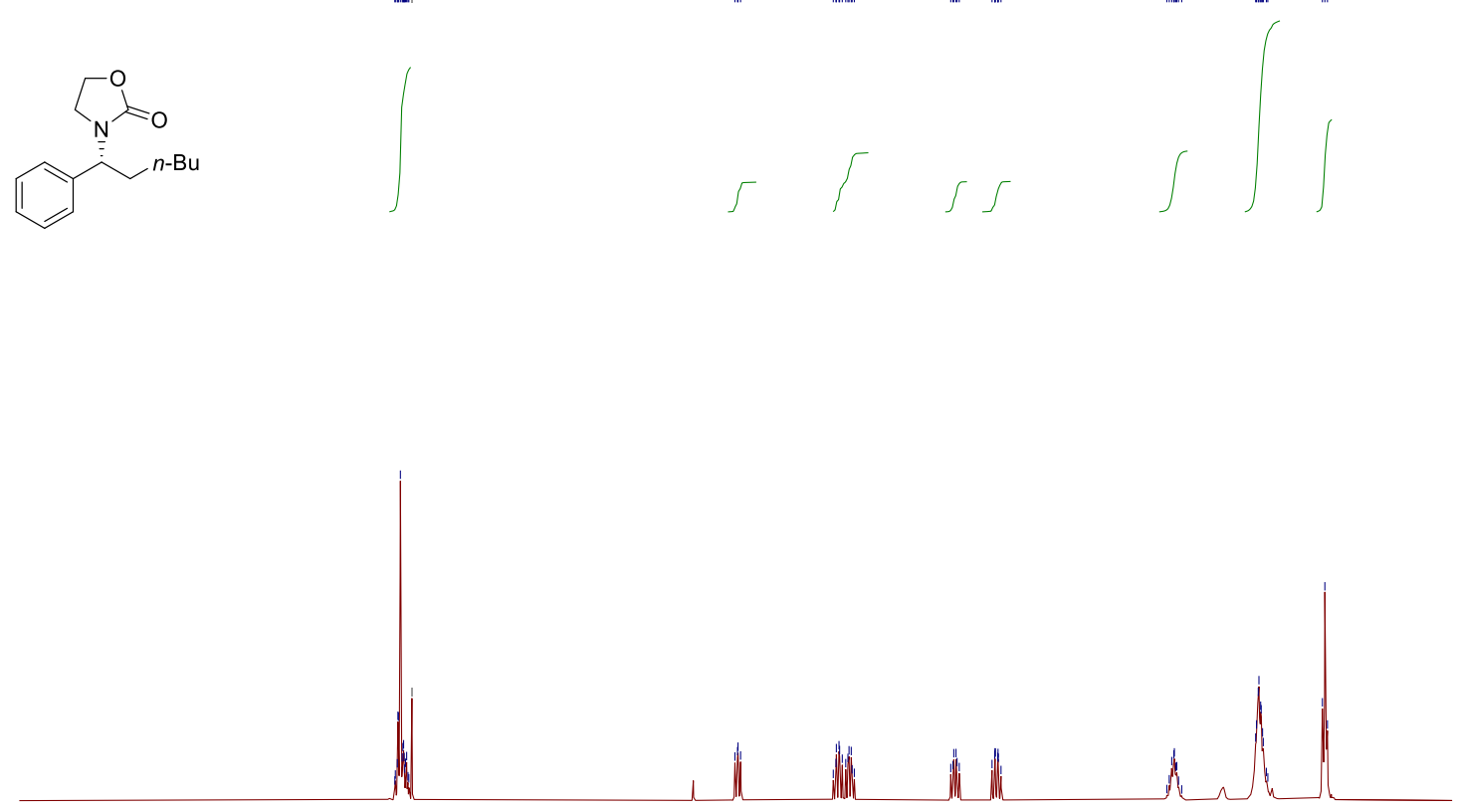

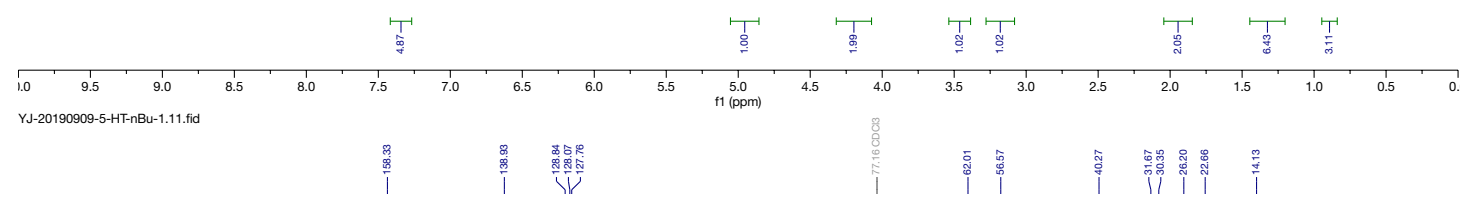<smiles>CCCCCC(Cc1ccccc1)N1CCOC1=O</smiles>
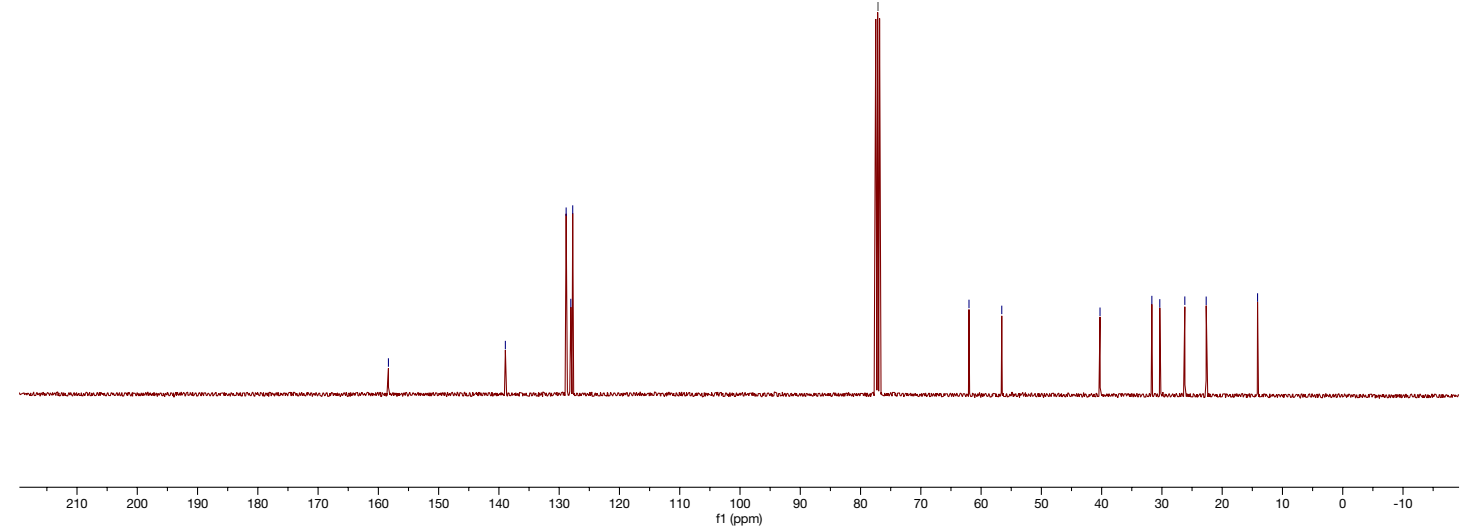


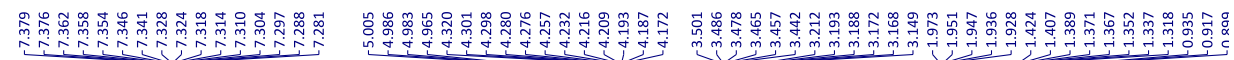<smiles>O=C1OCCN1C(CCP)c1ccccc1</smiles>
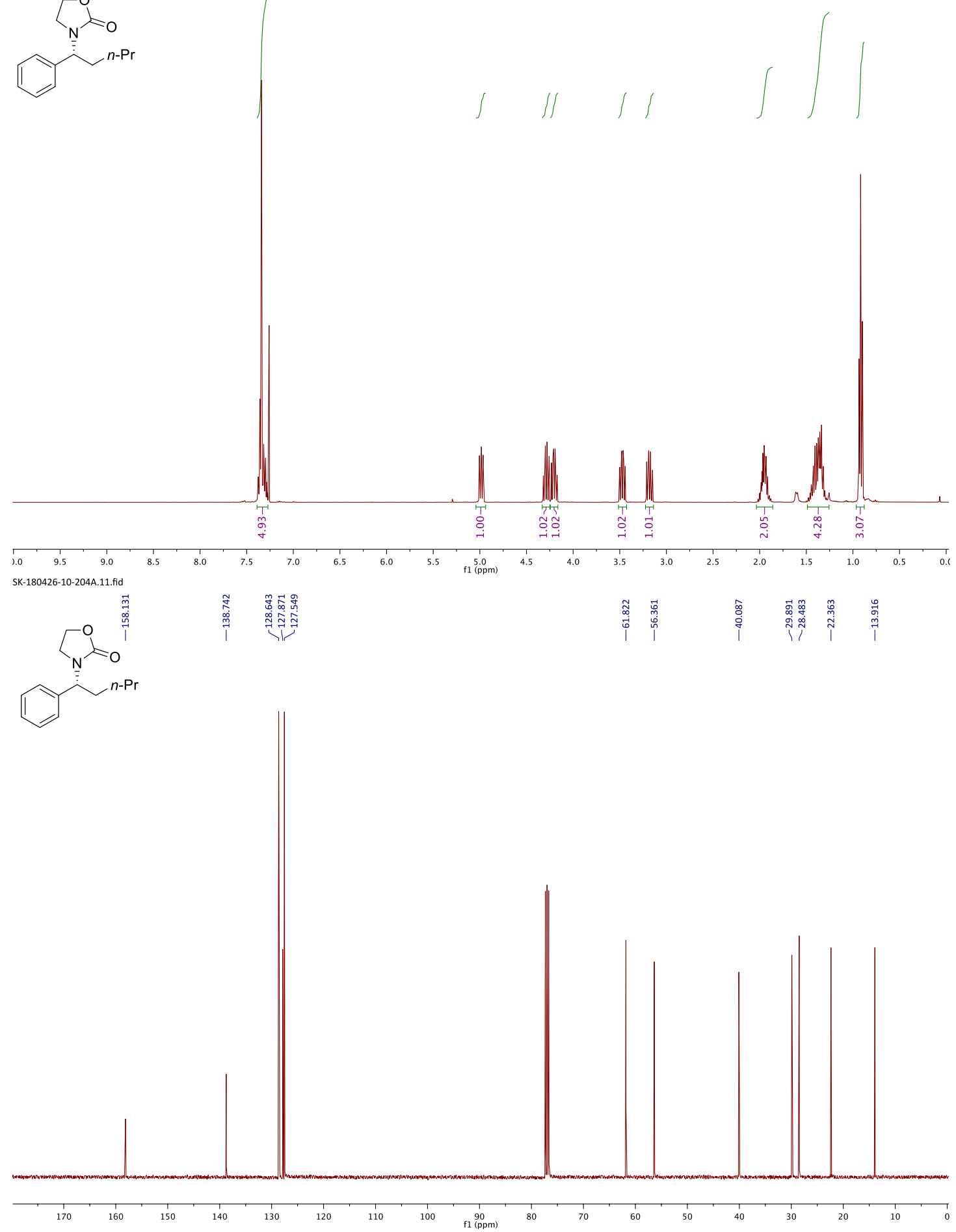

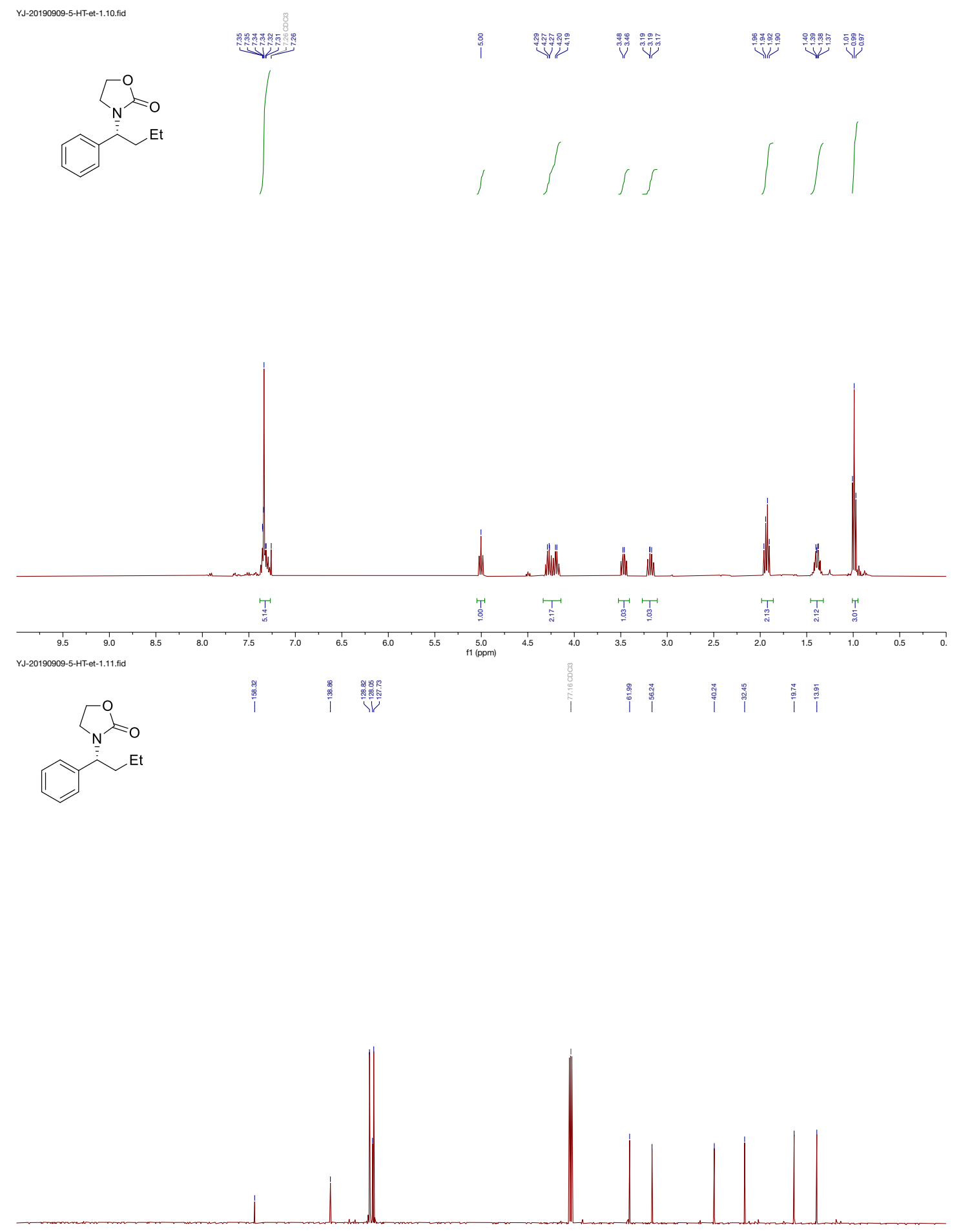

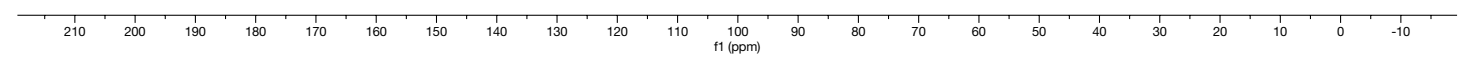



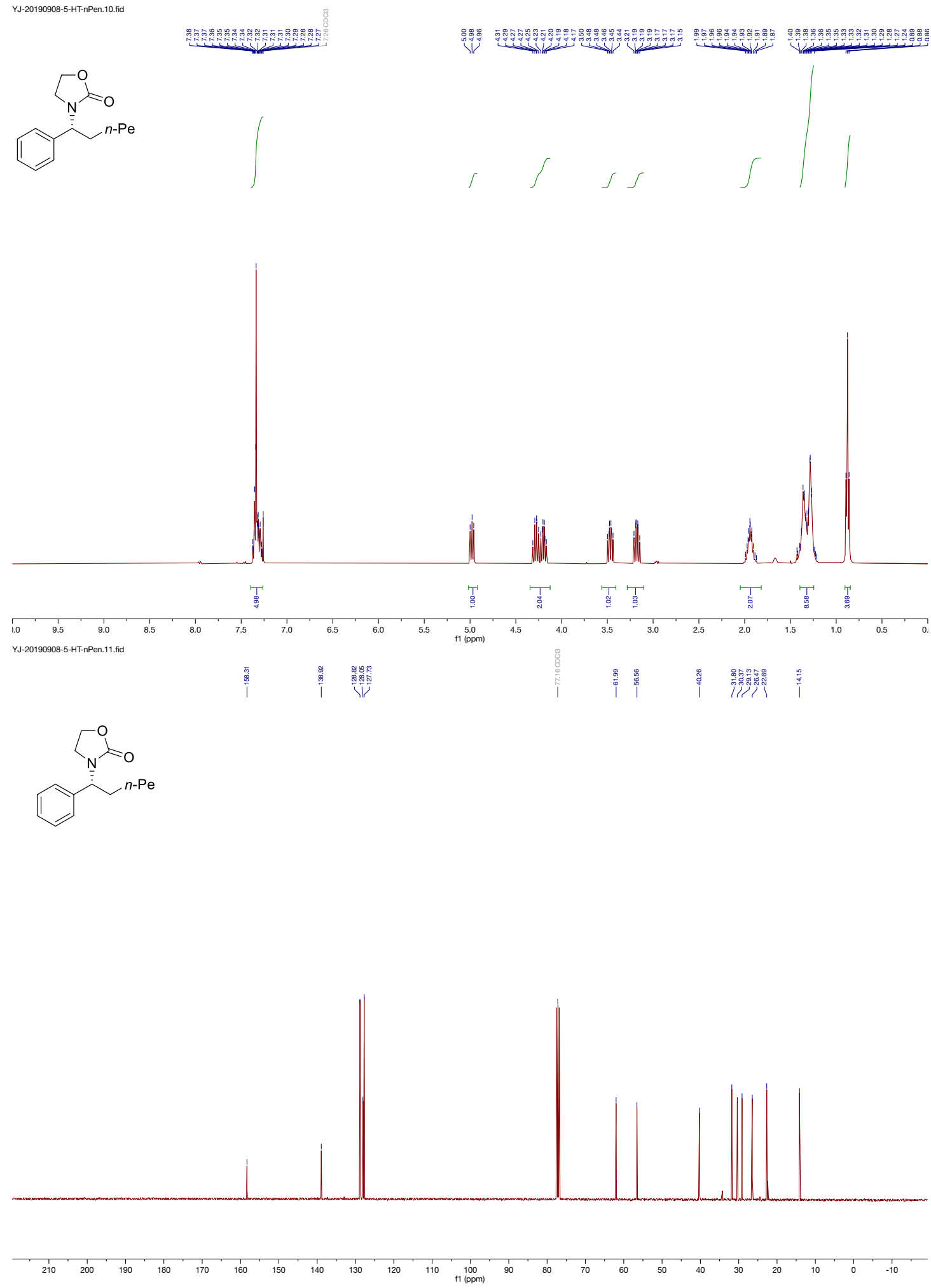

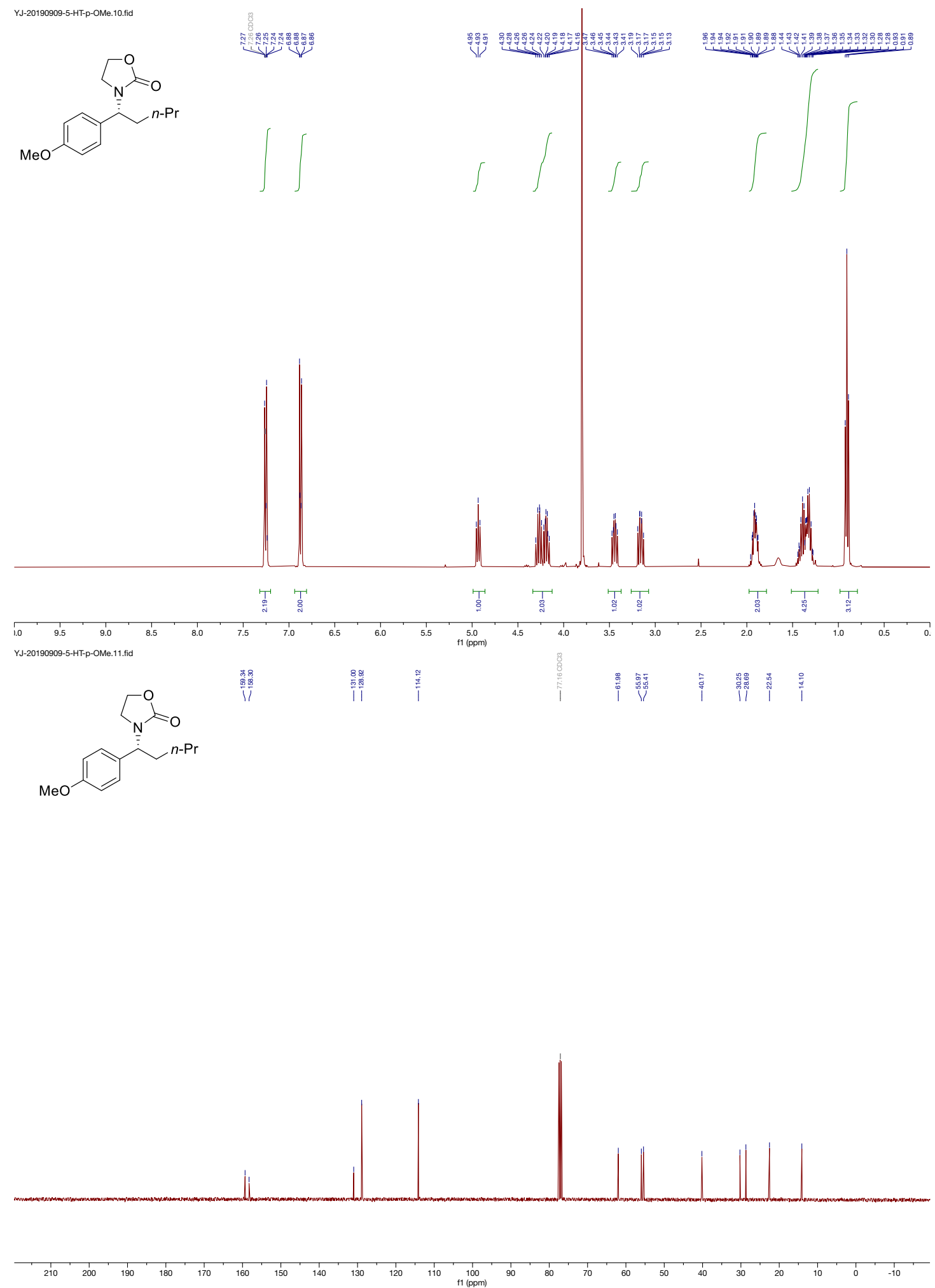

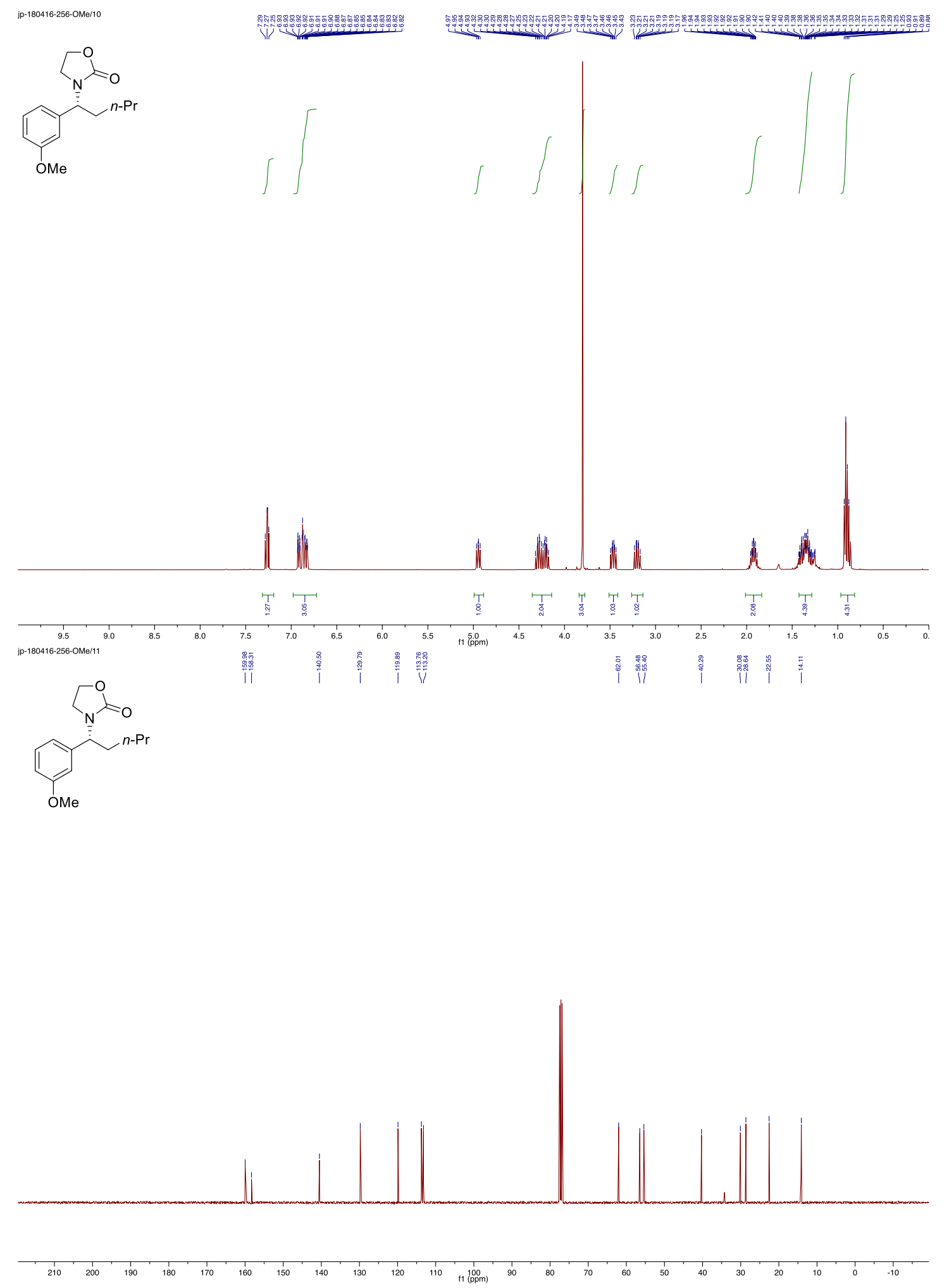

S62 

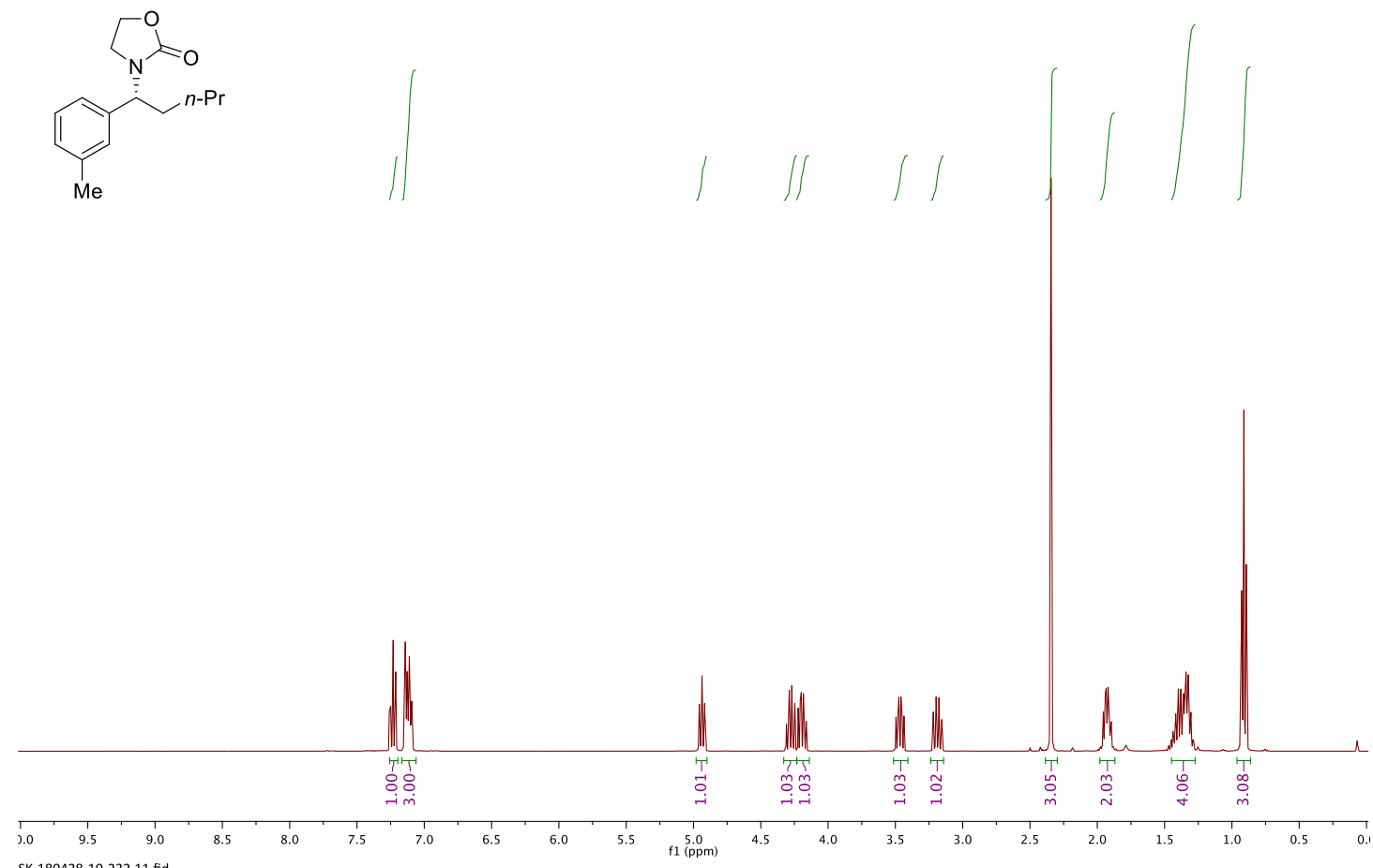

SK-180428-10-232.11.fid
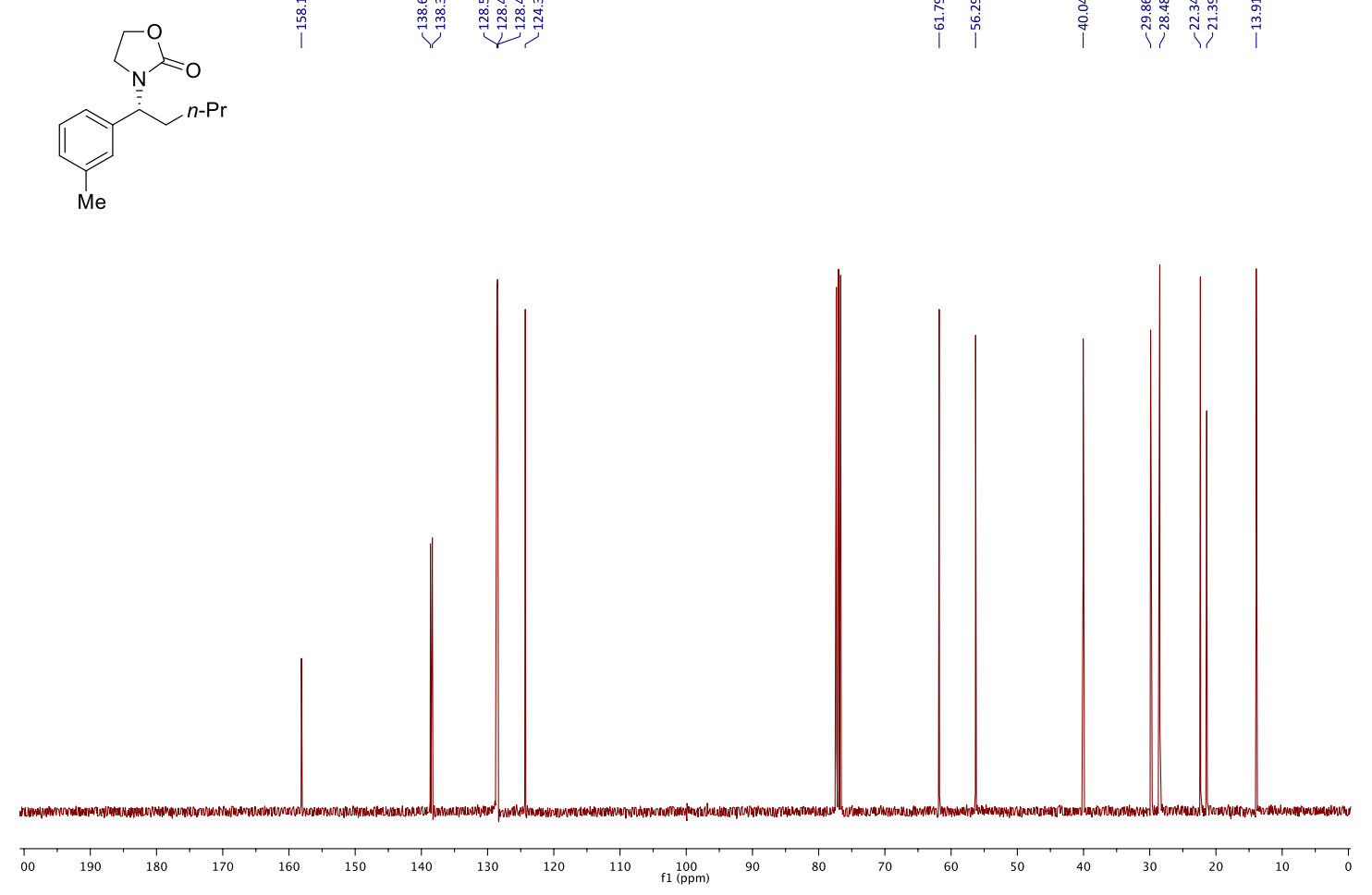
<smiles>O=C1OCCN1[C@H](C[18O])c1cccc(Br)c1</smiles>
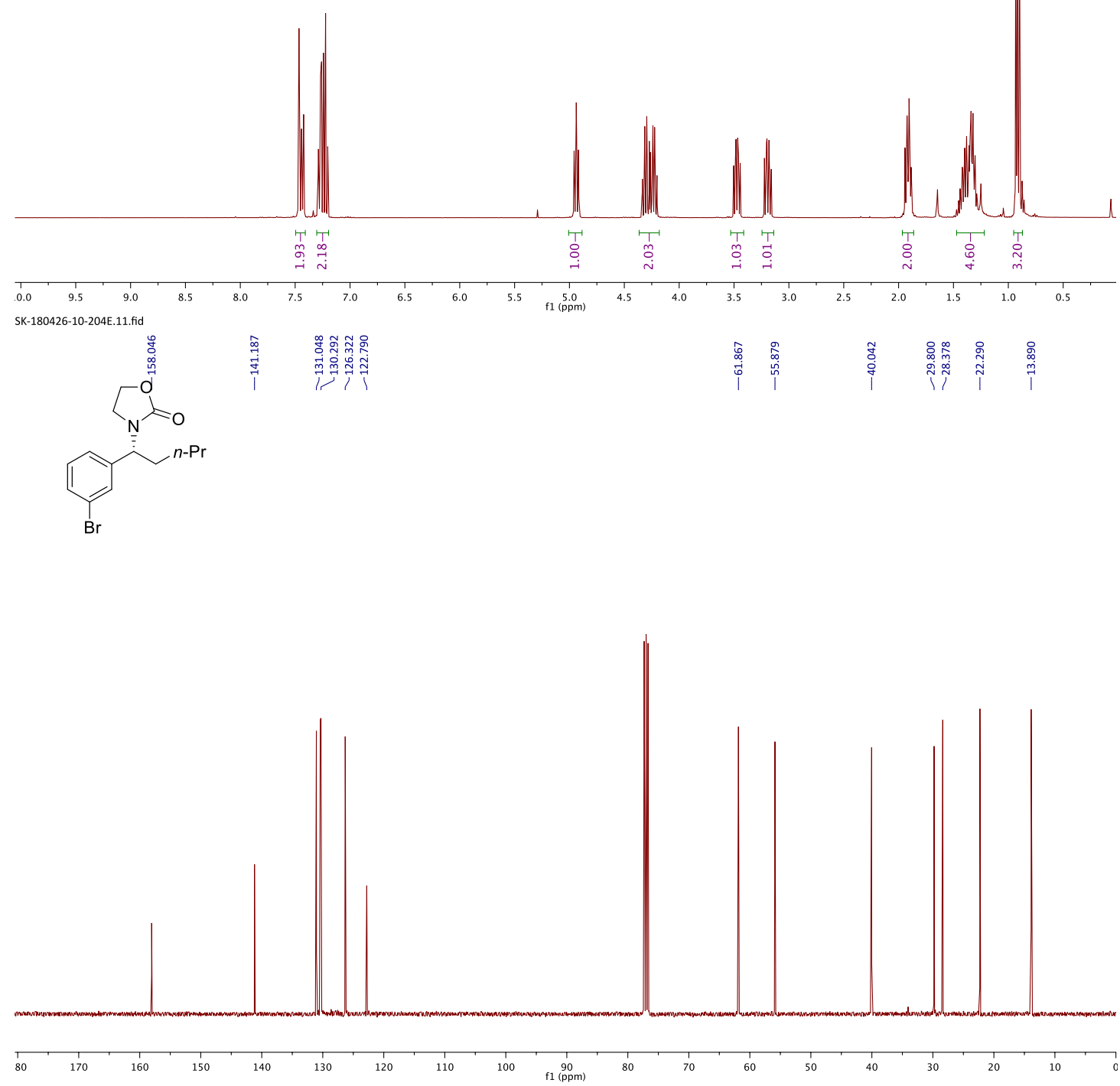


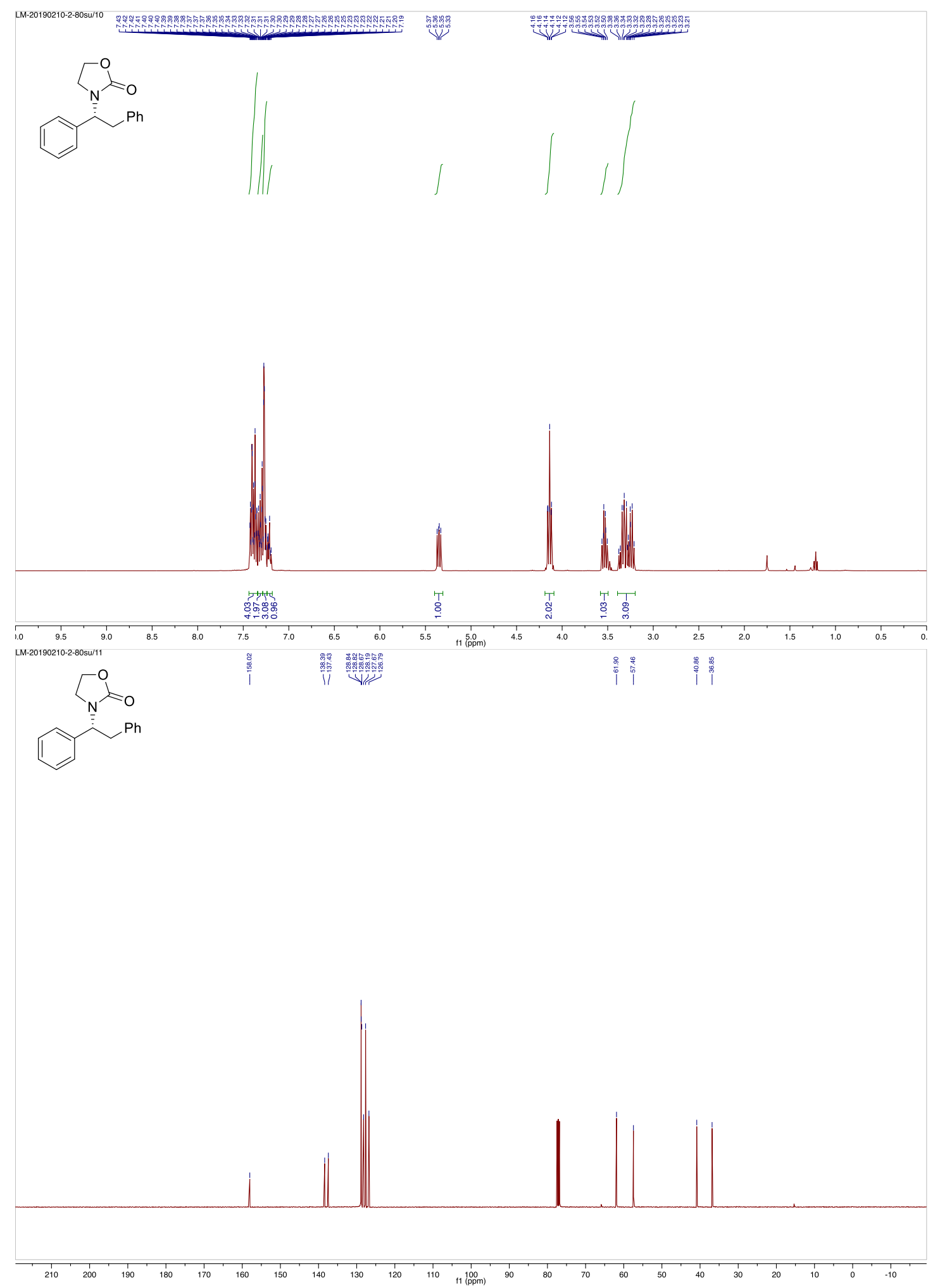

S65 


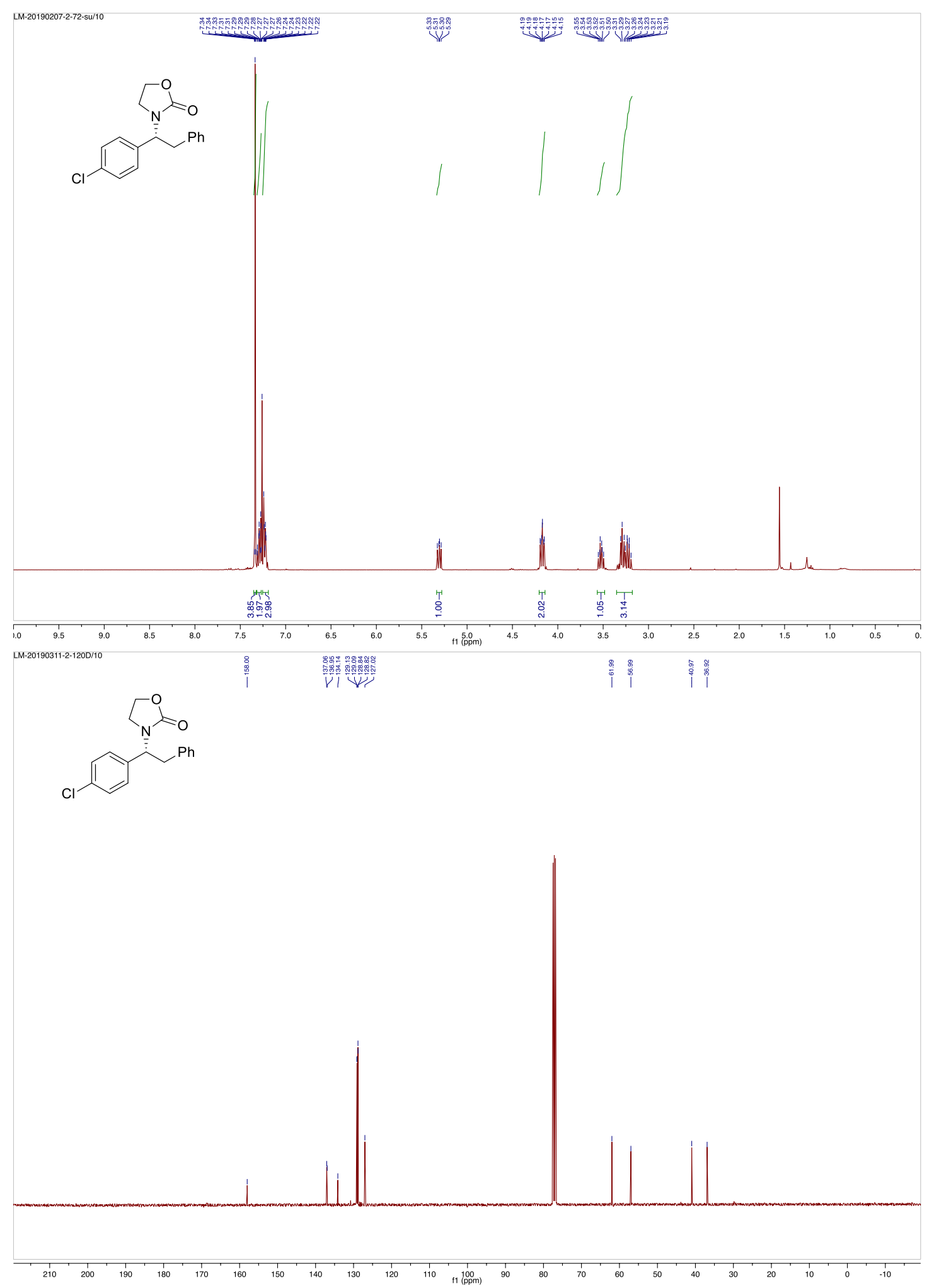

S66 


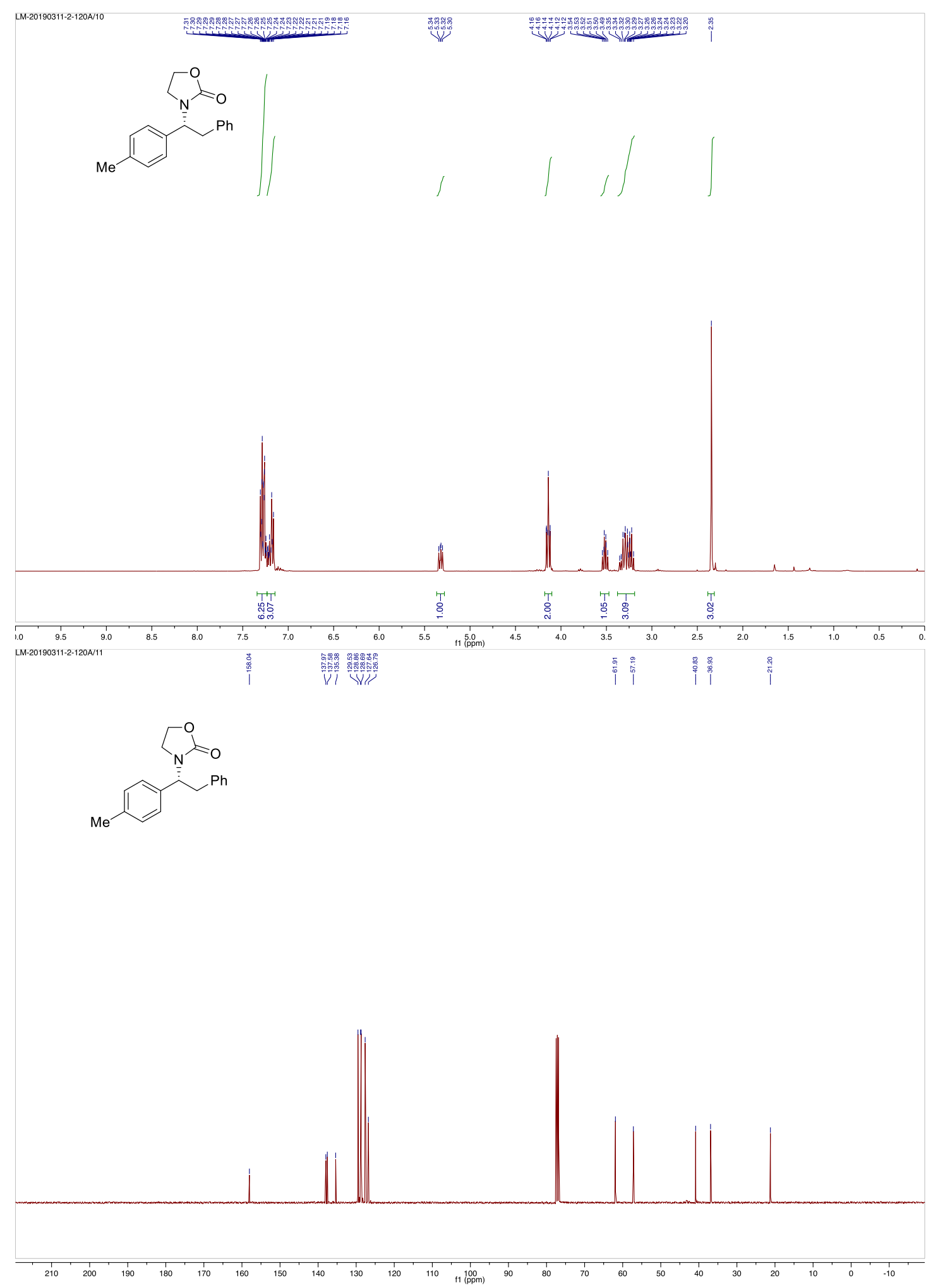

S67 


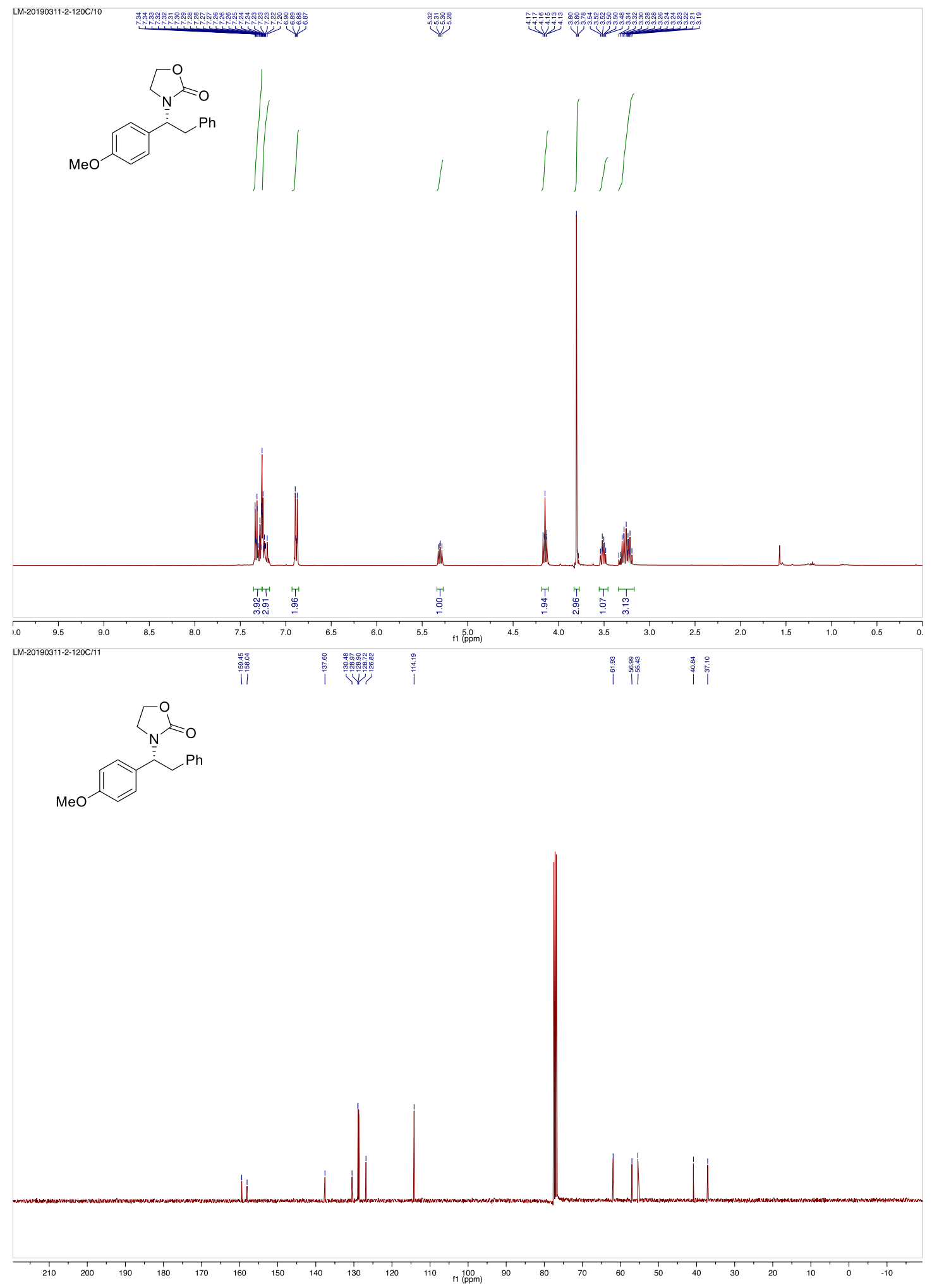

S68 


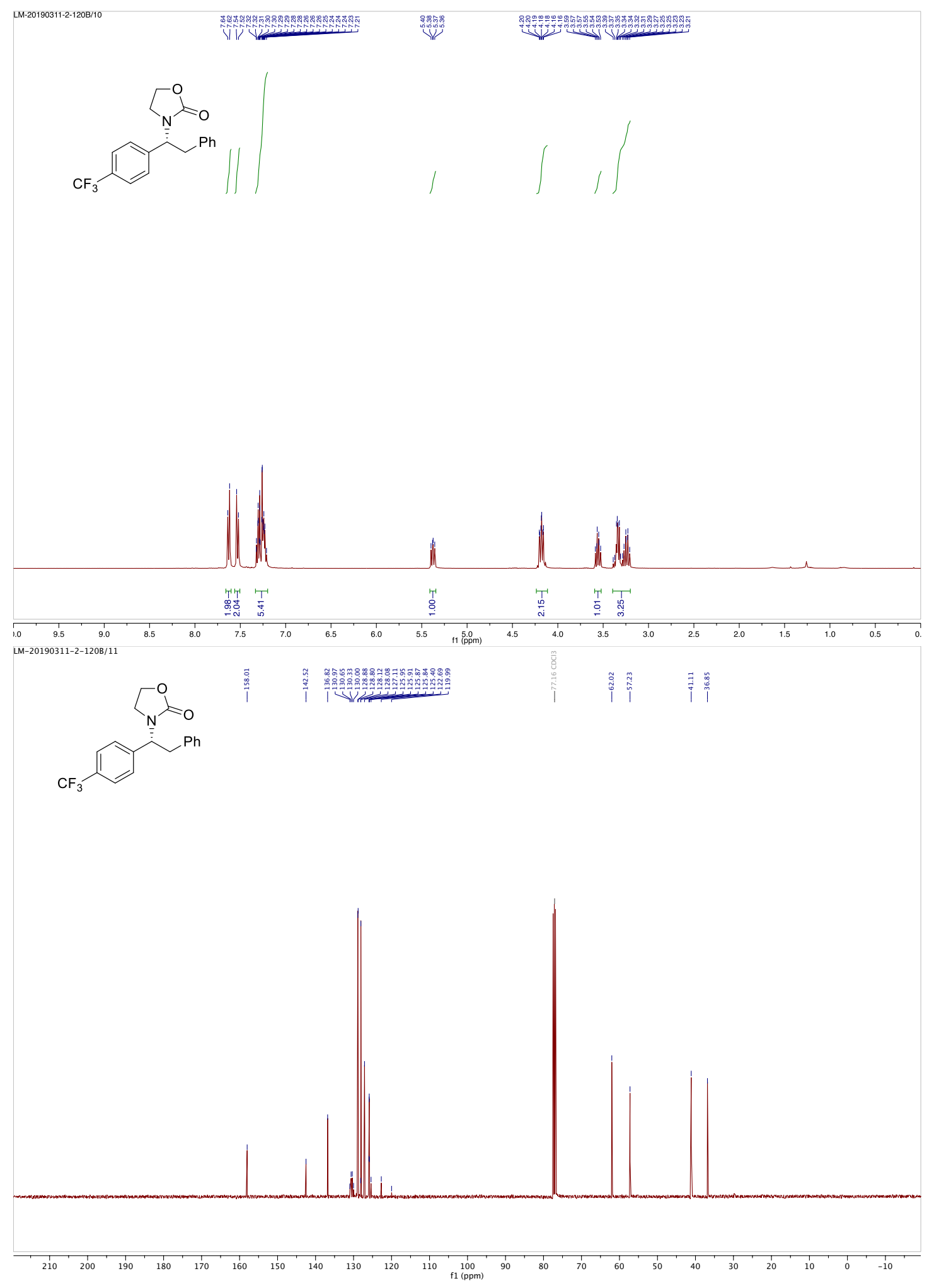

S69 
YJ-20190911-5-a-CF3-P.11.fid<smiles>[CH]c1ccc(C(Cc2ccccc2)N2CCOC2=O)cc1</smiles>

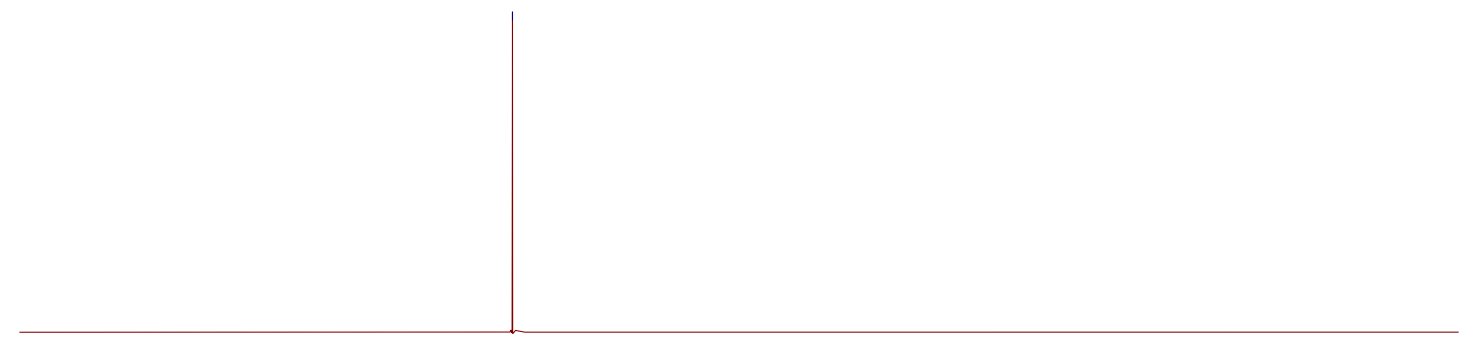

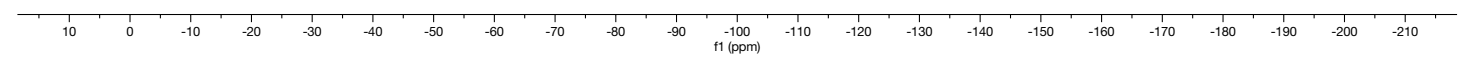



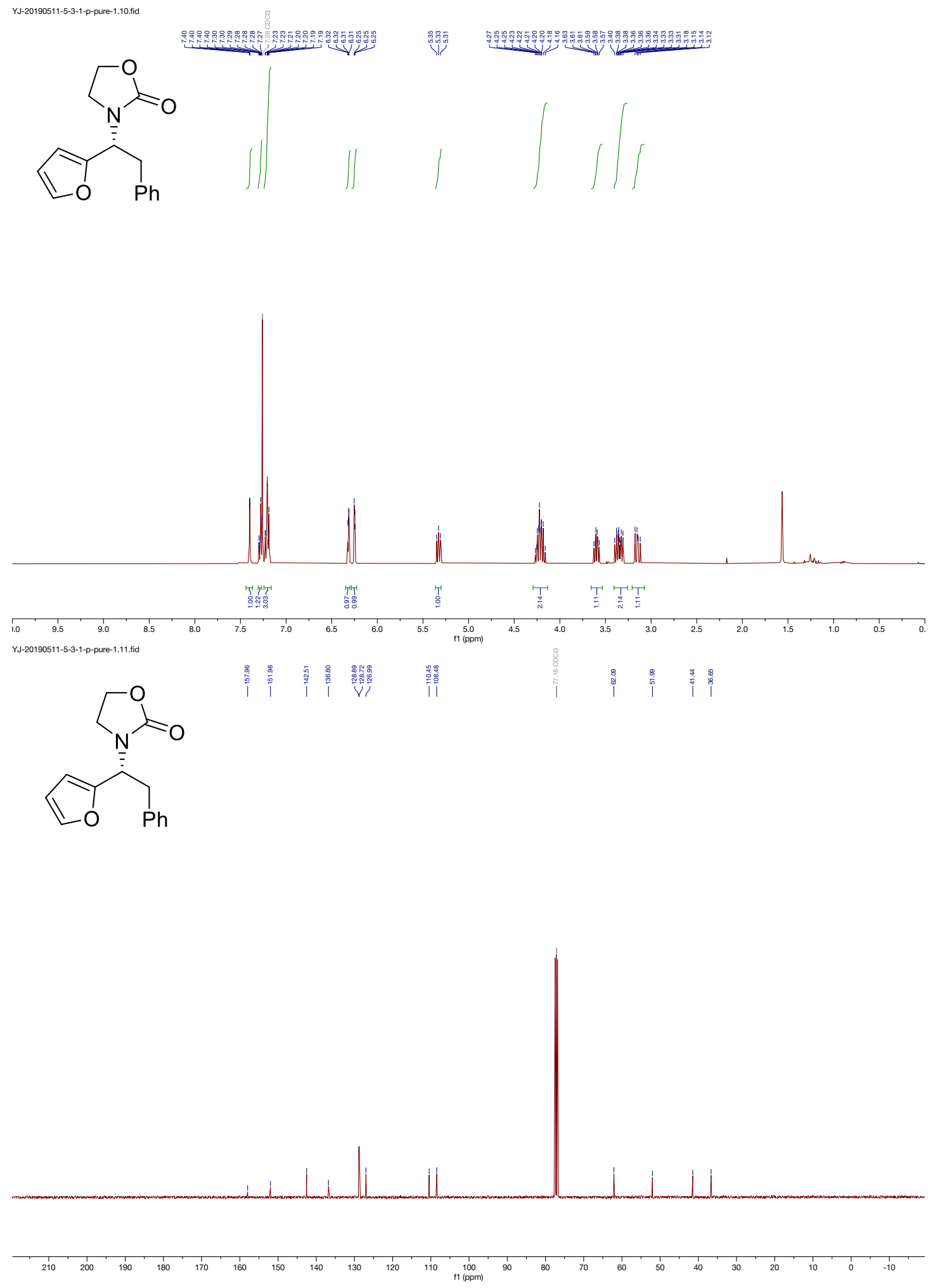


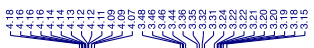<smiles>O=C1OCCN1[C@@H](Cc1ccccc1)c1ccsc1</smiles>
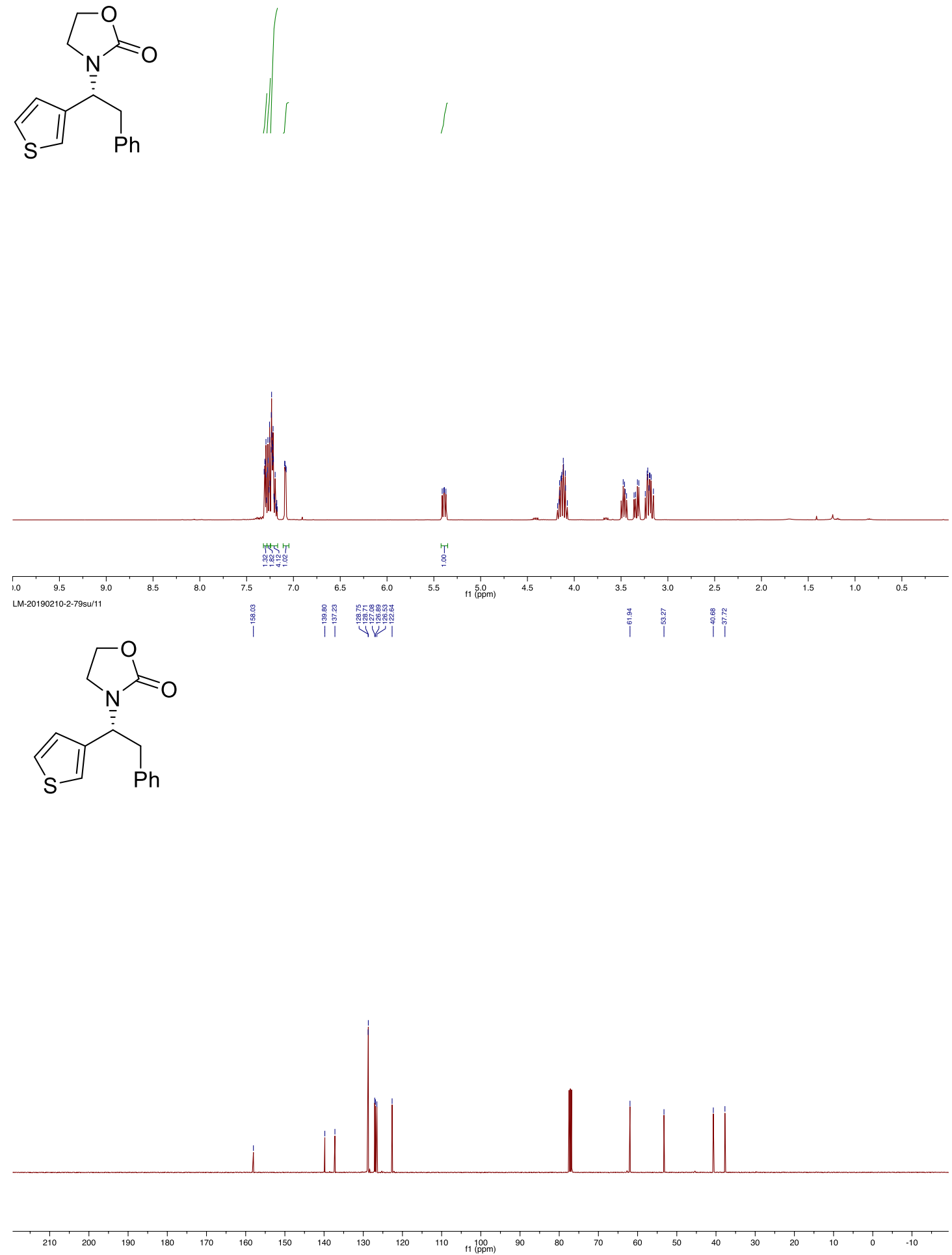


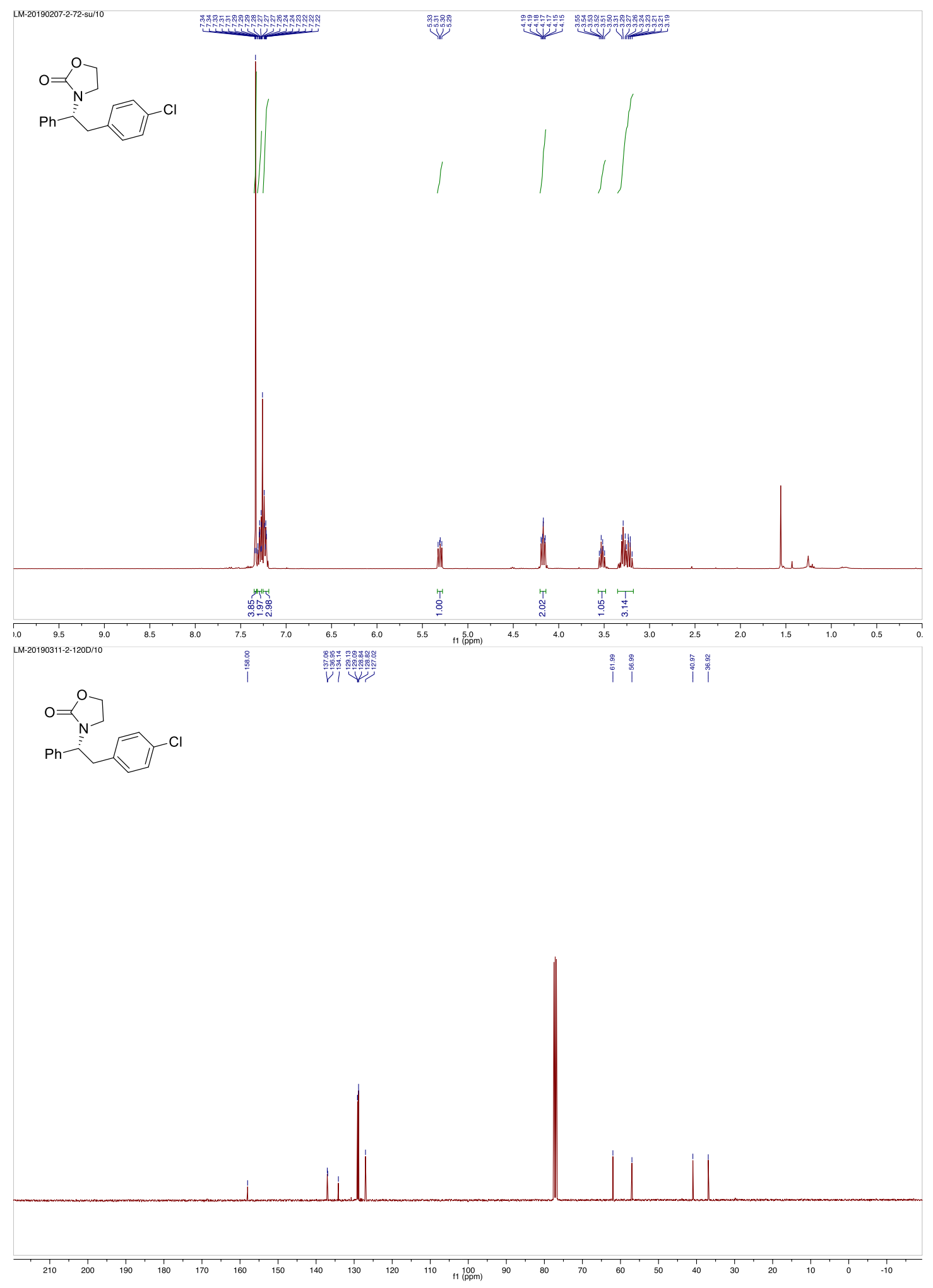

S73 

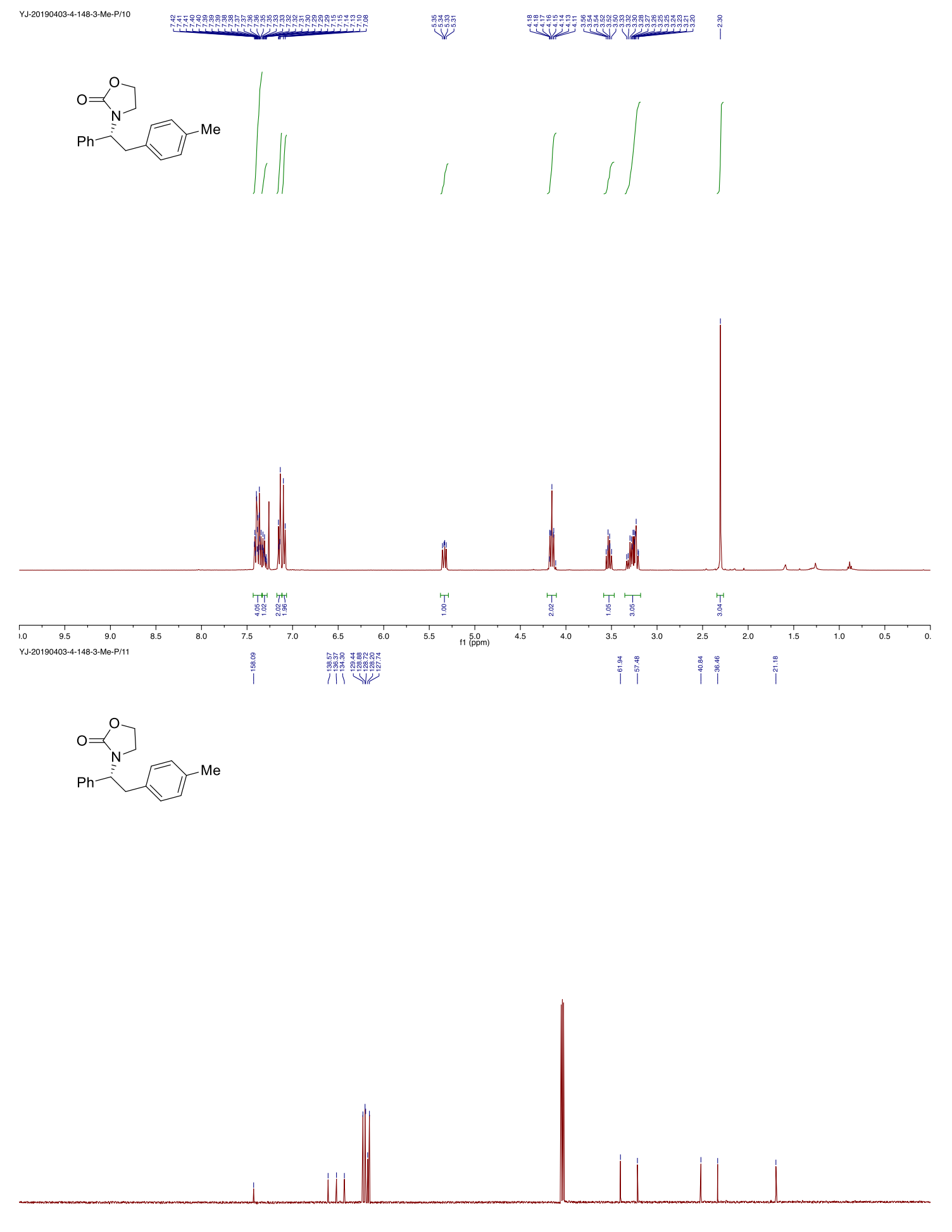

S74 


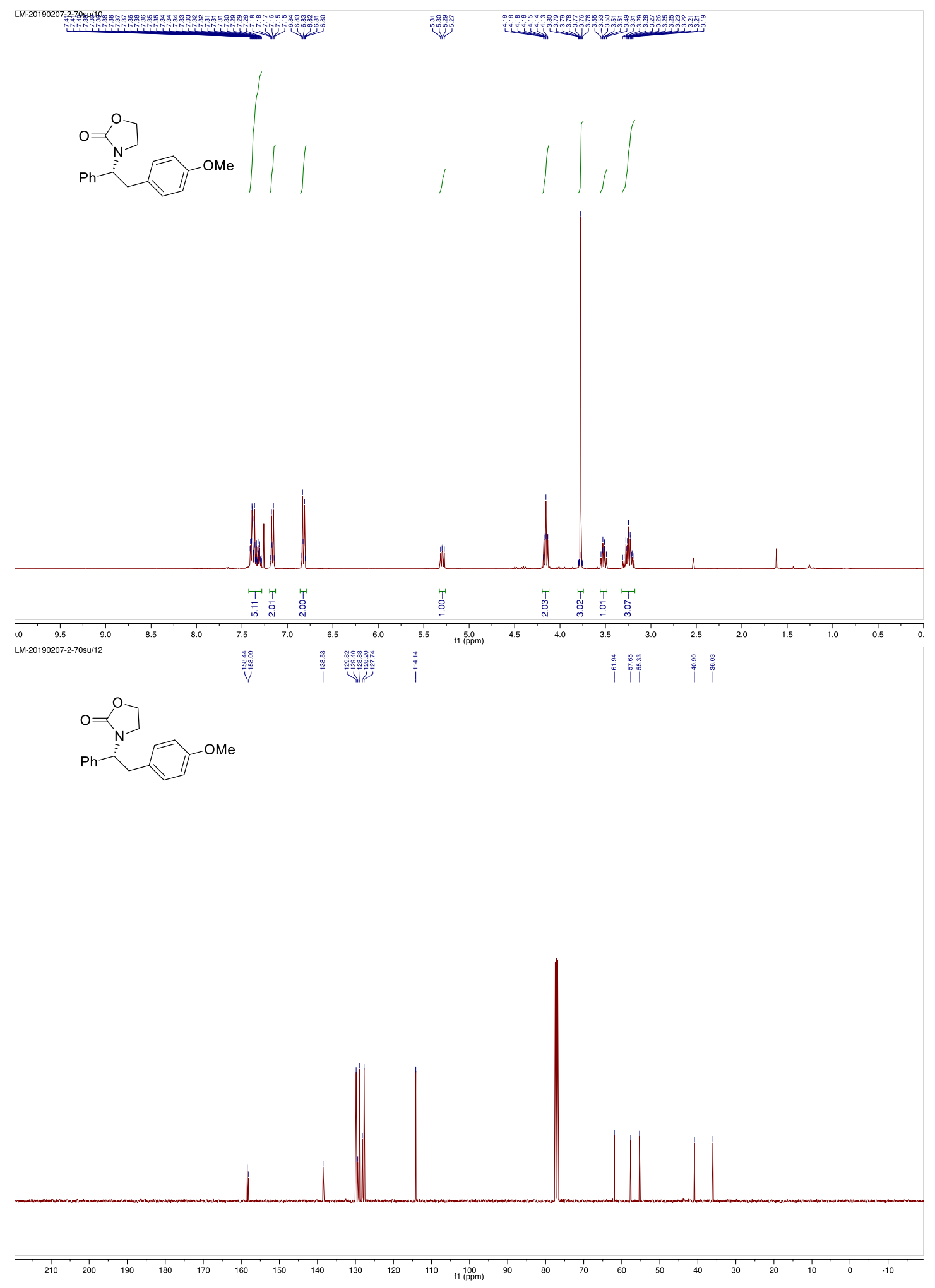

S75 
LM-20190207-2-71-su/10

留骂早

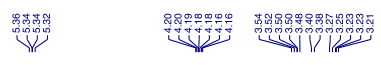
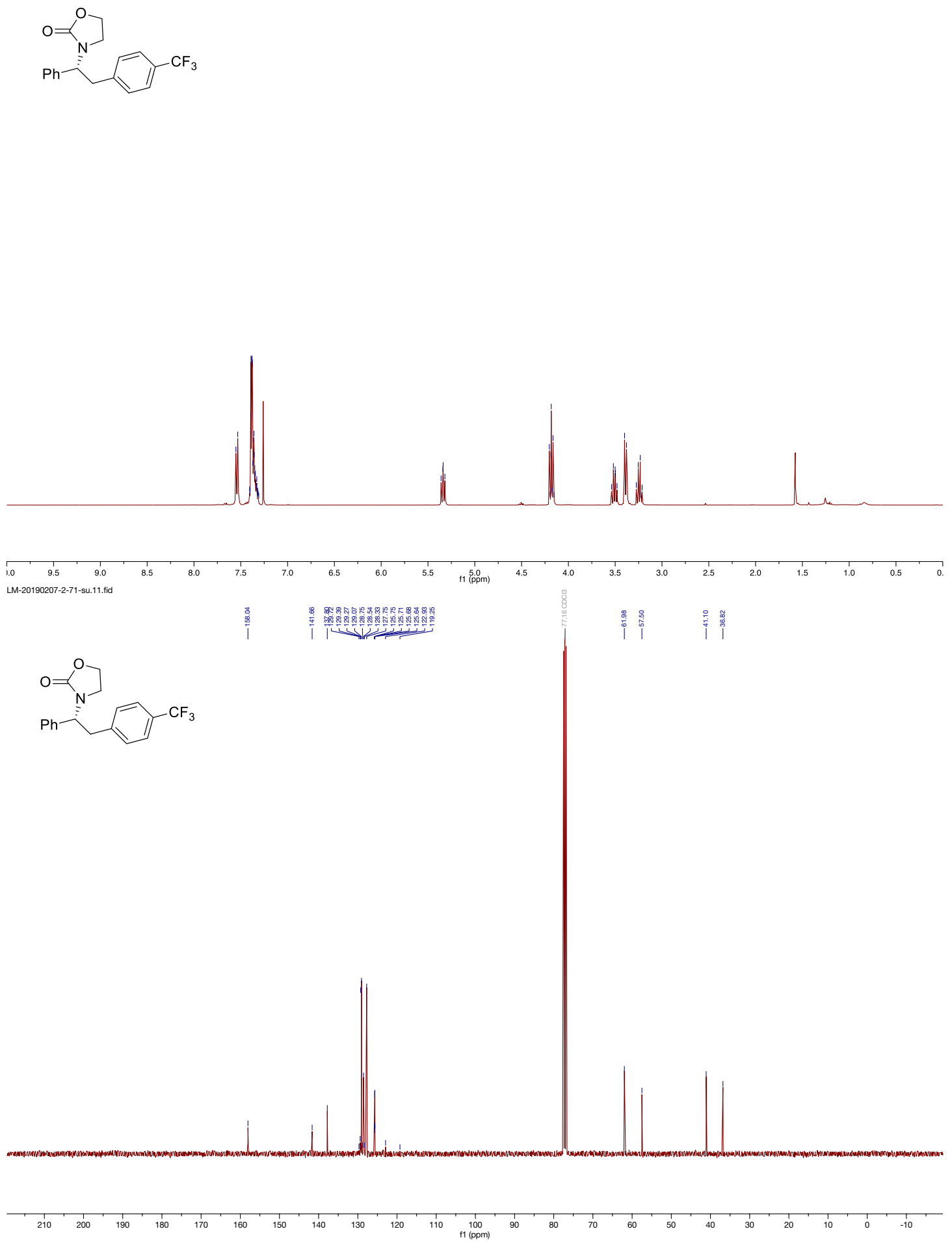

S76 

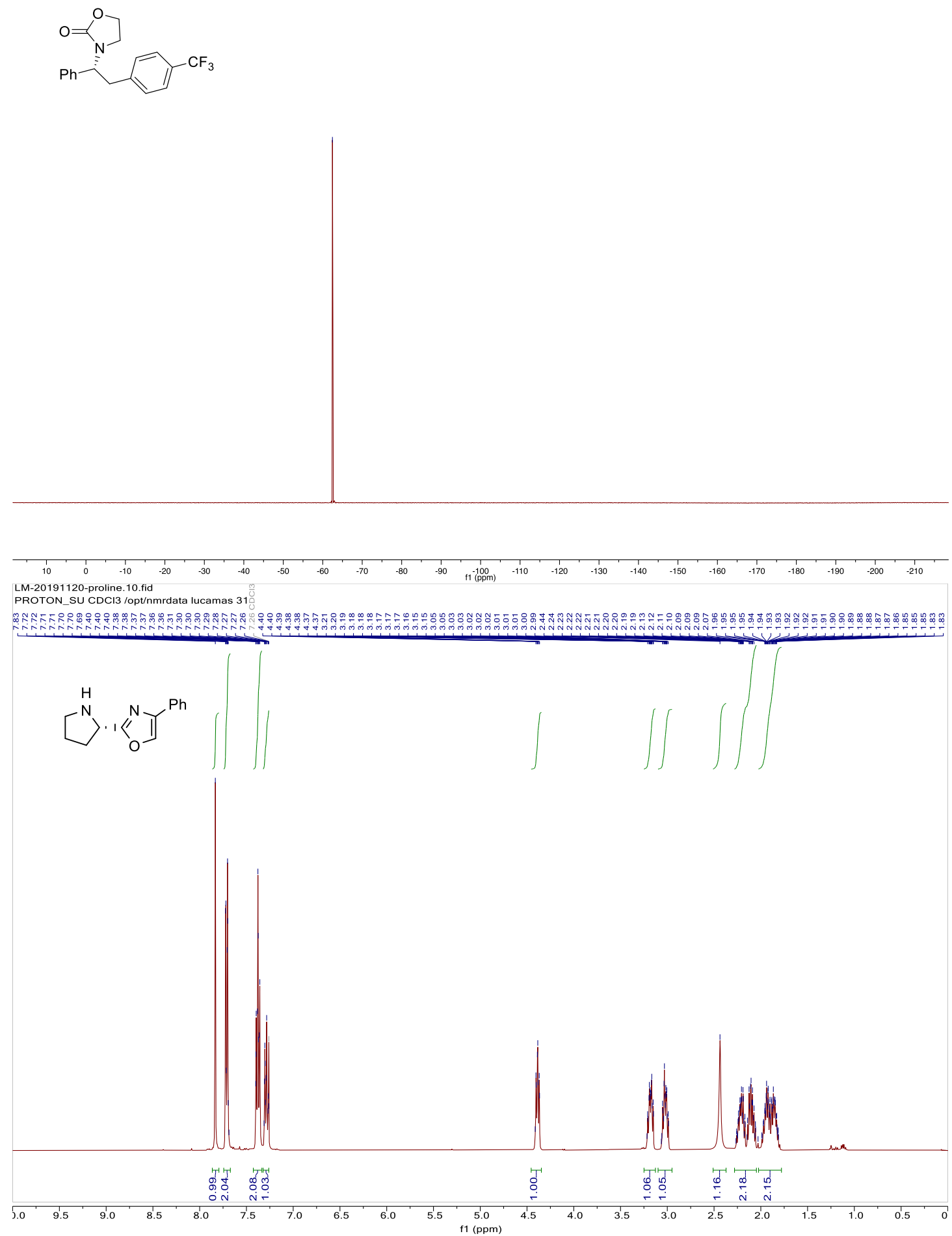

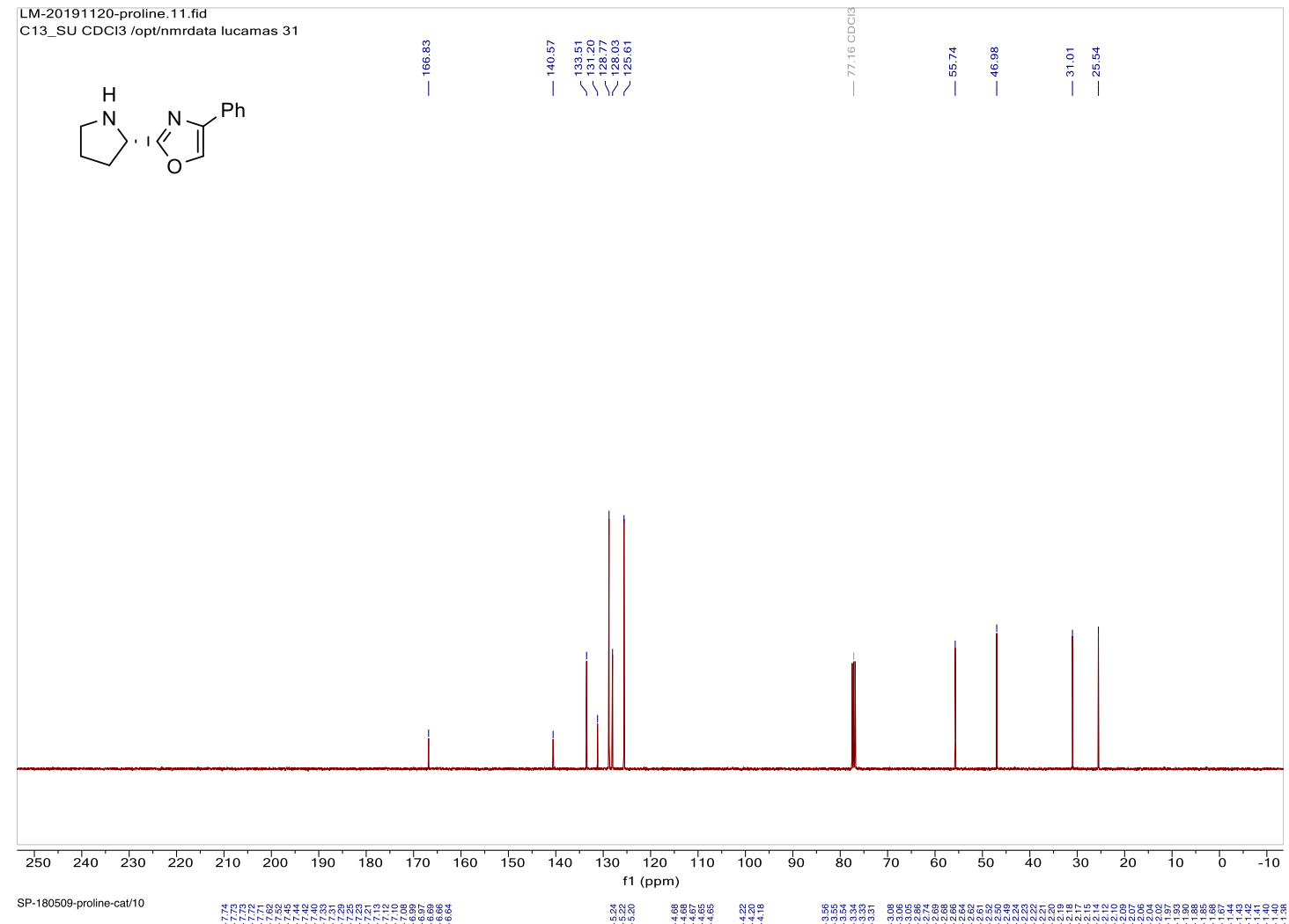
SP-180509-proline-cat10

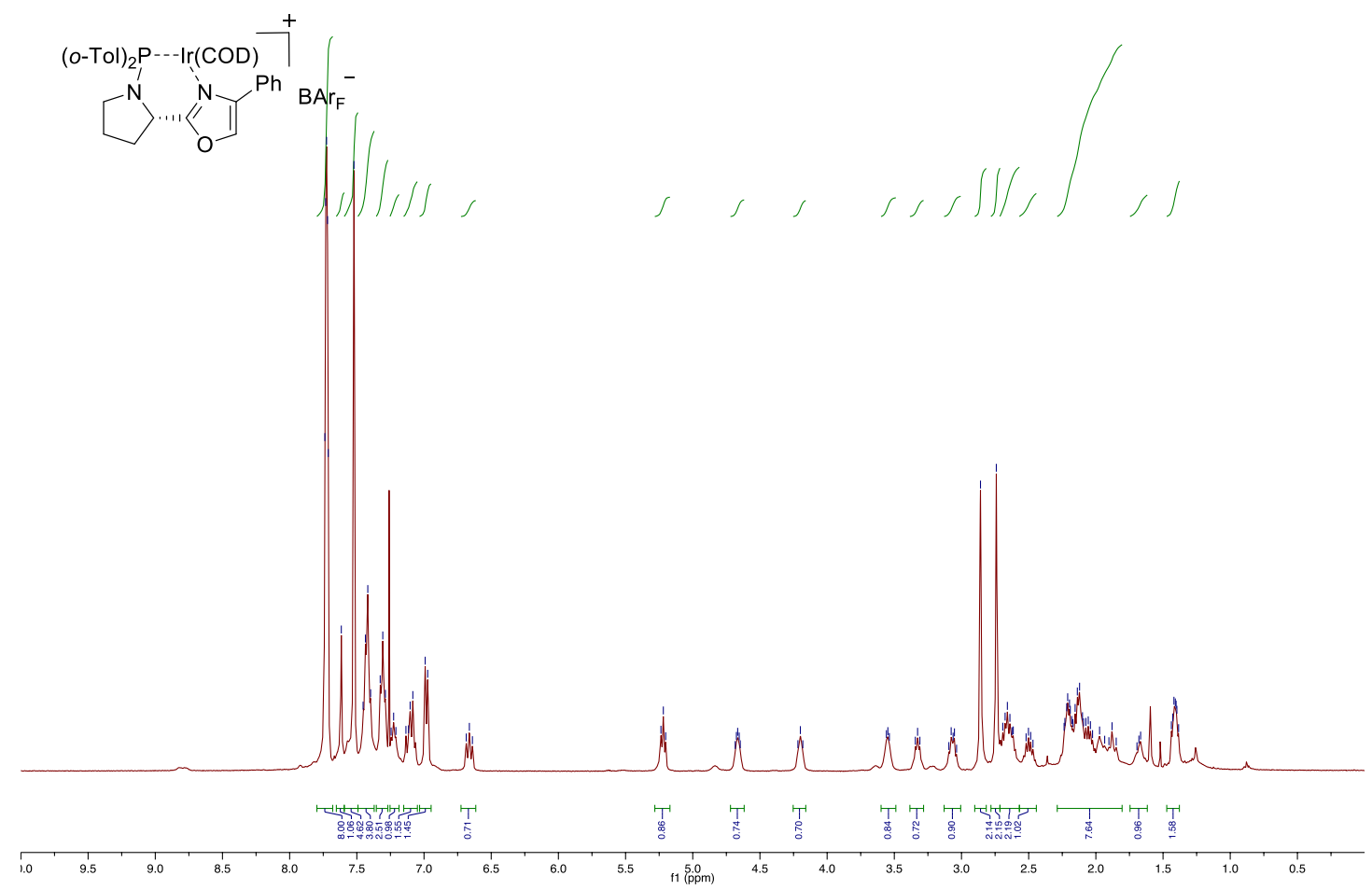



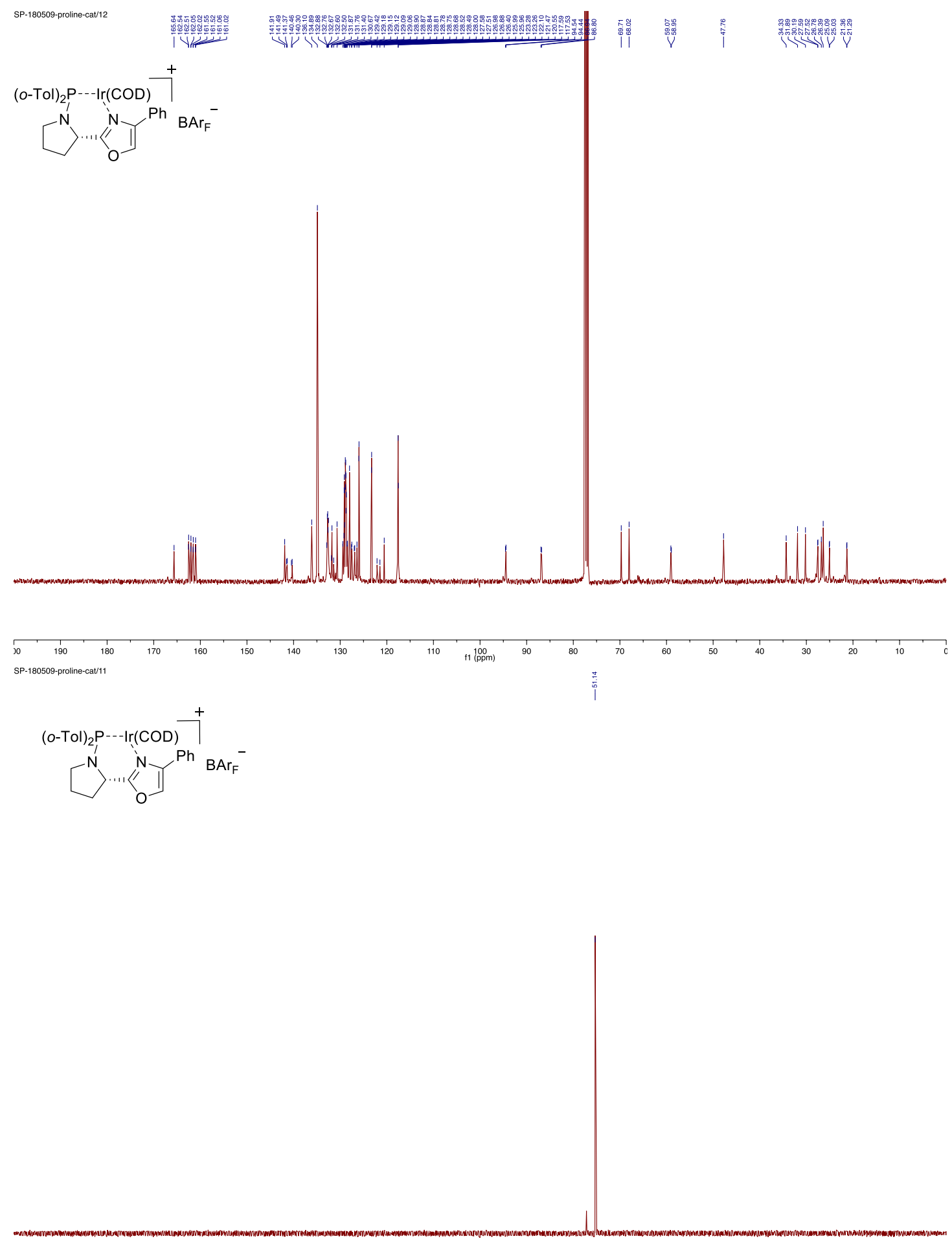

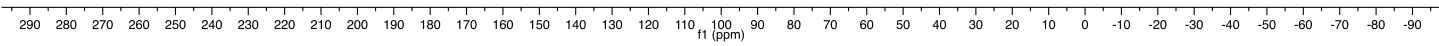




\section{Chromatogram of chiral compounds}
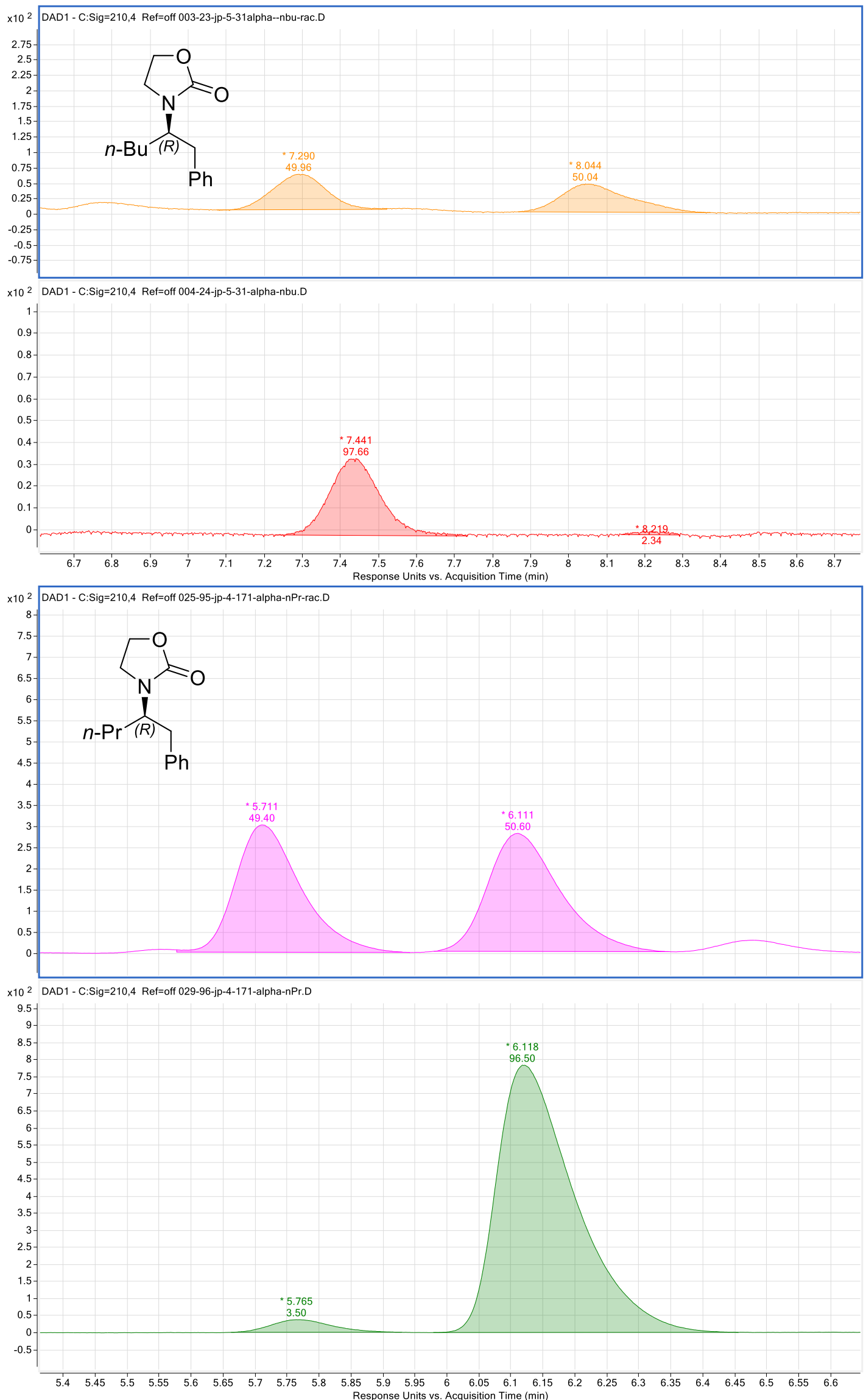


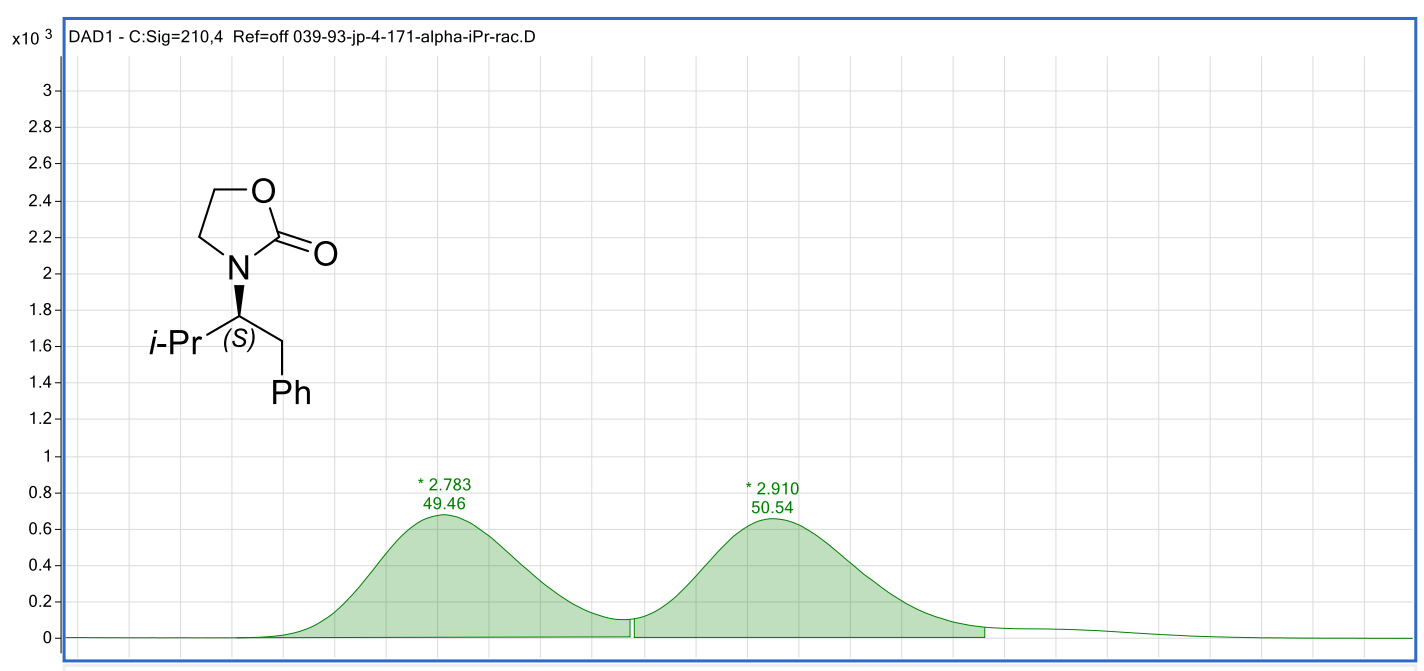

x10 3 DAD1 - C:Sig=210,4 Ref=off 040-94-jp-4-171-alpha-iPr.D

$$
\begin{aligned}
& 1.5 \\
& 4
\end{aligned}
$$

$0.3-2-1$
0.1

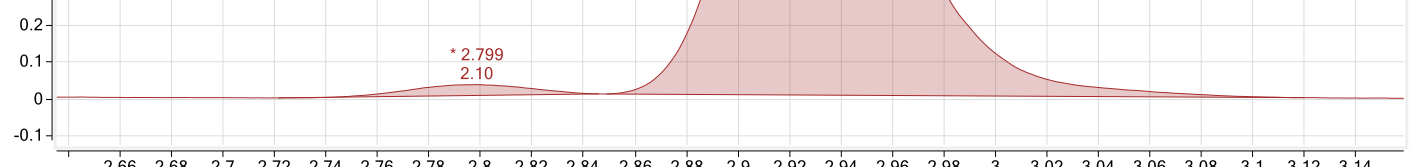

$-0.1$

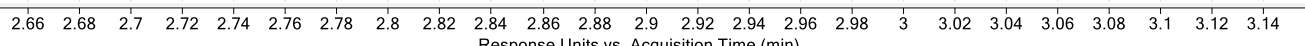

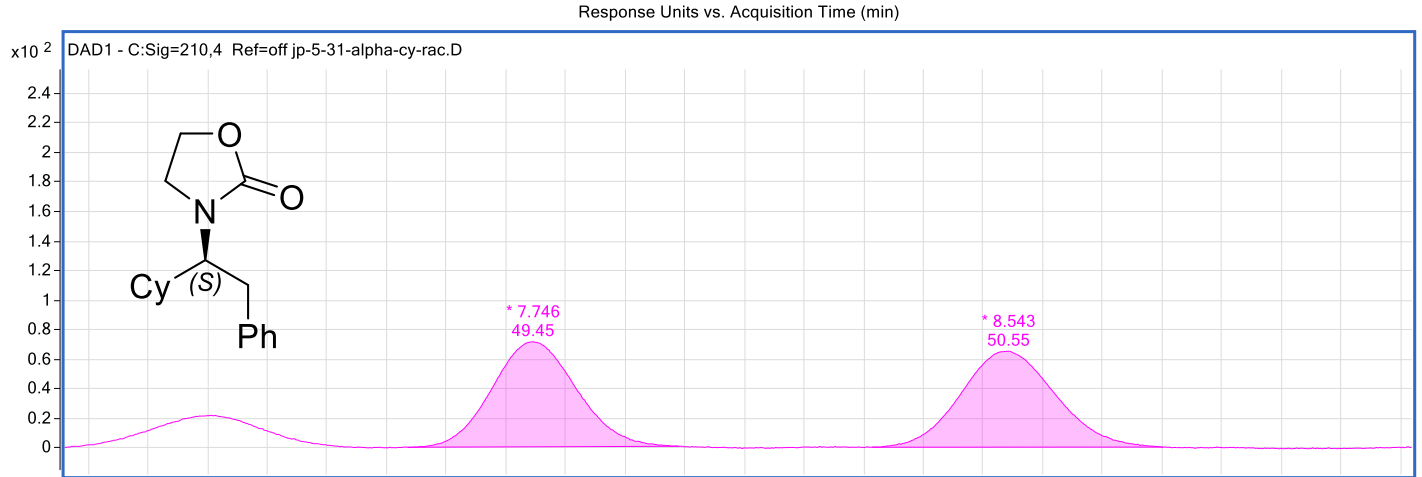

x10 3 DAD1 - C:Sig=210,4 Ref=off 002-100-jp-5-31-alpha-cy.D

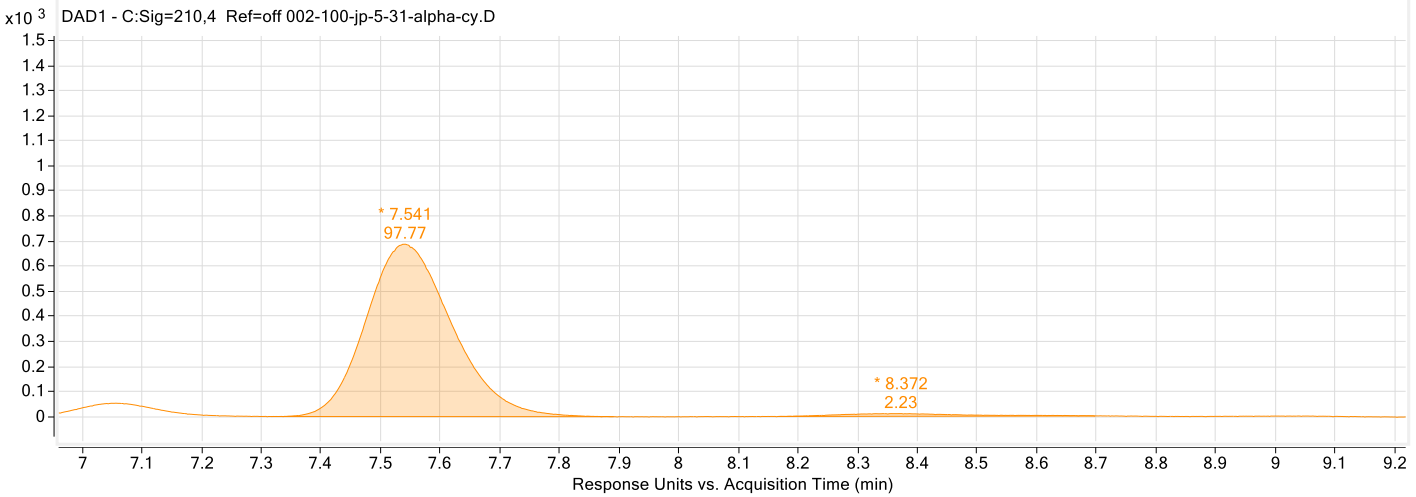




\section{$\underline{\text { Chromatogram Plots }}$}

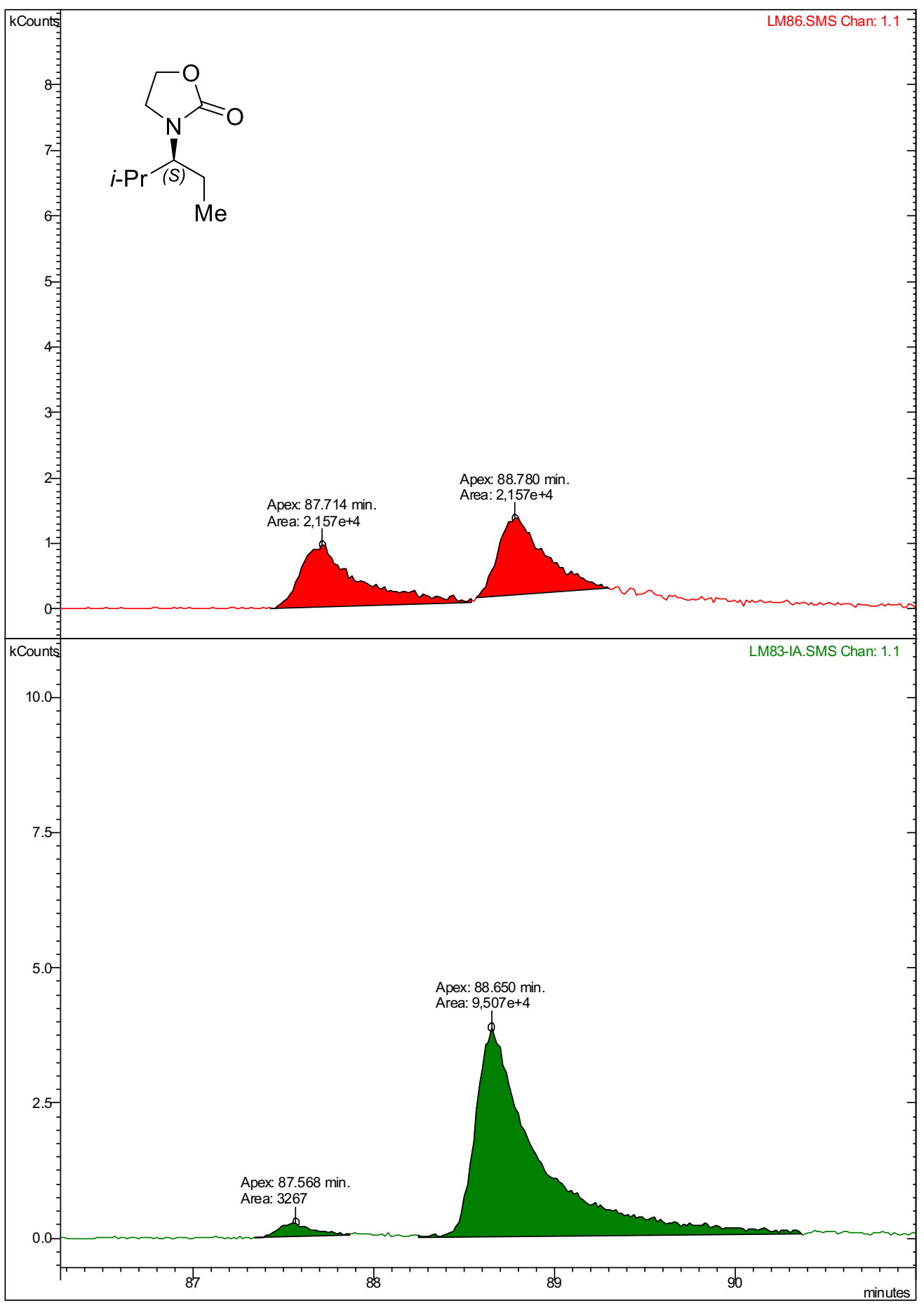




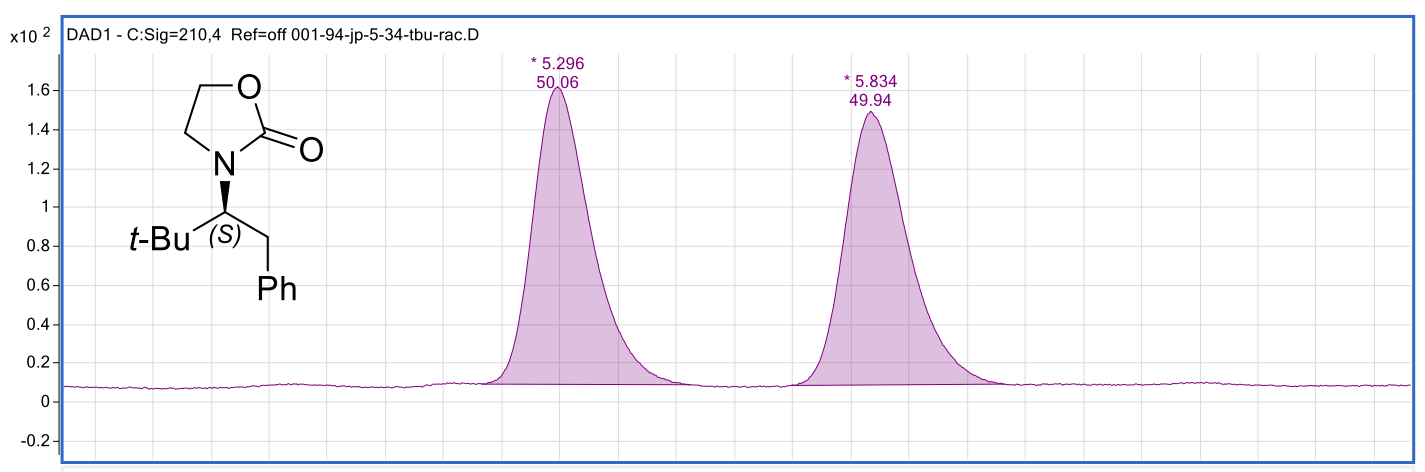

x10 2 DAD1 - C:Sig=210,4 Ref=off 001-97-jp-5-34-tbu-mix.D
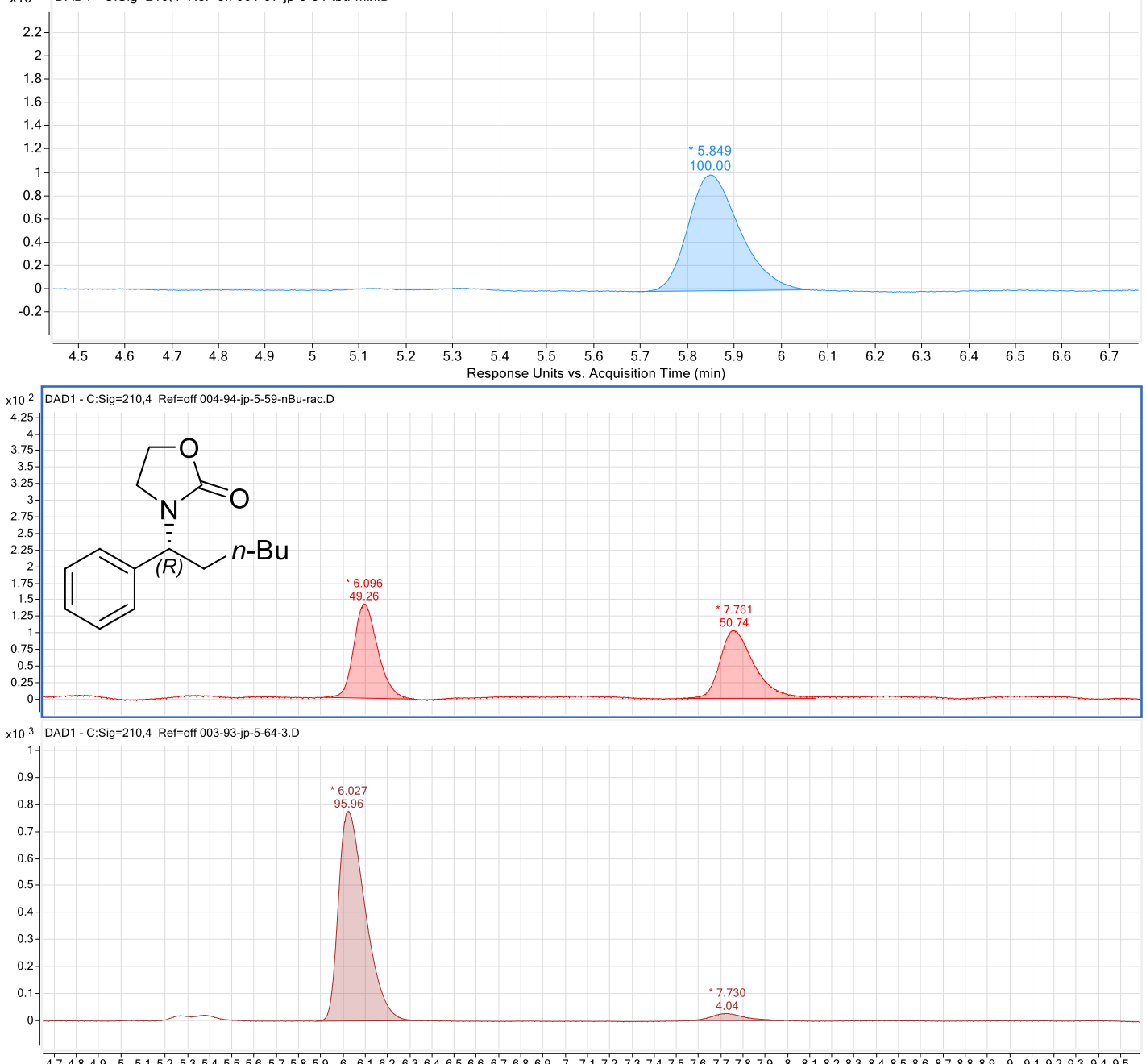

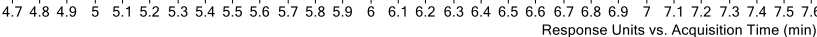



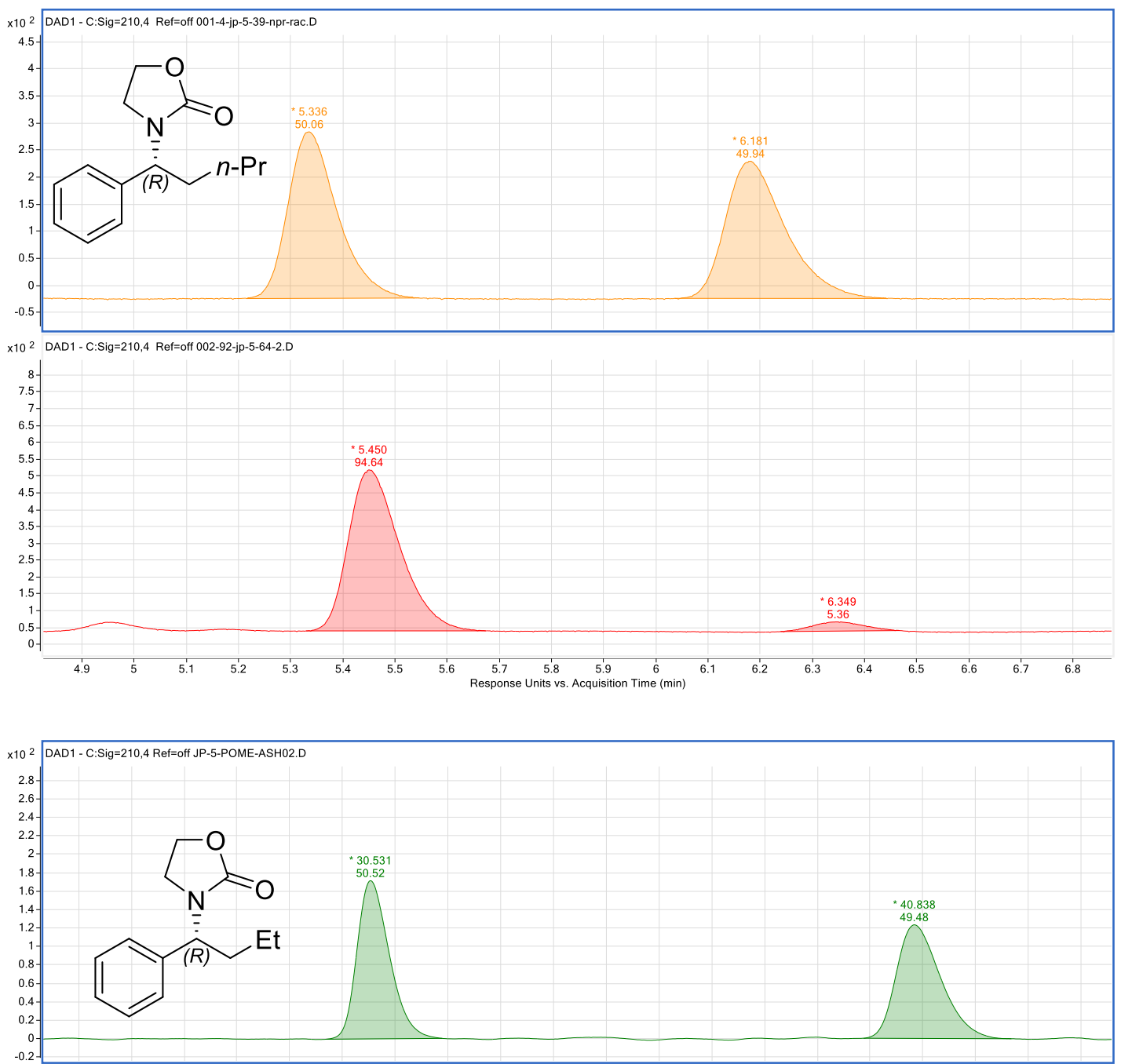

$\times 10^{2}$ DAD1 - C:Sig=210,4 Ref=off JP-5-POME-ASH03.D

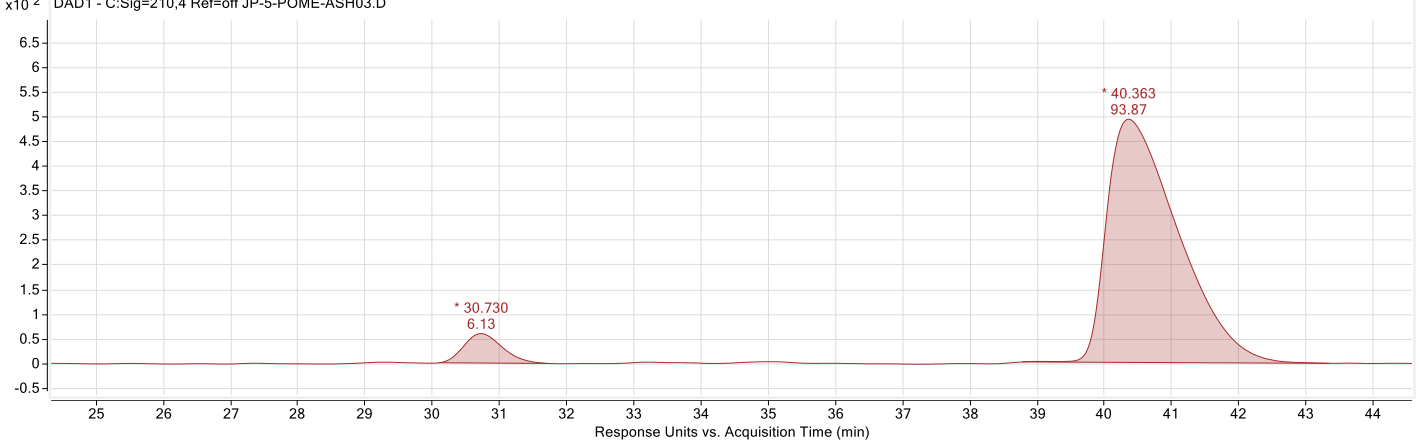



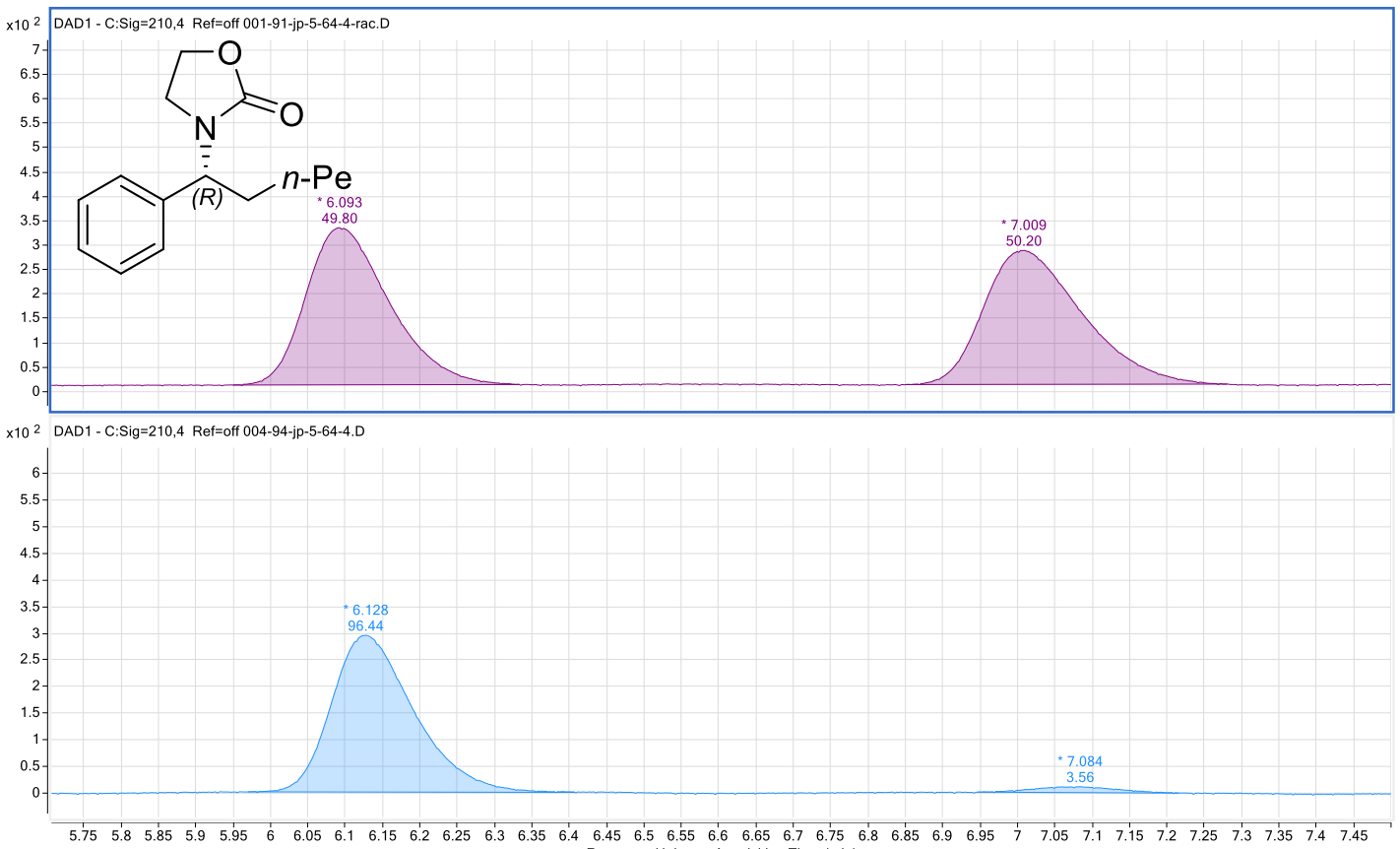

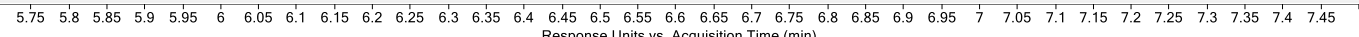

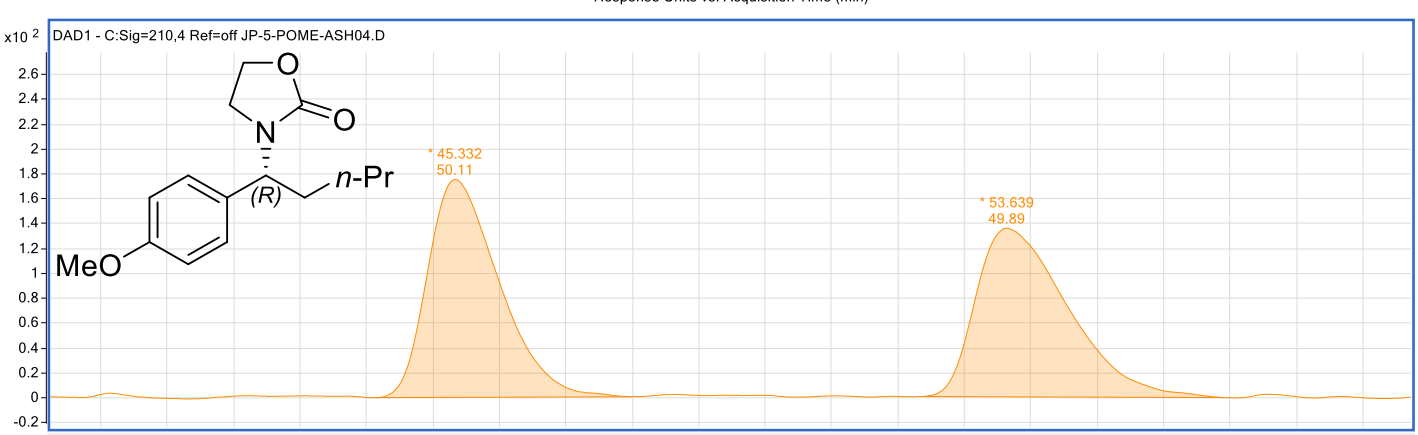

$\times 10^{2}$ DAD1 - B:Sig=230,4 Ref=off JP-5-POME-RT001.D

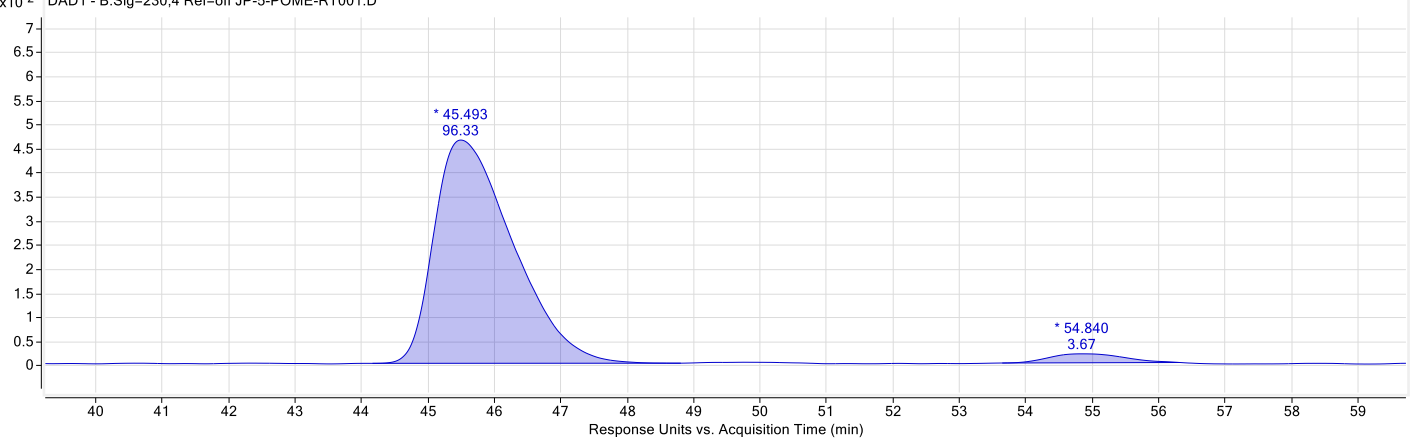



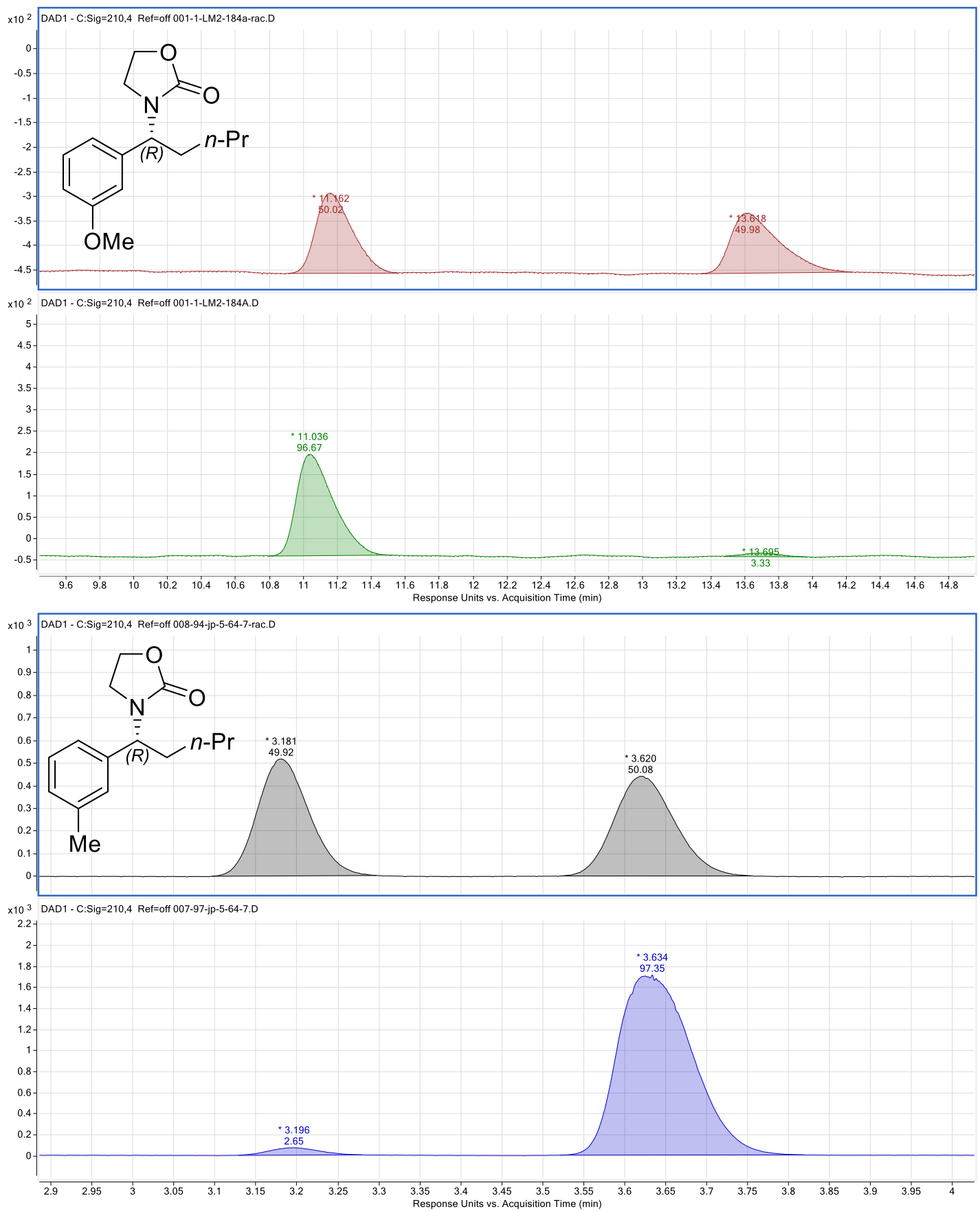


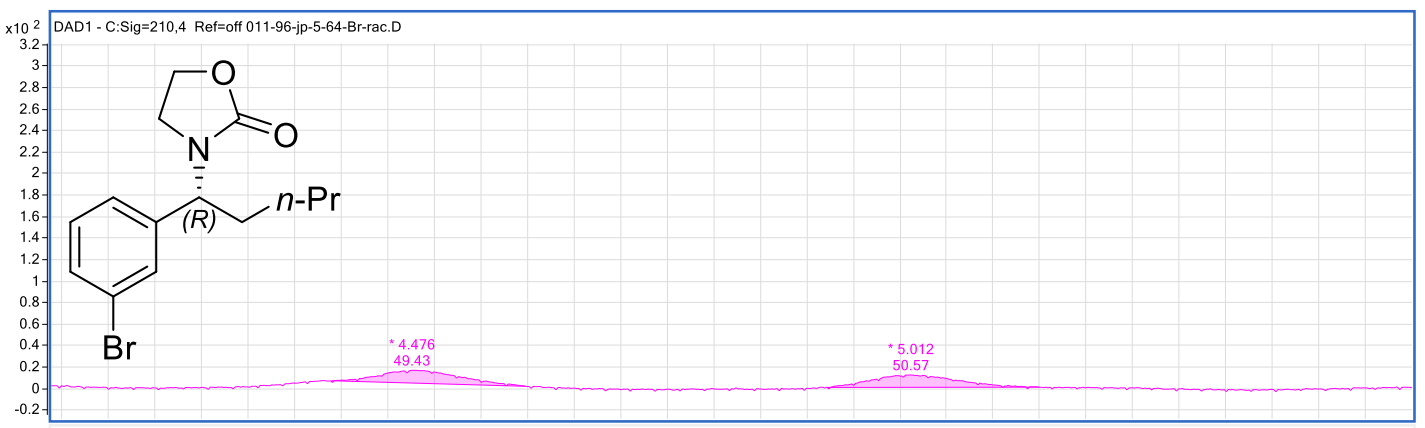

$\times 10^{3}$ DAD1 - C:Sig=210,4 Ref=off 010-82-jp-5-64-br.D
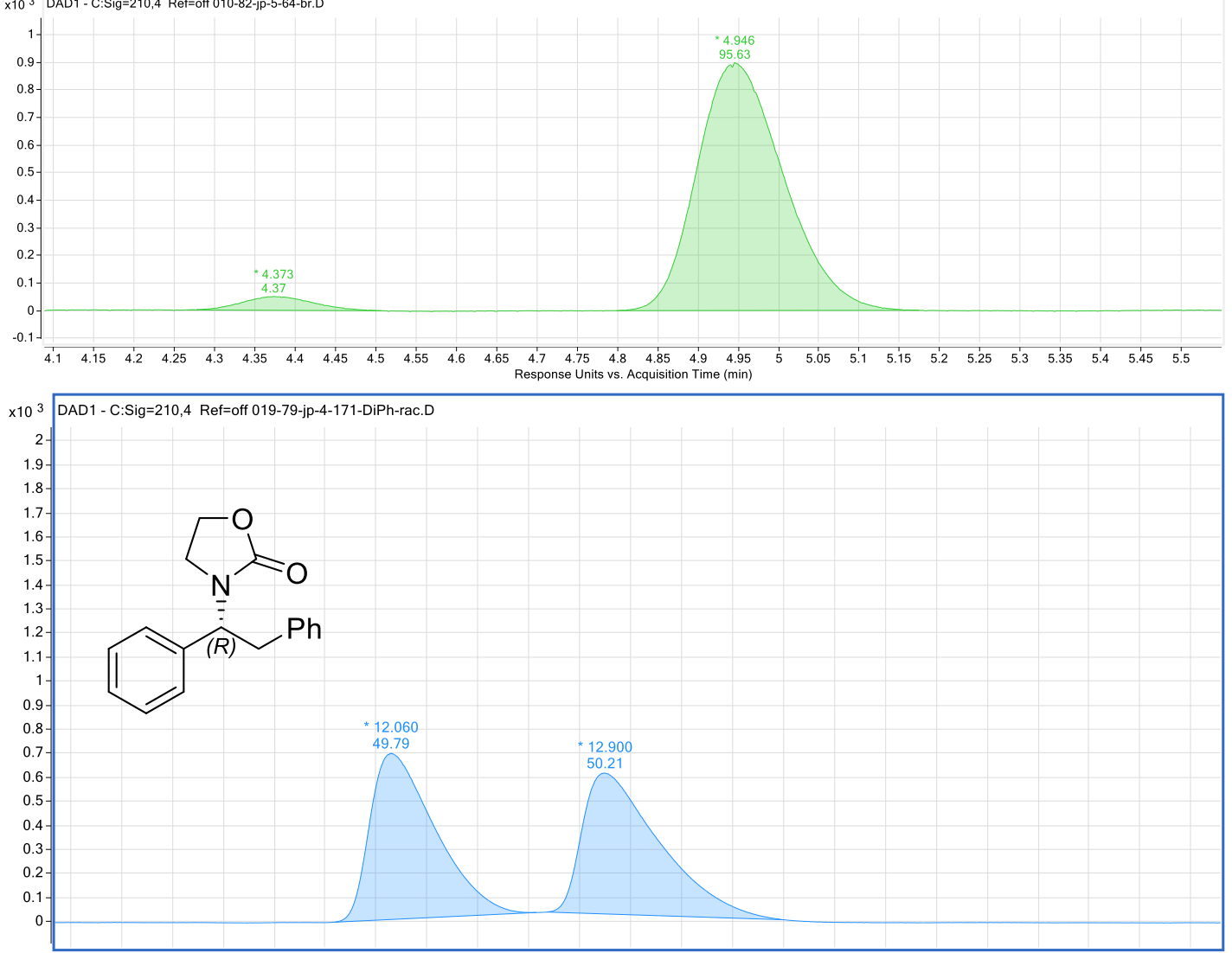

x10 2 DAD1 - C:Sig=210,4 Ref=off 020-80-jp-4-171-DiPh.D

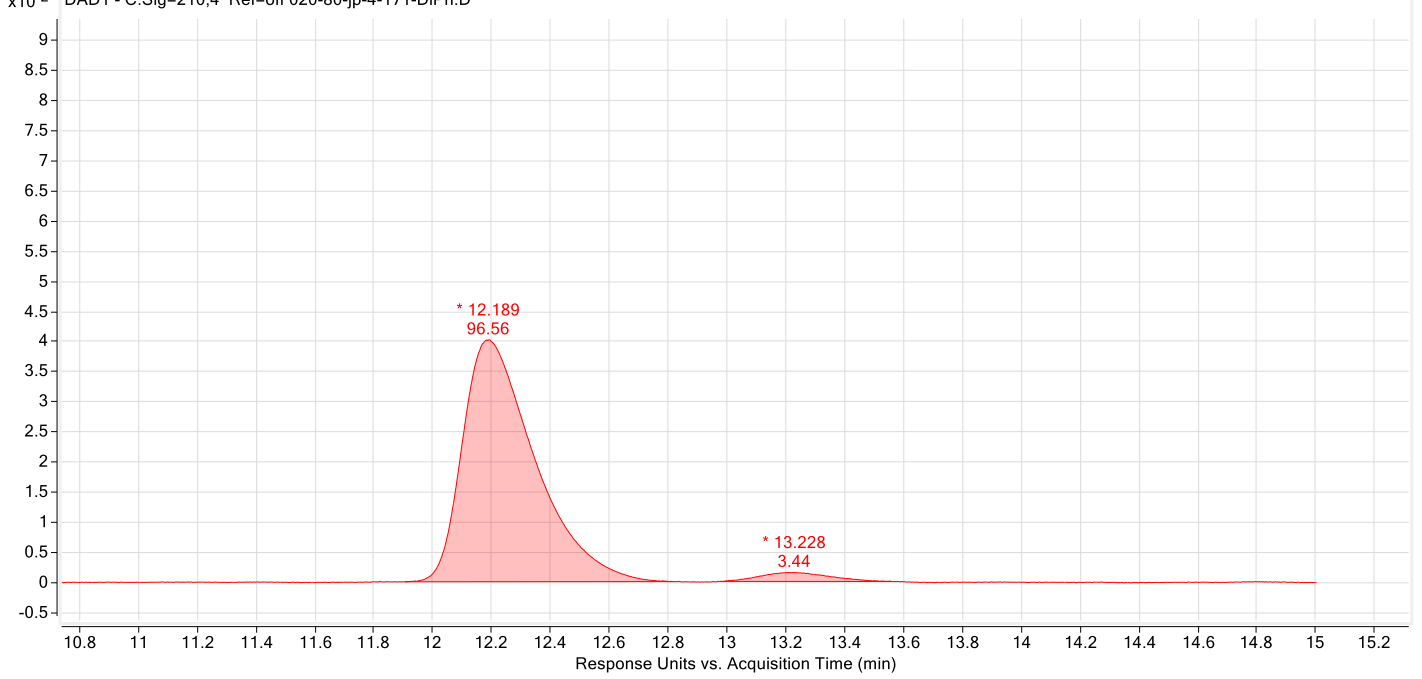




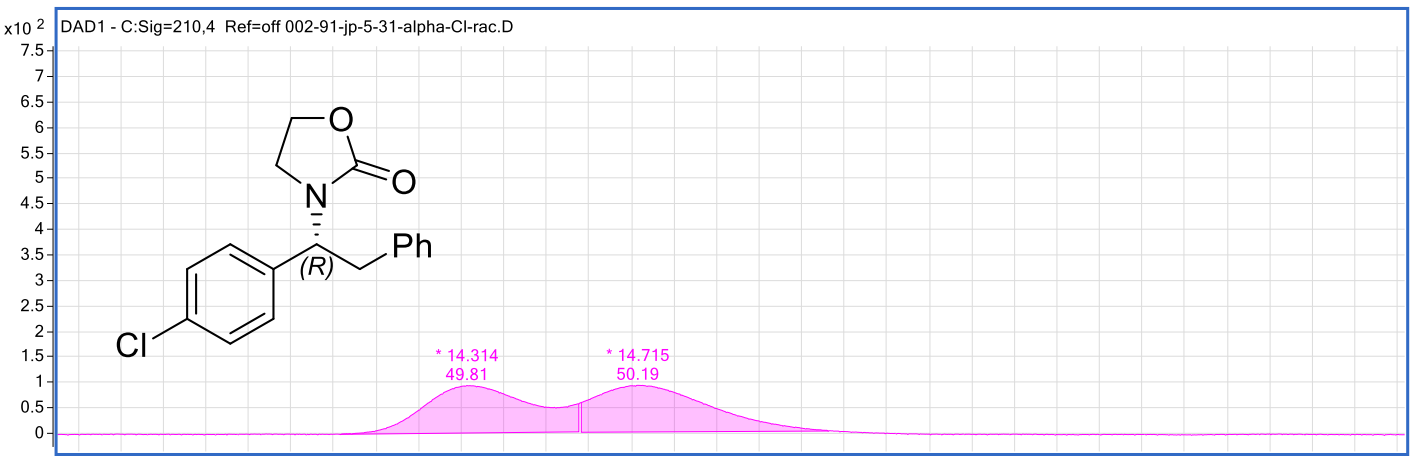

x10 2 DAD1 - C:Sig=210,4 Ref=off 003-92-jp-5-31-alpha-CI.D

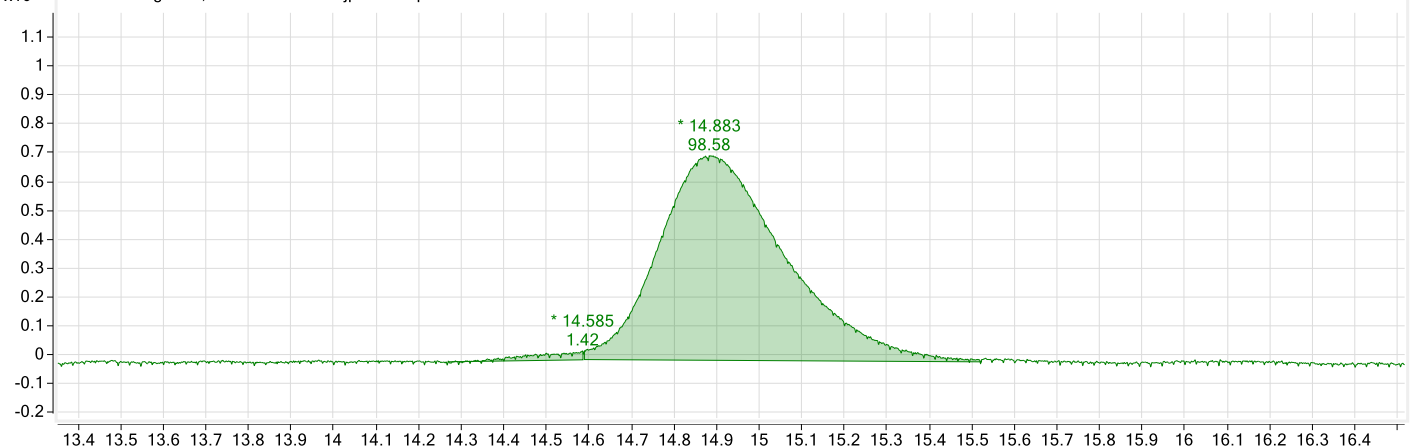

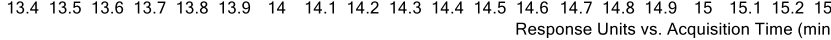
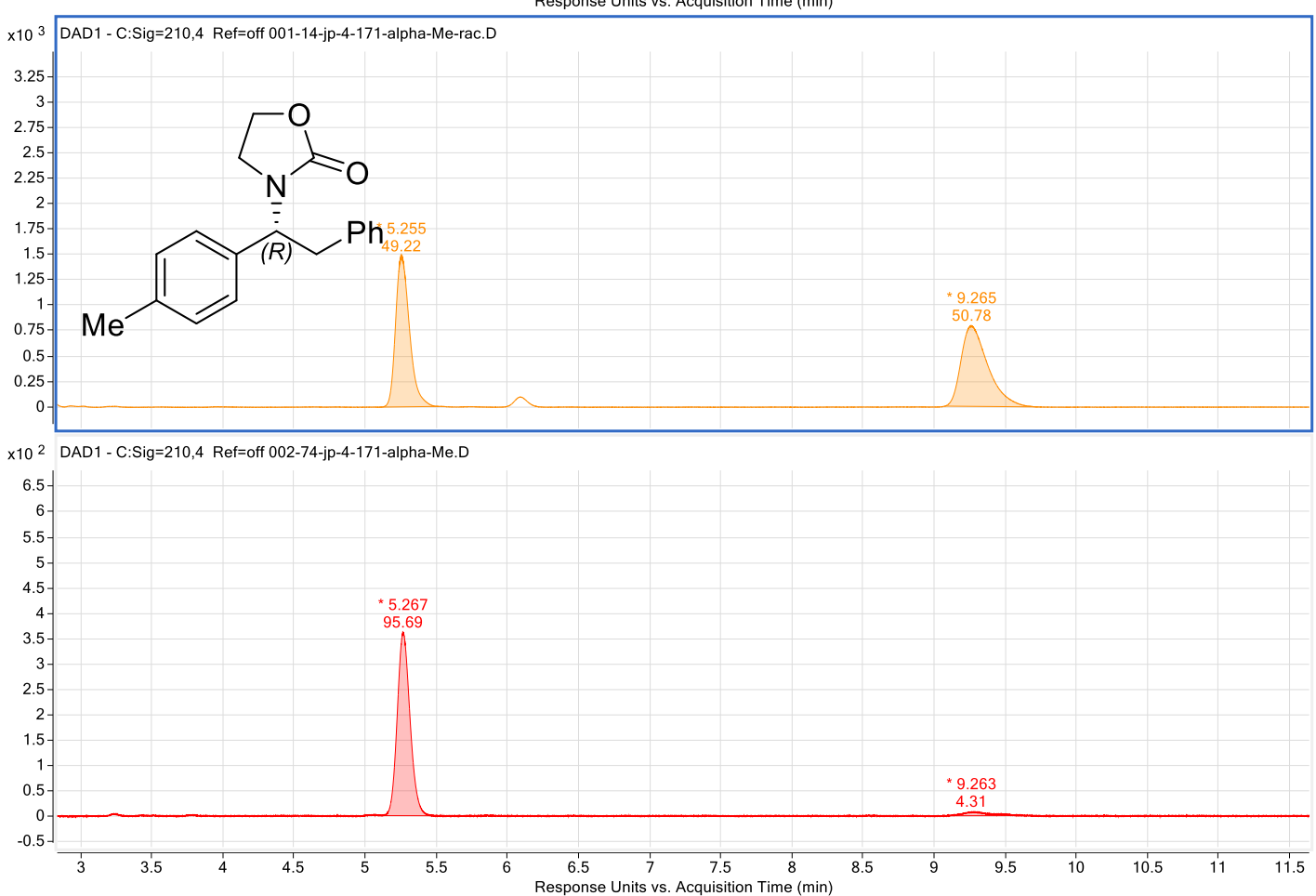

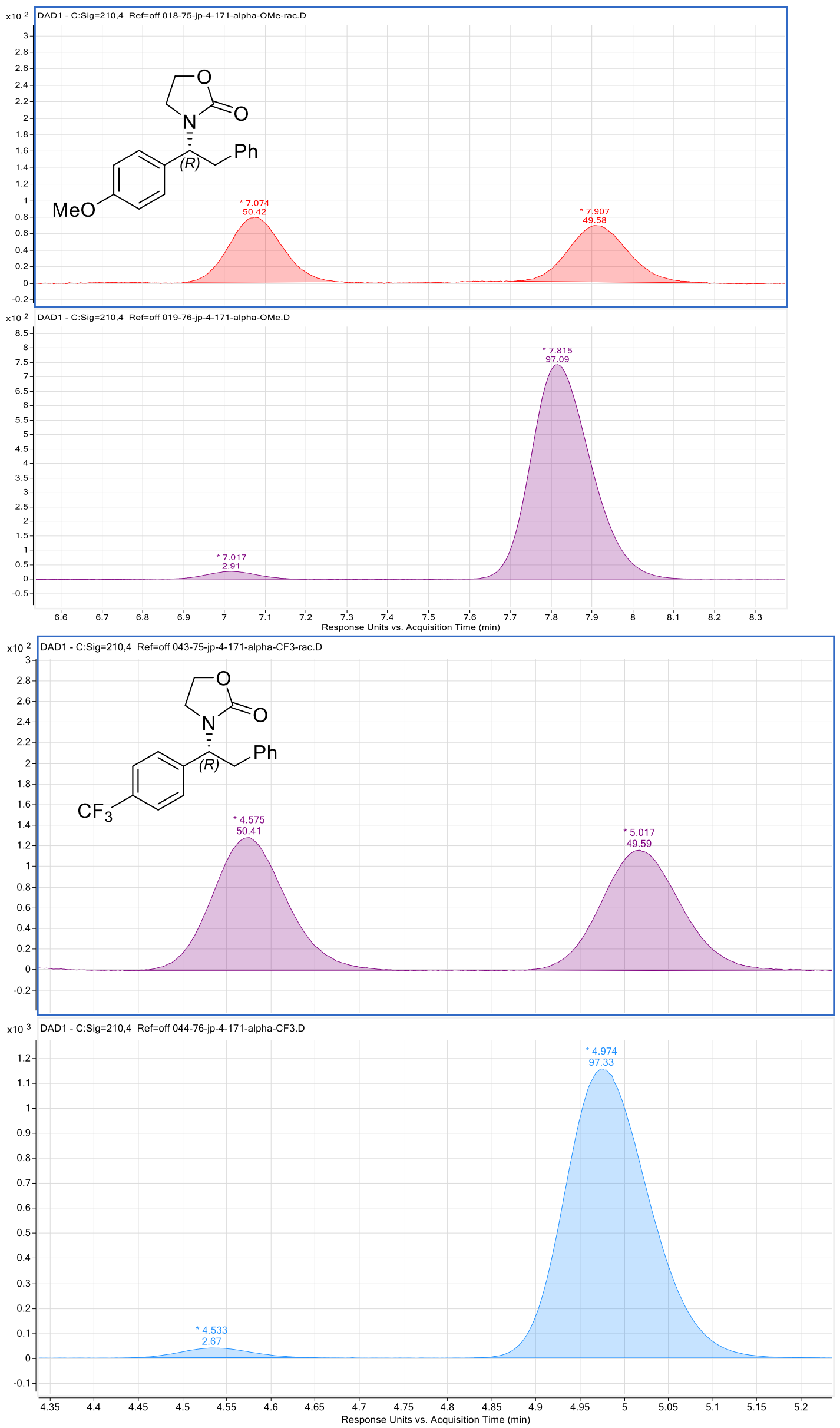

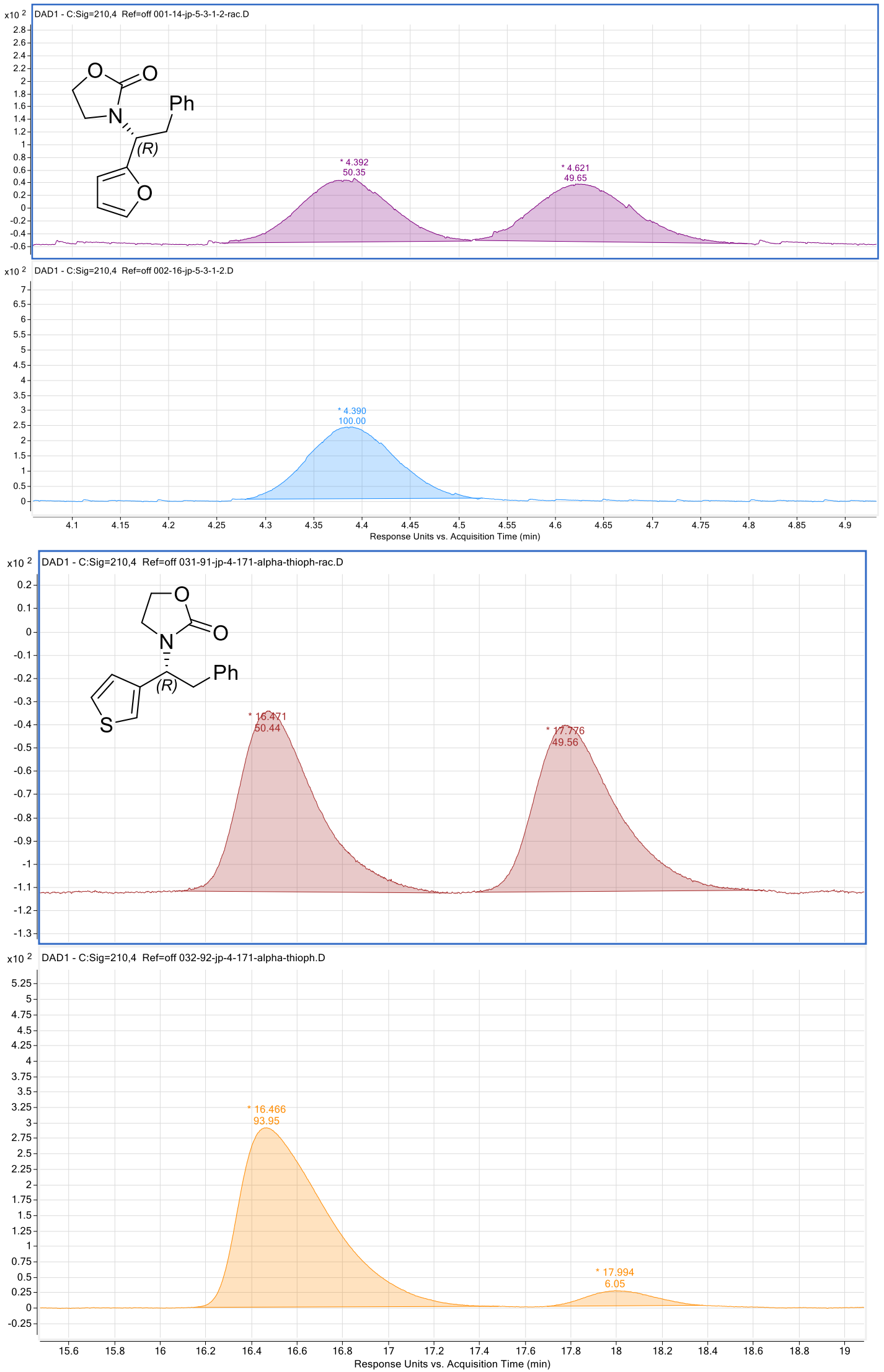

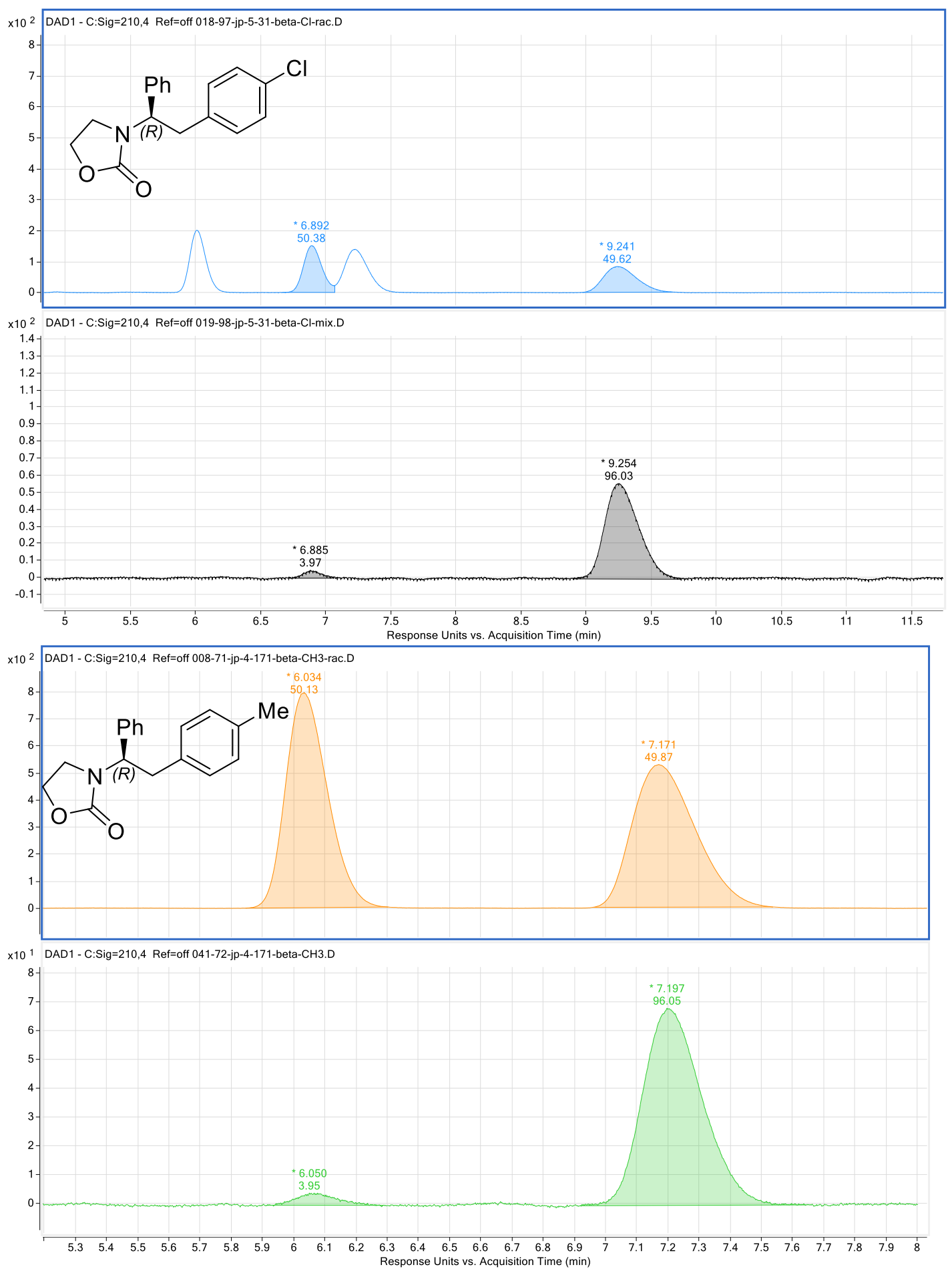

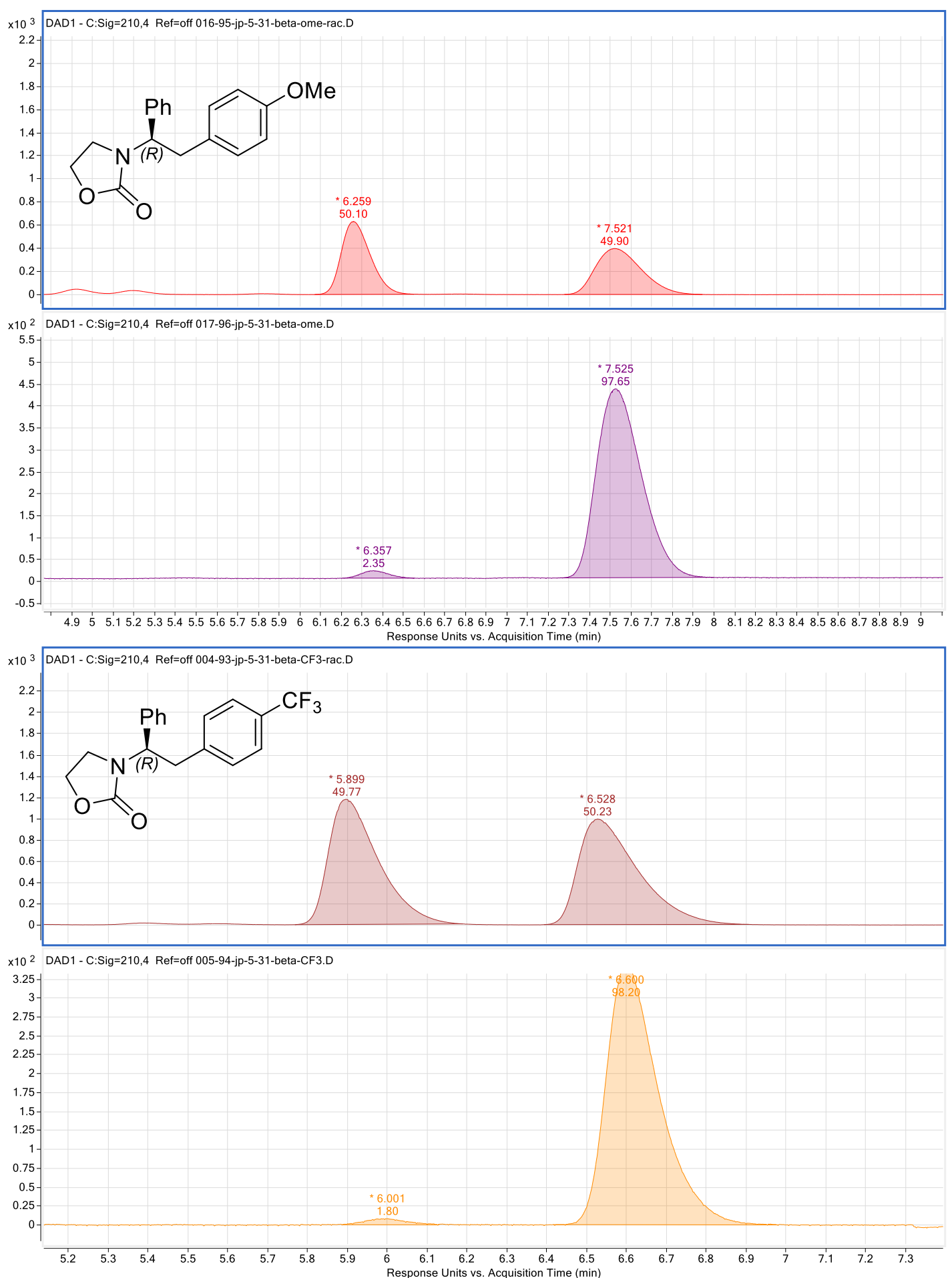

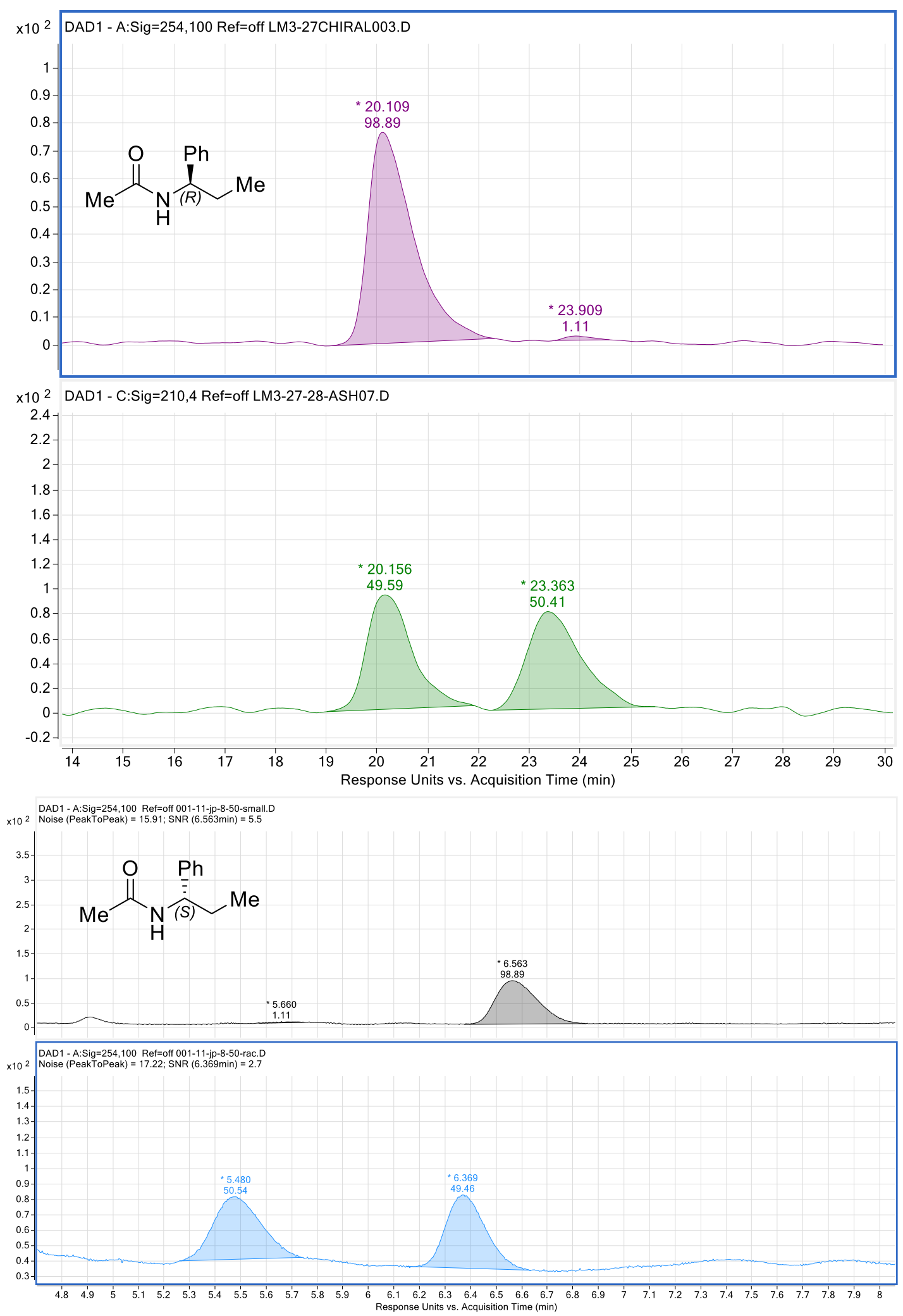

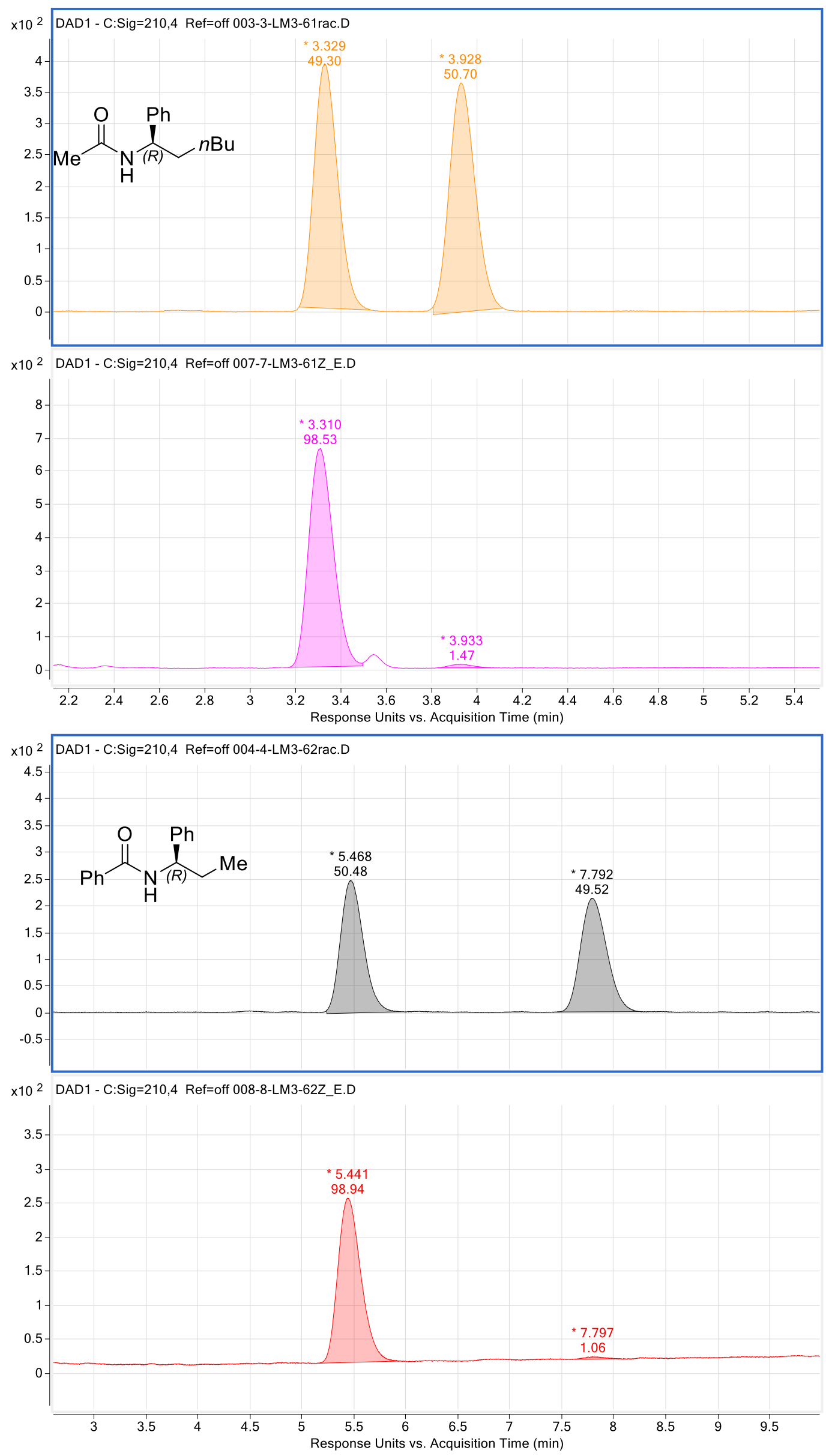

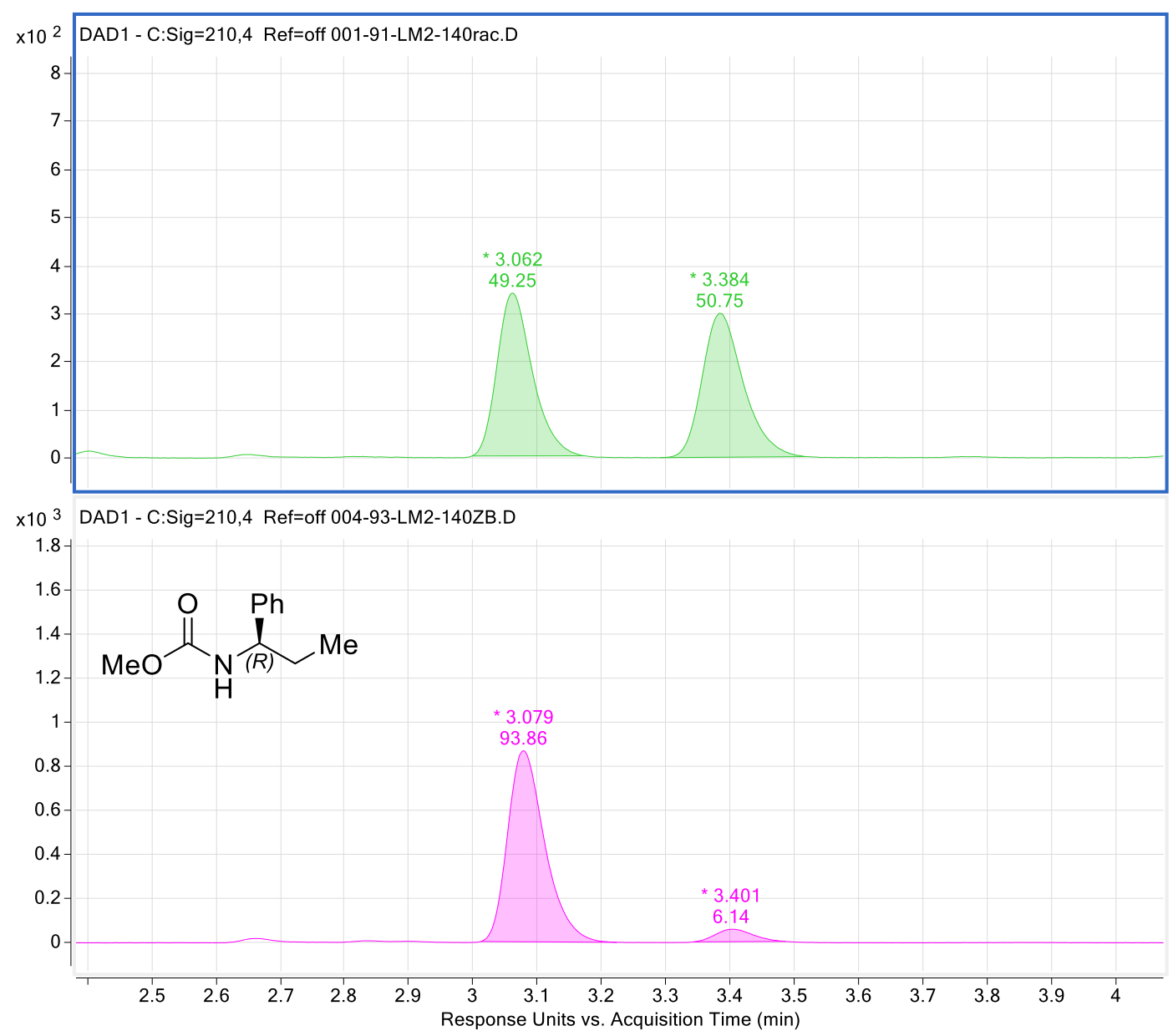


\section{C1_E R tN Chelbot}

E1

\begin{tabular}{lrr} 
C1 & $-4,217983$ & $-2,961081$ \\
C2 & $-2,146720$ & $-4,230141$ \\
C3 & $-3,448169$ & $-3,861797$ \\
C4 & $-3,244984$ & $-2,932842$ \\
H5 & $-5,174511$ & $-3,406053$ \\
H6 & $-3,242012$ & $-3,348974$ \\
H7 & $-3,666576$ & $-2,567680$ \\
H8 & $-4,416042$ & $-1,955037$ \\
H9 & $-1,599874$ & $-5,081628$ \\
H10 & $-4,011077$ & $-4,769262$ \\
C11 & $-2,661192$ & $-4,356709$ \\
H12 & $-1,867298$ & $-4,511814$ \\
H13 & $-3,420952$ & $-5,135101$ \\
C14 & 0,136670 & $-3,117726$ \\
N15 & 0,945008 & $-2,141631$ \\
C16 & 2,285599 & $-2,703712$ \\
C17 & 1,961868 & $-4,240930$ \\
P18 & $-1,642551$ & $-0,562963$ \\
Ir19 & 0,507575 & $-0,096073$ \\
C20 & $-1,584430$ & $-0,516713$ \\
C21 & $-1,306935$ & $-0,427523$ \\
C22 & $-1,562094$ & $-1,700793$ \\
C23 & $-1,444521$ & 0,714030 \\
C24 & $-1,308760$ & 0,754856 \\
C25 & $-1,429720$ & $-1,654058$ \\
H26 & $-1,439490$ & 1,642076 \\
H27 & $-1,199532$ & 1,715866 \\
H28 & $-1,412366$ & $-2,579102$ \\
H29 & $-1,202378$ & $-0,393318$ \\
O30 & 0,601784 & $-4,352360$ \\
C31 & 2,708978 & $-2,294362$ \\
C32 & 1,873687 & $-1,591198$ \\
C33 & 3,947759 & $-2,751317$ \\
C34 & 2,279970 & $-1,309031$ \\
H35 & 0,902173 & $-1,258948$ \\
C36 & 4,352384 & $-2,474159$ \\
H37 & 4,605795 & $-3,316608$ \\
H39 & 3,521596 & $-1,742162$ \\
H40 & 1,609716 & $-0,755633$ \\
& 5,318218 & $-2,828055$ \\
\hline
\end{tabular}

$-0,742644$
$-1,006060$
$-1,767178$
0,457047
$-0,449666$
$-2,711854$
1,394082
$-1,117025$
$-1,411687$
$-2,002925$
0,444933
1,181620
0,558334
$-0,470256$
$-0,217192$
0,189977
0,205644
0,482416
$-0,642132$
2,312500
5,105348
3,064011
2,976223
4,362232
4,452087
2,420113
4,856587
5,021146
6,186140
$-0,305102$
1,598708
2,467328
2,078196
3,773741
2,138525
3,381663
1,423298
4,233446
4,424736
3,730890

$\mathrm{TS}_{\mathrm{E} 1-2}$

\begin{tabular}{|c|c|c|c|}
\hline $\mathrm{C} 1$ & $-4,326353$ & $-3,069149$ & $-0,140508$ \\
\hline $\mathrm{C} 2$ & $-2,298479$ & $-4,317481$ & $-0,691570$ \\
\hline C3 & $-3,698641$ & $-3,965591$ & $-1,261091$ \\
\hline $\mathrm{C} 4$ & $-3,200364$ & $-3,040437$ & 0,917641 \\
\hline H5 & $-5,234702$ & $-3,516393$ & 0,276092 \\
\hline H6 & $-3,635005$ & $-3,454494$ & $-2,226967$ \\
\hline H7 & $-3,492970$ & $-2,686622$ & 1,906936 \\
\hline H8 & $-4,574771$ & $-2,062567$ & $-0,483851$ \\
\hline H9 & $-1,801689$ & $-5,158727$ & $-1,174628$ \\
\hline H10 & $-4,278898$ & $-4,880162$ & $-1,412470$ \\
\hline $\mathrm{C} 11$ & $-2,604801$ & $-4,455684$ & 0,816870 \\
\hline H12 & $-1,715398$ & $-4,594538$ & 1,439202 \\
\hline H13 & $-3,329531$ & $-5,246423$ & 1,030452 \\
\hline $\mathrm{C} 14$ & 0,029577 & $-3,170008$ & $-0,512794$ \\
\hline N15 & 0,845228 & $-2,194731$ & $-0,297325$ \\
\hline $\mathrm{C} 16$ & 2,230390 & $-2,744495$ & $-0,142560$ \\
\hline $\mathrm{C} 17$ & 1,989389 & $-4,264031$ & $-0,471878$ \\
\hline P18 & $-1,701858$ & $-0,619263$ & 0,613492 \\
\hline $\operatorname{Ir} 19$ & 0,268037 & $-0,103879$ & $-0,509097$ \\
\hline $\mathrm{C} 20$ & $-1,565712$ & $-0,388031$ & 2,416768 \\
\hline $\mathrm{C} 21$ & $-1,061501$ & 0,028596 & 5,139894 \\
\hline $\mathrm{C} 22$ & $-1,332524$ & $-1,477949$ & 3,266335 \\
\hline $\mathrm{C} 23$ & $-1,533391$ & 0,915829 & 2,938951 \\
\hline $\mathrm{C} 24$ & $-1,281444$ & 1,118262 & 4,293713 \\
\hline $\mathrm{C} 25$ & $-1,088141$ & $-1,267963$ & 4,624533 \\
\hline H26 & $-1,691820$ & 1,772571 & 2,293468 \\
\hline $\mathrm{H} 27$ & $-1,250387$ & 2,131733 & 4,682901 \\
\hline $\mathrm{H} 28$ & $-0,909408$ & $-2,118618$ & 5,276163 \\
\hline $\mathrm{H} 29$ & $-0,866253$ & 0,190082 & 6,196336 \\
\hline $\mathrm{O} 30$ & 0,544130 & $-4,397058$ & $-0,582575$ \\
\hline $\mathrm{C} 31$ & 2,730609 & $-2,593664$ & 1,294562 \\
\hline $\mathrm{C} 32$ & 2,010779 & $-1,902579$ & 2,271580 \\
\hline $\mathrm{C} 33$ & 3,928071 & $-3,226263$ & 1,666424 \\
\hline C34 & 2,489850 & $-1,814182$ & 3,580310 \\
\hline H35 & 1,070139 & $-1,435256$ & 2,022996 \\
\hline $\mathrm{C} 36$ & 4,409299 & $-3,136010$ & 2,970557 \\
\hline H37 & 4,496541 & $-3,788116$ & 0,929360 \\
\hline C38 & 3,693234 & $-2,421217$ & 3,933232 \\
\hline H39 & 1,905697 & $-1,272607$ & 4,318942 \\
\hline $\mathrm{H} 40$ & 5,342112 & $-3,626144$ & 3,234479 \\
\hline
\end{tabular}




\begin{tabular}{|c|c|c|c|c|c|c|c|}
\hline $\mathrm{H} 41$ & 3,839997 & $-1,521054$ & 5,248203 & $\mathrm{H} 41$ & 4,068228 & $-2,349612$ & 4,950148 \\
\hline $\mathrm{C} 42$ & $-3,105029$ & 0,438837 & 0,002537 & $\mathrm{C} 42$ & $-3,163881$ & 0,345875 & 0,082363 \\
\hline $\mathrm{C} 43$ & $-3,991432$ & 1,029815 & 0,910083 & $\mathrm{C} 43$ & $-4,137639$ & 0,818475 & 0,971414 \\
\hline $\mathrm{C} 44$ & $-3,335795$ & 0,582522 & $-1,377719$ & $\mathrm{C} 44$ & $-3,329497$ & 0,547047 & $-1,298585$ \\
\hline $\mathrm{C} 45$ & $-5,083130$ & 1,767663 & 0,444787 & $\mathrm{C} 45$ & $-5,252463$ & 1,503642 & 0,486761 \\
\hline H46 & $-3,836449$ & 0,921464 & 1,978259 & $\mathrm{H} 46$ & $-4,027051$ & 0,662314 & 2,039464 \\
\hline $\mathrm{C} 47$ & $-4,427992$ & 1,316089 & $-1,836038$ & $\mathrm{C} 47$ & $-4,449318$ & 1,223660 & $-1,777266$ \\
\hline $\mathrm{C} 48$ & $-5,302309$ & 1,917454 & $-0,924484$ & $\mathrm{C} 48$ & $-5,408674$ & 1,709532 & $-0,884080$ \\
\hline H49 & $-5,763259$ & 2,223892 & 1,158548 & $\mathrm{H} 49$ & $-5,999182$ & 1,875358 & 1,182631 \\
\hline H50 & $-4,598848$ & 1,419094 & $-2,903984$ & $\mathrm{H} 50$ & $-4,573866$ & 1,376074 & $-2,845695$ \\
\hline H51 & $-6,151228$ & 2,492966 & $-1,281399$ & H51 & $-6,277328$ & 2,244172 & $-1,257524$ \\
\hline H52 & 1,217781 & 0,301025 & 0,671998 & $\mathrm{H} 52$ & 0,809250 & 0,302155 & 0,885602 \\
\hline H53 & 1,966990 & $-0,193766$ & $-1,251191$ & $\mathrm{H} 53$ & 1,761691 & 0,324223 & $-1,126202$ \\
\hline H54 & $-1,621030$ & $-2,661793$ & 2,566426 & $\mathrm{H} 54$ & $-1,314295$ & $-2,485659$ & 2,864748 \\
\hline H55 & $-2,659288$ & 0,112099 & $-2,087244$ & $\mathrm{H} 55$ & $-2,580808$ & 0,172955 & $-1,992892$ \\
\hline H56 & 2,613839 & $-4,832065$ & $-0,438096$ & H56 & 2,421554 & $-4,567450$ & $-1,427849$ \\
\hline $\mathrm{C} 57$ & 5,054144 & $-1,695549$ & $-3,004939$ & C57 & 4,668804 & $-0,975381$ & $-3,284282$ \\
\hline C58 & 3,980620 & $-2,560454$ & $-3,230625$ & C58 & 3,413773 & $-1,524739$ & $-3,560180$ \\
\hline C59 & 3,115557 & $-2,883708$ & $-2,188385$ & C59 & 2,657527 & $-2,085738$ & $-2,535536$ \\
\hline C60 & 3,300606 & $-2,348532$ & $-0,905519$ & C60 & 3,130491 & $-2,104447$ & $-1,212755$ \\
\hline C61 & 4,362372 & $-1,462754$ & $-0,696142$ & C61 & 4,382883 & $-1,545776$ & $-0,945475$ \\
\hline C62 & 5,240365 & $-1,149591$ & $-1,736252$ & C62 & 5,148338 & $-0,991676$ & $-1,976867$ \\
\hline H63 & 5,737463 & $-1,449884$ & $-3,812761$ & $\mathrm{H} 63$ & 5,267369 & $-0,543320$ & $-4,081299$ \\
\hline H64 & 3,820805 & $-2,987374$ & $-4,216507$ & H64 & 3,024195 & $-1,526816$ & $-4,575128$ \\
\hline H65 & 2,278247 & $-3,548897$ & $-2,386337$ & $\mathrm{H} 65$ & 1,673687 & $-2,485027$ & $-2,762653$ \\
\hline H66 & 4,505742 & $-1,006868$ & 0,275629 & H66 & 4,767213 & $-1,526120$ & 0,067613 \\
\hline H67 & 6,066795 & $-0,469186$ & $-1,549668$ & H67 & 6,120499 & $-0,563825$ & $-1,748669$ \\
\hline C68 & 3,267813 & 4,712826 & $-1,323067$ & C68 & 3,347349 & 4,696527 & $-1,269022$ \\
\hline C69 & 2,107553 & 3,939122 & $-1,346076$ & C69 & 2,257195 & 3,863278 & $-1,506913$ \\
\hline $\mathrm{C} 70$ & 2,182263 & 2,550650 & $-1,537215$ & $\mathrm{C} 70$ & 2,361124 & 2,483024 & $-1,282835$ \\
\hline C71 & 3,434405 & 1,959059 & $-1,735215$ & C71 & 3,556407 & 1,952717 & $-0,797408$ \\
\hline $\mathrm{C} 72$ & 4,592860 & 2,732861 & $-1,711982$ & $\mathrm{C} 72$ & 4,645917 & 2,789070 & $-0,552133$ \\
\hline $\mathrm{C} 73$ & 4,514114 & 4,110933 & $-1,499640$ & $\mathrm{C} 73$ & 4,544937 & 4,159851 & $-0,790562$ \\
\hline H74 & 3,196343 & 5,785120 & $-1,164808$ & $\mathrm{H} 74$ & 3,257307 & 5,765118 & $-1,440894$ \\
\hline H75 & 1,142227 & 4,413318 & $-1,204033$ & $\mathrm{H} 75$ & 1,313108 & 4,283658 & $-1,842150$ \\
\hline H76 & 3,500934 & 0,889610 & $-1,896792$ & $\mathrm{H} 76$ & 3,628938 & 0,889274 & $-0,599538$ \\
\hline H77 & 5,558408 & 2,256440 & $-1,857889$ & $\mathrm{H} 77$ & 5,570902 & 2,368436 & $-0,168303$ \\
\hline H78 & 5,418000 & 4,713050 & $-1,475040$ & $\mathrm{H} 78$ & 5,391708 & 4,811301 & $-0,594551$ \\
\hline C79 & 0,902514 & 1,769095 & $-1,664965$ & C79 & 1,157428 & 1,631428 & $-1,598407$ \\
\hline $\mathrm{C} 80$ & $-0,237991$ & 1,965231 & $-0,820551$ & $\mathrm{C} 80$ & $-0,154618$ & 1,918012 & $-0,987593$ \\
\hline C81 & 0,558192 & 2,556093 & $-4,091901$ & C81 & 1,504788 & 2,137058 & $-4,129043$ \\
\hline C82 & $-0,096858$ & 0,424383 & $-3,470273$ & $\mathrm{C} 82$ & 0,319956 & 0,254852 & $-3,453272$ \\
\hline $\mathrm{C} 83$ & 0,155122 & 1,716382 & $-5,317908$ & $\mathrm{C} 83$ & 1,218739 & 1,194877 & $-5,315228$ \\
\hline H84 & 1,530633 & 3,038250 & $-4,202904$ & $\mathrm{H} 84$ & 2,559418 & 2,405080 & $-4,046797$ \\
\hline H85 & $-0,187469$ & 3,318441 & $-3,830072$ & H85 & 0,907966 & 3,057059 & $-4,159715$ \\
\hline H86 & $-0,575690$ & 2,199179 & $-5,967003$ & H86 & 0,743135 & 1,685709 & $-6,164536$ \\
\hline H87 & 1,022577 & 1,399900 & $-5,904479$ & H87 & 2,118496 & 0,667777 & $-5,644443$ \\
\hline N88 & 0,599315 & 1,508777 & $-3,069530$ & N88 & 1,085669 & 1,282304 & $-3,019554$ \\
\hline
\end{tabular}




\begin{tabular}{|c|c|c|c|c|c|c|c|}
\hline O89 & $-0,461746$ & 0,520574 & $-4,757112$ & O89 & 0,291728 & 0,200603 & $-4,792627$ \\
\hline O90 & $-0,389742$ & $-0,552526$ & $-2,762735$ & O90 & $-0,283618$ & $-0,560806$ & $-2,738448$ \\
\hline H91 & 1,964421 & $-4,648753$ & 1,216712 & H91 & 2,324442 & $-4,930267$ & 0,322429 \\
\hline N92 & $-2,035846$ & $-2,150666$ & 0,080105 & N92 & $-2,054302$ & $-2,251580$ & 0,395421 \\
\hline C93 & $-1,290393$ & $-2,942639$ & $-0,929703$ & C93 & $-1,458690$ & $-3,015969$ & $-0,725121$ \\
\hline H94 & $-1,249303$ & $-2,439083$ & $-1,900732$ & H94 & $-1,574455$ & $-2,503202$ & $-1,689593$ \\
\hline H96 & $-1,194468$ & 1,932784 & $-1,335777$ & H96 & $-0,962564$ & 1,948410 & $-1,718876$ \\
\hline C97 & $-0,498673$ & 4,443000 & 2,681663 & C97 & $-0,985647$ & 4,668138 & 2,219882 \\
\hline C98 & $-1,561021$ & 4,344187 & 1,783534 & C98 & $-1,913474$ & 4,439515 & 1,203315 \\
\hline C99 & $-1,450823$ & 3,527516 & 0,656440 & C99 & $-1,611458$ & 3,559742 & 0,162861 \\
\hline $\mathrm{C} 100$ & $-0,278246$ & 2,796547 & 0,409463 & $\mathrm{C} 100$ & $-0,382765$ & 2,880911 & 0,123258 \\
\hline C101 & 0,785585 & 2,902920 & 1,324251 & C101 & 0,536328 & 3,109490 & 1,162412 \\
\hline $\mathrm{C} 102$ & 0,674778 & 3,719417 & 2,444452 & C102 & 0,241342 & 3,999650 & 2,190582 \\
\hline H103 & $-0,581478$ & 5,077476 & 3,559638 & H103 & $-1,215570$ & 5,357422 & 3,027490 \\
\hline H104 & $-2,478782$ & 4,898225 & 1,958897 & H104 & $-2,875106$ & 4,945002 & 1,217179 \\
\hline H105 & $-2,288299$ & 3,445093 & $-0,031522$ & H105 & $-2,347117$ & 3,377852 & $-0,616807$ \\
\hline H106 & 1,697500 & 2,343717 & 1,156198 & H106 & 1,481502 & 2,578997 & 1,172862 \\
\hline H107 & 1,507146 & 3,789394 & 3,139051 & H107 & 0,969596 & 4,164491 & 2,980032 \\
\hline$E 2$ & & & & $\mathrm{TS}_{\mathrm{E} 2-3}$ & & & \\
\hline $\mathrm{C} 1$ & $-4,310252$ & $-2,924883$ & $-0,331866$ & $\mathrm{C} 1$ & $-4,379163$ & $-2,466986$ & $-0,570456$ \\
\hline $\mathrm{C} 2$ & $-2,335369$ & $-4,299957$ & $-0,749330$ & $\mathrm{C} 2$ & $-2,518987$ & $-3,920038$ & $-1,199674$ \\
\hline $\mathrm{C} 3$ & $-3,684718$ & $-3,888881$ & $-1,395958$ & $\mathrm{C} 3$ & $-3,806656$ & $-3,278377$ & $-1,780971$ \\
\hline $\mathrm{C} 4$ & $-3,240892$ & $-2,935730$ & 0,783455 & $\mathrm{C} 4$ & $-3,337855$ & $-2,755309$ & 0,533395 \\
\hline H5 & $-5,264549$ & $-3,303359$ & 0,048446 & H5 & $-5,365366$ & $-2,833067$ & $-0,266676$ \\
\hline H6 & $-3,547236$ & $-3,412233$ & $-2,371652$ & H6 & $-3,596981$ & $-2,648985$ & $-2,651604$ \\
\hline H7 & $-3,567149$ & $-2,543731$ & 1,747216 & $\mathrm{H} 7$ & $-3,649388$ & $-2,501741$ & 1,547016 \\
\hline H8 & $-4,477788$ & $-1,914490$ & $-0,711340$ & H8 & $-4,466252$ & $-1,397555$ & $-0,770684$ \\
\hline H9 & $-1,860365$ & $-5,177825$ & $-1,187676$ & H9 & $-2,117193$ & $-4,753144$ & $-1,776493$ \\
\hline H10 & $-4,309415$ & $-4,773470$ & $-1,548834$ & $\mathrm{H} 10$ & $-4,500832$ & $-4,060062$ & $-2,102078$ \\
\hline C11 & $-2,722906$ & $-4,384139$ & 0,743944 & $\mathrm{C} 11$ & $-2,950066$ & $-4,217981$ & 0,253925 \\
\hline H12 & $-1,875215$ & $-4,557498$ & 1,414056 & $\mathrm{H} 12$ & $-2,138595$ & $-4,581238$ & 0,892112 \\
\hline H13 & $-3,501852$ & $-5,127177$ & 0,937118 & H13 & $-3,797556$ & $-4,906863$ & 0,311831 \\
\hline C14 & 0,048710 & $-3,271948$ & $-0,515508$ & $\mathrm{C} 14$ & $-0,068588$ & $-3,203272$ & $-0,720364$ \\
\hline N15 & 0,867103 & $-2,323492$ & $-0,227775$ & N15 & 0,827096 & $-2,417788$ & $-0,237825$ \\
\hline $\mathrm{C} 16$ & 2,230002 & $-2,895045$ & $-0,033744$ & $\mathrm{C} 16$ & 2,125528 & $-3,148807$ & $-0,159069$ \\
\hline $\mathrm{C} 17$ & 2,004946 & $-4,364624$ & $-0,540303$ & $\mathrm{C} 17$ & 1,691392 & $-4,589260$ & $-0,623258$ \\
\hline P18 & $-1,598852$ & $-0,615465$ & 0,580967 & P18 & $-1,485328$ & $-0,605602$ & 0,761344 \\
\hline Ir19 & 0,316723 & $-0,213543$ & $-0,443297$ & Ir19 & 0,501156 & $-0,238348$ & $-0,128020$ \\
\hline $\mathrm{C} 20$ & $-1,518308$ & $-0,421521$ & 2,390162 & $\mathrm{C} 20$ & $-1,427804$ & $-0,744901$ & 2,576922 \\
\hline $\mathrm{C} 21$ & $-1,145881$ & $-0,071354$ & 5,141192 & $\mathrm{C} 21$ & $-1,077195$ & $-0,936324$ & 5,347086 \\
\hline $\mathrm{C} 22$ & $-1,456268$ & $-1,534034$ & 3,239332 & $\mathrm{C} 22$ & $-1,467774$ & $-1,995812$ & 3,207787 \\
\hline $\mathrm{C} 23$ & $-1,371556$ & 0,870271 & 2,924533 & $\mathrm{C} 23$ & $-1,189389$ & 0,411065 & 3,340824 \\
\hline $\mathrm{C} 24$ & $-1,187587$ & 1,039322 & 4,293923 & $\mathrm{C} 24$ & $-1,018596$ & 0,310591 & 4,718797 \\
\hline $\mathrm{C} 25$ & $-1,279222$ & $-1,355842$ & 4,612617 & $\mathrm{C} 25$ & $-1,299789$ & $-2,087288$ & 4,590690 \\
\hline $\mathrm{H} 26$ & $-1,385641$ & 1,740031 & 2,276880 & $\mathrm{H} 26$ & $-1,118755$ & 1,382259 & 2,864281 \\
\hline $\mathrm{H} 27$ & $-1,067555$ & 2,041546 & 4,694532 & $\mathrm{H} 27$ & $-0,828851$ & 1,209564 & 5,297719 \\
\hline $\mathrm{H} 28$ & $-1,234850$ & $-2,222899$ & 5,265627 & $\mathrm{H} 28$ & $-1,333497$ & $-3,060084$ & 5,072872 \\
\hline
\end{tabular}




\begin{tabular}{|c|c|c|c|c|c|c|c|}
\hline $\mathrm{H} 29$ & $-1,003119$ & 0,064363 & 6,209590 & $\mathrm{H} 29$ & $-0,942385$ & $-1,010820$ & 6,422489 \\
\hline $\mathrm{O} 30$ & 0,554934 & $-4,501211$ & $-0,641230$ & $\mathrm{O} 30$ & 0,297433 & $-4,451438$ & $-1,025002$ \\
\hline C31 & 2,620530 & $-2,888413$ & 1,445135 & $\mathrm{C} 31$ & 2,659326 & $-3,223281$ & 1,265878 \\
\hline C32 & 1,873682 & $-2,208993$ & 2,409726 & $\mathrm{C} 32$ & 1,942736 & $-2,720863$ & 2,353953 \\
\hline $\mathrm{C} 33$ & 3,758114 & $-3,602557$ & 1,851339 & $\mathrm{C} 33$ & 3,859123 & $-3,911626$ & 1,502969 \\
\hline $\mathrm{C} 34$ & 2,260830 & $-2,229865$ & 3,751081 & $\mathrm{C} 34$ & 2,430909 & $-2,872573$ & 3,653485 \\
\hline H35 & 0,989240 & $-1,660306$ & 2,121231 & H35 & 1,005222 & $-2,207361$ & 2,194083 \\
\hline C36 & 4,146315 & $-3,622584$ & 3,188999 & $\mathrm{C} 36$ & 4,345603 & $-4,063904$ & 2,799427 \\
\hline H37 & 4,356600 & $-4,135040$ & 1,115902 & H37 & 4,421272 & $-4,321764$ & 0,667303 \\
\hline C38 & 3,398394 & $-2,931582$ & 4,145199 & $\mathrm{C} 38$ & 3,634417 & $-3,537851$ & 3,880614 \\
\hline H39 & 1,660076 & $-1,695653$ & 4,481712 & H39 & 1,857784 & $-2,468659$ & 4,483561 \\
\hline $\mathrm{H} 40$ & 5,032614 & $-4,177043$ & 3,483833 & $\mathrm{H} 40$ & 5,279886 & $-4,592790$ & 2,964873 \\
\hline H41 & 3,700777 & $-2,946691$ & 5,188376 & $\mathrm{H} 41$ & 4,015727 & $-3,654000$ & 4,891040 \\
\hline $\mathrm{C} 42$ & $-2,988850$ & 0,424621 & 0,009737 & $\mathrm{C} 42$ & $-2,791648$ & 0,605202 & 0,352697 \\
\hline $\mathrm{C} 43$ & $-3,935209$ & 0,975723 & 0,883932 & $\mathrm{C} 43$ & $-3,664908$ & 1,143449 & 1,305132 \\
\hline $\mathrm{C} 44$ & $-3,135409$ & 0,608714 & $-1,375971$ & C44 & $-2,959896$ & 0,926050 & $-1,006044$ \\
\hline $\mathrm{C} 45$ & $-5,003560$ & 1,718615 & 0,379234 & $\mathrm{C} 45$ & $-4,685970$ & 2,007268 & 0,904484 \\
\hline $\mathrm{H} 46$ & $-3,841287$ & 0,835581 & 1,955323 & $\mathrm{H} 46$ & $-3,555323$ & 0,895374 & 2,355122 \\
\hline $\mathrm{C} 47$ & $-4,206658$ & 1,346558 & $-1,873335$ & $\mathrm{C} 47$ & $-3,979844$ & 1,787782 & $-1,398754$ \\
\hline $\mathrm{C} 48$ & $-5,138986$ & 1,908644 & $-0,995517$ & $\mathrm{C} 48$ & $-4,841875$ & 2,333960 & $-0,441942$ \\
\hline H49 & $-5,728862$ & 2,149065 & 1,063737 & $\mathrm{H} 49$ & $-5,357824$ & 2,424673 & 1,648854 \\
\hline H50 & $-4,315444$ & 1,487015 & $-2,945073$ & $\mathrm{H} 50$ & $-4,103403$ & 2,036957 & $-2,448738$ \\
\hline H51 & $-5,970279$ & 2,489610 & $-1,384446$ & H51 & $-5,635153$ & 3,009527 & $-0,748552$ \\
\hline H52 & 0,809053 & 0,157331 & 0,981979 & $\mathrm{H} 52$ & 0,968672 & $-0,141924$ & 1,352492 \\
\hline H53 & 2,047387 & 0,863061 & $-1,374622$ & $\mathrm{H} 53$ & 2,266332 & 2,540376 & $-0,831852$ \\
\hline H54 & $-1,516365$ & $-2,536595$ & 2,830006 & $\mathrm{H} 54$ & $-1,601152$ & $-2,898364$ & 2,621524 \\
\hline H55 & $-2,408008$ & 0,175781 & $-2,058066$ & $\mathrm{H} 55$ & $-2,285478$ & 0,505054 & $-1,748260$ \\
\hline H56 & 2,421253 & $-4,542872$ & $-1,535173$ & H56 & 2,255235 & $-4,962075$ & $-1,479695$ \\
\hline C57 & 4,834744 & $-0,651981$ & $-2,701715$ & $\mathrm{C} 57$ & 4,696218 & $-1,261266$ & $-3,114600$ \\
\hline C58 & 3,663794 & $-1,251717$ & $-3,170682$ & $\mathrm{C} 58$ & 3,509661 & $-1,896637$ & $-3,490989$ \\
\hline C59 & 2,853897 & $-1,975094$ & $-2,298984$ & C59 & 2,708411 & $-2,498927$ & $-2,525404$ \\
\hline C60 & 3,191436 & $-2,109314$ & $-0,940943$ & C60 & 3,076157 & $-2,486679$ & $-1,170520$ \\
\hline C61 & 4,356489 & $-1,491161$ & $-0,477299$ & C61 & 4,253264 & $-1,827376$ & $-0,799977$ \\
\hline C62 & 5,175415 & $-0,774653$ & $-1,355161$ & C62 & 5,060280 & $-1,224668$ & $-1,769188$ \\
\hline H63 & 5,473597 & $-0,092268$ & $-3,378792$ & $\mathrm{H} 63$ & 5,336226 & $-0,806461$ & $-3,866172$ \\
\hline H64 & 3,382810 & $-1,163289$ & $-4,216768$ & $\mathrm{H} 64$ & 3,206374 & $-1,926867$ & $-4,533200$ \\
\hline H65 & 1,932889 & $-2,413621$ & $-2,671358$ & $\mathrm{H} 65$ & 1,780565 & $-2,973331$ & $-2,832966$ \\
\hline H66 & 4,630280 & $-1,561623$ & 0,568838 & H66 & 4,546510 & $-1,782760$ & 0,241930 \\
\hline H67 & 6,082135 & $-0,309267$ & $-0,978912$ & H67 & 5,976253 & $-0,725560$ & $-1,465943$ \\
\hline C68 & 2,660470 & 5,313225 & $-1,876282$ & C68 & $-0,752253$ & 5,308402 & $-2,843922$ \\
\hline C69 & 1,819634 & 4,204236 & $-1,916397$ & C69 & $-0,260874$ & 4,033248 & $-2,573675$ \\
\hline $\mathrm{C} 70$ & 2,342395 & 2,916028 & $-1,740419$ & $\mathrm{C} 70$ & 0,831014 & 3,860769 & $-1,713484$ \\
\hline C71 & 3,711113 & 2,753727 & $-1,514358$ & C71 & 1,422272 & 4,981887 & $-1,127836$ \\
\hline $\mathrm{C} 72$ & 4,552174 & 3,867034 & $-1,465784$ & $\mathrm{C} 72$ & 0,926473 & 6,259920 & $-1,391208$ \\
\hline $\mathrm{C} 73$ & 4,028538 & 5,146673 & $-1,648049$ & $\mathrm{C} 73$ & $-0,160134$ & 6,425780 & $-2,249302$ \\
\hline H74 & 2,246482 & 6,308930 & $-2,005749$ & $\mathrm{H} 74$ & $-1,599398$ & 5,432459 & $-3,512889$ \\
\hline H75 & 0,750667 & 4,338862 & $-2,052288$ & $\mathrm{H} 75$ & $-0,731962$ & 3,162429 & $-3,025384$ \\
\hline H76 & 4,117708 & 1,756544 & $-1,369740$ & $\mathrm{H} 76$ & 2,256624 & 4,852811 & $-0,442675$ \\
\hline
\end{tabular}




\begin{tabular}{|c|c|c|c|c|c|c|c|}
\hline H77 & 5,614189 & 3,734129 & $-1,280425$ & H77 & 1,387934 & 7,124467 & $-0,922774$ \\
\hline $\mathrm{H} 78$ & 4,681263 & 6,013712 & $-1,603976$ & $\mathrm{H} 78$ & $-0,546675$ & 7,420000 & $-2,454012$ \\
\hline C79 & 1,422904 & 1,711106 & $-1,783340$ & C79 & 1,299681 & 2,461003 & $-1,338421$ \\
\hline $\mathrm{C} 80$ & 0,097319 & 1,789934 & $-0,975761$ & C80 & 0,252126 & 1,828277 & $-0,369658$ \\
\hline C81 & 1,327522 & 2,153929 & $-4,362892$ & C81 & 2,379657 & 2,255228 & $-3,635790$ \\
\hline $\mathrm{C} 82$ & 0,394676 & 0,227534 & $-3,477145$ & $\mathrm{C} 82$ & 0,942216 & 0,548706 & $-2,997785$ \\
\hline $\mathrm{C} 83$ & 0,951166 & 1,156350 & $-5,473193$ & $\mathrm{C} 83$ & 2,261227 & 1,192754 & $-4,746260$ \\
\hline H84 & 2,356316 & 2,509075 & $-4,441496$ & H84 & 3,408553 & 2,387213 & $-3,286018$ \\
\hline H85 & 0,661688 & 3,024430 & $-4,327564$ & H85 & 1,986055 & 3,230554 & $-3,935077$ \\
\hline H86 & 0,329850 & 1,584084 & $-6,260358$ & H86 & 1,932439 & 1,598781 & $-5,704309$ \\
\hline H87 & 1,830817 & 0,678451 & $-5,914814$ & H87 & 3,183567 & 0,623382 & $-4,881949$ \\
\hline N88 & 1,145242 & 1,309095 & $-3,182027$ & N88 & 1,549182 & 1,676539 & $-2,574485$ \\
\hline O89 & 0,177093 & 0,125476 & $-4,798603$ & O89 & 1,245437 & 0,270825 & $-4,276384$ \\
\hline O90 & $-0,060548$ & $-0,614869$ & $-2,682659$ & O90 & 0,194444 & $-0,223518$ & $-2,367461$ \\
\hline H91 & 2,359818 & $-5,120694$ & 0,158207 & H91 & 1,727602 & $-5,312094$ & 0,193050 \\
\hline N92 & $-2,022513$ & $-2,224649$ & 0,307754 & N92 & $-2,049981$ & $-2,086587$ & 0,189963 \\
\hline C93 & $-1,423224$ & $-3,048065$ & $-0,771947$ & C93 & $-1,490750$ & $-2,779336$ & $-0,995755$ \\
\hline H94 & $-1,477038$ & $-2,548494$ & $-1,749471$ & H94 & $-1,447903$ & $-2,124893$ & $-1,876957$ \\
\hline H96 & $-0,729762$ & 1,934787 & $-1,677822$ & H96 & $-0,704380$ & 1,951594 & $-0,883318$ \\
\hline C97 & $-0,414768$ & 4,575743 & 2,278842 & C97 & 0,021719 & 4,188704 & 3,252687 \\
\hline C98 & $-1,416475$ & 4,395741 & 1,324955 & C98 & $-1,028026$ & 4,195892 & 2,333744 \\
\hline C99 & $-1,221674$ & 3,512891 & 0,260294 & C99 & $-0,939815$ & 3,444468 & 1,160010 \\
\hline $\mathrm{C} 100$ & $-0,030623$ & 2,781645 & 0,135076 & $\mathrm{C} 100$ & 0,188995 & 2,658177 & 0,889353 \\
\hline C101 & 0,968399 & 2,972693 & 1,105483 & C101 & 1,239919 & 2,668108 & 1,819706 \\
\hline C102 & 0,781599 & 3,861168 & 2,159571 & C102 & 1,161865 & 3,424918 & 2,986472 \\
\hline H103 & $-0,560455$ & 5,266837 & 3,104342 & H103 & $-0,043594$ & 4,775173 & 4,164909 \\
\hline H104 & $-2,351666$ & 4,943510 & 1,405705 & H104 & $-1,916820$ & 4,790886 & 2,526293 \\
\hline $\mathrm{H} 105$ & $-2,012523$ & 3,372766 & $-0,473041$ & H105 & $-1,756673$ & 3,465031 & 0,443840 \\
\hline H106 & 1,897454 & 2,415045 & 1,033031 & H106 & 2,114056 & 2,050170 & 1,636152 \\
\hline \multirow[t]{3}{*}{ H107 } & 1,570145 & 3,995205 & 2,895200 & H107 & 1,986454 & 3,413178 & 3,694376 \\
\hline & & & & H108 & 3,293888 & 0,440742 & $-0,341385$ \\
\hline & & & & H109 & 3,385246 & 0,864873 & 0,262884 \\
\hline E3 & & & & $\mathrm{TS}_{\mathrm{E} 3-4}$ & & & \\
\hline $\mathrm{C} 1$ & $-4,355556$ & $-2,461423$ & $-0,796930$ & $\mathrm{C} 1$ & $-4,413515$ & $-2,302062$ & $-0,638785$ \\
\hline $\mathrm{C} 2$ & $-2,464517$ & $-3,925156$ & $-1,300068$ & $\mathrm{C} 2$ & $-2,573687$ & $-3,727476$ & $-1,382061$ \\
\hline $\mathrm{C} 3$ & $-3,708816$ & $-3,278811$ & $-1,965573$ & $\mathrm{C} 3$ & $-3,831807$ & $-2,993428$ & $-1,917442$ \\
\hline $\mathrm{C} 4$ & $-3,387604$ & $-2,750304$ & 0,372202 & $\mathrm{C} 4$ & $-3,398989$ & $-2,726113$ & 0,447706 \\
\hline H5 & $-5,361223$ & $-2,822084$ & $-0,557254$ & H5 & $-5,411763$ & $-2,679139$ & $-0,393277$ \\
\hline H6 & $-3,440042$ & $-2,652892$ & $-2,822424$ & H6 & $-3,586142$ & $-2,279212$ & $-2,710042$ \\
\hline $\mathrm{H} 7$ & $-3,760128$ & $-2,490877$ & 1,363835 & $\mathrm{H} 7$ & $-3,723856$ & $-2,569196$ & 1,476909 \\
\hline H8 & $-4,424135$ & $-1,392214$ & $-1,005325$ & H8 & $-4,479944$ & $-1,216799$ & $-0,729393$ \\
\hline H9 & $-2,031946$ & $-4,762519$ & $-1,847532$ & H9 & $-2,182898$ & $-4,507789$ & $-2,035118$ \\
\hline $\mathrm{H} 10$ & $-4,384329$ & $-4,058292$ & $-2,329190$ & $\mathrm{H} 10$ & $-4,536185$ & $-3,717304$ & $-2,336824$ \\
\hline $\mathrm{C} 11$ & $-2,991084$ & $-4,215905$ & 0,123019 & $\mathrm{C} 11$ & $-3,042881$ & $-4,161916$ & 0,025095 \\
\hline H12 & $-2,224535$ & $-4,581682$ & 0,813192 & H12 & $-2,253650$ & $-4,608941$ & 0,636961 \\
\hline H13 & $-3,844385$ & $-4,899983$ & 0,127859 & H13 & $-3,907675$ & $-4,830721$ & $-0,005156$ \\
\hline $\mathrm{C} 14$ & $-0,041523$ & $-3,216597$ & $-0,678653$ & $\mathrm{C} 14$ & $-0,120623$ & $-3,128987$ & $-0,781702$ \\
\hline
\end{tabular}




\begin{tabular}{|c|c|c|c|c|c|c|c|}
\hline N15 & 0,855807 & $-2,435612$ & $-0,188945$ & N15 & 0,842850 & $-2,407734$ & $-0,317074$ \\
\hline $\mathrm{C} 16$ & 2,150333 & $-3,183753$ & $-0,098660$ & $\mathrm{C} 16$ & 2,093723 & $-3,245358$ & $-0,251711$ \\
\hline $\mathrm{C} 17$ & 1,693364 & $-4,631656$ & $-0,500993$ & $\mathrm{C} 17$ & 1,513592 & $-4,657923$ & $-0,600204$ \\
\hline P18 & $-1,544302$ & $-0,606122$ & 0,700196 & P18 & $-1,514687$ & $-0,591532$ & 0,806256 \\
\hline Ir19 & 0,592166 & $-0,231970$ & $-0,063785$ & Ir19 & 0,759673 & $-0,294219$ & $-0,009525$ \\
\hline $\mathrm{C} 20$ & $-1,546094$ & $-0,756007$ & 2,518475 & $\mathrm{C} 20$ & $-1,527905$ & $-0,749885$ & 2,623980 \\
\hline $\mathrm{C} 21$ & $-1,268381$ & $-0,974106$ & 5,295448 & $\mathrm{C} 21$ & $-1,192945$ & $-0,974831$ & 5,402275 \\
\hline $\mathrm{C} 22$ & $-1,613054$ & $-2,010979$ & 3,136475 & $\mathrm{C} 22$ & $-1,582486$ & $-2,005246$ & 3,244667 \\
\hline $\mathrm{C} 23$ & $-1,320531$ & 0,390585 & 3,298644 & $\mathrm{C} 23$ & $-1,289125$ & 0,393669 & 3,406372 \\
\hline $\mathrm{C} 24$ & $-1,186329$ & 0,277843 & 4,679770 & $\mathrm{C} 24$ & $-1,127005$ & 0,277702 & 4,785551 \\
\hline $\mathrm{C} 25$ & $-1,480049$ & $-2,116059$ & 4,522682 & $\mathrm{C} 25$ & $-1,420279$ & $-2,113807$ & 4,628231 \\
\hline $\mathrm{H} 26$ & $-1,228715$ & 1,365464 & 2,832543 & $\mathrm{H} 26$ & $-1,211470$ & 1,371615 & 2,941757 \\
\hline $\mathrm{H} 27$ & $-1,006549$ & 1,170753 & 5,271393 & $\mathrm{H} 27$ & $-0,939308$ & 1,169871 & 5,376001 \\
\hline $\mathrm{H} 28$ & $-1,534139$ & $-3,092813$ & 4,994983 & $\mathrm{H} 28$ & $-1,468306$ & $-3,092136$ & 5,098143 \\
\hline $\mathrm{H} 29$ & $-1,161769$ & $-1,058831$ & 6,373224 & H29 & $-1,062974$ & $-1,061784$ & 6,476900 \\
\hline $\mathrm{O} 30$ & 0,315250 & $-4,477679$ & $-0,946080$ & O30 & 0,153901 & $-4,405804$ & $-1,055895$ \\
\hline $\mathrm{C} 31$ & 2,692002 & $-3,204161$ & 1,325974 & C31 & 2,656906 & $-3,306900$ & 1,165422 \\
\hline $\mathrm{C} 32$ & 1,933285 & $-2,734603$ & 2,402212 & C32 & 1,851683 & $-2,996121$ & 2,267468 \\
\hline $\mathrm{C} 33$ & 3,925829 & $-3,819134$ & 1,584584 & C33 & 3,933919 & $-3,837038$ & 1,392778 \\
\hline C34 & 2,414434 & $-2,841554$ & 3,707916 & C34 & 2,332424 & $-3,153284$ & 3,566629 \\
\hline H35 & 0,964536 & $-2,285964$ & 2,228755 & H35 & 0,845660 & $-2,624832$ & 2,118836 \\
\hline $\mathrm{C} 36$ & 4,404621 & $-3,928758$ & 2,888826 & C36 & 4,412205 & $-4,001835$ & 2,692640 \\
\hline H37 & 4,519070 & $-4,210503$ & 0,762383 & H37 & 4,562518 & $-4,118616$ & 0,553695 \\
\hline C38 & 3,652706 & $-3,432261$ & 3,955601 & C38 & 3,617779 & $-3,649556$ & 3,784303 \\
\hline H39 & 1,808509 & $-2,464015$ & 4,527096 & H39 & 1,692948 & $-2,885840$ & 4,403490 \\
\hline $\mathrm{H} 40$ & 5,364893 & $-4,402755$ & 3,070793 & $\mathrm{H} 40$ & 5,408485 & $-4,405297$ & 2,849429 \\
\hline $\mathrm{H} 41$ & 4,028208 & $-3,514824$ & 4,971468 & H41 & 3,995484 & $-3,770533$ & 4,795471 \\
\hline $\mathrm{C} 42$ & $-2,833127$ & 0,613549 & 0,264873 & $\mathrm{C} 42$ & $-2,782174$ & 0,663567 & 0,374632 \\
\hline $\mathrm{C} 43$ & $-3,739434$ & 1,151767 & 1,185436 & $\mathrm{C} 43$ & $-3,678867$ & 1,242747 & 1,280670 \\
\hline $\mathrm{C} 44$ & $-2,932165$ & 0,965633 & $-1,093502$ & $\mathrm{C} 44$ & $-2,844866$ & 1,033951 & $-0,982416$ \\
\hline $\mathrm{C} 45$ & $-4,723273$ & 2,044848 & 0,755652 & $\mathrm{C} 45$ & $-4,605088$ & 2,192258 & 0,842814 \\
\hline $\mathrm{H} 46$ & $-3,681164$ & 0,882844 & 2,234835 & $\mathrm{H} 46$ & $-3,651992$ & 0,963920 & 2,329021 \\
\hline $\mathrm{C} 47$ & $-3,915922$ & 1,855544 & $-1,516099$ & $\mathrm{C} 47$ & $-3,771994$ & 1,980211 & $-1,415246$ \\
\hline $\mathrm{C} 48$ & $-4,810581$ & 2,400715 & $-0,589921$ & $\mathrm{C} 48$ & $-4,650489$ & 2,568971 & $-0,500624$ \\
\hline $\mathrm{H} 49$ & $-5,421157$ & 2,461786 & 1,476048 & H49 & $-5,292730$ & 2,638111 & 1,555837 \\
\hline $\mathrm{H} 50$ & $-3,985367$ & 2,127252 & $-2,565685$ & $\mathrm{H} 50$ & $-3,805810$ & 2,262920 & $-2,463592$ \\
\hline H5 1 & $-5,575048$ & 3,098789 & $-0,918751$ & H51 & $-5,368613$ & 3,311869 & $-0,834699$ \\
\hline H52 & 0,938347 & $-0,159584$ & 1,456331 & H52 & 1,201376 & $-0,519862$ & 1,467952 \\
\hline H53 & 2,237083 & 2,572303 & $-0,914469$ & H53 & 2,235772 & 2,842658 & $-0,820397$ \\
\hline H54 & $-1,742447$ & $-2,906701$ & 2,538588 & H54 & $-1,725901$ & $-2,899988$ & 2,647340 \\
\hline $\mathrm{H} 55$ & $-2,232119$ & 0,545014 & $-1,811352$ & $\mathrm{H} 55$ & $-2,159470$ & 0,583233 & $-1,697304$ \\
\hline H56 & 2,268557 & $-5,060674$ & $-1,322576$ & H56 & 2,053272 & $-5,173120$ & $-1,394721$ \\
\hline C57 & 4,568871 & $-1,291076$ & $-3,176957$ & C57 & 4,634502 & $-1,691483$ & $-3,416030$ \\
\hline C58 & 3,404767 & $-1,992444$ & $-3,500726$ & C58 & 3,491573 & $-2,436607$ & $-3,713740$ \\
\hline C59 & 2,670007 & $-2,620247$ & $-2,498426$ & C59 & 2,705712 & $-2,947164$ & $-2,683056$ \\
\hline C60 & 3,079091 & $-2,565381$ & $-1,157038$ & C60 & 3,042282 & $-2,725863$ & $-1,340225$ \\
\hline C61 & 4,241555 & $-1,853755$ & $-0,842254$ & C61 & 4,169474 & $-1,947620$ & $-1,053712$ \\
\hline C62 & 4,982539 & $-1,223934$ & $-1,846404$ & C62 & 4,964774 & $-1,442494$ & $-2,083470$ \\
\hline
\end{tabular}




\begin{tabular}{|c|c|c|c|c|c|c|c|}
\hline H63 & 5,154549 & $-0,811033$ & $-3,956473$ & H63 & 5,265520 & $-1,314213$ & $-4,216714$ \\
\hline H64 & 3,065233 & $-2,051321$ & $-4,530629$ & H64 & 3,211678 & $-2,623443$ & $-4,746369$ \\
\hline H65 & 1,754383 & $-3,139489$ & $-2,767786$ & H65 & 1,809273 & $-3,506118$ & $-2,938483$ \\
\hline H66 & 4,568313 & $-1,776593$ & 0,188657 & H66 & 4,426158 & $-1,727026$ & $-0,024981$ \\
\hline H67 & 5,887109 & $-0,682849$ & $-1,583338$ & H67 & 5,845499 & $-0,854931$ & $-1,839697$ \\
\hline C68 & $-0,941122$ & 5,302451 & $-2,740422$ & C68 & $-1,239374$ & 4,946582 & $-2,928325$ \\
\hline C69 & $-0,431422$ & 4,033568 & $-2,472621$ & C69 & $-0,551899$ & 3,791548 & $-2,561081$ \\
\hline $\mathrm{C} 70$ & 0,727766 & 3,879871 & $-1,700349$ & C70 & 0,578611 & 3,869342 & $-1,737125$ \\
\hline C71 & 1,364559 & 5,016680 & $-1,197934$ & C71 & 1,010644 & 5,118463 & $-1,287847$ \\
\hline $\mathrm{C} 72$ & 0,851294 & 6,289036 & $-1,459581$ & $\mathrm{C} 72$ & 0,321163 & 6,276717 & $-1,653362$ \\
\hline $\mathrm{C} 73$ & $-0,300712$ & 6,436348 & $-2,232445$ & $\mathrm{C} 73$ & $-0,804963$ & 6,194623 & $-2,473050$ \\
\hline $\mathrm{H} 74$ & $-1,839673$ & 5,408351 & $-3,342105$ & $\mathrm{H} 74$ & $-2,114219$ & 4,874586 & $-3,568649$ \\
\hline $\mathrm{H} 75$ & $-0,939400$ & 3,152935 & $-2,860069$ & H75 & $-0,897795$ & 2,821789 & $-2,911454$ \\
\hline H76 & 2,254152 & 4,906666 & $-0,582773$ & H76 & 1,878616 & 5,186070 & $-0,636870$ \\
\hline H77 & 1,352876 & 7,164651 & $-1,057594$ & H77 & 0,664912 & 7,243025 & $-1,295989$ \\
\hline $\mathrm{H} 78$ & $-0,698665$ & 7,425733 & $-2,437694$ & $\mathrm{H} 78$ & $-1,340163$ & 7,095563 & $-2,757880$ \\
\hline C79 & 1,231840 & 2,485709 & $-1,345623$ & C79 & 1,274027 & 2,589293 & $-1,281250$ \\
\hline $\mathrm{C} 80$ & 0,279531 & 1,841264 & $-0,299986$ & $\mathrm{C} 80$ & 0,372635 & 1,899074 & $-0,228570$ \\
\hline C81 & 2,151619 & 2,261051 & $-3,712667$ & C81 & 2,400094 & 2,320958 & $-3,538157$ \\
\hline C82 & 0,782285 & 0,546597 & $-2,957598$ & $\mathrm{C} 82$ & 1,014190 & 0,596770 & $-2,844703$ \\
\hline $\mathrm{C} 83$ & 2,014814 & 1,157578 & $-4,780137$ & $\mathrm{C} 83$ & 2,468118 & 1,132737 & $-4,513051$ \\
\hline H84 & 3,191369 & 2,438017 & $-3,419097$ & H84 & 3,385466 & 2,620698 & $-3,168950$ \\
\hline H85 & 1,711908 & 3,213123 & $-4,023323$ & H85 & 1,902296 & 3,199144 & $-3,963897$ \\
\hline H86 & 1,646053 & 1,522587 & $-5,740083$ & H86 & 2,285224 & 1,405084 & $-5,553185$ \\
\hline H87 & 2,942325 & 0,598696 & $-4,927832$ & H87 & 3,403706 & 0,573880 & $-4,427801$ \\
\hline N88 & 1,393658 & 1,692068 & $-2,593554$ & N88 & 1,591135 & 1,753662 & $-2,455804$ \\
\hline O89 & 1,034454 & 0,235540 & $-4,241015$ & O89 & 1,400449 & 0,249112 & $-4,082562$ \\
\hline O90 & 0,078574 & $-0,220276$ & $-2,272604$ & O90 & 0,219975 & $-0,126608$ & $-2,215423$ \\
\hline H91 & 1,690070 & $-5,312458$ & 0,351887 & H91 & 1,447322 & $-5,296437$ & 0,281753 \\
\hline N92 & $-2,080084$ & $-2,087632$ & 0,108145 & N92 & $-2,087663$ & $-2,064249$ & 0,206832 \\
\hline C93 & $-1,446616$ & $-2,787698$ & $-1,033627$ & C93 & $-1,525662$ & $-2,638929$ & $-1,042371$ \\
\hline H94 & $-1,346212$ & $-2,138015$ & $-1,912527$ & H94 & $-1,450423$ & $-1,900535$ & $-1,850148$ \\
\hline H96 & $-0,716715$ & 1,933407 & $-0,735762$ & H96 & $-0,597644$ & 1,756589 & $-0,693654$ \\
\hline C97 & 0,235005 & 4,156271 & 3,364446 & C97 & $-0,236548$ & 4,407980 & 3,247056 \\
\hline C98 & $-0,859212$ & 4,173709 & 2,498619 & C98 & $-1,216081$ & 4,311537 & 2,257278 \\
\hline C99 & $-0,829433$ & 3,439081 & 1,310489 & C99 & $-1,010305$ & 3,503095 & 1,138514 \\
\hline C100 & 0,285664 & 2,660352 & 0,968642 & C100 & 0,175897 & 2,770876 & 0,994377 \\
\hline C101 & 1,384234 & 2,665596 & 1,842038 & C101 & 1,155033 & 2,879072 & 1,991695 \\
\hline C102 & 1,363454 & 3,404076 & 3,023469 & C102 & 0,954964 & 3,691472 & 3,106647 \\
\hline H103 & 0,214639 & 4,726860 & 4,288540 & H103 & $-0,398089$ & 5,034912 & 4,119123 \\
\hline H104 & $-1,739287$ & 4,762289 & 2,744004 & H104 & $-2,146081$ & 4,864790 & 2,353170 \\
\hline H105 & $-1,684065$ & 3,464982 & 0,640713 & H105 & $-1,775623$ & 3,439963 & 0,371914 \\
\hline H106 & 2,255799 & 2,060338 & 1,605442 & H106 & 2,075091 & 2,302378 & 1,912977 \\
\hline H107 & 2,225188 & 3,385879 & 3,685470 & H107 & 1,724494 & 3,756468 & 3,870542 \\
\hline H108 & 2,247137 & $-0,082602$ & $-0,743504$ & H108 & 1,441819 & 1,010523 & 0,528848 \\
\hline H109 & 2,300523 & 0,156327 & 0,049631 & H109 & 2,296573 & $-0,250062$ & $-0,468067$ \\
\hline
\end{tabular}

\section{C1_E_S_tN_Chelbot}


E4

\section{C1}

$\mathrm{C} 2$

C3

$\mathrm{C} 4$

H5

H6

$\mathrm{H} 7$

H8 $\quad-4,408994$

H9 $\quad-2,163541$

H10 - 4,489920

C11 $-3,091201$

H12 - $-2,335354$

H13 - -3,969194

C14 $-0,115842$

N15 $\quad 0,855719$

C16 2,088143

C17 1,496230

P18 $-1,463684$

Ir19 $\quad 0,764257$

C20 $-1,434252$

C21 - 1,050883

$\mathrm{C} 22$

$\mathrm{C} 23$

$\mathrm{C} 24$

$\mathrm{C} 25$

$\mathrm{H} 26$

H27

H28

H29

O30

C31

C32

C33

C34

H35

C36

H37

C38

H39

$\mathrm{H} 40$

H4 1

C42

C43

C44

$\mathrm{C} 45$

H46

C47
$-1,659851$

$-1,001241$

$-0,816415$

$-1,471666$

$-0,791768$

$-0,479380$

$-1,649047$

$-0,902197$

0,140693

2,575370

1,738916

3,818086

2,157313

0,757704

4,233500

4,468741

3,409275

1,494281

5,203891

3,738409

$-2,695400$

$-3,510995$

$-2,802919$

$-4,405507$

$-3,443706$

$-3,698230$
$-2,211852$

$-3,673862$

$-2,902100$

$-2,686235$

$-2,555375$

$-2,185626$

$-2,539275$

$-1,122843$

$-4,451804$

$-3,603704$

$-4,122596$

$-4,599039$

$-4,770443$

$-3,133136$

$-2,422475$

$-3,287961$

$-4,686332$

$-0,638096$

$-0,393387$

$-0,882337$

$-1,224185$

$-2,128835$

0,192121

0,020691

$-2,295649$

1,158814

0,858597

$-3,267840$

$-1,357017$

$-4,417845$

$-3,374404$

$-3,034309$

$-3,961689$

$-3,219027$

$-2,618897$

$-4,153228$

$-4,266833$

$-3,771222$

$-2,928672$

$-4,600364$

$-3,913053$

0,694027

1,248321

1,173814

2,274103

0,891041

2,196855
$-0,807837$

$-1,510030$

$-2,076284$

0,307894

$-0,607275$

$-2,852141$

1,326188

$-0,879941$

$-2,162495$

$-2,526086$

$-0,128994$

0,502410

$-0,204618$

$-0,819463$

$-0,344808$

$-0,208621$

$-0,596486$

0,799795

$-0,081898$

2,610002

5,370405

3,206427

3,407457

4,777355

4,581704

2,956523

5,381073

5,033054

6,438074

$-1,058913$

1,235853

2,303963

1,513001

3,621350

2,117960

2,830433

0,698631

3,889778

4,431452

3,026734

4,915210

0,507896

1,502547

$-0,810901$

1,188812

2,524994

$-1,119891$
ES_Chelbot_SM

$\begin{array}{ll}\mathrm{C} 1 & -3,836737 \\ \mathrm{C} 2 & -1,575812 \\ \mathrm{C} 3 & -2,779710 \\ \mathrm{C} 4 & -3,076036 \\ \mathrm{H} 5 & -4,735065 \\ \text { H6 } & -2,506930 \\ \text { H7 } & -3,689116 \\ \text { H8 } & -4,148384 \\ \text { H9 } & -0,850984 \\ \text { H10 } & -3,146271\end{array}$

$-3,471497$

$-1,259336$

$-4,394314 \quad-1,183623$

$-4,212660 \quad-2,145241$

$\begin{array}{ll}-3,299379 & 0,073748\end{array}$

$-4,078844 \quad-1,107657$

$-3,647510 \quad-3,042214$

$\begin{array}{ll}-3,013060 & 0,929069\end{array}$

$-2,510838-1,671522$

$-5,148978 \quad-1,489145$

$-5,189082 \quad-2,473613$

$-4,615981 \quad 0,171169$

$-4,654871 \quad 1,024821$

$-5,503106 \quad 0,175121$

$-2,993957 \quad-0,274591$

$-1,926316 \quad 0,128865$

$-2,307373 \quad 0,663661$

$-3,860688 \quad 0,717650$

$\begin{array}{ll}-0,707420 & 0,306425\end{array}$

$\begin{array}{ll}0,089759 & -0,263447\end{array}$

$-0,625658 \quad 2,115336$

$-0,404548 \quad 4,909137$

$\begin{array}{ll}-1,771869 & 2,918547\end{array}$

$0,635968 \quad 2,725764$

$0,742376 \quad 4,113113$

$-1,658999 \quad 4,309610$

$1,536426 \quad 2,124824$

$1,725373 \quad 4,568829$

\begin{tabular}{ll}
$-2,553441 \quad 4,923345$ \\
\hline
\end{tabular}

$-0,319762 \quad 5,991346$

$-4,156116 \quad-0,068915$

$-1,790388 \quad 2,078822$

$-1,419158 \quad 2,897940$

$-1,804741 \quad 2,623743$

$\begin{array}{ll}-1,008563 & 4,212969\end{array}$

$-1,440064 \quad 2,513029$

$-1,400935 \quad 3,940909$

$-2,119531 \quad 2,015629$

$-0,987775 \quad 4,735983$

$-0,707890 \quad 4,820268$

$-1,406836 \quad 4,342766$

$-0,663028 \quad 5,757954$

$0,037719 \quad-0,447270$

$0,582424 \quad 0,285180$

$0,047937-1,852055$

$1,159076 \quad-0,374869$

$0,570788 \quad 1,369333$

$0,627590 \quad-2,507199$ 


\begin{tabular}{|c|c|c|c|c|c|c|c|}
\hline $\mathrm{C} 48$ & $-4,498141$ & 2,755788 & $-0,118213$ & C48 & $-5,580224$ & 1,198237 & $-1,768330$ \\
\hline H49 & $-5,029501$ & 2,698374 & 1,970489 & $\mathrm{H} 49$ & $-6,353853$ & 1,580232 & 0,206638 \\
\hline H50 & $-3,761413$ & 2,569191 & $-2,137926$ & $\mathrm{H} 50$ & $-4,575811$ & 0,622694 & $-3,593265$ \\
\hline H51 & $-5,189586$ & 3,558702 & $-0,356404$ & H51 & $-6,425218$ & 1,652493 & $-2,277076$ \\
\hline H52 & 1,334877 & $-0,565343$ & 1,349212 & $\mathrm{H} 52$ & 0,608897 & 0,404722 & 1,229184 \\
\hline H53 & 2,149311 & 3,251278 & $-1,021340$ & $\mathrm{H} 53$ & 1,928447 & 0,164483 & $-0,493086$ \\
\hline H54 & $-1,951088$ & $-2,978885$ & 2,598262 & $\mathrm{H} 54$ & $-1,945313$ & $-2,749349$ & 2,464864 \\
\hline H55 & $-2,173458$ & 0,752234 & $-1,591435$ & $\mathrm{H} 55$ & $-2,663078$ & $-0,416282$ & $-2,432207$ \\
\hline H56 & 2,038645 & $-5,185579$ & $-1,399547$ & H56 & 3,053669 & $-4,399567$ & 0,282697 \\
\hline $\mathrm{C} 57$ & 4,834472 & $-1,733562$ & $-3,191210$ & C57 & 5,053766 & $-0,914149$ & $-2,449808$ \\
\hline C58 & 3,691967 & $-2,441800$ & $-3,568945$ & C58 & 4,295305 & $-2,073232$ & $-2,626408$ \\
\hline $\mathrm{C} 59$ & 2,840907 & $-2,960146$ & $-2,595211$ & C59 & 3,468129 & $-2,530832$ & $-1,603228$ \\
\hline C60 & 3,111117 & $-2,781081$ & $-1,232241$ & C60 & 3,386346 & $-1,846427$ & $-0,382129$ \\
\hline C61 & 4,239823 & $-2,039708$ & $-0,864913$ & C61 & 4,145640 & $-0,679903$ & $-0,219943$ \\
\hline C62 & 5,100784 & $-1,529550$ & $-1,836827$ & C62 & 4,974457 & $-0,219299$ & $-1,244106$ \\
\hline H63 & 5,514478 & $-1,348777$ & $-3,946903$ & $\mathrm{H} 63$ & 5,699427 & $-0,556992$ & $-3,247079$ \\
\hline H64 & 3,463428 & $-2,594027$ & $-4,619719$ & H64 & 4,344835 & $-2,619914$ & $-3,563588$ \\
\hline H65 & 1,944476 & $-3,487728$ & $-2,911461$ & H65 & 2,867147 & $-3,419729$ & $-1,776360$ \\
\hline H66 & 4,442397 & $-1,845599$ & 0,181491 & H66 & 4,088834 & $-0,114113$ & 0,700613 \\
\hline H67 & 5,980021 & $-0,969166$ & $-1,531299$ & $\mathrm{H} 67$ & 5,551694 & 0,688682 & $-1,093785$ \\
\hline C68 & $-1,619221$ & 4,680817 & $-3,163111$ & C68 & $-1,304366$ & 4,199888 & 2,244393 \\
\hline C69 & $-0,772133$ & 3,659344 & $-2,735448$ & C69 & $-0,506681$ & 3,450245 & 1,382024 \\
\hline $\mathrm{C} 70$ & 0,327739 & 3,948895 & $-1,918474$ & $\mathrm{C} 70$ & $-1,034630$ & 2,920125 & 0,190256 \\
\hline C71 & 0,564092 & 5,270574 & $-1,531943$ & C71 & $-2,392371$ & 3,156656 & $-0,086778$ \\
\hline $\mathrm{C} 72$ & $-0,288416$ & 6,292483 & $-1,953748$ & $\mathrm{C} 72$ & $-3,189872$ & 3,900135 & 0,783879 \\
\hline $\mathrm{C} 73$ & $-1,381457$ & 6,000909 & $-2,770938$ & $\mathrm{C} 73$ & $-2,651271$ & 4,432892 & 1,954603 \\
\hline H74 & $-2,465959$ & 4,447639 & $-3,802926$ & $\mathrm{H} 74$ & $-0,865607$ & 4,601276 & 3,153717 \\
\hline H75 & $-0,972236$ & 2,632587 & $-3,030194$ & $\mathrm{H} 75$ & 0,522738 & 3,272204 & 1,651035 \\
\hline H76 & 1,407548 & 5,500227 & $-0,885605$ & H76 & $-2,844845$ & 2,726444 & $-0,972318$ \\
\hline H77 & $-0,096951$ & 7,315657 & $-1,643653$ & $\mathrm{H} 77$ & $-4,238464$ & 4,049667 & 0,543237 \\
\hline H78 & $-2,042679$ & 6,796158 & $-3,102243$ & $\mathrm{H} 78$ & $-3,268447$ & 5,017175 & 2,630616 \\
\hline C79 & 1,213216 & 2,823567 & $-1,397356$ & C79 & $-0,212417$ & 2,109027 & $-0,765035$ \\
\hline $\mathrm{C} 80$ & 0,505030 & 2,121767 & $-0,211166$ & $\mathrm{C} 80$ & 1,236053 & 1,998019 & $-0,778653$ \\
\hline $\mathrm{C} 81$ & 2,505019 & 2,394979 & $-3,524387$ & C81 & $-0,935934$ & 3,245910 & $-2,968553$ \\
\hline $\mathrm{C} 82$ & 1,012506 & 0,747479 & $-2,861258$ & $\mathrm{C} 82$ & $-0,604715$ & 0,968105 & $-2,897709$ \\
\hline $\mathrm{C} 83$ & 2,672634 & 1,127555 & $-4,376003$ & $\mathrm{C} 83$ & $-1,484582$ & 2,573157 & $-4,233728$ \\
\hline H84 & 3,449881 & 2,743866 & $-3,097502$ & H84 & 0,035666 & 3,732796 & $-3,131542$ \\
\hline H85 & 2,035246 & 3,223588 & $-4,068778$ & H85 & $-1,626170$ & 3,968899 & $-2,532463$ \\
\hline H86 & 2,639344 & 1,308128 & $-5,450906$ & H86 & $-2,576900$ & 2,505584 & $-4,216071$ \\
\hline H87 & 3,572152 & 0,564111 & $-4,113036$ & H87 & $-1,148363$ & 3,022190 & $-5,168140$ \\
\hline N88 & 1,608002 & 1,904113 & $-2,475788$ & N88 & $-0,777338$ & 2,062452 & $-2,113428$ \\
\hline O89 & 1,530593 & 0,306335 & $-4,018970$ & O89 & $-0,953693$ & 1,217123 & $-4,168339$ \\
\hline O90 & 0,105644 & 0,116493 & $-2,294220$ & O90 & $-0,188645$ & $-0,141709$ & $-2,532029$ \\
\hline H91 & 1,418863 & $-5,346297$ & 0,268038 & H91 & 2,025618 & $-4,212674$ & 1,733630 \\
\hline N92 & $-2,097414$ & $-2,050395$ & 0,126010 & N92 & $-1,955636$ & $-2,335852$ & $-0,120541$ \\
\hline C93 & $-1,503158$ & $-2,612989$ & $-1,115709$ & C93 & $-0,939826$ & $-2,999620$ & $-0,973852$ \\
\hline H94 & $-1,388952$ & $-1,858094$ & $-1,903955$ & H94 & $-0,797152$ & $-2,470932$ & $-1,924990$ \\
\hline H96 & $-0,467384$ & 1,790031 & $-0,549387$ & H96 & 1,632593 & 1,919787 & $-1,792671$ \\
\hline
\end{tabular}




$\begin{array}{lrrrlllr}\text { C97 } & 0,042285 & 4,614948 & 3,271978 & \text { C97 } & 4,106730 & 4,162268 & 1,598419 \\ \text { C98 } & -1,019338 & 4,449636 & 2,380960 & \text { C98 } & 3,600068 & 4,678341 & 0,404511 \\ \text { C99 } & -0,869644 & 3,643445 & 1,252427 & \text { C99 } & 2,670158 & 3,947225 & -0,332235 \\ \text { C100 } & 0,346525 & 2,995341 & 1,008341 & \text { C100 } & 2,223482 & 2,693929 & 0,112234 \\ \text { C101 } & 1,407988 & 3,162052 & 1,906104 & \text { C101 } & 2,742664 & 2,181849 & 1,308224 \\ \text { C102 } & 1,258170 & 3,969559 & 3,032783 & \text { C102 } & 3,675540 & 2,912540 & 2,044777 \\ \text { H103 } & -0,077135 & 5,240585 & 4,152132 & \text { H103 } & 4,833841 & 4,729271 & 2,172496 \\ \text { H104 } & -1,968753 & 4,944422 & 2,564869 & \text { H104 } & 3,929354 & 5,649145 & 0,044810 \\ \text { H105 } & -1,697485 & 3,515661 & 0,562448 & \text { H105 } & 2,279596 & 4,355741 & -1,261870 \\ \text { H106 } & 2,347995 & 2,641552 & 1,733005 & \text { H106 } & 2,412168 & 1,214930 & 1,667817 \\ \text { H107 } & 2,085266 & 4,089246 & 3,726858 & \text { H107 } & 4,065613 & 2,496102 & 2,969302 \\ \text { H108 } & 1,259546 & 1,308447 & 0,135352 & & & & \\ \text { H109 } & 2,294829 & -0,311753 & -0,568377 & & & & \end{array}$

C1_ES_Chelbot_TS1

\begin{tabular}{|c|c|c|c|c|c|c|c|}
\hline $\mathrm{C} 1$ & $-3,890112$ & $-3,534552$ & $-1,110363$ & $\mathrm{C} 1$ & $-3,922167$ & $-3,535557$ & $-0,845760$ \\
\hline $\mathrm{C} 2$ & $-1,631229$ & $-4,463929$ & $-1,079330$ & $\mathrm{C} 2$ & $-1,682248$ & $-4,505975$ & $-0,875384$ \\
\hline $\mathrm{C} 3$ & $-2,860492$ & $-4,296359$ & $-2,010860$ & $\mathrm{C} 3$ & $-2,953609$ & $-4,365892$ & $-1,753156$ \\
\hline $\mathrm{C} 4$ & $-3,098485$ & $-3,354119$ & 0,203868 & $\mathrm{C} 4$ & $-3,070026$ & $-3,317475$ & 0,424945 \\
\hline H5 & $-4,791446$ & $-4,130297$ & $-0,932523$ & H5 & $-4,830153$ & $-4,096773$ & $-0,602200$ \\
\hline H6 & $-2,611115$ & $-3,749735$ & $-2,925971$ & H6 & $-2,742784$ & $-3,875484$ & $-2,708887$ \\
\hline H7 & $-3,692147$ & $-3,060030$ & 1,070139 & $\mathrm{H} 7$ & $-3,621589$ & $-2,980046$ & 1,302946 \\
\hline $\mathrm{H} 8$ & $-4,200129$ & $-2,574781$ & $-1,526957$ & H8 & $-4,225582$ & $-2,587775$ & $-1,293789$ \\
\hline H9 & $-0,912709$ & $-5,220574$ & $-1,394775$ & H9 & $-0,986636$ & $-5,283712$ & $-1,190540$ \\
\hline H10 & $-3,239313$ & $-5,277707$ & $-2,309765$ & H10 & $-3,364253$ & $-5,354445$ & $-1,977010$ \\
\hline $\mathrm{C} 11$ & $-2,305924$ & $-4,670014$ & 0,295949 & $\mathrm{C} 11$ & $-2,293896$ & $-4,641574$ & 0,537149 \\
\hline H12 & $-1,601790$ & $-4,695968$ & 1,133361 & H12 & $-1,553521$ & $-4,641675$ & 1,343164 \\
\hline H13 & $-2,943691$ & $-5,557847$ & 0,326556 & H13 & $-2,942847$ & $-5,516412$ & 0,634798 \\
\hline $\mathrm{C} 14$ & 0,375664 & $-3,038611$ & $-0,272659$ & $\mathrm{C} 14$ & 0,392823 & $-3,057206$ & $-0,284548$ \\
\hline N15 & 0,964758 & $-1,964872$ & 0,122388 & N15 & 0,973673 & $-1,977302$ & 0,101590 \\
\hline $\mathrm{C} 16$ & 2,361157 & $-2,312735$ & 0,580526 & $\mathrm{C} 16$ & 2,391639 & $-2,285109$ & 0,467499 \\
\hline $\mathrm{C} 17$ & 2,287653 & $-3,875147$ & 0,555195 & $\mathrm{C} 17$ & 2,451027 & $-3,830399$ & 0,178239 \\
\hline P18 & $-1,910208$ & $-0,754765$ & 0,349199 & P18 & $-1,853245$ & $-0,733679$ & 0,405834 \\
\hline Ir19 & 0,258869 & 0,046517 & $-0,229981$ & Ir19 & 0,162355 & 0,023971 & $-0,226352$ \\
\hline $\mathrm{C} 20$ & $-2,166115$ & $-0,608508$ & 2,153143 & $\mathrm{C} 20$ & $-2,106193$ & $-0,495120$ & 2,195510 \\
\hline $\mathrm{C} 21$ & $-2,285923$ & $-0,278419$ & 4,932011 & $\mathrm{C} 21$ & $-2,224971$ & $-0,020267$ & 4,948810 \\
\hline $\mathrm{C} 22$ & $-2,091489$ & $-1,722022$ & 2,998906 & $\mathrm{C} 22$ & $-1,997456$ & $-1,560878$ & 3,096608 \\
\hline $\mathrm{C} 23$ & $-2,282990$ & 0,676374 & 2,709582 & $\mathrm{C} 23$ & $-2,252957$ & 0,815868 & 2,679828 \\
\hline $\mathrm{C} 24$ & $-2,345880$ & 0,836609 & 4,091358 & $\mathrm{C} 24$ & $-2,315188$ & 1,047145 & 4,050899 \\
\hline $\mathrm{C} 25$ & $-2,155165$ & $-1,554879$ & 4,384161 & $\mathrm{C} 25$ & $-2,063542$ & $-1,321142$ & 4,470965 \\
\hline H26 & $-2,311218$ & 1,552412 & 2,071727 & $\mathrm{H} 26$ & $-2,299480$ & 1,654485 & 1,993543 \\
\hline $\mathrm{H} 27$ & $-2,433659$ & 1,835787 & 4,508617 & $\mathrm{H} 27$ & $-2,425078$ & 2,064209 & 4,415679 \\
\hline $\mathrm{H} 28$ & $-2,096141$ & $-2,423513$ & 5,033743 & $\mathrm{H} 28$ & $-1,981091$ & $-2,151921$ & 5,165988 \\
\hline $\mathrm{H} 29$ & $-2,334347$ & $-0,151092$ & 6,009859 & $\mathrm{H} 29$ & $-2,273650$ & 0,163365 & 6,018355 \\
\hline $\mathrm{O} 30$ & 1,043765 & $-4,187098$ & $-0,141976$ & $\mathrm{O} 30$ & 1,112447 & $-4,180872$ & $-0,282738$ \\
\hline C31 & 2,620273 & $-1,843382$ & 2,009016 & $\mathrm{C} 31$ & 2,653678 & $-2,025228$ & 1,948413 \\
\hline C32 & 1,561546 & $-1,505858$ & 2,859452 & $\mathrm{C} 32$ & 1,642403 & $-1,613607$ & 2,820671 \\
\hline $\mathrm{C} 33$ & 3,923944 & $-1,845388$ & 2,524295 & $\mathrm{C} 33$ & 3,933065 & $-2,277612$ & 2,466512 \\
\hline
\end{tabular}




\begin{tabular}{|c|c|c|c|c|c|c|c|}
\hline $\mathrm{C} 34$ & 1,802708 & $-1,114500$ & 4,176428 & C34 & 1,912668 & $-1,417012$ & 4,176514 \\
\hline H35 & 0,543045 & $-1,534022$ & 2,496000 & H35 & 0,641530 & $-1,442598$ & 2,449502 \\
\hline C36 & 4,165740 & $-1,459895$ & 3,842702 & $\mathrm{C} 36$ & 4,202199 & $-2,084241$ & 3,819623 \\
\hline H37 & 4,758040 & $-2,132333$ & 1,890729 & H37 & 4,726595 & $-2,614502$ & 1,803764 \\
\hline C38 & 3,107118 & $-1,078671$ & 4,669629 & $\mathrm{C} 38$ & 3,192788 & $-1,643410$ & 4,678766 \\
\hline H39 & 0,963357 & $-0,837997$ & 4,808594 & H39 & 1,110925 & $-1,089531$ & 4,832750 \\
\hline $\mathrm{H} 40$ & 5,184133 & $-1,453199$ & 4,220924 & $\mathrm{H} 40$ & 5,200432 & $-2,276392$ & 4,202194 \\
\hline H41 & 3,299200 & $-0,767310$ & 5,692646 & $\mathrm{H} 41$ & 3,404345 & $-1,488175$ & 5,732729 \\
\hline $\mathrm{C} 42$ & $-3,390656$ & $-0,008389$ & $-0,442763$ & $\mathrm{C} 42$ & $-3,313379$ & $-0,014336$ & $-0,432881$ \\
\hline $\mathrm{C} 43$ & $-4,477904$ & 0,520503 & 0,262061 & $\mathrm{C} 43$ & $-4,424227$ & 0,501728 & 0,245164 \\
\hline $\mathrm{C} 44$ & $-3,415408$ & 0,004708 & $-1,848767$ & $\mathrm{C} 44$ & $-3,327834$ & $-0,065129$ & $-1,838067$ \\
\hline $\mathrm{C} 45$ & $-5,557299$ & 1,080916 & $-0,426530$ & $\mathrm{C} 45$ & $-5,521627$ & 0,983296 & $-0,472164$ \\
\hline $\mathrm{H} 46$ & $-4,487374$ & 0,507958 & 1,346507 & $\mathrm{H} 46$ & $-4,441957$ & 0,533224 & 1,328818 \\
\hline $\mathrm{C} 47$ & $-4,490757$ & 0,567569 & $-2,532213$ & $\mathrm{C} 47$ & $-4,422684$ & 0,418417 & $-2,548940$ \\
\hline $\mathrm{C} 48$ & $-5,562904$ & 1,118700 & $-1,821105$ & $\mathrm{C} 48$ & $-5,520833$ & 0,951413 & $-1,865920$ \\
\hline H49 & $-6,394213$ & 1,489442 & 0,132900 & $\mathrm{H} 49$ & $-6,378083$ & 1,382686 & 0,063519 \\
\hline H50 & $-4,498692$ & 0,566789 & $-3,618943$ & $\mathrm{H} 50$ & $-4,425781$ & 0,369922 & $-3,634506$ \\
\hline H51 & $-6,401826$ & 1,559208 & $-2,351904$ & H51 & $-6,375877$ & 1,328324 & $-2,419691$ \\
\hline H52 & 0,457604 & 0,364248 & 1,272720 & $\mathrm{H} 52$ & 0,358663 & 0,395830 & 1,270501 \\
\hline H53 & 1,838351 & 0,335246 & $-0,465687$ & $\mathrm{H} 53$ & 1,871495 & 0,851245 & $-0,692610$ \\
\hline H54 & $-1,956438$ & $-2,714707$ & 2,583102 & $\mathrm{H} 54$ & $-1,834992$ & $-2,569632$ & 2,732095 \\
\hline H55 & $-2,598603$ & $-0,440567$ & $-2,407009$ & $\mathrm{H} 55$ & $-2,488276$ & $-0,498212$ & $-2,371281$ \\
\hline H56 & 3,109171 & $-4,345687$ & 0,014995 & H56 & 3,155528 & $-4,097031$ & $-0,611527$ \\
\hline $\mathrm{C} 57$ & 4,816541 & $-0,608844$ & $-2,574212$ & C57 & 4,704679 & 0,044809 & $-2,381224$ \\
\hline C58 & 4,066745 & $-1,766983$ & $-2,793793$ & C58 & 3,824536 & $-0,953112$ & $-2,809206$ \\
\hline C59 & 3,323707 & $-2,326082$ & $-1,757026$ & C59 & 3,120652 & $-1,706632$ & $-1,874740$ \\
\hline C60 & 3,317342 & $-1,747101$ & $-0,479742$ & C60 & 3,283051 & $-1,485582$ & $-0,496839$ \\
\hline C61 & 4,069059 & $-0,583788$ & $-0,272697$ & C61 & 4,162720 & $-0,481686$ & $-0,080571$ \\
\hline C62 & 4,814453 & $-0,020288$ & $-1,310833$ & C62 & 4,868908 & 0,277489 & $-1,017569$ \\
\hline H63 & 5,395670 & $-0,171067$ & $-3,382532$ & $\mathrm{H} 63$ & 5,255310 & 0,635584 & $-3,107678$ \\
\hline H64 & 4,056111 & $-2,231644$ & $-3,775444$ & $\mathrm{H} 64$ & 3,681512 & $-1,139944$ & $-3,869559$ \\
\hline H65 & 2,723676 & $-3,209141$ & $-1,961149$ & $\mathrm{H} 65$ & 2,414839 & $-2,454559$ & $-2,226424$ \\
\hline H66 & 4,069425 & $-0,096633$ & 0,693143 & H66 & 4,291531 & $-0,269082$ & 0,972870 \\
\hline H67 & 5,384835 & 0,885651 & $-1,124589$ & H67 & 5,537115 & 1,060542 & $-0,671961$ \\
\hline C68 & $-1,312077$ & 4,269219 & 2,125774 & C68 & $-1,293136$ & 4,392223 & 1,919171 \\
\hline C69 & $-0,481794$ & 3,501711 & 1,310432 & C69 & $-0,432849$ & 3,588225 & 1,170940 \\
\hline $\mathrm{C} 70$ & $-0,972984$ & 2,923672 & 0,126520 & $\mathrm{C} 70$ & $-0,869760$ & 2,960597 & $-0,006787$ \\
\hline $\mathrm{C} 71$ & $-2,326072$ & 3,134474 & $-0,194809$ & C71 & $-2,211631$ & 3,160841 & $-0,386631$ \\
\hline $\mathrm{C} 72$ & $-3,154931$ & 3,896090 & 0,627310 & $\mathrm{C} 72$ & $-3,072504$ & 3,957567 & 0,367018 \\
\hline $\mathrm{C} 73$ & $-2,652283$ & 4,473680 & 1,792664 & $\mathrm{C} 73$ & $-2,618370$ & 4,586561 & 1,527293 \\
\hline H74 & $-0,903382$ & 4,708587 & 3,031712 & $\mathrm{H} 74$ & $-0,917234$ & 4,866891 & 2,821494 \\
\hline H75 & 0,542235 & 3,349637 & 1,612343 & $\mathrm{H} 75$ & 0,574745 & 3,443768 & 1,527002 \\
\hline H76 & $-2,747985$ & 2,667276 & $-1,076987$ & $\mathrm{H} 76$ & $-2,602182$ & 2,651659 & $-1,260435$ \\
\hline H77 & $-4,198644$ & 4,025099 & 0,355404 & $\mathrm{H} 77$ & $-4,104223$ & 4,073485 & 0,046179 \\
\hline H78 & $-3,294276$ & 5,071701 & 2,433071 & $\mathrm{H} 78$ & $-3,283940$ & 5,212161 & 2,114844 \\
\hline C79 & $-0,141191$ & 2,059050 & $-0,773661$ & C79 & $-0,035033$ & 2,001046 & $-0,815950$ \\
\hline $\mathrm{C} 80$ & 1,325181 & 1,901088 & $-0,730143$ & $\mathrm{C} 80$ & 1,528273 & 1,952514 & $-0,804268$ \\
\hline C81 & $-0,738153$ & 3,220427 & $-3,014941$ & $\mathrm{C} 81$ & $-0,493325$ & 3,152482 & $-3,120438$ \\
\hline
\end{tabular}




\begin{tabular}{|c|c|c|c|c|c|c|c|}
\hline $\mathrm{C} 82$ & $-0,506030$ & 0,928816 & $-2,912554$ & C82 & $-0,366617$ & 0,858869 & $-2,949818$ \\
\hline C83 & $-1,264999$ & 2,554987 & $-4,294921$ & $\mathrm{C} 83$ & $-0,967209$ & 2,474263 & $-4,416301$ \\
\hline H84 & 0,249749 & 3,682628 & $-3,149341$ & H84 & 0,511373 & 3,588726 & $-3,211370$ \\
\hline H85 & $-1,424039$ & 3,962468 & $-2,603526$ & H85 & $-1,180186$ & 3,920758 & $-2,762166$ \\
\hline H86 & $-2,359110$ & 2,534707 & $-4,321333$ & H86 & $-2,056776$ & 2,502854 & $-4,517208$ \\
\hline H87 & $-0,874589$ & 2,979665 & $-5,219596$ & H87 & $-0,498017$ & 2,854180 & $-5,323827$ \\
\hline N88 & $-0,639548$ & 2,040288 & $-2,149315$ & N88 & $-0,472891$ & 1,992406 & $-2,224088$ \\
\hline O89 & $-0,796713$ & 1,177204 & $-4,197871$ & O89 & $-0,573248$ & 1,078384 & $-4,255755$ \\
\hline O90 & $-0,166263$ & $-0,196571$ & $-2,512724$ & O90 & $-0,111426$ & $-0,273208$ & $-2,497675$ \\
\hline H91 & 2,211398 & $-4,293652$ & 1,559984 & H91 & 2,653979 & $-4,414920$ & 1,075975 \\
\hline N92 & $-1,979476$ & $-2,397273$ & $-0,019156$ & N92 & $-1,941224$ & $-2,393571$ & 0,121692 \\
\hline C93 & $-0,994454$ & $-3,064104$ & $-0,905874$ & C93 & $-1,024587$ & $-3,105439$ & $-0,800368$ \\
\hline H94 & $-0,894851$ & $-2,547034$ & $-1,869506$ & H94 & $-0,992803$ & $-2,635734$ & $-1,793755$ \\
\hline H96 & 1,754188 & 1,850258 & $-1,733389$ & H96 & 1,890278 & 2,146172 & $-1,819924$ \\
\hline C97 & 4,039952 & 4,049582 & 1,832525 & $\mathrm{C} 97$ & 3,962379 & 4,173220 & 1,975169 \\
\hline C98 & 3,634125 & 4,563725 & 0,599762 & C98 & 3,602111 & 4,725439 & 0,744703 \\
\hline C99 & 2,760803 & 3,835634 & $-0,206261$ & C99 & 2,815826 & 3,994281 & $-0,145069$ \\
\hline $\mathrm{C} 100$ & 2,271669 & 2,589590 & 0,210919 & $\mathrm{C} 100$ & 2,375330 & 2,708806 & 0,187351 \\
\hline C101 & 2,690171 & 2,076926 & 1,444958 & C101 & 2,746334 & 2,156933 & 1,419282 \\
\hline $\mathrm{C} 102$ & 3,568065 & 2,804094 & 2,248849 & C102 & 3,533377 & 2,886670 & 2,309082 \\
\hline H103 & 4,721350 & 4,615800 & 2,460738 & H103 & 4,577018 & 4,741068 & 2,667633 \\
\hline H104 & 3,996747 & 5,531341 & 0,264487 & H104 & 3,934321 & 5,724264 & 0,476356 \\
\hline H105 & 2,444894 & 4,242523 & $-1,164574$ & H105 & 2,536109 & 4,428420 & $-1,102477$ \\
\hline H106 & 2,321234 & 1,115366 & 1,782109 & H106 & 2,409950 & 1,159499 & 1,684405 \\
\hline H107 & 3,881413 & 2,389230 & 3,202543 & H107 & 3,813598 & 2,446717 & 3,262111 \\
\hline \multicolumn{4}{|c|}{ C1_ES_Chelbot_TS2 } & \multicolumn{3}{|c|}{ C1_ES_Chelbot_INT2 } & \\
\hline $\mathrm{C} 1$ & $-3,650305$ & $-3,586452$ & $-1,241988$ & $\mathrm{C} 1$ & $-3,667825$ & $-3,630981$ & $-1,228670$ \\
\hline $\mathrm{C} 2$ & $-1,338706$ & $-4,369441$ & $-1,173943$ & $\mathrm{C} 2$ & $-1,348608$ & $-4,386477$ & $-1,123571$ \\
\hline $\mathrm{C} 3$ & $-2,561092$ & $-4,280839$ & $-2,125448$ & $\mathrm{C} 3$ & $-2,561129$ & $-4,325891$ & $-2,089436$ \\
\hline $\mathrm{C} 4$ & $-2,881591$ & $-3,320390$ & 0,069718 & $\mathrm{C} 4$ & $-2,915992$ & $-3,337030$ & 0,087052 \\
\hline H5 & $-4,498515$ & $-4,251430$ & $-1,048284$ & H5 & $-4,510983$ & $-4,302555$ & $-1,035418$ \\
\hline H6 & $-2,331831$ & $-3,717474$ & $-3,035668$ & H6 & $-2,327993$ & $-3,774555$ & $-3,005954$ \\
\hline H7 & $-3,499735$ & $-3,042241$ & 0,923327 & $\mathrm{H} 7$ & $-3,547028$ & $-3,054098$ & 0,929832 \\
\hline $\mathrm{H} 8$ & $-4,042814$ & $-2,667627$ & $-1,677501$ & $\mathrm{H} 8$ & $-4,064352$ & $-2,722299$ & $-1,681437$ \\
\hline H9 & $-0,576787$ & $-5,090752$ & $-1,467999$ & H9 & $-0,576528$ & $-5,104267$ & $-1,399279$ \\
\hline H10 & $-2,872577$ & $-5,283084$ & $-2,431975$ & H10 & $-2,857415$ & $-5,336666$ & $-2,383267$ \\
\hline $\mathrm{C} 11$ & $-2,018488$ & $-4,588765$ & 0,194891 & $\mathrm{C} 11$ & $-2,041194$ & $-4,594271$ & 0,240845 \\
\hline H12 & $-1,321679$ & $-4,571294$ & 1,038206 & H12 & $-1,354692$ & $-4,556401$ & 1,092076 \\
\hline H13 & $-2,612162$ & $-5,506219$ & 0,234050 & H13 & $-2,623771$ & $-5,518616$ & 0,285708 \\
\hline C14 & 0,563521 & $-2,848393$ & $-0,317652$ & $\mathrm{C} 14$ & 0,541263 & $-2,842588$ & $-0,285806$ \\
\hline N15 & 1,066515 & $-1,758079$ & 0,145894 & N15 & 1,050429 & $-1,751769$ & 0,170334 \\
\hline $\mathrm{C} 16$ & 2,426403 & $-2,073775$ & 0,726181 & $\mathrm{C} 16$ & 2,411576 & $-2,050986$ & 0,735784 \\
\hline $\mathrm{C} 17$ & 2,525042 & $-3,620846$ & 0,452218 & $\mathrm{C} 17$ & 2,521310 & $-3,597967$ & 0,457111 \\
\hline P18 & $-1,822959$ & $-0,694314$ & 0,327291 & P18 & $-1,893270$ & $-0,690939$ & 0,318551 \\
\hline Ir19 & 0,140518 & 0,230819 & $-0,282948$ & Ir19 & 0,193972 & 0,247753 & $-0,286031$ \\
\hline $\mathrm{C} 20$ & $-2,047449$ & $-0,716779$ & 2,138345 & $\mathrm{C} 20$ & $-2,101447$ & $-0,686860$ & 2,136290 \\
\hline $\mathrm{C} 21$ & $-2,265128$ & $-0,681916$ & 4,931522 & $\mathrm{C} 21$ & $-2,305027$ & $-0,609038$ & 4,931207 \\
\hline
\end{tabular}




\begin{tabular}{|c|c|c|c|c|c|c|c|}
\hline $\mathrm{C} 22$ & $-1,900591$ & $-1,901155$ & 2,873567 & $\mathrm{C} 22$ & $-1,967284$ & $-1,861006$ & 2,888711 \\
\hline $\mathrm{C} 23$ & $-2,283428$ & 0,488321 & 2,816920 & $\mathrm{C} 23$ & $-2,313545$ & 0,532084 & 2,798446 \\
\hline $\mathrm{C} 24$ & $-2,393678$ & 0,503574 & 4,204604 & $\mathrm{C} 24$ & $-2,416844$ & 0,567976 & 4,186990 \\
\hline $\mathrm{C} 25$ & $-2,016462$ & $-1,881495$ & 4,263482 & $\mathrm{C} 25$ & $-2,074431$ & $-1,820493$ & 4,279495 \\
\hline $\mathrm{H} 26$ & $-2,376489$ & 1,415156 & 2,268935 & $\mathrm{H} 26$ & $-2,391479$ & 1,454238 & 2,238376 \\
\hline $\mathrm{H} 27$ & $-2,574237$ & 1,445204 & 4,714993 & $\mathrm{H} 27$ & $-2,580896$ & 1,519488 & 4,684602 \\
\hline $\mathrm{H} 28$ & $-1,895201$ & $-2,803562$ & 4,824371 & $\mathrm{H} 28$ & $-1,964736$ & $-2,736621$ & 4,852600 \\
\hline $\mathrm{H} 29$ & $-2,351672$ & $-0,670895$ & 6,014354 & $\mathrm{H} 29$ & $-2,388260$ & $-0,580602$ & 6,013990 \\
\hline $\mathrm{O} 30$ & 1,274375 & $-3,969402$ & $-0,201321$ & $\mathrm{O} 30$ & 1,264423 & $-3,957179$ & $-0,179555$ \\
\hline C31 & 2,484549 & $-1,818452$ & 2,231097 & $\mathrm{C} 31$ & 2,484058 & $-1,804271$ & 2,241722 \\
\hline $\mathrm{C} 32$ & 1,434164 & $-1,267911$ & 2,962645 & $\mathrm{C} 32$ & 1,429094 & $-1,258855$ & 2,972583 \\
\hline C33 & 3,642894 & $-2,220551$ & 2,917874 & $\mathrm{C} 33$ & 3,641077 & $-2,209107$ & 2,927661 \\
\hline C34 & 1,541047 & $-1,102907$ & 4,346600 & $\mathrm{C} 34$ & 1,532206 & $-1,096807$ & 4,356498 \\
\hline H35 & 0,529187 & $-0,965066$ & 2,462850 & H35 & 0,519619 & $-0,965553$ & 2,473032 \\
\hline C36 & 3,753482 & $-2,052980$ & 4,294891 & $\mathrm{C} 36$ & 3,745402 & $-2,048587$ & 4,306629 \\
\hline H37 & 4,473781 & $-2,654762$ & 2,367637 & H37 & 4,472162 & $-2,644142$ & 2,377984 \\
\hline $\mathrm{C} 38$ & 2,699365 & $-1,488051$ & 5,016939 & C38 & 2,689383 & $-1,485091$ & 5,027158 \\
\hline H39 & 0,705529 & $-0,673753$ & 4,890985 & H39 & 0,693320 & $-0,673756$ & 4,900751 \\
\hline $\mathrm{H} 40$ & 4,660942 & $-2,365142$ & 4,803848 & $\mathrm{H} 40$ & 4,650409 & $-2,364096$ & 4,817885 \\
\hline H41 & 2,782321 & $-1,357183$ & 6,092093 & $\mathrm{H} 41$ & 2,770053 & $-1,358941$ & 6,103013 \\
\hline $\mathrm{C} 42$ & $-3,404295$ & $-0,073061$ & $-0,366221$ & $\mathrm{C} 42$ & $-3,494091$ & $-0,088258$ & $-0,358760$ \\
\hline $\mathrm{C} 43$ & $-4,459636$ & 0,406910 & 0,419526 & $\mathrm{C} 43$ & $-4,543050$ & 0,398174 & 0,430846 \\
\hline $\mathrm{C} 44$ & $-3,577133$ & $-0,170765$ & $-1,757214$ & C44 & $-3,682519$ & $-0,188532$ & $-1,748070$ \\
\hline $\mathrm{C} 45$ & $-5,652237$ & 0,815585 & $-0,179074$ & $\mathrm{C} 45$ & $-5,740956$ & 0,808633 & $-0,158935$ \\
\hline H46 & $-4,367942$ & 0,458161 & 1,497162 & $\mathrm{H} 46$ & $-4,438416$ & 0,462273 & 1,507323 \\
\hline $\mathrm{C} 47$ & $-4,767950$ & 0,238507 & $-2,350571$ & $\mathrm{C} 47$ & $-4,877671$ & 0,223313 & $-2,333728$ \\
\hline $\mathrm{C} 48$ & $-5,805962$ & 0,745849 & $-1,562382$ & $\mathrm{C} 48$ & $-5,909991$ & 0,735923 & $-1,540774$ \\
\hline H49 & $-6,461904$ & 1,185751 & 0,443037 & H49 & $-6,543170$ & 1,183473 & 0,470236 \\
\hline H50 & $-4,895209$ & 0,141859 & $-3,425338$ & $\mathrm{H} 50$ & $-5,012023$ & 0,123501 & $-3,407515$ \\
\hline H51 & $-6,735729$ & 1,064533 & $-2,024556$ & $\mathrm{H} 51$ & $-6,842909$ & 1,054844 & $-1,995864$ \\
\hline H52 & 0,426716 & 0,674167 & 1,182408 & $\mathrm{H} 52$ & 0,365066 & 0,684781 & 1,203127 \\
\hline H53 & 1,213594 & 2,719704 & $-2,168545$ & $\mathrm{H} 53$ & 1,258609 & 2,637417 & $-2,208456$ \\
\hline H54 & $-1,667540$ & $-2,831993$ & 2,371920 & H54 & $-1,750809$ & $-2,802608$ & 2,399629 \\
\hline H55 & $-2,798473$ & $-0,608262$ & $-2,372083$ & H55 & $-2,908530$ & $-0,622366$ & $-2,371948$ \\
\hline H56 & 3,338924 & $-3,887404$ & $-0,223646$ & H56 & 3,328707 & $-3,857095$ & $-0,229944$ \\
\hline $\mathrm{C} 57$ & 5,405154 & $-0,025842$ & $-1,682206$ & C57 & 5,315983 & 0,083749 & $-1,708907$ \\
\hline C58 & 4,498827 & $-0,919771$ & $-2,259289$ & C58 & 4,382249 & $-0,784697$ & $-2,283656$ \\
\hline C59 & 3,553345 & $-1,560772$ & $-1,463797$ & C59 & 3,473548 & $-1,464851$ & $-1,476530$ \\
\hline $\mathrm{C} 60$ & 3,490666 & $-1,322050$ & $-0,081959$ & C60 & 3,461690 & $-1,281469$ & $-0,083770$ \\
\hline C61 & 4,385196 & $-0,408945$ & 0,481979 & C61 & 4,380934 & $-0,392404$ & 0,479157 \\
\hline C62 & 5,345118 & 0,224225 & $-0,312992$ & C62 & 5,314018 & 0,267262 & $-0,327779$ \\
\hline H63 & 6,153177 & 0,467092 & $-2,296485$ & $\mathrm{H} 63$ & 6,036680 & 0,604917 & $-2,331797$ \\
\hline H64 & 4,531409 & $-1,118643$ & $-3,326671$ & H64 & 4,367594 & $-0,938371$ & $-3,358858$ \\
\hline H65 & 2,852872 & $-2,249858$ & $-1,929367$ & H65 & 2,753244 & $-2,133499$ & $-1,941444$ \\
\hline H66 & 4,341616 & $-0,185095$ & 1,541302 & H66 & 4,380101 & $-0,209390$ & 1,547170 \\
\hline H67 & 6,039158 & 0,922821 & 0,145341 & H67 & 6,039592 & 0,931012 & 0,132966 \\
\hline C68 & $-1,708231$ & 3,874909 & 2,305937 & C68 & $-1,604444$ & 3,941757 & 2,271165 \\
\hline C69 & $-0,916093$ & 3,206987 & 1,376789 & C69 & $-0,817438$ & 3,281424 & 1,331314 \\
\hline
\end{tabular}




\begin{tabular}{|c|c|c|c|c|c|c|c|}
\hline $\mathrm{C} 70$ & $-1,407634$ & 2,855384 & 0,105601 & $\mathrm{C} 70$ & $-1,340844$ & 2,870040 & 0,089846 \\
\hline C71 & $-2,758770$ & 3,122226 & $-0,156161$ & C71 & $-2,709334$ & 3,084892 & $-0,130233$ \\
\hline $\mathrm{C} 72$ & $-3,566779$ & 3,765842 & 0,786400 & $\mathrm{C} 72$ & $-3,508037$ & 3,726256 & 0,822209 \\
\hline $\mathrm{C} 73$ & $-3,043264$ & 4,168415 & 2,012511 & $\mathrm{C} 73$ & $-2,960138$ & 4,177228 & 2,021382 \\
\hline H74 & $-1,288139$ & 4,140072 & 3,272666 & H74 & $-1,158960$ & 4,253618 & 3,212055 \\
\hline H75 & 0,092987 & 2,923977 & 1,646266 & H75 & 0,214053 & 3,059932 & 1,571001 \\
\hline H76 & $-3,213191$ & 2,784044 & $-1,079480$ & H76 & $-3,181840$ & 2,707015 & $-1,028248$ \\
\hline H77 & $-4,612733$ & 3,944449 & 0,553383 & H77 & $-4,566872$ & 3,860000 & 0,619564 \\
\hline $\mathrm{H} 78$ & $-3,667794$ & 4,681722 & 2,737979 & $\mathrm{H} 78$ & $-3,577690$ & 4,685303 & 2,756068 \\
\hline C79 & $-0,465556$ & 2,198501 & $-0,888494$ & C79 & $-0,423112$ & 2,200669 & $-0,920572$ \\
\hline $\mathrm{C} 80$ & 0,718068 & 3,195837 & $-1,316237$ & $\mathrm{C} 80$ & 0,763888 & 3,153339 & $-1,375943$ \\
\hline C81 & $-1,611294$ & 3,016398 & $-3,162003$ & C81 & $-1,611731$ & 2,969333 & $-3,176808$ \\
\hline $\mathrm{C} 82$ & $-0,904734$ & 0,853407 & $-2,900058$ & C82 & $-0,947598$ & 0,798387 & $-2,876817$ \\
\hline $\mathrm{C} 83$ & $-2,174247$ & 2,123654 & $-4,271265$ & $\mathrm{C} 83$ & $-2,228857$ & 2,060141 & $-4,243783$ \\
\hline H84 & $-0,773607$ & 3,624654 & $-3,518881$ & H84 & $-0,771596$ & 3,548628 & $-3,574463$ \\
\hline H85 & $-2,356904$ & 3,674051 & $-2,717172$ & H85 & $-2,329147$ & 3,653815 & $-2,725338$ \\
\hline H86 & $-3,228900$ & 1,882907 & $-4,108992$ & H86 & $-3,282548$ & 1,847043 & $-4,041971$ \\
\hline H87 & $-2,024690$ & 2,511207 & $-5,278865$ & H87 & $-2,101622$ & 2,415230 & $-5,266264$ \\
\hline N88 & $-1,148301$ & 1,983514 & $-2,210043$ & N88 & $-1,143672$ & 1,951993 & $-2,212696$ \\
\hline O89 & $-1,409715$ & 0,889720 & $-4,142304$ & O89 & $-1,488899$ & 0,811922 & $-4,103280$ \\
\hline O90 & $-0,303024$ & $-0,157367$ & $-2,481060$ & O90 & $-0,362764$ & $-0,217232$ & $-2,446833$ \\
\hline H91 & 2,591048 & $-4,205527$ & 1,370044 & H91 & 2,605140 & $-4,182761$ & 1,373263 \\
\hline N92 & $-1,816098$ & $-2,298649$ & $-0,178865$ & N92 & $-1,862538$ & $-2,305979$ & $-0,162793$ \\
\hline C93 & $-0,772948$ & $-2,937490$ & $-1,009045$ & C93 & $-0,800090$ & $-2,945069$ & $-0,972224$ \\
\hline H94 & $-0,662468$ & $-2,435359$ & $-1,977645$ & H94 & $-0,687492$ & $-2,456342$ & $-1,947006$ \\
\hline H95 & 0,190929 & 4,070891 & $-1,718362$ & H95 & 0,289901 & 4,045841 & $-1,802597$ \\
\hline C96 & 3,883141 & 4,849470 & 1,165850 & C96 & 3,813558 & 4,653363 & 1,312632 \\
\hline C97 & 2,557910 & 5,228119 & 1,385219 & C97 & 2,584626 & 5,306426 & 1,201113 \\
\hline C98 & 1,532960 & 4,702389 & 0,599380 & C98 & 1,608592 & 4,816989 & 0,336351 \\
\hline C99 & 1,799578 & 3,768518 & $-0,414107$ & C99 & 1,825743 & 3,655514 & $-0,421688$ \\
\hline $\mathrm{C} 100$ & 3,143746 & 3,445489 & $-0,656257$ & $\mathrm{C} 100$ & 3,083396 & 3,042333 & $-0,337913$ \\
\hline C101 & 4,173660 & 3,968888 & 0,125231 & $\mathrm{C} 101$ & 4,063169 & 3,527855 & 0,529803 \\
\hline H102 & 4,680124 & 5,258241 & 1,780604 & $\mathrm{H} 102$ & 4,577037 & 5,033683 & 1,985255 \\
\hline $\mathrm{H} 103$ & 2,318096 & 5,944828 & 2,166170 & H103 & 2,389201 & 6,203623 & 1,781790 \\
\hline H104 & 0,519347 & 5,049690 & 0,759873 & H104 & 0,658570 & 5,336595 & 0,251996 \\
\hline H105 & 3,386092 & 2,823360 & $-1,513493$ & H105 & 3,327115 & 2,199766 & $-0,978698$ \\
\hline H106 & 5,203097 & 3,696802 & $-0,092055$ & H106 & 5,028947 & 3,034393 & 0,576921 \\
\hline H107 & 2,819279 & 1,196713 & $-0,936483$ & H107 & 1,766102 & 0,905772 & $-0,108723$ \\
\hline H108 & 2,611383 & 0,899730 & $-1,585830$ & H108 & 1,749935 & 0,617851 & $-0,922717$ \\
\hline \multicolumn{4}{|c|}{ C1_ES_Chelbot_TS3 } & \multicolumn{4}{|c|}{ C1_ES_Chelbot_PROD } \\
\hline $\mathrm{C} 1$ & $-3,687428$ & $-3,668740$ & $-1,267620$ & $\mathrm{C} 1$ & $-3,668498$ & $-3,640196$ & $-1,327243$ \\
\hline $\mathrm{C} 2$ & $-1,367048$ & $-4,416472$ & $-1,152490$ & $\mathrm{C} 2$ & $-1,348884$ & $-4,392078$ & $-1,239709$ \\
\hline $\mathrm{C} 3$ & $-2,569987$ & $-4,344974$ & $-2,129406$ & $\mathrm{C} 3$ & $-2,547434$ & $-4,272620$ & $-2,216826$ \\
\hline $\mathrm{C} 4$ & $-2,953396$ & $-3,403422$ & 0,065073 & $\mathrm{C} 4$ & $-2,943162$ & $-3,449399$ & 0,023486 \\
\hline H5 & $-4,533364$ & $-4,344078$ & $-1,101193$ & H5 & $-4,518301$ & $-4,319846$ & $-1,204179$ \\
\hline H6 & $-2,330437$ & $-3,778407$ & $-3,034973$ & H6 & $-2,304050$ & $-3,661673$ & $-3,092115$ \\
\hline $\mathrm{H} 7$ & $-3,599036$ & $-3,143257$ & 0,903864 & $\mathrm{H} 7$ & $-3,598002$ & $-3,236830$ & 0,868555 \\
\hline
\end{tabular}




\begin{tabular}{|c|c|c|c|c|c|c|c|}
\hline H8 & $-4,077587$ & $-2,749719$ & $-1,705134$ & H8 & $-4,051825$ & $-2,697125$ & $-1,717171$ \\
\hline H9 & $-0,586433$ & $-5,122722$ & $-1,434761$ & H9 & $-0,565011$ & $-5,080350$ & $-1,555409$ \\
\hline H10 & $-2,859116$ & $-5,352324$ & $-2,441811$ & $\mathrm{H} 10$ & $-2,834646$ & $-5,263213$ & $-2,580285$ \\
\hline $\mathrm{C} 11$ & $-2,073377$ & $-4,659283$ & 0,199984 & $\mathrm{C} 11$ & $-2,060452$ & $-4,708192$ & 0,096121 \\
\hline H12 & $-1,396594$ & $-4,634450$ & 1,059333 & H12 & $-1,389326$ & $-4,721618$ & 0,960119 \\
\hline H13 & $-2,649001$ & $-5,589064$ & 0,216555 & H13 & $-2,630677$ & $-5,640763$ & 0,059782 \\
\hline $\mathrm{C} 14$ & 0,498407 & $-2,865991$ & $-0,269807$ & C14 & 0,512630 & $-2,884525$ & $-0,283919$ \\
\hline N15 & 1,021788 & $-1,763271$ & 0,148763 & N15 & 1,065293 & $-1,796807$ & 0,148525 \\
\hline $\mathrm{C} 16$ & 2,375599 & $-2,056324$ & 0,751744 & $\mathrm{C} 16$ & 2,412885 & $-2,121786$ & 0,764372 \\
\hline $\mathrm{C} 17$ & 2,430662 & $-3,620133$ & 0,598191 & $\mathrm{C} 17$ & 2,411346 & $-3,684804$ & 0,620562 \\
\hline P18 & $-1,909134$ & $-0,750646$ & 0,359581 & P18 & $-1,891068$ & $-0,791254$ & 0,407180 \\
\hline Ir19 & 0,289021 & 0,185794 & $-0,314224$ & Ir19 & 0,317679 & 0,056497 & $-0,228298$ \\
\hline $\mathrm{C} 20$ & $-2,145908$ & $-0,768079$ & 2,174071 & $\mathrm{C} 20$ & $-2,134319$ & $-0,806354$ & 2,220584 \\
\hline $\mathrm{C} 21$ & $-2,409055$ & $-0,684076$ & 4,965811 & $\mathrm{C} 21$ & $-2,389691$ & $-0,676820$ & 5,010409 \\
\hline $\mathrm{C} 22$ & $-2,106517$ & $-1,950425$ & 2,925569 & $\mathrm{C} 22$ & $-2,230674$ & $-1,981153$ & 2,976998 \\
\hline $\mathrm{C} 23$ & $-2,285759$ & 0,459932 & 2,838697 & $\mathrm{C} 23$ & $-2,135527$ & 0,434892 & 2,878007 \\
\hline $\mathrm{C} 24$ & $-2,421918$ & 0,499979 & 4,224633 & $\mathrm{C} 24$ & $-2,269939$ & 0,498120 & 4,262803 \\
\hline $\mathrm{C} 25$ & $-2,241255$ & $-1,906163$ & 4,313784 & $\mathrm{C} 25$ & $-2,359925$ & $-1,913828$ & 4,365875 \\
\hline $\mathrm{H} 26$ & $-2,284737$ & 1,386587 & 2,279902 & $\mathrm{H} 26$ & $-2,023537$ & 1,352449 & 2,310469 \\
\hline $\mathrm{H} 27$ & $-2,533279$ & 1,459046 & 4,722523 & $\mathrm{H} 27$ & $-2,273184$ & 1,465273 & 4,757639 \\
\hline $\mathrm{H} 28$ & $-2,208107$ & $-2,829078$ & 4,885816 & $\mathrm{H} 28$ & $-2,432570$ & $-2,831010$ & 4,943433 \\
\hline H29 & $-2,517665$ & $-0,653759$ & 6,046309 & $\mathrm{H} 29$ & $-2,493653$ & $-0,628324$ & 6,090747 \\
\hline $\mathrm{O} 30$ & 1,197551 & $-3,982760$ & $-0,084106$ & $\mathrm{O} 30$ & 1,190506 & $-4,013289$ & $-0,103354$ \\
\hline $\mathrm{C} 31$ & 2,445341 & $-1,718915$ & 2,239428 & $\mathrm{C} 31$ & 2,482625 & $-1,799706$ & 2,256286 \\
\hline $\mathrm{C} 32$ & 1,340549 & $-1,280092$ & 2,969060 & $\mathrm{C} 32$ & 1,345087 & $-1,510966$ & 3,011922 \\
\hline C33 & 3,644548 & $-1,977221$ & 2,923686 & $\mathrm{C} 33$ & 3,710524 & $-1,959292$ & 2,917889 \\
\hline C34 & 1,435188 & $-1,069619$ & 4,346941 & $\mathrm{C} 34$ & 1,434808 & $-1,338281$ & 4,394449 \\
\hline H35 & 0,398415 & $-1,102562$ & 2,475472 & H35 & 0,382516 & $-1,416484$ & 2,533050 \\
\hline $\mathrm{C} 36$ & 3,739142 & $-1,772542$ & 4,297889 & $\mathrm{C} 36$ & 3,799360 & $-1,793770$ & 4,298335 \\
\hline H37 & 4,513646 & $-2,333218$ & 2,376924 & H37 & 4,603454 & $-2,207111$ & 2,350790 \\
\hline $\mathrm{C} 38$ & 2,633118 & $-1,310480$ & 5,015445 & $\mathrm{C} 38$ & 2,661413 & $-1,473352$ & 5,041447 \\
\hline H39 & 0,558631 & $-0,726193$ & 4,888061 & H39 & 0,535108 & $-1,103628$ & 4,955334 \\
\hline $\mathrm{H} 40$ & 4,676864 & $-1,974497$ & 4,807791 & $\mathrm{H} 40$ & 4,759153 & $-1,914711$ & 4,792593 \\
\hline H41 & 2,706881 & $-1,149009$ & 6,087142 & $\mathrm{H} 41$ & 2,732489 & $-1,339730$ & 6,117192 \\
\hline $\mathrm{C} 42$ & $-3,499164$ & $-0,108409$ & $-0,315476$ & $\mathrm{C} 42$ & $-3,477749$ & $-0,120284$ & $-0,248902$ \\
\hline $\mathrm{C} 43$ & $-4,535405$ & 0,417740 & 0,465720 & $\mathrm{C} 43$ & $-4,510618$ & 0,377337 & 0,554155 \\
\hline $\mathrm{C} 44$ & $-3,681914$ & $-0,199759$ & $-1,706441$ & C44 & $-3,652289$ & $-0,145407$ & $-1,643330$ \\
\hline $\mathrm{C} 45$ & $-5,715443$ & 0,868144 & $-0,132166$ & $\mathrm{C} 45$ & $-5,690774$ & 0,849856 & $-0,025180$ \\
\hline H46 & $-4,433417$ & 0,480714 & 1,542902 & $\mathrm{H} 46$ & $-4,402720$ & 0,399512 & 1,633005 \\
\hline $\mathrm{C} 47$ & $-4,860414$ & 0,248103 & $-2,300759$ & C47 & $-4,831216$ & 0,323598 & $-2,219111$ \\
\hline $\mathrm{C} 48$ & $-5,880446$ & 0,795578 & $-1,515028$ & $\mathrm{C} 48$ & $-5,853854$ & 0,829238 & $-1,409704$ \\
\hline H49 & $-6,508393$ & 1,271619 & 0,491200 & $\mathrm{H} 49$ & $-6,486131$ & 1,226850 & 0,611728 \\
\hline H50 & $-4,991284$ & 0,149411 & $-3,375210$ & $\mathrm{H} 50$ & $-4,962946$ & 0,272057 & $-3,296826$ \\
\hline H51 & $-6,800611$ & 1,142186 & $-1,975961$ & H51 & $-6,775759$ & 1,190249 & $-1,856377$ \\
\hline H52 & 0,667863 & 0,606360 & 1,130818 & H52 & 0,721904 & 0,403421 & 1,226187 \\
\hline H53 & 1,265819 & 3,002237 & $-2,324231$ & H53 & 1,221148 & 3,309406 & $-2,484816$ \\
\hline H54 & $-1,943914$ & $-2,904291$ & 2,438959 & $\mathrm{H} 54$ & $-2,177709$ & $-2,950241$ & 2,494881 \\
\hline H55 & $-2,914979$ & $-0,652929$ & $-2,325382$ & $\mathrm{H} 55$ & $-2,879010$ & $-0,564988$ & $-2,278457$ \\
\hline
\end{tabular}




\begin{tabular}{|c|c|c|c|c|c|c|c|}
\hline H56 & 3,265684 & $-3,970069$ & $-0,010106$ & H56 & 3,257058 & $-4,072403$ & 0,052128 \\
\hline C57 & 5,280433 & $-0,101817$ & $-1,828584$ & $\mathrm{C} 57$ & 5,348083 & $-0,187807$ & $-1,780960$ \\
\hline C58 & 4,469260 & $-1,134276$ & $-2,307325$ & $\mathrm{C} 58$ & 4,627738 & $-1,293994$ & $-2,234462$ \\
\hline C59 & 3,563824 & $-1,761152$ & $-1,454977$ & C59 & 3,710074 & $-1,919866$ & $-1,393617$ \\
\hline C60 & 3,441363 & $-1,368515$ & $-0,113453$ & C60 & 3,494140 & $-1,454267$ & $-0,090589$ \\
\hline C61 & 4,241259 & $-0,320120$ & 0,351824 & C61 & 4,199635 & $-0,326858$ & 0,345049 \\
\hline C62 & 5,163601 & 0,296891 & $-0,497722$ & C62 & 5,128501 & 0,293470 & $-0,490632$ \\
\hline H63 & 5,995917 & 0,383188 & $-2,485903$ & H63 & 6,071579 & 0,297345 & $-2,429795$ \\
\hline H64 & 4,544111 & $-1,453363$ & $-3,342841$ & H64 & 4,781460 & $-1,670725$ & $-3,241397$ \\
\hline H65 & 2,930343 & $-2,551600$ & $-1,850974$ & H65 & 3,142581 & $-2,765997$ & $-1,773905$ \\
\hline H66 & 4,142959 & 0,026255 & 1,373942 & H66 & 4,015822 & 0,078323 & 1,332532 \\
\hline H67 & 5,790719 & 1,096832 & $-0,114917$ & H67 & 5,679539 & 1,158640 & $-0,133056$ \\
\hline C68 & $-1,426527$ & 3,845266 & 2,321838 & C68 & $-1,519890$ & 3,977947 & 2,221694 \\
\hline C69 & $-0,654603$ & 3,239486 & 1,334035 & C69 & $-0,710806$ & 3,483084 & 1,201607 \\
\hline $\mathrm{C} 70$ & $-1,218045$ & 2,870185 & 0,097983 & $\mathrm{C} 70$ & $-1,265821$ & 3,122624 & $-0,037695$ \\
\hline C71 & $-2,593665$ & 3,068325 & $-0,080613$ & C71 & $-2,652063$ & 3,206724 & $-0,204105$ \\
\hline $\mathrm{C} 72$ & $-3,371042$ & 3,660026 & 0,919706 & $\mathrm{C} 72$ & $-3,461885$ & 3,693104 & 0,824737 \\
\hline $\mathrm{C} 73$ & $-2,792040$ & 4,069961 & 2,119339 & $\mathrm{C} 73$ & $-2,899285$ & 4,097625 & 2,033349 \\
\hline H74 & $-0,959115$ & 4,126389 & 3,261507 & H74 & $-1,070387$ & 4,258004 & 3,170237 \\
\hline H75 & 0,388940 & 3,031901 & 1,530275 & $\mathrm{H} 75$ & 0,350658 & 3,360152 & 1,375770 \\
\hline H76 & $-3,082050$ & 2,722559 & $-0,983313$ & H76 & $-3,120138$ & 2,848431 & $-1,113994$ \\
\hline H77 & $-4,436968$ & 3,786769 & 0,754053 & H77 & $-4,536702$ & 3,737216 & 0,678077 \\
\hline H78 & $-3,394630$ & 4,539036 & 2,891450 & $\mathrm{H} 78$ & $-3,529602$ & 4,482315 & 2,830001 \\
\hline C79 & $-0,326110$ & 2,322887 & $-1,000298$ & C79 & $-0,340696$ & 2,662307 & $-1,155593$ \\
\hline $\mathrm{C} 80$ & 0,758272 & 3,412119 & $-1,441227$ & $\mathrm{C} 80$ & 0,720735 & 3,729703 & $-1,604146$ \\
\hline C81 & $-1,552076$ & 3,017529 & $-3,204018$ & $\mathrm{C} 81$ & $-1,590754$ & 3,139172 & $-3,347643$ \\
\hline C82 & $-0,953385$ & 0,815901 & $-2,884879$ & $\mathrm{C} 82$ & $-0,937183$ & 0,961525 & $-2,908423$ \\
\hline $\mathrm{C} 83$ & $-2,280964$ & 2,105454 & $-4,192807$ & $\mathrm{C} 83$ & $-2,305273$ & 2,156577 & $-4,278207$ \\
\hline H84 & $-0,723859$ & 3,552046 & $-3,680956$ & H84 & $-0,784951$ & 3,677468 & $-3,858641$ \\
\hline H85 & $-2,208418$ & 3,739197 & $-2,717253$ & H85 & $-2,265631$ & 3,863031 & $-2,887773$ \\
\hline H86 & $-3,322510$ & 1,936663 & $-3,904859$ & H86 & $-3,343396$ & 1,989618 & $-3,976548$ \\
\hline H87 & $-2,219895$ & 2,430702 & $-5,231181$ & H87 & $-2,253954$ & 2,423932 & $-5,333489$ \\
\hline N88 & $-1,052446$ & 2,007897 & $-2,246997$ & N88 & $-1,048514$ & 2,199818 & $-2,351467$ \\
\hline O89 & $-1,575309$ & 0,838530 & $-4,073795$ & O89 & $-1,579943$ & 0,913007 & $-4,089447$ \\
\hline O90 & $-0,395683$ & $-0,213048$ & $-2,475731$ & O90 & $-0,369871$ & $-0,037953$ & $-2,459251$ \\
\hline H91 & 2,431416 & $-4,128781$ & 1,562492 & H91 & 2,354877 & $-4,180806$ & 1,589692 \\
\hline N92 & $-1,903346$ & $-2,362716$ & $-0,142698$ & N92 & $-1,892824$ & $-2,401175$ & $-0,112082$ \\
\hline C93 & $-0,835088$ & $-2,972932$ & $-0,968819$ & C93 & $-0,825192$ & $-2,957234$ & $-0,980759$ \\
\hline H94 & $-0,725099$ & $-2,461302$ & $-1,932216$ & H94 & $-0,724393$ & $-2,393324$ & $-1,916743$ \\
\hline H95 & 0,184935 & 4,284679 & $-1,772032$ & H95 & 0,165610 & 4,617095 & $-1,927353$ \\
\hline C96 & 3,668142 & 4,769177 & 1,453715 & C96 & 3,635672 & 4,854472 & 1,373627 \\
\hline C97 & 2,522566 & 5,524009 & 1,197068 & C97 & 2,617615 & 5,741429 & 1,021110 \\
\hline C98 & 1,594238 & 5,083460 & 0,255529 & C98 & 1,682494 & 5,379280 & 0,051612 \\
\hline C99 & 1,791114 & 3,884269 & $-0,443335$ & C99 & 1,755268 & 4,131695 & $-0,580224$ \\
\hline $\mathrm{C} 100$ & 2,962528 & 3,153858 & $-0,204520$ & $\mathrm{C} 100$ & 2,794314 & 3,255678 & $-0,237877$ \\
\hline C101 & 3,887137 & 3,587311 & 0,745375 & C101 & 3,721757 & 3,612871 & 0,739910 \\
\hline H102 & 4,391328 & 5,107164 & 2,190549 & H102 & 4,361467 & 5,131902 & 2,132960 \\
\hline H103 & 2,350569 & 6,454303 & 1,730972 & H103 & 2,548041 & 6,712422 & 1,503306 \\
\hline
\end{tabular}




$\begin{array}{lrrrrrrr}\text { H104 } & 0,694699 & 5,665834 & 0,073543 & \text { H104 } & 0,879439 & 6,065666 & -0,205829 \\ \text { H105 } & 3,168962 & 2,246564 & -0,763404 & \text { H105 } & 2,873728 & 2,287398 & -0,722489 \\ \text { H106 } & 4,786797 & 3,005785 & 0,923994 & \text { H106 } & 4,515941 & 2,920998 & 1,004532 \\ \text { H107 } & 1,817888 & 0,469221 & -0,698205 & \text { H107 } & 1,811776 & 0,456909 & -0,605517 \\ \text { H108 } & 0,978495 & 1,530354 & -0,784155 & \text { H108 } & 0,357523 & 1,838784 & -0,797711\end{array}$

\section{C1_E_R_tN_Cheltop}

C1_ER_Cheltop_SM

\begin{tabular}{|c|c|c|c|c|c|c|c|}
\hline $\mathrm{C} 1$ & $-4,302899$ & $-3,269123$ & 0,477358 & $\mathrm{C} 1$ & $-4,286837$ & $-3,352529$ & 0,426943 \\
\hline $\mathrm{C} 2$ & $-2,234129$ & $-4,424221$ & $-0,110304$ & $\mathrm{C} 2$ & $-2,199749$ & $-4,483993$ & $-0,140674$ \\
\hline C3 & $-3,701964$ & $-4,254214$ & $-0,579317$ & $\mathrm{C} 3$ & $-3,662853$ & $-4,325548$ & $-0,628286$ \\
\hline $\mathrm{C} 4$ & $-3,088001$ & $-2,998150$ & 1,395876 & $\mathrm{C} 4$ & $-3,090734$ & $-3,087898$ & 1,372293 \\
\hline H5 & $-5,110120$ & $-3,735254$ & 1,051931 & H5 & $-5,106897$ & $-3,824474$ & 0,977817 \\
\hline H6 & $-3,766758$ & $-3,866753$ & $-1,601135$ & H6 & $-3,718388$ & $-3,933490$ & $-1,648908$ \\
\hline $\mathrm{H} 7$ & $-3,316796$ & $-2,531709$ & 2,354787 & $\mathrm{H} 7$ & $-3,339635$ & $-2,637759$ & 2,334084 \\
\hline H8 & $-4,696438$ & $-2,354347$ & 0,033750 & $\mathrm{H} 8$ & $-4,669314$ & $-2,431553$ & $-0,014609$ \\
\hline H9 & $-1,714728$ & $-5,288447$ & $-0,523966$ & H9 & $-1,663649$ & $-5,335884$ & $-0,558576$ \\
\hline H10 & $-4,212548$ & $-5,221149$ & $-0,563506$ & H10 & $-4,164215$ & $-5,297420$ & $-0,623942$ \\
\hline $\mathrm{C} 11$ & $-2,389101$ & $-4,368486$ & 1,426287 & $\mathrm{C} 11$ & $-2,378708$ & $-4,451782$ & 1,393717 \\
\hline H12 & $-1,439335$ & $-4,356936$ & 1,969424 & H12 & $-1,437516$ & $-4,437401$ & 1,951435 \\
\hline H13 & $-3,024821$ & $-5,168384$ & 1,815710 & H13 & $-3,011708$ & $-5,263925$ & 1,761569 \\
\hline $\mathrm{C} 14$ & $-0,002198$ & $-3,133241$ & $-0,266159$ & C14 & 0,018307 & $-3,154722$ & $-0,270531$ \\
\hline N15 & 0,766005 & $-2,099433$ & $-0,226667$ & N15 & 0,762321 & $-2,105515$ & $-0,204842$ \\
\hline $\mathrm{C} 16$ & 2,205075 & $-2,573141$ & $-0,131995$ & C16 & 2,207523 & $-2,547578$ & $-0,132412$ \\
\hline $\mathrm{C} 17$ & 1,991230 & $-4,110631$ & 0,068169 & $\mathrm{C} 17$ & 2,031791 & $-4,093063$ & 0,037113 \\
\hline P18 & $-1,919840$ & $-0,525555$ & 0,560710 & P18 & $-1,923668$ & $-0,595165$ & 0,600194 \\
\hline Ir19 & 0,164061 & $-0,058183$ & $-0,624820$ & Ir19 & 0,085291 & $-0,060798$ & $-0,554580$ \\
\hline $\mathrm{C} 20$ & $-1,837053$ & 0,029039 & 2,300580 & $\mathrm{C} 20$ & $-1,860499$ & $-0,053368$ & 2,343610 \\
\hline $\mathrm{C} 21$ & $-1,361624$ & 1,003374 & 4,893823 & $\mathrm{C} 21$ & $-1,384675$ & 0,922331 & 4,931644 \\
\hline $\mathrm{C} 22$ & $-1,278160$ & $-0,801845$ & 3,281893 & $\mathrm{C} 22$ & $-1,308609$ & $-0,887565$ & 3,325153 \\
\hline $\mathrm{C} 23$ & $-2,164294$ & 1,352567 & 2,636372 & $\mathrm{C} 23$ & $-2,176064$ & 1,274694 & 2,671821 \\
\hline $\mathrm{C} 24$ & $-1,928352$ & 1,833539 & 3,924106 & $\mathrm{C} 24$ & $-1,939032$ & 1,756883 & 3,958363 \\
\hline $\mathrm{C} 25$ & $-1,044974$ & $-0,317082$ & 4,569458 & $\mathrm{C} 25$ & $-1,077303$ & $-0,401633$ & 4,612871 \\
\hline $\mathrm{H} 26$ & $-2,608373$ & 2,005351 & 1,892753 & $\mathrm{H} 26$ & $-2,614352$ & 1,927322 & 1,923877 \\
\hline $\mathrm{H} 27$ & $-2,194874$ & 2,857350 & 4,172941 & $\mathrm{H} 27$ & $-2,193318$ & 2,784536 & 4,203230 \\
\hline $\mathrm{H} 28$ & $-0,612812$ & $-0,973485$ & 5,319492 & $\mathrm{H} 28$ & $-0,653009$ & $-1,058844$ & 5,366510 \\
\hline $\mathrm{H} 29$ & $-1,174678$ & 1,380491 & 5,894729 & $\mathrm{H} 29$ & $-1,198058$ & 1,300967 & 5,932005 \\
\hline $\mathrm{O} 30$ & 0,574234 & $-4,332342$ & $-0,178612$ & $\mathrm{O} 30$ & 0,622321 & $-4,344541$ & $-0,225983$ \\
\hline C31 & 2,905725 & $-2,040141$ & 1,112898 & $\mathrm{C} 31$ & 2,911195 & $-2,017880$ & 1,110460 \\
\hline $\mathrm{C} 32$ & 2,168997 & $-1,569838$ & 2,201171 & $\mathrm{C} 32$ & 2,182989 & $-1,546120$ & 2,203911 \\
\hline $\mathrm{C} 33$ & 4,297294 & $-2,163935$ & 1,242284 & $\mathrm{C} 33$ & 4,304674 & $-2,136296$ & 1,223507 \\
\hline C34 & 2,808335 & $-1,170048$ & 3,373585 & C34 & 2,834980 & $-1,140024$ & 3,367954 \\
\hline H35 & 1,094785 & $-1,478376$ & 2,124841 & H35 & 1,107083 & $-1,457548$ & 2,138739 \\
\hline C36 & 4,936465 & $-1,774415$ & 2,417961 & $\mathrm{C} 36$ & 4,955145 & $-1,740646$ & 2,390215 \\
\hline H37 & 4,887805 & $-2,555183$ & 0,419791 & H37 & 4,885545 & $-2,524558$ & 0,391915 \\
\hline C38 & 4,194339 & $-1,265143$ & 3,485196 & C38 & 4,222534 & $-1,229909$ & 3,463697 \\
\hline H39 & 2,215171 & $-0,771340$ & 4,191732 & H39 & 2,249768 & $-0,740422$ & 4,191342 \\
\hline $\mathrm{H} 40$ & 6,016173 & $-1,864436$ & 2,496214 & $\mathrm{H} 40$ & 6,035925 & $-1,825643$ & 2,457256 \\
\hline
\end{tabular}




\begin{tabular}{|c|c|c|c|c|c|c|c|}
\hline H41 & 4,695087 & $-0,948253$ & 4,395547 & $\mathrm{H} 41$ & 4,732035 & $-0,907610$ & 4,367141 \\
\hline $\mathrm{C} 42$ & $-3,532178$ & 0,052655 & $-0,081620$ & $\mathrm{C} 42$ & $-3,510682$ & 0,013018 & $-0,067890$ \\
\hline $\mathrm{C} 43$ & $-4,625225$ & 0,333816 & 0,753062 & $\mathrm{C} 43$ & $-4,625385$ & 0,251716 & 0,750925 \\
\hline $\mathrm{C} 44$ & $-3,723611$ & 0,000009 & $-1,470139$ & C44 & $-3,652987$ & 0,070750 & $-1,462002$ \\
\hline $\mathrm{C} 45$ & $-5,885661$ & 0,564937 & 0,203069 & $\mathrm{C} 45$ & $-5,860621$ & 0,551748 & 0,179077 \\
\hline H46 & $-4,500499$ & 0,359873 & 1,830830 & $\mathrm{H} 46$ & $-4,533710$ & 0,198026 & 1,831134 \\
\hline $\mathrm{C} 47$ & $-4,986797$ & 0,224342 & $-2,014354$ & $\mathrm{C} 47$ & $-4,890852$ & 0,366154 & $-2,028320$ \\
\hline $\mathrm{C} 48$ & $-6,070883$ & 0,506633 & $-1,180224$ & $\mathrm{C} 48$ & $-5,995215$ & 0,607268 & $-1,209543$ \\
\hline H49 & $-6,725485$ & 0,781365 & 0,857342 & $\mathrm{H} 49$ & $-6,719412$ & 0,736629 & 0,817824 \\
\hline H50 & $-5,121517$ & 0,192381 & $-3,091235$ & $\mathrm{H} 50$ & $-4,988032$ & 0,425016 & $-3,107821$ \\
\hline H51 & $-7,054671$ & 0,682545 & $-1,605206$ & H51 & $-6,959235$ & 0,840868 & $-1,652079$ \\
\hline H52 & $-0,435034$ & $-0,548872$ & $-1,980053$ & H52 & $-0,539363$ & $-0,570115$ & $-1,890886$ \\
\hline H53 & 1,588385 & $-0,151212$ & $-1,322466$ & $\mathrm{H} 53$ & 1,512869 & 0,041373 & $-1,323708$ \\
\hline H54 & $-1,016799$ & $-1,825873$ & 3,034088 & $\mathrm{H} 54$ & $-1,050987$ & $-1,912725$ & 3,076942 \\
\hline H55 & $-2,879198$ & $-0,201801$ & $-2,121573$ & $\mathrm{H} 55$ & $-2,790241$ & $-0,095418$ & $-2,098070$ \\
\hline H56 & 2,563314 & $-4,730981$ & $-0,621481$ & $\mathrm{H} 56$ & 2,625534 & $-4,686835$ & $-0,657657$ \\
\hline $\mathrm{C} 57$ & 3,984224 & $-1,592429$ & $-3,974116$ & C57 & 3,945391 & $-1,409283$ & $-3,949645$ \\
\hline C58 & 3,103811 & $-2,668184$ & $-3,849196$ & C58 & 3,121858 & $-2,531247$ & $-3,850158$ \\
\hline C59 & 2,558973 & $-2,985306$ & $-2,605396$ & C59 & 2,585814 & $-2,899702$ & $-2,616403$ \\
\hline C60 & 2,889343 & $-2,243420$ & $-1,463048$ & C60 & 2,871367 & $-2,163345$ & $-1,458710$ \\
\hline C61 & 3,753906 & $-1,148967$ & $-1,607112$ & C61 & 3,679300 & $-1,023084$ & $-1,576255$ \\
\hline C62 & 4,300792 & $-0,830754$ & $-2,850506$ & C62 & 4,216194 & $-0,653660$ & $-2,810222$ \\
\hline H63 & 4,408542 & $-1,341302$ & $-4,941800$ & H63 & 4,360137 & $-1,117816$ & $-4,909909$ \\
\hline H64 & 2,838430 & $-3,260459$ & $-4,720246$ & H64 & 2,893728 & $-3,120702$ & $-4,733631$ \\
\hline H65 & 1,857482 & $-3,813115$ & $-2,537239$ & H65 & 1,928924 & $-3,764611$ & $-2,567585$ \\
\hline H66 & 3,999304 & $-0,537673$ & $-0,746867$ & H66 & 3,897414 & $-0,424724$ & $-0,698580$ \\
\hline H67 & 4,966835 & 0,022438 & $-2,940336$ & H67 & 4,839862 & 0,232782 & $-2,880176$ \\
\hline C68 & $-3,025228$ & 2,780749 & $-3,433654$ & C68 & $-2,969114$ & 2,940590 & $-3,459192$ \\
\hline C69 & $-1,850460$ & 2,287163 & $-2,872265$ & C69 & $-1,797577$ & 2,408809 & $-2,923319$ \\
\hline $\mathrm{C} 70$ & $-1,549668$ & 2,521044 & $-1,518909$ & $\mathrm{C} 70$ & $-1,506963$ & 2,540585 & $-1,555675$ \\
\hline C71 & $-2,495310$ & 3,218273 & $-0,748858$ & C71 & $-2,459706$ & 3,189512 & $-0,749076$ \\
\hline $\mathrm{C} 72$ & $-3,677904$ & 3,696992 & $-1,308849$ & $\mathrm{C} 72$ & $-3,635313$ & 3,710292 & $-1,283317$ \\
\hline $\mathrm{C} 73$ & $-3,943075$ & 3,493775 & $-2,661013$ & $\mathrm{C} 73$ & $-3,892418$ & 3,599989 & $-2,648672$ \\
\hline H74 & $-3,227502$ & 2,596650 & $-4,485181$ & $\mathrm{H} 74$ & $-3,159113$ & 2,832804 & $-4,523598$ \\
\hline H75 & $-1,177508$ & 1,710174 & $-3,488962$ & $\mathrm{H} 75$ & $-1,119592$ & 1,884817 & $-3,579757$ \\
\hline H76 & $-2,312078$ & 3,378234 & 0,307451 & $\mathrm{H} 76$ & $-2,280515$ & 3,279639 & 0,316375 \\
\hline H77 & $-4,391239$ & 4,225732 & $-0,683172$ & $\mathrm{H} 77$ & $-4,351075$ & 4,198500 & $-0,628110$ \\
\hline H78 & $-4,859258$ & 3,872621 & $-3,104347$ & $\mathrm{H} 78$ & $-4,803663$ & 4,011542 & $-3,072649$ \\
\hline C79 & $-0,272201$ & 2,065134 & $-0,890670$ & C79 & $-0,248410$ & 2,030883 & $-0,917981$ \\
\hline $\mathrm{C} 80$ & 0,942489 & 1,720350 & $-1,598378$ & $\mathrm{C} 80$ & 0,987982 & 1,642943 & $-1,615878$ \\
\hline C92 & 0,303878 & 4,150068 & 0,510510 & C92 & 0,356285 & 4,175216 & 0,399014 \\
\hline C93 & 0,734094 & 2,050287 & 1,326804 & C93 & 0,723904 & 2,099485 & 1,317144 \\
\hline C94 & 0,684668 & 4,208735 & 1,995722 & C94 & 0,711910 & 4,287502 & 1,888587 \\
\hline H95 & $-0,554609$ & 4,774028 & 0,260227 & H95 & $-0,497346$ & 4,790361 & 0,111642 \\
\hline H96 & 1,141261 & 4,415674 & $-0,150291$ & H96 & 1,204520 & 4,415047 & $-0,258004$ \\
\hline H97 & 1,468415 & 4,928285 & 2,232671 & H97 & 1,508312 & 4,998152 & 2,109684 \\
\hline H98 & $-0,186864$ & 4,375521 & 2,636899 & H98 & $-0,167089$ & 4,505497 & 2,503655 \\
\hline N99 & $-0,003521$ & 2,716083 & 0,395521 & N99 & 0,044321 & 2,741296 & 0,331961 \\
\hline
\end{tabular}




\begin{tabular}{|c|c|c|c|c|c|c|c|}
\hline $\mathrm{O} 100$ & 1,196364 & 2,874938 & 2,274277 & O100 & 1,184783 & 2,955432 & 2,239114 \\
\hline O101 & 0,989271 & 0,841848 & 1,310468 & O101 & 0,935149 & 0,882223 & 1,366944 \\
\hline H102 & 2,191756 & $-4,414172$ & 1,096744 & $\mathrm{H} 102$ & 2,231816 & $-4,409332$ & 1,062147 \\
\hline N103 & $-2,052431$ & $-2,210710$ & 0,669314 & N103 & $-2,052378$ & $-2,280894$ & 0,674570 \\
\hline C104 & $-1,503502$ & $-3,087580$ & $-0,393018$ & C104 & $-1,483954$ & $-3,134066$ & $-0,397517$ \\
\hline H105 & $-1,730096$ & $-2,716589$ & $-1,402352$ & H105 & $-1,713814$ & $-2,752235$ & $-1,402398$ \\
\hline C107 & 1,629127 & 2,213194 & $-5,816506$ & $\mathrm{C} 107$ & 1,557874 & 1,984005 & $-5,866112$ \\
\hline C108 & 1,455273 & 3,311866 & $-4,971562$ & C108 & 1,620856 & 3,100521 & $-5,028592$ \\
\hline C109 & 1,225948 & 3,113663 & $-3,612845$ & C109 & 1,443288 & 2,950676 & $-3,655794$ \\
\hline C110 & 1,159031 & 1,817117 & $-3,077706$ & $\mathrm{C} 110$ & 1,191868 & 1,686429 & $-3,10090$ \\
\hline C111 & 1,349096 & 0,723662 & $-3,925993$ & C111 & 1,154129 & 0,571361 & $-3,940716$ \\
\hline $\mathrm{C} 112$ & 1,581390 & 0,923648 & $-5,288464$ & $\mathrm{C} 112$ & 1,332593 & 0,722120 & $-5,317075$ \\
\hline H1 13 & 1,801211 & 2,364751 & $-6,878269$ & H113 & 1,688588 & 2,099489 & $-6,938266$ \\
\hline H1 14 & 1,490807 & 4,321282 & $-5,371578$ & H114 & 1,801026 & 4,087224 & $-5,445576$ \\
\hline H1 15 & 1,071771 & 3,970131 & $-2,960588$ & H115 & 1,472931 & 3,824330 & $-3,008669$ \\
\hline H116 & 1,316617 & $-0,280810$ & $-3,521626$ & H116 & 0,997732 & $-0,413452$ & $-3,516012$ \\
\hline H117 & 1,726390 & 0,063636 & $-5,936058$ & H117 & 1,299815 & $-0,153490$ & $-5,959196$ \\
\hline H107 & 1,843120 & 2,004118 & $-1,049859$ & H107 & 1,879356 & 1,990612 & $-1,08837$ \\
\hline \multicolumn{4}{|c|}{ C1_ER_Cheltop_INT1 } & \multicolumn{4}{|c|}{ C1_ER_Cheltop_TS2 } \\
\hline $\mathrm{C} 1$ & $-4,226375$ & $-3,382837$ & 0,456156 & $\mathrm{C} 1$ & $-4,086516$ & $-3,371088$ & 1,275450 \\
\hline $\mathrm{C} 2$ & $-2,132592$ & $-4,536453$ & $-0,041782$ & $\mathrm{C} 2$ & $-2,062815$ & $-4,535970$ & 0,579430 \\
\hline $\mathrm{C} 3$ & $-3,594362$ & $-4,407823$ & $-0,544119$ & $\mathrm{C} 3$ & $-3,588434$ & $-4,496900$ & 0,312334 \\
\hline $\mathrm{C} 4$ & $-3,041075$ & $-3,079813$ & 1,402035 & $\mathrm{C} 4$ & $-2,772404$ & $-2,906951$ & 1,946211 \\
\hline H5 & $-5,057226$ & $-3,822782$ & 1,017287 & H5 & $-4,765337$ & $-3,764769$ & 2,039330 \\
\hline H6 & $-3,646503$ & $-4,070964$ & $-1,584328$ & H6 & $-3,820306$ & $-4,284581$ & $-0,736405$ \\
\hline $\mathrm{H} 7$ & $-3,302762$ & $-2,590662$ & 2,341002 & $\mathrm{H} 7$ & $-2,900024$ & $-2,299575$ & 2,841851 \\
\hline $\mathrm{H} 8$ & $-4,595199$ & $-2,478438$ & $-0,030994$ & $\mathrm{H} 8$ & $-4,606441$ & $-2,565752$ & 0,760769 \\
\hline H9 & $-1,588617$ & $-5,402771$ & $-0,418041$ & H9 & $-1,557742$ & $-5,437680$ & 0,235240 \\
\hline $\mathrm{H} 10$ & $-4,093514$ & $-5,379433$ & $-0,489665$ & $\mathrm{H} 10$ & $-4,035975$ & $-5,464304$ & 0,55694 \\
\hline $\mathrm{C} 11$ & $-2,323620$ & $-4,438350$ & 1,488466 & $\mathrm{C} 11$ & $-2,001667$ & $-4,230710$ & 2,09302 \\
\hline H12 & $-1,387096$ & $-4,395063$ & 2,052795 & H12 & $-0,987339$ & $-4,099671$ & 2,482127 \\
\hline H13 & $-2,956543$ & $-5,236185$ & 1,886949 & H13 & $-2,534327$ & $-4,972525$ & 2,694591 \\
\hline $\mathrm{C} 14$ & 0,083680 & $-3,195037$ & $-0,253152$ & $\mathrm{C} 14$ & 0,050340 & $-3,236770$ & $-0,126110$ \\
\hline N15 & 0,799178 & $-2,128629$ & $-0,201023$ & N15 & 0,785475 & $-2,182928$ & $-0,24484$ \\
\hline $\mathrm{C} 16$ & 2,245320 & $-2,516010$ & $-0,094856$ & $\mathrm{C} 16$ & 2,221748 & $-2,650976$ & $-0,113575$ \\
\hline $\mathrm{C} 17$ & 2,142921 & $-4,084469$ & $-0,115820$ & $\mathrm{C} 17$ & 2,051479 & $-4,173112$ & $-0,39400$ \\
\hline P18 & $-1,853243$ & $-0,620424$ & 0,592018 & P18 & $-1,840601$ & $-0,570371$ & 0,590710 \\
\hline Ir19 & 0,007333 & $-0,096391$ & $-0,527968$ & Ir19 & $-0,112727$ & $-0,196721$ & $-0,77715^{\prime}$ \\
\hline $\mathrm{C} 20$ & $-1,735947$ & $-0,062844$ & 2,326915 & $\mathrm{C} 20$ & $-1,610779$ & 0,254558 & 2,19618 \\
\hline $\mathrm{C} 21$ & $-1,236858$ & 0,919029 & 4,906301 & $\mathrm{C} 21$ & $-1,097209$ & 1,601797 & 4,59789 \\
\hline $\mathrm{C} 22$ & $-1,272686$ & $-0,920661$ & 3,332725 & $\mathrm{C} 22$ & $-1,003467$ & $-0,416720$ & 3,26691 ( \\
\hline $\mathrm{C} 23$ & $-1,949935$ & 1,292630 & 2,624820 & $\mathrm{C} 23$ & $-1,958418$ & 1,606926 & 2,339199 \\
\hline $\mathrm{C} 24$ & $-1,701084$ & 1,778379 & 3,907158 & $\mathrm{C} 24$ & $-1,702117$ & 2,274956 & 3,53460 \\
\hline $\mathrm{C} 25$ & $-1,030395$ & $-0,430814$ & 4,617350 & $\mathrm{C} 25$ & $-0,755810$ & 0,255565 & 4,46294 \\
\hline $\mathrm{H} 26$ & $-2,315999$ & 1,963335 & 1,854179 & $\mathrm{H} 26$ & $-2,442721$ & 2,127775 & 1,52291 \\
\hline $\mathrm{H} 27$ & $-1,874559$ & 2,827747 & 4,129868 & $\mathrm{H} 27$ & $-1,986313$ & 3,318300 & 3,64125 \\
\hline $\mathrm{H} 28$ & $-0,677847$ & $-1,105909$ & 5,391962 & $\mathrm{H} 28$ & $-0,297668$ & $-0,276200$ & 5,29183 \\
\hline
\end{tabular}




\begin{tabular}{|c|c|c|c|c|c|c|c|}
\hline H29 & $-1,042987$ & 1,299594 & 5,904806 & H29 & $-0,900285$ & 2,122405 & 5,530456 \\
\hline $\mathrm{O} 30$ & 0,718341 & $-4,369738$ & $-0,229652$ & $\mathrm{O} 30$ & 0,656356 & $-4,424132$ & $-0,085279$ \\
\hline C31 & 2,847055 & $-2,100297$ & 1,247133 & $\mathrm{C} 31$ & 2,706219 & $-2,461753$ & 1,330983 \\
\hline C32 & 2,046770 & $-1,658930$ & 2,299660 & $\mathrm{C} 32$ & 2,002541 & $-1,677541$ & 2,243954 \\
\hline C33 & 4,221957 & $-2,277174$ & 1,464998 & $\mathrm{C} 33$ & 3,894877 & $-3,081733$ & 1,744446 \\
\hline C34 & 2,608846 & $-1,361891$ & 3,541426 & $\mathrm{C} 34$ & 2,471465 & $-1,515676$ & 3,549404 \\
\hline H35 & 0,988308 & $-1,510971$ & 2,143526 & $\mathrm{H} 35$ & 1,116219 & $-1,151546$ & 1,923350 \\
\hline C36 & 4,782643 & $-1,989958$ & 2,707449 & $\mathrm{C} 36$ & 4,363162 & $-2,921808$ & 3,045829 \\
\hline H37 & 4,857736 & $-2,626854$ & 0,655719 & H37 & 4,476273 & $-3,669095$ & 1,037738 \\
\hline C38 & 3,976670 & $-1,524529$ & 3,749793 & $\mathrm{C} 38$ & 3,651818 & $-2,134341$ & 3,954752 \\
\hline H39 & 1,969655 & $-0,990476$ & 4,337625 & H39 & 1,916856 & $-0,884914$ & 4,238223 \\
\hline $\mathrm{H} 40$ & 5,849751 & $-2,123661$ & 2,860341 & $\mathrm{H} 40$ & 5,287411 & $-3,405448$ & 3,348342 \\
\hline H41 & 4,416074 & $-1,290547$ & 4,715334 & $\mathrm{H} 41$ & 4,018843 & $-2,006387$ & 4,969012 \\
\hline $\mathrm{C} 42$ & $-3,440861$ & 0,021971 & $-0,039673$ & $\mathrm{C} 42$ & $-3,556290$ & $-0,253481$ & 0,054074 \\
\hline $\mathrm{C} 43$ & $-4,508889$ & 0,306159 & 0,826719 & $\mathrm{C} 43$ & $-4,542293$ & 0,178211 & 0,955420 \\
\hline $\mathrm{C} 44$ & $-3,645773$ & 0,062573 & $-1,426201$ & $\mathrm{C} 44$ & $-3,944806$ & $-0,770596$ & $-1,191245$ \\
\hline $\mathrm{C} 45$ & $-5,761066$ & 0,631086 & 0,307553 & $\mathrm{C} 45$ & $-5,890441$ & 0,101069 & 0,609063 \\
\hline H46 & $-4,370279$ & 0,268264 & 1,902391 & H46 & $-4,268916$ & 0,547954 & 1,937097 \\
\hline $\mathrm{C} 47$ & $-4,899485$ & 0,385540 & $-1,937899$ & $\mathrm{C} 47$ & $-5,293693$ & $-0,860868$ & $-1,524527$ \\
\hline $\mathrm{C} 48$ & $-5,958312$ & 0,669473 & $-1,073649$ & $\mathrm{C} 48$ & $-6,269090$ & $-0,421803$ & $-0,627677$ \\
\hline H49 & $-6,582942$ & 0,851412 & 0,982844 & $\mathrm{H} 49$ & $-6,645064$ & 0,439306 & 1,313209 \\
\hline H50 & $-5,044471$ & 0,432229 & $-3,012603$ & $\mathrm{H} 50$ & $-5,583049$ & $-1,261543$ & $-2,491659$ \\
\hline H51 & $-6,934698$ & 0,924609 & $-1,475574$ & H51 & $-7,320696$ & $-0,489244$ & $-0,891041$ \\
\hline H52 & $-0,694161$ & $-0,623209$ & $-1,828489$ & $\mathrm{H} 52$ & $-0,943801$ & $-0,903491$ & $-1,912028$ \\
\hline H53 & 1,543876 & 0,579390 & $-1,588868$ & $\mathrm{H} 53$ & $-0,195438$ & 1,338848 & $-3,550153$ \\
\hline H54 & $-1,092031$ & $-1,967195$ & 3,109914 & $\mathrm{H} 54$ & $-0,719257$ & $-1,457827$ & 3,165609 \\
\hline H55 & $-2,819050$ & $-0,134374$ & $-2,099433$ & $\mathrm{H} 55$ & $-3,189954$ & $-1,106243$ & $-1,894402$ \\
\hline H56 & 2,653276 & $-4,555840$ & $-0,956692$ & H56 & 2,211773 & $-4,446948$ & $-1,439700$ \\
\hline C57 & 4,124138 & $-0,762351$ & $-3,604468$ & C57 & 4,741670 & $-0,694513$ & $-3,061633$ \\
\hline C58 & 3,372615 & $-1,933947$ & $-3,713843$ & C58 & 3,716524 & $-1,554768$ & $-3,460454$ \\
\hline C59 & 2,787942 & $-2,500832$ & $-2,581756$ & C59 & 2,938817 & $-2,210018$ & $-2,504191$ \\
\hline C60 & 2,959692 & $-1,922758$ & $-1,315785$ & C60 & 3,159794 & $-2,008958$ & $-1,134274$ \\
\hline C61 & 3,701158 & $-0,737633$ & $-1,218836$ & C61 & 4,192534 & $-1,146333$ & $-0,745495$ \\
\hline C62 & 4,278107 & $-0,163361$ & $-2,354411$ & C62 & 4,981618 & $-0,502922$ & $-1,700266$ \\
\hline H63 & 4,571460 & $-0,314523$ & $-4,486531$ & H63 & 5,347446 & $-0,183895$ & $-3,804429$ \\
\hline H64 & 3,231155 & $-2,402424$ & $-4,683525$ & H64 & 3,517878 & $-1,715194$ & $-4,516311$ \\
\hline H65 & 2,181614 & $-3,395386$ & $-2,697828$ & $\mathrm{H} 65$ & 2,123046 & $-2,844081$ & $-2,840505$ \\
\hline H66 & 3,827704 & $-0,261101$ & $-0,252467$ & H66 & 4,383437 & $-0,968411$ & 0,305593 \\
\hline H67 & 4,850853 & 0,754814 & $-2,257765$ & H67 & 5,778139 & 0,160045 & $-1,374722$ \\
\hline C68 & $-2,934329$ & 2,874876 & $-3,497142$ & C68 & $-4,041492$ & 1,826175 & $-3,080540$ \\
\hline C69 & $-1,750384$ & 2,335358 & $-2,993803$ & C69 & $-2,717920$ & 1,518616 & $-2,789386$ \\
\hline $\mathrm{C} 70$ & $-1,389073$ & 2,509781 & $-1,647612$ & $\mathrm{C} 70$ & $-2,058827$ & 2,085463 & $-1,684199$ \\
\hline C71 & $-2,293993$ & 3,211893 & $-0,828239$ & C71 & $-2,806502$ & 2,937224 & $-0,862484$ \\
\hline $\mathrm{C} 72$ & $-3,484488$ & 3,735605 & $-1,326834$ & $\mathrm{C} 72$ & $-4,145195$ & 3,229903 & $-1,135430$ \\
\hline $\mathrm{C} 73$ & $-3,810851$ & 3,581138 & $-2,673509$ & $\mathrm{C} 73$ & $-4,767875$ & 2,688506 & $-2,255317$ \\
\hline H74 & $-3,172807$ & 2,729878 & $-4,547507$ & $\mathrm{H} 74$ & $-4,514529$ & 1,380263 & $-3,951085$ \\
\hline H75 & $-1,119530$ & 1,766732 & $-3,660144$ & $\mathrm{H} 75$ & $-2,184662$ & 0,827356 & $-3,433121$ \\
\hline H76 & $-2,070621$ & 3,335492 & 0,225776 & $\mathrm{H} 76$ & $-2,342625$ & 3,396362 & 0,001890 \\
\hline
\end{tabular}




\begin{tabular}{|c|c|c|c|c|c|c|c|}
\hline H77 & $-4,158382$ & 4,261209 & $-0,656109$ & H77 & $-4,693736$ & 3,893339 & $-0,472280$ \\
\hline H78 & $-4,732989$ & 3,994595 & $-3,070656$ & $\mathrm{H} 78$ & $-5,804364$ & 2,922800 & $-2,478209$ \\
\hline C79 & $-0,147961$ & 1,926460 & $-1,025081$ & C79 & $-0,585175$ & 1,798897 & $-1,467293$ \\
\hline $\mathrm{C} 80$ & 1,166380 & 1,642484 & $-1,823318$ & $\mathrm{C} 80$ & 0,190079 & 2,051477 & $-2,814758$ \\
\hline C92 & 0,575641 & 4,119874 & 0,206825 & $\mathrm{C} 81$ & 0,141036 & 4,157024 & $-0,403737$ \\
\hline C93 & 0,915896 & 2,057502 & 1,155916 & C82 & 0,782446 & 2,172208 & 0,552999 \\
\hline C94 & 1,020176 & 4,253510 & 1,671657 & $\mathrm{C} 83$ & 0,620462 & 4,368491 & 1,035163 \\
\hline H95 & $-0,274617$ & 4,754495 & $-0,045557$ & H84 & $-0,791063$ & 4,667928 & $-0,640373$ \\
\hline H96 & 1,391832 & 4,326682 & $-0,500415$ & H85 & 0,892005 & 4,461648 & $-1,134928$ \\
\hline H97 & 1,855753 & 4,935486 & 1,830121 & H86 & 1,346085 & 5,173608 & 1,151424 \\
\hline H98 & 0,187835 & 4,522773 & 2,329816 & H87 & $-0,206006$ & 4,503046 & 1,742125 \\
\hline N99 & 0,219934 & 2,695003 & 0,184487 & N88 & $-0,019093$ & 2,683735 & $-0,401437$ \\
\hline O100 & 1,458342 & 2,911360 & 2,032255 & O89 & 1,281759 & 3,113091 & 1,364387 \\
\hline O101 & 1,072987 & 0,829018 & 1,231394 & O90 & 1,074585 & 0,967979 & 0,707416 \\
\hline H102 & 2,490764 & $-4,525281$ & 0,818505 & H91 & 2,656972 & $-4,804926$ & 0,251933 \\
\hline N103 & $-1,998956$ & $-2,301271$ & 0,677675 & N92 & $-1,873660$ & $-2,220516$ & 0,964777 \\
\hline C104 & $-1,422776$ & $-3,194926$ & $-0,357408$ & C93 & $-1,449944$ & $-3,241134$ & $-0,017432$ \\
\hline H105 & $-1,663445$ & $-2,858762$ & $-1,376121$ & H94 & $-1,848542$ & $-3,050612$ & $-1,024395$ \\
\hline C107 & 1,317174 & 2,035884 & $-6,107680$ & C95 & 0,329240 & 6,067238 & $-4,488217$ \\
\hline C108 & 1,459600 & 3,157008 & $-5,287134$ & C96 & $-0,902931$ & 5,430563 & $-4,344037$ \\
\hline C109 & 1,419615 & 3,016250 & $-3,901196$ & C97 & $-0,981904$ & 4,140632 & $-3,815542$ \\
\hline C110 & 1,231318 & 1,756128 & $-3,321834$ & C98 & 0,175851 & 3,459546 & $-3,409518$ \\
\hline C111 & 1,104049 & 0,635701 & $-4,146704$ & C99 & 1,410084 & 4,108554 & $-3,578877$ \\
\hline $\mathrm{C} 112$ & 1,143949 & 0,775108 & $-5,533922$ & $\mathrm{C} 100$ & 1,491742 & 5,396034 & $-4,108651$ \\
\hline H113 & 1,344817 & 2,145131 & $-7,187957$ & H101 & 0,383605 & 7,071469 & $-4,898940$ \\
\hline H1 14 & 1,598540 & 4,140442 & $-5,726960$ & $\mathrm{H} 102$ & $-1,814790$ & 5,937712 & $-4,648186$ \\
\hline H1 15 & 1,518184 & 3,892782 & $-3,264536$ & H103 & $-1,952981$ & 3,671967 & $-3,728041$ \\
\hline H116 & 0,974268 & $-0,343535$ & $-3,696995$ & H104 & 2,323731 & 3,596935 & $-3,281781$ \\
\hline H1 17 & 1,043428 & $-0,102177$ & $-6,166878$ & H105 & 2,461211 & 5,872799 & $-4,224903$ \\
\hline \multirow[t]{3}{*}{ H107 } & 1,977254 & 2,227550 & $-1,373961$ & H106 & 1,237121 & 1,793709 & $-2,688421$ \\
\hline & & & & H107 & 2,418223 & 0,613369 & $-1,428334$ \\
\hline & & & & H108 & 2,158133 & 0,188329 & $-1,984950$ \\
\hline \multicolumn{4}{|c|}{ C1_ER_Cheltop_INT2 } & \multicolumn{3}{|c|}{ C1_ER_Cheltop_TS3 } & \\
\hline$\overline{\mathrm{C} 1}$ & $-4,137901$ & $-3,412395$ & 1,327088 & $\mathrm{C} 1$ & $-4,146374$ & $-3,505526$ & 1,373241 \\
\hline $\mathrm{C} 2$ & $-2,112310$ & $-4,596984$ & 0,674184 & $\mathrm{C} 2$ & $-2,052622$ & $-4,634007$ & 0,841155 \\
\hline $\mathrm{C} 3$ & $-3,636475$ & $-4,564864$ & 0,397333 & $\mathrm{C} 3$ & $-3,566742$ & $-4,677968$ & 0,514518 \\
\hline $\mathrm{C} 4$ & $-2,827482$ & $-2,936069$ & 1,998049 & $\mathrm{C} 4$ & $-2,882256$ & $-2,965302$ & 2,084887 \\
\hline H5 & $-4,826919$ & $-3,783078$ & 2,093276 & H5 & $-4,870290$ & $-3,864226$ & 2,112200 \\
\hline H6 & $-3,861024$ & $-4,381874$ & $-0,658446$ & H6 & $-3,763242$ & $-4,556906$ & $-0,555628$ \\
\hline $\mathrm{H} 7$ & $-2,964274$ & $-2,310099$ & 2,879279 & $\mathrm{H} 7$ & $-3,071033$ & $-2,316211$ & 2,940190 \\
\hline $\mathrm{H} 8$ & $-4,646105$ & $-2,615604$ & 0,786356 & $\mathrm{H} 8$ & $-4,636904$ & $-2,737795$ & 0,773902 \\
\hline H9 & $-1,602904$ & $-5,505572$ & 0,353605 & H9 & $-1,494544$ & $-5,528703$ & 0,566517 \\
\hline $\mathrm{H} 10$ & $-4,087041$ & $-5,524644$ & 0,665742 & $\mathrm{H} 10$ & $-3,985302$ & $-5,642295$ & 0,816320 \\
\hline C11 & $-2,059748$ & $-4,256894$ & 2,180524 & $\mathrm{C} 11$ & $-2,068956$ & $-4,246649$ & 2,336546 \\
\hline H12 & $-1,047597$ & $-4,117583$ & 2,572355 & H12 & $-1,078198$ & $-4,051071$ & 2,758071 \\
\hline H13 & $-2,596278$ & $-4,985283$ & 2,795005 & H13 & $-2,594007$ & $-4,980111$ & 2,954799 \\
\hline $\mathrm{C} 14$ & $-0,002780$ & $-3,313277$ & $-0,085491$ & $\mathrm{C} 14$ & 0,016324 & $-3,276251$ & 0,079785 \\
\hline
\end{tabular}




\begin{tabular}{|c|c|c|c|c|c|c|c|}
\hline N15 & 0,703751 & $-2,255374$ & $-0,270126$ & N15 & 0,692566 & $-2,210951$ & $-0,194348$ \\
\hline C16 & 2,157032 & $-2,655334$ & $-0,153609$ & $\mathrm{C} 16$ & 2,167227 & $-2,554129$ & $-0,087194$ \\
\hline $\mathrm{C} 17$ & 2,033550 & $-4,192889$ & $-0,352305$ & $\mathrm{C} 17$ & 2,088087 & $-4,107803$ & $-0,111774$ \\
\hline P18 & $-1,888504$ & $-0,613459$ & 0,629171 & P18 & $-1,975140$ & $-0,646416$ & 0,682235 \\
\hline Ir19 & $-0,118743$ & $-0,285076$ & $-0,853649$ & Ir19 & $-0,153699$ & $-0,399017$ & $-0,962993$ \\
\hline $\mathrm{C} 20$ & $-1,640841$ & 0,215022 & 2,241394 & $\mathrm{C} 20$ & $-1,746251$ & 0,191378 & 2,298251 \\
\hline $\mathrm{C} 21$ & $-1,102763$ & 1,580543 & 4,638277 & $\mathrm{C} 21$ & $-1,198572$ & 1,566444 & 4,688542 \\
\hline $\mathrm{C} 22$ & $-1,058253$ & $-0,455197$ & 3,326019 & $\mathrm{C} 22$ & $-1,102916$ & $-0,455931$ & 3,362191 \\
\hline $\mathrm{C} 23$ & $-1,956523$ & 1,576514 & 2,373807 & $\mathrm{C} 23$ & $-2,126191$ & 1,533891 & 2,455338 \\
\hline $\mathrm{C} 24$ & $-1,687339$ & 2,252799 & 3,562827 & $\mathrm{C} 24$ & $-1,853538$ & 2,215658 & 3,640238 \\
\hline $\mathrm{C} 25$ & $-0,797118$ & 0,224581 & 4,516414 & $\mathrm{C} 25$ & $-0,832435$ & 0,227938 & 4,547693 \\
\hline $\mathrm{H} 26$ & $-2,430018$ & 2,101064 & 1,552918 & $\mathrm{H} 26$ & $-2,667508$ & 2,036673 & 1,663151 \\
\hline $\mathrm{H} 27$ & $-1,948164$ & 3,303782 & 3,654420 & $\mathrm{H} 27$ & $-2,169111$ & 3,249803 & 3,752102 \\
\hline $\mathrm{H} 28$ & $-0,356542$ & $-0,311793$ & 5,351984 & $\mathrm{H} 28$ & $-0,340098$ & $-0,291456$ & 5,365032 \\
\hline H29 & $-0,896840$ & 2,106318 & 5,565608 & $\mathrm{H} 29$ & $-0,987787$ & 2,096359 & 5,612805 \\
\hline $\mathrm{O} 30$ & 0,647170 & $-4,476353$ & 0,000232 & $\mathrm{O} 30$ & 0,706799 & $-4,398478$ & 0,258024 \\
\hline C31 & 2,673939 & $-2,376229$ & 1,262950 & $\mathrm{C} 31$ & 2,728299 & $-2,126074$ & 1,274517 \\
\hline $\mathrm{C} 32$ & 1,931708 & $-1,669830$ & 2,205889 & $\mathrm{C} 32$ & 1,962830 & $-1,472422$ & 2,234983 \\
\hline $\mathrm{C} 33$ & 3,946116 & $-2,850435$ & 1,616263 & $\mathrm{C} 33$ & 4,065149 & $-2,436976$ & 1,567627 \\
\hline C34 & 2,443984 & $-1,442504$ & 3,484676 & C34 & 2,519707 & $-1,116123$ & 3,464524 \\
\hline H35 & 0,970290 & $-1,265072$ & 1,934442 & H35 & 0,942887 & $-1,204115$ & 2,012183 \\
\hline $\mathrm{C} 36$ & 4,455656 & $-2,630761$ & 2,892779 & $\mathrm{C} 36$ & 4,621192 & $-2,088614$ & 2,794894 \\
\hline H37 & 4,550971 & $-3,379265$ & 0,883488 & H37 & 4,680247 & $-2,931702$ & 0,820098 \\
\hline $\mathrm{C} 38$ & 3,702520 & $-1,924576$ & 3,834734 & $\mathrm{C} 38$ & 3,847959 & $-1,421411$ & 3,749188 \\
\hline H39 & 1,855045 & $-0,873352$ & 4,197719 & H39 & 1,909240 & $-0,584816$ & 4,188826 \\
\hline $\mathrm{H} 40$ & 5,442028 & $-3,005114$ & 3,150796 & $\mathrm{H} 40$ & 5,659724 & $-2,328750$ & 3,003364 \\
\hline H41 & 4,100749 & $-1,748706$ & 4,829759 & $\mathrm{H} 41$ & 4,283868 & $-1,139795$ & 4,703417 \\
\hline $\mathrm{C} 42$ & $-3,610511$ & $-0,254534$ & 0,132577 & $\mathrm{C} 42$ & $-3,704382$ & $-0,287209$ & 0,197183 \\
\hline $\mathrm{C} 43$ & $-4,581679$ & 0,171414 & 1,052952 & $\mathrm{C} 43$ & $-4,703533$ & 0,045067 & 1,124767 \\
\hline $\mathrm{C} 44$ & $-4,019422$ & $-0,708952$ & $-1,130099$ & C44 & $-4,066180$ & $-0,574675$ & $-1,126911$ \\
\hline $\mathrm{C} 45$ & $-5,933108$ & 0,145469 & 0,711257 & $\mathrm{C} 45$ & $-6,039455$ & 0,094302 & 0,729581 \\
\hline H46 & $-4,292580$ & 0,502874 & 2,044230 & $\mathrm{H} 46$ & $-4,445253$ & 0,253354 & 2,158067 \\
\hline $\mathrm{C} 47$ & $-5,371790$ & $-0,746039$ & $-1,461623$ & $\mathrm{C} 47$ & $-5,403748$ & $-0,536164$ & $-1,515370$ \\
\hline $\mathrm{C} 48$ & $-6,331650$ & $-0,320793$ & $-0,542262$ & $\mathrm{C} 48$ & $-6,392574$ & $-0,201516$ & $-0,588656$ \\
\hline H49 & $-6,675241$ & 0,477613 & 1,431557 & H49 & $-6,806248$ & 0,351959 & 1,454738 \\
\hline H50 & $-5,675557$ & $-1,097728$ & $-2,443199$ & $\mathrm{H} 50$ & $-5,672602$ & $-0,754662$ & $-2,544605$ \\
\hline H51 & $-7,385797$ & $-0,350852$ & $-0,802215$ & H51 & $-7,435032$ & $-0,169670$ & $-0,892337$ \\
\hline H52 & $-1,034947$ & $-0,968793$ & $-1,942098$ & H52 & $-1,000337$ & $-1,349643$ & $-1,898896$ \\
\hline H53 & 0,024973 & 1,335818 & $-3,585450$ & H53 & 0,069502 & 1,509152 & $-3,728913$ \\
\hline H54 & $-0,800788$ & $-1,504878$ & 3,241999 & $\mathrm{H} 54$ & $-0,810964$ & $-1,495565$ & 3,263632 \\
\hline H55 & $-3,278482$ & $-1,032441$ & $-1,853531$ & $\mathrm{H} 55$ & $-3,297430$ & $-0,816919$ & $-1,853324$ \\
\hline H56 & 2,179671 & $-4,524834$ & $-1,382743$ & H56 & 2,258868 & $-4,552800$ & $-1,093902$ \\
\hline $\mathrm{C} 57$ & 4,175405 & $-0,333916$ & $-3,208758$ & C57 & 4,146788 & $-0,674479$ & $-3,457336$ \\
\hline $\mathrm{C} 58$ & 3,498777 & $-1,505495$ & $-3,556312$ & C58 & 3,420459 & $-1,851951$ & $-3,640604$ \\
\hline C59 & 2,902963 & $-2,292078$ & $-2,569283$ & C59 & 2,817003 & $-2,478713$ & $-2,551419$ \\
\hline C60 & 2,976698 & $-1,926378$ & $-1,218510$ & C60 & 2,935555 & $-1,947801$ & $-1,260445$ \\
\hline C61 & 3,664423 & $-0,752422$ & $-0,876753$ & C61 & 3,658965 & $-0,760229$ & $-1,088318$ \\
\hline C62 & 4,258995 & 0,035361 & $-1,865034$ & C62 & 4,260861 & $-0,130294$ & $-2,177327$ \\
\hline
\end{tabular}




\begin{tabular}{|c|c|c|c|c|c|c|c|}
\hline H63 & 4,634765 & 0,280598 & $-3,977228$ & H63 & 4,618317 & $-0,185339$ & $-4,304996$ \\
\hline H64 & 3,427479 & $-1,805645$ & $-4,597709$ & H64 & 3,317139 & $-2,280990$ & $-4,632960$ \\
\hline H65 & 2,346538 & $-3,172592$ & $-2,875519$ & H65 & 2,221633 & $-3,368432$ & $-2,732456$ \\
\hline H66 & 3,707811 & $-0,436452$ & 0,158803 & H66 & 3,729623 & $-0,311905$ & $-0,104264$ \\
\hline H67 & 4,784431 & 0,942475 & $-1,580128$ & H67 & 4,820370 & 0,788493 & $-2,022221$ \\
\hline C68 & $-3,914812$ & 1,790012 & $-3,247800$ & C68 & $-3,914323$ & 2,033685 & $-3,378723$ \\
\hline C69 & $-2,593128$ & 1,500221 & $-2,930502$ & C69 & $-2,592686$ & 1,701803 & $-3,100763$ \\
\hline $\mathrm{C} 70$ & $-1,979443$ & 2,035092 & $-1,783357$ & $\mathrm{C} 70$ & $-1,988779$ & 2,073807 & $-1,887467$ \\
\hline C71 & $-2,766840$ & 2,839255 & $-0,951609$ & C71 & $-2,776559$ & 2,751736 & $-0,952723$ \\
\hline $\mathrm{C} 72$ & $-4,103235$ & 3,114623 & $-1,252926$ & C72 & $-4,107966$ & 3,073880 & $-1,220139$ \\
\hline $\mathrm{C} 73$ & $-4,682421$ & 2,603605 & $-2,409629$ & $\mathrm{C} 73$ & $-4,681233$ & 2,727828 & $-2,439520$ \\
\hline H74 & $-4,353308$ & 1,369748 & $-4,148838$ & H74 & $-4,349320$ & 1,744723 & $-4,331183$ \\
\hline H75 & $-2,025231$ & 0,847777 & $-3,585194$ & $\mathrm{H} 75$ & $-2,026136$ & 1,156075 & $-3,849847$ \\
\hline H76 & $-2,337484$ & 3,269554 & $-0,055119$ & H76 & $-2,350005$ & 3,033418 & 0,000389 \\
\hline H77 & $-4,685082$ & 3,739578 & $-0,580963$ & H77 & $-4,693273$ & 3,596660 & $-0,469191$ \\
\hline H78 & $-5,717546$ & 2,823945 & $-2,652161$ & $\mathrm{H} 78$ & $-5,714727$ & 2,982104 & $-2,653736$ \\
\hline C79 & $-0,515606$ & 1,746312 & $-1,523617$ & C79 & $-0,523615$ & 1,751958 & $-1,643533$ \\
\hline $\mathrm{C} 80$ & 0,349331 & 2,033137 & $-2,806166$ & $\mathrm{C} 80$ & 0,401596 & 2,100262 & $-2,867640$ \\
\hline C81 & 0,175431 & 4,059403 & $-0,355579$ & $\mathrm{C} 81$ & 0,105676 & 3,962135 & $-0,297585$ \\
\hline C82 & 0,760649 & 2,041831 & 0,565052 & $\mathrm{C} 82$ & 0,784381 & 1,903066 & 0,459558 \\
\hline $\mathrm{C} 83$ & 0,598437 & 4,222244 & 1,107423 & C83 & 0,557915 & 4,029752 & 1,163922 \\
\hline H84 & $-0,744295$ & 4,583915 & $-0,611564$ & H84 & $-0,836703$ & 4,472180 & $-0,491399$ \\
\hline H85 & 0,956446 & 4,380423 & $-1,047307$ & H85 & 0,860708 & 4,358991 & $-0,976132$ \\
\hline H86 & 1,324053 & 5,017562 & 1,278003 & H86 & 1,256274 & 4,839564 & 1,374935 \\
\hline H87 & $-0,254822$ & 4,339862 & 1,784476 & H87 & $-0,279860$ & 4,057787 & 1,868590 \\
\hline N88 & 0,004380 & 2,588999 & $-0,403900$ & N88 & $-0,020058$ & 2,490850 & $-0,448732$ \\
\hline O89 & 1,236923 & 2,951358 & 1,423841 & O89 & 1,253531 & 2,765727 & 1,364761 \\
\hline O90 & 1,024765 & 0,829422 & 0,699560 & O90 & 1,079925 & 0,696271 & 0,495664 \\
\hline H91 & 2,670577 & $-4,767546$ & 0,316595 & H91 & 2,735962 & $-4,575720$ & 0,626716 \\
\hline N92 & $-1,922931$ & $-2,268669$ & 1,011402 & N92 & $-1,981831$ & $-2,291878$ & 1,106799 \\
\hline $\mathrm{C} 93$ & $-1,502605$ & $-3,315699$ & 0,047759 & C93 & $-1,485171$ & $-3,344450$ & 0,190848 \\
\hline H94 & $-1,909820$ & $-3,145305$ & $-0,959594$ & H94 & $-1,874456$ & $-3,231130$ & $-0,831606$ \\
\hline C95 & 0,631385 & 6,053215 & $-4,429762$ & C95 & 0,924589 & 6,241306 & $-4,045216$ \\
\hline C96 & $-0,615476$ & 5,432918 & $-4,351029$ & C96 & $-0,354262$ & 5,683724 & $-4,044312$ \\
\hline C97 & $-0,736068$ & 4,140973 & $-3,836629$ & C97 & $-0,546177$ & 4,352229 & $-3,674313$ \\
\hline C98 & 0,391772 & 3,443747 & $-3,378748$ & C98 & 0,539847 & 3,553939 & $-3,285388$ \\
\hline C99 & 1,641494 & 4,075454 & $-3,481263$ & C99 & 1,822929 & 4,122143 & $-3,308902$ \\
\hline $\mathrm{C} 100$ & 1,765480 & 5,364782 & $-3,997758$ & $\mathrm{C} 100$ & 2,017024 & 5,451983 & $-3,683343$ \\
\hline H101 & 0,719551 & 7,058931 & $-4,830736$ & H101 & 1,069253 & 7,278626 & $-4,333133$ \\
\hline H102 & $-1,504255$ & 5,954319 & $-4,696466$ & H102 & $-1,209936$ & 6,286157 & $-4,337131$ \\
\hline H103 & $-1,714804$ & 3,681233 & $-3,798038$ & H103 & $-1,547552$ & 3,941233 & $-3,689893$ \\
\hline H104 & 2,531739 & 3,546938 & $-3,144689$ & H104 & 2,679224 & 3,514079 & $-3,024270$ \\
\hline H105 & 2,745370 & 5,829640 & $-4,063542$ & H105 & 3,019496 & 5,870697 & $-3,692331$ \\
\hline H106 & 1,382225 & 1,762122 & $-2,572066$ & H106 & 1,390385 & 1,704516 & $-2,618569$ \\
\hline H107 & 1,403385 & $-0,105371$ & $-1,725533$ & H107 & 1,025620 & $-0,408231$ & $-2,044249$ \\
\hline H108 & 0,873670 & $-0,454531$ & $-2,275755$ & H108 & $-0,572072$ & 0,292056 & $-2,34407$ \\
\hline & & & & C & $\begin{array}{l}\mathbf{E}-\mathbf{S} \text { tN_CCCh } \\
\text { heltop_SM }\end{array}$ & eltop & \\
\hline
\end{tabular}




\begin{tabular}{|c|c|c|c|c|c|c|c|}
\hline $\mathrm{C} 1$ & $-4,108083$ & $-4,025846$ & 0,538970 & $\mathrm{C} 1$ & $-4,058318$ & $-3,565409$ & 0,059551 \\
\hline $\mathrm{C} 2$ & $-1,876337$ & $-4,968475$ & 0,274129 & $\mathrm{C} 2$ & $-1,868330$ & $-4,632708$ & $-0,011502$ \\
\hline $\mathrm{C} 3$ & $-3,307108$ & $-5,064570$ & $-0,315496$ & $\mathrm{C} 3$ & $-3,240745$ & $-4,638964$ & $-0,730987$ \\
\hline $\mathrm{C} 4$ & $-3,034940$ & $-3,518627$ & 1,532057 & $\mathrm{C} 4$ & $-3,037534$ & $-3,090362$ & 1,121744 \\
\hline H5 & $-4,936027$ & $-4,496837$ & 1,078518 & H5 & $-4,929764$ & $-4,005838$ & 0,555172 \\
\hline H6 & $-3,330923$ & $-4,842357$ & $-1,387199$ & H6 & $-3,150955$ & $-4,400153$ & $-1,795681$ \\
\hline H7 & $-3,426050$ & $-2,996903$ & 2,405332 & $\mathrm{H} 7$ & $-3,462096$ & $-2,527225$ & 1,952839 \\
\hline H8 & $-4,520495$ & $-3,207605$ & $-0,054980$ & $\mathrm{H} 8$ & $-4,413431$ & $-2,749720$ & $-0,569850$ \\
\hline H9 & $-1,208476$ & $-5,786929$ & 0,004860 & $\mathrm{H} 9$ & $-1,238193$ & $-5,499413$ & $-0,210410$ \\
\hline H10 & $-3,697563$ & $-6,077595$ & $-0,182857$ & $\mathrm{H} 10$ & $-3,697880$ & $-5,629658$ & $-0,655333$ \\
\hline $\mathrm{C} 11$ & $-2,177600$ & $-4,772932$ & 1,777078 & $\mathrm{C} 11$ & $-2,285822$ & $-4,390691$ & 1,456776 \\
\hline H12 & $-1,290968$ & $-4,572712$ & 2,385619 & H12 & $-1,445218$ & $-4,238168$ & 2,140065 \\
\hline H13 & $-2,739814$ & $-5,608083$ & 2,204514 & $\mathrm{H} 13$ & $-2,938041$ & $-5,178931$ & 1,843028 \\
\hline $\mathrm{C} 14$ & 0,161224 & $-3,375687$ & $-0,015341$ & $\mathrm{C} 14$ & 0,289342 & $-3,206229$ & $-0,039854$ \\
\hline N15 & 0,765598 & $-2,245585$ & $-0,196307$ & N15 & 0,991342 & $-2,124014$ & $-0,061005$ \\
\hline $\mathrm{C} 16$ & 2,223677 & $-2,413628$ & 0,196293 & $\mathrm{C} 16$ & 2,355600 & $-2,450001$ & 0,516288 \\
\hline $\mathrm{C} 17$ & 2,316546 & $-3,962328$ & 0,124197 & $\mathrm{C} 17$ & 2,328384 & $-4,005280$ & 0,420952 \\
\hline P18 & $-2,038616$ & $-1,000412$ & 0,569323 & P18 & $-1,869994$ & $-0,672048$ & 0,114232 \\
\hline Ir19 & $-0,116039$ & $-0,525259$ & $-0,805459$ & Ir19 & 0,360007 & $-0,203232$ & $-0,780506$ \\
\hline $\mathrm{C} 20$ & $-2,099900$ & $-0,256912$ & 2,242292 & $\mathrm{C} 20$ & $-2,198309$ & 0,188538 & 1,693561 \\
\hline $\mathrm{C} 21$ & $-2,022986$ & 1,042067 & 4,731438 & $\mathrm{C} 21$ & $-2,436837$ & 1,663845 & 4,073781 \\
\hline $\mathrm{C} 22$ & $-1,844504$ & $-0,983150$ & 3,412929 & $\mathrm{C} 22$ & $-1,958259$ & $-0,419884$ & 2,933816 \\
\hline $\mathrm{C} 23$ & $-2,296229$ & 1,132088 & 2,328868 & $\mathrm{C} 23$ & $-2,564655$ & 1,544263 & 1,660659 \\
\hline $\mathrm{C} 24$ & $-2,258188$ & 1,774030 & 3,564149 & $\mathrm{C} 24$ & $-2,680997$ & 2,275058 & 2,841930 \\
\hline $\mathrm{C} 25$ & $-1,816078$ & $-0,335412$ & 4,650580 & $\mathrm{C} 25$ & $-2,082498$ & 0,314647 & 4,114505 \\
\hline $\mathrm{H} 26$ & $-2,485778$ & 1,715506 & 1,432818 & $\mathrm{H} 26$ & $-2,781135$ & 2,024243 & 0,711493 \\
\hline $\mathrm{H} 27$ & $-2,414126$ & 2,848066 & 3,612406 & $\mathrm{H} 27$ & $-2,969282$ & 3,321668 & 2,800132 \\
\hline $\mathrm{H} 28$ & $-1,625435$ & $-0,912015$ & 5,551468 & $\mathrm{H} 28$ & $-1,904638$ & $-0,172456$ & 5,069230 \\
\hline H29 & $-1,997584$ & 1,542293 & 5,694936 & $\mathrm{H} 29$ & $-2,528359$ & 2,233407 & 4,993652 \\
\hline $\mathrm{O} 30$ & 0,936993 & $-4,411064$ & 0,298800 & $\mathrm{O} 30$ & 0,912404 & $-4,326300$ & 0,323634 \\
\hline C31 & 2,449521 & $-1,978151$ & 1,650609 & $\mathrm{C} 31$ & 2,419714 & $-2,075277$ & 2,005020 \\
\hline C32 & 1,456690 & $-1,414826$ & 2,448738 & $\mathrm{C} 32$ & 1,323560 & $-1,571081$ & 2,700566 \\
\hline $\mathrm{C} 33$ & 3,722623 & $-2,190418$ & 2,203616 & $\mathrm{C} 33$ & 3,607148 & $-2,329355$ & 2,710048 \\
\hline C34 & 1,728665 & $-1,054800$ & 3,770777 & $\mathrm{C} 34$ & 1,413503 & $-1,294567$ & 4,066304 \\
\hline H35 & 0,472503 & $-1,229012$ & 2,045799 & H35 & 0,405599 & $-1,362006$ & 2,173449 \\
\hline C36 & 3,993782 & $-1,838665$ & 3,521934 & $\mathrm{C} 36$ & 3,697237 & $-2,059344$ & 4,073128 \\
\hline H37 & 4,513786 & $-2,612347$ & 1,589097 & H37 & 4,472926 & $-2,724655$ & 2,185070 \\
\hline C38 & 2,994017 & $-1,264613$ & 4,311595 & C38 & 2,598091 & $-1,533947$ & 4,758681 \\
\hline H39 & 0,941271 & $-0,603588$ & 4,366479 & H39 & 0,553126 & $-0,874223$ & 4,578375 \\
\hline $\mathrm{H} 40$ & 4,986150 & $-2,006081$ & 3,930510 & $\mathrm{H} 40$ & 4,627776 & $-2,254300$ & 4,598509 \\
\hline H41 & 3,205162 & $-0,983289$ & 5,339237 & $\mathrm{H} 41$ & 2,669848 & $-1,314269$ & 5,819743 \\
\hline $\mathrm{C} 42$ & $-3,667902$ & $-0,565728$ & $-0,170451$ & $\mathrm{C} 42$ & $-3,335599$ & $-0,372192$ & $-0,942283$ \\
\hline $\mathrm{C} 43$ & $-4,778100$ & $-0,172600$ & 0,590746 & $\mathrm{C} 43$ & $-4,581505$ & 0,022343 & $-0,433700$ \\
\hline $\mathrm{C} 44$ & $-3,812565$ & $-0,700021$ & $-1,562632$ & C44 & $-3,232178$ & $-0,748051$ & $-2,291199$ \\
\hline $\mathrm{C} 45$ & $-5,999969$ & 0,097119 & $-0,027783$ & $\mathrm{C} 45$ & $-5,702863$ & 0,043420 & $-1,263300$ \\
\hline H46 & $-4,688912$ & $-0,063927$ & 1,667032 & $\mathrm{H} 46$ & $-4,683579$ & 0,302499 & 0,609740 \\
\hline $\mathrm{C} 47$ & $-5,036585$ & $-0,436730$ & $-2,178014$ & $\mathrm{C} 47$ & $-4,356932$ & $-0,733646$ & $-3,113581$ \\
\hline $\mathrm{C} 48$ & $-6,133241$ & $-0,029785$ & $-1,412401$ & $\mathrm{C} 48$ & $-5,595286$ & $-0,338318$ & $-2,602300$ \\
\hline
\end{tabular}




\begin{tabular}{|c|c|c|c|c|c|c|c|}
\hline H49 & $-6,849756$ & 0,405528 & 0,574736 & H49 & $-6,663079$ & 0,352551 & $-0,860252$ \\
\hline H50 & $-5,133328$ & $-0,543575$ & $-3,254901$ & H50 & $-4,263008$ & $-1,014769$ & $-4,158277$ \\
\hline H51 & $-7,085484$ & 0,179528 & $-1,890842$ & H51 & $-6,470526$ & $-0,323187$ & $-3,245139$ \\
\hline H52 & $-0,743069$ & $-1,359252$ & $-1,966227$ & H52 & $-0,029588$ & $-0,825621$ & $-2,156933$ \\
\hline H53 & $-1,673549$ & 1,598960 & $-3,371206$ & H53 & 1,848624 & $-0,275747$ & $-1,317886$ \\
\hline H54 & $-1,639625$ & $-2,047044$ & 3,360552 & H54 & $-1,665664$ & $-1,463170$ & 2,979884 \\
\hline H55 & $-2,958898$ & $-0,999712$ & $-2,164479$ & H55 & $-2,268398$ & $-1,033893$ & $-2,697402$ \\
\hline H56 & 2,657731 & $-4,341792$ & $-0,841329$ & H56 & 2,816179 & $-4,409252$ & $-0,468605$ \\
\hline $\mathrm{C} 57$ & 4,612981 & $-0,181652$ & $-2,650617$ & $\mathrm{C} 57$ & 5,382240 & $-0,565831$ & $-1,966770$ \\
\hline C58 & 4,060871 & $-1,410630$ & $-3,014334$ & C58 & 4,689876 & $-1,687098$ & $-2,426679$ \\
\hline C59 & 3,319078 & $-2,145595$ & $-2,091060$ & C59 & 3,731871 & $-2,297686$ & $-1,618760$ \\
\hline C60 & 3,129309 & $-1,673263$ & $-0,786880$ & C60 & 3,460367 & $-1,814206$ & $-0,330529$ \\
\hline C61 & 3,679038 & $-0,434115$ & $-0,434076$ & C61 & 4,148599 & $-0,677413$ & 0,113064 \\
\hline C62 & 4,413801 & 0,305722 & $-1,358810$ & C62 & 5,101091 & $-0,060508$ & $-0,697951$ \\
\hline H63 & 5,193584 & 0,389499 & $-3,369849$ & H63 & 6,127337 & $-0,087427$ & $-2,595723$ \\
\hline H64 & 4,201311 & $-1,796091$ & $-4,019970$ & H64 & 4,888096 & $-2,083469$ & $-3,418514$ \\
\hline H65 & 2,862793 & $-3,075593$ & $-2,416078$ & H65 & 3,176876 & $-3,140338$ & $-2,019790$ \\
\hline H66 & 3,511150 & $-0,030488$ & 0,556826 & H66 & 3,932633 & $-0,267179$ & 1,092956 \\
\hline H67 & 4,830743 & 1,264470 & $-1,062513$ & H67 & 5,628753 & 0,816533 & $-0,332897$ \\
\hline C68 & $-4,159253$ & 3,354906 & $-0,659299$ & C68 & 1,860865 & 3,456766 & $-4,799203$ \\
\hline C69 & $-3,104192$ & 2,679817 & $-1,266512$ & C69 & 1,291795 & 3,152012 & $-3,564777$ \\
\hline $\mathrm{C} 70$ & $-1,774684$ & 2,988935 & $-0,943379$ & $\mathrm{C} 70$ & 1,585962 & 1,937533 & $-2,923334$ \\
\hline C71 & $-1,527086$ & 3,983033 & 0,008623 & C71 & 2,460105 & 1,037998 & $-3,537376$ \\
\hline $\mathrm{C} 72$ & $-2,584371$ & 4,671582 & 0,606901 & $\mathrm{C} 72$ & 3,027297 & 1,342565 & $-4,775486$ \\
\hline $\mathrm{C} 73$ & $-3,902422$ & 4,359567 & 0,276805 & $\mathrm{C} 73$ & 2,728899 & 2,548995 & $-5,409944$ \\
\hline H74 & $-5,179671$ & 3,084214 & $-0,911429$ & H74 & 1,618229 & 4,395663 & $-5,288653$ \\
\hline H75 & $-3,325310$ & 1,892757 & $-1,978033$ & H75 & 0,595267 & 3,845785 & $-3,102977$ \\
\hline H76 & $-0,511231$ & 4,221835 & 0,302356 & H76 & 2,685790 & 0,095998 & $-3,052421$ \\
\hline H77 & $-2,373476$ & 5,448067 & 1,336586 & H77 & 3,699967 & 0,630757 & $-5,246011$ \\
\hline H78 & $-4,724043$ & 4,889157 & 0,749881 & $\mathrm{H} 78$ & 3,165593 & 2,780374 & $-6,377513$ \\
\hline C79 & $-0,648162$ & 2,187287 & $-1,592308$ & C79 & 0,989849 & 1,719253 & $-1,559443$ \\
\hline $\mathrm{C} 80$ & $-0,710005$ & 2,061233 & $-3,135593$ & $\mathrm{C} 80$ & $-0,423190$ & 1,797097 & $-1,310796$ \\
\hline C81 & 1,421467 & 3,745750 & $-1,564667$ & C92 & 2,509508 & 3,580016 & $-0,575297$ \\
\hline $\mathrm{C} 82$ & 1,267776 & 2,010721 & $-0,014321$ & C93 & 1,780771 & 1,825754 & 0,751351 \\
\hline $\mathrm{C} 83$ & 2,352841 & 3,982307 & $-0,362194$ & C94 & 3,224552 & 3,570785 & 0,789922 \\
\hline H84 & 0,760743 & 4,590049 & $-1,769651$ & H95 & 1,769041 & 4,385811 & $-0,663939$ \\
\hline H85 & 1,967404 & 3,506937 & $-2,480342$ & H96 & 3,200759 & 3,645014 & $-1,417234$ \\
\hline H86 & 3,385152 & 4,189743 & $-0,644966$ & H97 & 4,260433 & 3,228188 & 0,706647 \\
\hline H87 & 1,989907 & 4,771637 & 0,304174 & H98 & 3,191474 & 4,526096 & 1,314229 \\
\hline N88 & 0,667051 & 2,574422 & $-1,096476$ & N99 & 1,854224 & 2,272999 & $-0,520568$ \\
\hline O89 & 2,332286 & 2,730892 & 0,373459 & O100 & 2,496866 & 2,592059 & 1,586792 \\
\hline O90 & 0,946351 & 0,972057 & 0,569397 & O101 & 1,146441 & 0,829601 & 1,122806 \\
\hline H91 & 2,910626 & $-4,391873$ & 0,927894 & H102 & 2,722379 & $-4,489422$ & 1,312457 \\
\hline N92 & $-2,034951$ & $-2,677397$ & 0,820548 & N103 & $-1,920954$ & $-2,326405$ & 0,491559 \\
\hline C93 & $-1,326036$ & $-3,576744$ & $-0,129100$ & C104 & $-1,178031$ & $-3,289805$ & $-0,362172$ \\
\hline H94 & $-1,579832$ & $-3,356304$ & $-1,175408$ & H105 & $-1,280921$ & $-3,067156$ & $-1,433986$ \\
\hline C95 & $-0,159791$ & 5,680951 & $-5,448716$ & C107 & $-3,381279$ & 2,529983 & $-4,341602$ \\
\hline C96 & $-1,266154$ & 5,558527 & $-4,606731$ & C108 & $-3,501692$ & 3,083403 & $-3,068010$ \\
\hline
\end{tabular}




$\begin{array}{lrrrlrrr}\text { C97 } & -1,457781 & 4,396612 & -3,858972 & \text { C109 } & -2,537109 & 2,815543 & -2,097277 \\ \text { C98 } & -0,538049 & 3,340068 & -3,932057 & \text { C110 } & -1,444398 & 1,980092 & -2,371756 \\ \text { C99 } & 0,561193 & 3,469812 & -4,791863 & \text { C111 } & -1,345484 & 1,416693 & -3,654692 \\ \text { C100 } & 0,751396 & 4,628775 & -5,544846 & \text { C112 } & -2,295819 & 1,699106 & -4,629750 \\ \text { H101 } & -0,014721 & 6,585607 & -6,032154 & \text { H113 } & -4,127328 & 2,740447 & -5,102555 \\ \text { H102 } & -1,988539 & 6,367045 & -4,536203 & \text { H114 } & -4,342083 & 3,728740 & -2,828799 \\ \text { H103 } & -2,330244 & 4,314555 & -3,220968 & \text { H115 } & -2,626239 & 3,277433 & -1,116801 \\ \text { H104 } & 1,274077 & 2,651777 & -4,873712 & \text { H116 } & -0,520271 & 0,754438 & -3,885867 \\ \text { H105 } & 1,608235 & 4,708023 & -6,207924 & \text { H117 } & -2,194238 & 1,260044 & -5,618562 \\ \text { H106 } & 0,054285 & 1,332848 & -3,417752 & \text { H107 } & -0,690930 & 2,271203 & -0,366928\end{array}$

H107 $\quad 1,102180 \quad-0,314064 \quad-1,831597$

H108 $\quad-0,890966 \quad 1,139564 \quad-1,213599$

C1 ES Cheltop TS1

\begin{tabular}{|c|c|c|c|c|c|c|c|}
\hline $\mathrm{C} 1$ & $-4,026752$ & $-3,759265$ & 0,025393 & $\mathrm{C} 1$ & $-4,236530$ & $-3,496566$ & 0,109269 \\
\hline $\mathrm{C} 2$ & $-1,807645$ & $-4,773433$ & 0,022319 & $\mathrm{C} 2$ & $-2,154753$ & $-4,671755$ & 0,595512 \\
\hline $\mathrm{C} 3$ & $-3,163969$ & $-4,825659$ & $-0,727846$ & $\mathrm{C} 3$ & $-3,432607$ & $-4,783393$ & $-0,276097$ \\
\hline $\mathrm{C} 4$ & $-3,051042$ & $-3,262224$ & 1,117920 & $\mathrm{C} 4$ & $-3,319204$ & $-2,843704$ & 1,169685 \\
\hline H5 & $-4,913263$ & $-4,206915$ & 0,486361 & H5 & $-5,208465$ & $-3,742612$ & 0,548955 \\
\hline H6 & $-3,056941$ & $-4,606043$ & $-1,794963$ & H6 & $-3,201833$ & $-4,834686$ & $-1,344954$ \\
\hline H7 & $-3,513456$ & $-2,707796$ & 1,934797 & $\mathrm{H} 7$ & $-3,790027$ & $-2,074813$ & 1,782508 \\
\hline H8 & $-4,362837$ & $-2,945323$ & $-0,619038$ & $\mathrm{H} 8$ & $-4,412890$ & $-2,830017$ & $-0,736778$ \\
\hline H9 & $-1,147652$ & $-5,622171$ & $-0,155863$ & H9 & $-1,574338$ & $-5,589957$ & 0,688158 \\
\hline H10 & $-3,598929$ & $-5,825482$ & $-0,641729$ & H10 & $-3,983226$ & $-5,692102$ & $-0,016767$ \\
\hline $\mathrm{C} 11$ & $-2,270461$ & $-4,538438$ & 1,478183 & $\mathrm{C} 11$ & $-2,722539$ & $-4,061643$ & 1,896217 \\
\hline H12 & $-1,452506$ & $-4,356539$ & 2,181538 & H12 & $-1,960304$ & $-3,790996$ & 2,633362 \\
\hline H13 & $-2,907943$ & $-5,344600$ & 1,851997 & H13 & $-3,478162$ & $-4,699038$ & 2,363917 \\
\hline $\mathrm{C} 14$ & 0,313774 & $-3,269555$ & $-0,002880$ & C14 & 0,117265 & $-3,413369$ & 0,460217 \\
\hline N15 & 0,969328 & $-2,159626$ & $-0,031176$ & N15 & 0,806376 & $-2,331031$ & 0,427287 \\
\hline $\mathrm{C} 16$ & 2,348363 & $-2,428026$ & 0,514782 & C16 & 2,171229 & $-2,615017$ & 0,961806 \\
\hline $\mathrm{C} 17$ & 2,389545 & $-3,986227$ & 0,432649 & $\mathrm{C} 17$ & 2,167200 & $-4,177844$ & 0,913184 \\
\hline P18 & $-1,903817$ & $-0,816213$ & 0,172941 & P18 & $-1,835972$ & $-0,740733$ & $-0,032032$ \\
\hline Ir19 & 0,176472 & $-0,244997$ & $-0,705693$ & Ir19 & 0,252906 & $-0,525898$ & $-0,678026$ \\
\hline $\mathrm{C} 20$ & $-2,184660$ & 0,023858 & 1,767467 & $\mathrm{C} 20$ & $-2,108180$ & 0,330749 & 1,417023 \\
\hline $\mathrm{C} 21$ & $-2,406447$ & 1,465068 & 4,160683 & $\mathrm{C} 21$ & $-2,276153$ & 2,062747 & 3,615447 \\
\hline $\mathrm{C} 22$ & $-2,013464$ & $-0,621301$ & 2,998441 & $\mathrm{C} 22$ & $-2,001991$ & $-0,170725$ & 2,721889 \\
\hline $\mathrm{C} 23$ & $-2,461278$ & 1,400700 & 1,745528 & $\mathrm{C} 23$ & $-2,284159$ & 1,710908 & 1,221379 \\
\hline $\mathrm{C} 24$ & $-2,571493$ & 2,115293 & 2,935269 & $\mathrm{C} 24$ & $-2,370496$ & 2,568761 & 2,315647 \\
\hline $\mathrm{C} 25$ & $-2,131425$ & 0,098130 & 4,189095 & $\mathrm{C} 25$ & $-2,092382$ & 0,693176 & 3,814449 \\
\hline $\mathrm{H} 26$ & $-2,603968$ & 1,909546 & 0,796176 & $\mathrm{H} 26$ & $-2,360309$ & 2,112427 & 0,214866 \\
\hline H27 & $-2,790565$ & 3,179026 & 2,907897 & $\mathrm{H} 27$ & $-2,513764$ & 3,633442 & 2,154849 \\
\hline $\mathrm{H} 28$ & $-2,008282$ & $-0,413212$ & 5,139826 & $\mathrm{H} 28$ & $-2,018181$ & 0,293115 & 4,821685 \\
\hline H29 & $-2,494978$ & 2,022634 & 5,088552 & $\mathrm{H} 29$ & $-2,345449$ & 2,733214 & 4,466864 \\
\hline $\mathrm{O} 30$ & 0,987883 & $-4,368593$ & 0,345093 & $\mathrm{O} 30$ & 0,749560 & $-4,528580$ & 0,837682 \\
\hline C31 & 2,427819 & $-2,033160$ & 1,996418 & $\mathrm{C} 31$ & 2,289397 & $-2,107729$ & 2,402599 \\
\hline C32 & 1,326685 & $-1,558584$ & 2,705633 & C32 & 1,359973 & $-1,218749$ & 2,948065 \\
\hline C33 & 3,640243 & $-2,226325$ & 2,677317 & $\mathrm{C} 33$ & 3,354237 & $-2,541153$ & 3,206120 \\
\hline C34 & 1,435801 & $-1,252576$ & 4,063289 & C34 & 1,494478 & $-0,766378$ & 4,260917 \\
\hline
\end{tabular}




\begin{tabular}{|c|c|c|c|c|c|c|c|}
\hline H35 & 0,388139 & $-1,397231$ & 2,197933 & H35 & 0,538453 & $-0,859953$ & 2,346253 \\
\hline C36 & 3,749161 & $-1,928024$ & 4,032454 & $\mathrm{C} 36$ & 3,491425 & $-2,087173$ & 4,517503 \\
\hline H37 & 4,508942 & $-2,595981$ & 2,138460 & H37 & 4,096419 & $-3,227013$ & 2,804494 \\
\hline C38 & 2,644209 & $-1,433273$ & 4,730545 & $\mathrm{C} 38$ & 2,559945 & $-1,194529$ & 5,051890 \\
\hline H39 & 0,570776 & $-0,856944$ & 4,587198 & H39 & 0,760531 & $-0,069345$ & 4,656177 \\
\hline $\mathrm{H} 40$ & 4,696835 & $-2,076370$ & 4,542142 & $\mathrm{H} 40$ & 4,326936 & $-2,431931$ & 5,119840 \\
\hline $\mathrm{H} 41$ & 2,730023 & $-1,191021$ & 5,785891 & $\mathrm{H} 41$ & 2,665880 & $-0,839622$ & 6,072703 \\
\hline $\mathrm{C} 42$ & $-3,379567$ & $-0,427293$ & $-0,832560$ & $\mathrm{C} 42$ & $-3,195709$ & $-0,406639$ & $-1,197071$ \\
\hline $\mathrm{C} 43$ & $-4,597641$ & $-0,039200$ & $-0,253156$ & $\mathrm{C} 43$ & $-4,384565$ & 0,215741 & $-0,787043$ \\
\hline $\mathrm{C} 44$ & $-3,317844$ & $-0,652276$ & $-2,215963$ & C44 & $-3,111253$ & $-0,947208$ & $-2,489952$ \\
\hline $\mathrm{C} 45$ & $-5,730119$ & 0,128573 & $-1,049135$ & $\mathrm{C} 45$ & $-5,464748$ & 0,307450 & $-1,665451$ \\
\hline H46 & $-4,666979$ & 0,131628 & 0,816375 & $\mathrm{H} 46$ & $-4,477263$ & 0,622673 & 0,214140 \\
\hline $\mathrm{C} 47$ & $-4,452266$ & $-0,485645$ & $-3,006366$ & $\mathrm{C} 47$ & $-4,191787$ & $-0,853882$ & $-3,360914$ \\
\hline $\mathrm{C} 48$ & $-5,659591$ & $-0,093918$ & $-2,425160$ & $\mathrm{C} 48$ & $-5,370345$ & $-0,225033$ & $-2,950990$ \\
\hline H49 & $-6,667896$ & 0,432379 & $-0,592380$ & $\mathrm{H} 49$ & $-6,380632$ & 0,793236 & $-1,341296$ \\
\hline H50 & $-4,387142$ & $-0,641848$ & $-4,078493$ & $\mathrm{H} 50$ & $-4,107395$ & $-1,254919$ & $-4,365968$ \\
\hline H51 & $-6,542481$ & 0,042371 & $-3,043236$ & H51 & $-6,211614$ & $-0,148483$ & $-3,633877$ \\
\hline H52 & $-0,230137$ & $-0,919524$ & $-2,052003$ & H52 & $-0,250375$ & $-1,412071$ & $-1,866816$ \\
\hline H53 & 1,642090 & 0,147778 & $-1,401239$ & $\mathrm{H} 53$ & 1,925585 & 1,117744 & $-2,875022$ \\
\hline H54 & $-1,775983$ & $-1,679299$ & 3,030751 & $\mathrm{H} 54$ & $-1,834204$ & $-1,230337$ & 2,882616 \\
\hline H55 & $-2,376687$ & $-0,931660$ & $-2,675942$ & $\mathrm{H} 55$ & $-2,190635$ & $-1,411962$ & $-2,823041$ \\
\hline H56 & 2,890705 & $-4,377735$ & $-0,455010$ & H56 & 2,650197 & $-4,571650$ & 0,013693 \\
\hline $\mathrm{C} 57$ & 5,289439 & $-0,436039$ & $-1,992737$ & C57 & 4,911375 & $-0,820029$ & $-1,904028$ \\
\hline C58 & 4,671404 & $-1,609231$ & $-2,428285$ & C58 & 3,819963 & $-1,579157$ & $-2,330983$ \\
\hline C59 & 3,724379 & $-2,242784$ & $-1,624599$ & C59 & 2,973109 & $-2,170369$ & $-1,392913$ \\
\hline C60 & 3,399517 & $-1,738107$ & $-0,357331$ & C60 & 3,187719 & $-1,996755$ & $-0,012385$ \\
\hline C61 & 4,010230 & $-0,547637$ & 0,059296 & C61 & 4,277392 & $-1,227273$ & 0,402542 \\
\hline C62 & 4,944382 & 0,097546 & $-0,752038$ & C62 & 5,137608 & $-0,654016$ & $-0,537894$ \\
\hline H63 & 6,023804 & 0,060734 & $-2,619843$ & H63 & 5,582517 & $-0,370810$ & $-2,630904$ \\
\hline H64 & 4,917159 & $-2,027869$ & $-3,400112$ & H64 & 3,632994 & $-1,724065$ & $-3,391346$ \\
\hline H65 & 3,230906 & $-3,130785$ & $-2,007206$ & $\mathrm{H} 65$ & 2,137415 & $-2,770589$ & $-1,739678$ \\
\hline H66 & 3,762609 & $-0,130714$ & 1,029014 & H66 & 4,446498 & $-1,048812$ & 1,457539 \\
\hline H67 & 5,413468 & 1,014846 & $-0,405614$ & H67 & 5,985676 & $-0,067933$ & $-0,194278$ \\
\hline C68 & 2,065579 & 3,392295 & $-4,763384$ & C68 & 0,674376 & 5,515007 & $-2,762253$ \\
\hline C69 & 1,512088 & 3,101018 & $-3,519468$ & C69 & 0,939545 & 4,304360 & $-2,125669$ \\
\hline $\mathrm{C} 70$ & 1,731941 & 1,848539 & $-2,925207$ & $\mathrm{C} 70$ & 1,290273 & 3,171416 & $-2,870900$ \\
\hline C71 & 2,490867 & 0,891460 & $-3,596382$ & C71 & 1,379420 & 3,271304 & $-4,260625$ \\
\hline $\mathrm{C} 72$ & 3,038800 & 1,182103 & $-4,846583$ & $\mathrm{C} 72$ & 1,106764 & 4,481095 & $-4,900785$ \\
\hline $\mathrm{C} 73$ & 2,829025 & 2,431190 & $-5,430724$ & $\mathrm{C} 73$ & 0,752124 & 5,604384 & $-4,154125$ \\
\hline H74 & 1,884977 & 4,360764 & $-5,220610$ & $\mathrm{H} 74$ & 0,401351 & 6,387615 & $-2,175121$ \\
\hline H75 & 0,881080 & 3,830891 & $-3,021486$ & $\mathrm{H} 75$ & 0,868577 & 4,232107 & $-1,042638$ \\
\hline H76 & 2,637661 & $-0,084617$ & $-3,147564$ & $\mathrm{H} 76$ & 1,631510 & 2,392401 & $-4,847458$ \\
\hline H77 & 3,622887 & 0,427614 & $-5,365610$ & $\mathrm{H} 77$ & 1,166985 & 4,543791 & $-5,983584$ \\
\hline H78 & 3,248246 & 2,653965 & $-6,407687$ & $\mathrm{H} 78$ & 0,537030 & 6,545301 & $-4,652562$ \\
\hline C79 & 1,116157 & 1,605328 & $-1,572825$ & C79 & 1,466707 & 1,827277 & $-2,172568$ \\
\hline $\mathrm{C} 80$ & $-0,340749$ & 1,713569 & $-1,383165$ & $\mathrm{C} 80$ & 0,059565 & 1,287973 & $-1,727192$ \\
\hline C92 & 2,689443 & 3,420946 & $-0,562317$ & C92 & 3,718276 & 2,642193 & $-1,259829$ \\
\hline C93 & 1,687283 & 1,817259 & 0,791545 & C93 & 2,340261 & 1,487518 & 0,186670 \\
\hline
\end{tabular}




\begin{tabular}{|c|c|c|c|c|c|c|c|}
\hline C94 & 3,251552 & 3,459270 & 0,874037 & C94 & 4,177869 & 2,826490 & 0,193662 \\
\hline H95 & 2,049246 & 4,282163 & $-0,790233$ & H95 & 3,611564 & 3,587728 & $-1,793709$ \\
\hline H96 & 3,470797 & 3,354222 & $-1,321456$ & H96 & 4,372968 & 1,974377 & $-1,832310$ \\
\hline H97 & 4,267560 & 3,057461 & 0,928581 & H97 & 5,227264 & 2,581196 & 0,360332 \\
\hline H98 & 3,221548 & 4,448715 & 1,331044 & H98 & 3,966818 & 3,830810 & 0,573717 \\
\hline N99 & 1,907108 & 2,188945 & $-0,492585$ & N99 & 2,413534 & 2,005931 & $-1,053818$ \\
\hline O100 & 2,386183 & 2,579115 & 1,645086 & O100 & 3,373213 & 1,883084 & 0,948665 \\
\hline O101 & 0,960452 & 0,887150 & 1,157636 & O101 & 1,486310 & 0,719863 & 0,665301 \\
\hline H102 & 2,808714 & $-4,445266$ & 1,326166 & $\mathrm{H} 102$ & 2,577948 & $-4,650626$ & 1,803012 \\
\hline N103 & $-1,944695$ & $-2,472467$ & 0,509032 & N103 & $-2,081141$ & $-2,319603$ & 0,523147 \\
\hline C104 & $-1,152276$ & $-3,411789$ & $-0,325411$ & C104 & $-1,323240$ & $-3,490878$ & 0,029357 \\
\hline H105 & $-1,254276$ & $-3,199388$ & $-1,399464$ & H105 & $-1,300870$ & $-3,537355$ & $-1,070525$ \\
\hline $\mathrm{C} 107$ & $-3,175575$ & 2,396908 & $-4,548633$ & C107 & $-2,433211$ & 1,481414 & $-5,258193$ \\
\hline $\mathrm{C} 108$ & $-3,331045$ & 2,993715 & $-3,298652$ & $\mathrm{C} 108$ & $-2,703069$ & 2,296030 & $-4,159063$ \\
\hline C109 & $-2,397218$ & 2,754981 & $-2,291275$ & C109 & $-1,906565$ & 2,217683 & $-3,017088$ \\
\hline $\mathrm{C} 110$ & $-1,299814$ & 1,907351 & $-2,502464$ & $\mathrm{C} 110$ & $-0,838693$ & 1,315391 & $-2,937578$ \\
\hline $\mathrm{C} 111$ & $-1,167473$ & 1,294427 & $-3,761210$ & C111 & $-0,587364$ & 0,493972 & $-4,047258$ \\
\hline C112 & $-2,089010$ & 1,547397 & $-4,773052$ & $\mathrm{C} 112$ & $-1,369002$ & 0,578415 & $-5,197462$ \\
\hline H1 13 & $-3,896103$ & 2,586057 & $-5,339253$ & H113 & $-3,045260$ & 1,548319 & $-6,153354$ \\
\hline H1 14 & $-4,176260$ & 3,648591 & $-3,106121$ & H114 & $-3,529202$ & 3,001140 & $-4,192874$ \\
\hline H1 15 & $-2,520468$ & 3,237733 & $-1,323814$ & H115 & $-2,102779$ & 2,884706 & $-2,180884$ \\
\hline H116 & $-0,342147$ & 0,616604 & $-3,945697$ & H116 & 0,230020 & $-0,222274$ & $-4,000589$ \\
\hline H1 17 & $-1,962775$ & 1,072715 & $-5,742507$ & H117 & $-1,150730$ & $-0,061957$ & $-6,048448$ \\
\hline H107 & $-0,605482$ & 2,315601 & $-0,512655$ & H107 & $-0,313217$ & 2,030575 & $-1,008705$ \\
\hline \multicolumn{2}{|c|}{ C1_ES_Cheltop_TS2 } & \multicolumn{6}{|c|}{ C1_ES_Cheltop_INT2 } \\
\hline $\mathrm{C} 1$ & $-4,270611$ & $-3,334181$ & 0,391654 & $\mathrm{C} 1$ & $-4,284394$ & $-3,315052$ & 0,401414 \\
\hline $\mathrm{C} 2$ & $-2,197265$ & $-4,561846$ & 0,760893 & $\mathrm{C} 2$ & $-2,221698$ & $-4,565507$ & 0,750002 \\
\hline $\mathrm{C} 3$ & $-3,535809$ & $-4,654367$ & $-0,014407$ & $\mathrm{C} 3$ & $-3,558142$ & $-4,631278$ & $-0,031672$ \\
\hline $\mathrm{C} 4$ & $-3,259085$ & $-2,680708$ & 1,363016 & $\mathrm{C} 4$ & $-3,270202$ & $-2,689578$ & 1,389504 \\
\hline $\mathrm{H} 5$ & $-5,212097$ & $-3,537685$ & 0,912211 & H5 & $-5,228525$ & $-3,523474$ & 0,915211 \\
\hline H6 & $-3,383710$ & $-4,738425$ & $-1,095097$ & H6 & $-3,403050$ & $-4,692145$ & $-1,113629$ \\
\hline $\mathrm{H} 7$ & $-3,660975$ & $-1,881134$ & 1,985466 & $\mathrm{H} 7$ & $-3,669331$ & $-1,901107$ & 2,027871 \\
\hline $\mathrm{H} 8$ & $-4,495254$ & $-2,689444$ & $-0,458966$ & H8 & $-4,502768$ & $-2,651283$ & $-0,435964$ \\
\hline H9 & $-1,643496$ & $-5,496912$ & 0,842171 & H9 & $-1,675084$ & $-5,506373$ & 0,812358 \\
\hline H10 & $-4,093285$ & $-5,538606$ & 0,307457 & H10 & $-4,123172$ & $-5,518561$ & 0,268003 \\
\hline $\mathrm{C} 11$ & $-2,649951$ & $-3,897644$ & 2,080542 & $\mathrm{C} 11$ & $-2,673588$ & $-3,926818$ & 2,082395 \\
\hline H12 & $-1,828602$ & $-3,632566$ & 2,753238 & H12 & $-1,851756$ & $-3,683603$ & 2,762510 \\
\hline H13 & $-3,390500$ & $-4,495283$ & 2,619450 & H13 & $-3,420330$ & $-4,530604$ & 2,605637 \\
\hline $\mathrm{C} 14$ & 0,096471 & $-3,389849$ & 0,460621 & $\mathrm{C} 14$ & 0,082356 & $-3,405084$ & 0,478186 \\
\hline N15 & 0,865908 & $-2,378601$ & 0,260947 & N15 & 0,862755 & $-2,404869$ & 0,270551 \\
\hline $\mathrm{C} 16$ & 2,199445 & $-2,697368$ & 0,881588 & $\mathrm{C} 16$ & 2,190204 & $-2,722838$ & 0,905151 \\
\hline $\mathrm{C} 17$ & 2,077814 & $-4,244128$ & 1,039371 & $\mathrm{C} 17$ & 2,056360 & $-4,265123$ & 1,082713 \\
\hline P18 & $-1,824999$ & $-0,694376$ & $-0,082670$ & P18 & $-1,826299$ & $-0,684601$ & $-0,026825$ \\
\hline Ir19 & 0,236056 & $-0,559728$ & $-0,859118$ & Ir19 & 0,290779 & $-0,602884$ & $-0,908234$ \\
\hline $\mathrm{C} 20$ & $-2,084959$ & 0,497509 & 1,271939 & $\mathrm{C} 20$ & $-2,068451$ & 0,505354 & 1,336931 \\
\hline $\mathrm{C} 21$ & $-2,226919$ & 2,416779 & 3,308323 & $\mathrm{C} 21$ & $-2,198114$ & 2,425606 & 3,381282 \\
\hline $\mathrm{C} 22$ & $-1,896915$ & 0,123948 & 2,609850 & $\mathrm{C} 22$ & $-1,894127$ & 0,128603 & 2,675438 \\
\hline
\end{tabular}




\begin{tabular}{|c|c|c|c|c|c|c|c|}
\hline $\mathrm{C} 23$ & $-2,353553$ & 1,841687 & 0,964297 & $\mathrm{C} 23$ & $-2,314294$ & 1,855004 & 1,032772 \\
\hline $\mathrm{C} 24$ & $-2,420865$ & 2,794458 & 1,977579 & $\mathrm{C} 24$ & $-2,375411$ & 2,806811 & 2,048745 \\
\hline $\mathrm{C} 25$ & $-1,973480$ & 1,080882 & 3,621945 & $\mathrm{C} 25$ & $-1,964516$ & 1,084622 & 3,690158 \\
\hline H26 & $-2,523263$ & 2,142083 & $-0,064254$ & $\mathrm{H} 26$ & $-2,476531$ & 2,161554 & 0,004224 \\
\hline $\mathrm{H} 27$ & $-2,631503$ & 3,830524 & 1,728353 & $\mathrm{H} 27$ & $-2,570916$ & 3,845823 & 1,799027 \\
\hline $\mathrm{H} 28$ & $-1,836113$ & 0,780624 & 4,656952 & $\mathrm{H} 28$ & $-1,839532$ & 0,777567 & 4,724818 \\
\hline H29 & $-2,282029$ & 3,160120 & 4,098355 & $\mathrm{H} 29$ & $-2,250083$ & 3,167201 & 4,172801 \\
\hline $\mathrm{O} 30$ & 0,641724 & $-4,482760$ & 1,005016 & $\mathrm{O} 30$ & 0,617049 & $-4,495101$ & 1,039013 \\
\hline C31 & 2,308524 & $-2,075141$ & 2,280275 & $\mathrm{C} 31$ & 2,298954 & $-2,074210$ & 2,291472 \\
\hline C32 & 1,320527 & $-1,250367$ & 2,812633 & $\mathrm{C} 32$ & 1,300584 & $-1,257340$ & 2,817166 \\
\hline $\mathrm{C} 33$ & 3,440156 & $-2,367598$ & 3,057120 & $\mathrm{C} 33$ & 3,435304 & $-2,342590$ & 3,070194 \\
\hline C34 & 1,459985 & $-0,713869$ & 4,093988 & $\mathrm{C} 34$ & 1,433107 & $-0,707105$ & 4,093958 \\
\hline H35 & 0,458170 & $-0,996558$ & 2,216348 & H35 & 0,432579 & $-1,023216$ & 2,221257 \\
\hline C36 & 3,580091 & $-1,835507$ & 4,335874 & $\mathrm{C} 36$ & 3,566831 & $-1,798518$ & 4,345432 \\
\hline H37 & 4,228411 & $-2,995874$ & 2,648334 & H37 & 4,230409 & $-2,966781$ & 2,667999 \\
\hline C38 & 2,587367 & $-1,002407$ & 4,858891 & $\mathrm{C} 38$ & 2,563092 & $-0,974446$ & 4,863718 \\
\hline H39 & 0,686438 & $-0,056312$ & 4,480808 & H39 & 0,649709 & $-0,057141$ & 4,473294 \\
\hline $\mathrm{H} 40$ & 4,465340 & $-2,065226$ & 4,921885 & $\mathrm{H} 40$ & 4,454885 & $-2,013548$ & 4,932838 \\
\hline H41 & 2,697921 & $-0,581277$ & 5,854073 & $\mathrm{H} 41$ & 2,667245 & $-0,545515$ & 5,855964 \\
\hline $\mathrm{C} 42$ & $-3,210043$ & $-0,449196$ & $-1,247607$ & $\mathrm{C} 42$ & $-3,224192$ & $-0,409461$ & $-1,173183$ \\
\hline $\mathrm{C} 43$ & $-4,421750$ & 0,117257 & $-0,820831$ & $\mathrm{C} 43$ & $-4,428471$ & 0,164547 & $-0,735699$ \\
\hline $\mathrm{C} 44$ & $-3,120075$ & $-0,991417$ & $-2,538353$ & C44 & $-3,147525$ & $-0,938432$ & $-2,470778$ \\
\hline $\mathrm{C} 45$ & $-5,518856$ & 0,151437 & $-1,680650$ & $\mathrm{C} 45$ & $-5,529848$ & 0,218861 & $-1,589255$ \\
\hline H46 & $-4,518632$ & 0,525051 & 0,179847 & $\mathrm{H} 46$ & $-4,513945$ & 0,566009 & 0,268841 \\
\hline $\mathrm{C} 47$ & $-4,218927$ & $-0,954936$ & $-3,391579$ & $\mathrm{C} 47$ & $-4,251325$ & $-0,885782$ & $-3,317873$ \\
\hline $\mathrm{C} 48$ & $-5,419161$ & $-0,382179$ & $-2,965790$ & $\mathrm{C} 48$ & $-5,443317$ & $-0,305435$ & $-2,879870$ \\
\hline H49 & $-6,451402$ & 0,594920 & $-1,343448$ & $\mathrm{H} 49$ & $-6,456037$ & 0,668890 & $-1,243264$ \\
\hline H50 & $-4,130626$ & $-1,356378$ & $-4,396163$ & H50 & $-4,171658$ & $-1,277735$ & $-4,327008$ \\
\hline H51 & $-6,273815$ & $-0,348704$ & $-3,635399$ & H51 & $-6,301924$ & $-0,258240$ & $-3,543636$ \\
\hline H52 & $-0,285583$ & $-1,454994$ & $-2,033056$ & H52 & $-0,294534$ & $-1,521870$ & $-2,040990$ \\
\hline H53 & 1,706015 & 1,610778 & $-2,985633$ & $\mathrm{H} 53$ & 1,763996 & 1,590537 & $-2,943609$ \\
\hline H54 & $-1,682918$ & $-0,909433$ & 2,860323 & $\mathrm{H} 54$ & $-1,695967$ & $-0,908064$ & 2,926061 \\
\hline H55 & $-2,182111$ & $-1,408102$ & $-2,885373$ & H55 & $-2,215490$ & $-1,358526$ & $-2,829946$ \\
\hline H56 & 2,523408 & $-4,812612$ & 0,219737 & H56 & 2,506177 & $-4,847656$ & 0,274816 \\
\hline $\mathrm{C} 57$ & 5,322285 & $-1,381730$ & $-1,837350$ & C57 & 5,085677 & $-1,290685$ & $-1,989207$ \\
\hline C58 & 4,550563 & $-2,505095$ & $-2,141787$ & $\mathrm{C} 58$ & 4,365455 & $-2,462661$ & $-2,232934$ \\
\hline C59 & 3,558603 & $-2,933160$ & $-1,260793$ & C59 & 3,478177 & $-2,950750$ & $-1,274716$ \\
\hline C60 & 3,325844 & $-2,255549$ & $-0,056020$ & C60 & 3,296110 & $-2,283494$ & $-0,055463$ \\
\hline C61 & 4,089838 & $-1,115445$ & 0,229904 & C61 & 4,018649 & $-1,106223$ & 0,178612 \\
\hline $\mathrm{C} 62$ & 5,083010 & $-0,686396$ & $-0,652575$ & C62 & 4,908890 & $-0,617425$ & $-0,780203$ \\
\hline H63 & 6,101058 & $-1,053006$ & $-2,519618$ & $\mathrm{H} 63$ & 5,779530 & $-0,910950$ & $-2,733818$ \\
\hline H64 & 4,719279 & $-3,048248$ & $-3,067044$ & $\mathrm{H} 64$ & 4,491000 & $-2,994848$ & $-3,171472$ \\
\hline H65 & 2,950531 & $-3,789222$ & $-1,536703$ & H65 & 2,897527 & $-3,838370$ & $-1,507120$ \\
\hline H66 & 3,907818 & $-0,555762$ & 1,139656 & H66 & 3,871881 & $-0,555842$ & 1,100738 \\
\hline H67 & 5,673313 & 0,192458 & $-0,406019$ & H67 & 5,470335 & 0,290381 & $-0,574921$ \\
\hline C68 & 0,056245 & 5,720461 & $-1,875603$ & C68 & $-0,007559$ & 5,679008 & $-1,921578$ \\
\hline C69 & 0,451845 & 4,430427 & $-1,527431$ & C69 & 0,402257 & 4,399047 & $-1,553533$ \\
\hline $\mathrm{C} 70$ & 0,859849 & 3,522664 & $-2,513347$ & $\mathrm{C} 70$ & 0,868320 & 3,497423 & $-2,519195$ \\
\hline
\end{tabular}




\begin{tabular}{|c|c|c|c|c|c|c|c|}
\hline C71 & 0,867548 & 3,925583 & $-3,850280$ & C71 & 0,919291 & 3,896545 & $-3,856210$ \\
\hline $\mathrm{C} 72$ & 0,466656 & 5,215663 & $-4,201249$ & $\mathrm{C} 72$ & 0,503957 & 5,176289 & $-4,227463$ \\
\hline $\mathrm{C} 73$ & 0,060692 & 6,115170 & $-3,215827$ & C73 & 0,040349 & 6,069650 & $-3,262004$ \\
\hline H74 & $-0,257728$ & 6,418251 & $-1,104072$ & H74 & $-0,367381$ & 6,371656 & $-1,165596$ \\
\hline H75 & 0,438038 & 4,120882 & $-0,484364$ & $\mathrm{H} 75$ & 0,352360 & 4,091932 & $-0,510894$ \\
\hline H76 & 1,159995 & 3,218602 & $-4,622255$ & H76 & 1,257146 & 3,194005 & $-4,613583$ \\
\hline H77 & 0,467710 & 5,515538 & $-5,245243$ & H77 & 0,539133 & 5,473071 & $-5,271747$ \\
\hline H78 & $-0,251885$ & 7,119000 & $-3,488865$ & $\mathrm{H} 78$ & $-0,283347$ & 7,065526 & $-3,550919$ \\
\hline C79 & 1,208645 & 2,088125 & $-2,132571$ & C79 & 1,220196 & 2,069598 & $-2,119000$ \\
\hline $\mathrm{C} 80$ & $-0,106432$ & 1,303807 & $-1,804859$ & $\mathrm{C} 80$ & $-0,087803$ & 1,265586 & $-1,841775$ \\
\hline C81 & 3,457737 & 2,838283 & $-1,164236$ & $\mathrm{C} 81$ & 3,406461 & 2,860647 & $-1,044793$ \\
\hline $\mathrm{C} 82$ & 2,084958 & 1,572292 & 0,197177 & $\mathrm{C} 82$ & 1,983023 & 1,586924 & 0,259823 \\
\hline C83 & 3,843637 & 3,012860 & 0,311323 & C83 & 3,717419 & 3,049666 & 0,446996 \\
\hline H84 & 3,315333 & 3,789740 & $-1,680429$ & H84 & 3,268576 & 3,807599 & $-1,570635$ \\
\hline H85 & 4,175844 & 2,225938 & $-1,724570$ & H85 & 4,163832 & 2,264574 & $-1,568998$ \\
\hline H86 & 4,901370 & 2,850268 & 0,519921 & H86 & 4,765716 & 2,902755 & 0,708502 \\
\hline H87 & 3,531753 & 3,983902 & 0,708714 & H87 & 3,374942 & 4,019817 & 0,820717 \\
\hline N88 & 2,187114 & 2,121613 & $-1,028658$ & N88 & 2,146509 & 2,117464 & $-0,968749$ \\
\hline O89 & 3,087374 & 1,980393 & 0,999836 & O89 & 2,941710 & 2,013081 & 1,106138 \\
\hline O90 & 1,249433 & 0,756181 & 0,617528 & O90 & 1,128129 & 0,776668 & 0,650839 \\
\hline H91 & 2,455710 & $-4,608715$ & 1,992641 & H91 & 2,422879 & $-4,619229$ & 2,044333 \\
\hline N92 & $-2,049495$ & $-2,214234$ & 0,622235 & N92 & $-2,055513$ & $-2,213198$ & 0,665236 \\
\hline C93 & $-1,367268$ & $-3,426609$ & 0,105935 & C93 & $-1,382088$ & $-3,422757$ & 0,122587 \\
\hline H94 & $-1,416899$ & $-3,494665$ & $-0,990914$ & H94 & $-1,431564$ & $-3,465963$ & $-0,975108$ \\
\hline C95 & $-2,496480$ & 1,482293 & $-5,406655$ & C95 & $-2,423782$ & 1,440246 & $-5,479051$ \\
\hline C96 & $-2,839568$ & 2,252882 & $-4,295885$ & C96 & $-2,783083$ & 2,205633 & $-4,369748$ \\
\hline C97 & $-2,070604$ & 2,186262 & $-3,135093$ & C97 & $-2,030378$ & 2,134208 & $-3,199113$ \\
\hline C98 & $-0,961685$ & 1,335117 & $-3,047745$ & C98 & $-0,916780$ & 1,289464 & $-3,101544$ \\
\hline C99 & $-0,623095$ & 0,573673 & $-4,175502$ & C99 & $-0,565161$ & 0,529594 & $-4,225935$ \\
\hline $\mathrm{C} 100$ & $-1,379427$ & 0,645499 & $-5,344056$ & $\mathrm{C} 100$ & $-1,307354$ & 0,603594 & $-5,403896$ \\
\hline H101 & $-3,090263$ & 1,535550 & $-6,314909$ & H101 & $-3,005541$ & 1,495559 & $-6,395040$ \\
\hline H102 & $-3,701741$ & 2,913017 & $-4,334669$ & H102 & $-3,647464$ & 2,862370 & $-4,416209$ \\
\hline H103 & $-2,322783$ & 2,821537 & $-2,289726$ & H103 & $-2,300711$ & 2,757683 & $-2,350660$ \\
\hline H104 & 0,231913 & $-0,096415$ & $-4,122521$ & H104 & 0,289632 & $-0,140033$ & $-4,167529$ \\
\hline H105 & $-1,100943$ & 0,045596 & $-6,206661$ & H105 & $-1,017951$ & 0,005467 & $-6,264103$ \\
\hline H106 & $-0,597482$ & 1,920986 & $-1,045311$ & H106 & $-0,615783$ & 1,854357 & $-1,085278$ \\
\hline H107 & 2,532304 & $-0,352007$ & $-1,755555$ & H107 & 2,027015 & $-0,529438$ & $-1,430504$ \\
\hline H108 & 2,514286 & $-0,506386$ & $-2,487906$ & H108 & 1,606264 & $-0,798939$ & $-2,085190$ \\
\hline \multicolumn{4}{|c|}{ C1_ES_Cheltop_TS3 } & \multicolumn{4}{|c|}{ C1_ES_Cheltop_PROD } \\
\hline $\mathrm{C} 1$ & $-4,312000$ & $-3,322437$ & 0,506855 & $\mathrm{C} 1$ & $-4,286520$ & $-3,433255$ & 0,557377 \\
\hline $\mathrm{C} 2$ & $-2,228695$ & $-4,536352$ & 0,867410 & $\mathrm{C} 2$ & $-2,187377$ & $-4,581184$ & 1,034519 \\
\hline $\mathrm{C} 3$ & $-3,570544$ & $-4,638056$ & 0,098770 & $\mathrm{C} 3$ & $-3,508585$ & $-4,754013$ & 0,241444 \\
\hline $\mathrm{C} 4$ & $-3,294897$ & $-2,656585$ & 1,464824 & $\mathrm{C} 4$ & $-3,314131$ & $-2,702360$ & 1,513435 \\
\hline H5 & $-5,246358$ & $-3,532051$ & 1,037909 & H5 & $-5,238264$ & $-3,633237$ & 1,060540 \\
\hline H6 & $-3,422934$ & $-4,722414$ & $-0,982623$ & H6 & $-3,334590$ & $-4,898461$ & $-0,829603$ \\
\hline H7 & $-3,695770$ & $-1,854008$ & 2,084342 & $\mathrm{H} 7$ & $-3,750533$ & $-1,881319$ & 2,082874 \\
\hline $\mathrm{H} 8$ & $-4,550559$ & $-2,683811$ & $-0,344254$ & $\mathrm{H} 8$ & $-4,499326$ & $-2,836608$ & $-0,331551$ \\
\hline
\end{tabular}




\begin{tabular}{|c|c|c|c|c|c|c|c|}
\hline H9 & $-1,671929$ & $-5,469413$ & 0,950086 & H9 & $-1,611043$ & $-5,495292$ & 1,176370 \\
\hline H10 & $-4,120002$ & $-5,526019$ & 0,424192 & $\mathrm{H} 10$ & $-4,048363$ & $-5,632590$ & 0,606416 \\
\hline $\mathrm{C} 11$ & $-2,676873$ & $-3,866879$ & 2,186180 & $\mathrm{C} 11$ & $-2,686442$ & $-3,861136$ & 2,307694 \\
\hline H12 & $-1,852297$ & $-3,595053$ & 2,851985 & H12 & $-1,885748$ & $-3,536658$ & 2,978804 \\
\hline H13 & $-3,410744$ & $-4,465824$ & 2,732755 & $\mathrm{H} 13$ & $-3,419062$ & $-4,452393$ & 2,864468 \\
\hline $\mathrm{C} 14$ & 0,052693 & $-3,345789$ & 0,563420 & C14 & 0,072331 & $-3,345417$ & 0,700789 \\
\hline N15 & 0,844791 & $-2,353677$ & 0,325540 & N15 & 0,866858 & $-2,381923$ & 0,356428 \\
\hline $\mathrm{C} 16$ & 2,161971 & $-2,643977$ & 1,012910 & $\mathrm{C} 16$ & 2,183249 & $-2,573539$ & 1,092725 \\
\hline $\mathrm{C} 17$ & 2,011777 & $-4,172752$ & 1,260762 & $\mathrm{C} 17$ & 2,035995 & $-4,068791$ & 1,495299 \\
\hline P18 & $-1,863207$ & $-0,693989$ & $-0,053029$ & P18 & $-1,851121$ & $-0,775982$ & $-0,038358$ \\
\hline $\operatorname{Ir} 19$ & 0,402377 & $-0,702897$ & $-0,978802$ & Ir19 & 0,389529 & $-0,865133$ & $-0,935307$ \\
\hline $\mathrm{C} 20$ & $-2,148262$ & 0,551585 & 1,251311 & $\mathrm{C} 20$ & $-2,152066$ & 0,511704 & 1,226906 \\
\hline $\mathrm{C} 21$ & $-2,299444$ & 2,580513 & 3,186648 & $\mathrm{C} 21$ & $-2,357452$ & 2,602339 & 3,092542 \\
\hline $\mathrm{C} 22$ & $-1,972204$ & 0,251648 & 2,609521 & $\mathrm{C} 22$ & $-2,056612$ & 0,249776 & 2,600108 \\
\hline $\mathrm{C} 23$ & $-2,408299$ & 1,880569 & 0,875739 & $\mathrm{C} 23$ & $-2,353195$ & 1,835586 & 0,800672 \\
\hline $\mathrm{C} 24$ & $-2,477294$ & 2,887660 & 1,836430 & $\mathrm{C} 24$ & $-2,447932$ & 2,873197 & 1,725296 \\
\hline $\mathrm{C} 25$ & $-2,055969$ & 1,260839 & 3,569479 & $\mathrm{C} 25$ & $-2,169428$ & 1,289336 & 3,525454 \\
\hline $\mathrm{H} 26$ & $-2,588091$ & 2,124018 & $-0,165777$ & $\mathrm{H} 26$ & $-2,468080$ & 2,053697 & $-0,256712$ \\
\hline $\mathrm{H} 27$ & $-2,682228$ & 3,909477 & 1,529525 & $\mathrm{H} 27$ & $-2,605738$ & 3,890634 & 1,377624 \\
\hline $\mathrm{H} 28$ & $-1,933705$ & 1,012924 & 4,620263 & $\mathrm{H} 28$ & $-2,109447$ & 1,070034 & 4,588038 \\
\hline $\mathrm{H} 29$ & $-2,360386$ & 3,363316 & 3,937030 & $\mathrm{H} 29$ & $-2,440473$ & 3,408565 & 3,815740 \\
\hline $\mathrm{O} 30$ & 0,573706 & $-4,398031$ & 1,190802 & $\mathrm{O} 30$ & 0,599225 & $-4,306768$ & 1,456946 \\
\hline $\mathrm{C} 31$ & 2,218164 & $-1,937711$ & 2,374916 & $\mathrm{C} 31$ & 2,215711 & $-1,731852$ & 2,374649 \\
\hline C32 & 1,211768 & $-1,085321$ & 2,823121 & $\mathrm{C} 32$ & 1,189879 & $-0,864957$ & 2,743461 \\
\hline C33 & 3,312842 & $-2,192426$ & 3,215421 & $\mathrm{C} 33$ & 3,308338 & $-1,893796$ & 3,241824 \\
\hline C34 & 1,299150 & $-0,483021$ & 4,079589 & $\mathrm{C} 34$ & 1,256822 & $-0,157227$ & 3,944959 \\
\hline H35 & 0,371162 & $-0,864556$ & 2,184224 & H35 & 0,344884 & $-0,715349$ & 2,089839 \\
\hline C36 & 3,400845 & $-1,595141$ & 4,469450 & $\mathrm{C} 36$ & 3,375585 & $-1,191504$ & 4,441489 \\
\hline H37 & 4,113106 & $-2,845885$ & 2,877271 & H37 & 4,121090 & $-2,561750$ & 2,966345 \\
\hline C38 & 2,391752 & $-0,732827$ & 4,905694 & $\mathrm{C} 38$ & 2,346653 & $-0,315376$ & 4,796618 \\
\hline H39 & 0,511703 & 0,193761 & 4,398184 & H39 & 0,452293 & 0,525520 & 4,200986 \\
\hline $\mathrm{H} 40$ & 4,259135 & $-1,797705$ & 5,103492 & $\mathrm{H} 40$ & 4,231366 & $-1,325717$ & 5,096986 \\
\hline H41 & 2,461736 & $-0,260851$ & 5,881514 & $\mathrm{H} 41$ & 2,398520 & 0,236985 & 5,730655 \\
\hline $\mathrm{C} 42$ & $-3,257445$ & $-0,480523$ & $-1,219502$ & $\mathrm{C} 42$ & $-3,218802$ & $-0,524706$ & $-1,233028$ \\
\hline $\mathrm{C} 43$ & $-4,490595$ & 0,061757 & $-0,824146$ & $\mathrm{C} 43$ & $-4,468126$ & $-0,011273$ & $-0,846436$ \\
\hline $\mathrm{C} 44$ & $-3,124688$ & $-1,010665$ & $-2,511487$ & $\mathrm{C} 44$ & $-3,028362$ & $-0,929162$ & $-2,562703$ \\
\hline $\mathrm{C} 45$ & $-5,565868$ & 0,080777 & $-1,711153$ & $\mathrm{C} 45$ & $-5,501289$ & 0,098967 & $-1,774729$ \\
\hline H46 & $-4,615026$ & 0,469342 & 0,174215 & $\mathrm{H} 46$ & $-4,632543$ & 0,310626 & 0,177587 \\
\hline $\mathrm{C} 47$ & $-4,202763$ & $-0,993441$ & $-3,393732$ & $\mathrm{C} 47$ & $-4,064628$ & $-0,819468$ & $-3,489327$ \\
\hline $\mathrm{C} 48$ & $-5,423972$ & $-0,446724$ & $-2,996077$ & $\mathrm{C} 48$ & $-5,300511$ & $-0,304041$ & $-3,097447$ \\
\hline H49 & $-6,515332$ & 0,505397 & $-1,397252$ & $\mathrm{H} 49$ & $-6,462844$ & 0,500076 & $-1,466574$ \\
\hline H50 & $-4,083037$ & $-1,390586$ & $-4,397037$ & $\mathrm{H} 50$ & $-3,898226$ & $-1,116038$ & $-4,520379$ \\
\hline H51 & $-6,262784$ & $-0,428759$ & $-3,686038$ & $\mathrm{H} 51$ & $-6,105841$ & $-0,211355$ & $-3,820714$ \\
\hline H52 & $-0,120143$ & $-1,747571$ & $-2,020912$ & $\mathrm{H} 52$ & $-0,045028$ & $-1,974537$ & $-1,943550$ \\
\hline H53 & 1,705924 & 1,797545 & $-3,103234$ & H53 & 1,637235 & 2,100797 & $-3,373237$ \\
\hline H54 & $-1,764667$ & $-0,767448$ & 2,918045 & H54 & $-1,881583$ & $-0,762643$ & 2,948867 \\
\hline H55 & $-2,169271$ & $-1,409881$ & $-2,833806$ & $\mathrm{H} 55$ & $-2,061142$ & $-1,308618$ & $-2,876142$ \\
\hline H56 & 2,477237 & $-4,795680$ & 0,493493 & H56 & 2,499788 & $-4,761899$ & 0,789564 \\
\hline
\end{tabular}




\begin{tabular}{|c|c|c|c|c|c|c|c|}
\hline C57 & 5,328281 & $-1,524135$ & $-1,731502$ & $\mathrm{C} 57$ & 5,398528 & $-1,813733$ & $-1,714134$ \\
\hline C58 & 4,529719 & $-2,639708$ & $-1,991381$ & $\mathrm{C} 58$ & 4,541385 & $-2,901847$ & $-1,893010$ \\
\hline C59 & 3,539230 & $-3,015127$ & $-1,086898$ & C59 & 3,530358 & $-3,151417$ & $-0,969517$ \\
\hline $\mathrm{C} 60$ & 3,331218 & $-2,289797$ & 0,094669 & $\mathrm{C} 60$ & 3,358892 & $-2,326012$ & 0,150235 \\
\hline C61 & 4,131181 & $-1,169197$ & 0,343319 & C61 & 4,212202 & $-1,231545$ & 0,315276 \\
\hline C62 & 5,122561 & $-0,791264$ & $-0,563076$ & C62 & 5,227107 & $-0,980674$ & $-0,609655$ \\
\hline H63 & 6,103613 & $-1,232088$ & $-2,434233$ & H63 & 6,192095 & $-1,620250$ & $-2,430599$ \\
\hline H64 & 4,673179 & $-3,214017$ & $-2,901951$ & H64 & 4,655580 & $-3,551625$ & $-2,755808$ \\
\hline H65 & 2,898854 & $-3,856806$ & $-1,334187$ & H65 & 2,844935 & $-3,973751$ & $-1,153281$ \\
\hline H66 & 3,969086 & $-0,572690$ & 1,232134 & H66 & 4,076826 & $-0,558155$ & 1,151787 \\
\hline H67 & 5,739420 & 0,077740 & $-0,348227$ & H67 & 5,891424 & $-0,133400$ & $-0,458319$ \\
\hline C68 & $-0,068364$ & 5,672034 & $-1,450077$ & C68 & $-0,218971$ & 5,476803 & $-0,903713$ \\
\hline C69 & 0,398999 & 4,371235 & $-1,272778$ & C69 & 0,258692 & 4,173788 & $-1,022535$ \\
\hline $\mathrm{C} 70$ & 0,811268 & 3,612060 & $-2,375447$ & $\mathrm{C} 70$ & 0,731629 & 3,705884 & $-2,257196$ \\
\hline C71 & 0,754237 & 4,171296 & $-3,653329$ & C71 & 0,721064 & 4,555210 & $-3,364454$ \\
\hline $\mathrm{C} 72$ & 0,284924 & 5,473545 & $-3,832018$ & $\mathrm{C} 72$ & 0,242057 & 5,861763 & $-3,244811$ \\
\hline $\mathrm{C} 73$ & $-0,128190$ & 6,224991 & $-2,731954$ & C73 & $-0,228814$ & 6,323424 & $-2,016294$ \\
\hline H74 & $-0,387405$ & 6,255175 & $-0,590556$ & H74 & $-0,583701$ & 5,833480 & 0,055783 \\
\hline H75 & 0,434376 & 3,938545 & $-0,275232$ & H75 & 0,251277 & 3,516648 & $-0,155635$ \\
\hline H76 & 1,054214 & 3,578804 & $-4,513925$ & H76 & 1,070741 & 4,190695 & $-4,327226$ \\
\hline H77 & 0,238452 & 5,898302 & $-4,830549$ & H77 & 0,235075 & 6,515816 & $-4,111856$ \\
\hline H78 & $-0,495151$ & 7,237846 & $-2,870978$ & $\mathrm{H} 78$ & $-0,602093$ & 7,339284 & $-1,923378$ \\
\hline $\mathrm{C} 79$ & 1,234331 & 2,160616 & $-2,180669$ & C79 & 1,202844 & 2,260438 & $-2,380135$ \\
\hline $\mathrm{C} 80$ & $-0,028667$ & 1,299995 & $-1,888564$ & $\mathrm{C} 80$ & $-0,023708$ & 1,324928 & $-2,245622$ \\
\hline C81 & 3,535771 & 2,779196 & $-1,281010$ & $\mathrm{C} 81$ & 3,589418 & 2,629000 & $-1,625785$ \\
\hline $\mathrm{C} 82$ & 2,163181 & 1,512559 & 0,089121 & $\mathrm{C} 82$ & 2,171248 & 1,509691 & $-0,161698$ \\
\hline $\mathrm{C} 83$ & 3,981630 & 2,883763 & 0,185494 & $\mathrm{C} 83$ & 4,134573 & 2,679300 & $-0,186041$ \\
\hline H84 & 3,400429 & 3,756041 & $-1,751579$ & H84 & 3,475853 & 3,625654 & $-2,062515$ \\
\hline H85 & 4,215492 & 2,172963 & $-1,892436$ & H85 & 4,201226 & 2,010446 & $-2,292167$ \\
\hline H86 & 5,037977 & 2,666304 & 0,345386 & H86 & 5,156227 & 2,309536 & $-0,093192$ \\
\hline H87 & 3,731636 & 3,853531 & 0,627148 & H87 & 4,061088 & 3,676941 & 0,256763 \\
\hline N88 & 2,252544 & 2,093131 & $-1,124848$ & N88 & 2,282584 & 2,003428 & $-1,416785$ \\
\hline O89 & 3,205303 & 1,864740 & 0,868029 & O89 & 3,268966 & 1,791633 & 0,564729 \\
\hline O90 & 1,293346 & 0,744768 & 0,522096 & O90 & 1,234611 & 0,872205 & 0,334947 \\
\hline H91 & 2,350490 & $-4,481807$ & 2,247540 & H91 & 2,381899 & $-4,274971$ & 2,506027 \\
\hline N92 & $-2,095817$ & $-2,186579$ & 0,714740 & N92 & $-2,112337$ & $-2,240940$ & 0,768604 \\
\hline C93 & $-1,409533$ & $-3,399006$ & 0,201883 & C93 & $-1,384040$ & $-3,457603$ & 0,327998 \\
\hline H94 & $-1,459573$ & $-3,478009$ & $-0,893623$ & H94 & $-1,414827$ & $-3,590080$ & $-0,762850$ \\
\hline C95 & $-2,728348$ & 1,712190 & $-5,232967$ & C95 & $-2,760058$ & 1,970142 & $-5,510160$ \\
\hline C96 & $-2,942639$ & 2,430669 & $-4,056686$ & C96 & $-2,965818$ & 2,608997 & $-4,286439$ \\
\hline C97 & $-2,073366$ & 2,278924 & $-2,979099$ & C97 & $-2,085697$ & 2,390993 & $-3,229156$ \\
\hline C98 & $-0,988349$ & 1,395038 & $-3,047184$ & C98 & $-0,996605$ & 1,526163 & $-3,381610$ \\
\hline C99 & $-0,782374$ & 0,681325 & $-4,234254$ & C99 & $-0,791615$ & 0,892765 & $-4,611354$ \\
\hline $\mathrm{C} 100$ & $-1,641106$ & 0,840875 & $-5,320824$ & C100 & $-1,669485$ & 1,113286 & $-5,672393$ \\
\hline H101 & $-3,404243$ & 1,828893 & $-6,075306$ & H101 & $-3,447105$ & 2,139060 & $-6,334508$ \\
\hline H102 & $-3,782996$ & 3,114304 & $-3,978144$ & H102 & $-3,811668$ & 3,277507 & $-4,154591$ \\
\hline H103 & $-2,218713$ & 2,879667 & $-2,086619$ & H103 & $-2,232506$ & 2,911938 & $-2,287743$ \\
\hline H104 & 0,042985 & $-0,024426$ & $-4,302397$ & H104 & 0,050277 & 0,213973 & $-4,734993$ \\
\hline
\end{tabular}




$\begin{array}{rrrrrrrr}\text { H105 } & -1,467376 & 0,277014 & -6,233173 & \text { H105 } & -1,505450 & 0,613999 & -6,623100 \\ \text { H106 } & -0,488911 & 1,729658 & -0,998898 & \text { H106 } & -0,507002 & 1,525374 & -1,295915 \\ \text { H107 } & 1,900232 & -0,915987 & -1,512540 & \text { H107 } & 1,854834 & -1,015969 & -1,578418 \\ \text { H108 } & 0,778736 & -0,055882 & -2,351456 & \text { H108 } & 0,372212 & 0,246418 & -2,390326\end{array}$

\section{C1_Z_S_tN_Chelbot}

C1_ZS_Chelbot_SM

\begin{tabular}{|c|c|c|c|c|c|c|c|}
\hline $\mathrm{C} 1$ & $-3,834726$ & $-3,440265$ & $-1,131187$ & $\mathrm{C} 1$ & $-3,839587$ & $-3,515745$ & $-1,066689$ \\
\hline $\mathrm{C} 2$ & $-1,572816$ & $-4,361917$ & $-1,123160$ & $\mathrm{C} 2$ & $-1,575933$ & $-4,433007$ & $-1,087895$ \\
\hline C3 & $-2,812893$ & $-4,200243$ & $-2,041485$ & $\mathrm{C} 3$ & $-2,829196$ & $-4,276061$ & $-1,989470$ \\
\hline $\mathrm{C} 4$ & $-3,021692$ & $-3,235666$ & 0,165912 & $\mathrm{C} 4$ & $-3,013826$ & $-3,318454$ & 0,223940 \\
\hline H5 & $-4,724173$ & $-4,045921$ & $-0,928194$ & H5 & $-4,729740$ & $-4,118214$ & $-0,857936$ \\
\hline H6 & $-2,575031$ & $-3,653776$ & $-2,959722$ & H6 & $-2,605583$ & $-3,731558$ & $-2,912478$ \\
\hline $\mathrm{H} 7$ & $-3,602548$ & $-2,928361$ & 1,036048 & $\mathrm{H} 7$ & $-3,587875$ & $-3,018608$ & 1,101266 \\
\hline H8 & $-4,166707$ & $-2,490636$ & $-1,552410$ & $\mathrm{H} 8$ & $-4,171427$ & $-2,562988$ & $-1,481519$ \\
\hline H9 & $-0,861328$ & $-5,124834$ & $-1,439320$ & H9 & $-0,863987$ & $-5,190538$ & $-1,415702$ \\
\hline H10 & $-3,191657$ & $-5,183073$ & $-2,335190$ & $\mathrm{H} 10$ & $-3,210727$ & $-5,260226$ & $-2,275194$ \\
\hline $\mathrm{C} 11$ & $-2,229218$ & $-4,551012$ & 0,263373 & $\mathrm{C} 11$ & $-2,214637$ & $-4,630962$ & 0,305835 \\
\hline H12 & $-1,512414$ & $-4,571388$ & 1,089879 & H12 & $-1,488869$ & $-4,648383$ & 1,124608 \\
\hline H13 & $-2,868602$ & $-5,436774$ & 0,313151 & H13 & $-2,848735$ & $-5,520335$ & 0,359032 \\
\hline $\mathrm{C} 14$ & 0,434905 & $-2,948415$ & $-0,321957$ & C14 & 0,444761 & $-2,993733$ & $-0,339298$ \\
\hline N15 & 1,051812 & $-1,877563$ & 0,044736 & N15 & 1,042912 & $-1,913942$ & 0,029475 \\
\hline $\mathrm{C} 16$ & 2,422087 & $-2,262006$ & 0,566694 & C16 & 2,428546 & $-2,265621$ & 0,510805 \\
\hline $\mathrm{C} 17$ & 2,271672 & $-3,816161$ & 0,630724 & $\mathrm{C} 17$ & 2,340385 & $-3,828621$ & 0,520510 \\
\hline P18 & $-1,808214$ & $-0,647503$ & 0,317043 & P18 & $-1,807734$ & $-0,724541$ & 0,353267 \\
\hline Ir19 & 0,421412 & 0,131843 & $-0,397577$ & $\operatorname{Ir} 19$ & 0,315968 & 0,087960 & $-0,358562$ \\
\hline $\mathrm{C} 20$ & $-1,965545$ & $-0,540345$ & 2,136538 & $\mathrm{C} 20$ & $-1,961349$ & $-0,571470$ & 2,168046 \\
\hline $\mathrm{C} 21$ & $-2,002193$ & $-0,281379$ & 4,929058 & $\mathrm{C} 21$ & $-1,982061$ & $-0,221112$ & 4,945809 \\
\hline $\mathrm{C} 22$ & $-1,928253$ & $-1,677905$ & 2,953141 & $\mathrm{C} 22$ & $-1,950994$ & $-1,683645$ & 3,017349 \\
\hline $\mathrm{C} 23$ & $-2,000260$ & 0,731780 & 2,732831 & $\mathrm{C} 23$ & $-1,960295$ & 0,721821 & 2,719103 \\
\hline $\mathrm{C} 24$ & $-2,023294$ & 0,857373 & 4,119459 & $\mathrm{C} 24$ & $-1,975636$ & 0,892298 & 4,100488 \\
\hline $\mathrm{C} 25$ & $-1,948875$ & $-1,546283$ & 4,343295 & $\mathrm{C} 25$ & $-1,964036$ & $-1,505812$ & 4,402952 \\
\hline H26 & $-2,004269$ & 1,625138 & 2,120603 & $\mathrm{H} 26$ & $-1,936613$ & 1,594132 & 2,074645 \\
\hline $\mathrm{H} 27$ & $-2,051464$ & 1,847789 & 4,564783 & $\mathrm{H} 27$ & $-1,974690$ & 1,896473 & 4,514620 \\
\hline $\mathrm{H} 28$ & $-1,917082$ & $-2,434786$ & 4,967306 & $\mathrm{H} 28$ & $-1,953283$ & $-2,373454$ & 5,056256 \\
\hline H29 & $-2,019406$ & $-0,182267$ & 6,010711 & $\mathrm{H} 29$ & $-1,991991$ & $-0,086167$ & 6,023621 \\
\hline $\mathrm{O} 30$ & 1,053825 & $-4,111652$ & $-0,113937$ & $\mathrm{O} 30$ & 1,105194 & $-4,144028$ & $-0,186793$ \\
\hline C31 & 2,677903 & $-1,750921$ & 1,979388 & $\mathrm{C} 31$ & 2,692664 & $-1,795814$ & 1,938142 \\
\hline C32 & 1,626172 & $-1,347827$ & 2,807157 & $\mathrm{C} 32$ & 1,649040 & $-1,420832$ & 2,788501 \\
\hline $\mathrm{C} 33$ & 3,971263 & $-1,819684$ & 2,517085 & $\mathrm{C} 33$ & 3,993903 & $-1,870573$ & 2,455769 \\
\hline $\mathrm{C} 34$ & 1,865360 & $-0,967490$ & 4,128013 & C34 & 1,904650 & $-1,072818$ & 4,115443 \\
\hline H35 & 0,614971 & $-1,322988$ & 2,426435 & H35 & 0,632755 & $-1,394040$ & 2,420868 \\
\hline C36 & 4,210325 & $-1,444381$ & 3,838607 & $\mathrm{C} 36$ & 4,248527 & $-1,530636$ & 3,783406 \\
\hline H37 & 4,798944 & $-2,155090$ & 1,899054 & H37 & 4,814654 & $-2,182067$ & 1,815950 \\
\hline C38 & 3,159461 & $-1,005775$ & 4,646024 & C 38 & 3,205636 & $-1,118599$ & 4,614962 \\
\hline H39 & 1,030845 & $-0,645326$ & 4,744237 & H39 & 1,077311 & $-0,770098$ & 4,750946 \\
\hline $\mathrm{H} 40$ & 5,220649 & $-1,492080$ & 4,234845 & $\mathrm{H} 40$ & 5,264009 & $-1,583484$ & 4,165411 \\
\hline H41 & 3,349343 & $-0,704970$ & 5,672467 & $\mathrm{H} 41$ & 3,407305 & $-0,844242$ & 5,646437 \\
\hline
\end{tabular}

C1_ZS_Chelbot_TS1 


\begin{tabular}{|c|c|c|c|c|c|c|c|}
\hline $\mathrm{C} 42$ & $-3,362171$ & 0,083848 & $-0,344786$ & $\mathrm{C} 42$ & $-3,342526$ & 0,019026 & $-0,328493$ \\
\hline $\mathrm{C} 43$ & $-4,366195$ & 0,644260 & 0,453989 & $\mathrm{C} 43$ & $-4,345788$ & 0,597627 & 0,457650 \\
\hline $\mathrm{C} 44$ & $-3,536228$ & 0,060501 & $-1,739925$ & C44 & $-3,502392$ & $-0,017791$ & $-1,725030$ \\
\hline $\mathrm{C} 45$ & $-5,505322$ & 1,202015 & $-0,132190$ & $\mathrm{C} 45$ & $-5,478335$ & 1,151405 & $-0,143700$ \\
\hline H46 & $-4,264363$ & 0,659166 & 1,533363 & $\mathrm{H} 46$ & $-4,249346$ & 0,626495 & 1,537248 \\
\hline $\mathrm{C} 47$ & $-4,670570$ & 0,622039 & $-2,322033$ & $\mathrm{C} 47$ & $-4,629942$ & 0,540329 & $-2,321803$ \\
\hline $\mathrm{C} 48$ & $-5,655535$ & 1,207577 & $-1,518702$ & $\mathrm{C} 48$ & $-5,619089$ & 1,134090 & $-1,530653$ \\
\hline H49 & $-6,275271$ & 1,633349 & 0,501262 & H49 & $-6,250610$ & 1,596775 & 0,476966 \\
\hline H50 & $-4,793466$ & 0,590078 & $-3,401353$ & $\mathrm{H} 50$ & $-4,744319$ & 0,500418 & $-3,401749$ \\
\hline H51 & $-6,540186$ & 1,646208 & $-1,970806$ & H51 & $-6,499687$ & 1,569145 & $-1,994428$ \\
\hline H52 & 0,808324 & 0,517362 & 1,054024 & H52 & 0,654856 & 0,462048 & 1,110068 \\
\hline H53 & 1,980098 & 0,209429 & $-0,675304$ & $\mathrm{H} 53$ & 1,879183 & 0,443791 & $-0,659854$ \\
\hline H54 & $-1,853471$ & $-2,665274$ & 2,511439 & $\mathrm{H} 54$ & $-1,902489$ & $-2,685759$ & 2,605304 \\
\hline H55 & $-2,793125$ & $-0,417423$ & $-2,369373$ & $\mathrm{H} 55$ & $-2,752396$ & $-0,500417$ & $-2,342400$ \\
\hline H56 & 3,097424 & $-4,358712$ & 0,171070 & $\mathrm{H} 56$ & 3,166532 & $-4,322317$ & 0,009072 \\
\hline $\mathrm{C} 57$ & 5,131769 & $-0,895235$ & $-2,541755$ & C57 & 5,106027 & $-0,719182$ & $-2,541423$ \\
\hline C58 & 4,366425 & $-2,048685$ & $-2,720289$ & C58 & 4,360659 & $-1,876818$ & $-2,768696$ \\
\hline C59 & 3,527259 & $-2,496641$ & $-1,700877$ & C59 & 3,519888 & $-2,373741$ & $-1,773715$ \\
\hline C60 & 3,442335 & $-1,807318$ & $-0,483315$ & C60 & 3,415804 & $-1,730493$ & $-0,532612$ \\
\hline C61 & 4,201725 & $-0,639806$ & $-0,323560$ & C61 & 4,145923 & $-0,552070$ & $-0,327148$ \\
\hline C62 & 5,043052 & $-0,191348$ & $-1,341975$ & C62 & 4,989452 & $-0,055144$ & $-1,321605$ \\
\hline H63 & 5,782992 & $-0,541604$ & $-3,335702$ & H63 & 5,760323 & $-0,328944$ & $-3,315256$ \\
\hline H64 & 4,419302 & $-2,599898$ & $-3,654736$ & H64 & 4,431878 & $-2,394229$ & $-3,721179$ \\
\hline H65 & 2,921882 & $-3,383012$ & $-1,873017$ & $\mathrm{H} 65$ & 2,932837 & $-3,265241$ & $-1,979851$ \\
\hline H66 & 4,131118 & $-0,070845$ & 0,595712 & H66 & 4,061018 & $-0,022215$ & 0,614939 \\
\hline H67 & 5,619348 & 0,718048 & $-1,198826$ & H67 & 5,547850 & 0,859183 & $-1,143519$ \\
\hline C68 & $-1,038242$ & 4,284989 & 2,059129 & C68 & $-0,997895$ & 4,388338 & 1,966126 \\
\hline C69 & $-0,328236$ & 3,512750 & 1,144220 & C69 & $-0,275695$ & 3,589226 & 1,083006 \\
\hline $\mathrm{C} 70$ & $-0,968516$ & 2,947614 & 0,025963 & $\mathrm{C} 70$ & $-0,905394$ & 2,964960 & $-0,006302$ \\
\hline C71 & $-2,342387$ & 3,181662 & $-0,135903$ & C71 & $-2,283569$ & 3,173987 & $-0,178956$ \\
\hline $\mathrm{C} 72$ & $-3,053196$ & 3,953527 & 0,784255 & $\mathrm{C} 72$ & $-3,005924$ & 3,974075 & 0,705983 \\
\hline $\mathrm{C} 73$ & $-2,406683$ & 4,511475 & 1,885187 & $\mathrm{C} 73$ & $-2,368563$ & 4,586817 & 1,783752 \\
\hline H74 & $-0,519827$ & 4,711517 & 2,913202 & H74 & $-0,484962$ & 4,860748 & 2,799164 \\
\hline $\mathrm{H} 75$ & 0,727512 & 3,342432 & 1,318330 & $\mathrm{H} 75$ & 0,784620 & 3,447288 & 1,262401 \\
\hline H76 & $-2,871526$ & 2,731724 & $-0,967181$ & $\mathrm{H} 76$ & $-2,804020$ & 2,679889 & $-0,991040$ \\
\hline H77 & $-4,118696$ & 4,105315 & 0,638271 & H77 & $-4,073110$ & 4,105740 & 0,552366 \\
\hline $\mathrm{H} 78$ & $-2,958164$ & 5,115122 & 2,600003 & $\mathrm{H} 78$ & $-2,928829$ & 5,212893 & 2,471882 \\
\hline $\mathrm{C} 79$ & $-0,214418$ & 2,117247 & $-0,965847$ & C79 & $-0,161051$ & 2,072606 & $-0,951256$ \\
\hline $\mathrm{C} 80$ & 1,228678 & 2,056915 & $-0,985404$ & $\mathrm{C} 80$ & 1,305669 & 1,972765 & $-0,937986$ \\
\hline H81 & 1,732139 & 2,471968 & $-0,118569$ & H81 & 1,785944 & 2,397967 & $-0,061938$ \\
\hline $\mathrm{C} 82$ & 2,004538 & 2,282510 & $-2,252542$ & $\mathrm{C} 82$ & 2,089233 & 2,223535 & $-2,193803$ \\
\hline C83 & 2,363752 & 3,616100 & $-2,516649$ & $\mathrm{C} 83$ & 2,526959 & 3,542133 & $-2,398084$ \\
\hline C84 & 2,366656 & 1,294030 & $-3,171265$ & C84 & 2,355981 & 1,259032 & $-3,167415$ \\
\hline $\mathrm{C} 85$ & 3,043921 & 3,954199 & $-3,685337$ & $\mathrm{C} 85$ & 3,196148 & 3,891980 & $-3,569282$ \\
\hline H86 & 2,099964 & 4,391750 & $-1,800550$ & H86 & 2,332324 & 4,296180 & $-1,638479$ \\
\hline $\mathrm{C} 87$ & 3,046943 & 1,634824 & $-4,343199$ & C87 & 3,021141 & 1,612678 & $-4,342848$ \\
\hline H88 & 2,122759 & 0,258243 & $-2,969180$ & $\mathrm{H} 88$ & 2,055327 & 0,231377 & $-3,000767$ \\
\hline C89 & 3,382493 & 2,961741 & $-4,609626$ & C89 & 3,437333 & 2,926892 & $-4,550783$ \\
\hline
\end{tabular}




\begin{tabular}{|c|c|c|c|c|c|c|c|}
\hline H90 & 3,310174 & 4,990809 & $-3,872581$ & H90 & 3,528618 & 4,915853 & $-3,715551$ \\
\hline H91 & 3,318514 & 0,852444 & $-5,045966$ & H91 & 3,220341 & 0,852245 & $-5,092339$ \\
\hline H92 & 3,910057 & 3,222872 & $-5,522271$ & H92 & 3,954929 & 3,199227 & $-5,466134$ \\
\hline C93 & $-1,125803$ & 3,157944 & $-3,157111$ & C93 & $-0,989990$ & 3,153900 & $-3,169383$ \\
\hline C94 & $-0,747045$ & 0,882959 & $-3,005556$ & C94 & $-0,710500$ & 0,865578 & $-3,000873$ \\
\hline C95 & $-1,743835$ & 2,412567 & $-4,349893$ & C95 & $-1,623077$ & 2,426759 & $-4,366593$ \\
\hline H96 & $-0,194523$ & 3,674919 & $-3,412413$ & H96 & $-0,040547$ & 3,639187 & $-3,417788$ \\
\hline H97 & $-1,816589$ & 3,862358 & $-2,692558$ & H97 & $-1,660992$ & 3,883060 & $-2,712641$ \\
\hline H98 & $-2,832711$ & 2,338941 & $-4,267697$ & H98 & $-2,715191$ & 2,398751 & $-4,299517$ \\
\hline H99 & $-1,464594$ & 2,816339 & $-5,322827$ & H99 & $-1,314991$ & 2,812743 & $-5,338104$ \\
\hline N100 & $-0,848723$ & 2,017238 & $-2,271389$ & N100 & $-0,759634$ & 2,007861 & $-2,278074$ \\
\hline O101 & $-1,202356$ & 1,062326 & $-4,251434$ & O101 & $-1,140133$ & 1,054879 & $-4,254258$ \\
\hline O102 & $-0,319315$ & $-0,213301$ & $-2,608826$ & O102 & $-0,338831$ & $-0,247097$ & $-2,588449$ \\
\hline H103 & 2,118656 & $-4,163603$ & 1,653599 & $\mathrm{H} 103$ & 2,242713 & $-4,219143$ & 1,534474 \\
\hline N104 & $-1,909566$ & $-2,277621$ & $-0,095536$ & N104 & $-1,905234$ & $-2,358794$ & $-0,037298$ \\
\hline C105 & $-0,927416$ & $-2,963677$ & $-0,971016$ & $\mathrm{C} 105$ & $-0,938625$ & $-3,029816$ & $-0,943366$ \\
\hline H106 & $-0,822518$ & $-2,457924$ & $-1,938426$ & H106 & $-0,869127$ & $-2,520223$ & $-1,912415$ \\
\hline \multicolumn{4}{|c|}{ C1_ZS_Chelbot_INT1 } & \multicolumn{3}{|c|}{ C1_ZS_Chelbot_TS2 } & \\
\hline $\mathrm{C} 1$ & $-3,837347$ & $-3,522150$ & $-0,960991$ & $\mathrm{C} 1$ & $-3,837789$ & $-3,593314$ & $-0,714641$ \\
\hline $\mathrm{C} 2$ & $-1,577798$ & $-4,444827$ & $-0,996165$ & $\mathrm{C} 2$ & $-1,564852$ & $-4,485327$ & $-0,782117$ \\
\hline $\mathrm{C} 3$ & $-2,846078$ & $-4,311452$ & $-1,879954$ & $\mathrm{C} 3$ & $-2,865712$ & $-4,414655$ & $-1,625164$ \\
\hline $\mathrm{C} 4$ & $-2,994721$ & $-3,304616$ & 0,315783 & $\mathrm{C} 4$ & $-2,938812$ & $-3,254563$ & 0,493634 \\
\hline H5 & $-4,730618$ & $-4,111033$ & $-0,728504$ & H5 & $-4,691368$ & $-4,196052$ & $-0,386981$ \\
\hline H6 & $-2,638445$ & $-3,794510$ & $-2,822435$ & H6 & $-2,698177$ & $-3,943045$ & $-2,598883$ \\
\hline $\mathrm{H} 7$ & $-3,558584$ & $-2,991075$ & 1,194818 & $\mathrm{H} 7$ & $-3,459616$ & $-2,871908$ & 1,371848 \\
\hline $\mathrm{H} 8$ & $-4,166186$ & $-2,575666$ & $-1,392669$ & $\mathrm{H} 8$ & $-4,230519$ & $-2,698194$ & $-1,196010$ \\
\hline H9 & $-0,867489$ & $-5,205364$ & $-1,320568$ & H9 & $-0,868271$ & $-5,270108$ & $-1,076028$ \\
\hline H10 & $-3,235218$ & $-5,302323$ & $-2,130114$ & H10 & $-3,246983$ & $-5,422190$ & $-1,812515$ \\
\hline $\mathrm{C} 11$ & $-2,194007$ & $-4,615715$ & 0,410416 & $\mathrm{C} 11$ & $-2,123785$ & $-4,549971$ & 0,656300 \\
\hline H12 & $-1,456471$ & $-4,615400$ & 1,218924 & H12 & $-1,353407$ & $-4,492479$ & 1,430922 \\
\hline H13 & $-2,826998$ & $-5,503814$ & 0,490653 & $\mathrm{H} 13$ & $-2,750074$ & $-5,429238$ & 0,830697 \\
\hline $\mathrm{C} 14$ & 0,469288 & $-2,972915$ & $-0,364508$ & C14 & 0,474126 & $-3,004590$ & $-0,224180$ \\
\hline N15 & 1,035737 & $-1,884271$ & 0,018868 & N15 & 1,082707 & $-1,910842$ & 0,081487 \\
\hline $\mathrm{C} 16$ & 2,457731 & $-2,171467$ & 0,385715 & $\mathrm{C} 16$ & 2,461772 & $-2,270252$ & 0,585263 \\
\hline $\mathrm{C} 17$ & 2,494496 & $-3,734084$ & 0,232243 & $\mathrm{C} 17$ & 2,387306 & $-3,843960$ & 0,585499 \\
\hline P18 & $-1,802125$ & $-0,710518$ & 0,394982 & P18 & $-1,767841$ & $-0,663363$ & 0,356286 \\
\hline Ir19 & 0,167131 & 0,087492 & $-0,298276$ & Ir19 & 0,175377 & 0,074241 & $-0,508614$ \\
\hline $\mathrm{C} 20$ & $-1,975078$ & $-0,523245$ & 2,198599 & $\mathrm{C} 20$ & $-1,830650$ & $-0,432306$ & 2,165414 \\
\hline $\mathrm{C} 21$ & $-2,013227$ & $-0,122602$ & 4,966202 & $\mathrm{C} 21$ & $-1,721250$ & $-0,005526$ & 4,934029 \\
\hline $\mathrm{C} 22$ & $-1,937078$ & $-1,619906$ & 3,066637 & $\mathrm{C} 22$ & $-1,634747$ & $-1,510546$ & 3,038478 \\
\hline $\mathrm{C} 23$ & $-2,007600$ & 0,781062 & 2,723329 & $\mathrm{C} 23$ & $-1,963092$ & 0,863515 & 2,689047 \\
\hline $\mathrm{C} 24$ & $-2,032739$ & 0,975654 & 4,100855 & $\mathrm{C} 24$ & $-1,909497$ & 1,072634 & 4,064739 \\
\hline $\mathrm{C} 25$ & $-1,960534$ & $-1,416572$ & 4,448488 & $\mathrm{C} 25$ & $-1,586712$ & $-1,295306$ & 4,417061 \\
\hline $\mathrm{H} 26$ & $-1,991213$ & 1,640252 & 2,059728 & $\mathrm{H} 26$ & $-2,095532$ & 1,710303 & 2,029405 \\
\hline $\mathrm{H} 27$ & $-2,056371$ & 1,986385 & 4,497870 & $\mathrm{H} 27$ & $-2,009507$ & 2,081840 & 4,453808 \\
\hline $\mathrm{H} 28$ & $-1,928516$ & $-2,271166$ & 5,118102 & $\mathrm{H} 28$ & $-1,428657$ & $-2,136741$ & 5,085210 \\
\hline $\mathrm{H} 29$ & $-2,029689$ & 0,032236 & 6,041196 & $\mathrm{H} 29$ & $-1,678418$ & 0,158901 & 6,006819 \\
\hline
\end{tabular}




\begin{tabular}{|c|c|c|c|c|c|c|c|}
\hline $\mathrm{O} 30$ & 1,199418 & $-4,091119$ & $-0,336952$ & $\mathrm{O} 30$ & 1,112646 & $-4,159262$ & $-0,031778$ \\
\hline $\mathrm{C} 31$ & 2,759205 & $-1,807739$ & 1,835646 & C31 & 2,716199 & $-1,806941$ & 2,015858 \\
\hline C32 & 1,745117 & $-1,476803$ & 2,737663 & $\mathrm{C} 32$ & 1,754528 & $-1,134707$ & 2,769821 \\
\hline C33 & 4,077543 & $-1,911777$ & 2,302589 & $\mathrm{C} 33$ & 3,925082 & $-2,169038$ & 2,633272 \\
\hline C34 & 2,047419 & $-1,211038$ & 4,074211 & $\mathrm{C} 34$ & 2,003562 & $-0,797606$ & 4,102323 \\
\hline H35 & 0,718739 & $-1,415311$ & 2,402083 & H35 & 0,809241 & $-0,869664$ & 2,323173 \\
\hline C36 & 4,378189 & $-1,652038$ & 3,637941 & C36 & 4,176749 & $-1,827097$ & 3,959325 \\
\hline H37 & 4,875053 & $-2,180279$ & 1,614597 & H37 & 4,676836 & $-2,718833$ & 2,072729 \\
\hline C38 & 3,363473 & $-1,291093$ & 4,527004 & C38 & 3,216779 & $-1,132773$ & 4,699304 \\
\hline H39 & 1,244250 & $-0,941931$ & 4,754652 & H39 & 1,237158 & $-0,272820$ & 4,664622 \\
\hline $\mathrm{H} 40$ & 5,405907 & $-1,726610$ & 3,981615 & $\mathrm{H} 40$ & 5,122155 & $-2,106682$ & 4,415400 \\
\hline $\mathrm{H} 41$ & 3,600024 & $-1,079900$ & 5,565818 & $\mathrm{H} 41$ & 3,413805 & $-0,864970$ & 5,733297 \\
\hline $\mathrm{C} 42$ & $-3,315511$ & 0,019933 & $-0,342815$ & $\mathrm{C} 42$ & $-3,371443$ & $-0,056801$ & $-0,297562$ \\
\hline $\mathrm{C} 43$ & $-4,334176$ & 0,617176 & 0,409333 & $\mathrm{C} 43$ & $-4,370444$ & 0,507813 & 0,504961 \\
\hline $\mathrm{C} 44$ & $-3,449595$ & $-0,058037$ & $-1,740609$ & C44 & $-3,635932$ & $-0,286621$ & $-1,658530$ \\
\hline $\mathrm{C} 45$ & $-5,456940$ & 1,149547 & $-0,228269$ & $\mathrm{C} 45$ & $-5,599769$ & 0,865253 & $-0,051648$ \\
\hline H46 & $-4,259051$ & 0,675075 & 1,489335 & $\mathrm{H} 46$ & $-4,202633$ & 0,671441 & 1,562513 \\
\hline $\mathrm{C} 47$ & $-4,566524$ & 0,481233 & $-2,372733$ & $\mathrm{C} 47$ & $-4,861131$ & 0,075653 & $-2,210954$ \\
\hline $\mathrm{C} 48$ & $-5,571391$ & 1,093592 & $-1,616330$ & $\mathrm{C} 48$ & $-5,844630$ & 0,662930 & $-1,408792$ \\
\hline $\mathrm{H} 49$ & $-6,242012$ & 1,609147 & 0,365332 & H49 & $-6,366699$ & 1,301083 & 0,581988 \\
\hline H50 & $-4,661563$ & 0,410963 & $-3,452989$ & $\mathrm{H} 50$ & $-5,058169$ & $-0,122308$ & $-3,261030$ \\
\hline H5 1 & $-6,444508$ & 1,512722 & $-2,108110$ & H51 & $-6,802655$ & 0,942046 & $-1,837729$ \\
\hline H52 & 0,462729 & 0,495796 & 1,179312 & H52 & 0,578113 & 0,656845 & 0,883632 \\
\hline H53 & 1,861260 & 1,043524 & $-0,849721$ & H53 & 1,465355 & 2,075189 & $-2,364341$ \\
\hline H54 & $-1,859291$ & $-2,627244$ & 2,672564 & H54 & $-1,487478$ & $-2,510454$ & 2,646801 \\
\hline H55 & $-2,687822$ & $-0,556120$ & $-2,330672$ & H55 & $-2,902092$ & $-0,790054$ & $-2,277264$ \\
\hline H56 & 3,272865 & $-4,094156$ & $-0,441231$ & H56 & 3,175801 & $-4,311039$ & $-0,006108$ \\
\hline C57 & 4,847820 & $-0,136681$ & $-2,624073$ & C57 & 5,267274 & $-0,907435$ & $-2,443701$ \\
\hline C58 & 4,124017 & $-1,286590$ & $-2,943968$ & C58 & 4,188968 & $-1,732908$ & $-2,772732$ \\
\hline C59 & 3,371984 & $-1,933191$ & $-1,965124$ & C59 & 3,322853 & $-2,176948$ & $-1,776615$ \\
\hline C60 & 3,340941 & $-1,455107$ & $-0,646628$ & C60 & 3,503959 & $-1,796062$ & $-0,438524$ \\
\hline C61 & 4,048334 & $-0,284893$ & $-0,343871$ & C61 & 4,581429 & $-0,963909$ & $-0,121026$ \\
\hline C62 & 4,798190 & 0,366815 & $-1,325495$ & C62 & 5,461628 & $-0,532064$ & $-1,116492$ \\
\hline H63 & 5,428347 & 0,374294 & $-3,386314$ & H63 & 5,947351 & $-0,562061$ & $-3,216918$ \\
\hline H64 & 4,139309 & $-1,677536$ & $-3,957445$ & H64 & 4,023807 & $-2,032829$ & $-3,803722$ \\
\hline $\mathrm{H} 65$ & 2,794487 & $-2,811871$ & $-2,241629$ & H65 & 2,487064 & $-2,813848$ & $-2,053946$ \\
\hline H66 & 4,019274 & 0,119438 & 0,661801 & H66 & 4,736720 & $-0,632155$ & 0,898209 \\
\hline H67 & 5,339541 & 1,273895 & $-1,072915$ & H67 & 6,292305 & 0,115175 & $-0,850086$ \\
\hline C68 & $-1,026272$ & 4,593725 & 1,768782 & C68 & $-1,314100$ & 4,197665 & 1,693451 \\
\hline C69 & $-0,279187$ & 3,743541 & 0,954655 & C69 & $-0,606850$ & 3,392347 & 0,806064 \\
\hline $\mathrm{C} 70$ & $-0,879905$ & 3,029475 & $-0,094707$ & $\mathrm{C} 70$ & $-1,229477$ & 2,809279 & $-0,315883$ \\
\hline C71 & $-2,261880$ & 3,204115 & $-0,292602$ & C71 & $-2,603208$ & 3,028650 & $-0,474750$ \\
\hline $\mathrm{C} 72$ & $-3,009120$ & 4,052891 & 0,521691 & C72 & $-3,320320$ & 3,827433 & 0,422175 \\
\hline $\mathrm{C} 73$ & $-2,396205$ & 4,756068 & 1,558492 & C73 & $-2,681938$ & 4,428811 & 1,504484 \\
\hline H74 & $-0,530829$ & 5,133590 & 2,570976 & H74 & $-0,796551$ & 4,642900 & 2,538761 \\
\hline $\mathrm{H} 75$ & 0,778932 & 3,633701 & 1,164900 & $\mathrm{H} 75$ & 0,447262 & 3,211035 & 0,986698 \\
\hline H76 & $-2,766475$ & 2,640968 & $-1,069049$ & $\mathrm{H} 76$ & $-3,136556$ & 2,553772 & $-1,288661$ \\
\hline $\mathrm{H} 77$ & $-4,076519$ & 4,153406 & 0,346039 & H77 & $-4,385770$ & 3,971783 & 0,266144 \\
\hline
\end{tabular}




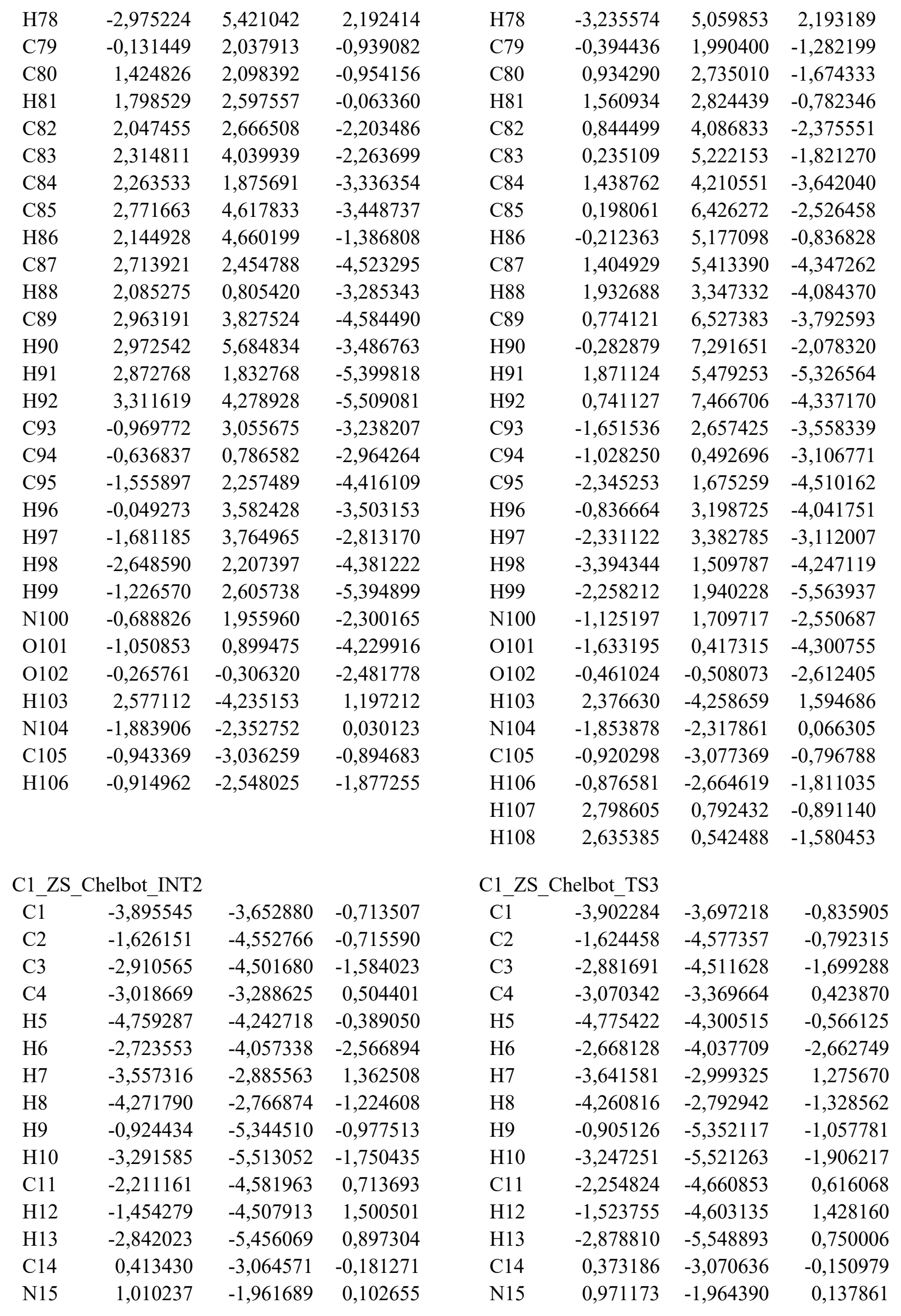




\begin{tabular}{|c|c|c|c|c|c|c|c|}
\hline C16 & 2,407627 & $-2,272591$ & 0,569457 & $\mathrm{C} 16$ & 2,368904 & $-2,278364$ & 0,625623 \\
\hline $\mathrm{C} 17$ & 2,376714 & $-3,843388$ & 0,580535 & $\mathrm{C} 17$ & 2,272664 & $-3,832500$ & 0,768619 \\
\hline P18 & $-1,850054$ & $-0,697769$ & 0,340132 & P18 & $-1,872667$ & $-0,768230$ & 0,391167 \\
\hline $\operatorname{Ir} 19$ & 0,230695 & 0,027190 & $-0,502825$ & Ir19 & 0,310792 & $-0,037404$ & $-0,464531$ \\
\hline $\mathrm{C} 20$ & $-1,933083$ & $-0,449981$ & 2,151729 & $\mathrm{C} 20$ & $-1,963254$ & $-0,568273$ & 2,207142 \\
\hline $\mathrm{C} 21$ & $-1,837496$ & 0,010991 & 4,919394 & $\mathrm{C} 21$ & $-1,829772$ & $-0,135324$ & 4,978274 \\
\hline $\mathrm{C} 22$ & $-1,757045$ & $-1,517716$ & 3,041778 & $\mathrm{C} 22$ & $-1,880591$ & $-1,653008$ & 3,089354 \\
\hline $\mathrm{C} 23$ & $-2,053569$ & 0,852434 & 2,660388 & $\mathrm{C} 23$ & $-1,969117$ & 0,736699 & 2,725344 \\
\hline $\mathrm{C} 24$ & $-2,007009$ & 1,078762 & 4,033933 & $\mathrm{C} 24$ & $-1,907562$ & 0,950008 & 4,100482 \\
\hline $\mathrm{C} 25$ & $-1,715042$ & $-1,285940$ & 4,418401 & $\mathrm{C} 25$ & $-1,816000$ & $-1,434337$ & 4,467795 \\
\hline $\mathrm{H} 26$ & $-2,175141$ & 1,692178 & 1,990550 & $\mathrm{H} 26$ & $-2,006775$ & 1,588260 & 2,057528 \\
\hline $\mathrm{H} 27$ & $-2,097807$ & 2,094599 & 4,407569 & $\mathrm{H} 27$ & $-1,910422$ & 1,966837 & 4,482954 \\
\hline $\mathrm{H} 28$ & $-1,572515$ & $-2,121796$ & 5,097229 & $\mathrm{H} 28$ & $-1,745190$ & $-2,283084$ & 5,141876 \\
\hline H29 & $-1,800681$ & 0,188472 & 5,990252 & $\mathrm{H} 29$ & $-1,776786$ & 0,030755 & 6,050238 \\
\hline $\mathrm{O} 30$ & 1,085315 & $-4,201461$ & 0,010998 & $\mathrm{O} 30$ & 1,033922 & $-4,201704$ & 0,095424 \\
\hline C31 & 2,677509 & $-1,767254$ & 1,982263 & $\mathrm{C} 31$ & 2,666172 & $-1,686209$ & 1,997037 \\
\hline C32 & 1,685643 & $-1,180173$ & 2,768317 & $\mathrm{C} 32$ & 1,655712 & $-1,175713$ & 2,815815 \\
\hline $\mathrm{C} 33$ & 3,944974 & $-1,984719$ & 2,545124 & $\mathrm{C} 33$ & 3,970070 & $-1,772112$ & 2,506936 \\
\hline C34 & 1,960057 & $-0,780987$ & 4,077975 & C34 & 1,948423 & $-0,712697$ & 4,099759 \\
\hline H35 & 0,694774 & $-1,033296$ & 2,367365 & $\mathrm{H} 35$ & 0,637161 & $-1,132140$ & 2,458594 \\
\hline C36 & 4,219400 & $-1,590081$ & 3,852437 & $\mathrm{C} 36$ & 4,260951 & $-1,317594$ & 3,791865 \\
\hline H37 & 4,727371 & $-2,451534$ & 1,952592 & H37 & 4,766454 & $-2,180831$ & 1,891798 \\
\hline $\mathrm{C} 38$ & 3,227659 & $-0,977410$ & 4,622285 & $\mathrm{C} 38$ & 3,251877 & $-0,775092$ & 4,589844 \\
\hline H39 & 1,169792 & $-0,322655$ & 4,665187 & H39 & 1,145800 & $-0,309440$ & 4,710056 \\
\hline $\mathrm{H} 40$ & 5,208737 & $-1,758474$ & 4,268057 & $\mathrm{H} 40$ & 5,278593 & $-1,382693$ & 4,166079 \\
\hline $\mathrm{H} 41$ & 3,443466 & $-0,664020$ & 5,639669 & $\mathrm{H} 41$ & 3,481499 & $-0,411723$ & 5,587442 \\
\hline $\mathrm{C} 42$ & $-3,456961$ & $-0,102161$ & $-0,324571$ & $\mathrm{C} 42$ & $-3,458720$ & $-0,105878$ & $-0,260876$ \\
\hline $\mathrm{C} 43$ & $-4,461872$ & 0,469654 & 0,464540 & $\mathrm{C} 43$ & $-4,439501$ & 0,508904 & 0,524130 \\
\hline $\mathrm{C} 44$ & $-3,702352$ & $-0,323572$ & $-1,690841$ & C44 & $-3,692014$ & $-0,279853$ & $-1,636389$ \\
\hline $\mathrm{C} 45$ & $-5,680596$ & 0,838815 & $-0,108637$ & $\mathrm{C} 45$ & $-5,625991$ & 0,960424 & $-0,059281$ \\
\hline H46 & $-4,306063$ & 0,629092 & 1,525177 & $\mathrm{H} 46$ & $-4,286294$ & 0,640900 & 1,589498 \\
\hline $\mathrm{C} 47$ & $-4,917030$ & 0,051141 & $-2,259664$ & $\mathrm{C} 47$ & $-4,874612$ & 0,173497 & $-2,215858$ \\
\hline $\mathrm{C} 48$ & $-5,908408$ & 0,640941 & $-1,469522$ & $\mathrm{C} 48$ & $-5,844096$ & 0,802114 & $-1,427234$ \\
\hline H49 & $-6,453393$ & 1,278745 & 0,515101 & H49 & $-6,381889$ & 1,433181 & 0,561281 \\
\hline H50 & $-5,097872$ & $-0,137720$ & $-3,314384$ & $\mathrm{H} 50$ & $-5,048751$ & 0,018469 & $-3,277422$ \\
\hline H51 & $-6,858174$ & 0,928404 & $-1,911057$ & $\mathrm{H} 51$ & $-6,768783$ & 1,152952 & $-1,876328$ \\
\hline H52 & 0,549595 & 0,583644 & 0,923200 & $\mathrm{H} 52$ & 0,769510 & 0,510379 & 0,914300 \\
\hline H53 & 1,642442 & 2,071860 & $-2,289987$ & $\mathrm{H} 53$ & 1,824742 & 2,359993 & $-2,342432$ \\
\hline H54 & $-1,619531$ & $-2,524785$ & 2,665738 & H54 & $-1,831826$ & $-2,666321$ & 2,707266 \\
\hline H55 & $-2,959913$ & $-0,821968$ & $-2,303915$ & H55 & $-2,961431$ & $-0,796678$ & $-2,249286$ \\
\hline H56 & 3,154592 & $-4,299422$ & $-0,033262$ & H56 & 3,093835 & $-4,369551$ & 0,294082 \\
\hline $\mathrm{C} 57$ & 4,912859 & $-0,644450$ & $-2,583132$ & C57 & 4,921648 & $-1,009112$ & $-2,650457$ \\
\hline C58 & 4,045626 & $-1,711795$ & $-2,831034$ & C58 & 4,141257 & $-2,161741$ & $-2,758407$ \\
\hline C59 & 3,283857 & $-2,245157$ & $-1,793961$ & C59 & 3,356239 & $-2,575939$ & $-1,684429$ \\
\hline $\mathrm{C} 60$ & 3,372675 & $-1,727830$ & $-0,493333$ & C60 & 3,336841 & $-1,850973$ & $-0,484847$ \\
\hline C61 & 4,241099 & $-0,656987$ & $-0,255385$ & C61 & 4,112208 & $-0,688712$ & $-0,392000$ \\
\hline C62 & 5,008311 & $-0,121859$ & $-1,293914$ & C62 & 4,903685 & $-0,275128$ & $-1,465020$ \\
\hline H63 & 5,508097 & $-0,225779$ & $-3,389467$ & $\mathrm{H} 63$ & 5,538606 & $-0,687116$ & $-3,484605$ \\
\hline
\end{tabular}




\begin{tabular}{|c|c|c|c|c|c|c|c|}
\hline H64 & 3,959906 & $-2,125754$ & $-3,831595$ & H64 & 4,143235 & $-2,738437$ & $-3,678837$ \\
\hline H65 & 2,595913 & $-3,058738$ & $-2,011280$ & H65 & 2,739464 & $-3,464016$ & $-1,798671$ \\
\hline H66 & 4,314751 & $-0,226163$ & 0,736530 & H66 & 4,091517 & $-0,096501$ & 0,515549 \\
\hline H67 & 5,678123 & 0,708887 & $-1,090726$ & H67 & 5,504456 & 0,625312 & $-1,371579$ \\
\hline C68 & $-1,188624$ & 4,134686 & 1,750431 & C68 & $-1,125948$ & 4,053193 & 1,738528 \\
\hline C69 & $-0,478259$ & 3,349420 & 0,847963 & C69 & $-0,383698$ & 3,313117 & 0,823553 \\
\hline $\mathrm{C} 70$ & $-1,098358$ & 2,781603 & $-0,283206$ & $\mathrm{C} 70$ & $-0,979974$ & 2,780156 & $-0,335914$ \\
\hline C71 & $-2,475114$ & 2,991253 & $-0,432705$ & C71 & $-2,356381$ & 2,964756 & $-0,508322$ \\
\hline $\mathrm{C} 72$ & $-3,195723$ & 3,770062 & 0,479378 & C72 & $-3,103664$ & 3,701497 & 0,415835 \\
\hline $\mathrm{C} 73$ & $-2,558234$ & 4,360044 & 1,568702 & C73 & $-2,494798$ & 4,264208 & 1,535379 \\
\hline $\mathrm{H} 74$ & $-0,672280$ & 4,567114 & 2,602948 & $\mathrm{H} 74$ & $-0,634122$ & 4,461959 & 2,616689 \\
\hline $\mathrm{H} 75$ & 0,577330 & 3,173251 & 1,025213 & H75 & 0,670434 & 3,143767 & 1,015188 \\
\hline H76 & $-3,006968$ & 2,523965 & $-1,252136$ & H76 & $-2,862287$ & 2,518227 & $-1,355206$ \\
\hline H77 & $-4,263141$ & 3,906525 & 0,329946 & H77 & $-4,169641$ & 3,826377 & 0,250844 \\
\hline $\mathrm{H} 78$ & $-3,114799$ & 4,975627 & 2,268852 & $\mathrm{H} 78$ & $-3,073863$ & 4,847961 & 2,244311 \\
\hline C79 & $-0,261928$ & 1,982961 & $-1,267334$ & C79 & $-0,117395$ & 2,087879 & $-1,373971$ \\
\hline $\mathrm{C} 80$ & 1,066715 & 2,737411 & $-1,639757$ & $\mathrm{C} 80$ & 1,158127 & 2,970867 & $-1,720780$ \\
\hline H81 & 1,657667 & 2,849603 & $-0,724864$ & H81 & 1,697544 & 3,127270 & $-0,782101$ \\
\hline $\mathrm{C} 82$ & 0,979396 & 4,079841 & $-2,351848$ & C82 & 0,953839 & 4,298715 & $-2,428380$ \\
\hline $\mathrm{C} 83$ & 0,356910 & 5,212611 & $-1,806403$ & $\mathrm{C} 83$ & 0,199081 & 5,350603 & $-1,888879$ \\
\hline $\mathrm{C} 84$ & 1,571383 & 4,199504 & $-3,619230$ & C84 & 1,576133 & 4,495239 & $-3,670693$ \\
\hline $\mathrm{C} 85$ & 0,307690 & 6,411315 & $-2,519822$ & $\mathrm{C} 85$ & 0,054727 & 6,550326 & $-2,587596$ \\
\hline H86 & $-0,091959$ & 5,167363 & $-0,821936$ & H86 & $-0,274443$ & 5,242720 & $-0,921057$ \\
\hline $\mathrm{C} 87$ & 1,525846 & 5,396901 & $-4,332983$ & C87 & 1,435896 & 5,695081 & $-4,367427$ \\
\hline H88 & 2,070755 & 3,336558 & $-4,056117$ & H88 & 2,176342 & 3,695152 & $-4,099608$ \\
\hline $\mathrm{C} 89$ & 0,885166 & 6,509085 & $-3,785940$ & C89 & 0,667352 & 6,727353 & $-3,828538$ \\
\hline $\mathrm{H} 90$ & $-0,182826$ & 7,275014 & $-2,078934$ & H90 & $-0,535117$ & 7,353264 & $-2,154006$ \\
\hline H91 & 1,990505 & 5,460564 & $-5,313162$ & H91 & 1,928189 & 5,823484 & $-5,327369$ \\
\hline H92 & 0,843245 & 7,444087 & $-4,337341$ & H92 & 0,553233 & 7,663768 & $-4,367105$ \\
\hline C93 & $-1,528772$ & 2,680757 & $-3,518840$ & C93 & $-1,341858$ & 2,681314 & $-3,628340$ \\
\hline C94 & $-1,014776$ & 0,484801 & $-3,066485$ & C94 & $-0,932904$ & 0,472894 & $-3,080181$ \\
\hline C95 & $-2,321423$ & 1,732242 & $-4,424867$ & C95 & $-2,225823$ & 1,738818 & $-4,447768$ \\
\hline H96 & $-0,708413$ & 3,170032 & $-4,046193$ & H96 & $-0,516773$ & 3,087644 & $-4,215855$ \\
\hline H97 & $-2,142492$ & 3,447702 & $-3,046605$ & H97 & $-1,882605$ & 3,510921 & $-3,173386$ \\
\hline H98 & $-3,363627$ & 1,624807 & $-4,108139$ & H98 & $-3,256016$ & 1,704722 & $-4,078956$ \\
\hline H99 & $-2,272265$ & 1,981692 & $-5,484804$ & H99 & $-2,213992$ & 1,936920 & $-5,519546$ \\
\hline N100 & $-1,010084$ & 1,713204 & $-2,526117$ & N100 & $-0,842162$ & 1,737788 & $-2,604328$ \\
\hline O101 & $-1,670572$ & 0,439441 & $-4,235244$ & O101 & $-1,633299$ & 0,426785 & $-4,224314$ \\
\hline O102 & $-0,516313$ & $-0,551943$ & $-2,574121$ & O102 & $-0,478751$ & $-0,564211$ & $-2,569995$ \\
\hline H103 & 2,412300 & $-4,247834$ & 1,593029 & H103 & 2,178844 & $-4,136949$ & 1,812087 \\
\hline N104 & $-1,926512$ & $-2,362183$ & 0,077006 & N104 & $-1,981827$ & $-2,418072$ & 0,059691 \\
\hline C105 & $-0,981312$ & $-3,145705$ & $-0,753144$ & $\mathrm{C} 105$ & $-1,001148$ & $-3,160780$ & $-0,767315$ \\
\hline H106 & $-0,922814$ & $-2,756209$ & $-1,776906$ & H106 & $-0,920236$ & $-2,739986$ & $-1,776791$ \\
\hline H107 & 1,884302 & 0,518632 & $-0,527380$ & H107 & 1,844101 & 0,190167 & $-0,909082$ \\
\hline H108 & 1,756145 & 0,220554 & $-1,314889$ & H108 & 1,016851 & 1,141453 & $-1,252165$ \\
\hline \multicolumn{8}{|c|}{ C1_Z_R_tN_Chelbot } \\
\hline & - 1 & & & 1 & lbot_SM & & \\
\hline
\end{tabular}




\begin{tabular}{|c|c|c|c|c|c|c|c|}
\hline $\mathrm{C} 1$ & $-3,895922$ & $-3,761888$ & $-0,807289$ & $\mathrm{C} 1$ & $-3,911023$ & $-3,442904$ & $-0,965329$ \\
\hline $\mathrm{C} 2$ & $-1,611516$ & $-4,628001$ & $-0,814296$ & $\mathrm{C} 2$ & $-1,654917$ & $-4,372785$ & $-1,080668$ \\
\hline $\mathrm{C} 3$ & $-2,879615$ & $-4,544402$ & $-1,704816$ & $\mathrm{C} 3$ & $-2,937462$ & $-4,192420$ & $-1,935271$ \\
\hline $\mathrm{C} 4$ & $-3,056682$ & $-3,478251$ & 0,458669 & $\mathrm{C} 4$ & $-3,035457$ & $-3,261598$ & 0,294444 \\
\hline H5 & $-4,768217$ & $-4,374019$ & $-0,555182$ & H5 & $-4,791402$ & $-4,049259$ & $-0,728091$ \\
\hline H6 & $-2,680477$ & $-4,042137$ & $-2,656970$ & H6 & $-2,742000$ & $-3,632442$ & $-2,855544$ \\
\hline H7 & $-3,625711$ & $-3,144465$ & 1,326852 & $\mathrm{H} 7$ & $-3,571256$ & $-2,967028$ & 1,197291 \\
\hline H8 & $-4,255978$ & $-2,839789$ & $-1,265641$ & $\mathrm{H} 8$ & $-4,260560$ & $-2,487612$ & $-1,358033$ \\
\hline H9 & $-0,887972$ & $-5,386720$ & $-1,112821$ & $\mathrm{H} 9$ & $-0,962714$ & $-5,132937$ & $-1,442878$ \\
\hline H10 & $-3,240886$ & $-5,550362$ & $-1,935843$ & $\mathrm{H} 10$ & $-3,334149$ & $-5,169111$ & $-2,225796$ \\
\hline $\mathrm{C} 11$ & $-2,227490$ & $-4,766916$ & 0,597420 & $\mathrm{C} 11$ & $-2,244867$ & $-4,580882$ & 0,332946 \\
\hline H12 & $-1,490650$ & $-4,726185$ & 1,405041 & H12 & $-1,490737$ & $-4,614585$ & 1,124972 \\
\hline H13 & $-2,839974$ & $-5,666642$ & 0,704666 & H13 & $-2,884962$ & $-5,464971$ & 0,399432 \\
\hline $\mathrm{C} 14$ & 0,379637 & $-3,118052$ & $-0,154831$ & $\mathrm{C} 14$ & 0,390990 & $-2,971424$ & $-0,353773$ \\
\hline N15 & 1,009889 & $-2,014295$ & 0,088871 & N15 & 1,022528 & $-1,909009$ & 0,013916 \\
\hline $\mathrm{C} 16$ & 2,406287 & $-2,342350$ & 0,597189 & $\mathrm{C} 16$ & 2,407443 & $-2,307558$ & 0,479052 \\
\hline $\mathrm{C} 17$ & 2,238712 & $-3,874569$ & 0,846809 & $\mathrm{C} 17$ & 2,261580 & $-3,864107$ & 0,505018 \\
\hline P18 & $-1,884586$ & $-0,848083$ & 0,416895 & P18 & $-1,838355$ & $-0,660972$ & 0,350615 \\
\hline Ir19 & 0,301293 & $-0,167029$ & $-0,395677$ & Ir19 & 0,382327 & 0,114335 & $-0,361366$ \\
\hline $\mathrm{C} 20$ & $-2,006830$ & $-0,593820$ & 2,221393 & $\mathrm{C} 20$ & $-1,997047$ & $-0,498259$ & 2,163913 \\
\hline $\mathrm{C} 21$ & $-1,965504$ & $-0,049291$ & 4,970549 & $\mathrm{C} 21$ & $-1,988179$ & $-0,148502$ & 4,943616 \\
\hline $\mathrm{C} 22$ & $-1,993666$ & $-1,643067$ & 3,147286 & $\mathrm{C} 22$ & $-1,869959$ & $-1,604093$ & 3,013718 \\
\hline $\mathrm{C} 23$ & $-1,976377$ & 0,732811 & 2,683147 & $\mathrm{C} 23$ & $-2,104079$ & 0,789101 & 2,717042 \\
\hline $\mathrm{C} 24$ & $-1,962631$ & 1,001893 & 4,048719 & $\mathrm{C} 24$ & $-2,102112$ & 0,958920 & 4,099060 \\
\hline $\mathrm{C} 25$ & $-1,974665$ & $-1,368628$ & 4,517379 & $\mathrm{C} 25$ & $-1,869399$ & $-1,427157$ & 4,398861 \\
\hline $\mathrm{H} 26$ & $-1,950887$ & 1,556347 & 1,976973 & $\mathrm{H} 26$ & $-2,181556$ & 1,659302 & 2,075570 \\
\hline $\mathrm{H} 27$ & $-1,939915$ & 2,032487 & 4,391844 & $\mathrm{H} 27$ & $-2,183760$ & 1,959649 & 4,513403 \\
\hline $\mathrm{H} 28$ & $-1,962148$ & $-2,188561$ & 5,229816 & $\mathrm{H} 28$ & $-1,769541$ & $-2,290276$ & 5,050508 \\
\hline H29 & $-1,951166$ & 0,160294 & 6,036375 & $\mathrm{H} 29$ & $-1,985690$ & $-0,013659$ & 6,021385 \\
\hline $\mathrm{O} 30$ & 1,016120 & $-4,249661$ & 0,145983 & $\mathrm{O} 30$ & 1,016107 & $-4,139259$ & $-0,198722$ \\
\hline C31 & 2,719210 & $-1,696690$ & 1,942185 & $\mathrm{C} 31$ & 2,706913 & $-1,836703$ & 1,896549 \\
\hline C32 & 1,687964 & $-1,343276$ & 2,821944 & $\mathrm{C} 32$ & 1,686681 & $-1,425759$ & 2,759101 \\
\hline $\mathrm{C} 33$ & 4,043923 & $-1,614331$ & 2,390364 & $\mathrm{C} 33$ & 4,010630 & $-1,953214$ & 2,400467 \\
\hline C34 & 1,975249 & $-0,857107$ & 4,096288 & $\mathrm{C} 34$ & 1,968943 & $-1,081671$ & 4,081188 \\
\hline H35 & 0,654114 & $-1,438278$ & 2,515764 & H35 & 0,666868 & $-1,367783$ & 2,404916 \\
\hline C36 & 4,330796 & $-1,136369$ & 3,669555 & $\mathrm{C} 36$ & 4,292062 & $-1,615249$ & 3,723370 \\
\hline H37 & 4,857998 & $-1,919662$ & 1,740737 & H37 & 4,813358 & $-2,297232$ & 1,754612 \\
\hline C38 & 3,299247 & $-0,743910$ & 4,522226 & C38 & 3,273673 & $-1,166387$ & 4,565712 \\
\hline H39 & 1,156348 & $-0,571742$ & 4,750527 & H39 & 1,159050 & $-0,751167$ & 4,725345 \\
\hline $\mathrm{H} 40$ & 5,364684 & $-1,070759$ & 3,996312 & $\mathrm{H} 40$ & 5,309927 & $-1,699832$ & 4,093027 \\
\hline H41 & 3,526146 & $-0,363505$ & 5,514109 & $\mathrm{H} 41$ & 3,496627 & $-0,894002$ & 5,593210 \\
\hline $\mathrm{C} 42$ & $-3,466202$ & $-0,190101$ & $-0,258407$ & $\mathrm{C} 42$ & $-3,386154$ & 0,036679 & $-0,351752$ \\
\hline $\mathrm{C} 43$ & $-4,478542$ & 0,383015 & 0,519570 & $\mathrm{C} 43$ & $-4,437412$ & 0,550272 & 0,415747 \\
\hline $\mathrm{C} 44$ & $-3,656243$ & $-0,309531$ & $-1,646717$ & C44 & $-3,513005$ & 0,003555 & $-1,751945$ \\
\hline $\mathrm{C} 45$ & $-5,654466$ & 0,840743 & $-0,080383$ & $\mathrm{C} 45$ & $-5,583539$ & 1,049693 & $-0,206959$ \\
\hline H46 & $-4,356063$ & 0,477679 & 1,593200 & $\mathrm{H} 46$ & $-4,367779$ & 0,571412 & 1,497470 \\
\hline $\mathrm{C} 47$ & $-4,828453$ & 0,150374 & $-2,243062$ & $\mathrm{C} 47$ & $-4,654560$ & 0,507128 & $-2,370444$ \\
\hline $\mathrm{C} 48$ & $-5,830807$ & 0,732526 & $-1,459732$ & $\mathrm{C} 48$ & $-5,691209$ & 1,040649 & $-1,597060$ \\
\hline
\end{tabular}




\begin{tabular}{|c|c|c|c|c|c|c|c|}
\hline H49 & $-6,435376$ & 1,278214 & 0,535531 & H49 & $-6,392840$ & 1,446364 & 0,399296 \\
\hline H50 & $-4,968018$ & 0,036990 & $-3,314963$ & $\mathrm{H} 50$ & $-4,742459$ & 0,471032 & $-3,453101$ \\
\hline H51 & $-6,747229$ & 1,088094 & $-1,921989$ & $\mathrm{H} 51$ & $-6,582448$ & 1,434197 & $-2,077303$ \\
\hline H52 & 0,768505 & 0,254352 & 1,017848 & $\mathrm{H} 52$ & 0,738871 & 0,482595 & 1,102281 \\
\hline H53 & 1,724053 & 2,564102 & $-2,332798$ & $\mathrm{H} 53$ & 1,947942 & 0,211072 & $-0,596350$ \\
\hline H54 & $-1,970052$ & $-2,672660$ & 2,806899 & H54 & $-1,742559$ & $-2,598109$ & 2,598445 \\
\hline H55 & $-2,895684$ & $-0,783385$ & $-2,258687$ & $\mathrm{H} 55$ & $-2,728184$ & $-0,439748$ & $-2,355317$ \\
\hline H56 & 3,059862 & $-4,475110$ & 0,457501 & H56 & 3,068590 & $-4,391006$ & $-0,003620$ \\
\hline C57 & 4,991660 & $-1,403990$ & $-2,761781$ & $\mathrm{C} 57$ & 5,092901 & $-0,927283$ & $-2,646196$ \\
\hline C58 & 4,256115 & $-2,589353$ & $-2,740235$ & C58 & 4,269375 & $-2,035193$ & $-2,851029$ \\
\hline C59 & 3,455715 & $-2,894935$ & $-1,640725$ & C59 & 3,429223 & $-2,479230$ & $-1,831037$ \\
\hline C60 & 3,377565 & $-2,026672$ & $-0,543052$ & C60 & 3,401087 & $-1,830790$ & $-0,588050$ \\
\hline C61 & 4,102149 & $-0,828965$ & $-0,586424$ & C61 & 4,211959 & $-0,702676$ & $-0,404312$ \\
\hline C62 & 4,909951 & $-0,525254$ & $-1,682057$ & C62 & 5,055295 & $-0,259453$ & $-1,423603$ \\
\hline H63 & 5,621101 & $-1,166851$ & $-3,614760$ & $\mathrm{H} 63$ & 5,748754 & $-0,579033$ & $-3,438674$ \\
\hline H64 & 4,304155 & $-3,277726$ & $-3,578967$ & H64 & 4,279660 & $-2,555028$ & $-3,804731$ \\
\hline H65 & 2,875290 & $-3,813680$ & $-1,659638$ & H65 & 2,782162 & $-3,332403$ & $-2,019582$ \\
\hline H66 & 4,028270 & $-0,125036$ & 0,233279 & H66 & 4,183688 & $-0,163721$ & 0,535666 \\
\hline H67 & 5,471954 & 0,404514 & $-1,691203$ & H67 & 5,674687 & 0,617815 & $-1,262399$ \\
\hline C68 & $-1,402244$ & 4,278878 & 1,708394 & C68 & $-1,258348$ & 4,359429 & 1,974947 \\
\hline C69 & $-0,582999$ & 3,674960 & 0,757209 & C69 & $-0,494232$ & 3,571748 & 1,117336 \\
\hline $\mathrm{C} 70$ & $-1,131351$ & 3,080542 & $-0,390773$ & $\mathrm{C} 70$ & $-1,072780$ & 2,945900 & $-0,000629$ \\
\hline C71 & $-2,522014$ & 3,068860 & $-0,537507$ & C71 & $-2,444334$ & 3,144234 & $-0,226629$ \\
\hline $\mathrm{C} 72$ & $-3,343085$ & 3,676067 & 0,416119 & $\mathrm{C} 72$ & $-3,209369$ & 3,933587 & 0,633692 \\
\hline $\mathrm{C} 73$ & $-2,790032$ & 4,292247 & 1,537199 & $\mathrm{C} 73$ & $-2,623732$ & 4,546338 & 1,740727 \\
\hline H74 & $-0,954303$ & 4,739342 & 2,584305 & $\mathrm{H} 74$ & $-0,782168$ & 4,831908 & 2,829406 \\
\hline H75 & 0,490097 & 3,667517 & 0,917340 & $\mathrm{H} 75$ & 0,559887 & 3,440894 & 1,335398 \\
\hline H76 & $-2,979220$ & 2,560615 & $-1,379011$ & $\mathrm{H} 76$ & $-2,927555$ & 2,657604 & $-1,065294$ \\
\hline H77 & $-4,419607$ & 3,649942 & 0,279652 & $\mathrm{H} 77$ & $-4,270045$ & 4,057061 & 0,435296 \\
\hline H78 & $-3,428908$ & 4,769492 & 2,274167 & $\mathrm{H} 78$ & $-3,217025$ & 5,163940 & 2,408168 \\
\hline C79 & $-0,205978$ & 2,450869 & $-1,423057$ & $\mathrm{C} 79$ & $-0,259672$ & 2,105563 & $-0,938625$ \\
\hline $\mathrm{C} 80$ & 1,099436 & 3,244404 & $-1,745554$ & $\mathrm{C} 80$ & 1,183331 & 2,057881 & $-0,890153$ \\
\hline H81 & 1,628893 & 3,388465 & $-0,798352$ & H81 & 1,640315 & 2,470704 & 0,003330 \\
\hline $\mathrm{C} 82$ & 0,958265 & 4,564407 & $-2,477095$ & C82 & 2,017395 & 2,295927 & $-2,116026$ \\
\hline $\mathrm{C} 83$ & 0,269826 & 5,658769 & $-1,934677$ & $\mathrm{C} 83$ & 2,420158 & 3,625387 & $-2,330373$ \\
\hline $\mathrm{C} 84$ & 1,559312 & 4,710447 & $-3,735968$ & C84 & 2,388708 & 1,320183 & $-3,045345$ \\
\hline $\mathrm{C} 85$ & 0,169959 & 6,856313 & $-2,643929$ & $\mathrm{C} 85$ & 3,153505 & 3,973944 & $-3,462733$ \\
\hline H86 & $-0,186952$ & 5,583352 & $-0,954823$ & H86 & 2,149118 & 4,389477 & $-1,604886$ \\
\hline $\mathrm{C} 87$ & 1,463297 & 5,908410 & $-4,444405$ & C87 & 3,120243 & 1,672015 & $-4,181764$ \\
\hline H88 & 2,109587 & 3,875735 & $-4,165320$ & H88 & 2,119311 & 0,284205 & $-2,876634$ \\
\hline $\mathrm{C} 89$ & 0,761165 & 6,984962 & $-3,901313$ & C89 & 3,499620 & 2,995596 & $-4,398317$ \\
\hline H90 & $-0,367228$ & 7,693980 & $-2,207789$ & $\mathrm{H} 90$ & 3,455805 & 5,006587 & $-3,613165$ \\
\hline H91 & 1,938853 & 6,000545 & $-5,416795$ & H91 & 3,400783 & 0,900464 & $-4,892908$ \\
\hline H92 & 0,683352 & 7,919529 & $-4,449309$ & H92 & 4,069419 & 3,265086 & $-5,283021$ \\
\hline C93 & $-1,440324$ & 2,931061 & $-3,643066$ & C93 & $-1,052933$ & 3,149969 & $-3,170443$ \\
\hline C94 & $-0,959587$ & 0,726682 & $-3,089247$ & C94 & $-0,699927$ & 0,871607 & $-3,004040$ \\
\hline C95 & $-2,302487$ & 1,959610 & $-4,451759$ & C95 & $-1,608374$ & 2,412051 & $-4,399016$ \\
\hline H96 & $-0,635059$ & 3,368874 & $-4,236449$ & H96 & $-0,106583$ & 3,663042 & $-3,372847$ \\
\hline
\end{tabular}




$\begin{array}{lrrrlrrr}\text { H97 } & -2,007170 & 3,738898 & -3,178476 & \text { H97 } & -1,764476 & 3,857272 & -2,741883 \\ \text { H98 } & -3,328601 & 1,898876 & -4,074182 & \text { H98 } & -2,701184 & 2,352893 & -4,384631 \\ \text { H99 } & -2,305464 & 2,156233 & -5,523808 & \text { H99 } & -1,266011 & 2,813458 & -5,352711 \\ \text { N100 } & -0,898764 & 2,007069 & -2,628628 & \text { N100 } & -0,828599 & 2,005139 & -2,274738 \\ \text { O101 } & -1,671851 & 0,669221 & -4,230032 & \text { O101 } & -1,091024 & 1,055182 & -4,271082 \\ \text { O102 } & -0,486221 & -0,299965 & -2,592687 & \text { O102 } & -0,298411 & -0,227067 & -2,588307 \\ \text { H103 } & 2,081190 & -4,093352 & 1,904450 & \text { H103 } & 2,150079 & -4,241970 & 1,522430 \\ \text { N104 } & -1,977605 & -2,508617 & 0,128879 & \text { N104 } & -1,928044 & -2,307721 & 0,003075 \\ \text { C105 } & -1,005052 & -3,204737 & -0,750252 & \text { C105 } & -0,998812 & -2,978426 & -0,939137 \\ \text { H106 } & -0,951399 & -2,745269 & -1,746428 & \text { H106 } & -0,941017 & -2,458745 & -1,903402 \\ \text { H107 } & 1,792663 & 0,188149 & -0,856433 & & & & \end{array}$

C1 ZR Chelbot TS1

$\begin{array}{lrr}\text { C1 } & -3,864759 & -3,516923 \\ \text { C2 } & -1,600828 & -4,431882 \\ \text { C3 } & -2,854837 & -4,278750 \\ \text { C4 } & -3,039585 & -3,319404 \\ \text { H5 } & -4,755778 & -4,118286 \\ \text { H6 } & -2,633169 & -3,736841 \\ \text { H7 } & -3,614560 & -3,021147 \\ \text { H8 } & -4,195639 & -2,564345 \\ \text { H9 } & -0,887309 & -5,187953 \\ \text { H10 } & -3,235561 & -5,264266 \\ \text { C11 } & -2,238363 & -4,630723 \\ \text { H12 } & -1,512358 & -4,646825 \\ \text { H13 } & -2,871083 & -5,521105 \\ \text { C14 } & 0,418433 & -2,990358 \\ \text { N15 } & 1,017392 & -1,909778 \\ \text { C16 } & 2,403520 & -2,259484 \\ \text { C17 } & 2,321688 & -3,823667 \\ \text { P18 } & -1,840371 & -0,721124 \\ \text { Ir19 } & 0,287431 & 0,092211 \\ \text { C20 } & -1,999137 & -0,560474 \\ \text { C21 } & -2,024060 & -0,198613 \\ \text { C22 } & -1,988501 & -1,668839 \\ \text { C23 } & -2,001892 & 0,734789 \\ \text { C24 } & -2,018990 & 0,910976 \\ \text { C25 } & -2,003735 & -1,485359 \\ \text { H26 } & -1,979577 & 1,604742 \\ \text { H27 } & -2,020633 & 1,917032 \\ \text { H28 } & -1,992779 & -2,350452 \\ \text { H29 } & -2,035222 & -0,059195 \\ \text { O30 } & 1,079275 & -4,140173 \\ \text { C31 } & 2,661574 & -1,796163 \\ \text { C32 } & 1,612886 & -1,432147 \\ \text { C33 } & 3,960813 & -1,870081 \\ \text { C34 } & 1,861288 & -1,094446\end{array}$

$-1,067888$

$-1,089187$

$-1,989746$

0,223327

$-0,859452$

$-2,914779$

1,100525

$-1,483698$

$-1,417097$

$-2,271972$

0,304979

1,123588

0,358469

$-0,344426$

0,019982

0,500815

0,501877

0,345760

$-0,357916$

2,157947

4,935738

3,012759

2,704783

4,085486

4,397808

2,057421

4,495065

5,054541

6,012991

$-0,191907$

1,931509

2,780458

2,454397

4,111329
C1 ZR Chelbot INT1

$\mathrm{C} 1$

$\mathrm{C} 2$

$\mathrm{C} 3$

C4

H5

H6

$\mathrm{H} 7$

H8

H9

H10

C11

H12

H13

C14

N15

C16

C17

P18

Ir19

C20

C21

$\mathrm{C} 22$

$\mathrm{C} 23$

C24

C25

H26

H27

H28

H29

O30

C31

C32

C 33

C34
$-3,907882$

$-1,664930$

$-2,921843$

$-3,074916$

$-4,814509$

$-2,696241$

$-3,642533$

$-4,213623$

$-0,965170$

$-3,325877$

$-2,298196$

$-1,568846$

$-2,947156$

0,396178

0,983531

2,379121

2,363393

$-1,828624$

0,167751

$-2,002419$

$-2,058397$

$-2,016855$

$-1,992600$

$-2,025358$

$-2,049241$

$-1,941128$

$-2,015420$

$-2,057770$

$-2,082024$

1,086804

2,638638

1,599236

3,937840

1,857026
$-3,536817$

$-4,499785$

$-4,361916$

$-3,310526$

$-4,104491$

$-3,868595$

$-2,971044$

$-2,592149$

$-5,279244$

$-5,351089$

$-4,633339$

$-4,631249$

$-5,508962$

$-3,060515$

$-1,977408$

$-2,301557$

$-3,868664$

$-0,739123$

0,014471

$-0,533823$

$-0,113456$

$-1,624699$

0,773519

0,978352

$-1,411643$

1,627695

1,992319

$-2,262793$

0,048871

$-4,200282$

$-1,904851$

$-1,544732$

$-2,021226$

$-1,261635$
$-0,962417$

$-0,956801$

$-1,855378$

0,318844

$-0,728611$

$-2,806159$

1,185743

$-1,414809$

$-1,258447$

$-2,088262$

0,446644

1,262586

0,536972

$-0,312579$

0,055406

0,494194

0,400799

0,373067

$-0,322023$

2,177854

4,944521

3,054456

2,695643

4,071684

4,434894

2,028393

4,460903

5,109657

6,018357

$-0,220805$

1,944521

2,805097

2,460233

4,147457 


\begin{tabular}{|c|c|c|c|c|c|c|c|}
\hline H35 & 0,598045 & $-1,405448$ & 2,408868 & H35 & 0,586751 & $-1,475410$ & 2,432622 \\
\hline $\mathrm{C} 36$ & 4,208285 & $-1,539470$ & 3,785712 & C36 & 4,194305 & $-1,745099$ & 3,801673 \\
\hline H37 & 4,785743 & $-2,174077$ & 1,816353 & H37 & 4,755284 & $-2,313681$ & 1,806563 \\
\hline C38 & 3,160205 & $-1,139032$ & 4,616179 & $\mathrm{C} 38$ & 3,154611 & $-1,354681$ & 4,648461 \\
\hline H39 & 1,030150 & $-0,799896$ & 4,745698 & H39 & 1,033963 & $-0,970065$ & 4,793890 \\
\hline $\mathrm{H} 40$ & 5,222331 & $-1,591504$ & 4,171615 & $\mathrm{H} 40$ & 5,207649 & $-1,830791$ & 4,183464 \\
\hline $\mathrm{H} 41$ & 3,356319 & $-0,872528$ & 5,650769 & H41 & 3,357226 & $-1,131538$ & 5,692032 \\
\hline $\mathrm{C} 42$ & $-3,376310$ & 0,015579 & $-0,341393$ & $\mathrm{C} 42$ & $-3,329952$ & 0,015568 & $-0,362756$ \\
\hline $\mathrm{C} 43$ & $-4,386627$ & 0,582865 & 0,444082 & $\mathrm{C} 43$ & $-4,341373$ & 0,618623 & 0,394769 \\
\hline $\mathrm{C} 44$ & $-3,533888$ & $-0,020954$ & $-1,738013$ & $\mathrm{C} 44$ & $-3,468612$ & $-0,059094$ & $-1,759878$ \\
\hline $\mathrm{C} 45$ & $-5,524547$ & 1,124844 & $-0,157832$ & $\mathrm{C} 45$ & $-5,464259$ & 1,157167 & $-0,237127$ \\
\hline $\mathrm{H} 46$ & $-4,291642$ & 0,611643 & 1,523803 & H46 & $-4,261148$ & 0,674214 & 1,474579 \\
\hline $\mathrm{C} 47$ & $-4,666591$ & 0,525989 & $-2,335582$ & $\mathrm{C} 47$ & $-4,586227$ & 0,486031 & $-2,386269$ \\
\hline $\mathrm{C} 48$ & $-5,663649$ & 1,107125 & $-1,545062$ & $\mathrm{C} 48$ & $-5,585451$ & 1,101433 & $-1,624833$ \\
\hline $\mathrm{H} 49$ & $-6,302475$ & 1,560747 & 0,462581 & H49 & $-6,244482$ & 1,619913 & 0,360472 \\
\hline $\mathrm{H} 50$ & $-4,778785$ & 0,486388 & $-3,415759$ & $\mathrm{H} 50$ & $-4,685660$ & 0,418113 & $-3,466218$ \\
\hline H51 & $-6,548757$ & 1,532405 & $-2,009430$ & H51 & $-6,459502$ & 1,523607 & $-2,112357$ \\
\hline H52 & 0,626889 & 0,460564 & 1,112018 & H52 & 0,467885 & 0,413201 & 1,153718 \\
\hline $\mathrm{H} 53$ & 1,852467 & 0,443352 & $-0,648809$ & $\mathrm{H} 53$ & 1,877691 & 0,879817 & $-0,787723$ \\
\hline H54 & $-1,936881$ & $-2,672771$ & 2,605577 & H54 & $-1,971385$ & $-2,637384$ & 2,669513 \\
\hline H55 & $-2,778672$ & $-0,495492$ & $-2,354946$ & H55 & $-2,710435$ & $-0,559092$ & $-2,352973$ \\
\hline H56 & 3,143119 & $-4,310474$ & $-0,023792$ & H56 & 3,162040 & $-4,284680$ & $-0,213991$ \\
\hline C57 & 5,093236 & $-0,702864$ & $-2,536596$ & C57 & 4,968388 & $-0,444405$ & $-2,462052$ \\
\hline C58 & 4,339454 & $-1,852997$ & $-2,773952$ & C58 & 4,245945 & $-1,597677$ & $-2,771766$ \\
\hline C59 & 3,493654 & $-2,351350$ & $-1,784179$ & C59 & 3,428778 & $-2,189766$ & $-1,810405$ \\
\hline C60 & 3,392427 & $-1,717475$ & $-0,537950$ & C60 & 3,329812 & $-1,651331$ & $-0,519367$ \\
\hline C61 & 4,129472 & $-0,544932$ & $-0,323087$ & C61 & 4,036636 & $-0,477562$ & $-0,228499$ \\
\hline C62 & 4,978448 & $-0,046620$ & $-1,312524$ & C62 & 4,852481 & 0,118460 & $-1,192204$ \\
\hline H63 & 5,751918 & $-0,311848$ & $-3,306256$ & H63 & 5,600883 & 0,022483 & $-3,211021$ \\
\hline H64 & 4,408031 & $-2,363266$ & $-3,730465$ & H64 & 4,314195 & $-2,035279$ & $-3,763716$ \\
\hline $\mathrm{H} 65$ & 2,900697 & $-3,237236$ & $-1,997937$ & H65 & 2,855710 & $-3,073592$ & $-2,079709$ \\
\hline H66 & 4,045526 & $-0,020054$ & 0,621909 & H66 & 3,957107 & $-0,029321$ & 0,755770 \\
\hline H67 & 5,542323 & 0,862867 & $-1,126899$ & H67 & 5,393680 & 1,028120 & $-0,948713$ \\
\hline C68 & $-1,018546$ & 4,399570 & 1,971311 & C68 & $-0,860448$ & 4,488106 & 1,817789 \\
\hline C69 & $-0,294585$ & 3,603150 & 1,086653 & C69 & $-0,142710$ & 3,641548 & 0,973494 \\
\hline $\mathrm{C} 70$ & $-0,925015$ & 2,973972 & 0,000843 & $\mathrm{C} 70$ & $-0,772418$ & 2,969592 & $-0,085844$ \\
\hline C71 & $-2,304763$ & 3,176522 & $-0,168261$ & C71 & $-2,151909$ & 3,181903 & $-0,264534$ \\
\hline $\mathrm{C} 72$ & $-3,028405$ & 3,973632 & 0,717655 & $\mathrm{C} 72$ & $-2,869800$ & 4,026042 & 0,580158 \\
\hline $\mathrm{C} 73$ & $-2,390716$ & 4,590415 & 1,793004 & $\mathrm{C} 73$ & $-2,228626$ & 4,687129 & 1,628035 \\
\hline $\mathrm{H} 74$ & $-0,505783$ & 4,875888 & 2,802251 & $\mathrm{H} 74$ & $-0,343671$ & 4,994852 & 2,628131 \\
\hline H75 & 0,767356 & 3,467625 & 1,261818 & $\mathrm{H} 75$ & 0,914481 & 3,499336 & 1,169473 \\
\hline H76 & $-2,824680$ & 2,679595 & $-0,978842$ & H76 & $-2,677011$ & 2,648398 & $-1,048554$ \\
\hline H77 & $-4,096673$ & 4,100017 & 0,567126 & H77 & $-3,936643$ & 4,155340 & 0,420760 \\
\hline H78 & $-2,952219$ & 5,214076 & 2,482271 & $\mathrm{H} 78$ & $-2,784978$ & 5,347762 & 2,286392 \\
\hline C79 & $-0,182349$ & 2,081541 & $-0,945151$ & C79 & $-0,063987$ & 1,974434 & $-0,959600$ \\
\hline $\mathrm{C} 80$ & 1,283682 & 1,971606 & $-0,934368$ & $\mathrm{C} 80$ & 1,491258 & 1,952635 & $-0,971161$ \\
\hline H81 & 1,769847 & 2,396435 & $-0,061141$ & H81 & 1,895851 & 2,481755 & $-0,110271$ \\
\hline C82 & 2,063191 & 2,209391 & $-2,195562$ & $\mathrm{C} 82$ & 2,165732 & 2,387421 & $-2,246967$ \\
\hline
\end{tabular}




\begin{tabular}{|c|c|c|c|c|c|c|c|}
\hline $\mathrm{C} 83$ & 2,503305 & 3,524422 & $-2,416308$ & C83 & 2,552612 & 3,726008 & $-2,389767$ \\
\hline C84 & 2,322208 & 1,233306 & $-3,160021$ & C84 & 2,348640 & 1,502999 & $-3,314159$ \\
\hline C85 & 3,168371 & 3,859371 & $-3,594266$ & $\mathrm{C} 85$ & 3,097648 & 4,177344 & $-3,592193$ \\
\hline H86 & 2,313641 & 4,287365 & $-1,664301$ & H86 & 2,414463 & 4,417475 & $-1,561678$ \\
\hline C87 & 2,983193 & 1,572368 & $-4,342021$ & $\mathrm{C} 87$ & 2,886986 & 1,956389 & $-4,518997$ \\
\hline H88 & 2,019283 & 0,207858 & $-2,981141$ & H88 & 2,079360 & 0,457401 & $-3,196619$ \\
\hline C89 & 3,402531 & 2,882899 & $-4,566045$ & C89 & 3,258731 & 3,294329 & $-4,662679$ \\
\hline H90 & 3,503197 & 4,880574 & $-3,753224$ & H90 & 3,394057 & 5,217541 & $-3,693830$ \\
\hline H91 & 3,176953 & 0,803356 & $-5,084104$ & H91 & 3,021086 & 1,261541 & $-5,343245$ \\
\hline H92 & 3,916947 & 3,143612 & $-5,486523$ & H92 & 3,678367 & 3,647114 & $-5,600420$ \\
\hline C93 & $-1,010792$ & 3,172859 & $-3,158726$ & C93 & $-0,860106$ & 3,074323 & $-3,222287$ \\
\hline C94 & $-0,751064$ & 0,881822 & $-2,993883$ & C94 & $-0,641907$ & 0,785407 & $-3,004086$ \\
\hline C95 & $-1,660842$ & 2,452266 & $-4,351066$ & C95 & $-1,508255$ & 2,335988 & $-4,405981$ \\
\hline H96 & $-0,059061$ & 3,650487 & $-3,415649$ & H96 & 0,088668 & 3,549379 & $-3,488266$ \\
\hline H97 & $-1,671709$ & 3,906852 & $-2,694850$ & H97 & $-1,521969$ & 3,815820 & $-2,772391$ \\
\hline H98 & $-2,752608$ & 2,434966 & $-4,275335$ & H98 & $-2,601267$ & 2,338919 & $-4,347608$ \\
\hline H99 & $-1,356996$ & 2,836020 & $-5,324804$ & H99 & $-1,183279$ & 2,691472 & $-5,383603$ \\
\hline N100 & $-0,782210$ & 2,023887 & $-2,271215$ & N100 & $-0,633035$ & 1,941099 & $-2,312165$ \\
\hline O101 & $-1,190225$ & 1,075696 & $-4,243615$ & O101 & $-1,066275$ & 0,952252 & $-4,261755$ \\
\hline O102 & $-0,384354$ & $-0,233173$ & $-2,584822$ & O102 & $-0,313698$ & $-0,332843$ & $-2,558744$ \\
\hline H103 & 2,239179 & $-4,221656$ & 1,514152 & H103 & 2,375905 & $-4,332420$ & 1,387800 \\
\hline N104 & $-1,931401$ & $-2,358329$ & $-0,035909$ & N104 & $-1,945580$ & $-2,383268$ & 0,025971 \\
\hline C105 & $-0,966151$ & $-3,027444$ & $-0,945347$ & $\mathrm{C} 105$ & $-1,005525$ & $-3,100915$ & $-0,873520$ \\
\hline H106 & $-0,899334$ & $-2,517034$ & $-1,914169$ & H106 & $-0,951887$ & $-2,629077$ & $-1,863264$ \\
\hline \multicolumn{4}{|c|}{ C1_ZR_Chelbot_TS2 } & \multicolumn{3}{|c|}{ C1_ZR_Chelbot_INT2 } & \\
\hline $\mathrm{C} 1$ & $-4,265594$ & $-2,911447$ & 0,903202 & $\mathrm{C} 1$ & $-4,350695$ & $-2,897125$ & 0,679996 \\
\hline $\mathrm{C} 2$ & $-2,396518$ & $-4,291031$ & 0,165889 & $\mathrm{C} 2$ & $-2,435451$ & $-4,275968$ & 0,077365 \\
\hline $\mathrm{C} 3$ & $-3,898367$ & $-4,035131$ & $-0,120366$ & $\mathrm{C} 3$ & $-3,911982$ & $-4,018420$ & $-0,316786$ \\
\hline $\mathrm{C} 4$ & $-2,917310$ & $-2,661695$ & 1,617148 & $\mathrm{C} 4$ & $-3,055677$ & $-2,647477$ & 1,487462 \\
\hline H5 & $-5,011552$ & $-3,251399$ & 1,629273 & H5 & $-5,143350$ & $-3,240323$ & 1,353281 \\
\hline H6 & $-4,075305$ & $-3,733867$ & $-1,157684$ & H6 & $-4,014260$ & $-3,714519$ & $-1,363519$ \\
\hline $\mathrm{H} 7$ & $-2,982646$ & $-2,098612$ & 2,545761 & $\mathrm{H} 7$ & $-3,189140$ & $-2,087283$ & 2,410874 \\
\hline H8 & $-4,654698$ & $-2,013698$ & 0,423532 & H8 & $-4,710281$ & $-2,001195$ & 0,176063 \\
\hline H9 & $-2,006614$ & $-5,230070$ & $-0,225639$ & H9 & $-2,020161$ & $-5,214869$ & $-0,287350$ \\
\hline H10 & $-4,474875$ & $-4,946764$ & 0,060265 & $\mathrm{H} 10$ & $-4,499997$ & $-4,930582$ & $-0,180516$ \\
\hline $\mathrm{C} 11$ & $-2,326206$ & $-4,078948$ & 1,693140 & $\mathrm{C} 11$ & $-2,476944$ & $-4,067165$ & 1,604947 \\
\hline H12 & $-1,311902$ & $-4,097489$ & 2,103589 & H12 & $-1,495429$ & $-4,089749$ & 2,088350 \\
\hline H13 & $-2,960318$ & $-4,777223$ & 2,246747 & $\mathrm{H} 13$ & $-3,152024$ & $-4,764194$ & 2,109684 \\
\hline C14 & $-0,121802$ & $-3,250116$ & $-0,470988$ & $\mathrm{C} 14$ & $-0,121439$ & $-3,245725$ & $-0,430460$ \\
\hline N15 & 0,759980 & $-2,320511$ & $-0,391729$ & N15 & 0,775480 & $-2,331484$ & $-0,330880$ \\
\hline $\mathrm{C} 16$ & 2,114756 & $-2,922675$ & $-0,616041$ & $\mathrm{C} 16$ & 2,119214 & $-2,954018$ & $-0,597479$ \\
\hline $\mathrm{C} 17$ & 1,758373 & $-4,447585$ & $-0,738622$ & $\mathrm{C} 17$ & 1,743215 & $-4,473308$ & $-0,677998$ \\
\hline P18 & $-1,704937$ & $-0,396317$ & 0,284150 & P18 & $-1,772386$ & $-0,376043$ & 0,245233 \\
\hline Ir19 & 0,414820 & $-0,129807$ & $-0,293349$ & Ir19 & 0,492499 & $-0,117294$ & $-0,203332$ \\
\hline $\mathrm{C} 20$ & $-2,153345$ & 0,511328 & 1,792027 & $\mathrm{C} 20$ & $-2,264261$ & 0,509633 & 1,759838 \\
\hline $\mathrm{C} 21$ & $-2,628221$ & 1,934978 & 4,154547 & $\mathrm{C} 21$ & $-2,825968$ & 1,892088 & 4,135498 \\
\hline $\mathrm{C} 22$ & $-1,647200$ & 0,042319 & 3,016967 & $\mathrm{C} 22$ & $-1,815056$ & 0,012627 & 2,995650 \\
\hline
\end{tabular}




\begin{tabular}{|c|c|c|c|c|c|c|c|}
\hline $\mathrm{C} 23$ & $-2,855496$ & 1,722228 & 1,754785 & $\mathrm{C} 23$ & $-2,957587$ & 1,725415 & 1,722722 \\
\hline $\mathrm{C} 24$ & $-3,096422$ & 2,424091 & 2,937023 & $\mathrm{C} 24$ & $-3,240208$ & 2,406704 & 2,908567 \\
\hline $\mathrm{C} 25$ & $-1,894255$ & 0,744713 & 4,191699 & $\mathrm{C} 25$ & $-2,103228$ & 0,694946 & 4,174363 \\
\hline H26 & $-3,190591$ & 2,141036 & 0,814286 & $\mathrm{H} 26$ & $-3,254808$ & 2,165057 & 0,778921 \\
\hline $\mathrm{H} 27$ & $-3,637962$ & 3,364273 & 2,894569 & $\mathrm{H} 27$ & $-3,774664$ & 3,350875 & 2,861401 \\
\hline $\mathrm{H} 28$ & $-1,508953$ & 0,370069 & 5,135530 & $\mathrm{H} 28$ & $-1,760463$ & 0,295188 & 5,124404 \\
\hline H29 & $-2,819112$ & 2,483928 & 5,072179 & $\mathrm{H} 29$ & $-3,049933$ & 2,423756 & 5,055574 \\
\hline $\mathrm{O} 30$ & 0,305350 & $-4,492009$ & $-0,736667$ & $\mathrm{O} 30$ & 0,291449 & $-4,493476$ & $-0,696141$ \\
\hline C31 & 3,013919 & $-2,741220$ & 0,604716 & $\mathrm{C} 31$ & 3,082186 & $-2,756101$ & 0,568552 \\
\hline C32 & 2,486431 & $-2,362827$ & 1,843855 & $\mathrm{C} 32$ & 2,636806 & $-2,310285$ & 1,817822 \\
\hline $\mathrm{C} 33$ & 4,371621 & $-3,082155$ & 0,524347 & $\mathrm{C} 33$ & 4,422356 & $-3,147379$ & 0,429557 \\
\hline C34 & 3,312138 & $-2,267381$ & 2,964965 & $\mathrm{C} 34$ & 3,524897 & $-2,205613$ & 2,890021 \\
\hline H35 & 1,432463 & $-2,126057$ & 1,927854 & H35 & 1,596985 & $-2,037029$ & 1,950500 \\
\hline C36 & 5,194641 & $-2,991378$ & 1,645861 & $\mathrm{C} 36$ & 5,306179 & $-3,049492$ & 1,502664 \\
\hline H37 & 4,793178 & $-3,409481$ & $-0,421897$ & H37 & 4,780048 & $-3,522362$ & $-0,525416$ \\
\hline C38 & 4,669799 & $-2,570908$ & 2,868809 & C38 & 4,862554 & $-2,566443$ & 2,735042 \\
\hline H39 & 2,889564 & $-1,955903$ & 3,916297 & H39 & 3,164857 & $-1,844209$ & 3,849244 \\
\hline $\mathrm{H} 40$ & 6,246843 & $-3,247737$ & 1,562029 & $\mathrm{H} 40$ & 6,342390 & $-3,348501$ & 1,373983 \\
\hline H41 & 5,312287 & $-2,491767$ & 3,740816 & $\mathrm{H} 41$ & 5,553018 & $-2,481575$ & 3,569021 \\
\hline $\mathrm{C} 42$ & $-2,973783$ & $-0,087851$ & $-1,007621$ & $\mathrm{C} 42$ & $-3,032918$ & $-0,069026$ & $-1,053153$ \\
\hline $\mathrm{C} 43$ & $-4,295991$ & 0,248800 & $-0,671824$ & $\mathrm{C} 43$ & $-4,361120$ & 0,277140 & $-0,755436$ \\
\hline $\mathrm{C} 44$ & $-2,661149$ & $-0,369479$ & $-2,347382$ & C44 & $-2,689244$ & $-0,366205$ & $-2,382935$ \\
\hline $\mathrm{C} 45$ & $-5,272282$ & 0,340194 & $-1,661981$ & $\mathrm{C} 45$ & $-5,313326$ & 0,358437 & $-1,769687$ \\
\hline H46 & $-4,574170$ & 0,436366 & 0,358944 & $\mathrm{H} 46$ & $-4,662587$ & 0,478847 & 0,266223 \\
\hline $\mathrm{C} 47$ & $-3,646470$ & $-0,285959$ & $-3,331568$ & $\mathrm{C} 47$ & $-3,649617$ & $-0,292760$ & $-3,392860$ \\
\hline $\mathrm{C} 48$ & $-4,950611$ & 0,077146 & $-2,994703$ & $\mathrm{C} 48$ & $-4,960820$ & 0,076072 & $-3,091107$ \\
\hline H49 & $-6,286984$ & 0,614535 & $-1,388165$ & $\mathrm{H} 49$ & $-6,334094$ & 0,637972 & $-1,524827$ \\
\hline H50 & $-3,388687$ & $-0,498364$ & $-4,365255$ & H50 & $-3,367659$ & $-0,518296$ & $-4,417493$ \\
\hline H51 & $-5,714413$ & 0,149897 & $-3,763393$ & $\mathrm{H} 51$ & $-5,706445$ & 0,139150 & $-3,878501$ \\
\hline H52 & 0,606833 & $-0,127122$ & 1,257815 & $\mathrm{H} 52$ & 0,533159 & $-0,112644$ & 1,364152 \\
\hline H53 & 2,634819 & 1,945031 & $-0,943192$ & $\mathrm{H} 53$ & 2,684589 & 2,064176 & $-0,876188$ \\
\hline H54 & $-1,050745$ & $-0,865083$ & 3,043481 & $\mathrm{H} 54$ & $-1,235837$ & $-0,905682$ & 3,030013 \\
\hline H55 & $-1,643052$ & $-0,612305$ & $-2,629515$ & H55 & $-1,664799$ & $-0,614881$ & $-2,636296$ \\
\hline H56 & 2,119295 & $-4,912752$ & $-1,656913$ & H56 & 2,107254 & $-4,969371$ & $-1,578426$ \\
\hline $\mathrm{C} 57$ & 3,511446 & $-1,200657$ & $-4,357973$ & C57 & 3,350259 & $-1,387115$ & $-4,467707$ \\
\hline C58 & 2,474400 & $-2,135331$ & $-4,340007$ & $\mathrm{C} 58$ & 2,293432 & $-2,292249$ & $-4,358928$ \\
\hline C59 & 2,052283 & $-2,686615$ & $-3,134034$ & C59 & 1,927199 & $-2,791158$ & $-3,112388$ \\
\hline C60 & 2,663359 & $-2,329325$ & $-1,923427$ & C60 & 2,612117 & $-2,410442$ & $-1,949155$ \\
\hline C61 & 3,691820 & $-1,380250$ & $-1,952144$ & C61 & 3,660718 & $-1,490777$ & $-2,069358$ \\
\hline C62 & 4,113054 & $-0,819165$ & $-3,160500$ & C62 & 4,027226 & $-0,983295$ & $-3,318984$ \\
\hline H63 & 3,843884 & $-0,773419$ & $-5,299945$ & $\mathrm{H} 63$ & 3,639856 & $-1,000653$ & $-5,440788$ \\
\hline H64 & 1,986066 & $-2,424813$ & $-5,265524$ & $\mathrm{H} 64$ & 1,747070 & $-2,601439$ & $-5,244831$ \\
\hline H65 & 1,223660 & $-3,390285$ & $-3,137938$ & H65 & 1,085129 & $-3,475511$ & $-3,047752$ \\
\hline H66 & 4,179450 & $-1,089097$ & $-1,029604$ & H66 & 4,215498 & $-1,179199$ & $-1,191809$ \\
\hline H67 & 4,925727 & $-0,097317$ & $-3,160090$ & H67 & 4,857071 & $-0,284759$ & $-3,389187$ \\
\hline C68 & 1,343299 & 6,233598 & $-1,654507$ & C68 & 1,198887 & 6,248088 & $-1,818479$ \\
\hline C69 & 1,152961 & 4,867377 & $-1,850035$ & C69 & 1,072993 & 4,865594 & $-1,937354$ \\
\hline $\mathrm{C} 70$ & 1,945659 & 3,934266 & $-1,166486$ & $\mathrm{C} 70$ & 1,895505 & 4,006316 & $-1,194044$ \\
\hline
\end{tabular}




\begin{tabular}{|c|c|c|c|c|c|c|c|}
\hline C71 & 2,925684 & 4,397748 & $-0,283650$ & C71 & 2,843583 & 4,564459 & $-0,331596$ \\
\hline $\mathrm{C} 72$ & 3,110953 & 5,766193 & $-0,079721$ & $\mathrm{C} 72$ & 2,965641 & 5,949964 & $-0,204699$ \\
\hline $\mathrm{C} 73$ & 2,320715 & 6,687526 & $-0,766586$ & $\mathrm{C} 73$ & 2,144353 & 6,797086 & $-0,949106$ \\
\hline H74 & 0,718517 & 6,944692 & $-2,187600$ & H74 & 0,549215 & 6,897440 & $-2,398688$ \\
\hline $\mathrm{H} 75$ & 0,368533 & 4,520631 & $-2,515903$ & $\mathrm{H} 75$ & 0,314634 & 4,448413 & $-2,592111$ \\
\hline H76 & 3,546461 & 3,683638 & 0,253600 & H76 & 3,491200 & 3,911784 & 0,250496 \\
\hline H77 & 3,875136 & 6,110952 & 0,611055 & $\mathrm{H} 77$ & 3,706982 & 6,365945 & 0,471504 \\
\hline $\mathrm{H} 78$ & 2,464311 & 7,753070 & $-0,611529$ & $\mathrm{H} 78$ & 2,239627 & 7,874800 & $-0,854434$ \\
\hline C79 & 1,714698 & 2,434511 & $-1,284130$ & C79 & 1,737191 & 2,490357 & $-1,233232$ \\
\hline $\mathrm{C} 80$ & 0,545071 & 1,985334 & $-0,320043$ & $\mathrm{C} 80$ & 0,605102 & 2,015829 & $-0,242737$ \\
\hline H81 & 0,942294 & 2,258358 & 0,664124 & H81 & 1,015116 & 2,301070 & 0,731798 \\
\hline $\mathrm{C} 82$ & $-0,690208$ & 2,826273 & $-0,538697$ & $\mathrm{C} 82$ & $-0,653959$ & 2,824311 & $-0,436937$ \\
\hline $\mathrm{C} 83$ & $-1,526114$ & 2,661356 & $-1,652311$ & C83 & $-1,499662$ & 2,647572 & $-1,541205$ \\
\hline $\mathrm{C} 84$ & $-0,996731$ & 3,859736 & 0,360279 & $\mathrm{C} 84$ & $-0,959783$ & 3,860860 & 0,458732 \\
\hline $\mathrm{C} 85$ & $-2,640039$ & 3,477883 & $-1,849368$ & $\mathrm{C} 85$ & $-2,619370$ & 3,456782 & $-1,735135$ \\
\hline H86 & $-1,321245$ & 1,867815 & $-2,358688$ & H86 & $-1,294833$ & 1,854915 & $-2,247392$ \\
\hline C87 & $-2,100389$ & 4,688124 & 0,163844 & $\mathrm{C} 87$ & $-2,066832$ & 4,684801 & 0,263435 \\
\hline H88 & $-0,352781$ & 4,019057 & 1,221034 & H88 & $-0,308817$ & 4,031783 & 1,312063 \\
\hline C89 & $-2,932126$ & 4,497760 & $-0,941536$ & C89 & $-2,907136$ & 4,483349 & $-0,833900$ \\
\hline H90 & $-3,282961$ & 3,309496 & $-2,709210$ & H90 & $-3,268798$ & 3,277223 & $-2,587854$ \\
\hline H91 & $-2,310313$ & 5,483549 & 0,874007 & H91 & $-2,274331$ & 5,484553 & 0,969317 \\
\hline H92 & $-3,796525$ & 5,137996 & $-1,094649$ & H92 & $-3,774612$ & 5,119832 & $-0,985318$ \\
\hline C93 & 2,391051 & 2,611983 & $-3,766876$ & C93 & 2,423898 & 2,556477 & $-3,713159$ \\
\hline C94 & 0,975105 & 0,915680 & $-3,159240$ & C94 & 0,963038 & 0,910143 & $-3,070061$ \\
\hline C95 & 1,705344 & 2,022441 & $-5,001092$ & C95 & 1,715806 & 1,967326 & $-4,934575$ \\
\hline H96 & 3,422897 & 2,255237 & $-3,646798$ & H96 & 3,446740 & 2,174282 & $-3,594589$ \\
\hline H97 & 2,388376 & 3,700905 & $-3,756395$ & H97 & 2,448771 & 3,645321 & $-3,718143$ \\
\hline H98 & 0,929669 & 2,684612 & $-5,399548$ & H98 & 0,959177 & 2,645886 & $-5,342335$ \\
\hline H99 & 2,390499 & 1,729226 & $-5,796980$ & H99 & 2,388717 & 1,639762 & $-5,727673$ \\
\hline N100 & 1,538125 & 2,056308 & $-2,703999$ & N100 & 1,565199 & 2,040366 & $-2,634777$ \\
\hline O101 & 1,061658 & 0,823442 & $-4,497743$ & O101 & 1,038656 & 0,798095 & $-4,408679$ \\
\hline O102 & 0,424807 & 0,003379 & $-2,523802$ & O102 & 0,379081 & 0,024021 & $-2,428622$ \\
\hline H103 & 2,105455 & $-5,015496$ & 0,125902 & H103 & 2,071683 & $-5,020397$ & 0,207144 \\
\hline N104 & $-1,926185$ & $-2,036466$ & 0,685811 & N104 & $-1,986555$ & $-2,025764$ & 0,644742 \\
\hline C105 & $-1,611257$ & $-3,054354$ & $-0,345297$ & C105 & $-1,613213$ & $-3,037865$ & $-0,370724$ \\
\hline H106 & $-1,976861$ & $-2,762982$ & $-1,342890$ & H106 & $-1,917141$ & $-2,742822$ & $-1,388324$ \\
\hline H107 & 3,184696 & 0,236705 & 0,010217 & H107 & 2,235104 & $-0,062561$ & $-0,578998$ \\
\hline H108 & 3,035165 & 0,236918 & 0,740057 & H108 & 2,180693 & $-0,027832$ & 0,254869 \\
\hline \multicolumn{2}{|c|}{ C1_ZR_Chelbot_TS3 } & \multicolumn{6}{|c|}{ C1_ZR_Chelbot_PROD } \\
\hline $\mathrm{C} 1$ & $-4,378606$ & $-3,027690$ & 0,449091 & $\mathrm{C} 1$ & $-4,378606$ & $-3,027690$ & 0,449091 \\
\hline $\mathrm{C} 2$ & $-2,399283$ & $-4,287599$ & $-0,212623$ & $\mathrm{C} 2$ & $-2,399283$ & $-4,287599$ & $-0,212623$ \\
\hline $\mathrm{C} 3$ & $-3,873445$ & $-4,043290$ & $-0,626116$ & $\mathrm{C} 3$ & $-3,873445$ & $-4,043290$ & $-0,626116$ \\
\hline $\mathrm{C} 4$ & $-3,113888$ & $-2,807595$ & 1,310806 & $\mathrm{C} 4$ & $-3,113888$ & $-2,807595$ & 1,310806 \\
\hline H5 & $-5,176735$ & $-3,454033$ & 1,066162 & H5 & $-5,176735$ & $-3,454033$ & 1,066162 \\
\hline H6 & $-3,958454$ & $-3,653164$ & $-1,645464$ & H6 & $-3,958454$ & $-3,653164$ & $-1,645464$ \\
\hline H7 & $-3,289096$ & $-2,328297$ & 2,273199 & $\mathrm{H} 7$ & $-3,289096$ & $-2,328297$ & 2,273199 \\
\hline $\mathrm{H} 8$ & $-4,755086$ & $-2,102015$ & 0,015707 & $\mathrm{H} 8$ & $-4,755086$ & $-2,102015$ & 0,015707 \\
\hline
\end{tabular}




\begin{tabular}{|c|c|c|c|c|c|c|c|}
\hline H9 & $-1,945859$ & $-5,179554$ & $-0,643236$ & H9 & $-1,945859$ & $-5,179554$ & $-0,643236$ \\
\hline $\mathrm{H} 10$ & $-4,431990$ & $-4,982843$ & $-0,586428$ & $\mathrm{H} 10$ & $-4,431990$ & $-4,982843$ & $-0,586428$ \\
\hline C11 & $-2,489473$ & $-4,213635$ & 1,326896 & C11 & $-2,489473$ & $-4,213635$ & 1,32689 \\
\hline H12 & $-1,520946$ & $-4,245842$ & 1,835469 & H12 & $-1,520946$ & $-4,245842$ & 1,83546 \\
\hline H13 & $-3,152278$ & $-4,974493$ & 1,748723 & H13 & $-3,152278$ & $-4,974493$ & 1,748723 \\
\hline C14 & $-0,106290$ & $-3,144510$ & $-0,569121$ & C14 & $-0,106290$ & $-3,144510$ & $-0,56912$ \\
\hline N15 & 0,777196 & $-2,219449$ & $-0,410801$ & N15 & 0,777196 & $-2,219449$ & $-0,4108$ \\
\hline $\mathrm{C} 16$ & 2,151192 & $-2,817823$ & $-0,582275$ & C16 & 2,151192 & $-2,817823$ & $-0,5822^{\prime}$ \\
\hline $\mathrm{C} 17$ & 1,795730 & $-4,331617$ & $-0,776587$ & $\mathrm{C} 17$ & 1,795730 & $-4,331617$ & $-0,7765$ \\
\hline P18 & $-1,884972$ & $-0,401510$ & 0,276436 & P18 & $-1,884972$ & $-0,401510$ & 0,2764 \\
\hline Ir19 & 0,516610 & $-0,091166$ & $-0,174670$ & Ir19 & 0,516610 & $-0,091166$ & $-0,1746^{\circ}$ \\
\hline $\mathrm{C} 20$ & $-2,254069$ & 0,359488 & 1,891262 & $\mathrm{C} 20$ & $-2,254069$ & 0,359488 & 1,8912 \\
\hline $\mathrm{C} 21$ & $-2,491479$ & 1,645197 & 4,370627 & $\mathrm{C} 21$ & $-2,491479$ & 1,645197 & 4,37062 \\
\hline $\mathrm{C} 22$ & $-1,798057$ & $-0,267141$ & 3,062846 & $\mathrm{C} 22$ & $-1,798057$ & $-0,267141$ & 3,0628 \\
\hline $\mathrm{C} 23$ & $-2,801552$ & 1,647029 & 1,970346 & $\mathrm{C} 23$ & $-2,801552$ & 1,647029 & 1,9703 \\
\hline $\mathrm{C} 24$ & $-2,920912$ & 2,282236 & 3,207318 & $\mathrm{C} 24$ & $-2,920912$ & 2,282236 & 3,2073 \\
\hline $\mathrm{C} 25$ & $-1,925921$ & 0,369488 & 4,294891 & $\mathrm{C} 25$ & $-1,925921$ & 0,369488 & 4,2948 \\
\hline H26 & $-3,114960$ & 2,168770 & 1,073816 & $\mathrm{H} 26$ & $-3,114960$ & 2,168770 & 1,0738 \\
\hline $\mathrm{H} 27$ & $-3,344445$ & 3,281438 & 3,254547 & $\mathrm{H} 27$ & $-3,344445$ & 3,281438 & 3,2545 \\
\hline $\mathrm{H} 28$ & $-1,579811$ & $-0,127462$ & 5,196822 & $\mathrm{H} 28$ & $-1,579811$ & $-0,127462$ & 5,1968 \\
\hline $\mathrm{H} 29$ & $-2,587528$ & 2,141654 & 5,331994 & $\mathrm{H} 29$ & $-2,587528$ & 2,141654 & 5,3319 \\
\hline $\mathrm{O} 30$ & 0,344110 & $-4,370816$ & $-0,844640$ & $\mathrm{O} 30$ & 0,344110 & $-4,370816$ & $-0,8446$ \\
\hline C31 & 2,985822 & $-2,697162$ & 0,692122 & $\mathrm{C} 31$ & 2,985822 & $-2,697162$ & 0,6921 \\
\hline $\mathrm{C} 32$ & 2,380252 & $-2,471807$ & 1,932871 & $\mathrm{C} 32$ & 2,380252 & $-2,471807$ & $1,9328^{\prime}$ \\
\hline $\mathrm{C} 33$ & 4,361191 & $-2,970373$ & 0,649928 & $\mathrm{C} 33$ & 4,361191 & $-2,970373$ & 0,6499 \\
\hline $\mathrm{C} 34$ & 3,141876 & $-2,459749$ & 3,101307 & $\mathrm{C} 34$ & 3,141876 & $-2,459749$ & 3,1013 \\
\hline H35 & 1,311520 & $-2,300800$ & 1,986672 & H35 & 1,311520 & $-2,300800$ & $1,9866^{\circ}$ \\
\hline $\mathrm{C} 36$ & 5,120158 & $-2,964980$ & 1,819660 & C36 & 5,120158 & $-2,964980$ & 1,8196 \\
\hline H37 & 4,844079 & $-3,184622$ & $-0,298603$ & H37 & 4,844079 & $-3,184622$ & $-0,2986$ \\
\hline C38 & 4,515376 & $-2,696982$ & 3,048172 & $\mathrm{C} 38$ & 4,515376 & $-2,696982$ & 3,04817 \\
\hline H39 & 2,657789 & $-2,268118$ & 4,054873 & H39 & 2,657789 & $-2,268118$ & $4,0548^{\prime}$ \\
\hline $\mathrm{H} 40$ & 6,185759 & $-3,169420$ & 1,768577 & $\mathrm{H} 40$ & 6,185759 & $-3,169420$ & $1,7685^{\circ}$ \\
\hline $\mathrm{H} 41$ & 5,108889 & $-2,684373$ & 3,957697 & $\mathrm{H} 41$ & 5,108889 & $-2,684373$ & 3,9576 \\
\hline $\mathrm{C} 42$ & $-3,269587$ & 0,017680 & $-0,861700$ & $\mathrm{C} 42$ & $-3,269587$ & 0,017680 & $-0,8617$ \\
\hline $\mathrm{C} 43$ & $-4,551839$ & 0,398706 & $-0,437945$ & $\mathrm{C} 43$ & $-4,551839$ & 0,398706 & $-0,43794$ \\
\hline $\mathrm{C} 44$ & $-3,039119$ & $-0,187254$ & $-2,233093$ & $\mathrm{C} 44$ & $-3,039119$ & $-0,187254$ & $-2,2330 s$ \\
\hline $\mathrm{C} 45$ & $-5,572120$ & 0,598045 & $-1,367679$ & $\mathrm{C} 45$ & $-5,572120$ & 0,598045 & $-1,36767$ \\
\hline $\mathrm{H} 46$ & $-4,760450$ & 0,538173 & 0,617685 & $\mathrm{H} 46$ & $-4,760450$ & 0,538173 & 0,61768 \\
\hline $\mathrm{C} 47$ & $-4,066751$ & $-0,000257$ & $-3,159052$ & $\mathrm{C} 47$ & $-4,066751$ & $-0,000257$ & $-3,1590$ \\
\hline $\mathrm{C} 48$ & $-5,333664$ & 0,401748 & $-2,730334$ & $\mathrm{C} 48$ & $-5,333664$ & 0,401748 & $-2,73033$ \\
\hline $\mathrm{H} 49$ & $-6,557223$ & 0,902835 & $-1,025546$ & $\mathrm{H} 49$ & $-6,557223$ & 0,902835 & $-1,0255$ \\
\hline $\mathrm{H} 50$ & $-3,874253$ & $-0,158979$ & $-4,216495$ & $\mathrm{H} 50$ & $-3,874253$ & $-0,158979$ & $-4,2164$ \\
\hline H5 1 & $-6,131488$ & 0,556649 & $-3,450720$ & H51 & $-6,131488$ & 0,556649 & $-3,45072$ \\
\hline H52 & 0,562366 & $-0,390766$ & 1,365799 & H52 & 0,562366 & $-0,390766$ & $1,3657 \mathrm{c}$ \\
\hline H53 & 2,685002 & 1,971802 & $-1,117626$ & H53 & 2,685002 & 1,971802 & $-1,11762$ \\
\hline H54 & $-1,333226$ & $-1,247151$ & 3,005697 & H54 & $-1,333226$ & $-1,247151$ & $3,0056 \mathrm{C}$ \\
\hline H55 & $-2,045266$ & $-0,458891$ & $-2,578724$ & $\mathrm{H} 55$ & $-2,045266$ & $-0,458891$ & $-2,57872$ \\
\hline H56 & 2,195709 & $-4,769962$ & $-1,691014$ & H56 & 2,195709 & $-4,769962$ & $-1,69101$ \\
\hline
\end{tabular}




\begin{tabular}{|c|c|c|c|c|c|c|c|}
\hline $\mathrm{C} 57$ & 3,858221 & $-1,042968$ & $-4,169130$ & $\mathrm{C} 57$ & 3,858221 & $-1,042968$ & $-4,169130$ \\
\hline C58 & 2,867649 & $-2,021125$ & $-4,258645$ & $\mathrm{C} 58$ & 2,867649 & $-2,021125$ & $-4,258645$ \\
\hline C59 & 2,336077 & $-2,583068$ & $-3,099465$ & $\mathrm{C} 59$ & 2,336077 & $-2,583068$ & $-3,099465$ \\
\hline C60 & 2,790417 & $-2,194554$ & $-1,832636$ & C60 & 2,790417 & $-2,194554$ & $-1,832636$ \\
\hline C61 & 3,760650 & $-1,185846$ & $-1,754136$ & C61 & 3,760650 & $-1,185846$ & $-1,754136$ \\
\hline C62 & 4,292003 & $-0,617820$ & $-2,912825$ & C62 & 4,292003 & $-0,617820$ & $-2,912825$ \\
\hline H63 & 4,289609 & $-0,616598$ & $-5,071041$ & H63 & 4,289609 & $-0,616598$ & $-5,071041$ \\
\hline H64 & 2,501754 & $-2,342710$ & $-5,228881$ & H64 & 2,501754 & $-2,342710$ & $-5,228881$ \\
\hline H65 & 1,544020 & $-3,321161$ & $-3,193693$ & H65 & 1,544020 & $-3,321161$ & $-3,193693$ \\
\hline H66 & 4,113765 & $-0,848815$ & $-0,787401$ & H66 & 4,113765 & $-0,848815$ & $-0,787401$ \\
\hline H67 & 5,061072 & 0,145725 & $-2,829192$ & H67 & 5,061072 & 0,145725 & $-2,829192$ \\
\hline C68 & 1,267425 & 6,168859 & $-2,182380$ & C68 & 1,267425 & 6,168859 & $-2,182380$ \\
\hline C69 & 1,089014 & 4,787794 & $-2,213848$ & C69 & 1,089014 & 4,787794 & $-2,213848$ \\
\hline $\mathrm{C} 70$ & 1,966483 & 3,943095 & $-1,519425$ & $\mathrm{C} 70$ & 1,966483 & 3,943095 & $-1,519425$ \\
\hline C71 & 3,020425 & 4,506718 & $-0,794617$ & C71 & 3,020425 & 4,506718 & $-0,794617$ \\
\hline $\mathrm{C} 72$ & 3,195776 & 5,891311 & $-0,756804$ & $\mathrm{C} 72$ & 3,195776 & 5,891311 & $-0,756804$ \\
\hline $\mathrm{C} 73$ & 2,319642 & 6,724788 & $-1,451321$ & C73 & 2,319642 & 6,724788 & $-1,451321$ \\
\hline H74 & 0,578686 & 6,812693 & $-2,722001$ & H74 & 0,578686 & 6,812693 & $-2,722001$ \\
\hline H75 & 0,255430 & 4,360912 & $-2,762851$ & $\mathrm{H} 75$ & 0,255430 & 4,360912 & $-2,762851$ \\
\hline H76 & 3,710018 & 3,860502 & $-0,255127$ & H76 & 3,710018 & 3,860502 & $-0,255127$ \\
\hline H77 & 4,019319 & 6,316482 & $-0,190399$ & H77 & 4,019319 & 6,316482 & $-0,190399$ \\
\hline H78 & 2,455740 & 7,802153 & $-1,425316$ & $\mathrm{H} 78$ & 2,455740 & 7,802153 & $-1,425316$ \\
\hline $\mathrm{C} 79$ & 1,750251 & 2,436729 & $-1,455192$ & C79 & 1,750251 & 2,436729 & $-1,455192$ \\
\hline $\mathrm{C} 80$ & 0,673460 & 2,128733 & $-0,360302$ & $\mathrm{C} 80$ & 0,673460 & 2,128733 & $-0,360302$ \\
\hline H81 & 1,143442 & 2,615515 & 0,503572 & H81 & 1,143442 & 2,615515 & 0,503572 \\
\hline C82 & $-0,636732$ & 2,856515 & $-0,521587$ & $\mathrm{C} 82$ & $-0,636732$ & 2,856515 & $-0,521587$ \\
\hline $\mathrm{C} 83$ & $-1,526536$ & 2,597372 & $-1,571205$ & $\mathrm{C} 83$ & $-1,526536$ & 2,597372 & $-1,571205$ \\
\hline C84 & $-0,945937$ & 3,899458 & 0,364781 & C84 & $-0,945937$ & 3,899458 & 0,364781 \\
\hline $\mathrm{C} 85$ & $-2,694719$ & 3,342192 & $-1,723735$ & $\mathrm{C} 85$ & $-2,694719$ & 3,342192 & $-1,723735$ \\
\hline H86 & $-1,318852$ & 1,790119 & $-2,258489$ & H86 & $-1,318852$ & 1,790119 & $-2,258489$ \\
\hline $\mathrm{C} 87$ & $-2,106949$ & 4,655056 & 0,211602 & $\mathrm{C} 87$ & $-2,106949$ & 4,655056 & 0,211602 \\
\hline H88 & $-0,262408$ & 4,128945 & 1,178352 & H88 & $-0,262408$ & 4,128945 & 1,178352 \\
\hline $\mathrm{C} 89$ & $-2,989624$ & 4,376662 & $-0,833697$ & C89 & $-2,989624$ & 4,376662 & $-0,833697$ \\
\hline H90 & $-3,381904$ & 3,098187 & $-2,528898$ & H90 & $-3,381904$ & 3,098187 & $-2,528898$ \\
\hline H91 & $-2,321268$ & 5,460701 & 0,908448 & H91 & $-2,321268$ & 5,460701 & 0,908448 \\
\hline H92 & $-3,899068$ & 4,958890 & $-0,952251$ & H92 & $-3,899068$ & 4,958890 & $-0,952251$ \\
\hline C93 & 2,301501 & 2,339536 & $-3,949388$ & C93 & 2,301501 & 2,339536 & $-3,949388$ \\
\hline C94 & 0,873166 & 0,744320 & $-3,122369$ & C94 & 0,873166 & 0,744320 & $-3,122369$ \\
\hline C95 & 1,598722 & 1,605072 & $-5,095028$ & C95 & 1,598722 & 1,605072 & $-5,095028$ \\
\hline H96 & 3,335848 & 2,002917 & $-3,805729$ & H96 & 3,335848 & 2,002917 & $-3,805729$ \\
\hline H97 & 2,289182 & 3,422271 & $-4,068268$ & H97 & 2,289182 & 3,422271 & $-4,068268$ \\
\hline H98 & 0,843021 & 2,225603 & $-5,586954$ & H98 & 0,843021 & 2,225603 & $-5,586954$ \\
\hline H99 & 2,279890 & 1,187310 & $-5,836870$ & H99 & 2,279890 & 1,187310 & $-5,836870$ \\
\hline N100 & 1,474473 & 1,913520 & $-2,808324$ & N100 & 1,474473 & 1,913520 & $-2,808324$ \\
\hline O101 & 0,919527 & 0,503331 & $-4,441405$ & O101 & 0,919527 & 0,503331 & $-4,441405$ \\
\hline O102 & 0,289191 & $-0,064390$ & $-2,385866$ & O102 & 0,289191 & $-0,064390$ & $-2,385866$ \\
\hline H103 & 2,097937 & $-4,931829$ & 0,082197 & H103 & 2,097937 & $-4,931829$ & 0,082197 \\
\hline N104 & $-2,045885$ & $-2,084187$ & 0,558289 & N104 & $-2,045885$ & $-2,084187$ & 0,558289 \\
\hline
\end{tabular}




$\begin{array}{lrrrrrrr}\text { C105 } & -1,610030 & -2,987370 & -0,532290 & \text { C105 } & -1,610030 & -2,987370 & -0,532290 \\ \text { H106 } & -1,900084 & -2,619624 & -1,528870 & \text { H106 } & -1,900084 & -2,619624 & -1,528870 \\ \text { H107 } & 2,062945 & 0,012316 & -0,199976 & \text { H107 } & 2,062945 & 0,012316 & -0,199976 \\ \text { H108 } & 1,172634 & 1,006051 & 0,770765 & \text { H108 } & 1,172634 & 1,006051 & 0,770765\end{array}$

\section{C1_Z_R_tN_Cheltop}

C1_ZR_Cheltop_SM

\begin{tabular}{|c|c|c|c|c|c|c|c|}
\hline $\mathrm{C} 1$ & $-4,163822$ & $-3,413246$ & 0,500286 & $\mathrm{C} 1$ & $-3,985440$ & $-3,688238$ & $-0,507837$ \\
\hline $\mathrm{C} 2$ & $-2,022887$ & $-4,484285$ & 0,029911 & $\mathrm{C} 2$ & $-1,734715$ & $-4,631017$ & $-0,543501$ \\
\hline $\mathrm{C} 3$ & $-3,482708$ & $-4,418509$ & $-0,485656$ & $\mathrm{C} 3$ & $-3,047673$ & $-4,600110$ & $-1,368599$ \\
\hline $\mathrm{C} 4$ & $-2,990179$ & $-3,023927$ & 1,429779 & $\mathrm{C} 4$ & $-3,087472$ & $-3,351155$ & 0,705208 \\
\hline H5 & $-4,960655$ & $-3,890715$ & 1,080214 & H5 & $-4,883957$ & $-4,222408$ & $-0,182525$ \\
\hline H6 & $-3,537678$ & $-4,090098$ & $-1,528487$ & H6 & $-2,892273$ & $-4,214193$ & $-2,381196$ \\
\hline $\mathrm{H} 7$ & $-3,266325$ & $-2,517402$ & 2,355083 & $\mathrm{H} 7$ & $-3,608539$ & $-2,947615$ & 1,573525 \\
\hline H8 & $-4,593539$ & $-2,548370$ & $-0,004974$ & $\mathrm{H} 8$ & $-4,305188$ & $-2,784063$ & $-1,030201$ \\
\hline H9 & $-1,449989$ & $-5,341411$ & $-0,322379$ & H9 & $-1,034948$ & $-5,420752$ & $-0,816730$ \\
\hline $\mathrm{H} 10$ & $-3,942435$ & $-5,409219$ & $-0,430607$ & $\mathrm{H} 10$ & $-3,451332$ & $-5,612221$ & $-1,462982$ \\
\hline C11 & $-2,222742$ & $-4,350826$ & 1,555913 & $\mathrm{C} 11$ & $-2,280997$ & $-4,646727$ & 0,901562 \\
\hline H12 & $-1,291846$ & $-4,256405$ & 2,123303 & H12 & $-1,506873$ & $-4,558960$ & 1,669698 \\
\hline H13 & $-2,827439$ & $-5,159721$ & 1,975102 & H13 & $-2,906425$ & $-5,520161$ & 1,106645 \\
\hline C14 & 0,151678 & $-3,109060$ & $-0,165865$ & $\mathrm{C} 14$ & 0,326072 & $-3,079454$ & $-0,205062$ \\
\hline N15 & 0,893574 & $-2,055927$ & $-0,124078$ & N15 & 0,939385 & $-1,957501$ & $-0,044093$ \\
\hline $\mathrm{C} 16$ & 2,348581 & $-2,500516$ & $-0,199586$ & C16 & 2,312987 & $-2,246486$ & 0,502841 \\
\hline $\mathrm{C} 17$ & 2,186909 & $-4,027765$ & 0,061654 & $\mathrm{C} 17$ & 2,395602 & $-3,792228$ & 0,269504 \\
\hline P18 & $-1,924114$ & $-0,542219$ & 0,464727 & P18 & $-1,959485$ & $-0,752685$ & 0,333158 \\
\hline Ir19 & 0,274759 & $-0,006737$ & $-0,426515$ & $\operatorname{Ir} 19$ & 0,057576 & $-0,027395$ & $-0,574160$ \\
\hline $\mathrm{C} 20$ & $-2,162716$ & 0,089448 & 2,164020 & $\mathrm{C} 20$ & $-2,264643$ & $-0,340436$ & 2,085234 \\
\hline $\mathrm{C} 21$ & $-2,175954$ & 1,166236 & 4,755891 & $\mathrm{C} 21$ & $-2,578878$ & 0,396040 & 4,770759 \\
\hline $\mathrm{C} 22$ & $-1,462221$ & $-0,543592$ & 3,203525 & $\mathrm{C} 22$ & $-2,042342$ & $-1,275781$ & 3,105793 \\
\hline $\mathrm{C} 23$ & $-2,866203$ & 1,270886 & 2,439398 & $\mathrm{C} 23$ & $-2,636043$ & 0,971563 & 2,422520 \\
\hline $\mathrm{C} 24$ & $-2,872043$ & 1,804441 & 3,729486 & $\mathrm{C} 24$ & $-2,786680$ & 1,335525 & 3,758526 \\
\hline $\mathrm{C} 25$ & $-1,474891$ & $-0,012104$ & 4,489994 & $\mathrm{C} 25$ & $-2,207644$ & $-0,908526$ & 4,441686 \\
\hline H26 & $-3,422200$ & 1,770175 & 1,654765 & $\mathrm{H} 26$ & $-2,813548$ & 1,707959 & 1,646633 \\
\hline $\mathrm{H} 27$ & $-3,430909$ & 2,714014 & 3,931950 & $\mathrm{H} 27$ & $-3,069697$ & 2,354762 & 4,005273 \\
\hline $\mathrm{H} 28$ & $-0,929103$ & $-0,512075$ & 5,284699 & $\mathrm{H} 28$ & $-2,040677$ & $-1,643151$ & 5,224339 \\
\hline $\mathrm{H} 29$ & $-2,180416$ & 1,582866 & 5,758834 & $\mathrm{H} 29$ & $-2,703695$ & 0,679968 & 5,811790 \\
\hline $\mathrm{O} 30$ & 0,770845 & $-4,292897$ & $-0,146065$ & $\mathrm{O} 30$ & 1,018348 & $-4,196436$ & 0,026023 \\
\hline $\mathrm{C} 31$ & 3,198648 & $-1,909446$ & 0,917743 & C31 & 2,383891 & $-1,973353$ & 2,009128 \\
\hline $\mathrm{C} 32$ & 2,602939 & $-1,431603$ & 2,087810 & $\mathrm{C} 32$ & 1,328318 & $-1,423166$ & 2,731866 \\
\hline $\mathrm{C} 33$ & 4,598555 & $-1,958000$ & 0,841655 & $\mathrm{C} 33$ & 3,556796 & $-2,332258$ & 2,692828 \\
\hline C34 & 3,385224 & $-0,937864$ & 3,131366 & C34 & 1,440773 & $-1,218145$ & 4,108391 \\
\hline H35 & 1,525079 & $-1,409146$ & 2,174545 & H35 & 0,423527 & $-1,125694$ & 2,225214 \\
\hline C36 & 5,380990 & $-1,479057$ & 1,891304 & $\mathrm{C} 36$ & 3,671468 & $-2,130130$ & 4,065202 \\
\hline H37 & 5,081246 & $-2,358149$ & $-0,044546$ & H37 & 4,395118 & $-2,753869$ & 2,142682 \\
\hline C38 & 4,776182 & $-0,952864$ & 3,034442 & C38 & 2,610036 & $-1,567748$ & 4,779095 \\
\hline H39 & 2,900404 & $-0,533988$ & 4,015167 & H39 & 0,606278 & $-0,778066$ & 4,646106 \\
\hline $\mathrm{H} 40$ & 6,463771 & $-1,512751$ & 1,811564 & $\mathrm{H} 40$ & 4,589323 & $-2,406990$ & 4,575872 \\
\hline $\mathrm{H} 41$ & 5,386241 & $-0,565986$ & 3,845709 & $\mathrm{H} 41$ & 2,698798 & $-1,404638$ & 5,849405 \\
\hline
\end{tabular}

C1_ZR_Cheltop_TS1

C1 $-3,985440$

C2 $-1,734715$

C3 $-3,047673$

$-3,087472$

$-4,883957$

$-2,892273$

$-3,608539$

$-4,305188$

,034948

451332

$-2,280997$

, 506873

, 770765 


\begin{tabular}{|c|c|c|c|c|c|c|c|}
\hline $\mathrm{C} 42$ & $-3,402936$ & $-0,059681$ & $-0,500964$ & $\mathrm{C} 42$ & $-3,461673$ & $-0,149575$ & $-0,545760$ \\
\hline $\mathrm{C} 43$ & $-4,683414$ & 0,031264 & 0,073048 & $\mathrm{C} 43$ & $-4,679724$ & 0,053980 & 0,121837 \\
\hline $\mathrm{C} 44$ & $-3,265983$ & 0,099566 & $-1,887507$ & C44 & $-3,424286$ & $-0,001169$ & $-1,942236$ \\
\hline $\mathrm{C} 45$ & $-5,795759$ & 0,294955 & $-0,724477$ & $\mathrm{C} 45$ & $-5,823651$ & 0,422240 & $-0,587352$ \\
\hline H46 & $-4,815878$ & $-0,109619$ & 1,141559 & $\mathrm{H} 46$ & $-4,740781$ & $-0,068436$ & 1,198095 \\
\hline $\mathrm{C} 47$ & $-4,382791$ & 0,355496 & $-2,682549$ & $\mathrm{C} 47$ & $-4,570765$ & 0,359940 & $-2,64914$ \\
\hline $\mathrm{C} 48$ & $-5,648482$ & 0,457646 & $-2,104662$ & $\mathrm{C} 48$ & $-5,773174$ & 0,580210 & $-1,97291$ C \\
\hline H49 & $-6,778963$ & 0,367892 & $-0,268299$ & H49 & $-6,755678$ & 0,583359 & $-0,05329$ \\
\hline H50 & $-4,258583$ & 0,492985 & $-3,752287$ & $\mathrm{H} 50$ & $-4,523722$ & 0,472884 & $-3,728586$ \\
\hline H51 & $-6,516452$ & 0,664815 & $-2,723537$ & H51 & $-6,664589$ & 0,867691 & $-2,52239$ \\
\hline H52 & $-0,118265$ & $-0,426696$ & $-1,876820$ & H52 & $-0,510252$ & $-0,644608$ & $-1,88781$ \\
\hline H53 & 1,756879 & $-0,019484$ & $-0,994288$ & $\mathrm{H} 53$ & 1,397277 & 0,442583 & $-1,47452$ \\
\hline H54 & $-0,898290$ & $-1,447363$ & 2,994827 & $\mathrm{H} 54$ & $-1,721794$ & $-2,282353$ & 2,85990 \\
\hline H55 & $-2,280543$ & 0,049830 & $-2,337724$ & $\mathrm{H} 55$ & $-2,495843$ & $-0,169604$ & $-2,47789$ \\
\hline H56 & 2,759819 & $-4,655219$ & $-0,620771$ & $\mathrm{H} 56$ & 2,982088 & $-4,077008$ & $-0,60685$ \\
\hline $\mathrm{C} 57$ & 3,440060 & $-1,516173$ & $-4,289974$ & C57 & 5,278874 & $-0,073434$ & $-1,81818$ \\
\hline C58 & 2,651651 & $-2,634007$ & $-4,010154$ & C58 & 4,545152 & $-1,111458$ & $-2,39665$ \\
\hline C59 & 2,347692 & $-2,964294$ & $-2,691094$ & C59 & 3,583240 & $-1,788578$ & $-1,64868$ \\
\hline C60 & 2,827657 & $-2,190005$ & $-1,625024$ & C60 & 3,359116 & $-1,467652$ & $-0,3024$ \\
\hline C61 & 3,616145 & $-1,069096$ & $-1,919436$ & C61 & 4,079120 & $-0,405985$ & 0,25942 \\
\hline C62 & 3,920989 & $-0,736308$ & $-3,239883$ & C62 & 5,028943 & 0,286194 & $-0,49390$ \\
\hline H63 & 3,677595 & $-1,258404$ & $-5,318104$ & H63 & 6,032949 & 0,452262 & $-2,39708$ \\
\hline H64 & 2,270926 & $-3,249316$ & $-4,820205$ & H64 & 4,715479 & $-1,390389$ & $-3,43258$ \\
\hline H65 & 1,711277 & $-3,824846$ & $-2,502155$ & $\mathrm{H} 65$ & 2,999655 & $-2,568986$ & $-2,1288 \mathrm{c}$ \\
\hline H66 & 3,992277 & $-0,439695$ & $-1,123062$ & H66 & 3,898699 & $-0,116723$ & 1,28781 \\
\hline H67 & 4,533014 & 0,138668 & $-3,440284$ & H67 & 5,587728 & 1,096835 & $-0,03323$ \\
\hline C68 & $-2,453897$ & 3,302128 & $-3,398380$ & C68 & 0,931160 & 3,343253 & $-5,05346$ \\
\hline C69 & $-1,343744$ & 2,760440 & $-2,754242$ & C69 & 0,511598 & 3,021901 & $-3,76165$ \\
\hline $\mathrm{C} 70$ & $-1,295880$ & 2,674830 & $-1,354769$ & $\mathrm{C} 70$ & 1,250817 & 2,119419 & $-2,98734$ \\
\hline C71 & $-2,394101$ & 3,159978 & $-0,628321$ & C71 & 2,416733 & 1,552185 & $-3,51236$ \\
\hline $\mathrm{C} 72$ & $-3,505105$ & 3,700735 & $-1,270648$ & $\mathrm{C} 72$ & 2,833070 & 1,873488 & $-4,80141$ \\
\hline $\mathrm{C} 73$ & $-3,541160$ & 3,774906 & $-2,662024$ & $\mathrm{C} 73$ & 2,089144 & 2,768352 & $-5,57592$ \\
\hline H74 & $-2,464601$ & 3,362781 & $-4,483077$ & $\mathrm{H} 74$ & 0,352978 & 4,044331 & $-5,64820$ \\
\hline H75 & $-0,513075$ & 2,408586 & $-3,356781$ & $\mathrm{H} 75$ & $-0,378273$ & 3,487901 & $-3,34875$ \\
\hline H76 & $-2,371831$ & 3,109198 & 0,453696 & $\mathrm{H} 76$ & 2,989997 & 0,854594 & $-2,91069$ \\
\hline H77 & $-4,342910$ & 4,062848 & $-0,681596$ & $\mathrm{H} 77$ & 3,735000 & 1,422223 & $-5,20490$ \\
\hline $\mathrm{H} 78$ & $-4,403005$ & 4,199897 & $-3,167737$ & $\mathrm{H} 78$ & 2,411881 & 3,015712 & $-6,58303$ \\
\hline $\mathrm{C} 79$ & $-0,105718$ & 2,135046 & $-0,625453$ & C79 & 0,832097 & 1,869472 & $-1,56228$ \\
\hline $\mathrm{C} 80$ & 1,125894 & 1,794340 & $-1,301997$ & $\mathrm{C} 80$ & $-0,606508$ & 1,880847 & $-1,2385$ \\
\hline H81 & 1,049895 & 1,681344 & $-2,378278$ & $\mathrm{H} 81$ & $-1,193797$ & 1,718687 & $-2,13894$ \\
\hline $\mathrm{C} 82$ & 2,448627 & 2,374831 & $-0,891429$ & $\mathrm{C} 82$ & $-1,284687$ & 2,908453 & $-0,38814$ \\
\hline $\mathrm{C} 83$ & 3,258886 & 1,897898 & 0,145576 & $\mathrm{C} 83$ & $-2,452759$ & 3,484804 & $-0,92339$ \\
\hline $\mathrm{C} 84$ & 2,869544 & 3,501593 & $-1,616833$ & C84 & $-0,870269$ & 3,344976 & 0,88260 \\
\hline $\mathrm{C} 85$ & 4,457747 & 2,542401 & 0,457150 & $\mathrm{C} 85$ & $-3,160040$ & 4,470630 & $-0,23867$ \\
\hline H86 & 2,963486 & 1,017598 & 0,704094 & H86 & $-2,818511$ & 3,139636 & $-1,88718$ \\
\hline $\mathrm{C} 87$ & 4,065072 & 4,145460 & $-1,302243$ & C87 & $-1,566655$ & 4,346704 & 1,55931 \\
\hline H88 & 2,252098 & 3,875455 & $-2,430776$ & $\mathrm{H} 88$ & $-0,052512$ & 2,862983 & 1,39618 \\
\hline $\mathrm{C} 89$ & 4,863186 & 3,667668 & $-0,260299$ & C89 & $-2,711491$ & 4,918294 & 1,00483 \\
\hline
\end{tabular}




\begin{tabular}{|c|c|c|c|c|c|c|c|}
\hline H90 & 5,074813 & 2,151098 & 1,260956 & H90 & $-4,061421$ & 4,888643 & $-0,678137$ \\
\hline H91 & 4,375024 & 5,015702 & $-1,874073$ & H91 & $-1,221336$ & 4,661866 & 2,540376 \\
\hline H92 & 5,797544 & 4,165669 & $-0,017714$ & H92 & $-3,254796$ & 5,691960 & 1,539509 \\
\hline C93 & 0,253719 & 4,152200 & 0,963127 & C93 & 2,382860 & 3,788424 & $-0,767224$ \\
\hline C94 & 0,565857 & 1,992161 & 1,718499 & C94 & 1,894209 & 1,996902 & 0,636393 \\
\hline C95 & 0,396116 & 4,111600 & 2,494225 & C95 & 3,297123 & 3,777524 & 0,475681 \\
\hline H96 & $-0,600665$ & 4,744427 & 0,631138 & H96 & 1,629901 & 4,584521 & $-0,736266$ \\
\hline H97 & 1,160012 & 4,506365 & 0,458763 & H97 & 2,941272 & 3,854964 & $-1,702679$ \\
\hline H98 & 1,140008 & 4,802905 & 2,890317 & H98 & 4,319853 & 3,477591 & 0,230771 \\
\hline H99 & $-0,562381$ & 4,255793 & 3,002762 & H99 & 3,305754 & 4,720004 & 1,023389 \\
\hline N100 & 0,050859 & 2,720383 & 0,699010 & N100 & 1,746671 & 2,480445 & $-0,618967$ \\
\hline O101 & 0,845388 & 2,755521 & 2,780340 & O101 & 2,735547 & 2,756401 & 1,350476 \\
\hline O102 & 0,775563 & 0,772568 & 1,698640 & O102 & 1,327054 & 1,001924 & 1,100646 \\
\hline $\mathrm{H} 103$ & 2,418786 & $-4,287215$ & 1,096734 & H103 & 2,750948 & $-4,333692$ & 1,144298 \\
\hline N104 & $-1,976677$ & $-2,223764$ & 0,684957 & N104 & $-1,989625$ & $-2,441503$ & 0,280211 \\
\hline $\mathrm{C} 105$ & $-1,350842$ & $-3,127565$ & $-0,305465$ & C105 & $-1,113954$ & $-3,213520$ & $-0,634717$ \\
\hline H106 & $-1,566896$ & $-2,828804$ & $-1,342630$ & H106 & $-1,162456$ & $-2,841813$ & $-1,667793$ \\
\hline \multicolumn{4}{|c|}{ C1_ZR_Cheltop_INT1 } & \multicolumn{3}{|c|}{ C1_ZR_Cheltop_TS2 } & \\
\hline $\mathrm{C} 1$ & $-3,965371$ & $-3,603242$ & 0,894195 & $\mathrm{C} 1$ & $-4,124279$ & $-3,124928$ & 0,159666 \\
\hline $\mathrm{C} 2$ & $-1,807226$ & $-4,586670$ & 0,328842 & $\mathrm{C} 2$ & $-2,071143$ & $-4,280357$ & $-0,462415$ \\
\hline $\mathrm{C} 3$ & $-3,308515$ & $-4,668443$ & $-0,044655$ & $\mathrm{C} 3$ & $-3,526487$ & $-4,057611$ & $-0,943572$ \\
\hline $\mathrm{C} 4$ & $-2,746872$ & $-3,064935$ & 1,680435 & $\mathrm{C} 4$ & $-2,921098$ & $-2,931449$ & 1,113872 \\
\hline H5 & $-4,688654$ & $-4,056746$ & 1,579988 & H5 & $-4,943845$ & $-3,611002$ & 0,699304 \\
\hline H6 & $-3,482983$ & $-4,463705$ & $-1,105671$ & H6 & $-3,568062$ & $-3,608201$ & $-1,940951$ \\
\hline $\mathrm{H} 7$ & $-2,981112$ & $-2,496035$ & 2,580074 & H7 & $-3,164399$ & $-2,523652$ & 2,094954 \\
\hline $\mathrm{H} 8$ & $-4,477660$ & $-2,811469$ & 0,345697 & $\mathrm{H} 8$ & $-4,504241$ & $-2,184666$ & $-0,236744$ \\
\hline H9 & $-1,203606$ & $-5,431669$ & $-0,001192$ & H9 & $-1,558463$ & $-5,125849$ & $-0,919556$ \\
\hline $\mathrm{H} 10$ & $-3,688950$ & $-5,672947$ & 0,161330 & $\mathrm{H} 10$ & $-4,054480$ & $-5,014082$ & $-0,992957$ \\
\hline $\mathrm{C} 11$ & $-1,878268$ & $-4,322733$ & 1,849076 & $\mathrm{C} 11$ & $-2,250662$ & $-4,315372$ & 1,071898 \\
\hline H12 & $-0,909819$ & $-4,114419$ & 2,314479 & H12 & $-1,311238$ & $-4,357489$ & 1,631160 \\
\hline H13 & $-2,383090$ & $-5,125848$ & 2,393340 & H13 & $-2,911579$ & $-5,122046$ & 1,401071 \\
\hline $\mathrm{C} 14$ & 0,233641 & $-3,113761$ & $-0,248708$ & $\mathrm{C} 14$ & 0,190230 & $-3,038574$ & $-0,521293$ \\
\hline N15 & 0,898406 & $-2,015381$ & $-0,211861$ & N15 & 0,982463 & $-2,039426$ & $-0,338734$ \\
\hline $\mathrm{C} 16$ & 2,362284 & $-2,337685$ & $-0,304198$ & $\mathrm{C} 16$ & 2,375695 & $-2,606747$ & $-0,134967$ \\
\hline $\mathrm{C} 17$ & 2,306569 & $-3,856450$ & $-0,681605$ & $\mathrm{C} 17$ & 2,158346 & $-4,105806$ & $-0,527743$ \\
\hline P18 & $-1,877231$ & $-0,606696$ & 0,489458 & $\mathrm{P} 18$ & $-1,691060$ & $-0,440560$ & 0,483200 \\
\hline Ir19 & 0,052281 & $-0,009391$ & $-0,441195$ & Ir19 & 0,241647 & 0,088237 & $-0,520707$ \\
\hline $\mathrm{C} 20$ & $-2,044303$ & 0,117001 & 2,161606 & $\mathrm{C} 20$ & $-1,692013$ & $-0,024819$ & 2,259580 \\
\hline $\mathrm{C} 21$ & $-1,972406$ & 1,380194 & 4,668642 & $\mathrm{C} 21$ & $-1,509535$ & 0,659845 & 4,970534 \\
\hline $\mathrm{C} 22$ & $-1,302231$ & $-0,436064$ & 3,217533 & $\mathrm{C} 22$ & $-1,219419$ & $-0,956484$ & 3,195247 \\
\hline $\mathrm{C} 23$ & $-2,746097$ & 1,312634 & 2,375558 & $\mathrm{C} 23$ & $-2,068589$ & 1,255335 & 2,694128 \\
\hline $\mathrm{C} 24$ & $-2,710630$ & 1,937755 & 3,623393 & $\mathrm{C} 24$ & $-1,982942$ & 1,590901 & 4,044238 \\
\hline $\mathrm{C} 25$ & $-1,273186$ & 0,188122 & 4,462641 & $\mathrm{C} 25$ & $-1,131467$ & $-0,613943$ & 4,543471 \\
\hline $\mathrm{H} 26$ & $-3,332616$ & 1,749932 & 1,575627 & H26 & $-2,443652$ & 1,980147 & 1,982429 \\
\hline $\mathrm{H} 27$ & $-3,270119$ & 2,856042 & 3,779407 & $\mathrm{H} 27$ & $-2,296176$ & 2,577192 & 4,375737 \\
\hline $\mathrm{H} 28$ & $-0,697809$ & $-0,251712$ & 5,271768 & $\mathrm{H} 28$ & $-0,771434$ & $-1,345983$ & 5,260452 \\
\hline $\mathrm{H} 29$ & $-1,945254$ & 1,867889 & 5,638509 & H29 & $-1,442334$ & 0,923942 & 6,021850 \\
\hline
\end{tabular}




\begin{tabular}{|c|c|c|c|c|c|c|c|}
\hline $\mathrm{O} 30$ & 0,926436 & $-4,244285$ & $-0,422043$ & O30 & 0,717182 & $-4,262340$ & $-0,571687$ \\
\hline C31 & 3,041728 & $-2,165921$ & 1,055857 & $\mathrm{C} 31$ & 2,773039 & $-2,551243$ & 1,345502 \\
\hline C32 & 2,350929 & $-1,707868$ & 2,178550 & $\mathrm{C} 32$ & 1,977616 & $-1,946896$ & 2,314873 \\
\hline C33 & 4,394523 & $-2,519961$ & 1,188266 & $\mathrm{C} 33$ & 3,973539 & $-3,163827$ & 1,740280 \\
\hline C34 & 3,003761 & $-1,578190$ & 3,406301 & $\mathrm{C} 34$ & 2,375367 & $-1,932983$ & 3,653712 \\
\hline H35 & 1,311543 & $-1,426684$ & 2,093481 & H35 & 1,063006 & $-1,456726$ & 2,025763 \\
\hline $\mathrm{C} 36$ & 5,043550 & $-2,395515$ & 2,413868 & $\mathrm{C} 36$ & 4,369878 & $-3,155506$ & 3,074522 \\
\hline H37 & 4,947073 & $-2,880981$ & 0,324681 & H37 & 4,612916 & $-3,631150$ & 0,994762 \\
\hline C38 & 4,349258 & $-1,917785$ & 3,528417 & $\mathrm{C} 38$ & 3,571038 & $-2,533467$ & 4,038366 \\
\hline H39 & 2,453574 & $-1,203494$ & 4,265085 & H39 & 1,747252 & $-1,436035$ & 4,387808 \\
\hline $\mathrm{H} 40$ & 6,091784 & $-2,667457$ & 2,498531 & $\mathrm{H} 40$ & 5,305137 & $-3,627295$ & 3,361900 \\
\hline H41 & 4,856531 & $-1,814740$ & 4,483423 & $\mathrm{H} 41$ & 3,883834 & $-2,518877$ & 5,078564 \\
\hline $\mathrm{C} 42$ & $-3,410829$ & $-0,181838$ & $-0,416465$ & $\mathrm{C} 42$ & $-3,280746$ & 0,135662 & $-0,205942$ \\
\hline $\mathrm{C} 43$ & $-4,651108$ & $-0,192902$ & 0,248425 & $\mathrm{C} 43$ & $-4,388259$ & 0,383808 & 0,621354 \\
\hline $\mathrm{C} 44$ & $-3,379940$ & 0,066275 & $-1,795605$ & C44 & $-3,470630$ & 0,020260 & $-1,59066$ \\
\hline $\mathrm{C} 45$ & $-5,829808$ & 0,047598 & $-0,455155$ & $\mathrm{C} 45$ & $-5,658406$ & 0,530328 & 0,066376 \\
\hline H46 & $-4,698822$ & $-0,384023$ & 1,316619 & $\mathrm{H} 46$ & $-4,273720$ & 0,436284 & 1,69799 \\
\hline $\mathrm{C} 47$ & $-4,563028$ & 0,303476 & $-2,495379$ & $\mathrm{C} 47$ & $-4,744342$ & 0,154177 & $-2,137193$ \\
\hline $\mathrm{C} 48$ & $-5,788971$ & 0,296108 & $-1,829957$ & $\mathrm{C} 48$ & $-5,840110$ & 0,412826 & $-1,312053$ \\
\hline H49 & $-6,780164$ & 0,040644 & 0,070752 & $\mathrm{H} 49$ & $-6,507920$ & 0,722742 & 0,71530 \\
\hline H50 & $-4,519784$ & 0,512808 & $-3,559690$ & $\mathrm{H} 50$ & $-4,878716$ & 0,069294 & $-3,211426$ \\
\hline H51 & $-6,708399$ & 0,486914 & $-2,375627$ & H51 & $-6,831837$ & 0,519912 & $-1,74165$ \\
\hline H52 & $-0,499029$ & $-0,476815$ & $-1,835112$ & H52 & $-0,460208$ & $-0,276032$ & $-1,88113$ \\
\hline H53 & 1,689038 & 0,816110 & $-1,337113$ & $\mathrm{H} 53$ & 2,059941 & 2,372488 & $-1,29992$ \\
\hline H54 & $-0,742926$ & $-1,352240$ & 3,058460 & $\mathrm{H} 54$ & $-0,920574$ & $-1,946289$ & 2,86888 \\
\hline H55 & $-2,429294$ & 0,107492 & $-2,313095$ & $\mathrm{H} 55$ & $-2,621805$ & $-0,170656$ & $-2,23749$ \\
\hline H56 & 2,511863 & $-4,048623$ & $-1,737491$ & $\mathrm{H} 56$ & 2,549328 & $-4,370108$ & $-1,51181$ \\
\hline $\mathrm{C} 57$ & 3,867317 & 0,164987 & $-3,532433$ & C57 & 5,158228 & $-0,529783$ & $-2,74321$ \\
\hline C58 & 2,847442 & $-0,765338$ & $-3,753614$ & C58 & 4,260433 & $-1,461032$ & $-3,26794$ \\
\hline C59 & 2,403420 & $-1,577635$ & $-2,712860$ & C59 & 3,389585 & $-2,146684$ & $-2,42234$ \\
\hline C60 & 2,966512 & $-1,483406$ & $-1,428689$ & C60 & 3,404316 & $-1,924236$ & $-1,03834$ \\
\hline C61 & 3,984541 & $-0,547275$ & $-1,219068$ & C61 & 4,302632 & $-0,980139$ & $-0,52282$ \\
\hline C62 & 4,432934 & 0,267083 & $-2,263058$ & C62 & 5,168410 & $-0,284895$ & $-1,369847$ \\
\hline H63 & 4,211869 & 0,804624 & $-4,339553$ & H63 & 5,836225 & 0,007455 & $-3,39981$ \\
\hline H64 & 2,392485 & $-0,854220$ & $-4,736138$ & H64 & 4,232658 & $-1,650516$ & $-4,33700$ \\
\hline H65 & 1,582436 & $-2,265987$ & $-2,893925$ & $\mathrm{H} 65$ & 2,667664 & $-2,827673$ & $-2,86376$ \\
\hline H66 & 4,420072 & $-0,429997$ & $-0,234874$ & H66 & 4,336193 & $-0,793349$ & 0,54450 \\
\hline H67 & 5,210415 & 0,999553 & $-2,068916$ & H67 & 5,865262 & 0,434807 & $-0,94945$ \\
\hline C68 & $-2,424056$ & 3,268821 & $-3,607780$ & C68 & $-2,906270$ & 2,929868 & $-3,09196$ \\
\hline C69 & $-1,309563$ & 2,726601 & $-2,966457$ & C69 & $-1,709520$ & 2,469765 & $-2,556167$ \\
\hline $\mathrm{C} 70$ & $-1,245890$ & 2,651581 & $-1,567599$ & $\mathrm{C} 70$ & $-1,389169$ & 2,663024 & $-1,19953$ \\
\hline C71 & $-2,346575$ & 3,139198 & $-0,840517$ & C71 & $-2,349622$ & 3,290150 & $-0,39844$ \\
\hline $\mathrm{C} 72$ & $-3,458254$ & 3,682495 & $-1,477034$ & $\mathrm{C} 72$ & $-3,562865$ & 3,738518 & $-0,927104$ \\
\hline $\mathrm{C} 73$ & $-3,503651$ & 3,752468 & $-2,869559$ & $\mathrm{C} 73$ & $-3,842072$ & 3,577863 & $-2,28028$ \\
\hline H74 & $-2,442109$ & 3,318209 & $-4,693081$ & $\mathrm{H} 74$ & $-3,113906$ & 2,776989 & $-4,14755$ \\
\hline H75 & $-0,493242$ & 2,358373 & $-3,578541$ & $\mathrm{H} 75$ & $-1,007090$ & 1,952684 & $-3,20137$ \\
\hline H76 & $-2,329196$ & 3,075794 & 0,241464 & $\mathrm{H} 76$ & $-2,158260$ & 3,439189 & 0,65679 \\
\hline H77 & $-4,292745$ & 4,045800 & $-0,883513$ & $\mathrm{H} 77$ & $-4,285481$ & 4,219204 & $-0,27345$ \\
\hline
\end{tabular}




\begin{tabular}{|c|c|c|c|c|c|c|c|}
\hline H78 & $-4,366815$ & 4,178710 & $-3,372117$ & $\mathrm{H} 78$ & $-4,778638$ & 3,936459 & $-2,696539$ \\
\hline $\mathrm{C} 79$ & $-0,093832$ & 2,039747 & $-0,824433$ & C79 & $-0,029569$ & 2,227352 & $-0,697608$ \\
\hline $\mathrm{C} 80$ & 1,247613 & 1,836162 & $-1,588043$ & $\mathrm{C} 80$ & 1,102930 & 2,767369 & $-1,644807$ \\
\hline H81 & 1,072242 & 1,759075 & $-2,657857$ & H81 & 0,950791 & 2,328878 & $-2,635364$ \\
\hline C82 & 2,347522 & 2,822712 & $-1,288230$ & $\mathrm{C} 82$ & 1,273868 & 4,275258 & $-1,797267$ \\
\hline $\mathrm{C} 83$ & 3,198653 & 2,659181 & $-0,188976$ & C83 & 2,518075 & 4,834894 & $-1,463912$ \\
\hline $\mathrm{C} 84$ & 2,472330 & 3,966178 & $-2,085879$ & C84 & 0,272066 & 5,136045 & $-2,269301$ \\
\hline $\mathrm{C} 85$ & 4,147058 & 3,634875 & 0,119601 & $\mathrm{C} 85$ & 2,751471 & 6,205992 & $-1,570353$ \\
\hline H86 & 3,118931 & 1,765432 & 0,423230 & H86 & 3,314955 & 4,184711 & $-1,107691$ \\
\hline C87 & 3,425807 & 4,938979 & $-1,781682$ & C87 & 0,502362 & 6,508558 & $-2,373769$ \\
\hline H88 & 1,810970 & 4,100688 & $-2,938456$ & H88 & $-0,693117$ & 4,744111 & $-2,560874$ \\
\hline C89 & 4,261765 & 4,778211 & $-0,674464$ & C89 & 1,737337 & 7,052208 & $-2,020228$ \\
\hline H90 & 4,798463 & 3,500396 & 0,978552 & H90 & 3,723183 & 6,611196 & $-1,301455$ \\
\hline H91 & 3,513367 & 5,823056 & $-2,406983$ & H91 & $-0,291972$ & 7,154971 & $-2,737452$ \\
\hline H92 & 5,001354 & 5,537014 & $-0,435650$ & H92 & 1,909433 & 8,121722 & $-2,100760$ \\
\hline C93 & 0,229008 & 4,166322 & 0,734180 & C93 & 0,342734 & 4,149922 & 1,149049 \\
\hline C94 & 0,650785 & 2,029694 & 1,487534 & C94 & 0,928539 & 1,965472 & 1,537772 \\
\hline C95 & 0,431488 & 4,137670 & 2,259971 & C95 & 0,635785 & 3,913148 & 2,635092 \\
\hline H96 & $-0,672629$ & 4,704801 & 0,439315 & H96 & $-0,575311$ & 4,708366 & 0,971136 \\
\hline H97 & 1,088431 & 4,585541 & 0,203589 & H97 & 1,161814 & 4,666400 & 0,646886 \\
\hline H98 & 1,163227 & 4,859632 & 2,622522 & H98 & 1,326996 & 4,634651 & 3,071246 \\
\hline H99 & $-0,509794$ & 4,245409 & 2,808366 & H99 & $-0,273279$ & 3,849496 & 3,241941 \\
\hline N100 & 0,105592 & 2,723211 & 0,469756 & N100 & 0,240239 & 2,745849 & 0,681762 \\
\hline $\mathrm{O} 101$ & 0,944106 & 2,800521 & 2,536980 & O101 & 1,272573 & 2,604302 & 2,661610 \\
\hline $\mathrm{O} 102$ & 0,884939 & 0,805960 & 1,470536 & $\mathrm{O} 102$ & 1,244135 & 0,773003 & 1,350070 \\
\hline H103 & 2,950629 & $-4,473271$ & $-0,057339$ & H103 & 2,544312 & $-4,797287$ & 0,219226 \\
\hline N104 & $-1,859897$ & $-2,275589$ & 0,778054 & N104 & $-1,842443$ & $-2,125764$ & 0,460872 \\
\hline $\mathrm{C} 105$ & $-1,271037$ & $-3,225788$ & $-0,192738$ & $\mathrm{C} 105$ & $-1,307514$ & $-2,942947$ & $-0,651962$ \\
\hline \multirow[t]{3}{*}{ H106 } & $-1,623720$ & $-3,044151$ & $-1,220328$ & H106 & $-1,522293$ & $-2,504147$ & $-1,636701$ \\
\hline & & & & H107 & 2,650155 & 0,421250 & $-1,775188$ \\
\hline & & & & H108 & 2,146376 & 0,370561 & $-2,334344$ \\
\hline \multicolumn{2}{|c|}{ C1_ZR_Cheltop_INT2 } & \multicolumn{6}{|c|}{ C1_ZR_Cheltop_TS3 } \\
\hline $\mathrm{C} 1$ & $-4,167331$ & $-3,142396$ & 0,230583 & $\mathrm{C} 1$ & $-4,121495$ & $-3,584966$ & $-0,338868$ \\
\hline $\mathrm{C} 2$ & $-2,120207$ & $-4,319958$ & $-0,368700$ & $\mathrm{C} 2$ & $-1,920310$ & $-4,598277$ & $-0,615310$ \\
\hline $\mathrm{C} 3$ & $-3,574095$ & $-4,097924$ & $-0,855289$ & $\mathrm{C} 3$ & $-3,301718$ & $-4,495156$ & $-1,312578$ \\
\hline $\mathrm{C} 4$ & $-2,963069$ & $-2,937606$ & 1,181343 & $\mathrm{C} 4$ & $-3,098773$ & $-3,308374$ & 0,787814 \\
\hline H5 & $-4,990534$ & $-3,613905$ & 0,777507 & H5 & $-4,995754$ & $-4,106900$ & 0,063850 \\
\hline H6 & $-3,611611$ & $-3,666187$ & $-1,860660$ & H6 & $-3,225791$ & $-4,076935$ & $-2,321620$ \\
\hline $\mathrm{H} 7$ & $-3,206608$ & $-2,511553$ & 2,154529 & $\mathrm{H} 7$ & $-3,524812$ & $-2,913053$ & 1,709774 \\
\hline $\mathrm{H} 8$ & $-4,540155$ & $-2,206299$ & $-0,182838$ & H8 & $-4,470339$ & $-2,661805$ & $-0,804314$ \\
\hline H9 & $-1,610507$ & $-5,175872$ & $-0,810352$ & H9 & $-1,279640$ & $-5,402957$ & $-0,975753$ \\
\hline H10 & $-4,108041$ & $-5,051783$ & $-0,888602$ & $\mathrm{H} 10$ & $-3,748720$ & $-5,489164$ & $-1,404042$ \\
\hline $\mathrm{C} 11$ & $-2,300112$ & $-4,325805$ & 1,165775 & $\mathrm{C} 11$ & $-2,328372$ & $-4,637079$ & 0,874493 \\
\hline H12 & $-1,360844$ & $-4,361659$ & 1,726012 & H12 & $-1,483219$ & $-4,599438$ & 1,568575 \\
\hline H13 & $-2,964177$ & $-5,123839$ & 1,509209 & H13 & $-2,964832$ & $-5,493754$ & 1,113631 \\
\hline C14 & 0,146935 & $-3,089053$ & $-0,471259$ & C14 & 0,224093 & $-3,145207$ & $-0,445415$ \\
\hline N15 & 0,931379 & $-2,083208$ & $-0,313602$ & N15 & 0,903229 & $-2,061157$ & $-0,268789$ \\
\hline
\end{tabular}




\begin{tabular}{|c|c|c|c|c|c|c|c|}
\hline $\mathrm{C} 16$ & 2,333920 & $-2,614577$ & $-0,131000$ & $\mathrm{C} 16$ & 2,318326 & $-2,448174$ & 0,115215 \\
\hline $\mathrm{C} 17$ & 2,142515 & $-4,106971$ & $-0,551656$ & $\mathrm{C} 17$ & 2,318386 & $-3,934280$ & $-0,344257$ \\
\hline P18 & $-1,721689$ & $-0,457490$ & 0,521379 & P18 & $-1,929437$ & $-0,728332$ & 0,358471 \\
\hline Ir19 & 0,295014 & 0,053284 & $-0,531703$ & Ir19 & 0,197622 & $-0,117626$ & $-0,671197$ \\
\hline $\mathrm{C} 20$ & $-1,727228$ & $-0,025904$ & 2,298803 & $\mathrm{C} 20$ & $-2,113877$ & $-0,352477$ & 2,140993 \\
\hline $\mathrm{C} 21$ & $-1,544160$ & 0,697333 & 5,002364 & $\mathrm{C} 21$ & $-2,191517$ & 0,348189 & 4,859360 \\
\hline $\mathrm{C} 22$ & $-1,260312$ & $-0,944768$ & 3,249369 & $\mathrm{C} 22$ & $-1,860449$ & $-1,313139$ & 3,130245 \\
\hline $\mathrm{C} 23$ & $-2,102608$ & 1,259967 & 2,717323 & $\mathrm{C} 23$ & $-2,399041$ & 0,965678 & 2,53026 \\
\hline $\mathrm{C} 24$ & $-2,015790$ & 1,615021 & 4,062524 & $\mathrm{C} 24$ & $-2,436957$ & 1,311914 & 3,87949 \\
\hline $\mathrm{C} 25$ & $-1,170112$ & $-0,582833$ & 4,592883 & $\mathrm{C} 25$ & $-1,905859$ & $-0,963457$ & 4,480273 \\
\hline $\mathrm{H} 26$ & $-2,477884$ & 1,976285 & 1,996279 & $\mathrm{H} 26$ & $-2,602078$ & 1,721886 & 1,780870 \\
\hline $\mathrm{H} 27$ & $-2,327062$ & 2,606661 & 4,379640 & $\mathrm{H} 27$ & $-2,665016$ & 2,335394 & 4,16509 \\
\hline $\mathrm{H} 28$ & $-0,811567$ & $-1,305714$ & 5,320036 & $\mathrm{H} 28$ & $-1,719882$ & $-1,721085$ & 5,23651 \\
\hline H29 & $-1,475801$ & 0,976409 & 6,049642 & $\mathrm{H} 29$ & $-2,225268$ & 0,617060 & 5,91118 \\
\hline $\mathrm{O} 30$ & 0,699674 & $-4,302332$ & $-0,537972$ & $\mathrm{O} 30$ & 0,902961 & $-4,288804$ & $-0,397266$ \\
\hline C31 & 2,754898 & $-2,555384$ & 1,338414 & $\mathrm{C} 31$ & 2,479782 & $-2,420228$ & 1,641431 \\
\hline C32 & 1,952259 & $-1,988914$ & 2,325148 & $\mathrm{C} 32$ & 1,442956 & $-2,078213$ & 2,50626 \\
\hline $\mathrm{C} 33$ & 3,985499 & $-3,121324$ & 1,706841 & $\mathrm{C} 33$ & 3,711068 & $-2,818651$ & 2,184372 \\
\hline $\mathrm{C} 34$ & 2,372220 & $-1,968822$ & 3,656748 & C34 & 1,631994 & $-2,113368$ & 3,88896 \\
\hline H35 & 1,014189 & $-1,532347$ & 2,055051 & H35 & 0,494635 & $-1,752053$ & 2,10801 \\
\hline C36 & 4,403314 & $-3,108147$ & 3,034566 & $\mathrm{C} 36$ & 3,900250 & $-2,859391$ & 3,56284 \\
\hline H37 & 4,629938 & $-3,556707$ & 0,946893 & H37 & 4,532873 & $-3,079900$ & 1,522528 \\
\hline C38 & 3,596164 & $-2,527025$ & 4,016744 & $\mathrm{C} 38$ & 2,857824 & $-2,502975$ & 4,42262 \\
\hline H39 & 1,737722 & $-1,501584$ & 4,404695 & H39 & 0,814851 & $-1,820907$ & 4,54173 \\
\hline $\mathrm{H} 40$ & 5,361152 & $-3,544804$ & 3,302191 & $\mathrm{H} 40$ & 4,861921 & $-3,164119$ & 3,96575 \\
\hline H41 & 3,925081 & $-2,509685$ & 5,051782 & $\mathrm{H} 41$ & 3,005324 & $-2,528356$ & 5,49857 \\
\hline $\mathrm{C} 42$ & $-3,314848$ & 0,127499 & $-0,159162$ & $\mathrm{C} 42$ & $-3,482109$ & $-0,140448$ & $-0,431548$ \\
\hline $\mathrm{C} 43$ & $-4,431264$ & 0,370387 & 0,657377 & $\mathrm{C} 43$ & $-4,631542$ & 0,244516 & 0,27005 \\
\hline $\mathrm{C} 44$ & $-3,491909$ & 0,044539 & $-1,548380$ & C44 & $-3,529677$ & $-0,192304$ & $-1,83549$ \\
\hline $\mathrm{C} 45$ & $-5,694622$ & 0,537279 & 0,091236 & $\mathrm{C} 45$ & $-5,795953$ & 0,592205 & $-0,41842$ \\
\hline H46 & $-4,327489$ & 0,408672 & 1,736023 & H46 & $-4,625522$ & 0,278216 & 1,35445 \\
\hline $\mathrm{C} 47$ & $-4,758606$ & 0,199519 & $-2,108092$ & $\mathrm{C} 47$ & $-4,697992$ & 0,135933 & $-2,520128$ \\
\hline $\mathrm{C} 48$ & $-5,864683$ & 0,444709 & $-1,291441$ & $\mathrm{C} 48$ & $-5,835227$ & 0,538207 & $-1,81242$ \\
\hline H49 & $-6,549065$ & 0,725545 & 0,735021 & $\mathrm{H} 49$ & $-6,676834$ & 0,897612 & 0,139357 \\
\hline $\mathrm{H} 50$ & $-4,879460$ & 0,140351 & $-3,185676$ & $\mathrm{H} 50$ & $-4,720024$ & 0,087414 & $-3,605367$ \\
\hline H51 & $-6,851077$ & 0,565263 & $-1,729275$ & H51 & $-6,744892$ & 0,801923 & $-2,34413$ \\
\hline H52 & $-0,430829$ & $-0,307752$ & $-1,884546$ & H52 & $-0,275949$ & $-0,697879$ & $-2,058817$ \\
\hline H53 & 2,163318 & 2,376647 & $-1,207793$ & $\mathrm{H} 53$ & 1,571784 & 2,111698 & $-2,46217$ \\
\hline H54 & $-0,964553$ & $-1,940735$ & 2,938582 & H54 & $-1,616013$ & $-2,331718$ & 2,85055 \\
\hline H55 & $-2,636712$ & $-0,129670$ & $-2,192239$ & H55 & $-2,641774$ & $-0,480784$ & $-2,39223$ \\
\hline H56 & 2,497371 & $-4,332806$ & $-1,559657$ & H56 & 2,728151 & $-4,094984$ & $-1,34381$ \\
\hline C57 & 4,691268 & $-0,156526$ & $-2,812719$ & C57 & 4,957007 & 0,270457 & $-2,00464$ \\
\hline C58 & 3,812398 & $-1,117050$ & $-3,320125$ & C58 & 4,357674 & $-0,795060$ & $-2,67956$ \\
\hline $\mathrm{C} 59$ & 3,107298 & $-1,947570$ & $-2,450606$ & C59 & 3,547137 & $-1,696520$ & $-1,989785$ \\
\hline $\mathrm{C} 60$ & 3,266918 & $-1,836901$ & $-1,061985$ & C60 & 3,327256 & $-1,558162$ & $-0,61352$ \\
\hline C61 & 4,148770 & $-0,870997$ & $-0,562625$ & C61 & 3,933787 & $-0,486633$ & 0,05464 \\
\hline C62 & 4,858022 & $-0,039053$ & $-1,433118$ & C62 & 4,740374 & 0,418702 & $-0,63348$ \\
\hline H63 & 5,238483 & 0,494492 & $-3,488241$ & H63 & 5,590123 & 0,971869 & $-2,54091$ \\
\hline
\end{tabular}




\begin{tabular}{|c|c|c|c|c|c|c|c|}
\hline H64 & 3,671094 & $-1,214816$ & $-4,392620$ & H64 & 4,513341 & $-0,921663$ & $-3,747117$ \\
\hline H65 & 2,394357 & $-2,654043$ & $-2,866361$ & H65 & 3,055847 & $-2,485134$ & $-2,551775$ \\
\hline H66 & 4,267262 & $-0,749384$ & 0,507867 & H66 & 3,752957 & $-0,340661$ & 1,112220 \\
\hline H67 & 5,536867 & 0,705619 & $-1,027192$ & H67 & 5,201233 & 1,241011 & $-0,092441$ \\
\hline C68 & $-2,747438$ & 2,847795 & $-3,230580$ & C68 & $-3,543250$ & 3,354554 & $-1,335046$ \\
\hline C69 & $-1,556215$ & 2,420189 & $-2,655721$ & C69 & $-2,310112$ & 2,748477 & $-1,546375$ \\
\hline $\mathrm{C} 70$ & $-1,290833$ & 2,613972 & $-1,287574$ & $\mathrm{C} 70$ & $-1,268525$ & 2,875854 & $-0,614451$ \\
\hline $\mathrm{C} 71$ & $-2,298593$ & 3,204708 & $-0,517348$ & C71 & $-1,510962$ & 3,619170 & 0,546282 \\
\hline $\mathrm{C} 72$ & $-3,504173$ & 3,622573 & $-1,085748$ & C72 & $-2,745277$ & 4,238194 & 0,757875 \\
\hline $\mathrm{C} 73$ & $-3,730178$ & 3,462897 & $-2,448791$ & $\mathrm{C} 73$ & $-3,765526$ & 4,110895 & $-0,181821$ \\
\hline H74 & $-2,913282$ & 2,696410 & $-4,293750$ & $\mathrm{H} 74$ & $-4,335918$ & 3,220443 & $-2,063647$ \\
\hline $\mathrm{H} 75$ & $-0,815526$ & 1,930092 & $-3,279253$ & H75 & $-2,164493$ & 2,160094 & $-2,446379$ \\
\hline H76 & $-2,150677$ & 3,344787 & 0,545501 & H76 & $-0,746648$ & 3,707611 & 1,308161 \\
\hline H77 & $-4,263516$ & 4,076283 & $-0,454953$ & H77 & $-2,905462$ & 4,815646 & 1,664108 \\
\hline $\mathrm{H} 78$ & $-4,661596$ & 3,796784 & $-2,895926$ & $\mathrm{H} 78$ & $-4,727147$ & 4,587120 & $-0,015909$ \\
\hline C79 & 0,055921 & 2,204535 & $-0,732279$ & C79 & 0,089119 & 2,258797 & $-0,920868$ \\
\hline $\mathrm{C} 80$ & 1,227284 & 2,759930 & $-1,624911$ & $\mathrm{C} 80$ & 0,661459 & 2,696300 & $-2,315090$ \\
\hline H81 & 1,133091 & 2,307975 & $-2,617502$ & H81 & $-0,043760$ & 2,347956 & $-3,077034$ \\
\hline C82 & 1,395878 & 4,267404 & $-1,771264$ & $\mathrm{C} 82$ & 0,953046 & 4,168194 & $-2,547633$ \\
\hline $\mathrm{C} 83$ & 2,617232 & 4,838930 & $-1,379540$ & $\mathrm{C} 83$ & 2,268305 & 4,555980 & $-2,841512$ \\
\hline $\mathrm{C} 84$ & 0,407507 & 5,120230 & $-2,285922$ & $\mathrm{C} 84$ & $-0,042096$ & 5,156735 & $-2,520278$ \\
\hline $\mathrm{C} 85$ & 2,840169 & 6,213120 & $-1,469608$ & $\mathrm{C} 85$ & 2,591093 & 5,892927 & $-3,072396$ \\
\hline H86 & 3,403347 & 4,196109 & $-0,987705$ & H86 & 3,048730 & 3,798748 & $-2,885772$ \\
\hline C87 & 0,626489 & 6,495883 & $-2,373016$ & C87 & 0,279931 & 6,495558 & $-2,746363$ \\
\hline H88 & $-0,539970$ & 4,718861 & $-2,620267$ & H88 & $-1,073121$ & 4,887400 & $-2,324982$ \\
\hline C89 & 1,838077 & 7,050499 & $-1,960721$ & C89 & 1,596621 & 6,869900 & $-3,016898$ \\
\hline H90 & 3,793895 & 6,628250 & $-1,155230$ & H90 & 3,617028 & 6,170474 & $-3,297258$ \\
\hline H91 & $-0,157221$ & 7,136479 & $-2,768593$ & H91 & $-0,504199$ & 7,247092 & $-2,717130$ \\
\hline H92 & 2,002243 & 8,122265 & $-2,027678$ & H92 & 1,843324 & 7,912928 & $-3,192971$ \\
\hline C93 & 0,323720 & 4,117108 & 1,133968 & C93 & 1,734702 & 3,873847 & 0,336456 \\
\hline C94 & 0,874088 & 1,931409 & 1,562927 & C94 & 1,402777 & 1,734554 & 1,145503 \\
\hline C95 & 0,528788 & 3,883566 & 2,635121 & C95 & 2,124845 & 3,768592 & 1,818127 \\
\hline H96 & $-0,582740$ & 4,674733 & 0,900810 & H96 & 1,066762 & 4,708503 & 0,123653 \\
\hline H97 & 1,170022 & 4,634125 & 0,681580 & H97 & 2,604297 & 3,953271 & $-0,317753$ \\
\hline H98 & 1,195492 & 4,604109 & 3,109300 & H98 & 3,114541 & 4,167232 & 2,042286 \\
\hline H99 & $-0,413627$ & 3,823642 & 3,188553 & H99 & 1,385450 & 4,225748 & 2,484651 \\
\hline N100 & 0,249495 & 2,714356 & 0,662047 & N100 & 1,063341 & 2,571666 & 0,143243 \\
\hline O101 & 1,158301 & 2,572558 & 2,701524 & O101 & 2,142175 & 2,339305 & 2,082120 \\
\hline $\mathrm{O} 102$ & 1,175888 & 0,731286 & 1,406825 & O102 & 1,116572 & 0,532517 & 1,225294 \\
\hline H103 & 2,579859 & $-4,805299$ & 0,159903 & H103 & 2,802108 & $-4,598458$ & 0,369136 \\
\hline N104 & $-1,880640$ & $-2,147989$ & 0,518467 & N104 & $-2,006714$ & $-2,424261$ & 0,292122 \\
\hline $\mathrm{C} 105$ & $-1,352098$ & $-2,989662$ & $-0,581753$ & $\mathrm{C} 105$ & $-1,257369$ & $-3,200046$ & $-0,724320$ \\
\hline H106 & $-1,570160$ & $-2,567614$ & $-1,573579$ & H106 & $-1,387310$ & $-2,797159$ & $-1,739490$ \\
\hline H107 & 1,956990 & 0,305012 & $-1,078480$ & H107 & 1,588143 & 0,144206 & $-1,404078$ \\
\hline H108 & 1,512272 & 0,162463 & $-1,775920$ & H108 & $-0,370591$ & 1,038500 & $-1,603213$ \\
\hline \multicolumn{8}{|c|}{ C1_Z_S_tN_Cheltop } \\
\hline $\mathrm{C} 1$ & $-4,025728$ & $-3,711207$ & $-0,533282$ & $\mathrm{C} 1$ & $-3,899940$ & $-3,462945$ & $-0,928246$ \\
\hline
\end{tabular}




\begin{tabular}{|c|c|c|c|c|c|c|c|}
\hline $\mathrm{C} 2$ & $-1,807264$ & $-4,712122$ & $-0,673039$ & $\mathrm{C} 2$ & $-1,668150$ & $-4,447952$ & $-0,847333$ \\
\hline $\mathrm{C} 3$ & $-3,148361$ & $-4,630704$ & $-1,446877$ & $\mathrm{C} 3$ & $-2,908687$ & $-4,333484$ & $-1,771731$ \\
\hline $\mathrm{C} 4$ & $-3,078959$ & $-3,432975$ & 0,658050 & $\mathrm{C} 4$ & $-3,090599$ & $-3,210004$ & 0,365116 \\
\hline H5 & $-4,930521$ & $-4,223701$ & $-0,190831$ & H5 & $-4,823053$ & $-4,005966$ & $-0,701228$ \\
\hline H6 & $-3,021104$ & $-4,229752$ & $-2,457491$ & H6 & $-2,664269$ & $-3,882194$ & $-2,738946$ \\
\hline H7 & $-3,565364$ & $-3,043351$ & 1,552091 & $\mathrm{H} 7$ & $-3,666474$ & $-2,842694$ & 1,214719 \\
\hline H8 & $-4,332845$ & $-2,784916$ & $-1,023194$ & H8 & $-4,174585$ & $-2,527456$ & $-1,418895$ \\
\hline H9 & $-1,136493$ & $-5,509633$ & $-0,992897$ & H9 & $-0,964753$ & $-5,237641$ & $-1,112651$ \\
\hline H10 & $-3,583097$ & $-5,629721$ & $-1,543812$ & H10 & $-3,318084$ & $-5,328024$ & $-1,970189$ \\
\hline $\mathrm{C} 11$ & $-2,301168$ & $-4,754309$ & 0,790645 & $\mathrm{C} 11$ & $-2,327892$ & $-4,533824$ & 0,546328 \\
\hline H12 & $-1,498472$ & $-4,705996$ & 1,532418 & H12 & $-1,614970$ & $-4,513656$ & 1,375746 \\
\hline H13 & $-2,940741$ & $-5,618219$ & 0,993068 & $\mathrm{H} 13$ & $-2,986109$ & $-5,401193$ & 0,648800 \\
\hline $\mathrm{C} 14$ & 0,301125 & $-3,214794$ & $-0,406079$ & C14 & 0,370360 & $-2,969254$ & $-0,211017$ \\
\hline N15 & 0,965741 & $-2,110595$ & $-0,284999$ & N15 & 0,988499 & $-1,863789$ & 0,036766 \\
\hline $\mathrm{C} 16$ & 2,355818 & $-2,442367$ & 0,238217 & $\mathrm{C} 16$ & 2,304697 & $-2,178942$ & 0,710469 \\
\hline $\mathrm{C} 17$ & 2,393949 & $-3,956947$ & $-0,111306$ & $\mathrm{C} 17$ & 2,329803 & $-3,740657$ & 0,563276 \\
\hline P18 & $-1,886818$ & $-0,845470$ & 0,285838 & P18 & $-1,899614$ & $-0,617089$ & 0,268061 \\
\hline $\operatorname{Ir} 19$ & 0,248955 & $-0,248985$ & $-0,656488$ & Ir19 & 0,309386 & 0,048477 & $-0,606920$ \\
\hline $\mathrm{C} 20$ & $-2,140431$ & $-0,418736$ & 2,045887 & $\mathrm{C} 20$ & $-2,310644$ & $-0,370818$ & 2,034744 \\
\hline $\mathrm{C} 21$ & $-2,350192$ & 0,392929 & 4,722770 & $\mathrm{C} 21$ & $-2,684252$ & 0,073870 & 4,785902 \\
\hline $\mathrm{C} 22$ & $-2,029939$ & $-1,356699$ & 3,080893 & $\mathrm{C} 22$ & $-2,077025$ & $-1,398318$ & 2,962364 \\
\hline $\mathrm{C} 23$ & $-2,338958$ & 0,934464 & 2,366200 & $\mathrm{C} 23$ & $-2,726802$ & 0,886496 & 2,504566 \\
\hline $\mathrm{C} 24$ & $-2,442645$ & 1,335066 & 3,695343 & $\mathrm{C} 24$ & $-2,909102$ & 1,103280 & 3,869272 \\
\hline $\mathrm{C} 25$ & $-2,143725$ & $-0,951072$ & 4,412294 & $\mathrm{C} 25$ & $-2,269806$ & $-1,177798$ & 4,326294 \\
\hline $\mathrm{H} 26$ & $-2,421185$ & 1,676513 & 1,579077 & $\mathrm{H} 26$ & $-2,910648$ & 1,699578 & 1,813308 \\
\hline $\mathrm{H} 27$ & $-2,597511$ & 2,385252 & 3,926506 & $\mathrm{H} 27$ & $-3,225406$ & 2,084042 & 4,212163 \\
\hline $\mathrm{H} 28$ & $-2,066162$ & $-1,688867$ & 5,206264 & $\mathrm{H} 28$ & $-2,084218$ & $-1,984585$ & 5,029492 \\
\hline $\mathrm{H} 29$ & $-2,435405$ & 0,705233 & 5,759706 & $\mathrm{H} 29$ & $-2,829001$ & 0,245551 & 5,848438 \\
\hline $\mathrm{O} 30$ & 0,987451 & $-4,337825$ & $-0,205646$ & $\mathrm{O} 30$ & 0,993913 & $-4,099585$ & 0,103606 \\
\hline C31 & 2,409873 & $-2,302231$ & 1,765189 & $\mathrm{C} 31$ & 2,289491 & $-1,814237$ & 2,197503 \\
\hline C32 & 1,329469 & $-1,879714$ & 2,535930 & $\mathrm{C} 32$ & 1,248064 & $-1,117821$ & 2,806107 \\
\hline $\mathrm{C} 33$ & 3,599331 & $-2,673048$ & 2,413392 & $\mathrm{C} 33$ & 3,371952 & $-2,231393$ & 2,988953 \\
\hline $\mathrm{C} 34$ & 1,435093 & $-1,810941$ & 3,926933 & C34 & 1,282491 & $-0,836438$ & 4,173890 \\
\hline H35 & 0,406172 & $-1,579376$ & 2,063881 & H35 & 0,412056 & $-0,769021$ & 2,219705 \\
\hline $\mathrm{C} 36$ & 3,704490 & $-2,611083$ & 3,799256 & $\mathrm{C} 36$ & 3,410757 & $-1,949644$ & 4,351999 \\
\hline H37 & 4,455477 & $-2,994767$ & 1,826034 & H37 & 4,203187 & $-2,763099$ & 2,531884 \\
\hline $\mathrm{C} 38$ & 2,618625 & $-2,175109$ & 4,563199 & C38 & 2,361902 & $-1,247629$ & 4,952731 \\
\hline H39 & 0,583589 & $-1,462166$ & 4,503088 & H39 & 0,454965 & $-0,293642$ & 4,620360 \\
\hline $\mathrm{H} 40$ & 4,634190 & $-2,898258$ & 4,282096 & $\mathrm{H} 40$ & 4,261564 & $-2,275693$ & 4,943400 \\
\hline H41 & 2,699680 & $-2,120353$ & 5,645068 & $\mathrm{H} 41$ & 2,390688 & $-1,025775$ & 6,015430 \\
\hline $\mathrm{C} 42$ & $-3,401752$ & $-0,207894$ & $-0,543923$ & $\mathrm{C} 42$ & $-3,345893$ & 0,023878 & $-0,679990$ \\
\hline $\mathrm{C} 43$ & $-4,597138$ & 0,050606 & 0,141999 & $\mathrm{C} 43$ & $-4,582329$ & 0,324457 & $-0,092610$ \\
\hline $\mathrm{C} 44$ & $-3,365291$ & $-0,036256$ & $-1,937711$ & C44 & $-3,225158$ & 0,105335 & $-2,079509$ \\
\hline $\mathrm{C} 45$ & $-5,727357$ & 0,489291 & $-0,549415$ & $\mathrm{C} 45$ & $-5,661634$ & 0,727949 & $-0,882137$ \\
\hline H46 & $-4,645541$ & $-0,076188$ & 1,218955 & $\mathrm{H} 46$ & $-4,712070$ & 0,246973 & 0,981260 \\
\hline $\mathrm{C} 47$ & $-4,497072$ & 0,399416 & $-2,627799$ & $\mathrm{C} 47$ & $-4,305171$ & 0,503342 & $-2,865420$ \\
\hline $\mathrm{C} 48$ & $-5,680581$ & 0,669962 & $-1,933964$ & $\mathrm{C} 48$ & $-5,527331$ & 0,824184 & $-2,267477$ \\
\hline H49 & $-6,645849$ & 0,689646 & $-0,004745$ & $\mathrm{H} 49$ & $-6,610820$ & 0,963565 & $-0,409445$ \\
\hline
\end{tabular}




\begin{tabular}{|c|c|c|c|c|c|c|c|}
\hline H50 & $-4,454895$ & 0,533685 & $-3,705100$ & H50 & $-4,191816$ & 0,563227 & $-3,944302$ \\
\hline H51 & $-6,561052$ & 1,012675 & $-2,469670$ & $\mathrm{H} 51$ & $-6,368294$ & 1,139251 & $-2,878042$ \\
\hline H52 & $-0,197633$ & $-0,791208$ & $-2,051810$ & $\mathrm{H} 52$ & $-0,265516$ & $-0,559521$ & $-1,919076$ \\
\hline H53 & 1,328472 & 2,542302 & $-2,714972$ & $\mathrm{H} 53$ & 1,715643 & $-0,015333$ & $-1,341261$ \\
\hline H54 & $-1,831776$ & $-2,398739$ & 2,854276 & $\mathrm{H} 54$ & $-1,721178$ & $-2,364000$ & 2,620082 \\
\hline H55 & $-2,441941$ & $-0,231626$ & $-2,477157$ & H55 & $-2,282625$ & $-0,144566$ & $-2,558524$ \\
\hline H56 & 2,853002 & $-4,180348$ & $-1,076569$ & H56 & 3,041120 & $-4,098945$ & $-0,183341$ \\
\hline $\mathrm{C} 57$ & 5,174412 & 0,087456 & $-1,870824$ & C57 & 5,365918 & $-0,184809$ & $-1,638215$ \\
\hline C58 & 4,596081 & $-1,006562$ & $-2,516046$ & $\mathrm{C} 58$ & 4,614325 & $-1,227355$ & $-2,185385$ \\
\hline C59 & 3,716546 & $-1,840461$ & $-1,827851$ & C59 & 3,657574 & $-1,876826$ & $-1,408726$ \\
\hline C60 & 3,408994 & $-1,603773$ & $-0,482614$ & C60 & 3,437291 & $-1,505394$ & $-0,074208$ \\
\hline C61 & 3,985906 & $-0,497108$ & 0,153203 & C61 & 4,191208 & $-0,457844$ & 0,463037 \\
\hline C62 & 4,861156 & 0,341000 & $-0,535144$ & C62 & 5,148282 & 0,197153 & $-0,314396$ \\
\hline H63 & 5,863398 & 0,734940 & $-2,406074$ & $\mathrm{H} 63$ & 6,117034 & 0,320482 & $-2,239194$ \\
\hline H64 & 4,823435 & $-1,208339$ & $-3,558751$ & $\mathrm{H} 64$ & 4,768641 & $-1,531178$ & $-3,216852$ \\
\hline H65 & 3,246060 & $-2,655854$ & $-2,368622$ & H65 & 3,054294 & $-2,657610$ & $-1,865254$ \\
\hline H66 & 3,733298 & $-0,272324$ & 1,181879 & H66 & 4,014980 & $-0,130184$ & 1,479628 \\
\hline H67 & 5,301348 & 1,191386 & $-0,020742$ & H67 & 5,725647 & 1,006967 & 0,123274 \\
\hline C68 & $-3,474147$ & 3,527540 & $-0,366109$ & C68 & 0,130934 & 2,435432 & $-5,297935$ \\
\hline C69 & $-2,297175$ & 3,008367 & $-0,897531$ & C69 & $-0,107627$ & 2,425524 & $-3,922096$ \\
\hline $\mathrm{C} 70$ & $-1,064058$ & 3,251792 & $-0,278975$ & $\mathrm{C} 70$ & 0,879381 & 1,976976 & $-3,035478$ \\
\hline $\mathrm{C} 71$ & $-1,029942$ & 4,029798 & 0,881576 & C71 & 2,118938 & 1,564644 & $-3,547949$ \\
\hline $\mathrm{C} 72$ & $-2,207908$ & 4,563477 & 1,409366 & $\mathrm{C} 72$ & 2,358885 & 1,587203 & $-4,918048$ \\
\hline $\mathrm{C} 73$ & $-3,431838$ & 4,312299 & 0,790325 & $\mathrm{C} 73$ & 1,361903 & 2,016342 & $-5,799828$ \\
\hline H74 & $-4,421530$ & 3,300699 & $-0,844668$ & $\mathrm{H} 74$ & $-0,643063$ & 2,786226 & $-5,974650$ \\
\hline H75 & $-2,345121$ & 2,386749 & $-1,785047$ & $\mathrm{H} 75$ & $-1,057181$ & 2,791352 & $-3,544137$ \\
\hline H76 & $-0,089614$ & 4,220703 & 1,387102 & $\mathrm{H} 76$ & 2,884404 & 1,204904 & $-2,865238$ \\
\hline H77 & $-2,165753$ & 5,170683 & 2,309172 & $\mathrm{H} 77$ & 3,321380 & 1,261014 & $-5,302301$ \\
\hline H78 & $-4,348133$ & 4,718486 & 1,208553 & $\mathrm{H} 78$ & 1,548152 & 2,028794 & $-6,869894$ \\
\hline C79 & 0,195135 & 2,675298 & $-0,901786$ & C79 & 0,645922 & 1,978365 & $-1,553598$ \\
\hline $\mathrm{C} 80$ & 0,483066 & 3,137736 & $-2,357131$ & $\mathrm{C} 80$ & $-0,679448$ & 1,986896 & $-1,018926$ \\
\hline H81 & $-0,385474$ & 2,828300 & $-2,948148$ & $\mathrm{H} 81$ & $-1,426494$ & 1,761687 & $-1,768261$ \\
\hline C82 & 0,745800 & 4,616374 & $-2,571059$ & C82 & $-1,250093$ & 2,870914 & 0,034383 \\
\hline $\mathrm{C} 83$ & 1,985200 & 5,034546 & $-3,073480$ & $\mathrm{C} 83$ & $-2,503414$ & 3,439309 & $-0,268464$ \\
\hline $\mathrm{C} 84$ & $-0,230265$ & 5,588154 & $-2,303258$ & $\mathrm{C} 84$ & $-0,664684$ & 3,196604 & 1,269417 \\
\hline $\mathrm{C} 85$ & 2,256213 & 6,387370 & $-3,280946$ & $\mathrm{C} 85$ & $-3,130471$ & 4,321406 & 0,609194 \\
\hline H86 & 2,745917 & 4,292210 & $-3,306367$ & H86 & $-2,995079$ & 3,176174 & $-1,201554$ \\
\hline $\mathrm{C} 87$ & 0,041564 & 6,941422 & $-2,503585$ & C87 & $-1,288367$ & 4,089290 & 2,141216 \\
\hline H88 & $-1,207800$ & 5,291057 & $-1,941767$ & $\mathrm{H} 88$ & 0,235253 & 2,701757 & 1,598506 \\
\hline $\mathrm{C} 89$ & 1,286262 & 7,346520 & $-2,987740$ & C89 & $-2,519754$ & 4,661096 & 1,818717 \\
\hline H90 & 3,222597 & 6,691134 & $-3,673151$ & $\mathrm{H} 90$ & $-4,097111$ & 4,741884 & 0,346709 \\
\hline H91 & $-0,725201$ & 7,680073 & $-2,287223$ & H91 & $-0,814224$ & 4,318097 & 3,091567 \\
\hline H92 & 1,494627 & 8,400956 & $-3,144402$ & H92 & $-3,002538$ & 5,352045 & 2,503443 \\
\hline C93 & 2,308075 & 3,895438 & $-0,002084$ & C93 & 2,278112 & 3,933621 & $-1,094251$ \\
\hline C94 & 1,618688 & 1,894630 & 0,974436 & C94 & 2,127988 & 2,147301 & 0,383256 \\
\hline C95 & 2,931424 & 3,705319 & 1,392575 & C95 & 3,448174 & 3,931379 & $-0,091131$ \\
\hline H96 & 1,801672 & 4,855115 & $-0,112208$ & H96 & 1,538700 & 4,717168 & $-0,889796$ \\
\hline H97 & 3,039883 & 3,797663 & $-0,808085$ & H97 & 2,609428 & 4,010113 & $-2,132018$ \\
\hline
\end{tabular}




\begin{tabular}{|c|c|c|c|c|c|c|c|}
\hline H98 & 4,015912 & 3,817263 & 1,405341 & H98 & 4,384992 & 3,607142 & $-0,554384$ \\
\hline H99 & 2,486390 & 4,365538 & 2,144087 & H99 & 3,594003 & 4,881060 & 0,423496 \\
\hline N100 & 1,357144 & 2,775736 & $-0,028851$ & N100 & 1,715003 & 2,612258 & $-0,815776$ \\
\hline O101 & 2,616095 & 2,337024 & 1,756080 & O101 & 3,081656 & 2,931881 & 0,905300 \\
\hline $\mathrm{O} 102$ & 1,071150 & 0,811152 & 1,188685 & O102 & 1,685716 & 1,144473 & 0,960867 \\
\hline H103 & 2,850827 & $-4,561010$ & 0,669708 & H103 & 2,497492 & $-4,249692$ & 1,510825 \\
\hline N104 & $-1,969066$ & $-2,539188$ & 0,230697 & N104 & $-1,950436$ & $-2,298604$ & 0,069645 \\
\hline $\mathrm{C} 105$ & $-1,161648$ & $-3,304292$ & $-0,753602$ & $\mathrm{C} 105$ & $-1,012849$ & $-3,045795$ & $-0,801422$ \\
\hline H106 & $-1,254334$ & $-2,898491$ & $-1,771112$ & H106 & $-0,946036$ & $-2,619786$ & $-1,811643$ \\
\hline H107 & 1,640099 & 0,070385 & $-1,392492$ & & & & \\
\hline H108 & $-0,005514$ & 1,573306 & $-1,104460$ & & & & \\
\hline $\mathrm{TS}_{\mathrm{Z1-2}}$ & & & & E2 & & & \\
\hline $\mathrm{C} 1$ & $-3,985439$ & $-3,688239$ & $-0,507839$ & $\mathrm{C} 1$ & $-3,953441$ & $-3,737875$ & $-0,490279$ \\
\hline $\mathrm{C} 2$ & $-1,734714$ & $-4,631018$ & $-0,543502$ & $\mathrm{C} 2$ & $-1,738154$ & $-4,720753$ & $-0,164229$ \\
\hline $\mathrm{C} 3$ & $-3,047672$ & $-4,600110$ & $-1,368600$ & $\mathrm{C} 3$ & $-2,974412$ & $-4,797818$ & $-1,099743$ \\
\hline $\mathrm{C} 4$ & $-3,087472$ & $-3,351156$ & 0,705207 & $\mathrm{C} 4$ & $-3,138153$ & $-3,203440$ & 0,708846 \\
\hline H5 & $-4,883956$ & $-4,222408$ & $-0,182527$ & H5 & $-4,887552$ & $-4,196450$ & $-0,149715$ \\
\hline H6 & $-2,892271$ & $-4,214192$ & $-2,381197$ & H6 & $-2,718083$ & $-4,588487$ & $-2,143136$ \\
\hline H7 & $-3,608538$ & $-2,947617$ & 1,573524 & $\mathrm{H} 7$ & $-3,699464$ & $-2,631179$ & 1,447934 \\
\hline $\mathrm{H} 8$ & $-4,305187$ & $-2,784063$ & $-1,030202$ & $\mathrm{H} 8$ & $-4,208213$ & $-2,935662$ & $-1,186266$ \\
\hline H9 & $-1,034947$ & $-5,420752$ & $-0,816732$ & H9 & $-1,054985$ & $-5,567017$ & $-0,232123$ \\
\hline H10 & $-3,451331$ & $-5,612221$ & $-1,462984$ & H10 & $-3,403487$ & $-5,803143$ & $-1,065182$ \\
\hline $\mathrm{C} 11$ & $-2,280996$ & $-4,646728$ & 0,901560 & $\mathrm{C} 11$ & $-2,403616$ & $-4,460701$ & 1,204462 \\
\hline H12 & $-1,506873$ & $-4,558962$ & 1,669697 & H12 & $-1,695799$ & $-4,257593$ & 2,013833 \\
\hline H13 & $-2,906425$ & $-5,520162$ & 1,106643 & H13 & $-3,080965$ & $-5,265083$ & 1,504614 \\
\hline $\mathrm{C} 14$ & 0,326072 & $-3,079454$ & $-0,205062$ & $\mathrm{C} 14$ & 0,352381 & $-3,208348$ & 0,132350 \\
\hline N15 & 0,939385 & $-1,957501$ & $-0,044093$ & N15 & 0,938930 & $-2,084612$ & 0,353717 \\
\hline $\mathrm{C} 16$ & 2,312987 & $-2,246486$ & 0,502841 & $\mathrm{C} 16$ & 2,312079 & $-2,357608$ & 0,896507 \\
\hline $\mathrm{C} 17$ & 2,395602 & $-3,792227$ & 0,269505 & $\mathrm{C} 17$ & 2,399386 & $-3,912739$ & 0,712715 \\
\hline P18 & $-1,959484$ & $-0,752686$ & 0,333158 & $\mathrm{P} 18$ & $-1,888703$ & $-0,737937$ & 0,044494 \\
\hline Ir19 & 0,057576 & $-0,027396$ & $-0,574160$ & Ir19 & 0,187428 & $-0,161789$ & $-0,454795$ \\
\hline $\mathrm{C} 20$ & $-2,264643$ & $-0,340436$ & 2,085234 & $\mathrm{C} 20$ & $-2,299889$ & $-0,139517$ & 1,721759 \\
\hline $\mathrm{C} 21$ & $-2,578879$ & 0,396040 & 4,770758 & $\mathrm{C} 21$ & $-2,717579$ & 0,786808 & 4,333224 \\
\hline $\mathrm{C} 22$ & $-2,042342$ & $-1,275782$ & 3,105793 & $\mathrm{C} 22$ & $-1,938307$ & $-0,932229$ & 2,824504 \\
\hline $\mathrm{C} 23$ & $-2,636043$ & 0,971562 & 2,422520 & $\mathrm{C} 23$ & $-2,851515$ & 1,132469 & 1,941525 \\
\hline $\mathrm{C} 24$ & $-2,786681$ & 1,335525 & 3,758525 & $\mathrm{C} 24$ & $-3,062560$ & 1,586152 & 3,242312 \\
\hline $\mathrm{C} 25$ & $-2,207645$ & $-0,908526$ & 4,441686 & $\mathrm{C} 25$ & $-2,151059$ & $-0,472729$ & 4,122167 \\
\hline $\mathrm{H} 26$ & $-2,813547$ & 1,707959 & 1,646632 & $\mathrm{H} 26$ & $-3,111424$ & 1,772299 & 1,106948 \\
\hline $\mathrm{H} 27$ & $-3,069698$ & 2,354762 & 4,005272 & $\mathrm{H} 27$ & $-3,489709$ & 2,572316 & 3,397605 \\
\hline $\mathrm{H} 28$ & $-2,040678$ & $-1,643151$ & 5,224340 & $\mathrm{H} 28$ & $-1,870762$ & $-1,096577$ & 4,965992 \\
\hline H29 & $-2,703697$ & 0,679968 & 5,811790 & $\mathrm{H} 29$ & $-2,885693$ & 1,144881 & 5,345105 \\
\hline $\mathrm{O} 30$ & 1,018349 & $-4,196435$ & 0,026023 & $\mathrm{O} 30$ & 1,044412 & $-4,324334$ & 0,372189 \\
\hline $\mathrm{C} 31$ & 2,383889 & $-1,973352$ & 2,009129 & $\mathrm{C} 31$ & 2,380339 & $-1,948186$ & 2,370590 \\
\hline $\mathrm{C} 32$ & 1,328316 & $-1,423165$ & 2,731866 & $\mathrm{C} 32$ & 1,487057 & $-1,022863$ & 2,919123 \\
\hline $\mathrm{C} 33$ & 3,556794 & $-2,332257$ & 2,692830 & C33 & 3,378638 & $-2,482996$ & 3,198089 \\
\hline C34 & 1,440769 & $-1,218143$ & 4,108391 & C34 & 1,580104 & $-0,650105$ & 4,25994 \\
\hline H35 & 0,423525 & $-1,125693$ & 2,225213 & H35 & 0,710247 & $-0,587287$ & 2,30569 \\
\hline
\end{tabular}




\begin{tabular}{|c|c|c|c|c|c|c|c|}
\hline C36 & 3,671464 & $-2,130128$ & 4,065205 & $\mathrm{C} 36$ & 3,477552 & $-2,106914$ & 4,536833 \\
\hline H37 & 4,395116 & $-2,753868$ & 2,142685 & H37 & 4,105214 & $-3,184048$ & 2,796142 \\
\hline C38 & 2,610031 & $-1,567746$ & 4,779096 & $\mathrm{C} 38$ & 2,575112 & $-1,188640$ & 5,074459 \\
\hline H39 & 0,606274 & $-0,778064$ & 4,646105 & H39 & 0,865005 & 0,061946 & 4,662431 \\
\hline $\mathrm{H} 40$ & 4,589318 & $-2,406988$ & 4,575876 & $\mathrm{H} 40$ & 4,261272 & $-2,531691$ & 5,157336 \\
\hline H41 & 2,698792 & $-1,404636$ & 5,849406 & $\mathrm{H} 41$ & 2,648653 & $-0,897423$ & 6,118272 \\
\hline $\mathrm{C} 42$ & $-3,461673$ & $-0,149576$ & $-0,545760$ & $\mathrm{C} 42$ & $-3,261470$ & $-0,210928$ & $-1,045786$ \\
\hline $\mathrm{C} 43$ & $-4,679724$ & 0,053978 & 0,121837 & $\mathrm{C} 43$ & $-4,553910$ & 0,010705 & $-0,542540$ \\
\hline $\mathrm{C} 44$ & $-3,424286$ & $-0,001170$ & $-1,942236$ & C44 & $-3,050097$ & $-0,132183$ & $-2,431178$ \\
\hline $\mathrm{C} 45$ & $-5,823651$ & 0,422238 & $-0,587352$ & $\mathrm{C} 45$ & $-5,601979$ & 0,333179 & $-1,404339$ \\
\hline H46 & $-4,740781$ & $-0,068438$ & 1,198095 & $\mathrm{H} 46$ & $-4,746681$ & $-0,060747$ & 0,522902 \\
\hline $\mathrm{C} 47$ & $-4,570765$ & 0,359940 & $-2,649149$ & $\mathrm{C} 47$ & $-4,100752$ & 0,187547 & $-3,290547$ \\
\hline $\mathrm{C} 48$ & $-5,773174$ & 0,580210 & $-1,972919$ & $\mathrm{C} 48$ & $-5,378494$ & 0,428415 & $-2,779273$ \\
\hline H49 & $-6,755678$ & 0,583357 & $-0,053294$ & $\mathrm{H} 49$ & $-6,593981$ & 0,509800 & $-0,998717$ \\
\hline H50 & $-4,523722$ & 0,472884 & $-3,728585$ & $\mathrm{H} 50$ & $-3,920001$ & 0,253308 & $-4,359546$ \\
\hline H51 & $-6,664589$ & 0,867691 & $-2,522395$ & H51 & $-6,194848$ & 0,684656 & $-3,447917$ \\
\hline H52 & $-0,510252$ & $-0,644608$ & $-1,887816$ & H52 & $-0,210303$ & $-0,892075$ & $-1,766382$ \\
\hline H53 & 1,397277 & 0,442584 & $-1,474526$ & $\mathrm{H} 53$ & 1,502939 & 1,415293 & $-2,584303$ \\
\hline H54 & $-1,721795$ & $-2,282353$ & 2,859900 & $\mathrm{H} 54$ & $-1,482651$ & $-1,902800$ & 2,663060 \\
\hline H55 & $-2,495843$ & $-0,169605$ & $-2,477892$ & $\mathrm{H} 55$ & $-2,060850$ & $-0,315823$ & $-2,838128$ \\
\hline H56 & 2,982090 & $-4,077008$ & $-0,606848$ & $\mathrm{H} 56$ & 3,048340 & $-4,200828$ & $-0,118019$ \\
\hline $\mathrm{C} 57$ & 5,278873 & $-0,073433$ & $-1,818179$ & C57 & 5,095729 & $-0,261516$ & $-1,699143$ \\
\hline C58 & 4,545151 & $-1,111456$ & $-2,396650$ & C58 & 4,071229 & $-1,048389$ & $-2,231162$ \\
\hline C59 & 3,583240 & $-1,788577$ & $-1,648683$ & C59 & 3,210425 & $-1,737586$ & $-1,379360$ \\
\hline C60 & 3,359116 & $-1,467651$ & $-0,302455$ & C60 & 3,347437 & $-1,643033$ & 0,013897 \\
\hline C61 & 4,079121 & $-0,405985$ & 0,259428 & C61 & 4,380192 & $-0,862303$ & 0,536560 \\
\hline C62 & 5,028944 & 0,286194 & $-0,493899$ & C62 & 5,250693 & $-0,179801$ & $-0,315432$ \\
\hline H63 & 6,032949 & 0,452263 & $-2,397081$ & H63 & 5,771836 & 0,274035 & $-2,359980$ \\
\hline H64 & 4,715477 & $-1,390387$ & $-3,432588$ & H64 & 3,945300 & $-1,129814$ & $-3,307375$ \\
\hline H65 & 2,999654 & $-2,568983$ & $-2,128895$ & H65 & 2,406824 & $-2,333045$ & $-1,803902$ \\
\hline H66 & 3,898700 & $-0,116724$ & 1,287817 & H66 & 4,489380 & $-0,752496$ & 1,608837 \\
\hline H67 & 5,587729 & 1,096834 & $-0,033231$ & H67 & 6,046153 & 0,425585 & 0,110067 \\
\hline C68 & 0,931158 & 3,343255 & $-5,053463$ & C68 & $-0,096490$ & 5,541896 & $-3,622395$ \\
\hline C69 & 0,511597 & 3,021903 & $-3,761656$ & C69 & 0,233323 & 4,590673 & $-2,658792$ \\
\hline $\mathrm{C} 70$ & 1,250815 & 2,119419 & $-2,987346$ & $\mathrm{C} 70$ & 0,752785 & 3,342021 & $-3,038055$ \\
\hline C71 & 2,416730 & 1,552183 & $-3,512371$ & C71 & 0,922666 & 3,071713 & $-4,400955$ \\
\hline $\mathrm{C} 72$ & 2,833066 & 1,873487 & $-4,801416$ & $\mathrm{C} 72$ & 0,584494 & 4,021052 & $-5,366442$ \\
\hline $\mathrm{C} 73$ & 2,089140 & 2,768352 & $-5,575930$ & $\mathrm{C} 73$ & 0,075345 & 5,260200 & $-4,978737$ \\
\hline H74 & 0,352975 & 4,044333 & $-5,648204$ & $\mathrm{H} 74$ & $-0,497683$ & 6,502327 & $-3,311211$ \\
\hline H75 & $-0,378273$ & 3,487903 & $-3,348752$ & $\mathrm{H} 75$ & 0,067290 & 4,815320 & $-1,611076$ \\
\hline H76 & 2,989994 & 0,854591 & $-2,910695$ & $\mathrm{H} 76$ & 1,321711 & 2,108200 & $-4,710440$ \\
\hline H77 & 3,734994 & 1,422221 & $-5,204911$ & $\mathrm{H} 77$ & 0,723160 & 3,793130 & $-6,419343$ \\
\hline H78 & 2,411876 & 3,015713 & $-6,583040$ & $\mathrm{H} 78$ & $-0,186907$ & 6,002171 & $-5,727390$ \\
\hline C79 & 0,832097 & 1,869472 & $-1,562286$ & C79 & 1,044248 & 2,245448 & $-2,031076$ \\
\hline $\mathrm{C} 80$ & $-0,606508$ & 1,880847 & $-1,238591$ & $\mathrm{C} 80$ & $-0,265587$ & 1,673943 & $-1,389529$ \\
\hline H81 & $-1,193798$ & 1,718687 & $-2,138941$ & $\mathrm{C} 82$ & $-1,024984$ & 2,666259 & $-0,543314$ \\
\hline $\mathrm{C} 82$ & $-1,284687$ & 2,908453 & $-0,388148$ & $\mathrm{C} 83$ & $-2,245642$ & 3,184831 & $-1,009500$ \\
\hline $\mathrm{C} 83$ & $-2,452759$ & 3,484804 & $-0,923393$ & C84 & $-0,556433$ & 3,127941 & 0,700680 \\
\hline
\end{tabular}




\begin{tabular}{|c|c|c|c|c|c|c|c|}
\hline $\mathrm{C} 84$ & $-0,870267$ & 3,344976 & 0,882603 & C85 & $-2,957773$ & 4,137519 & $-0,279817$ \\
\hline $\mathrm{C} 85$ & $-3,160040$ & 4,470629 & $-0,238676$ & H86 & $-2,636470$ & 2,837610 & $-1,961654$ \\
\hline H86 & $-2,818511$ & 3,139636 & $-1,887184$ & C87 & $-1,254269$ & 4,095756 & 1,422746 \\
\hline $\mathrm{C} 87$ & $-1,566653$ & 4,346704 & 1,559319 & H88 & 0,337837 & 2,698355 & 1,137423 \\
\hline H88 & $-0,052510$ & 2,862983 & 1,396188 & C89 & $-2,457290$ & 4,609929 & 0,935008 \\
\hline $\mathrm{C} 89$ & $-2,711489$ & 4,918293 & 1,004836 & H90 & $-3,898704$ & 4,518811 & $-0,667116$ \\
\hline H90 & $-4,061420$ & 4,888642 & $-0,678134$ & H91 & $-0,866349$ & 4,434102 & 2,379931 \\
\hline H91 & $-1,221333$ & 4,661866 & 2,540377 & H92 & $-3,002307$ & 5,361779 & 1,498799 \\
\hline H92 & $-3,254794$ & 5,691960 & 1,539512 & C93 & 2,819586 & 3,935730 & $-1,053641$ \\
\hline C93 & 2,382863 & 3,788423 & $-0,767227$ & C94 & 2,402990 & 1,940863 & 0,020020 \\
\hline C94 & 1,894211 & 1,996902 & 0,636392 & C95 & 3,929974 & 3,603574 & $-0,043900$ \\
\hline C95 & 3,297126 & 3,777523 & 0,475678 & H96 & 2,187015 & 4,765531 & $-0,724590$ \\
\hline H96 & 1,629906 & 4,584521 & $-0,736269$ & H97 & 3,201241 & 4,162454 & $-2,050567$ \\
\hline H97 & 2,941275 & 3,854960 & $-1,702682$ & H98 & 4,844259 & 3,248017 & $-0,529630$ \\
\hline H98 & 4,319856 & 3,477588 & 0,230767 & H99 & 4,163537 & 4,417729 & 0,642112 \\
\hline H99 & 3,305758 & 4,720002 & 1,023385 & N100 & 2,062432 & 2,677108 & $-1,043492$ \\
\hline N100 & 1,746671 & 2,480445 & $-0,618968$ & O101 & 3,390188 & 2,498530 & 0,733012 \\
\hline O101 & 2,735550 & 2,756400 & 1,350474 & O102 & 1,912842 & 0,853126 & 0,391025 \\
\hline O102 & 1,327056 & 1,001924 & 1,100645 & H103 & 2,684994 & $-4,448523$ & 1,616242 \\
\hline $\mathrm{H} 103$ & 2,750948 & $-4,333692$ & 1,144300 & N104 & $-1,982215$ & $-2,416679$ & 0,197585 \\
\hline N104 & $-1,989624$ & $-2,441503$ & 0,280211 & C105 & $-1,042152$ & $-3,360401$ & $-0,435020$ \\
\hline $\mathrm{C} 105$ & $-1,113953$ & $-3,213521$ & $-0,634717$ & H106 & $-0,945914$ & $-3,193184$ & $-1,518434$ \\
\hline H106 & $-1,162455$ & $-2,841813$ & $-1,667793$ & H107 & $-0,880113$ & 1,465577 & $-2,269023$ \\
\hline $\mathrm{TS}_{\mathrm{Z} 2-3}$ & & & & $Z 3$ & & & \\
\hline $\mathrm{C} 1$ & $-3,784872$ & $-3,856984$ & $-0,824496$ & $\mathrm{C} 1$ & $-3,856889$ & $-3,856465$ & $-0,553987$ \\
\hline $\mathrm{C} 2$ & $-1,569341$ & $-4,766921$ & $-0,344530$ & $\mathrm{C} 2$ & $-1,628280$ & $-4,774443$ & $-0,179079$ \\
\hline $\mathrm{C} 3$ & $-2,715698$ & $-4,853284$ & $-1,386188$ & $\mathrm{C} 3$ & $-2,833221$ & $-4,880180$ & $-1,148429$ \\
\hline $\mathrm{C} 4$ & $-3,098714$ & $-3,339821$ & 0,460695 & $\mathrm{C} 4$ & $-3,096015$ & $-3,315245$ & 0,679386 \\
\hline H5 & $-4,723555$ & $-4,364540$ & $-0,579080$ & H5 & $-4,786109$ & $-4,345424$ & $-0,243383$ \\
\hline H6 & $-2,380221$ & $-4,590021$ & $-2,394433$ & H6 & $-2,554541$ & $-4,649644$ & $-2,181748$ \\
\hline $\mathrm{H} 7$ & $-3,746237$ & $-2,822051$ & 1,168502 & $\mathrm{H} 7$ & $-3,703007$ & $-2,779465$ & 1,409012 \\
\hline H8 & $-4,016533$ & $-3,041789$ & $-1,512317$ & H8 & $-4,117588$ & $-3,057258$ & $-1,249608$ \\
\hline H9 & $-0,848680$ & $-5,583639$ & $-0,382257$ & H9 & $-0,913511$ & $-5,595105$ & $-0,238761$ \\
\hline $\mathrm{H} 10$ & $-3,103853$ & $-5,874842$ & $-1,428056$ & $\mathrm{H} 10$ & $-3,232298$ & $-5,898465$ & $-1,137363$ \\
\hline C11 & $-2,358647$ & $-4,587910$ & 0,970445 & $\mathrm{C} 11$ & $-2,337480$ & $-4,557487$ & 1,175392 \\
\hline H12 & $-1,730163$ & $-4,390773$ & 1,843949 & H12 & $-1,658438$ & $-4,344296$ & 2,006428 \\
\hline H13 & $-3,026571$ & $-5,427764$ & 1,181450 & H13 & $-2,997146$ & $-5,387381$ & 1,443955 \\
\hline $\mathrm{C} 14$ & 0,408191 & $-3,184663$ & 0,211601 & $\mathrm{C} 14$ & 0,394613 & $-3,204907$ & 0,200224 \\
\hline N15 & 0,963961 & $-2,043648$ & 0,424959 & N15 & 0,964978 & $-2,064246$ & 0,360772 \\
\hline $\mathrm{C} 16$ & 2,234757 & $-2,277792$ & 1,195318 & $\mathrm{C} 16$ & 2,270616 & $-2,287010$ & 1,077682 \\
\hline $\mathrm{C} 17$ & 2,317787 & $-3,844050$ & 1,185816 & $\mathrm{C} 17$ & 2,390061 & $-3,844310$ & 0,996259 \\
\hline P18 & $-1,927185$ & $-0,803073$ & $-0,036520$ & P18 & $-1,951647$ & $-0,790950$ & 0,060752 \\
\hline Ir19 & 0,150558 & $-0,146561$ & $-0,464542$ & Ir19 & 0,181004 & $-0,192588$ & $-0,581390$ \\
\hline $\mathrm{C} 20$ & $-2,562811$ & $-0,283833$ & 1,596689 & $\mathrm{C} 20$ & $-2,560999$ & $-0,235709$ & 1,694978 \\
\hline $\mathrm{C} 21$ & $-3,378933$ & 0,513153 & 4,155455 & $\mathrm{C} 21$ & $-3,334231$ & 0,660460 & 4,243187 \\
\hline $\mathrm{C} 22$ & $-2,260265$ & $-1,074369$ & 2,718773 & $\mathrm{C} 22$ & $-2,235254$ & $-0,978315$ & 2,842144 \\
\hline $\mathrm{C} 23$ & $-3,275373$ & 0,911749 & 1,770899 & $\mathrm{C} 23$ & $-3,291242$ & 0,952905 & 1,839028 \\
\hline
\end{tabular}




\begin{tabular}{|c|c|c|c|c|c|c|c|}
\hline $\mathrm{C} 24$ & $-3,682050$ & 1,302050 & 3,045624 & $\mathrm{C} 24$ & $-3,670455$ & 1,396542 & 3,106302 \\
\hline $\mathrm{C} 25$ & $-2,666817$ & $-0,677168$ & 3,990146 & $\mathrm{C} 25$ & $-2,619812$ & $-0,532462$ & 4,105728 \\
\hline $\mathrm{H} 26$ & $-3,514773$ & 1,540266 & 0,922877 & $\mathrm{H} 26$ & $-3,568347$ & 1,535444 & 0,969954 \\
\hline $\mathrm{H} 27$ & $-4,231236$ & 2,231075 & 3,165647 & $\mathrm{H} 27$ & $-4,230128$ & 2,322998 & 3,197845 \\
\hline $\mathrm{H} 28$ & $-2,428892$ & $-1,298223$ & 4,848976 & $\mathrm{H} 28$ & $-2,362485$ & $-1,121331$ & 4,981692 \\
\hline H29 & $-3,698175$ & 0,822053 & 5,146911 & $\mathrm{H} 29$ & $-3,633378$ & 1,007546 & 5,228153 \\
\hline $\mathrm{O} 30$ & 1,037374 & $-4,277720$ & 0,649649 & $\mathrm{O} 30$ & 1,063457 & $-4,291504$ & 0,594409 \\
\hline C31 & 2,121461 & $-1,783421$ & 2,639149 & $\mathrm{C} 31$ & 2,186846 & $-1,851379$ & 2,542679 \\
\hline C32 & 1,055323 & $-1,009538$ & 3,093943 & $\mathrm{C} 32$ & 1,109868 & $-1,131274$ & 3,053083 \\
\hline C33 & 3,146234 & $-2,119910$ & 3,536720 & $\mathrm{C} 33$ & 3,247294 & $-2,184252$ & 3,400119 \\
\hline C34 & 0,999821 & $-0,591463$ & 4,425336 & $\mathrm{C} 34$ & 1,081330 & $-0,753876$ & 4,397088 \\
\hline H35 & 0,275396 & $-0,709005$ & 2,410679 & H35 & 0,304162 & $-0,834685$ & 2,401377 \\
\hline C36 & 3,096714 & $-1,698859$ & 4,862403 & $\mathrm{C} 36$ & 3,222430 & $-1,807167$ & 4,740292 \\
\hline H37 & 4,000595 & $-2,697652$ & 3,190527 & H37 & 4,109551 & $-2,722997$ & 3,012661 \\
\hline C38 & 2,017347 & $-0,934197$ & 5,312969 & $\mathrm{C} 38$ & 2,134379 & $-1,089355$ & 5,244731 \\
\hline H39 & 0,156362 & 0,007346 & 4,757301 & H39 & 0,230522 & $-0,190280$ & 4,769109 \\
\hline $\mathrm{H} 40$ & 3,900508 & $-1,964860$ & 5,542748 & $\mathrm{H} 40$ & 4,053200 & $-2,068959$ & 5,389546 \\
\hline H41 & 1,976513 & $-0,606295$ & 6,347806 & H41 & 2,114510 & $-0,793096$ & 6,289748 \\
\hline $\mathrm{C} 42$ & $-3,227567$ & $-0,375815$ & $-1,264093$ & $\mathrm{C} 42$ & $-3,294248$ & $-0,407222$ & $-1,132691$ \\
\hline $\mathrm{C} 43$ & $-4,579077$ & $-0,300979$ & $-0,885696$ & $\mathrm{C} 43$ & $-4,637592$ & $-0,359212$ & $-0,724268$ \\
\hline $\mathrm{C} 44$ & $-2,902639$ & $-0,282369$ & $-2,626649$ & C44 & $-3,002002$ & $-0,307137$ & $-2,501511$ \\
\hline $\mathrm{C} 45$ & $-5,573284$ & $-0,101766$ & $-1,843873$ & $\mathrm{C} 45$ & $-5,657091$ & $-0,185109$ & $-1,659605$ \\
\hline H46 & $-4,864270$ & $-0,396829$ & 0,156077 & $\mathrm{H} 46$ & $-4,896745$ & $-0,455904$ & 0,324835 \\
\hline $\mathrm{C} 47$ & $-3,899580$ & $-0,085494$ & $-3,582593$ & C47 & $-4,024330$ & $-0,141085$ & $-3,435431$ \\
\hline $\mathrm{C} 48$ & $-5,237705$ & 0,012566 & $-3,194218$ & $\mathrm{C} 48$ & $-5,355083$ & $-0,071617$ & $-3,017851$ \\
\hline H49 & $-6,612007$ & $-0,037879$ & $-1,532655$ & $\mathrm{H} 49$ & $-6,689294$ & $-0,140942$ & $-1,323859$ \\
\hline H50 & $-3,630380$ & $-0,005726$ & $-4,631850$ & $\mathrm{H} 50$ & $-3,778913$ & $-0,057252$ & $-4,490425$ \\
\hline H51 & $-6,013455$ & 0,171711 & $-3,937445$ & H51 & $-6,150555$ & 0,065958 & $-3,744228$ \\
\hline H52 & $-0,178846$ & $-0,932762$ & $-1,773420$ & H52 & $-0,253707$ & $-1,007853$ & $-1,852492$ \\
\hline H53 & 1,417830 & 1,999250 & $-2,474531$ & $\mathrm{H} 53$ & 1,625402 & 2,047717 & $-2,297429$ \\
\hline H54 & $-1,708400$ & $-1,999381$ & 2,594531 & $\mathrm{H} 54$ & $-1,683996$ & $-1,907067$ & 2,746089 \\
\hline H55 & $-1,867634$ & $-0,358005$ & $-2,940213$ & $\mathrm{H} 55$ & $-1,971226$ & $-0,343954$ & $-2,835865$ \\
\hline H56 & 3,099690 & $-4,243234$ & 0,537836 & H56 & 3,098841 & $-4,196180$ & 0,243070 \\
\hline $\mathrm{C} 57$ & 5,417760 & $-0,357543$ & $-1,055047$ & C57 & 5,156417 & $-0,021172$ & $-1,243785$ \\
\hline C58 & 4,844092 & $-1,543879$ & $-1,516524$ & C58 & 4,640071 & $-1,221891$ & $-1,738922$ \\
\hline C59 & 3,834855 & $-2,162917$ & $-0,781265$ & C59 & 3,753152 & $-1,972233$ & $-0,967909$ \\
\hline C60 & 3,393807 & $-1,621641$ & 0,434641 & C60 & 3,368536 & $-1,542072$ & 0,310287 \\
\hline C61 & 3,966722 & $-0,427242$ & 0,884233 & C61 & 3,889611 & $-0,337266$ & 0,797647 \\
\hline C62 & 4,968039 & 0,202075 & 0,141254 & C62 & 4,777490 & 0,415551 & 0,025862 \\
\hline H63 & 6,206043 & 0,125908 & $-1,625169$ & H63 & 5,850697 & 0,563039 & $-1,841451$ \\
\hline H64 & 5,174593 & $-1,982062$ & $-2,453705$ & H64 & 4,925614 & $-1,572297$ & $-2,726731$ \\
\hline H65 & 3,375076 & $-3,062201$ & $-1,182077$ & H65 & 3,332514 & $-2,880566$ & $-1,390911$ \\
\hline H66 & 3,621842 & 0,025615 & 1,805281 & H66 & 3,585446 & 0,030906 & 1,769956 \\
\hline H67 & 5,403709 & 1,125267 & 0,513258 & H67 & 5,177826 & 1,342755 & 0,427841 \\
\hline C68 & $-0,338272$ & 6,178271 & $-2,151870$ & C68 & $-0,140097$ & 6,219147 & $-2,100726$ \\
\hline C69 & 0,171865 & 5,024770 & $-1,559865$ & C69 & 0,269783 & 5,044523 & $-1,473087$ \\
\hline $\mathrm{C} 70$ & 0,438801 & 3,886833 & $-2,331875$ & $\mathrm{C} 70$ & 0,626590 & 3,920436 & $-2,228875$ \\
\hline C71 & 0,194030 & 3,930504 & $-3,707664$ & C71 & 0,575491 & 4,000906 & $-3,624296$ \\
\hline
\end{tabular}




\begin{tabular}{|c|c|c|c|c|c|c|c|}
\hline $\mathrm{C} 72$ & $-0,322333$ & 5,082803 & $-4,301545$ & $\mathrm{C} 72$ & 0,160622 & 5,174091 & $-4,255184$ \\
\hline $\mathrm{C} 73$ & $-0,591723$ & 6,209518 & $-3,523887$ & C73 & $-0,200388$ & 6,286549 & $-3,493775$ \\
\hline H74 & $-0,549836$ & 7,049373 & $-1,538059$ & H74 & $-0,422835$ & 7,079149 & $-1,499705$ \\
\hline H75 & 0,329234 & 4,994483 & $-0,486377$ & $\mathrm{H} 75$ & 0,280368 & 4,988785 & $-0,389596$ \\
\hline H76 & 0,399072 & 3,053353 & $-4,318272$ & H76 & 0,854391 & 3,135934 & $-4,223399$ \\
\hline $\mathrm{H} 77$ & $-0,512062$ & 5,101426 & $-5,370994$ & $\mathrm{H} 77$ & 0,122779 & 5,220915 & $-5,340168$ \\
\hline H78 & $-0,995834$ & 7,106847 & $-3,983804$ & $\mathrm{H} 78$ & $-0,525082$ & 7,200919 & $-3,982182$ \\
\hline C79 & 0,870885 & 2,564541 & $-1,709824$ & C79 & 0,965065 & 2,576034 & $-1,596113$ \\
\hline $\mathrm{C} 80$ & $-0,390125$ & 1,708108 & $-1,328323$ & $\mathrm{C} 80$ & $-0,327551$ & 1,699787 & $-1,427245$ \\
\hline C81 & $-1,408711$ & 2,547235 & $-0,578130$ & $\mathrm{C} 81$ & $-1,440174$ & 2,517312 & $-0,793926$ \\
\hline $\mathrm{C} 82$ & $-2,579322$ & 2,950786 & $-1,242120$ & $\mathrm{C} 82$ & $-2,542237$ & 2,888274 & $-1,583666$ \\
\hline $\mathrm{C} 83$ & $-1,203460$ & 3,036118 & 0,724127 & $\mathrm{C} 83$ & $-1,378260$ & 3,046641 & 0,507188 \\
\hline $\mathrm{C} 84$ & $-3,489703$ & 3,825094 & $-0,650110$ & $\mathrm{C} 84$ & $-3,527117$ & 3,753223 & $-1,109837$ \\
\hline H85 & $-2,770196$ & 2,586363 & $-2,247004$ & H85 & $-2,620601$ & 2,502516 & $-2,595805$ \\
\hline $\mathrm{C} 86$ & $-2,098210$ & 3,931876 & 1,311315 & C86 & $-2,340355$ & 3,943435 & 0,973046 \\
\hline H87 & $-0,355370$ & 2,695743 & 1,303006 & H87 & $-0,592788$ & 2,741910 & 1,183003 \\
\hline $\mathrm{C} 88$ & $-3,241657$ & 4,339313 & 0,623696 & C88 & $-3,421465$ & 4,303193 & 0,169464 \\
\hline H89 & $-4,382814$ & 4,120691 & $-1,193890$ & H89 & $-4,366036$ & 4,014228 & $-1,749664$ \\
\hline H90 & $-1,906032$ & 4,299293 & 2,316058 & H90 & $-2,253981$ & 4,340675 & 1,981251 \\
\hline H91 & $-3,938105$ & 5,037612 & 1,079725 & H91 & $-4,174299$ & 4,996558 & 0,534567 \\
\hline C92 & 3,006057 & 3,660722 & $-0,798582$ & C92 & 2,953821 & 3,607009 & $-0,345477$ \\
\hline C93 & 1,950171 & 2,118464 & 0,541939 & C93 & 1,689001 & 2,052610 & 0,790714 \\
\hline C94 & 3,485129 & 3,790304 & 0,649468 & C94 & 3,208088 & 3,704574 & 1,160966 \\
\hline H95 & 2,737245 & 4,617234 & $-1,247008$ & H95 & 2,766177 & 4,575036 & $-0,810113$ \\
\hline H96 & 3,734115 & 3,152568 & $-1,444263$ & H96 & 3,766242 & 3,100081 & $-0,882699$ \\
\hline H97 & 4,568776 & 3,784792 & 0,767737 & H97 & 4,261043 & 3,685333 & 1,442886 \\
\hline H98 & 3,059328 & 4,665816 & 1,150360 & H98 & 2,719879 & 4,576847 & 1,607869 \\
\hline N99 & 1,822127 & 2,809784 & $-0,608577$ & N99 & 1,746854 & 2,768046 & $-0,354989$ \\
\hline $\mathrm{O} 100$ & 2,955222 & 2,601043 & 1,294723 & O100 & 2,577639 & 2,510518 & 1,694860 \\
\hline O101 & 1,300125 & 1,141541 & 0,955497 & O101 & 0,964152 & 1,087891 & 1,086083 \\
\hline H102 & 2,416310 & $-4,267053$ & 2,184523 & H102 & 2,615897 & $-4,303519$ & 1,957208 \\
\hline N103 & $-1,932908$ & $-2,488360$ & 0,088996 & N103 & $-1,945661$ & $-2,478546$ & 0,233283 \\
\hline C104 & $-0,909328$ & $-3,373464$ & $-0,503324$ & C104 & $-0,972728$ & $-3,388514$ & $-0,411890$ \\
\hline H105 & $-0,725668$ & $-3,150942$ & $-1,563692$ & H105 & $-0,861804$ & $-3,188748$ & $-1,487319$ \\
\hline H106 & $-0,822903$ & 1,508685 & $-2,312001$ & H106 & $-0,627785$ & 1,522449 & $-2,464089$ \\
\hline H107 & 2,625373 & 0,300477 & $-1,375021$ & H107 & 1,939844 & 0,093834 & $-0,980810$ \\
\hline H108 & 2,220954 & 0,038109 & $-1,947079$ & H108 & 1,579833 & $-0,115056$ & $-1,684124$ \\
\hline $\mathrm{TS}_{\mathrm{Z3-4}}$ & & & & Z4 & & & \\
\hline $\mathrm{C} 1$ & $-3,825379$ & $-3,978950$ & $-0,556223$ & $\mathrm{C} 1$ & $-3,724188$ & $-4,145535$ & $-0,601233$ \\
\hline $\mathrm{C} 2$ & $-1,576345$ & $-4,798897$ & $-0,085304$ & $\mathrm{C} 2$ & $-1,431953$ & $-4,908094$ & $-0,254999$ \\
\hline $\mathrm{C} 3$ & $-2,744638$ & $-4,978256$ & $-1,089017$ & $\mathrm{C} 3$ & $-2,624689$ & $-5,062497$ & $-1,234931$ \\
\hline $\mathrm{C} 4$ & $-3,128953$ & $-3,382549$ & 0,689612 & $\mathrm{C} 4$ & $-3,018518$ & $-3,624586$ & 0,673326 \\
\hline H5 & $-4,748573$ & $-4,493732$ & $-0,270380$ & H5 & $-4,623784$ & $-4,712282$ & $-0,340194$ \\
\hline H6 & $-2,441784$ & $-4,767119$ & $-2,119629$ & H6 & $-2,363736$ & $-4,769768$ & $-2,257076$ \\
\hline $\mathrm{H} 7$ & $-3,781483$ & $-2,856239$ & 1,385886 & $\mathrm{H} 7$ & $-3,675693$ & $-3,181735$ & 1,421898 \\
\hline H8 & $-4,085984$ & $-3,202514$ & $-1,277820$ & H8 & $-4,025449$ & $-3,322328$ & $-1,252660$ \\
\hline H9 & $-0,826793$ & $-5,589871$ & $-0,103490$ & H9 & $-0,651200$ & $-5,662247$ & $-0,355034$ \\
\hline
\end{tabular}




\begin{tabular}{|c|c|c|c|c|c|c|c|}
\hline H10 & $-3,104497$ & $-6,010701$ & $-1,059569$ & $\mathrm{H} 10$ & $-2,945925$ & $-6,107585$ & $-1,266573$ \\
\hline $\mathrm{C} 11$ & $-2,339022$ & $-4,582128$ & 1,239238 & $\mathrm{C} 11$ & $-2,162442$ & $-4,828263$ & 1,104139 \\
\hline H12 & $-1,697124$ & $-4,324657$ & 2,086894 & $\mathrm{H} 12$ & $-1,504510$ & $-4,606817$ & 1,949678 \\
\hline H13 & $-2,972779$ & $-5,433253$ & 1,504184 & $\mathrm{H} 13$ & $-2,750512$ & $-5,725124$ & 1,319190 \\
\hline $\mathrm{C} 14$ & 0,368465 & $-3,124441$ & 0,292926 & C14 & 0,455248 & $-3,163681$ & 0,156530 \\
\hline N15 & 0,940273 & $-1,967862$ & 0,366923 & N15 & 0,995364 & $-1,988681$ & 0,220941 \\
\hline $\mathrm{C} 16$ & 2,224506 & $-2,122128$ & 1,152780 & $\mathrm{C} 16$ & 2,303748 & $-2,096125$ & 0,978172 \\
\hline $\mathrm{C} 17$ & 2,351690 & $-3,680109$ & 1,176471 & $\mathrm{C} 17$ & 2,491733 & $-3,646664$ & 0,958792 \\
\hline P18 & $-2,029434$ & $-0,819072$ & 0,075898 & P18 & $-2,006428$ & $-0,987823$ & 0,159494 \\
\hline $\operatorname{Ir} 19$ & 0,234097 & $-0,237977$ & $-0,717338$ & Ir19 & 0,176343 & $-0,294797$ & $-0,592589$ \\
\hline $\mathrm{C} 20$ & $-2,704035$ & $-0,284353$ & 1,693296 & $\mathrm{C} 20$ & $-2,580001$ & $-0,432357$ & 1,804169 \\
\hline $\mathrm{C} 21$ & $-3,611371$ & 0,585711 & 4,204250 & $\mathrm{C} 21$ & $-3,269108$ & 0,564140 & 4,339074 \\
\hline $\mathrm{C} 22$ & $-2,417799$ & $-1,024897$ & 2,851873 & $\mathrm{C} 22$ & $-2,444862$ & $-1,237277$ & 2,944719 \\
\hline $\mathrm{C} 23$ & $-3,447714$ & 0,898084 & 1,810267 & $\mathrm{C} 23$ & $-3,050131$ & 0,881667 & 1,949782 \\
\hline $\mathrm{C} 24$ & $-3,896438$ & 1,328246 & 3,058224 & $\mathrm{C} 24$ & $-3,391119$ & 1,374245 & 3,207159 \\
\hline $\mathrm{C} 25$ & $-2,870377$ & $-0,592937$ & 4,097316 & $\mathrm{C} 25$ & $-2,796029$ & $-0,742295$ & 4,202257 \\
\hline $\mathrm{H} 26$ & $-3,681044$ & 1,485676 & 0,930558 & $\mathrm{H} 26$ & $-3,158460$ & 1,520852 & 1,082310 \\
\hline $\mathrm{H} 27$ & $-4,469102$ & 2,248508 & 3,129380 & $\mathrm{H} 27$ & $-3,752669$ & 2,394561 & 3,297321 \\
\hline $\mathrm{H} 28$ & $-2,644592$ & $-1,180420$ & 4,982800 & $\mathrm{H} 28$ & $-2,692055$ & $-1,380042$ & 5,075851 \\
\hline $\mathrm{H} 29$ & $-3,966761$ & 0,920605 & 5,174686 & $\mathrm{H} 29$ & $-3,540048$ & 0,947236 & 5,318632 \\
\hline $\mathrm{O} 30$ & 1,027842 & $-4,160221$ & 0,804419 & $\mathrm{O} 30$ & 1,170030 & $-4,174026$ & 0,646572 \\
\hline C31 & 2,052798 & $-1,611318$ & 2,586572 & $\mathrm{C} 31$ & 2,134933 & $-1,627453$ & 2,426105 \\
\hline C32 & 0,919375 & $-0,925262$ & 3,018126 & $\mathrm{C} 32$ & 0,958853 & $-1,060130$ & 2,911785 \\
\hline $\mathrm{C} 33$ & 3,071951 & $-1,877513$ & 3,514847 & $\mathrm{C} 33$ & 3,205847 & $-1,825326$ & 3,314232 \\
\hline C34 & 0,801191 & $-0,508870$ & 4,345291 & $\mathrm{C} 34$ & 0,854210 & $-0,678978$ & 4,251352 \\
\hline H35 & 0,128679 & $-0,692624$ & 2,322030 & H35 & 0,119707 & $-0,893153$ & 2,255043 \\
\hline C36 & 2,959711 & $-1,459072$ & 4,838030 & $\mathrm{C} 36$ & 3,103441 & $-1,446772$ & 4,649307 \\
\hline H37 & 3,971421 & $-2,399270$ & 3,195803 & H37 & 4,133812 & $-2,262880$ & 2,953144 \\
\hline C38 & 1,819162 & $-0,771136$ & 5,259057 & $\mathrm{C} 38$ & 1,923176 & $-0,866953$ & 5,122816 \\
\hline H39 & $-0,095297$ & 0,020811 & 4,654131 & H39 & $-0,072961$ & $-0,234544$ & 4,600040 \\
\hline $\mathrm{H} 40$ & 3,762356 & $-1,669459$ & 5,538986 & $\mathrm{H} 40$ & 3,944292 & $-1,603281$ & 5,319026 \\
\hline H41 & 1,728416 & $-0,445221$ & 6,291344 & $\mathrm{H} 41$ & 1,841782 & $-0,568693$ & 6,164165 \\
\hline $\mathrm{C} 42$ & $-3,385106$ & $-0,460426$ & $-1,120661$ & $\mathrm{C} 42$ & $-3,396488$ & $-0,592776$ & $-0,978443$ \\
\hline $\mathrm{C} 43$ & $-4,736926$ & $-0,485769$ & $-0,738734$ & $\mathrm{C} 43$ & $-4,716497$ & $-0,401762$ & $-0,545497$ \\
\hline $\mathrm{C} 44$ & $-3,075320$ & $-0,278911$ & $-2,477171$ & C44 & $-3,119902$ & $-0,563499$ & $-2,355554$ \\
\hline $\mathrm{C} 45$ & $-5,746566$ & $-0,310151$ & $-1,684454$ & $\mathrm{C} 45$ & $-5,734605$ & $-0,167900$ & $-1,469867$ \\
\hline $\mathrm{H} 46$ & $-5,007744$ & $-0,637126$ & 0,301230 & $\mathrm{H} 46$ & $-4,951345$ & $-0,424557$ & 0,513704 \\
\hline $\mathrm{C} 47$ & $-4,086245$ & $-0,107751$ & $-3,423553$ & $\mathrm{C} 47$ & $-4,140777$ & $-0,338903$ & $-3,278760$ \\
\hline $\mathrm{C} 48$ & $-5,425625$ & $-0,116949$ & $-3,029855$ & $\mathrm{C} 48$ & $-5,449801$ & $-0,134676$ & $-2,836335$ \\
\hline H49 & $-6,785848$ & $-0,327174$ & $-1,368527$ & H49 & $-6,752623$ & $-0,015893$ & $-1,122323$ \\
\hline H50 & $-3,826509$ & 0,035619 & $-4,468699$ & $\mathrm{H} 50$ & $-3,915097$ & $-0,319656$ & $-4,341367$ \\
\hline H51 & $-6,213194$ & 0,020297 & $-3,764815$ & $\mathrm{H} 51$ & $-6,245267$ & 0,045668 & $-3,553756$ \\
\hline H52 & $-0,210499$ & $-1,255857$ & $-1,826913$ & $\mathrm{H} 52$ & 0,024073 & $-1,144867$ & $-1,892784$ \\
\hline H53 & 1,555422 & 2,119155 & $-2,491652$ & H53 & 1,525102 & 2,075720 & $-2,241352$ \\
\hline H54 & $-1,840819$ & $-1,940154$ & 2,779619 & $\mathrm{H} 54$ & $-2,044189$ & $-2,241678$ & 2,856304 \\
\hline H55 & $-2,038607$ & $-0,271529$ & $-2,795138$ & $\mathrm{H} 55$ & $-2,101054$ & $-0,713552$ & $-2,701930$ \\
\hline H56 & 3,059101 & $-4,070821$ & 0,441499 & H56 & 3,173124 & $-3,991654$ & 0,177702 \\
\hline $\mathrm{C} 57$ & 5,423022 & $-0,256283$ & $-1,114905$ & C57 & 5,396341 & $-0,049999$ & $-1,280131$ \\
\hline
\end{tabular}




\begin{tabular}{|c|c|c|c|c|c|c|c|}
\hline C58 & 4,690083 & $-1,317715$ & $-1,652307$ & C58 & 4,692204 & $-1,119556$ & $-1,838605$ \\
\hline C59 & 3,679599 & $-1,914083$ & $-0,903705$ & C59 & 3,713598 & $-1,771950$ & $-1,095099$ \\
\hline C60 & 3,382766 & $-1,469006$ & 0,393169 & C60 & 3,416362 & $-1,372721$ & 0,216041 \\
\hline C61 & 4,115293 & $-0,403166$ & 0,919624 & C61 & 4,109796 & $-0,288863$ & 0,760279 \\
\hline C62 & 5,129488 & 0,197410 & 0,169748 & C62 & 5,099276 & 0,361251 & 0,018187 \\
\hline H63 & 6,217297 & 0,207245 & $-1,693505$ & H63 & 6,170940 & 0,453099 & $-1,852148$ \\
\hline H64 & 4,902721 & $-1,676915$ & $-2,655068$ & H64 & 4,904792 & $-1,443622$ & $-2,853274$ \\
\hline H65 & 3,092411 & $-2,711368$ & $-1,351616$ & H65 & 3,149595 & $-2,576766$ & $-1,558513$ \\
\hline H66 & 3,884092 & $-0,017208$ & 1,903865 & H66 & 3,874899 & 0,061389 & 1,757652 \\
\hline H67 & 5,695080 & 1,019084 & 0,601448 & H67 & 5,650083 & 1,184497 & 0,466205 \\
\hline C68 & $-0,154047$ & 6,292520 & $-1,925787$ & C68 & 0,564137 & 6,521623 & $-2,256494$ \\
\hline C69 & 0,293322 & 5,082247 & $-1,399706$ & C69 & 0,665451 & 5,306467 & $-1,579982$ \\
\hline $\mathrm{C} 70$ & 0,592160 & 4,009622 & $-2,248211$ & $\mathrm{C} 70$ & 0,902002 & 4,121891 & $-2,286465$ \\
\hline C71 & 0,443625 & 4,171401 & $-3,629110$ & C71 & 1,041090 & 4,175192 & $-3,678027$ \\
\hline $\mathrm{C} 72$ & $-0,010282$ & 5,380510 & $-4,156680$ & $\mathrm{C} 72$ & 0,932811 & 5,388999 & $-4,356417$ \\
\hline $\mathrm{C} 73$ & $-0,311338$ & 6,443592 & $-3,304867$ & $\mathrm{C} 73$ & 0,692384 & 6,565482 & $-3,645707$ \\
\hline H74 & $-0,389179$ & 7,115755 & $-1,257266$ & H74 & 0,379615 & 7,434674 & $-1,697721$ \\
\hline H75 & 0,384427 & 4,958186 & $-0,325385$ & H75 & 0,557109 & 5,278825 & $-0,500936$ \\
\hline H76 & 0,680619 & 3,345609 & $-4,297449$ & H76 & 1,237158 & 3,261106 & $-4,235424$ \\
\hline H77 & $-0,123623$ & 5,493128 & $-5,231051$ & $\mathrm{H} 77$ & 1,042281 & 5,416194 & $-5,436682$ \\
\hline H78 & $-0,664460$ & 7,386026 & $-3,713399$ & $\mathrm{H} 78$ & 0,609390 & 7,512461 & $-4,171029$ \\
\hline C79 & 0,973034 & 2,633749 & $-1,715121$ & C79 & 0,938567 & 2,753291 & $-1,609507$ \\
\hline $\mathrm{C} 80$ & $-0,324363$ & 1,790153 & $-1,491813$ & $\mathrm{C} 80$ & $-0,500876$ & 2,188391 & $-1,621974$ \\
\hline C81 & $-1,376192$ & 2,505756 & $-0,653054$ & $\mathrm{C} 81$ & $-1,623274$ & 2,876605 & $-0,877962$ \\
\hline C82 & $-2,535834$ & 2,947807 & $-1,312657$ & C82 & $-2,905501$ & 2,807284 & $-1,446959$ \\
\hline $\mathrm{C} 83$ & $-1,200065$ & 2,901029 & 0,683563 & C83 & $-1,467679$ & 3,592655 & 0,316400 \\
\hline $\mathrm{C} 84$ & $-3,458783$ & 3,783652 & $-0,684941$ & $\mathrm{C} 84$ & $-3,999342$ & 3,421390 & $-0,840359$ \\
\hline H85 & $-2,709510$ & 2,648571 & $-2,342312$ & H85 & $-3,053510$ & 2,253419 & $-2,369557$ \\
\hline $\mathrm{C} 86$ & $-2,106987$ & 3,761109 & 1,303069 & C86 & $-2,557452$ & 4,229488 & 0,912564 \\
\hline H87 & $-0,370707$ & 2,522211 & 1,260981 & H87 & $-0,500497$ & 3,666445 & 0,791389 \\
\hline $\mathrm{C} 88$ & $-3,237602$ & 4,215998 & 0,623966 & $\mathrm{C} 88$ & $-3,829085$ & 4,142116 & 0,343774 \\
\hline H89 & $-4,340851$ & 4,110186 & $-1,228383$ & H89 & $-4,980951$ & 3,336620 & $-1,296630$ \\
\hline H90 & $-1,935223$ & 4,057254 & 2,334134 & H90 & $-2,409163$ & 4,788470 & 1,832243 \\
\hline H91 & $-3,940728$ & 4,886962 & 1,108887 & H91 & $-4,675937$ & 4,633481 & 0,813899 \\
\hline C92 & 3,095934 & 3,541071 & $-0,638378$ & C92 & 3,010360 & 3,358518 & $-0,302808$ \\
\hline C93 & 1,900929 & 1,977082 & 0,553064 & C93 & 1,445737 & 2,065438 & 0,790836 \\
\hline C94 & 3,520453 & 3,549604 & 0,833087 & C94 & 3,298930 & 3,327357 & 1,199419 \\
\hline H95 & 2,905675 & 4,539019 & $-1,034557$ & H95 & 3,057612 & 4,362133 & $-0,726572$ \\
\hline H96 & 3,818289 & 3,024534 & $-1,283904$ & H96 & 3,669594 & 2,688389 & $-0,870052$ \\
\hline H97 & 4,595278 & 3,454299 & 0,988146 & H97 & 4,321216 & 3,043962 & 1,450322 \\
\hline H98 & 3,140975 & 4,424625 & 1,370450 & H98 & 3,041025 & 4,269817 & 1,693501 \\
\hline N99 & 1,855376 & 2,758419 & $-0,547637$ & N99 & 1,636308 & 2,834913 & $-0,315855$ \\
\hline O100 & 2,881273 & 2,369690 & 1,385141 & O100 & 2,410085 & 2,297530 & 1,700432 \\
\hline O101 & 1,171547 & 1,028199 & 0,875838 & O101 & 0,523274 & 1,282289 & 1,044818 \\
\hline H102 & 2,581328 & $-4,076257$ & 2,163749 & H102 & 2,791400 & $-4,051417$ & 1,923361 \\
\hline N103 & $-2,002558$ & $-2,508662$ & 0,260001 & N103 & $-1,938419$ & $-2,675849$ & 0,295397 \\
\hline C104 & $-0,977166$ & $-3,389805$ & $-0,337436$ & C104 & $-0,905285$ & $-3,459454$ & $-0,422409$ \\
\hline H105 & $-0,850098$ & $-3,221421$ & $-1,416639$ & H105 & $-0,846076$ & $-3,196765$ & $-1,488015$ \\
\hline
\end{tabular}




$\begin{array}{rrrrrrrr}\text { H106 } & -0,761489 & 1,776629 & -2,495548 & \text { H106 } & -0,756238 & 2,197980 & -2,687412 \\ \text { H107 } & 1,679775 & -0,119202 & -1,392547 & \text { H107 } & 1,628191 & 0,057484 & -1,189735 \\ \text { H108 } & 0,236253 & 0,431305 & -2,148581 & \text { H108 } & -0,616550 & 1,043242 & -1,516089\end{array}$

Isomerization

Iso1

$\mathrm{C} 1$

$-4,163957$

$-3,413621$

0,495949

TS 1

$\mathrm{C} 2$

$-2,022982$

$-4,485459$

0,026619

$\mathrm{C} 2$

$-4,418747$

$-0,490090$

$\mathrm{C} 3$

$\mathrm{C} 4$

$-2,991128$

$-3,026092$

1,427059

$\mathrm{C} 4$

H5 $\quad-4,961675$

$-3,891034$

1,074636

H5

H6

$-3,536093$

$-4,089495$

$-1,532711$

H6

$\mathrm{H} 7 \quad-3,267742$

$-2,520272$

2,352589

$\mathrm{H} 7$

H8 $\quad-4,592510$

$-2,548004$

$-0,008998$

$\mathrm{H} 8$

H9 $-1,449996$

$-5,342382$

$-0,325984$

H9

H10 $-3,942675$

$-5,409269$

$-0,436314$

H10

1,552521

$\begin{array}{ll}\text { C11 } & -2,224355 \\ \text { H12 } & -1,293876\end{array}$

$-4,353581$

2,120793

C11

$-4,260150$

1,970202

H13 - $-2,829643$

$-5,162804$

$-0,165796$

$\mathrm{H} 12$

C14 0,152012

$-3,110362$

$-2,057120$

$-0,123457$

H13

C14

N15

C16 2,348877

$-2,501544$

$-0,197728$

C16

C17 2,186916

$-4,028465$

0,065158

C17

P18 $-1,924278$

$-0,543643$

0,464644

P18

Ir19 $\quad 0,274502$

$-0,008131$

$-0,426442$

Ir19

2,164131

C20

$\mathrm{C} 21$

$-2,161935$

4,755967

C21

3,203504

$\mathrm{C} 22$

$\mathrm{C} 23$

$-1,463363$

$-0,548212$

2,439483

$\mathrm{C} 23$

$\mathrm{C} 24$

$-2,861686$

1,270604

3,729439

$\mathrm{C} 24$

$\mathrm{C} 25$

H26

$-1,474395$

$-0,016881$

4,490101

C25

1,771534

1,654956

H26

H27 - $-3,421403$

2,715740

3,931945

H27

H28 $\quad-0,930393$

$-0,518863$

5,284880

1,580659

5,758915

H28

H29 - $-2,174407$

$-4,294209$

$-0,144853$

H29

O30 0,771150

$-1,908942$

0,919348

O30

C31 3,198321

$-1,431515$

2,089597

C33

2,602482

$-1,955256$

0,842561

C34

4,598267

$-0,936438$

3,132724

$-1,410267$

2,176880

$-1,474994$

1,891534

$-2,354774$

$-0,043770$

C31

$\mathrm{C} 32$

C33

C34

C36 5,380638

H35

H37 $\quad 5,081068$

$-0,949494$

3,034920

C36

C38 4,775849

H39 2,899988

$-0,532960$

$\mathrm{H} 40 \quad 6,463394$

$-1,507060$

4,016736

H37

C38

1,811104

H39

$-0,561588$

3,845728

H40

4,346353

3,217037

0,263892

2,259239

4,384293

$-0,227178$

3,701615

4,202789

$-0,765849$

3,176618

2,961192

1,243615

5,189183

3,675193

0,791983

3,714378

3,813073

$-1,788864$

3,447576

2,499120

2,193547

4,704699

2,294690

$-0,194802$

1,725944

5,248340

$-0,622460$

4,218791

5,166392

$-0,777937$

2,489743

4,336607

1,299949

1,569152

4,334075

1,891728

3,149606

5,133868

1,653067

0,007517

3,108169

$-0,317945$

$-0,776244$

2,091120

$-0,209954$

$-2,208721$

2,585513

$-0,307392$

$-1,986872$

4,122475

$-0,162750$

1,940575

0,495614

0,482512

$-0,226986$

$-0,002426$

$-0,404242$

2,094370

1,929672

$-0,042618$

2,218503

1,506843

$-0,988937$

4,852181

2,591663

0,743900

3,220487

2,509920

$-1,312005$

2,548385

1,432581

$-1,780063$

3,860198

3,047757

0,275533

4,529763

$-1,933487$

1,786119

2,908258

$-2,760313$

4,107698

0,978728

0,894090

5,298525

1,865129

$-1,355148$

5,872519

$-0,561993$

4,315914

$-0,381884$

$-3,068113$

2,107681

0,855317

$-2,482060$

1,634149

2,031931

$-4,462657$

2,248537

0,800891

$-3,275588$

1,242064

3,110371

$-1,406851$

1,537510

2,100836

$-5,254385$

1,872044

1,884198

$-4,933773$

2,640566

$-0,095448$

$-4,663589$

1,353918

3,038720

$-2,801210$

0,841484

4,001305

$-6,333562$

1,978923

1,822859

H41

$-5,282228$

1,048285

3,877653

C42 $-3,402865$

$-0,059708$

$-0,500188$

C42

3,400544

$-0,148548$

$-0,410044$ 


\begin{tabular}{|c|c|c|c|c|c|c|c|}
\hline $\mathrm{C} 43$ & $-4,682540$ & 0,032883 & 0,074487 & $\mathrm{C} 43$ & 4,637022 & $-0,363737$ & 0,220583 \\
\hline $\mathrm{C} 44$ & $-3,266370$ & 0,099402 & $-1,886597$ & $\mathrm{C} 44$ & 3,305935 & $-0,287794$ & $-1,802633$ \\
\hline $\mathrm{C} 45$ & $-5,794990$ & 0,298304 & $-0,722171$ & $\mathrm{C} 45$ & 5,753766 & $-0,723571$ & $-0,531561$ \\
\hline $\mathrm{H} 46$ & $-4,814556$ & $-0,107826$ & 1,142984 & $\mathrm{H} 46$ & 4,733825 & $-0,242250$ & 1,294890 \\
\hline $\mathrm{C} 47$ & $-4,383129$ & 0,357195 & $-2,681144$ & $\mathrm{C} 47$ & 4,427149 & $-0,640176$ & $-2,551205$ \\
\hline $\mathrm{C} 48$ & $-5,648418$ & 0,461097 & $-2,102500$ & $\mathrm{C} 48$ & 5,650327 & $-0,861592$ & $-1,917425$ \\
\hline $\mathrm{H} 49$ & $-6,777827$ & 0,372730 & $-0,265463$ & $\mathrm{H} 49$ & 6,706050 & $-0,890578$ & $-0,036308$ \\
\hline H50 & $-4,259303$ & 0,494953 & $-3,750946$ & $\mathrm{H} 50$ & 4,340139 & $-0,759443$ & $-3,626752$ \\
\hline H51 & $-6,516448$ & 0,669770 & $-2,720887$ & $\mathrm{H} 51$ & 6,522661 & $-1,141944$ & $-2,500838$ \\
\hline H52 & $-0,118809$ & $-0,428638$ & $-1,876377$ & H52 & 0,213168 & 0,348314 & $-1,859339$ \\
\hline H53 & 1,756460 & $-0,020859$ & $-0,994801$ & $\mathrm{H} 53$ & $-1,709866$ & $-0,069468$ & $-1,031224$ \\
\hline H54 & $-0,902186$ & $-1,453808$ & 2,994702 & H54 & 1,095098 & 1,715715 & 2,965622 \\
\hline H55 & $-2,281344$ & 0,048333 & $-2,337410$ & H55 & 2,348110 & $-0,150459$ & $-2,292681$ \\
\hline H56 & 2,761357 & $-4,656663$ & $-0,615218$ & H56 & $-2,534652$ & 4,718544 & $-0,892680$ \\
\hline C57 & 3,441736 & $-1,519157$ & $-4,288053$ & C57 & $-3,380542$ & 1,313445 & $-4,294481$ \\
\hline C58 & 2,656782 & $-2,639437$ & $-4,007968$ & C58 & $-2,534468$ & 2,409817 & $-4,114400$ \\
\hline C59 & 2,351987 & $-2,968942$ & $-2,688848$ & C59 & $-2,204142$ & 2,835734 & $-2,829575$ \\
\hline C60 & 2,828100 & $-2,191625$ & $-1,623160$ & $\mathrm{C} 60$ & $-2,714522$ & 2,181545 & $-1,699572$ \\
\hline C61 & 3,612994 & $-1,068426$ & $-1,918028$ & C61 & $-3,563190$ & 1,083499 & $-1,893208$ \\
\hline C62 & 3,918381 & $-0,736228$ & $-3,238228$ & C62 & $-3,893035$ & 0,652750 & $-3,179390$ \\
\hline $\mathrm{H} 63$ & 3,679810 & $-1,262062$ & $-5,316241$ & H63 & $-3,638810$ & 0,981313 & $-5,295981$ \\
\hline H64 & 2,279453 & $-3,257252$ & $-4,817746$ & H64 & $-2,129848$ & 2,933481 & $-4,975774$ \\
\hline H65 & 1,718127 & $-3,831351$ & $-2,499850$ & H65 & $-1,524674$ & 3,676047 & $-2,716567$ \\
\hline H66 & 3,986116 & $-0,436856$ & $-1,122065$ & H66 & $-3,969731$ & 0,549772 & $-1,043602$ \\
\hline H67 & 4,527618 & 0,140556 & $-3,439156$ & H67 & $-4,550803$ & $-0,203286$ & $-3,301388$ \\
\hline C68 & $-2,454159$ & 3,304004 & $-3,397543$ & C68 & 2,183042 & $-3,383957$ & $-3,440746$ \\
\hline C69 & $-1,344000$ & 2,761373 & $-2,754102$ & C69 & 1,118782 & $-2,796816$ & $-2,759807$ \\
\hline $\mathrm{C} 70$ & $-1,296252$ & 2,673925 & $-1,354775$ & $\mathrm{C} 70$ & 1,101550 & $-2,745230$ & $-1,357878$ \\
\hline C71 & $-2,394445$ & 3,157909 & $-0,627800$ & C71 & 2,189606 & $-3,303290$ & $-0,668332$ \\
\hline $\mathrm{C} 72$ & $-3,505325$ & 3,699669 & $-1,269321$ & $\mathrm{C} 72$ & 3,255000 & $-3,889432$ & $-1,347517$ \\
\hline $\mathrm{C} 73$ & $-3,541384$ & 3,775807 & $-2,660551$ & $\mathrm{C} 73$ & 3,257004 & $-3,935284$ & $-2,740328$ \\
\hline $\mathrm{H} 74$ & $-2,464845$ & 3,366183 & $-4,482193$ & $\mathrm{H} 74$ & 2,169500 & $-3,414206$ & $-4,526705$ \\
\hline $\mathrm{H} 75$ & $-0,513264$ & 2,410466 & $-3,357111$ & H75 & 0,302662 & $-2,375385$ & $-3,337364$ \\
\hline $\mathrm{H} 76$ & $-2,372119$ & 3,105654 & 0,454095 & H76 & 2,202863 & $-3,265504$ & 0,415075 \\
\hline H77 & $-4,343115$ & 4,061073 & $-0,679835$ & H77 & 4,086985 & $-4,303722$ & $-0,785321$ \\
\hline $\mathrm{H} 78$ & $-4,403228$ & 4,201513 & $-3,165751$ & $\mathrm{H} 78$ & 4,084166 & $-4,393350$ & $-3,274316$ \\
\hline C79 & $-0,106033$ & 2,133807 & $-0,626018$ & C79 & $-0,029502$ & $-2,139255$ & $-0,585748$ \\
\hline $\mathrm{C} 80$ & 1,125307 & 1,792608 & $-1,303040$ & $\mathrm{C} 80$ & $-1,278532$ & $-1,740727$ & $-1,230887$ \\
\hline H81 & 1,048777 & 1,679271 & $-2,379219$ & H81 & $-1,234841$ & $-1,675966$ & $-2,313437$ \\
\hline $\mathrm{C} 82$ & 2,448229 & 2,373226 & $-0,893344$ & $\mathrm{C} 82$ & $-2,608088$ & $-2,259811$ & $-0,762282$ \\
\hline $\mathrm{C} 83$ & 3,259374 & 1,896668 & 0,143166 & $\mathrm{C} 83$ & $-3,366989$ & $-1,707009$ & 0,274847 \\
\hline C84 & 2,868132 & 3,500193 & $-1,619064$ & C84 & $-3,090482$ & $-3,398122$ & $-1,427581$ \\
\hline $\mathrm{C} 85$ & 4,457768 & 2,542121 & 0,454324 & $\mathrm{C} 85$ & $-4,579246$ & $-2,290845$ & 0,647646 \\
\hline H86 & 2,964816 & 1,016009 & 0,701672 & H86 & $-3,018353$ & $-0,817984$ & 0,787117 \\
\hline $\mathrm{C} 87$ & 4,063310 & 4,144893 & $-1,304865$ & C87 & $-4,299106$ & $-3,981243$ & $-1,051220$ \\
\hline H88 & 2,250148 & 3,873636 & $-2,432802$ & H88 & $-2,512526$ & $-3,827686$ & $-2,243129$ \\
\hline $\mathrm{C} 89$ & 4,862027 & 3,667689 & $-0,263125$ & C89 & $-5,046992$ & $-3,429131$ & $-0,008597$ \\
\hline H90 & 5,075384 & 2,151381 & 1,257963 & H90 & $-5,156448$ & $-1,843327$ & 1,451478 \\
\hline
\end{tabular}




\begin{tabular}{|c|c|c|c|c|c|c|c|}
\hline H91 & 4,372375 & 5,015353 & $-1,876865$ & H91 & $-4,658645$ & $-4,861684$ & $-1,576393$ \\
\hline H92 & 5,795992 & 4,166521 & $-0,020807$ & H92 & $-5,991259$ & $-3,880471$ & 0,281643 \\
\hline C93 & 0,254094 & 4,151686 & 0,961488 & C93 & $-0,441600$ & $-4,136937$ & 1,023317 \\
\hline C94 & 0,565770 & 1,991957 & 1,718085 & C94 & $-0,644979$ & $-1,957904$ & 1,764513 \\
\hline C95 & 0,397076 & 4,111983 & 2,492557 & C95 & $-0,572213$ & $-4,076367$ & 2,555191 \\
\hline H96 & $-0,600219$ & 4,743908 & 0,629460 & H96 & 0,381277 & $-4,771882$ & 0,690919 \\
\hline H97 & 1,160255 & 4,505547 & 0,456694 & H97 & $-1,366196$ & $-4,460016$ & 0,531103 \\
\hline H98 & 1,141533 & 4,803136 & 2,887851 & H98 & $-1,344412$ & $-4,730195$ & 2,960863 \\
\hline H99 & $-0,561121$ & 4,257198 & 3,001336 & H99 & 0,380953 & $-4,266026$ & 3,059316 \\
\hline N100 & 0,050990 & 2,719689 & 0,698213 & N100 & $-0,181224$ & $-2,718297$ & 0,742361 \\
\hline O101 & 0,845583 & 2,755845 & 2,779472 & O101 & $-0,953012$ & $-2,700218$ & 2,833473 \\
\hline O102 & 0,775321 & 0,772262 & 1,698901 & O102 & $-0,798476$ & $-0,729771$ & 1,735516 \\
\hline H103 & 2,416940 & $-4,286348$ & 1,101050 & $\mathrm{H} 103$ & $-2,213139$ & 4,468237 & 0,848174 \\
\hline N104 & $-1,976927$ & $-2,225396$ & 0,683726 & N104 & 2,103820 & 2,178035 & 0,571835 \\
\hline C105 & $-1,350348$ & $-3,128567$ & $-0,306966$ & $\mathrm{C} 105$ & 1,508408 & 3,047827 & $-0,464122$ \\
\hline H106 & $-1,565209$ & $-2,828735$ & $-1,344085$ & H106 & 1,696789 & 2,674951 & $-1,483108$ \\
\hline Iso2 & & & & $\mathrm{TS}_{\text {Iso2-3 }}$ & & & \\
\hline $\mathrm{C} 1$ & 4,843561 & 2,498820 & $-0,482967$ & $\mathrm{C} 1$ & $-4,338247$ & 2,694784 & $-1,634939$ \\
\hline $\mathrm{C} 2$ & 2,981888 & 3,839251 & $-1,315714$ & $\mathrm{C} 2$ & $-4,312143$ & 0,407061 & $-2,480902$ \\
\hline $\mathrm{C} 3$ & 4,382473 & 3,321595 & $-1,731988$ & $\mathrm{C} 3$ & $-4,451374$ & 1,874037 & $-2,962482$ \\
\hline $\mathrm{C} 4$ & 3,642901 & 2,666109 & 0,476882 & $\mathrm{C} 4$ & $-4,170576$ & 1,580282 & $-0,575436$ \\
\hline H5 & 5,750371 & 2,916876 & $-0,033693$ & H5 & $-5,246363$ & 3,273413 & $-1,436174$ \\
\hline H6 & 4,346168 & 2,720039 & $-2,645717$ & H6 & $-3,682692$ & 2,143279 & $-3,693827$ \\
\hline $\mathrm{H} 7$ & 3,829001 & 2,398250 & 1,517273 & $\mathrm{H} 7$ & $-4,339121$ & 1,887039 & 0,457034 \\
\hline $\mathrm{H} 8$ & 5,037176 & 1,448989 & $-0,709024$ & H8 & $-3,495340$ & 3,388306 & $-1,629491$ \\
\hline H9 & 2,595418 & 4,660659 & $-1,918503$ & H9 & $-4,629488$ & $-0,349141$ & $-3,199008$ \\
\hline H10 & 5,049894 & 4,166554 & $-1,923308$ & $\mathrm{H} 10$ & $-5,423860$ & 2,018465 & $-3,441791$ \\
\hline $\mathrm{C} 11$ & 3,185366 & 4,106635 & 0,191923 & $\mathrm{C} 11$ & $-5,059647$ & 0,454014 & $-1,129735$ \\
\hline H12 & 2,270165 & 4,379259 & 0,726734 & H12 & $-4,993582$ & $-0,470937$ & $-0,548078$ \\
\hline H13 & 3,960976 & 4,852394 & 0,387020 & H13 & $-6,108235$ & 0,745263 & $-1,238163$ \\
\hline C14 & 0,555778 & 2,940029 & $-1,236661$ & $\mathrm{C} 14$ & $-2,381232$ & $-1,207984$ & $-1,850879$ \\
\hline N15 & $-0,358347$ & 2,145518 & $-0,806548$ & N15 & $-1,396209$ & $-1,553909$ & $-1,104642$ \\
\hline $\mathrm{C} 16$ & $-1,690595$ & 2,816668 & $-0,959944$ & $\mathrm{C} 16$ & $-1,238082$ & $-3,038369$ & $-1,168230$ \\
\hline $\mathrm{C} 17$ & $-1,289397$ & 4,113461 & $-1,748727$ & $\mathrm{C} 17$ & $-2,186359$ & $-3,381344$ & $-2,366780$ \\
\hline P18 & 1,991962 & 0,342789 & 0,307287 & $\mathrm{P} 18$ & $-1,414846$ & 1,349289 & 0,095074 \\
\hline Ir19 & $-0,073215$ & $-0,003165$ & $-0,435216$ & Ir19 & 0,106655 & $-0,202197$ & $-0,291685$ \\
\hline $\mathrm{C} 20$ & 2,018273 & 0,280332 & 2,128194 & $\mathrm{C} 20$ & $-1,889612$ & 1,357047 & 1,852694 \\
\hline $\mathrm{C} 21$ & 1,641370 & 0,120230 & 4,900222 & $\mathrm{C} 21$ & $-2,380294$ & 1,086581 & 4,597613 \\
\hline $\mathrm{C} 22$ & 1,608052 & 1,408195 & 2,854719 & $\mathrm{C} 22$ & $-2,881016$ & 0,477863 & 2,311952 \\
\hline $\mathrm{C} 23$ & 2,239199 & $-0,929057$ & 2,804339 & $\mathrm{C} 23$ & $-1,142188$ & 2,098289 & 2,782086 \\
\hline $\mathrm{C} 24$ & 2,052507 & $-1,005522$ & 4,184384 & $\mathrm{C} 24$ & $-1,391777$ & 1,964028 & 4,147019 \\
\hline $\mathrm{C} 25$ & 1,426065 & 1,327932 & 4,233494 & $\mathrm{C} 25$ & $-3,126111$ & 0,348046 & 3,677748 \\
\hline $\mathrm{H} 26$ & 2,568168 & $-1,805532$ & 2,256557 & $\mathrm{H} 26$ & $-0,369203$ & 2,777857 & 2,439124 \\
\hline $\mathrm{H} 27$ & 2,234305 & $-1,943571$ & 4,701675 & $\mathrm{H} 27$ & $-0,819442$ & 2,550926 & 4,860545 \\
\hline $\mathrm{H} 28$ & 1,109658 & 2,207252 & 4,787112 & $\mathrm{H} 28$ & $-3,898394$ & $-0,332829$ & 4,024012 \\
\hline $\mathrm{H} 29$ & 1,491790 & 0,057316 & 5,974100 & $\mathrm{H} 29$ & $-2,570066$ & 0,981790 & 5,661885 \\
\hline $\mathrm{O} 30$ & 0,167030 & 4,115478 & $-1,737839$ & $\mathrm{O} 30$ & $-2,980892$ & $-2,171094$ & $-2,556556$ \\
\hline
\end{tabular}




\begin{tabular}{|c|c|c|c|c|c|c|c|}
\hline C31 & $-2,278706$ & 3,197707 & 0,396966 & C31 & $-1,758951$ & $-3,693655$ & 0,112502 \\
\hline C32 & $-1,658360$ & 2,861172 & 1,599704 & $\mathrm{C} 32$ & $-2,139546$ & $-2,948186$ & 1,228139 \\
\hline C33 & $-3,466153$ & 3,945586 & 0,429776 & $\mathrm{C} 33$ & $-1,858315$ & $-5,092088$ & 0,161802 \\
\hline C34 & $-2,227680$ & 3,239694 & 2,817435 & C34 & $-2,611304$ & $-3,586560$ & 2,376801 \\
\hline H35 & $-0,746941$ & 2,282426 & 1,593911 & H35 & $-2,044052$ & $-1,872766$ & 1,215750 \\
\hline C36 & $-4,031252$ & 4,327533 & 1,643615 & $\mathrm{C} 36$ & $-2,331096$ & $-5,728997$ & 1,306237 \\
\hline H37 & $-3,960194$ & 4,215570 & $-0,500960$ & H37 & $-1,544776$ & $-5,687768$ & $-0,693003$ \\
\hline C38 & $-3,413882$ & 3,969676 & 2,845044 & $\mathrm{C} 38$ & $-2,710158$ & $-4,975562$ & 2,420491 \\
\hline H39 & $-1,739223$ & 2,951131 & 3,744044 & H39 & $-2,890739$ & $-2,988564$ & 3,240044 \\
\hline $\mathrm{H} 40$ & $-4,954625$ & 4,899653 & 1,651929 & $\mathrm{H} 40$ & $-2,400185$ & $-6,812753$ & 1,330297 \\
\hline H41 & $-3,857450$ & 4,261052 & 3,792803 & $\mathrm{H} 41$ & $-3,076130$ & $-5,472077$ & 3,314604 \\
\hline $\mathrm{C} 42$ & 3,311368 & $-0,776198$ & $-0,282387$ & $\mathrm{C} 42$ & $-1,043174$ & 3,074582 & $-0,369582$ \\
\hline $\mathrm{C} 43$ & 4,489556 & $-0,947278$ & 0,465789 & $\mathrm{C} 43$ & $-1,601144$ & 4,154602 & 0,334706 \\
\hline $\mathrm{C} 44$ & 3,213191 & $-1,370297$ & $-1,548818$ & $\mathrm{C} 44$ & $-0,346045$ & 3,316340 & $-1,562379$ \\
\hline $\mathrm{C} 45$ & 5,541302 & $-1,707847$ & $-0,042925$ & $\mathrm{C} 45$ & $-1,450888$ & 5,454781 & $-0,145483$ \\
\hline H46 & 4,589032 & $-0,488319$ & 1,444955 & $\mathrm{H} 46$ & $-2,162357$ & 3,988169 & 1,248218 \\
\hline $\mathrm{C} 47$ & 4,270648 & $-2,125073$ & $-2,054765$ & $\mathrm{C} 47$ & $-0,204075$ & 4,616890 & $-2,038597$ \\
\hline $\mathrm{C} 48$ & 5,434979 & $-2,298252$ & $-1,304711$ & $\mathrm{C} 48$ & $-0,755164$ & 5,687715 & $-1,332579$ \\
\hline H49 & 6,444348 & $-1,837384$ & 0,546767 & $\mathrm{H} 49$ & $-1,883394$ & 6,284576 & 0,406479 \\
\hline H50 & 4,173041 & $-2,595672$ & $-3,028103$ & $\mathrm{H} 50$ & 0,353092 & 4,795075 & $-2,953047$ \\
\hline H51 & 6,254528 & $-2,892943$ & $-1,697183$ & $\mathrm{H} 51$ & $-0,639272$ & 6,701928 & $-1,704031$ \\
\hline H52 & 0,512792 & $-0,095084$ & $-1,888116$ & $\mathrm{H} 52$ & 0,264459 & 0,385997 & $-1,737970$ \\
\hline H53 & $-1,908184$ & $-0,614304$ & $-1,123946$ & H53 & 2,453381 & $-1,490840$ & 0,680694 \\
\hline H54 & 1,421649 & 2,343264 & 2,336171 & $\mathrm{H} 54$ & $-3,450937$ & $-0,110971$ & 1,600720 \\
\hline H55 & 2,297886 & $-1,271168$ & $-2,120767$ & $\mathrm{H} 55$ & 0,111077 & 2,491977 & $-2,097377$ \\
\hline H56 & $-1,613290$ & 4,105252 & $-2,791941$ & $\mathrm{H} 56$ & $-1,658086$ & $-3,582087$ & $-3,302155$ \\
\hline $\mathrm{C} 57$ & $-4,049059$ & 0,075552 & $-3,372072$ & C57 & 2,992897 & $-3,673457$ & $-1,945403$ \\
\hline C58 & $-2,848751$ & 0,607543 & $-3,850578$ & C58 & 2,176119 & $-3,067224$ & $-2,902874$ \\
\hline C59 & $-2,124452$ & 1,504140 & $-3,070110$ & C59 & 0,817147 & $-2,890511$ & $-2,650107$ \\
\hline C60 & $-2,581430$ & 1,889875 & $-1,798802$ & C60 & 0,245390 & $-3,325960$ & $-1,442849$ \\
\hline C61 & $-3,779899$ & 1,345487 & $-1,327514$ & C61 & 1,076485 & $-3,917714$ & $-0,483601$ \\
\hline C62 & $-4,509387$ & 0,447392 & $-2,111173$ & C62 & 2,438960 & $-4,092721$ & $-0,736746$ \\
\hline H63 & $-4,614752$ & $-0,628902$ & $-3,974851$ & H63 & 4,054442 & $-3,800782$ & $-2,134572$ \\
\hline H64 & $-2,475100$ & 0,320525 & $-4,829484$ & H64 & 2,598442 & $-2,725899$ & $-3,843795$ \\
\hline H65 & $-1,176175$ & 1,882766 & $-3,442187$ & H65 & 0,203778 & $-2,386686$ & $-3,392295$ \\
\hline H66 & $-4,142737$ & 1,605442 & $-0,340817$ & H66 & 0,662461 & $-4,237491$ & 0,465926 \\
\hline H67 & $-5,428105$ & 0,022187 & $-1,719355$ & $\mathrm{H} 67$ & 3,069105 & $-4,552585$ & 0,019190 \\
\hline C68 & 1,381926 & $-4,589811$ & $-2,459505$ & C68 & 3,252546 & 3,348508 & $-1,799379$ \\
\hline C69 & 0,469924 & $-3,614166$ & $-2,053382$ & C69 & 2,858619 & 2,099851 & $-1,326414$ \\
\hline $\mathrm{C} 70$ & 0,486154 & $-3,110351$ & $-0,745354$ & $\mathrm{C} 70$ & 2,338287 & 1,939718 & $-0,033293$ \\
\hline C71 & 1,460406 & $-3,617419$ & 0,133444 & C71 & 2,172664 & 3,099609 & 0,741889 \\
\hline $\mathrm{C} 72$ & 2,367854 & $-4,592608$ & $-0,266440$ & $\mathrm{C} 72$ & 2,556656 & 4,353692 & 0,269307 \\
\hline $\mathrm{C} 73$ & 2,331801 & $-5,089302$ & $-1,569700$ & $\mathrm{C} 73$ & 3,117462 & 4,484516 & $-0,999096$ \\
\hline H74 & 1,340046 & $-4,967045$ & $-3,477565$ & $\mathrm{H} 74$ & 3,663822 & 3,434227 & $-2,801634$ \\
\hline H75 & $-0,255023$ & $-3,255223$ & $-2,776100$ & $\mathrm{H} 75$ & 2,934463 & 1,242648 & $-1,980858$ \\
\hline H76 & 1,506444 & $-3,226956$ & 1,144191 & H76 & 1,711460 & 3,031246 & 1,720527 \\
\hline H77 & 3,109519 & $-4,957749$ & 0,438523 & $\mathrm{H} 77$ & 2,407258 & 5,229278 & 0,895075 \\
\hline H78 & 3,034275 & $-5,854302$ & $-1,886850$ & $\mathrm{H} 78$ & 3,427578 & 5,458307 & $-1,366766$ \\
\hline
\end{tabular}




\begin{tabular}{|c|c|c|c|c|c|c|c|}
\hline C79 & $-0,455128$ & $-2,050446$ & $-0,249052$ & C79 & 1,900289 & 0,594367 & 0,463107 \\
\hline $\mathrm{C} 80$ & $-1,724319$ & $-1,738381$ & $-1,097761$ & $\mathrm{C} 80$ & 2,677043 & $-0,691837$ & $-0,032987$ \\
\hline H81 & $-1,551770$ & $-1,976878$ & $-2,144944$ & H81 & 2,236952 & $-1,030630$ & $-0,992783$ \\
\hline C82 & $-3,023016$ & $-2,335138$ & $-0,614414$ & C82 & 4,182318 & $-0,672268$ & $-0,244258$ \\
\hline $\mathrm{C} 83$ & $-3,793648$ & $-1,707996$ & 0,371142 & $\mathrm{C} 83$ & 5,051211 & $-0,878796$ & 0,834740 \\
\hline C84 & $-3,430210$ & $-3,576776$ & $-1,115837$ & C84 & 4,740418 & $-0,529958$ & $-1,520965$ \\
\hline C85 & $-4,947932$ & $-2,321437$ & 0,859073 & C85 & 6,434129 & $-0,885105$ & 0,656417 \\
\hline H86 & $-3,491226$ & $-0,735734$ & 0,750028 & H86 & 4,643803 & $-1,076527$ & 1,822409 \\
\hline C87 & $-4,587886$ & $-4,187742$ & $-0,632244$ & $\mathrm{C} 87$ & 6,122422 & $-0,536553$ & $-1,707448$ \\
\hline H88 & $-2,831370$ & $-4,071392$ & $-1,876943$ & H88 & 4,087903 & $-0,435125$ & $-2,384391$ \\
\hline C89 & $-5,347332$ & $-3,563095$ & 0,359302 & C89 & 6,975628 & $-0,704329$ & $-0,616525$ \\
\hline H90 & $-5,538025$ & $-1,827586$ & 1,626036 & H90 & 7,087177 & $-1,047014$ & 1,509564 \\
\hline H91 & $-4,895104$ & $-5,151805$ & $-1,027957$ & H91 & 6,531464 & $-0,418820$ & $-2,707065$ \\
\hline H92 & $-6,247557$ & $-4,039725$ & 0,736492 & H92 & 8,052357 & $-0,709386$ & $-0,759101$ \\
\hline C93 & $-1,250301$ & $-3,555380$ & 1,783132 & C93 & 2,821267 & 0,923180 & 2,940787 \\
\hline C94 & $-1,114377$ & $-1,255243$ & 1,953235 & C94 & 0,961978 & $-0,352135$ & 2,470866 \\
\hline C95 & $-1,457783$ & $-3,084619$ & 3,235876 & C95 & 2,104312 & 0,481807 & 4,228532 \\
\hline H96 & $-0,497522$ & $-4,340545$ & 1,699009 & H96 & 3,053016 & 1,987921 & 2,930821 \\
\hline H97 & $-2,176498$ & $-3,896790$ & 1,314752 & H97 & 3,750056 & 0,375376 & 2,765908 \\
\hline H98 & $-2,367533$ & $-3,470552$ & 3,696385 & H98 & 2,759294 & 0,017511 & 4,966488 \\
\hline H99 & $-0,595346$ & $-3,301705$ & 3,873754 & H99 & 1,545903 & 1,300918 & 4,692902 \\
\hline N100 & $-0,787489$ & $-2,300227$ & 1,168516 & N100 & 1,798829 & 0,566746 & 1,941505 \\
\hline O101 & $-1,580779$ & $-1,635455$ & 3,146055 & O101 & 1,139593 & $-0,512747$ & 3,786849 \\
\hline $\mathrm{O} 102$ & $-1,023991$ & $-0,058569$ & 1,623522 & O102 & 0,128047 & $-1,013839$ & 1,825621 \\
\hline H103 & $-1,626603$ & 5,026165 & $-1,259088$ & H103 & $-2,872777$ & $-4,197104$ & $-2,146128$ \\
\hline N104 & 2,462291 & 1,926124 & $-0,051385$ & N104 & $-2,836648$ & 0,931216 & $-0,722518$ \\
\hline $\mathrm{C} 105$ & 2,028368 & 2,615268 & $-1,285238$ & $\mathrm{C} 105$ & $-2,843037$ & 0,216407 & $-2,018754$ \\
\hline H106 & 2,171109 & 1,994454 & $-2,183379$ & H106 & $-2,153691$ & 0,670207 & $-2,748212$ \\
\hline Iso3 & & & & $\mathrm{TS}_{\text {Iso3-4 }}$ & & & \\
\hline $\mathrm{C} 1$ & 0,752502 & $-4,421648$ & $-2,686798$ & $\mathrm{C} 1$ & $-2,811440$ & $-3,038130$ & $-3,146331$ \\
\hline $\mathrm{C} 2$ & 2,233990 & $-2,621454$ & $-3,403070$ & $\mathrm{C} 2$ & $-0,422953$ & $-2,999946$ & $-3,658226$ \\
\hline $\mathrm{C} 3$ & 1,197550 & $-3,629712$ & $-3,961557$ & $\mathrm{C} 3$ & $-1,805447$ & $-2,708276$ & $-4,299074$ \\
\hline $\mathrm{C} 4$ & 1,624601 & $-3,779605$ & $-1,582144$ & $\mathrm{C} 4$ & $-1,862659$ & $-3,459388$ & $-2,000405$ \\
\hline H5 & 0,975938 & $-5,489776$ & $-2,773877$ & H5 & $-3,473487$ & $-3,868878$ & $-3,411446$ \\
\hline H6 & 0,362918 & $-3,130510$ & $-4,463699$ & H6 & $-1,888101$ & $-1,674101$ & $-4,648384$ \\
\hline $\mathrm{H} 7$ & 1,692563 & $-4,347080$ & $-0,653879$ & $\mathrm{H7}$ & $-2,337359$ & $-3,973386$ & $-1,163952$ \\
\hline $\mathrm{H} 8$ & $-0,312051$ & $-4,321303$ & $-2,467938$ & H8 & $-3,437549$ & $-2,189735$ & $-2,866396$ \\
\hline H9 & 2,868743 & $-2,146360$ & $-4,150831$ & H9 & 0,398516 & $-3,118128$ & $-4,364443$ \\
\hline $\mathrm{H} 10$ & 1,674306 & $-4,286817$ & $-4,694343$ & $\mathrm{H} 10$ & $-1,959646$ & $-3,358154$ & $-5,164907$ \\
\hline $\mathrm{C} 11$ & 2,938163 & $-3,472267$ & $-2,322073$ & C11 & $-0,749461$ & $-4,209618$ & $-2,753001$ \\
\hline H12 & 3,654048 & $-2,914746$ & $-1,710427$ & H12 & 0,078246 & $-4,516938$ & $-2,106781$ \\
\hline H13 & 3,420670 & $-4,361940$ & $-2,736115$ & H13 & $-1,114975$ & $-5,072785$ & $-3,315899$ \\
\hline $\mathrm{C} 14$ & 2,181002 & $-0,324762$ & $-2,190834$ & $\mathrm{C} 14$ & 1,238004 & $-1,797616$ & $-2,070486$ \\
\hline N15 & 1,821642 & 0,469845 & $-1,246896$ & N15 & 1,583019 & $-1,104094$ & $-1,041237$ \\
\hline $\mathrm{C} 16$ & 2,856950 & 1,553201 & $-1,125031$ & $\mathrm{C} 16$ & 3,069013 & $-1,264845$ & $-0,826640$ \\
\hline $\mathrm{C} 17$ & 3,621835 & 1,367451 & $-2,472698$ & $\mathrm{C} 17$ & 3,419875 & $-2,311481$ & $-1,941339$ \\
\hline P18 & 0,044718 & $-1,886140$ & $-0,156067$ & P18 & $-1,488143$ & $-1,209507$ & $-0,280571$ \\
\hline
\end{tabular}




\begin{tabular}{|c|c|c|c|c|c|c|c|}
\hline Ir19 & $-0,074724$ & 0,341953 & $-0,157424$ & Ir19 & 0,309495 & 0,332786 & $-0,010396$ \\
\hline $\mathrm{C} 20$ & 0,682992 & $-2,523155$ & 1,434225 & $\mathrm{C} 20$ & $-1,698765$ & $-2,253244$ & 1,199683 \\
\hline $\mathrm{C} 21$ & 1,639218 & $-3,226468$ & 3,978610 & $\mathrm{C} 21$ & $-1,741791$ & $-3,673678$ & 3,616442 \\
\hline $\mathrm{C} 22$ & 2,039424 & $-2,816486$ & 1,624770 & $\mathrm{C} 22$ & $-1,053890$ & $-3,492444$ & 1,303526 \\
\hline $\mathrm{C} 23$ & $-0,192584$ & $-2,592966$ & 2,529981 & $\mathrm{C} 23$ & $-2,370524$ & $-1,733691$ & 2,317915 \\
\hline $\mathrm{C} 24$ & 0,284490 & $-2,939047$ & 3,792915 & $\mathrm{C} 24$ & $-2,389612$ & $-2,440332$ & 3,518308 \\
\hline $\mathrm{C} 25$ & 2,510866 & $-3,172251$ & 2,890131 & $\mathrm{C} 25$ & $-1,079542$ & $-4,199287$ & 2,506598 \\
\hline $\mathrm{H} 26$ & $-1,248236$ & $-2,389593$ & 2,388334 & $\mathrm{H} 26$ & $-2,875000$ & $-0,775287$ & 2,248834 \\
\hline $\mathrm{H} 27$ & $-0,403611$ & $-2,995231$ & 4,631866 & $\mathrm{H} 27$ & $-2,913151$ & $-2,031021$ & 4,377922 \\
\hline $\mathrm{H} 28$ & 3,562927 & $-3,407277$ & 3,023701 & H28 & $-0,578278$ & $-5,160402$ & 2,575778 \\
\hline H29 & 2,009899 & $-3,499342$ & 4,962152 & $\mathrm{H} 29$ & $-1,755886$ & $-4,222692$ & 4,553195 \\
\hline $\mathrm{O} 30$ & 3,255391 & 0,021584 & $-2,906000$ & O30 & 2,190533 & $-2,487053$ & $-2,699188$ \\
\hline C31 & 3,816903 & 1,266225 & 0,035096 & $\mathrm{C} 31$ & 3,384238 & $-1,902589$ & 0,523235 \\
\hline C32 & 3,659372 & 0,175601 & 0,886411 & C32 & 2,396300 & $-2,534812$ & 1,277849 \\
\hline C33 & 4,904755 & 2,130254 & 0,232025 & C33 & 4,718005 & $-1,975879$ & 0,952909 \\
\hline C34 & 4,559130 & $-0,044223$ & 1,931082 & C34 & 2,722366 & $-3,200069$ & 2,459501 \\
\hline H35 & 2,816625 & $-0,484733$ & 0,759355 & H35 & 1,363858 & $-2,488442$ & 0,963637 \\
\hline $\mathrm{C} 36$ & 5,806121 & 1,909509 & 1,269906 & C36 & 5,045839 & $-2,648684$ & 2,128015 \\
\hline $\mathrm{H} 37$ & 5,031990 & 2,994164 & $-0,416312$ & H37 & 5,501197 & $-1,496122$ & 0,372534 \\
\hline $\mathrm{C} 38$ & 5,632652 & 0,819755 & 2,127614 & C38 & 4,046318 & $-3,259604$ & 2,889181 \\
\hline H39 & 4,402513 & $-0,887934$ & 2,596876 & H39 & 1,930472 & $-3,661023$ & 3,042925 \\
\hline $\mathrm{H} 40$ & 6,638287 & 2,591598 & 1,415486 & $\mathrm{H} 40$ & 6,081928 & $-2,691728$ & 2,451024 \\
\hline H41 & 6,329608 & 0,652230 & 2,943749 & $\mathrm{H} 41$ & 4,302200 & $-3,776494$ & 3,809525 \\
\hline $\mathrm{C} 42$ & $-1,497643$ & $-2,815638$ & $-0,457087$ & $\mathrm{C} 42$ & $-3,148968$ & $-0,577093$ & $-0,700174$ \\
\hline $\mathrm{C} 43$ & $-1,671097$ & $-4,107415$ & 0,066948 & $\mathrm{C} 43$ & $-4,329557$ & $-1,188110$ & $-0,253027$ \\
\hline $\mathrm{C} 44$ & $-2,452213$ & $-2,299453$ & $-1,344980$ & $\mathrm{C} 44$ & $-3,219881$ & 0,447134 & $-1,656942$ \\
\hline $\mathrm{C} 45$ & $-2,784695$ & $-4,864564$ & $-0,290885$ & $\mathrm{C} 45$ & $-5,563565$ & $-0,774846$ & $-0,755278$ \\
\hline H46 & $-0,939110$ & $-4,526214$ & 0,750063 & $\mathrm{H} 46$ & $-4,290618$ & $-1,992333$ & 0,474240 \\
\hline $\mathrm{C} 47$ & $-3,564097$ & $-3,061113$ & $-1,697823$ & $\mathrm{C} 47$ & $-4,453222$ & 0,849289 & $-2,161579$ \\
\hline $\mathrm{C} 48$ & $-3,732155$ & $-4,343415$ & $-1,173619$ & $\mathrm{C} 48$ & $-5,626629$ & 0,240377 & $-1,710553$ \\
\hline $\mathrm{H} 49$ & $-2,911220$ & $-5,862219$ & 0,119389 & $\mathrm{H} 49$ & $-6,474366$ & $-1,252133$ & $-0,405164$ \\
\hline H50 & $-4,307574$ & $-2,644960$ & $-2,370480$ & H50 & $-4,500632$ & 1,650091 & $-2,892728$ \\
\hline H51 & $-4,601242$ & $-4,934202$ & $-1,448395$ & H51 & $-6,588719$ & 0,558342 & $-2,101597$ \\
\hline H52 & $-0,831413$ & 0,233738 & $-1,530023$ & H52 & $-0,012877$ & 0,952030 & $-1,406386$ \\
\hline H53 & $-1,147446$ & 2,917124 & 1,330328 & H53 & 1,347700 & 2,264984 & 1,677842 \\
\hline H54 & 2,729667 & $-2,758143$ & 0,790274 & $\mathrm{H} 54$ & $-0,519949$ & $-3,896714$ & 0,449707 \\
\hline H55 & $-2,337525$ & $-1,295229$ & $-1,735696$ & H55 & $-2,310870$ & 0,938235 & $-1,988788$ \\
\hline H56 & 3,315367 & 2,061206 & $-3,258926$ & H56 & 4,204264 & $-1,988179$ & $-2,625661$ \\
\hline $\mathrm{C} 57$ & 0,589788 & 5,230008 & $-0,684315$ & C57 & 4,790170 & 2,664396 & $-1,511134$ \\
\hline C58 & 0,661250 & 4,590010 & $-1,922130$ & C58 & 4,415431 & 1,858109 & $-2,586533$ \\
\hline C59 & 1,416480 & 3,427944 & $-2,066560$ & C59 & 3,881329 & 0,590448 & $-2,358162$ \\
\hline C60 & 2,129181 & 2,892781 & $-0,983609$ & C60 & 3,726878 & 0,099275 & $-1,054940$ \\
\hline C61 & 2,041888 & 3,536775 & 0,257661 & C61 & 4,085367 & 0,926790 & 0,018399 \\
\hline C62 & 1,279490 & 4,697307 & 0,403694 & C62 & 4,616218 & 2,197451 & $-0,209049$ \\
\hline $\mathrm{H} 63$ & $-0,009204$ & 6,128293 & $-0,568648$ & H63 & 5,200456 & 3,654195 & $-1,686624$ \\
\hline H64 & 0,108745 & 4,982971 & $-2,769577$ & H64 & 4,533613 & 2,217026 & $-3,604795$ \\
\hline H65 & 1,419355 & 2,922980 & $-3,027940$ & H65 & 3,572990 & $-0,008711$ & $-3,211000$ \\
\hline H66 & 2,553748 & 3,121326 & 1,117332 & H66 & 3,951473 & 0,575039 & 1,035530 \\
\hline
\end{tabular}




\begin{tabular}{|c|c|c|c|c|c|c|c|}
\hline H67 & 1,225812 & 5,183365 & 1,373764 & H67 & 4,889726 & 2,823672 & 0,635193 \\
\hline C68 & $-5,187056$ & $-0,203434$ & $-0,391292$ & C68 & $-3,273770$ & 3,931915 & $-0,739652$ \\
\hline C69 & $-3,969106$ & 0,464308 & $-0,270602$ & C69 & $-2,050629$ & 3,369276 & $-0,379862$ \\
\hline $\mathrm{C} 70$ & $-3,097531$ & 0,197801 & 0,797671 & C70 & $-1,964398$ & 2,458589 & 0,685894 \\
\hline C71 & $-3,491735$ & $-0,799949$ & 1,709194 & C71 & $-3,158808$ & 2,104656 & 1,338148 \\
\hline $\mathrm{C} 72$ & $-4,699831$ & $-1,480005$ & 1,580278 & $\mathrm{C} 72$ & $-4,382114$ & 2,658216 & 0,969973 \\
\hline $\mathrm{C} 73$ & $-5,565984$ & $-1,176555$ & 0,531856 & C73 & $-4,445391$ & 3,589508 & $-0,064515$ \\
\hline H74 & $-5,840730$ & 0,039617 & $-1,224591$ & H74 & $-3,307485$ & 4,643123 & $-1,560350$ \\
\hline H75 & $-3,703388$ & 1,186518 & $-1,026165$ & H75 & $-1,167554$ & 3,640644 & $-0,940228$ \\
\hline H76 & $-2,833402$ & $-1,059756$ & 2,529947 & $\mathrm{H} 76$ & $-3,133806$ & 1,373037 & 2,138029 \\
\hline H77 & $-4,960665$ & $-2,249343$ & 2,301584 & $\mathrm{H} 77$ & $-5,285620$ & 2,354049 & 1,490350 \\
\hline $\mathrm{H} 78$ & $-6,513874$ & $-1,696850$ & 0,430651 & $\mathrm{H} 78$ & $-5,395651$ & 4,030484 & $-0,350528$ \\
\hline C79 & $-1,754181$ & 0,855415 & 0,975294 & C79 & $-0,674308$ & 1,848670 & 1,142784 \\
\hline $\mathrm{C} 80$ & $-1,433311$ & 2,303456 & 0,470158 & $\mathrm{C} 80$ & 0,677078 & 2,333190 & 0,817837 \\
\hline H81 & $-0,442221$ & 2,301483 & $-0,111507$ & H81 & 1,674286 & 1,210019 & 0,023134 \\
\hline $\mathrm{C} 82$ & $-2,397498$ & 3,068608 & $-0,396509$ & $\mathrm{C} 82$ & 1,002453 & 3,541341 & $-0,010799$ \\
\hline $\mathrm{C} 83$ & $-3,443430$ & 3,769730 & 0,212006 & $\mathrm{C} 83$ & 0,849930 & 4,786776 & 0,616974 \\
\hline $\mathrm{C} 84$ & $-2,284835$ & 3,080946 & $-1,789627$ & $\mathrm{C} 84$ & 1,461723 & 3,495144 & $-1,328820$ \\
\hline $\mathrm{C} 85$ & $-4,374479$ & 4,461708 & $-0,561081$ & $\mathrm{C} 85$ & 1,131741 & 5,965214 & $-0,068793$ \\
\hline H86 & $-3,539360$ & 3,760263 & 1,295517 & H86 & 0,489269 & 4,830366 & 1,642118 \\
\hline $\mathrm{C} 87$ & $-3,216017$ & 3,771514 & $-2,564848$ & $\mathrm{C} 87$ & 1,744624 & 4,677226 & $-2,015476$ \\
\hline H88 & $-1,470901$ & 2,536644 & $-2,259117$ & H88 & 1,604079 & 2,535368 & $-1,811644$ \\
\hline C89 & $-4,264283$ & 4,461779 & $-1,952618$ & C89 & 1,576531 & 5,913232 & $-1,392087$ \\
\hline H90 & $-5,187530$ & 4,995948 & $-0,078129$ & H90 & 1,000739 & 6,922706 & 0,426909 \\
\hline H91 & $-3,124619$ & 3,770741 & $-3,647405$ & H91 & 2,103075 & 4,627843 & $-3,039582$ \\
\hline H92 & $-4,991317$ & 4,997147 & $-2,556197$ & H92 & 1,792903 & 6,831451 & $-1,930231$ \\
\hline C93 & $-2,069338$ & 1,373048 & 3,527580 & C93 & $-0,988452$ & 2,236675 & 3,683775 \\
\hline C94 & $-0,020531$ & 0,780498 & 2,680540 & C94 & 0,043969 & 0,333235 & 2,908198 \\
\hline C95 & $-1,068001$ & 1,085416 & 4,656564 & C95 & $-0,792624$ & 1,216578 & 4,814844 \\
\hline H96 & $-3,040047$ & 0,901017 & 3,681966 & H96 & $-1,985230$ & 2,678622 & 3,668672 \\
\hline H97 & $-2,223220$ & 2,449446 & 3,365771 & H97 & $-0,244580$ & 3,045726 & 3,712420 \\
\hline H98 & $-1,006403$ & 1,863886 & 5,416919 & H98 & $-0,321846$ & 1,622484 & 5,710269 \\
\hline H99 & $-1,250712$ & 0,114678 & 5,128533 & H99 & $-1,728991$ & 0,715662 & 5,081658 \\
\hline N100 & $-1,343287$ & 0,778175 & 2,396599 & N100 & $-0,754382$ & 1,364552 & 2,524922 \\
\hline O101 & 0,219484 & 1,014942 & 3,976008 & O101 & 0,102043 & 0,222485 & 4,242407 \\
\hline O102 & 0,887860 & 0,618303 & 1,848544 & O102 & 0,675679 & $-0,406576$ & 2,144371 \\
\hline H103 & 4,703366 & 1,395318 & $-2,353093$ & H103 & 3,681303 & $-3,280425$ & $-1,514458$ \\
\hline N104 & 1,169658 & $-2,386403$ & $-1,314869$ & N104 & $-1,085086$ & $-2,285135$ & $-1,519589$ \\
\hline C105 & 1,451136 & $-1,597058$ & $-2,540445$ & C105 & $-0,166208$ & $-1,887841$ & $-2,610269$ \\
\hline H106 & 0,528312 & $-1,287902$ & $-3,053367$ & H106 & $-0,410488$ & $-0,899243$ & $-3,024358$ \\
\hline
\end{tabular}

Iso4

$\begin{array}{llll}\text { C1 } & -2,836136 & -2,994277 & -3,124542 \\ \text { C2 } & -0,450569 & -2,963668 & -3,644260 \\ \text { C3 } & -1,833267 & -2,668976 & -4,280964 \\ \text { C4 } & -1,884882 & -3,414609 & -1,979532 \\ \text { H5 } & -3,498509 & -3,826252 & -3,385556 \\ \text { H6 } & -1,914933 & -1,635029 & -4,631322\end{array}$




\begin{tabular}{|c|c|c|c|}
\hline H7 & $-2,356025$ & $-3,925955$ & $-1,139378$ \\
\hline H8 & $-3,462063$ & $-2,145142$ & $-2,849296$ \\
\hline H9 & 0,367323 & $-3,086668$ & $-4,353920$ \\
\hline H10 & $-1,991487$ & $-3,320158$ & $-5,145221$ \\
\hline C11 & $-0,777723$ & $-4,170020$ & $-2,735348$ \\
\hline H12 & 0,050888 & $-4,480338$ & $-2,091780$ \\
\hline H13 & $-1,149393$ & $-5,032159$ & $-3,296011$ \\
\hline $\mathrm{C} 14$ & 1,222434 & $-1,798046$ & $-2,055600$ \\
\hline N15 & 1,591139 & $-1,119294$ & $-1,023561$ \\
\hline $\mathrm{C} 16$ & 3,085163 & $-1,303914$ & $-0,838600$ \\
\hline $\mathrm{C} 17$ & 3,374985 & $-2,412577$ & $-1,903151$ \\
\hline P18 & $-1,512349$ & $-1,146720$ & $-0,278427$ \\
\hline $\operatorname{Ir} 19$ & 0,385131 & 0,355230 & 0,015098 \\
\hline $\mathrm{C} 20$ & $-1,759174$ & $-2,193443$ & 1,195869 \\
\hline $\mathrm{C} 21$ & $-1,842191$ & $-3,619662$ & 3,609432 \\
\hline $\mathrm{C} 22$ & $-1,065253$ & $-3,403670$ & 1,328428 \\
\hline $\mathrm{C} 23$ & $-2,501900$ & $-1,707756$ & 2,283579 \\
\hline $\mathrm{C} 24$ & $-2,542070$ & $-2,418204$ & 3,481844 \\
\hline $\mathrm{C} 25$ & $-1,109920$ & $-4,112866$ & 2,528905 \\
\hline H26 & $-3,053803$ & $-0,778735$ & 2,189890 \\
\hline $\mathrm{H} 27$ & $-3,125487$ & $-2,037096$ & 4,315687 \\
\hline $\mathrm{H} 28$ & $-0,569820$ & $-5,051091$ & 2,619469 \\
\hline $\mathrm{H} 29$ & $-1,871682$ & $-4,170441$ & 4,544929 \\
\hline $\mathrm{O} 30$ & 2,153004 & $-2,516198$ & $-2,685065$ \\
\hline C31 & 3,431866 & $-1,877448$ & 0,529428 \\
\hline C32 & 2,462520 & $-2,502201$ & 1,315113 \\
\hline C33 & 4,771822 & $-1,921453$ & 0,943624 \\
\hline C34 & 2,809266 & $-3,108498$ & 2,521850 \\
\hline H35 & 1,426669 & $-2,489493$ & 1,007157 \\
\hline C36 & 5,120455 & $-2,537190$ & 2,144093 \\
\hline H37 & 5,544465 & $-1,464235$ & 0,332374 \\
\hline C38 & 4,137412 & $-3,124466$ & 2,943437 \\
\hline H39 & 2,030365 & $-3,556081$ & 3,132705 \\
\hline $\mathrm{H} 40$ & 6,160572 & $-2,552496$ & 2,456912 \\
\hline H41 & 4,408855 & $-3,592054$ & 3,885554 \\
\hline $\mathrm{C} 42$ & $-3,161180$ & $-0,518910$ & $-0,748129$ \\
\hline $\mathrm{C} 43$ & $-4,351369$ & $-1,160134$ & $-0,373554$ \\
\hline $\mathrm{C} 44$ & $-3,199992$ & 0,509402 & $-1,701321$ \\
\hline $\mathrm{C} 45$ & $-5,563476$ & $-0,769334$ & $-0,941032$ \\
\hline $\mathrm{H} 46$ & $-4,335220$ & $-1,970438$ & 0,347993 \\
\hline $\mathrm{C} 47$ & $-4,412078$ & 0,890008 & $-2,271523$ \\
\hline $\mathrm{C} 48$ & $-5,594989$ & 0,251682 & $-1,892604$ \\
\hline H49 & $-6,481759$ & $-1,269867$ & $-0,647230$ \\
\hline H50 & $-4,435678$ & 1,693868 & $-3,000905$ \\
\hline H51 & $-6,540056$ & 0,550150 & $-2,337131$ \\
\hline H52 & 0,123999 & 1,014749 & $-1,373579$ \\
\hline H53 & 1,300700 & 2,218456 & 1,738425 \\
\hline H54 & $-0,481667$ & $-3,783790$ & 0,495881 \\
\hline
\end{tabular}




\begin{tabular}{|c|c|c|c|}
\hline H55 & $-2,282803$ & 1,017716 & $-1,982130$ \\
\hline H56 & 4,198267 & $-2,176342$ & $-2,576931$ \\
\hline $\mathrm{C} 57$ & 4,861690 & 2,526948 & $-1,860351$ \\
\hline C58 & 4,381949 & 1,675848 & $-2,856953$ \\
\hline C59 & 3,832401 & 0,440389 & $-2,516553$ \\
\hline $\mathrm{C} 60$ & 3,762625 & 0,027138 & $-1,179094$ \\
\hline C61 & 4,225034 & 0,901022 & $-0,185296$ \\
\hline C62 & 4,774442 & 2,138128 & $-0,524585$ \\
\hline H63 & 5,287987 & 3,490745 & $-2,122616$ \\
\hline H64 & 4,433255 & 1,973200 & $-3,900497$ \\
\hline H65 & 3,444393 & $-0,196141$ & $-3,307995$ \\
\hline H66 & 4,154411 & 0,615915 & 0,858308 \\
\hline H67 & 5,126530 & 2,801523 & 0,259915 \\
\hline C68 & $-3,333282$ & 3,848014 & $-0,699825$ \\
\hline C69 & $-2,107575$ & 3,292534 & $-0,340852$ \\
\hline $\mathrm{C} 70$ & $-2,012999$ & 2,397543 & 0,737557 \\
\hline C71 & $-3,201390$ & 2,047870 & 1,401614 \\
\hline $\mathrm{C} 72$ & $-4,428979$ & 2,592048 & 1,032392 \\
\hline $\mathrm{C} 73$ & $-4,500033$ & 3,509328 & $-0,013789$ \\
\hline H74 & $-3,374321$ & 4,547696 & $-1,530091$ \\
\hline H75 & $-1,227523$ & 3,554685 & $-0,910090$ \\
\hline H76 & $-3,167602$ & 1,330507 & 2,213504 \\
\hline H77 & $-5,328838$ & 2,292690 & 1,562047 \\
\hline H78 & $-5,453296$ & 3,943468 & $-0,300625$ \\
\hline C79 & $-0,713307$ & 1,823017 & 1,205902 \\
\hline $\mathrm{C} 80$ & 0,610364 & 2,326599 & 0,899325 \\
\hline H81 & 1,825470 & 1,026720 & $-0,007609$ \\
\hline $\mathrm{C} 82$ & 0,941900 & 3,540869 & 0,082884 \\
\hline $\mathrm{C} 83$ & 0,641554 & 4,784539 & 0,661890 \\
\hline $\mathrm{C} 84$ & 1,567600 & 3,509123 & $-1,166016$ \\
\hline $\mathrm{C} 85$ & 0,940059 & 5,970062 & $-0,004644$ \\
\hline H86 & 0,147672 & 4,818704 & 1,630193 \\
\hline $\mathrm{C} 87$ & 1,869364 & 4,698634 & $-1,832049$ \\
\hline H88 & 1,824146 & 2,556698 & $-1,613793$ \\
\hline $\mathrm{C} 89$ & 1,551966 & 5,929622 & $-1,259931$ \\
\hline H90 & 0,688676 & 6,923681 & 0,450897 \\
\hline H91 & 2,356015 & 4,656683 & $-2,802375$ \\
\hline H92 & 1,779603 & 6,852897 & $-1,785106$ \\
\hline C93 & $-1,034213$ & 2,138634 & 3,742234 \\
\hline C94 & 0,008614 & 0,257199 & 2,933546 \\
\hline C95 & $-0,849707$ & 1,095411 & 4,852201 \\
\hline H96 & $-2,028107$ & 2,587372 & 3,732191 \\
\hline H97 & $-0,282415$ & 2,939368 & 3,785461 \\
\hline H98 & $-0,387717$ & 1,481131 & 5,761044 \\
\hline H99 & $-1,787233$ & 0,584716 & 5,094972 \\
\hline N100 & $-0,804562$ & 1,285238 & 2,566529 \\
\hline O101 & 0,054176 & 0,117640 & 4,265373 \\
\hline $\mathrm{O} 102$ & 0,656841 & $-0,458017$ & 2,163041 \\
\hline
\end{tabular}




$\begin{array}{lrrr}\text { H103 } & 3,544406 & -3,383175 & -1,435082 \\ \text { N104 } & -1,099178 & -2,242343 & -1,501082 \\ \text { C105 } & -0,182291 & -1,853139 & -2,597381 \\ \text { H106 } & -0,411950 & -0,859171 & -3,006370\end{array}$

\section{C3_ES_Cheltop}

C3_ES_Cheltop_SM

\begin{tabular}{|c|c|c|c|}
\hline $\mathrm{C} 1$ & -4.0724 & -3.51299 & 0.036369 \\
\hline $\mathrm{C} 2$ & -1.8955 & -4.6104 & -0.0578 \\
\hline $\mathrm{C} 3$ & -3.2708 & -4.58461 & -0.77373 \\
\hline 4 & -3.0409 & -3.0685 & 1.099679 \\
\hline $\mathrm{H} 5$ & -4.9481 & -3.95009 & 0.527236 \\
\hline H6 & -3.1799 & -4.33063 & -1.83487 \\
\hline H7 & -3.4541 & -2.50923 & 1.939156 \\
\hline 18 & -4.4174 & -2.68016 & -0.57691 \\
\hline H9 & -1.2803 & -5.48425 & -0.27085 \\
\hline $\mathrm{H} 10$ & -3.7418 & -5.56971 & -0.7131 \\
\hline C11 & -2.3053 & -4.38271 & 1.41487 \\
\hline$[12$ & -1.4597 & -4.25058 & 2.09635 \\
\hline $\mathrm{H} 13$ & -2.9662 & -5.16734 & 1.793514 \\
\hline $\mathrm{C} 14$ & 0.2855 & -3.21475 & -0.07198 \\
\hline 15 & 1.00283 & -2.14212 & -0.06856 \\
\hline C16 & 2.36915 & -2.50715 & 0.481172 \\
\hline $\mathrm{C} 17$ & 2.31978 & -4.05556 & 0.332861 \\
\hline P18 & -1.8319 & -0.65359 & 0.136554 \\
\hline Ir19 & 0.39762 & -0.21244 & -0.78742 \\
\hline $\mathrm{C} 20$ & -2.0534 & 0.18371 & 1.747998 \\
\hline $\mathrm{C} 21$ & -2.1596 & 1.602723 & 4.171654 \\
\hline $\mathrm{C} 22$ & -1.8664 & -0.47916 & 2.967765 \\
\hline $\mathrm{C} 23$ & -2.2946 & 1.568215 & 1.757012 \\
\hline $\mathrm{C} 24$ & -2.3451 & 2.270794 & 2.959086 \\
\hline $\mathrm{C} 25$ & -1.926 & 0.227567 & 4.170467 \\
\hline H26 & -2.4684 & 2.096825 & 0.823661 \\
\hline $\mathrm{H} 27$ & -2.5383 & 3.339764 & 2.949911 \\
\hline H28 & -1.7927 & -0.3024 & 5.109459 \\
\hline H29 & -2.2026 & 2.149461 & 5.108652 \\
\hline $\mathrm{O} 30$ & 0.89751 & -4.35409 & 0.249968 \\
\hline $\mathrm{C} 31$ & 2.45183 & -2.17837 & 1.977896 \\
\hline $\mathrm{C} 32$ & 1.37517 & -1.6637 & 2.695179 \\
\hline $\mathrm{C} 33$ & 3.64318 & -2.47288 & 2.659895 \\
\hline C34 & 1.48863 & -1.41958 & 4.065322 \\
\hline H35 & 0.45567 & -1.42313 & 2.184695 \\
\hline C36 & 3.75582 & -2.23582 & 4.02645 \\
\hline H37 & 4.49366 & -2.87101 & 2.112608 \\
\hline & 2.67593 & -1.70178 & 4.7348 \\
\hline
\end{tabular}

C3_ES_Cheltop_INT2

$\begin{array}{lrrr}\text { C1 } & -4.296 & -3.09198 & 0.982633 \\ \text { C2 } & -2.2841 & -4.45618 & 0.821148 \\ \text { C3 } & -3.7472 & -4.39582 & 0.316365 \\ \text { C4 } & -3.0709 & -2.59069 & 1.783265 \\ \text { H5 } & -5.1262 & -3.30296 & 1.664684 \\ \text { H6 } & -3.8076 & -4.36995 & -0.77618 \\ \text { H7 } & -3.2878 & -1.83811 & 2.541337 \\ \text { H8 } & -4.643 & -2.35523 & 0.258351 \\ \text { H9 } & -1.7969 & -5.42435 & 0.710107 \\ \text { H10 } & -4.2955 & -5.27988 & 0.653826 \\ \text { C11 } & -2.4264 & -3.90436 & 2.257373 \\ \text { H12 } & -1.4743 & -3.75305 & 2.775052 \\ \text { H13 } & -3.0917 & -4.51103 & 2.877991 \\ \text { C14 } & -0.0022 & -3.40527 & 0.212148 \\ \text { N15 } & 0.80884 & -2.43403 & -0.01197 \\ \text { C16 } & 2.19654 & -2.90991 & 0.332237 \\ \text { C17 } & 1.97075 & -4.45157 & 0.379789 \\ \text { P18 } & -1.7918 & -0.5564 & 0.233929 \\ \text { Ir19 } & 0.20783 & -0.45887 & -0.85362 \\ \text { C20 } & -1.8784 & 0.533078 & 1.699952 \\ \text { C21 } & -1.8774 & 2.251068 & 3.918953 \\ \text { C22 } & -1.4465 & 0.075739 & 2.953735 \\ \text { C23 } & -2.2857 & 1.870496 & 1.564177 \\ \text { C24 } & -2.2954 & 2.718936 & 2.671326 \\ \text { C25 } & -1.4459 & 0.930976 & 4.054683 \\ \text { H26 } & -2.6228 & 2.244792 & 0.602152 \\ \text { H27 } & -2.6435 & 3.742448 & 2.560719 \\ \text { H28 } & -1.1148 & 0.561978 & 5.020978 \\ \text { H29 } & -1.8942 & 2.910789 & 4.781901 \\ \text { O30 } & 0.52926 & -4.58752 & 0.54402 \\ \text { C31 } 38 & 2.5885 & -2.42971 & 1.733473 \\ \text { C32 } & 1.70798 & -1.71826 & 2.546477 \\ \text { C33 } & 3.85136 & -2.7709 & 2.239724 \\ \text { C34 } & 2.08722 & -1.32248 & 3.829412 \\ & 0.74018 & -1.43794 & 2.161025 \\ \text { H3 } & 4.22961 & -2.38392 & 3.522989 \\ \text { H.55006 } & -3.32621 & 1.618962 \\ \text { H } 34796 & -1.6513 & 4.322025\end{array}$




\begin{tabular}{|c|c|c|c|c|c|c|c|}
\hline H39 & 0.64488 & -0.9919 & 4.598414 & H39 & 1.3961 & -0.74054 & 4.43307 \\
\hline $\mathrm{H} 40$ & 4.6875 & -2.46151 & 4.537066 & H40 & 5.21459 & -2.64833 & 3.896729 \\
\hline H41 & 2.76496 & -1.50769 & 5.799832 & H41 & 3.64663 & -1.34047 & 5.31905 \\
\hline $\mathrm{C} 42$ & -3.3366 & -0.25293 & -0.82492 & $\mathrm{C} 42$ & -3.2935 & -0.16732 & -0.74574 \\
\hline $\mathrm{C} 43$ & -4.5077 & 0.269182 & -0.25842 & $\mathrm{C} 43$ & -4.4733 & 0.284508 & -0.1253 \\
\hline $\mathrm{C} 44$ & -3.3393 & -0.64039 & -2.17425 & $\mathrm{C} 44$ & -3.3284 & -0.48705 & -2.11096 \\
\hline $\mathrm{C} 45$ & -5.6562 & 0.415519 & -1.03712 & $\mathrm{C} 45$ & -5.65 & 0.423664 & -0.85889 \\
\hline $\mathrm{H} 46$ & -4.5322 & 0.553861 & 0.788137 & H46 & -4.4811 & 0.521324 & 0.933909 \\
\hline $\mathrm{C} 47$ & -4.4888 & -0.49943 & -2.94592 & $\mathrm{C} 47$ & -4.5108 & -0.35291 & -2.84015 \\
\hline $\mathrm{C} 48$ & -5.6489 & 0.03278 & -2.37867 & $\mathrm{C} 48$ & -5.6736 & 0.103163 & -2.21927 \\
\hline H49 & -6.5594 & 0.822749 & -0.59161 & H49 & -6.5505 & 0.778504 & -0.36569 \\
\hline H50 & -4.4744 & -0.78734 & -3.99244 & H50 & -4.5122 & -0.59018 & -3.89972 \\
\hline H5 1 & -6.5449 & 0.148404 & -2.98167 & H51 & -6.5919 & 0.212335 & -2.78858 \\
\hline H52 & 0.07278 & -0.86118 & -2.16901 & H52 & -0.555 & -1.17989 & -2.01873 \\
\hline H53 & 1.90809 & -0.24684 & -1.26165 & H53 & 1.88357 & 2.066437 & -1.50151 \\
\hline H54 & -1.6612 & -1.54403 & 2.982211 & H54 & -1.1179 & -0.95091 & 3.070574 \\
\hline H55 & -2.4339 & -1.03695 & -2.62163 & H55 & -2.426 & -0.81333 & -2.61379 \\
\hline H56 & 2.78496 & -4.43454 & -0.57938 & H56 & 2.24879 & -4.9714 & -0.54017 \\
\hline $\mathrm{C} 57$ & 5.36018 & -0.5051 & -1.9464 & $\mathrm{C} 57$ & 4.5597 & -1.35704 & -2.95078 \\
\hline C58 & 4.70474 & -1.63696 & -2.43238 & $\mathrm{C} 58$ & 3.69354 & -2.43533 & -3.14809 \\
\hline C59 & 3.76353 & -2.29444 & -1.64176 & C59 & 2.98925 & -2.97099 & -2.07109 \\
\hline $\mathrm{C} 60$ & 3.47169 & -1.84552 & -0.34648 & $\mathrm{C} 60$ & 3.1384 & -2.44621 & -0.77945 \\
\hline C61 & 4.12651 & -0.70015 & 0.126049 & C61 & 4.00547 & -1.36318 & -0.59212 \\
\hline C62 & 5.06208 & -0.03638 & -0.66684 & C62 & 4.71354 & -0.82563 & -1.67003 \\
\hline H63 & 6.0927 & 0.008702 & -2.56228 & H63 & 5.10793 & -0.93597 & -3.78865 \\
\hline H64 & 4.91611 & -2.00276 & -3.43288 & H64 & 3.55946 & -2.85438 & -4.14125 \\
\hline H65 & 3.23529 & -3.14309 & -2.06502 & H65 & 2.285 & -3.77627 & -2.25852 \\
\hline H66 & 3.89157 & -0.31695 & 1.112322 & H66 & 4.10684 & -0.91681 & 0.390129 \\
\hline H67 & 5.561 & 0.848177 & -0.28036 & H67 & 5.38169 & 0.01528 & -1.50583 \\
\hline C68 & 1.93349 & 1.965337 & -2.77994 & C68 & 0.9006 & 3.912055 & -1.96872 \\
\hline C69 & 1.02357 & 1.732293 & -1.54504 & C69 & 0.90492 & 2.510067 & -1.28081 \\
\hline $\mathrm{C} 70$ & -0.4129 & 1.716096 & -1.49386 & $\mathrm{C} 70$ & -0.2166 & 1.493179 & -1.67255 \\
\hline $\mathrm{C} 71$ & 1.90531 & 3.837858 & -0.31361 & C71 & 0.55144 & 3.929205 & 0.961451 \\
\hline $\mathrm{C} 72$ & 1.45963 & 1.912349 & 0.873904 & C72 & 1.18452 & 1.720334 & 1.055645 \\
\hline $\mathrm{C} 73$ & 2.40814 & 3.951041 & 1.136305 & $\mathrm{C} 73$ & 1.12996 & 3.581632 & 2.342373 \\
\hline H74 & 0.98897 & 4.416642 & -0.49282 & H74 & -0.5337 & 4.064278 & 0.998729 \\
\hline H75 & 2.6623 & 4.142064 & -1.03811 & H75 & 1.00409 & 4.828546 & 0.544802 \\
\hline H76 & 3.498 & 3.875966 & 1.199972 & H76 & 2.12813 & 4.004117 & 2.496873 \\
\hline H77 & 2.06841 & 4.848112 & 1.654792 & H77 & 0.47448 & 3.857779 & 3.168377 \\
\hline N78 & 1.62659 & 2.398673 & -0.37843 & N78 & 0.89963 & 2.719439 & 0.201999 \\
\hline O79 & 1.83816 & 2.795953 & 1.808198 & O79 & 1.24665 & 2.1386 & 2.331739 \\
\hline $\mathrm{O} 80$ & 1.01104 & 0.796259 & 1.159321 & O80 & 1.38949 & 0.52762 & 0.782372 \\
\hline H81 & 2.7232 & -4.57591 & 1.19933 & H81 & 2.4523 & -4.92467 & 1.233927 \\
\hline N82 & -1.9205 & -2.31229 & 0.468105 & N82 & -1.9946 & -2.11531 & 0.864439 \\
\hline $\mathrm{C} 83$ & -1.185 & -3.27418 & -0.39165 & $\mathrm{C} 83$ & -1.5052 & -3.30924 & 0.124773 \\
\hline
\end{tabular}




\begin{tabular}{|c|c|c|c|c|c|c|c|}
\hline H84 & -1.2822 & -3.03959 & -1.46126 & $\mathrm{H} 84$ & -1.7551 & -3.26332 & -0.94538 \\
\hline $\mathrm{C} 85$ & -3.1362 & 2.229675 & -4.78445 & $\mathrm{C} 85$ & -1.0422 & 1.173228 & -5.92307 \\
\hline C86 & -3.2778 & 2.951964 & -3.60017 & $\mathrm{C} 86$ & -2.0173 & 1.621529 & -5.03434 \\
\hline $\mathrm{C} 87$ & -2.3899 & 2.744747 & -2.54629 & C87 & -1.7262 & 1.734964 & -3.67359 \\
\hline $\mathrm{C} 88$ & -1.3477 & 1.809618 & -2.64569 & $\mathrm{C} 88$ & -0.466 & 1.395222 & -3.15998 \\
\hline C89 & -1.243 & 1.06398 & -3.829 & C89 & 0.50628 & 0.953458 & -4.07494 \\
\hline C90 & -2.1161 & 1.282377 & -4.89112 & C90 & 0.22617 & 0.846751 & -5.43443 \\
\hline H91 & -3.8223 & 2.392279 & -5.61051 & H91 & -1.262 & 1.084877 & -6.98299 \\
\hline H92 & -4.0785 & 3.678302 & -3.49481 & H92 & -3.0067 & 1.889193 & -5.39599 \\
\hline H93 & -2.5044 & 3.321872 & -1.63128 & H93 & -2.4964 & 2.08598 & -2.99232 \\
\hline H94 & -0.4953 & 0.287295 & -3.90029 & H94 & 1.50704 & 0.712717 & -3.72822 \\
\hline H95 & -2.0095 & 0.697646 & -5.80077 & H95 & 1.00176 & 0.511658 & -6.11771 \\
\hline H96 & -0.8069 & 2.234557 & -0.62012 & H96 & -1.1348 & 1.888391 & -1.22404 \\
\hline C97 & 2.18427 & 0.805459 & -3.75435 & C97 & 1.54501 & 3.922157 & -3.36618 \\
\hline H98 & 3.00212 & 1.089173 & -4.42542 & H98 & 1.74703 & 4.957562 & -3.66039 \\
\hline H99 & 2.47506 & -0.10467 & -3.2305 & H99 & 2.49809 & 3.38187 & -3.37756 \\
\hline $\mathrm{H} 100$ & 1.31774 & 0.591059 & -4.37995 & H100 & 0.89757 & 3.481589 & -4.12383 \\
\hline $\mathrm{C} 101$ & 1.49124 & 3.221134 & -3.56327 & C101 & -0.4781 & 4.590549 & -2.02084 \\
\hline H102 & 2.28103 & 3.519195 & -4.25978 & H102 & -0.3691 & 5.637388 & -2.32172 \\
\hline H103 & 0.58888 & 3.020677 & -4.14375 & H103 & -1.1172 & 4.100269 & -2.75763 \\
\hline H104 & 1.27717 & 4.075145 & -2.91508 & H104 & -1.0058 & 4.576261 & -1.06305 \\
\hline H105 & 2.91225 & 2.168069 & -2.32793 & H105 & 1.56698 & 4.527413 & -1.35049 \\
\hline \multirow[t]{2}{*}{$\mathrm{X}$} & 0.28333 & 1.758175 & -1.43512 & H106 & 1.87986 & -0.2998 & -1.55546 \\
\hline & & & & H107 & 1.43672 & -0.66616 & -2.13286 \\
\hline \multicolumn{8}{|c|}{ C3_ES_Cheltop_TS1 } \\
\hline $\mathrm{C} 1$ & -3.9716 & -3.84047 & -0.22732 & \multicolumn{4}{|c|}{ C3_ES_Cheltop_TS3 } \\
\hline $\mathrm{C} 2$ & -1.7434 & -4.82485 & -0.09421 & $\mathrm{C} 1$ & -4.2969 & -3.17722 & 0.770562 \\
\hline $\mathrm{C} 3$ & -3.0499 & -4.89436 & -0.927 & $\mathrm{C} 2$ & -2.2627 & -4.5163 & 0.671137 \\
\hline $\mathrm{C} 4$ & -3.0726 & -3.33449 & 0.924586 & $\mathrm{C} 3$ & -3.697 & -4.45095 & 0.088825 \\
\hline H5 & -4.8806 & -4.29993 & 0.174117 & $\mathrm{C} 4$ & -3.1256 & -2.69894 & 1.662421 \\
\hline H6 & -2.8789 & -4.67259 & -1.98543 & H5 & -5.1655 & -3.42072 & 1.391329 \\
\hline $\mathrm{H} 7$ & -3.5945 & -2.78729 & 1.709782 & H6 & -3.6985 & -4.38606 & -1.00388 \\
\hline H8 & -4.2737 & -3.02599 & -0.88813 & $\mathrm{H} 7$ & -3.3927 & -1.97884 & 2.436389 \\
\hline H9 & -1.061 & -5.66305 & -0.23328 & $\mathrm{H} 8$ & -4.6052 & -2.41508 & 0.054253 \\
\hline H10 & -3.476 & -5.89997 & -0.86912 & H9 & -1.7576 & -5.47402 & 0.548636 \\
\hline C11 & -2.2984 & -4.60056 & 1.330739 & $\mathrm{H} 10$ & -4.2524 & -5.35222 & 0.363885 \\
\hline H12 & -1.5272 & -4.40965 & 2.08309 & $\mathrm{C} 11$ & -2.4933 & -4.02561 & 2.118346 \\
\hline H13 & -2.9452 & -5.41734 & 1.663052 & H12 & -1.5739 & -3.88736 & 2.694892 \\
\hline $\mathrm{C} 14$ & 0.35836 & -3.29588 & -0.00243 & H13 & -3.1851 & -4.66536 & 2.673612 \\
\hline N15 & 0.99191 & -2.17409 & 0.02348 & $\mathrm{C} 14$ & 0.02766 & -3.39217 & 0.223953 \\
\hline C16 & 2.36691 & -2.43008 & 0.588551 & N15 & 0.84302 & -2.41704 & 0.001216 \\
\hline $\mathrm{C} 17$ & 2.43741 & -3.98411 & 0.464518 & $\mathrm{C} 16$ & 2.22063 & -2.85388 & 0.451641 \\
\hline P18 & -1.8699 & -0.85554 & 0.159474 & $\mathrm{C} 17$ & 2.00124 & -4.39214 & 0.574999 \\
\hline Ir19 & 0.21484 & -0.29456 & -0.75023 & P18 & -1.7776 & -0.60947 & 0.230384 \\
\hline $\mathrm{C} 20$ & -2.0886 & -0.11032 & 1.817309 & Ir19 & 0.34883 & -0.55441 & -0.93573 \\
\hline
\end{tabular}




\begin{tabular}{|c|c|c|c|c|c|c|c|}
\hline $\mathrm{C} 21$ & -2.2847 & 1.161643 & 4.311486 & $\mathrm{C} 20$ & -1.8999 & 0.466114 & 1.704466 \\
\hline $\mathrm{C} 22$ & -2.0581 & -0.86154 & 2.998594 & $\mathrm{C} 21$ & -1.8669 & 2.193601 & 3.921646 \\
\hline $\mathrm{C} 23$ & -2.1996 & 1.287729 & 1.898078 & $\mathrm{C} 22$ & -1.5333 & -0.00759 & 2.973085 \\
\hline $\mathrm{C} 24$ & -2.2986 & 1.917674 & 3.136015 & $\mathrm{C} 23$ & -2.2446 & 1.821271 & 1.560673 \\
\hline $\mathrm{C} 25$ & -2.1624 & -0.22611 & 4.23858 & $\mathrm{C} 24$ & -2.2346 & 2.675238 & 2.663863 \\
\hline H26 & -2.2171 & 1.88275 & 0.988634 & $\mathrm{C} 25$ & -1.5161 & 0.851101 & 4.071213 \\
\hline $\mathrm{H} 27$ & -2.3876 & 2.999155 & 3.18524 & $\mathrm{H} 26$ & -2.5574 & 2.206711 & 0.594505 \\
\hline $\mathrm{H} 28$ & -2.1467 & -0.82065 & 5.147644 & $\mathrm{H} 27$ & -2.532 & 3.713673 & 2.542584 \\
\hline H29 & -2.3653 & 1.652995 & 5.276446 & $\mathrm{H} 28$ & -1.2386 & 0.466128 & 5.048259 \\
\hline $\mathrm{O} 30$ & 1.04446 & -4.39188 & 0.329487 & $\mathrm{H} 29$ & -1.8676 & 2.856944 & 4.781884 \\
\hline C31 & 2.41909 & -2.05501 & 2.073619 & $\mathrm{O} 30$ & 0.55416 & -4.53716 & 0.655517 \\
\hline C32 & 1.32909 & -1.51875 & 2.755174 & $\mathrm{C} 31$ & 2.52175 & -2.30706 & 1.853458 \\
\hline C33 & 3.60457 & -2.30859 & 2.782131 & $\mathrm{C} 32$ & 1.59517 & -1.56411 & 2.580919 \\
\hline C34 & 1.42384 & -1.21525 & 4.115006 & $\mathrm{C} 33$ & 3.75018 & -2.62674 & 2.450624 \\
\hline H35 & 0.41065 & -1.31082 & 2.228522 & $\mathrm{C} 34$ & 1.8934 & -1.1173 & 3.868419 \\
\hline C36 & 3.69923 & -2.01125 & 4.138392 & H35 & 0.65276 & -1.29968 & 2.128198 \\
\hline H37 & 4.4656 & -2.72241 & 2.262148 & $\mathrm{C} 36$ & 4.048 & -2.19009 & 3.738933 \\
\hline $\mathrm{C} 38$ & 2.60593 & -1.45684 & 4.809953 & H37 & 4.48606 & -3.20322 & 1.89611 \\
\hline H39 & 0.56648 & -0.77921 & 4.61894 & $\mathrm{C} 38$ & 3.11953 & -1.42693 & 4.45214 \\
\hline $\mathrm{H} 40$ & 4.62609 & -2.20695 & 4.669889 & H39 & 1.16707 & -0.51052 & 4.401548 \\
\hline H41 & 2.67987 & -1.21718 & 5.866859 & $\mathrm{H} 40$ & 5.00748 & -2.4393 & 4.183255 \\
\hline $\mathrm{C} 42$ & -3.3695 & -0.37018 & -0.76992 & $\mathrm{H} 41$ & 3.35581 & -1.07727 & 5.453192 \\
\hline $\mathrm{C} 43$ & -4.4969 & 0.209728 & -0.17081 & $\mathrm{C} 42$ & -3.254 & -0.20105 & -0.77829 \\
\hline $\mathrm{C} 44$ & -3.4003 & -0.67598 & -2.13931 & $\mathrm{C} 43$ & -4.4454 & 0.282086 & -0.20825 \\
\hline $\mathrm{C} 45$ & -5.6279 & 0.493786 & -0.93698 & $\mathrm{C} 44$ & -3.2433 & -0.55299 & -2.1366 \\
\hline $\mathrm{H} 46$ & -4.4984 & 0.441256 & 0.888989 & $\mathrm{C} 45$ & -5.5944 & 0.415703 & -0.98481 \\
\hline $\mathrm{C} 47$ & -4.5328 & -0.39683 & -2.89852 & $\mathrm{H} 46$ & -4.483 & 0.544322 & 0.84443 \\
\hline $\mathrm{C} 48$ & -5.6469 & 0.194288 & -2.29907 & $\mathrm{C} 47$ & -4.3996 & -0.43135 & -2.90712 \\
\hline H49 & -6.4953 & 0.947555 & -0.46613 & $\mathrm{C} 48$ & -5.5748 & 0.054631 & -2.33502 \\
\hline H50 & -4.5371 & -0.61909 & -3.96082 & H49 & -6.5082 & 0.792762 & -0.53406 \\
\hline H51 & -6.5271 & 0.421404 & -2.89347 & $\mathrm{H} 50$ & -4.3718 & -0.69657 & -3.95962 \\
\hline H52 & -0.1712 & -1.04069 & -2.06716 & H51 & -6.4736 & 0.15507 & -2.93688 \\
\hline H53 & 1.70962 & 0.029888 & -1.39183 & H52 & -0.3351 & -1.35333 & -2.08891 \\
\hline H54 & -1.9303 & -1.9377 & 2.957132 & $\mathrm{H} 53$ & 1.9093 & 2.047066 & -1.57735 \\
\hline H55 & -2.5286 & -1.11529 & -2.61208 & H54 & -1.2634 & -1.04978 & 3.102968 \\
\hline H56 & 2.96926 & -4.33648 & -0.42227 & H55 & -2.3254 & -0.90007 & -2.59754 \\
\hline $\mathrm{C} 57$ & 5.22545 & -0.28049 & -1.87891 & H56 & 2.33884 & -4.96377 & -0.2917 \\
\hline C58 & 4.58167 & -1.41767 & -2.37027 & C57 & 4.9915 & -1.65554 & -2.66098 \\
\hline C59 & 3.67651 & -2.11074 & -1.5688 & C58 & 4.11707 & -2.72466 & -2.86615 \\
\hline C60 & 3.41081 & -1.69616 & -0.25613 & C59 & 3.25457 & -3.12462 & -1.84803 \\
\hline C61 & 4.04682 & -0.54245 & 0.219896 & C60 & 3.25447 & -2.47267 & -0.60679 \\
\hline C62 & 4.94723 & 0.157656 & -0.58433 & C61 & 4.12044 & -1.38986 & -0.41773 \\
\hline H63 & 5.93625 & 0.256804 & -2.50039 & C62 & 4.98543 & -0.98797 & -1.43605 \\
\hline H64 & 4.77694 & -1.76097 & -3.38186 & $\mathrm{H} 63$ & 5.66776 & -1.34363 & -3.452 \\
\hline H65 & 3.15795 & -2.9677 & -1.98837 & H64 & 4.10185 & -3.24424 & -3.81998 \\
\hline
\end{tabular}




\begin{tabular}{|c|c|c|c|c|c|c|c|}
\hline H66 & 3.83825 & -0.18886 & 1.223246 & H65 & 2.55447 & -3.93056 & -2.04795 \\
\hline H67 & 5.44019 & 1.043113 & -0.19106 & H66 & 4.10263 & -0.84298 & 0.517314 \\
\hline C68 & 2.0338 & 1.6051 & -3.00445 & H67 & 5.65427 & -0.14781 & -1.27046 \\
\hline C69 & 1.18782 & 1.494998 & -1.70724 & C68 & 1.04971 & 3.919447 & -2.09962 \\
\hline $\mathrm{C} 70$ & -0.2759 & 1.612147 & -1.58891 & C69 & 0.96041 & 2.551368 & -1.36479 \\
\hline C71 & 2.47026 & 3.511606 & -0.65039 & $\mathrm{C} 70$ & -0.21 & 1.553455 & -1.70808 \\
\hline $\mathrm{C} 72$ & 1.61071 & 1.840935 & 0.690614 & C71 & 0.56876 & 3.95237 & 0.859355 \\
\hline $\mathrm{C} 73$ & 2.9891 & 3.632853 & 0.791963 & C72 & 1.26956 & 1.753956 & 0.95501 \\
\hline H74 & 1.70199 & 4.260161 & -0.8841 & C73 & 1.13553 & 3.612246 & 2.248243 \\
\hline H75 & 3.27423 & 3.584578 & -1.38389 & H74 & -0.5221 & 4.051729 & 0.878148 \\
\hline H76 & 4.03148 & 3.311369 & 0.880791 & $\mathrm{H} 75$ & 1.00098 & 4.867278 & 0.452636 \\
\hline H77 & 2.86862 & 4.626473 & 1.224117 & H76 & 2.11767 & 4.06476 & 2.418486 \\
\hline N78 & 1.88932 & 2.165835 & -0.59849 & $\mathrm{H} 77$ & 0.45931 & 3.863606 & 3.065402 \\
\hline O79 & 2.16814 & 2.705323 & 1.549753 & N78 & 0.9749 & 2.762593 & 0.104361 \\
\hline $\mathrm{O} 80$ & 0.93699 & 0.876355 & 1.067473 & O79 & 1.29638 & 2.173504 & 2.233194 \\
\hline H81 & 2.84166 & -4.46317 & 1.354248 & O80 & 1.50393 & 0.574799 & 0.668829 \\
\hline N82 & -1.9461 & -2.52795 & 0.378479 & H81 & 2.43029 & -4.80858 & 1.484463 \\
\hline $\mathrm{C} 83$ & -1.0877 & -3.45325 & -0.40004 & N82 & -2.0064 & -2.17609 & 0.828351 \\
\hline H84 & -1.1262 & -3.24292 & -1.47882 & C83 & -1.4686 & -3.33114 & 0.061657 \\
\hline $\mathrm{C} 85$ & -3.0469 & 2.291296 & -4.82306 & H84 & -1.667 & -3.24417 & -1.01618 \\
\hline $\mathrm{C} 86$ & -3.0363 & 3.103206 & -3.68948 & $\mathrm{C} 85$ & -2.1183 & 1.710536 & -5.59002 \\
\hline C87 & -2.1341 & 2.851538 & -2.65689 & C86 & -2.7241 & 2.279431 & -4.47034 \\
\hline $\mathrm{C} 88$ & -1.2321 & 1.777746 & -2.72055 & $\mathrm{C} 87$ & -2.0957 & 2.21098 & -3.22749 \\
\hline C89 & -1.2909 & 0.943001 & -3.84653 & C88 & -0.8467 & 1.593212 & -3.0773 \\
\hline $\mathrm{C} 90$ & -2.1698 & 1.207523 & -4.8938 & C89 & -0.2611 & 1.004428 & -4.2079 \\
\hline H91 & -3.7395 & 2.491665 & -5.63531 & C90 & -0.8853 & 1.067274 & -5.4524 \\
\hline H92 & -3.7251 & 3.939541 & -3.61036 & H91 & -2.6066 & 1.754475 & -6.55948 \\
\hline H93 & -2.124 & 3.50449 & -1.78674 & H92 & -3.694 & 2.760861 & -4.55748 \\
\hline H94 & -0.6633 & 0.062566 & -3.88473 & H93 & -2.5906 & 2.632302 & -2.35614 \\
\hline H95 & -2.1837 & 0.551273 & -5.76007 & H94 & 0.68585 & 0.478613 & -4.11404 \\
\hline H96 & -0.547 & 2.28378 & -0.77153 & H95 & -0.4125 & 0.606637 & -6.31516 \\
\hline C97 & 2.01109 & 0.40436 & -3.96409 & H96 & -0.9951 & 1.774794 & -0.98674 \\
\hline H98 & 2.89172 & 0.458274 & -4.6119 & C97 & 1.64794 & 3.774623 & -3.50914 \\
\hline H99 & 2.04447 & -0.54517 & -3.42681 & H98 & 1.86282 & 4.76653 & -3.91949 \\
\hline H100 & 1.13408 & 0.415496 & -4.61069 & H99 & 2.58708 & 3.210564 & -3.49326 \\
\hline $\mathrm{C} 101$ & 1.70977 & 2.89827 & -3.78073 & H100 & 0.96012 & 3.274521 & -4.19256 \\
\hline H102 & 2.44062 & 3.03112 & -4.58456 & C101 & -0.2393 & 4.758856 & -2.15478 \\
\hline H103 & 0.71803 & 2.837916 & -4.2325 & H102 & 0.01023 & 5.798225 & -2.39101 \\
\hline H104 & 1.73472 & 3.796926 & -3.15982 & H103 & -0.907 & 4.395999 & -2.93674 \\
\hline H105 & 3.0639 & 1.659055 & -2.63114 & H104 & -0.8024 & 4.760881 & -1.21759 \\
\hline \multirow[t]{2}{*}{$\mathrm{X}$} & 0.28333 & 1.758175 & -1.43512 & H105 & 1.79522 & 4.478239 & -1.51703 \\
\hline & & & & H106 & 1.73418 & -0.76873 & -1.72083 \\
\hline \multicolumn{4}{|c|}{ C3_ES_Cheltop_INT1 } & H107 & 0.80616 & 0.406179 & -2.08986 \\
\hline $\mathrm{C} 1$ & -4.2887 & -3.09818 & 0.869072 & & & & \\
\hline $\mathrm{C} 2$ & -2.2605 & -4.44892 & 0.851244 & $\mathrm{C3} \mathrm{ES}$ & heltop $\mathrm{P}$ & & \\
\hline
\end{tabular}




\begin{tabular}{|c|c|c|c|c|c|c|c|}
\hline $\mathrm{C} 3$ & -3.712 & -4.44089 & 0.309091 & $\mathrm{C} 1$ & -4.2226 & -3.44318 & 0.909874 \\
\hline $\mathrm{C} 4$ & -3.0956 & -2.54123 & 1.682443 & $\mathrm{C} 2$ & -2.1136 & -4.6673 & 0.87345 \\
\hline H5 & -5.1478 & -3.26836 & 1.526023 & $\mathrm{C} 3$ & -3.5567 & -4.72665 & 0.312056 \\
\hline H6 & -3.747 & -4.49469 & -0.78348 & $\mathrm{C} 4$ & -3.0717 & -2.83842 & 1.747925 \\
\hline H7 & -3.3427 & -1.74932 & 2.389882 & H5 & -5.0735 & -3.68934 & 1.553586 \\
\hline $\mathrm{H} 8$ & -4.6024 & -2.4071 & 0.084879 & H6 & -3.5755 & -4.74437 & -0.78215 \\
\hline H9 & -1.7538 & -5.41309 & 0.805324 & $\mathrm{H} 7$ & -3.3704 & -2.08059 & 2.473023 \\
\hline H10 & -4.2598 & -5.30432 & 0.697088 & $\mathrm{H} 8$ & -4.5737 & -2.74775 & 0.145653 \\
\hline $\mathrm{C} 11$ & -2.449 & -3.81708 & 2.248336 & H9 & -1.5561 & -5.60142 & 0.810693 \\
\hline H12 & -1.5144 & -3.6214 & 2.783 & $\mathrm{H} 10$ & -4.0542 & -5.63543 & 0.662617 \\
\hline H13 & -3.1238 & -4.39762 & 2.883751 & $\mathrm{C} 11$ & -2.3546 & -4.08876 & 2.286179 \\
\hline $\mathrm{C} 14$ & 0.01735 & -3.40518 & 0.187164 & H12 & -1.4374 & -3.85433 & 2.834697 \\
\hline N15 & 0.78625 & -2.38156 & 0.11211 & H13 & -2.9979 & -4.72758 & 2.897866 \\
\hline $\mathrm{C} 16$ & 2.19563 & -2.82656 & 0.311455 & $\mathrm{C} 14$ & 0.10437 & -3.45875 & 0.31115 \\
\hline $\mathrm{C} 17$ & 2.03718 & -4.35243 & 0.033904 & N15 & 0.88217 & -2.47209 & -0.00108 \\
\hline P18 & -1.7689 & -0.56058 & 0.109939 & C16 & 2.27999 & -2.80503 & 0.503924 \\
\hline Ir19 & 0.24654 & -0.46169 & -0.72223 & $\mathrm{C} 17$ & 2.13293 & -4.34277 & 0.657935 \\
\hline $\mathrm{C} 20$ & -1.8838 & 0.589153 & 1.525037 & P18 & -1.83 & -0.7752 & 0.167791 \\
\hline $\mathrm{C} 21$ & -1.7337 & 2.453166 & 3.618267 & $\operatorname{Ir} 19$ & 0.29932 & -0.75864 & -0.95688 \\
\hline $\mathrm{C} 22$ & -1.4461 & 0.194344 & 2.797795 & $\mathrm{C} 20$ & -1.9933 & 0.383525 & 1.579379 \\
\hline $\mathrm{C} 23$ & -2.2315 & 1.933685 & 1.30663 & $\mathrm{C} 21$ & -1.9587 & 2.28838 & 3.649577 \\
\hline $\mathrm{C} 24$ & -2.1616 & 2.856345 & 2.350502 & $\mathrm{C} 22$ & -1.6122 & 0.019712 & 2.878895 \\
\hline $\mathrm{C} 25$ & -1.3769 & 1.121446 & 3.836931 & $\mathrm{C} 23$ & -2.361 & 1.718558 & 1.332848 \\
\hline $\mathrm{H} 26$ & -2.5721 & 2.256652 & 0.327216 & $\mathrm{C} 24$ & -2.3421 & 2.662481 & 2.359841 \\
\hline $\mathrm{H} 27$ & -2.4539 & 3.888127 & 2.174886 & $\mathrm{C} 25$ & -1.5981 & 0.964542 & 3.9045 \\
\hline H28 & -1.042 & 0.802343 & 4.819226 & $\mathrm{H} 26$ & -2.702 & 2.014873 & 0.344499 \\
\hline H29 & -1.687 & 3.170824 & 4.43206 & $\mathrm{H} 27$ & -2.6506 & 3.684987 & 2.156997 \\
\hline $\mathrm{O} 30$ & 0.61293 & -4.60071 & 0.276796 & $\mathrm{H} 28$ & -1.3116 & 0.662647 & 4.907954 \\
\hline C31 & 2.60818 & -2.59932 & 1.768861 & $\mathrm{H} 29$ & -1.9563 & 3.019468 & 4.453202 \\
\hline $\mathrm{C} 32$ & 1.80981 & -1.87379 & 2.655348 & O30 & 0.69735 & -4.54033 & 0.816277 \\
\hline $\mathrm{C} 33$ & 3.81001 & -3.1499 & 2.234439 & $\mathrm{C} 31$ & 2.50009 & -2.2213 & 1.90987 \\
\hline C34 & 2.20688 & -1.69649 & 3.981142 & $\mathrm{C} 32$ & 1.50297 & -1.55338 & 2.616415 \\
\hline H35 & 0.89452 & -1.4226 & 2.300153 & $\mathrm{C} 33$ & 3.73739 & -2.44 & 2.535502 \\
\hline C36 & 4.20715 & -2.97452 & 3.558416 & C34 & 1.73943 & -1.08023 & 3.908242 \\
\hline H37 & 4.45031 & -3.70844 & 1.555879 & H35 & 0.55062 & -1.3664 & 2.145425 \\
\hline C38 & 3.4042 & -2.24514 & 4.43809 & $\mathrm{C} 36$ & 3.97275 & -1.97796 & 3.82831 \\
\hline H39 & 1.57863 & -1.11935 & 4.65434 & H37 & 4.5284 & -2.95766 & 1.998687 \\
\hline $\mathrm{H} 40$ & 5.14249 & -3.40626 & 3.902484 & C38 & 2.97322 & -1.28851 & 4.520964 \\
\hline $\mathrm{H} 41$ & 3.7122 & -2.10717 & 5.470548 & H39 & 0.9562 & -0.53256 & 4.424139 \\
\hline $\mathrm{C} 42$ & -3.1978 & -0.20304 & -0.96946 & $\mathrm{H} 40$ & 4.93981 & -2.15053 & 4.292156 \\
\hline $\mathrm{C} 43$ & -4.4048 & 0.27124 & -0.42185 & H41 & 3.15949 & -0.91898 & 5.525171 \\
\hline $\mathrm{C} 44$ & -3.1535 & -0.55379 & -2.327 & $\mathrm{C} 42$ & -3.3052 & -0.38891 & -0.85908 \\
\hline $\mathrm{C} 45$ & -5.5367 & 0.399286 & -1.22337 & $\mathrm{C} 43$ & -4.5464 & -0.05935 & -0.28438 \\
\hline $\mathrm{H} 46$ & -4.4654 & 0.534278 & 0.629909 & $\mathrm{C} 44$ & -3.2055 & -0.47118 & -2.25575 \\
\hline $\mathrm{C} 47$ & -4.2928 & -0.43061 & -3.12122 & $\mathrm{C} 45$ & -5.6569 & 0.180191 & -1.09075 \\
\hline
\end{tabular}




\begin{tabular}{|c|c|c|c|c|c|c|c|}
\hline $\mathrm{C} 48$ & -5.4827 & 0.046799 & -2.57403 & H46 & -4.6427 & 0.018874 & 0.795126 \\
\hline H49 & -6.4615 & 0.771322 & -0.79202 & $\mathrm{C} 47$ & -4.3191 & -0.22718 & -3.06176 \\
\hline H50 & -4.2379 & -0.68898 & -4.17406 & $\mathrm{C} 48$ & -5.5456 & 0.097778 & -2.48289 \\
\hline H51 & -6.3667 & 0.148412 & -3.19657 & $\mathrm{H} 49$ & -6.6094 & 0.434081 & -0.63393 \\
\hline H52 & -0.4056 & -1.18071 & -1.94516 & $\mathrm{H} 50$ & -4.218 & -0.27412 & -4.14182 \\
\hline H53 & 2.19711 & 1.507362 & -1.53638 & H51 & -6.4115 & 0.291187 & -3.10932 \\
\hline H54 & -1.1575 & -0.8371 & 2.972243 & H52 & -0.2991 & -1.64707 & -2.0953 \\
\hline H55 & -2.2247 & -0.89347 & -2.77046 & $\mathrm{H} 53$ & 1.95181 & 1.709941 & -1.61163 \\
\hline H56 & 2.24539 & -4.62643 & -1.00474 & H54 & -1.328 & -1.00509 & 3.091615 \\
\hline C57 & 4.3878 & -0.50862 & -2.6467 & $\mathrm{H} 55$ & -2.2516 & -0.70747 & -2.71474 \\
\hline C58 & 3.36216 & -1.3746 & -3.03228 & H56 & 2.44209 & -4.91357 & -0.22007 \\
\hline C59 & 2.69127 & -2.13721 & -2.07366 & C57 & 5.1334 & -1.47704 & -2.47679 \\
\hline C60 & 3.048 & -2.06419 & -0.71456 & C58 & 4.38666 & -2.63666 & -2.68828 \\
\hline C61 & 4.07857 & -1.19608 & -0.34102 & C59 & 3.49146 & -3.07853 & -1.71592 \\
\hline C62 & 4.7426 & -0.42741 & -1.29907 & $\mathrm{C} 60$ & 3.33395 & -2.37774 & -0.51358 \\
\hline H63 & 4.9086 & 0.087731 & -3.39016 & C61 & 4.07319 & -1.2048 & -0.3196 \\
\hline H64 & 3.08076 & -1.45922 & -4.07807 & C62 & 4.96857 & -0.76072 & -1.29114 \\
\hline H65 & 1.87816 & -2.78446 & -2.3892 & H63 & 5.83474 & -1.1336 & -3.23214 \\
\hline H66 & 4.34905 & -1.0986 & 0.703146 & H64 & 4.4957 & -3.19625 & -3.61283 \\
\hline H67 & 5.53877 & 0.24223 & -0.98656 & H65 & 2.89333 & -3.95946 & -1.92725 \\
\hline C68 & 1.73891 & 3.446856 & -2.29745 & H66 & 3.93053 & -0.62396 & 0.583503 \\
\hline C69 & 1.35772 & 2.207514 & -1.42684 & H67 & 5.53817 & 0.148754 & -1.12023 \\
\hline $\mathrm{C} 70$ & 0.01132 & 1.44765 & -1.67268 & C68 & 1.55821 & 3.682572 & -2.30062 \\
\hline C71 & 0.95755 & 3.875681 & 0.593364 & C69 & 1.13433 & 2.426881 & -1.49399 \\
\hline $\mathrm{C} 72$ & 1.47698 & 1.674373 & 0.972223 & $\mathrm{C} 70$ & -0.1853 & 1.64719 & -1.86493 \\
\hline $\mathrm{C} 73$ & 1.35276 & 3.65142 & 2.06242 & C71 & 0.69355 & 3.935947 & 0.633225 \\
\hline H74 & -0.1254 & 4.004701 & 0.475823 & $\mathrm{C} 72$ & 1.22131 & 1.698357 & 0.854679 \\
\hline H75 & 1.46985 & 4.731639 & 0.152773 & $\mathrm{C} 73$ & 1.10604 & 3.605285 & 2.078696 \\
\hline H76 & 2.34374 & 4.054454 & 2.292877 & H74 & -0.3903 & 4.084448 & 0.539147 \\
\hline H77 & 0.61816 & 4.025331 & 2.77545 & H75 & 1.20954 & 4.816931 & 0.248842 \\
\hline N78 & 1.39728 & 2.605518 & 0.006932 & H76 & 2.09882 & 3.995537 & 2.324011 \\
\hline O79 & 1.40956 & 2.207839 & 2.201961 & H77 & 0.37937 & 3.927256 & 2.824517 \\
\hline $\mathrm{O} 80$ & 1.60127 & 0.446749 & 0.812955 & N78 & 1.11838 & 2.709479 & -0.04458 \\
\hline H81 & 2.60273 & -4.98606 & 0.713875 & O79 & 1.16556 & 2.158513 & 2.116751 \\
\hline N82 & -2.0016 & -2.10573 & 0.765805 & O80 & 1.35567 & 0.496284 & 0.615768 \\
\hline $\mathrm{C} 83$ & -1.4853 & -3.33066 & 0.103218 & H81 & 2.62519 & -4.72899 & 1.54824 \\
\hline H84 & -1.7293 & -3.35541 & -0.97057 & N82 & -2.0041 & -2.31227 & 0.855074 \\
\hline $\mathrm{C} 85$ & -1.2722 & 0.973297 & -5.78468 & $\mathrm{C} 83$ & -1.3976 & -3.48474 & 0.168485 \\
\hline $\mathrm{C} 86$ & -2.0209 & 1.737683 & -4.8916 & H84 & -1.609 & -3.48841 & -0.91022 \\
\hline $\mathrm{C} 87$ & -1.5912 & 1.89701 & -3.57252 & $\mathrm{C} 85$ & -2.7345 & 2.650835 & -5.19567 \\
\hline C88 & -0.4063 & 1.305098 & -3.1136 & C86 & -3.0151 & 3.136699 & -3.91728 \\
\hline C89 & 0.33495 & 0.538516 & -4.0288 & C87 & -2.1807 & 2.810211 & -2.85106 \\
\hline $\mathrm{C} 90$ & -0.0884 & 0.37327 & -5.34475 & $\mathrm{C} 88$ & -1.0497 & 2.00881 & -3.04858 \\
\hline H91 & -1.603 & 0.846689 & -6.81145 & C89 & -0.7881 & 1.506574 & -4.32832 \\
\hline H92 & -2.9452 & 2.208055 & -5.21639 & C90 & -1.6225 & 1.830979 & -5.39815 \\
\hline
\end{tabular}




\begin{tabular}{|c|c|c|c|c|c|c|c|}
\hline H93 & -2.1925 & 2.484682 & -2.88235 & H91 & -3.3856 & 2.901023 & -6.02817 \\
\hline H94 & 1.2536 & 0.067191 & -3.69478 & H92 & -3.8886 & 3.759775 & -3.749 \\
\hline H95 & 0.50584 & -0.22291 & -6.03247 & H93 & -2.4071 & 3.183842 & -1.85502 \\
\hline H96 & -0.7573 & 2.050167 & -1.1726 & H94 & 0.06227 & 0.846359 & -4.48388 \\
\hline C97 & 2.46702 & 3.049723 & -3.59239 & H95 & -1.4087 & 1.437376 & -6.38757 \\
\hline H98 & 2.88561 & 3.942112 & -4.06913 & H96 & -0.8217 & 1.674974 & -0.98522 \\
\hline H99 & 3.2926 & 2.357658 & -3.39165 & C97 & 2.19618 & 3.280483 & -3.64076 \\
\hline H100 & 1.79227 & 2.575037 & -4.30616 & H98 & 2.59896 & 4.166252 & -4.14209 \\
\hline C101 & 0.58469 & 4.413211 & -2.61118 & H99 & 3.01717 & 2.568907 & -3.50215 \\
\hline H102 & 0.98401 & 5.348959 & -3.01587 & H100 & 1.46075 & 2.830635 & -4.31243 \\
\hline H103 & -0.085 & 3.986989 & -3.35987 & C101 & 0.48917 & 4.767987 & -2.52982 \\
\hline H104 & -0.0208 & 4.664822 & -1.73582 & H102 & 0.9802 & 5.715454 & -2.77394 \\
\hline H105 & 2.48301 & 3.98452 & -1.69237 & H103 & -0.1631 & 4.511255 & -3.36524 \\
\hline \multirow[t]{2}{*}{$\mathrm{X}$} & 0.28333 & 1.758175 & -1.43512 & H104 & -0.1515 & 4.942863 & -1.66137 \\
\hline & & & & $\mathrm{H} 105$ & 2.3607 & 4.120634 & -1.69076 \\
\hline \multicolumn{4}{|c|}{ C3_ES_Cheltop_TS2 } & H106 & 1.66898 & -0.82986 & -1.79182 \\
\hline $\mathrm{C} 1$ & -4.297 & -3.13353 & 0.572658 & H107 & 0.1548 & 0.579674 & -2.16827 \\
\hline $\mathrm{C} 2$ & -2.261 & -4.47681 & 0.547246 & & & & \\
\hline $\mathrm{C} 3$ & -3.6737 & -4.40787 & -0.08718 & & & & \\
\hline $\mathrm{C} 4$ & -3.1563 & -2.65575 & 1.502212 & & & & \\
\hline H5 & -5.185 & -3.37673 & 1.165185 & & & & \\
\hline H6 & -3.6346 & -4.34135 & -1.17904 & & & & \\
\hline $\mathrm{H} 7$ & -3.4472 & -1.93238 & 2.264404 & & & & \\
\hline H8 & -4.5822 & -2.37148 & -0.15352 & & & & \\
\hline H9 & -1.7548 & -5.43618 & 0.445561 & & & & \\
\hline H10 & -4.2411 & -5.30804 & 0.16545 & & & & \\
\hline $\mathrm{C} 11$ & -2.5422 & -3.98184 & 1.983488 & & & & \\
\hline H12 & -1.6435 & -3.84445 & 2.592057 & & & & \\
\hline H13 & -3.255 & -4.61759 & 2.516006 & & & & \\
\hline C14 & 0.05239 & -3.3602 & 0.183945 & & & & \\
\hline N15 & 0.85883 & -2.37189 & 0.013934 & & & & \\
\hline $\mathrm{C} 16$ & 2.22965 & -2.81867 & 0.447998 & & & & \\
\hline $\mathrm{C} 17$ & 2.01637 & -4.36618 & 0.561849 & & & & \\
\hline P18 & -1.7687 & -0.5562 & 0.167501 & & & & \\
\hline Ir19 & 0.19377 & -0.39543 & -0.80124 & & & & \\
\hline $\mathrm{C} 20$ & -1.8301 & 0.451363 & 1.692376 & & & & \\
\hline $\mathrm{C} 21$ & -1.7448 & 2.039978 & 4.003726 & & & & \\
\hline $\mathrm{C} 22$ & -1.4901 & -0.11299 & 2.9305 & & & & \\
\hline $\mathrm{C} 23$ & -2.1173 & 1.824591 & 1.622403 & & & & \\
\hline $\mathrm{C} 24$ & -2.0839 & 2.60993 & 2.773907 & & & & \\
\hline $\mathrm{C} 25$ & -1.4456 & 0.678874 & 4.077815 & & & & \\
\hline H26 & -2.3883 & 2.278589 & 0.674003 & & & & \\
\hline $\mathrm{H} 27$ & -2.3342 & 3.665761 & 2.712956 & & & & \\
\hline $\mathrm{H} 28$ & -1.186 & 0.228186 & 5.031213 & & & & \\
\hline $\mathrm{H} 29$ & -1.7241 & 2.652354 & 4.900684 & & & & \\
\hline
\end{tabular}




\begin{tabular}{|c|c|c|c|}
\hline $\mathrm{O} 30$ & 0.56974 & -4.52575 & 0.58197 \\
\hline C31 & 2.56953 & -2.26758 & 1.835551 \\
\hline C32 & 1.70509 & -1.43373 & 2.541956 \\
\hline C33 & 3.76833 & -2.66539 & 2.446553 \\
\hline C34 & 2.03512 & -0.98152 & 3.819648 \\
\hline H35 & 0.78722 & -1.10609 & 2.079702 \\
\hline $\mathrm{C} 36$ & 4.1 & -2.21866 & 3.723263 \\
\hline H37 & 4.45691 & -3.31544 & 1.912834 \\
\hline $\mathrm{C} 38$ & 3.23308 & -1.3695 & 4.415003 \\
\hline H39 & 1.35517 & -0.3098 & 4.335723 \\
\hline $\mathrm{H} 40$ & 5.03709 & -2.53026 & 4.17544 \\
\hline H41 & 3.49434 & -1.01483 & 5.407901 \\
\hline $\mathrm{C} 42$ & -3.2653 & -0.1181 & -0.79062 \\
\hline $\mathrm{C} 43$ & -4.3998 & 0.435964 & -0.17241 \\
\hline $\mathrm{C} 44$ & -3.3588 & -0.55733 & -2.12047 \\
\hline $\mathrm{C} 45$ & -5.5955 & 0.559855 & -0.87831 \\
\hline H46 & -4.3631 & 0.756535 & 0.863112 \\
\hline $\mathrm{C} 47$ & -4.5608 & -0.44506 & -2.81636 \\
\hline $\mathrm{C} 48$ & -5.6797 & 0.115874 & -2.19942 \\
\hline H49 & -6.4639 & 0.994379 & -0.39157 \\
\hline H50 & -4.6142 & -0.77857 & -3.84815 \\
\hline H51 & -6.6143 & 0.208069 & -2.74506 \\
\hline H52 & -0.5612 & -1.11977 & -1.95976 \\
\hline H53 & 1.80926 & 2.099095 & -1.7609 \\
\hline H54 & -1.2572 & -1.17014 & 2.995755 \\
\hline H55 & -2.4877 & -0.96664 & -2.61967 \\
\hline H56 & 2.39973 & -4.92418 & -0.29542 \\
\hline $\mathrm{C} 57$ & 4.98382 & -1.82318 & -2.75871 \\
\hline C58 & 3.9189 & -2.70024 & -2.97358 \\
\hline $\mathrm{C} 59$ & 3.05283 & -3.0113 & -1.92794 \\
\hline $\mathrm{C} 60$ & 3.23588 & -2.46174 & -0.65006 \\
\hline C61 & 4.29033 & -1.56665 & -0.4507 \\
\hline C62 & 5.16064 & -1.25425 & -1.49855 \\
\hline H63 & 5.66373 & -1.57985 & -3.57007 \\
\hline H64 & 3.76037 & -3.1391 & -3.95453 \\
\hline H65 & 2.21237 & -3.67303 & -2.12049 \\
\hline H66 & 4.42853 & -1.09801 & 0.516123 \\
\hline H67 & 5.97765 & -0.55952 & -1.32523 \\
\hline C68 & 0.78369 & 3.955274 & -2.06348 \\
\hline C69 & 0.87697 & 2.545255 & -1.39924 \\
\hline $\mathrm{C} 70$ & -0.2934 & 1.53335 & -1.63383 \\
\hline C71 & 0.69693 & 3.853125 & 0.91878 \\
\hline $\mathrm{C} 72$ & 1.51243 & 1.702277 & 0.82215 \\
\hline $\mathrm{C} 73$ & 1.35373 & 3.450031 & 2.253027 \\
\hline H74 & -0.391 & 3.921546 & 1.006419 \\
\hline
\end{tabular}




\begin{tabular}{|c|c|c|c|c|c|c|c|}
\hline H75 & 1.07967 & 4.802444 & 0.54149 & & & & \\
\hline H76 & 2.30549 & 3.961607 & 2.424164 & & & & \\
\hline H77 & 0.69712 & 3.582201 & 3.113323 & & & & \\
\hline N78 & 1.08365 & 2.723545 & 0.066815 & & & & \\
\hline O79 & 1.62542 & 2.031423 & 2.117594 & & & & \\
\hline O80 & 1.79616 & 0.553856 & 0.426818 & & & & \\
\hline H81 & 2.41173 & -4.78414 & 1.486162 & & & & \\
\hline N82 & -2.0089 & -2.14095 & 0.698381 & & & & \\
\hline $\mathrm{C} 83$ & -1.4385 & -3.29801 & -0.03524 & & & & \\
\hline H84 & -1.5934 & -3.21741 & -1.12069 & & & & \\
\hline $\mathrm{C} 85$ & -1.5106 & 1.146554 & -5.77937 & & & & \\
\hline C86 & -2.3822 & 1.674082 & -4.82801 & & & & \\
\hline $\mathrm{C} 87$ & -1.9754 & 1.801174 & -3.49897 & & & & \\
\hline C88 & -0.6949 & 1.406252 & -3.08397 & & & & \\
\hline C89 & 0.17208 & 0.883495 & -4.05963 & & & & \\
\hline C90 & -0.2274 & 0.754202 & -5.3873 & & & & \\
\hline H91 & -1.8225 & 1.047378 & -6.81503 & & & & \\
\hline H92 & -3.3822 & 1.987475 & -5.11574 & & & & \\
\hline H93 & -2.6708 & 2.201501 & -2.76591 & & & & \\
\hline H94 & 1.17878 & 0.601135 & -3.77538 & & & & \\
\hline H95 & 0.46648 & 0.353698 & -6.12133 & & & & \\
\hline H96 & -1.1522 & 1.964551 & -1.10668 & & & & \\
\hline C97 & 1.25282 & 3.966439 & -3.52888 & & & & \\
\hline H98 & 1.3788 & 5.002295 & -3.86099 & & & & \\
\hline H99 & 2.21559 & 3.458305 & -3.64978 & & & & \\
\hline H100 & 0.5326 & 3.488679 & -4.19355 & & & & \\
\hline C101 & -0.5917 & 4.633716 & -1.95204 & & & & \\
\hline H102 & -0.5142 & 5.683457 & -2.25243 & & & & \\
\hline H103 & -1.3102 & 4.153623 & -2.6188 & & & & \\
\hline H104 & -1.0104 & 4.612424 & -0.94203 & & & & \\
\hline H105 & 1.51639 & 4.567496 & -1.51991 & & & & \\
\hline H106 & 2.68306 & -0.01544 & -1.97187 & & & & \\
\hline H107 & 2.31957 & -0.39918 & -2.49978 & & & & \\
\hline \multicolumn{8}{|c|}{ C3_ES_Chelbot } \\
\hline \multicolumn{4}{|c|}{ C3_ES_Chelbot_SM } & \multicolumn{4}{|c|}{ C3_ES_Chelbot_int2 } \\
\hline $\mathrm{C} 1$ & -3.92334 & -3.52563 & -0.95247 & $\mathrm{C} 1$ & -3.64301 & -3.342 & -1.96871 \\
\hline $\mathrm{C} 2$ & -1.6669 & -4.46398 & -0.96739 & $\mathrm{C} 2$ & -1.38485 & -4.21093 & -1.64029 \\
\hline $\mathrm{C} 3$ & -2.92551 & -4.31793 & -1.86331 & $\mathrm{C} 3$ & -2.43784 & -3.95995 & -2.75276 \\
\hline $\mathrm{C} 4$ & -3.09225 & -3.32181 & 0.333851 & $\mathrm{C} 4$ & -3.07706 & -3.23895 & -0.53632 \\
\hline H5 & -4.82519 & -4.10781 & -0.73666 & H5 & -4.51478 & -4.00482 & -1.97916 \\
\hline H6 & -2.70519 & -3.79957 & -2.80185 & H6 & -2.05735 & -3.29438 & -3.53389 \\
\hline H7 & -3.65581 & -3.0096 & 1.213869 & $\mathrm{H} 7$ & -3.81167 & -3.02735 & 0.241043 \\
\hline H8 & -4.23468 & -2.57194 & -1.38269 & $\mathrm{H} 8$ & -3.95996 & -2.37275 & -2.3521 \\
\hline H9 & -0.95787 & -5.22735 & -1.2881 & H9 & -0.60686 & -4.92936 & -1.89761 \\
\hline H10 & -3.31721 & -5.30626 & -2.1198 & $\mathrm{H} 10$ & -2.71222 & -4.90405 & -3.23129 \\
\hline
\end{tabular}




\begin{tabular}{|c|c|c|c|c|c|c|c|}
\hline $\mathrm{C} 11$ & -2.3003 & -4.63802 & 0.430447 & $\mathrm{C} 11$ & -2.27591 & -4.5511 & -0.42848 \\
\hline H12 & -1.57272 & -4.65033 & 1.247961 & H12 & -1.71426 & -4.66434 & 0.503192 \\
\hline H13 & -2.94055 & -5.52192 & 0.499462 & H13 & -2.89355 & -5.43976 & -0.58551 \\
\hline $\mathrm{C} 14$ & 0.369131 & -3.00871 & -0.27084 & C14 & 0.404818 & -2.89054 & -0.32633 \\
\hline N15 & 0.92404 & -1.93078 & 0.158627 & N15 & 0.87795 & -1.88895 & 0.332738 \\
\hline $\mathrm{C} 16$ & 2.347562 & -2.22891 & 0.526082 & $\mathrm{C} 16$ & 2.162049 & -2.31954 & 0.997783 \\
\hline $\mathrm{C} 17$ & 2.388381 & -3.78733 & 0.327028 & $\mathrm{C} 17$ & 2.190737 & -3.85033 & 0.634477 \\
\hline P18 & -1.92296 & -0.72309 & 0.399554 & P18 & -1.9584 & -0.74854 & 0.288742 \\
\hline Ir19 & 0.099792 & 0.075575 & -0.19174 & Ir19 & 0.164935 & 0.204216 & -0.13281 \\
\hline $\mathrm{C} 20$ & -2.18718 & -0.6099 & 2.204439 & $\mathrm{C} 20$ & -2.10105 & -1.0866 & 2.091348 \\
\hline $\mathrm{C} 21$ & -2.3016 & -0.37148 & 4.996218 & $\mathrm{C} 21$ & -2.24105 & -1.58468 & 4.852575 \\
\hline $\mathrm{C} 22$ & -1.91962 & -1.71597 & 3.022034 & $\mathrm{C} 22$ & -2.1699 & -2.39773 & 2.575872 \\
\hline $\mathrm{C} 23$ & -2.50669 & 0.621292 & 2.800085 & $\mathrm{C} 23$ & -2.08061 & -0.02465 & 3.011334 \\
\hline $\mathrm{C} 24$ & -2.5647 & 0.73708 & 4.187008 & $\mathrm{C} 24$ & -2.16112 & -0.27208 & 4.379964 \\
\hline $\mathrm{C} 25$ & -1.97939 & -1.59624 & 4.41165 & $\mathrm{C} 25$ & -2.23653 & -2.64456 & 3.948152 \\
\hline H26 & -2.70141 & 1.493899 & 2.185441 & $\mathrm{H} 26$ & -1.98644 & 0.998335 & 2.665624 \\
\hline $\mathrm{H} 27$ & -2.81177 & 1.69432 & 4.636825 & $\mathrm{H} 27$ & -2.14932 & 0.56071 & 5.077464 \\
\hline $\mathrm{H} 28$ & -1.76902 & -2.46078 & 5.0347 & $\mathrm{H} 28$ & -2.2776 & -3.66877 & 4.307221 \\
\hline H29 & -2.34532 & -0.27826 & 6.077518 & $\mathrm{H} 29$ & -2.29267 & -1.77798 & 5.92009 \\
\hline $\mathrm{O} 30$ & 1.098317 & -4.12724 & -0.26138 & $\mathrm{O} 30$ & 1.068448 & -4.04604 & -0.26674 \\
\hline C31 & 2.640009 & -1.88956 & 1.983252 & $\mathrm{C} 31$ & 2.173263 & -2.16289 & 2.516515 \\
\hline C32 & 1.638424 & -1.45846 & 2.857001 & $\mathrm{C} 32$ & 1.238365 & -1.41497 & 3.228407 \\
\hline $\mathrm{C} 33$ & 3.931119 & -2.10161 & 2.490286 & $\mathrm{C} 33$ & 3.20723 & -2.80017 & 3.223892 \\
\hline $\mathrm{C} 34$ & 1.926937 & -1.20446 & 4.199261 & $\mathrm{C} 34$ & 1.337898 & -1.29482 & 4.618064 \\
\hline H35 & 0.630036 & -1.3172 & 2.495765 & H35 & 0.430534 & -0.92738 & 2.707074 \\
\hline C36 & 4.219535 & -1.84991 & 3.830055 & $\mathrm{C} 36$ & 3.303741 & -2.68547 & 4.607849 \\
\hline H37 & 4.720206 & -2.45339 & 1.831239 & H37 & 3.960922 & -3.37007 & 2.684816 \\
\hline C38 & 3.21848 & -1.39083 & 4.689012 & $\mathrm{C} 38$ & 2.367035 & -1.92532 & 5.313566 \\
\hline H39 & 1.130455 & -0.86488 & 4.855716 & H39 & 0.59684 & -0.70666 & 5.149342 \\
\hline $\mathrm{H} 40$ & 5.226995 & -2.01201 & 4.202302 & $\mathrm{H} 40$ & 4.114522 & -3.18221 & 5.133048 \\
\hline H41 & 3.444835 & -1.19092 & 5.732406 & $\mathrm{H} 41$ & 2.44303 & -1.82865 & 6.392534 \\
\hline $\mathrm{C} 42$ & -3.41981 & -0.01954 & -0.39514 & $\mathrm{C} 42$ & -3.59498 & -0.03835 & -0.16662 \\
\hline $\mathrm{C} 43$ & -4.58916 & 0.304597 & 0.305617 & $\mathrm{C} 43$ & -4.55131 & 0.333242 & 0.787611 \\
\hline $\mathrm{C} 44$ & -3.4058 & 0.096919 & -1.79534 & C44 & -3.9035 & 0.107644 & -1.52884 \\
\hline $\mathrm{C} 45$ & -5.71257 & 0.771721 & -0.38099 & $\mathrm{C} 45$ & -5.78061 & 0.861629 & 0.388538 \\
\hline H46 & -4.63462 & 0.193591 & 1.383547 & $\mathrm{H} 46$ & -4.34809 & 0.212828 & 1.845716 \\
\hline $\mathrm{C} 47$ & -4.53065 & 0.555811 & -2.47584 & $\mathrm{C} 47$ & -5.13497 & 0.626976 & -1.92358 \\
\hline $\mathrm{C} 48$ & -5.68554 & 0.905913 & -1.76893 & $\mathrm{C} 48$ & -6.07584 & 1.015932 & -0.96532 \\
\hline H49 & -6.61134 & 1.024906 & 0.17397 & $\mathrm{H} 49$ & -6.5099 & 1.144413 & 1.142094 \\
\hline $\mathrm{H} 50$ & -4.51022 & 0.635703 & -3.55923 & $\mathrm{H} 50$ & -5.37182 & 0.709254 & -2.98109 \\
\hline H51 & -6.56144 & 1.268497 & -2.29858 & H51 & -7.03563 & 1.419723 & -1.2735 \\
\hline H52 & 0.269764 & 0.481403 & 1.299186 & $\mathrm{H} 52$ & 0.247393 & 0.567546 & 1.376663 \\
\hline H53 & 1.855511 & 0.762868 & -0.591 & $\mathrm{H} 53$ & 1.342812 & 2.041705 & -2.28691 \\
\hline H54 & -1.64145 & -2.66388 & 2.574681 & H54 & -2.14988 & -3.23434 & 1.891416 \\
\hline H55 & -2.52153 & -0.1967 & -2.34931 & H55 & -3.19864 & -0.22024 & -2.28441 \\
\hline
\end{tabular}




\begin{tabular}{|c|c|c|c|c|c|c|c|}
\hline H56 & 3.168344 & -4.11754 & -0.36028 & H56 & 3.098247 & -4.15753 & 0.113064 \\
\hline C57 & 4.670766 & -0.13685 & -2.49537 & $\mathrm{C} 57$ & 5.450083 & -0.28326 & -0.98842 \\
\hline C58 & 3.784599 & -1.16014 & -2.84473 & $\mathrm{C} 58$ & 4.573699 & -1.10012 & -1.70844 \\
\hline C59 & 3.074856 & -1.83394 & -1.85571 & C59 & 3.523591 & -1.74052 & -1.05405 \\
\hline $\mathrm{C} 60$ & 3.235688 & -1.50546 & -0.49948 & $\mathrm{C} 60$ & 3.325286 & -1.57287 & 0.325557 \\
\hline C61 & 4.123618 & -0.47986 & -0.16227 & C61 & 4.185331 & -0.72515 & 1.030878 \\
\hline C62 & 4.83581 & 0.2 & -1.15369 & C62 & 5.248825 & -0.09694 & 0.377753 \\
\hline H63 & 5.226377 & 0.390975 & -3.26526 & H63 & 6.277528 & 0.207292 & -1.49292 \\
\hline H64 & 3.643591 & -1.43026 & -3.88732 & H64 & 4.713525 & -1.24508 & -2.77584 \\
\hline H65 & 2.372592 & -2.60977 & -2.14909 & H65 & 2.854014 & -2.37803 & -1.62674 \\
\hline H66 & 4.254274 & -0.18689 & 0.870528 & H66 & 4.041203 & -0.56239 & 2.091621 \\
\hline H67 & 5.510014 & 1.002074 & -0.86793 & H67 & 5.918106 & 0.542018 & 0.945789 \\
\hline C68 & -0.62429 & 3.259812 & -0.19303 & C68 & -0.8588 & 3.214511 & 0.184472 \\
\hline C69 & 0.024602 & 2.032898 & -0.8655 & C69 & -0.25795 & 2.195762 & -0.81744 \\
\hline $\mathrm{C} 70$ & 1.579927 & 1.862547 & -0.82691 & $\mathrm{C} 70$ & 0.991321 & 2.808932 & -1.5869 \\
\hline C71 & -0.28766 & 3.105033 & -3.24037 & C71 & -1.87967 & 3.005975 & -2.76986 \\
\hline $\mathrm{C} 72$ & -0.29748 & 0.824549 & -2.96531 & $\mathrm{C} 72$ & -0.99679 & 0.882997 & -2.71721 \\
\hline $\mathrm{C} 73$ & -0.71953 & 2.395815 & -4.53505 & $\mathrm{C} 73$ & -2.39057 & 2.167577 & -3.95803 \\
\hline H74 & 0.742489 & 3.48602 & -3.28762 & H74 & -1.17039 & 3.779501 & -3.08386 \\
\hline H75 & -0.95366 & 3.926479 & -2.97187 & $\mathrm{H} 75$ & -2.69378 & 3.484488 & -2.22395 \\
\hline H76 & -1.7962 & 2.485915 & -4.71018 & H76 & -3.44511 & 1.904876 & -3.85014 \\
\hline H77 & -0.17106 & 2.706907 & -5.42425 & H77 & -2.21992 & 2.628417 & -4.9315 \\
\hline N78 & -0.38344 & 1.990335 & -2.29227 & N78 & -1.21933 & 1.971741 & -1.95805 \\
\hline O79 & -0.4233 & 0.990938 & -4.28982 & O79 & -1.61939 & 0.93375 & -3.9033 \\
\hline $\mathrm{O} 80$ & -0.12168 & -0.29793 & -2.45422 & O80 & -0.3115 & -0.10484 & -2.38448 \\
\hline H81 & 2.470472 & -4.32189 & 1.274348 & H81 & 2.024153 & -4.48548 & 1.505407 \\
\hline N82 & -1.97825 & -2.37383 & 0.054894 & N82 & -1.97523 & -2.22442 & -0.52942 \\
\hline $\mathrm{C} 83$ & -1.02543 & -3.05957 & -0.84711 & $\mathrm{C} 83$ & -0.81266 & -2.83566 & -1.22049 \\
\hline H84 & -0.96204 & -2.57133 & -1.82906 & H84 & -0.52645 & -2.2433 & -2.09527 \\
\hline H85 & 1.981595 & 1.925169 & -1.84452 & H85 & 0.593505 & 3.619824 & -2.20985 \\
\hline $\mathrm{C} 86$ & 4.055047 & 4.211489 & 1.8045 & C86 & 4.451846 & 4.573964 & 0.330182 \\
\hline $\mathrm{C} 87$ & 3.697967 & 4.693083 & 0.543954 & $\mathrm{C} 87$ & 3.261144 & 5.295305 & 0.214795 \\
\hline $\mathrm{C} 88$ & 2.898972 & 3.919948 & -0.29866 & $\mathrm{C} 88$ & 2.150006 & 4.714793 & -0.38973 \\
\hline $\mathrm{C} 89$ & 2.439582 & 2.664388 & 0.113423 & C89 & 2.189726 & 3.397545 & -0.87533 \\
\hline C90 & 2.805336 & 2.185018 & 1.376311 & $\mathrm{C} 90$ & 3.399925 & 2.698549 & -0.77949 \\
\hline C91 & 3.608374 & 2.954134 & 2.216858 & C91 & 4.51859 & 3.278304 & -0.1774 \\
\hline H92 & 4.680635 & 4.811273 & 2.459089 & H92 & 5.323446 & 5.027757 & 0.793108 \\
\hline H93 & 4.04499 & 5.668248 & 0.21427 & H93 & 3.203195 & 6.315 & 0.585274 \\
\hline H94 & 2.629798 & 4.295591 & -1.28377 & H94 & 1.234519 & 5.292507 & -0.48808 \\
\hline H95 & 2.446525 & 1.215099 & 1.706133 & H95 & 3.493864 & 1.705805 & -1.21118 \\
\hline H96 & 3.884096 & 2.569337 & 3.194691 & H96 & 5.447337 & 2.718932 & -0.12652 \\
\hline C97 & -0.36298 & 3.398097 & 1.316545 & C97 & -0.06893 & 3.303609 & 1.506533 \\
\hline H98 & -0.61659 & 2.47241 & 1.839296 & H98 & -0.32646 & 2.465495 & 2.160047 \\
\hline H99 & 0.670866 & 3.642688 & 1.55095 & H99 & 1.010091 & 3.286186 & 1.365043 \\
\hline H100 & -0.99114 & 4.200457 & 1.718792 & H100 & -0.32412 & 4.229422 & 2.033062 \\
\hline
\end{tabular}




\begin{tabular}{|c|c|c|c|c|c|c|c|}
\hline $\mathrm{C} 101$ & -2.14562 & 3.273673 & -0.42767 & $\mathrm{C} 101$ & -2.33603 & 2.996794 & 0.536178 \\
\hline H102 & -2.43258 & 3.138174 & -1.47336 & $\mathrm{H} 102$ & -2.99866 & 2.905767 & -0.32561 \\
\hline H103 & -2.62218 & 2.465125 & 0.129834 & H103 & -2.46209 & 2.087424 & 1.120759 \\
\hline H104 & -2.57044 & 4.220022 & -0.07832 & $\mathrm{H} 104$ & -2.6946 & 3.829948 & 1.149624 \\
\hline H105 & -0.19455 & 4.154821 & -0.67461 & H105 & -0.79168 & 4.200818 & -0.30435 \\
\hline \multirow[t]{2}{*}{$\mathrm{X}$} & 0.511818 & 2.053523 & -0.77184 & H106 & 1.739713 & 0.822751 & 0.183774 \\
\hline & & & & H107 & 1.806645 & 0.475789 & -0.59764 \\
\hline \multicolumn{8}{|c|}{ C3_ES_Chelbot_TS1 } \\
\hline $\mathrm{C} 1$ & -3.93912 & -3.46052 & -0.95048 & \multicolumn{4}{|c|}{ C3_ES_Chelbot_TS3 } \\
\hline $\mathrm{C} 2$ & -1.68073 & -4.389 & -1.04942 & $\mathrm{C} 1$ & -3.70265 & -3.39788 & -1.93728 \\
\hline $\mathrm{C} 3$ & -2.95225 & -4.19683 & -1.91795 & $\mathrm{C} 2$ & -1.41948 & -4.22001 & -1.6525 \\
\hline $\mathrm{C} 4$ & -3.08363 & -3.30895 & 0.328034 & $\mathrm{C} 3$ & -2.49522 & -3.98019 & -2.74619 \\
\hline H5 & -4.82802 & -4.0651 & -0.74251 & $\mathrm{C} 4$ & -3.11803 & -3.30314 & -0.51145 \\
\hline H6 & -2.74585 & -3.62404 & -2.82787 & H5 & -4.56159 & -4.07722 & -1.94773 \\
\hline H7 & -3.63021 & -3.03743 & 1.231817 & H6 & -2.1408 & -3.29882 & -3.52618 \\
\hline H8 & -4.274 & -2.49489 & -1.3317 & $\mathrm{H} 7$ & -3.84532 & -3.11669 & 0.279092 \\
\hline H9 & -0.97927 & -5.13821 & -1.41719 & H8 & -4.04179 & -2.42755 & -2.30007 \\
\hline H10 & -3.34461 & -5.16992 & -2.22635 & H9 & -0.62895 & -4.9167 & -1.93069 \\
\hline $\mathrm{C} 11$ & -2.29073 & -4.62743 & 0.349901 & $\mathrm{H} 10$ & -2.75553 & -4.92527 & -3.23072 \\
\hline H12 & -1.54949 & -4.6759 & 1.153507 & $\mathrm{C} 11$ & -2.2856 & -4.59739 & -0.4326 \\
\hline H13 & -2.93011 & -5.51374 & 0.388837 & H12 & -1.70877 & -4.70864 & 0.489979 \\
\hline $\mathrm{C} 14$ & 0.353981 & -2.98157 & -0.28065 & H13 & -2.88261 & -5.49938 & -0.59397 \\
\hline N15 & 0.943549 & -1.91756 & 0.139939 & $\mathrm{C} 14$ & 0.349895 & -2.86209 & -0.34533 \\
\hline $\mathrm{C} 16$ & 2.349481 & -2.27218 & 0.560244 & N15 & 0.825786 & -1.85013 & 0.302742 \\
\hline $\mathrm{C} 17$ & 2.313694 & -3.83328 & 0.416056 & $\mathrm{C} 16$ & 2.117715 & -2.27304 & 0.971746 \\
\hline P18 & -1.94784 & -0.68812 & 0.391717 & $\mathrm{C} 17$ & 2.138079 & -3.80774 & 0.630135 \\
\hline $\operatorname{Ir} 19$ & 0.239724 & 0.115333 & -0.21513 & P18 & -2.01136 & -0.79277 & 0.319203 \\
\hline $\mathrm{C} 20$ & -2.21427 & -0.56727 & 2.200572 & $\operatorname{Ir} 19$ & 0.218239 & 0.201783 & -0.11591 \\
\hline $\mathrm{C} 21$ & -2.29716 & -0.30982 & 4.999506 & $\mathrm{C} 20$ & -2.17321 & -1.15057 & 2.117368 \\
\hline $\mathrm{C} 22$ & -1.90103 & -1.65609 & 3.025823 & $\mathrm{C} 21$ & -2.3223 & -1.65812 & 4.87935 \\
\hline $\mathrm{C} 23$ & -2.57703 & 0.652043 & 2.795097 & $\mathrm{C} 22$ & -2.21903 & -2.46333 & 2.600717 \\
\hline $\mathrm{C} 24$ & -2.61699 & 0.778198 & 4.182915 & $\mathrm{C} 23$ & -2.17818 & -0.0919 & 3.041904 \\
\hline $\mathrm{C} 25$ & -1.9442 & -1.52758 & 4.415856 & $\mathrm{C} 24$ & -2.26471 & -0.34359 & 4.409907 \\
\hline H26 & -2.82507 & 1.510643 & 2.180522 & $\mathrm{C} 25$ & -2.28987 & -2.71509 & 3.97166 \\
\hline $\mathrm{H} 27$ & -2.89632 & 1.729658 & 4.626431 & $\mathrm{H} 26$ & -2.09856 & 0.934774 & 2.701866 \\
\hline $\mathrm{H} 28$ & -1.69671 & -2.38114 & 5.040668 & $\mathrm{H} 27$ & -2.27583 & 0.487615 & 5.109484 \\
\hline H29 & -2.32663 & -0.20886 & 6.080452 & $\mathrm{H} 28$ & -2.31622 & -3.74083 & 4.327993 \\
\hline $\mathrm{O} 30$ & 1.029682 & -4.129 & -0.20866 & $\mathrm{H} 29$ & -2.37884 & -1.85488 & 5.946002 \\
\hline C31 & 2.621108 & -1.90086 & 2.014627 & $\mathrm{O} 30$ & 1.01236 & -4.01344 & -0.26408 \\
\hline C32 & 1.604092 & -1.46639 & 2.867621 & $\mathrm{C} 31$ & 2.122689 & -2.10584 & 2.489756 \\
\hline $\mathrm{C} 33$ & 3.910489 & -2.07663 & 2.538807 & $\mathrm{C} 32$ & 1.16943 & -1.38156 & 3.203055 \\
\hline $\mathrm{C} 34$ & 1.875217 & -1.16388 & 4.202957 & $\mathrm{C} 33$ & 3.168383 & -2.72118 & 3.200545 \\
\hline H35 & 0.596151 & -1.35583 & 2.495609 & $\mathrm{C} 34$ & 1.266119 & -1.25701 & 4.592397 \\
\hline C36 & 4.181858 & -1.78024 & 3.873324 & H35 & 0.341678 & -0.92258 & 2.686928 \\
\hline H37 & 4.712657 & -2.43006 & 1.896988 & $\mathrm{C} 36$ & 3.260986 & -2.60397 & 4.584802 \\
\hline
\end{tabular}




\begin{tabular}{|c|c|c|c|c|c|c|c|}
\hline $\mathrm{C} 38$ & 3.165914 & -1.31024 & 4.708664 & H37 & 3.933107 & -3.27802 & 2.663294 \\
\hline H39 & 1.066866 & -0.8164 & 4.840453 & C38 & 2.309129 & -1.8621 & 5.289641 \\
\hline $\mathrm{H} 40$ & 5.189204 & -1.9116 & 4.258047 & H39 & 0.508499 & -0.68862 & 5.122132 \\
\hline H41 & 3.380371 & -1.07008 & 5.746084 & $\mathrm{H} 40$ & 4.080878 & -3.08492 & 5.110691 \\
\hline $\mathrm{C} 42$ & -3.47249 & -0.03508 & -0.39704 & $\mathrm{H} 41$ & 2.381956 & -1.76347 & 6.368644 \\
\hline $\mathrm{C} 43$ & -4.66907 & 0.188969 & 0.296533 & $\mathrm{C} 42$ & -3.64008 & -0.06587 & -0.14136 \\
\hline $\mathrm{C} 44$ & -3.45054 & 0.125701 & -1.79218 & $\mathrm{C} 43$ & -4.60816 & 0.318435 & 0.794268 \\
\hline $\mathrm{C} 45$ & -5.81378 & 0.599273 & -0.39078 & C44 & -3.91598 & 0.092109 & -1.50976 \\
\hline H46 & -4.71571 & 0.047969 & 1.371117 & $\mathrm{C} 45$ & -5.82072 & 0.866333 & 0.371135 \\
\hline $\mathrm{C} 47$ & -4.59679 & 0.525205 & -2.47563 & $\mathrm{H} 46$ & -4.42836 & 0.188418 & 1.855508 \\
\hline $\mathrm{C} 48$ & -5.78131 & 0.772946 & -1.77492 & C47 & -5.13168 & 0.62797 & -1.92929 \\
\hline H49 & -6.73412 & 0.776015 & 0.158482 & $\mathrm{C} 48$ & -6.08528 & 1.025504 & -0.9876 \\
\hline H50 & -4.56919 & 0.637667 & -3.55623 & $\mathrm{H} 49$ & -6.56228 & 1.158335 & 1.10906 \\
\hline H51 & -6.6744 & 1.089229 & -2.30586 & $\mathrm{H} 50$ & -5.34782 & 0.716476 & -2.99094 \\
\hline H52 & 0.440795 & 0.470646 & 1.277764 & H51 & -7.03318 & 1.44295 & -1.31421 \\
\hline H53 & 1.827948 & 0.267238 & -0.41732 & $\mathrm{H} 52$ & 0.404685 & 0.542426 & 1.380155 \\
\hline H54 & -1.60126 & -2.59925 & 2.581476 & $\mathrm{H} 53$ & 1.381403 & 2.223882 & -2.38209 \\
\hline H55 & -2.54418 & -0.09506 & -2.34329 & H54 & -2.17864 & -3.2981 & 1.914452 \\
\hline H56 & 3.09997 & -4.23074 & -0.22647 & $\mathrm{H} 55$ & -3.19568 & -0.2427 & -2.24929 \\
\hline $\mathrm{C} 57$ & 4.811202 & -0.34089 & -2.45531 & H56 & 3.042282 & -4.12477 & 0.109227 \\
\hline $\mathrm{C} 58$ & 3.973102 & -1.41001 & -2.7855 & C57 & 5.415678 & -0.30581 & -1.062 \\
\hline C59 & 3.223371 & -2.03928 & -1.79597 & C58 & 4.521045 & -1.11792 & -1.76489 \\
\hline $\mathrm{C} 60$ & 3.298979 & -1.62224 & -0.45796 & C59 & 3.468644 & -1.73484 & -1.09447 \\
\hline C61 & 4.141095 & -0.55073 & -0.13912 & C60 & 3.282982 & -1.54653 & 0.284037 \\
\hline C62 & 4.89061 & 0.085869 & -1.13135 & C61 & 4.162905 & -0.70735 & 0.972182 \\
\hline H63 & 5.395223 & 0.153347 & -3.22671 & C62 & 5.230726 & -0.10332 & 0.30413 \\
\hline H64 & 3.898705 & -1.74818 & -3.81509 & H63 & 6.246651 & 0.165637 & -1.57884 \\
\hline H65 & 2.554201 & -2.84802 & -2.07919 & H64 & 4.649095 & -1.27736 & -2.83165 \\
\hline H66 & 4.201935 & -0.18647 & 0.877702 & H65 & 2.782203 & -2.36479 & -1.65513 \\
\hline H67 & 5.526882 & 0.923674 & -0.86101 & H66 & 4.028656 & -0.52938 & 2.032488 \\
\hline C68 & -0.77662 & 3.269234 & -0.11252 & H67 & 5.917714 & 0.526824 & 0.861312 \\
\hline C69 & -0.05998 & 2.129942 & -0.85972 & C68 & -0.78431 & 3.197684 & 0.206473 \\
\hline $\mathrm{C} 70$ & 1.387426 & 1.900124 & -0.77461 & C69 & -0.15936 & 2.199771 & -0.81446 \\
\hline C71 & -0.47046 & 3.197312 & -3.18824 & $\mathrm{C} 70$ & 1.036423 & 2.955678 & -1.64312 \\
\hline $\mathrm{C} 72$ & -0.37428 & 0.911093 & -2.96299 & C71 & -1.8223 & 2.945431 & -2.73113 \\
\hline $\mathrm{C} 73$ & -0.91455 & 2.499704 & -4.48285 & $\mathrm{C} 72$ & -0.88198 & 0.836466 & -2.71686 \\
\hline H74 & 0.548165 & 3.605702 & -3.25223 & $\mathrm{C} 73$ & -2.33998 & 2.100282 & -3.91093 \\
\hline H75 & -1.15282 & 3.993635 & -2.8869 & $\mathrm{H} 74$ & -1.14477 & 3.737279 & -3.0649 \\
\hline H76 & -2.0002 & 2.54132 & -4.61692 & $\mathrm{H} 75$ & -2.63483 & 3.399817 & -2.16412 \\
\hline H77 & -0.41388 & 2.85718 & -5.38276 & $\mathrm{H} 76$ & -3.38517 & 1.813991 & -3.78079 \\
\hline N78 & -0.51008 & 2.060421 & -2.26151 & $\mathrm{H} 77$ & -2.20122 & 2.572888 & -4.88378 \\
\hline O79 & -0.54108 & 1.107856 & -4.27999 & N78 & -1.11895 & 1.919991 & -1.93939 \\
\hline $\mathrm{O} 80$ & -0.1299 & -0.21074 & -2.48836 & O79 & -1.53912 & 0.887129 & -3.88226 \\
\hline H81 & 2.329541 & -4.33606 & 1.38428 & O80 & -0.16315 & -0.12451 & -2.4048 \\
\hline N82 & -1.97318 & -2.34877 & 0.07771 & $\mathrm{H} 81$ & 1.971845 & -4.43048 & 1.509982 \\
\hline
\end{tabular}




\begin{tabular}{|c|c|c|c|c|c|c|c|}
\hline $\mathrm{C} 83$ & -1.03473 & -2.99457 & -0.87061 & N82 & -2.04307 & -2.26408 & -0.50943 \\
\hline H84 & -0.9708 & -2.4579 & -1.8265 & $\mathrm{C} 83$ & -0.87777 & -2.83401 & -1.22635 \\
\hline H85 & 1.855524 & 1.751697 & -1.75075 & H84 & -0.61562 & -2.22713 & -2.09898 \\
\hline $\mathrm{C} 86$ & 4.155679 & 4.045976 & 1.72781 & H85 & 0.548282 & 3.770826 & -2.18092 \\
\hline C87 & 3.755381 & 4.539545 & 0.484603 & C86 & 4.450712 & 4.65706 & 0.382524 \\
\hline $\mathrm{C} 88$ & 2.85456 & 3.815572 & -0.29483 & C87 & 3.288276 & 5.410707 & 0.208427 \\
\hline $\mathrm{C} 89$ & 2.332918 & 2.595239 & 0.158598 & C88 & 2.189689 & 4.854968 & -0.44238 \\
\hline C90 & 2.743471 & 2.105408 & 1.403216 & C89 & 2.227351 & 3.537071 & -0.92249 \\
\hline C91 & 3.648534 & 2.82725 & 2.181093 & C90 & 3.403644 & 2.79506 & -0.75472 \\
\hline H92 & 4.859471 & 4.607171 & 2.335643 & C91 & 4.506287 & 3.35222 & -0.10558 \\
\hline H93 & 4.145381 & 5.485777 & 0.120094 & H92 & 5.311831 & 5.090509 & 0.882878 \\
\hline H94 & 2.550732 & 4.201207 & -1.2661 & H93 & 3.24188 & 6.433397 & 0.571413 \\
\hline H95 & 2.350579 & 1.162868 & 1.765479 & H94 & 1.292342 & 5.452421 & -0.58505 \\
\hline H96 & 3.955959 & 2.431117 & 3.144755 & H95 & 3.469052 & 1.783275 & -1.14562 \\
\hline C97 & -0.46543 & 3.355589 & 1.389859 & H96 & 5.411688 & 2.764719 & 0.006815 \\
\hline H98 & -0.66727 & 2.403298 & 1.885921 & C97 & 0.017014 & 3.294268 & 1.521415 \\
\hline H99 & 0.570257 & 3.624309 & 1.587866 & H98 & -0.20496 & 2.438105 & 2.164083 \\
\hline H100 & -1.10109 & 4.121628 & 1.846742 & H99 & 1.095802 & 3.323015 & 1.370755 \\
\hline C101 & -2.29977 & 3.232371 & -0.3164 & $\mathrm{H} 100$ & -0.26978 & 4.204365 & 2.057732 \\
\hline H102 & -2.59912 & 3.142018 & -1.36368 & C101 & -2.24939 & 2.922729 & 0.565286 \\
\hline H103 & -2.74336 & 2.384278 & 0.206604 & H102 & -2.92111 & 2.838773 & -0.28938 \\
\hline H104 & -2.74903 & 4.146284 & 0.085089 & $\mathrm{H} 103$ & -2.33625 & 1.991034 & 1.119844 \\
\hline H105 & -0.40041 & 4.201564 & -0.56716 & H104 & -2.62221 & 3.725831 & 1.209351 \\
\hline \multirow[t]{2}{*}{$\mathrm{X}$} & 0.511818 & 2.053523 & -0.77184 & H105 & -0.74317 & 4.184796 & -0.27785 \\
\hline & & & & H106 & 1.765166 & 0.539234 & -0.26164 \\
\hline \multicolumn{4}{|c|}{ C3_ES_Chelbot_int1 } & H107 & 0.924033 & 1.60068 & -0.38607 \\
\hline $\mathrm{C} 1$ & -3.91123 & -3.50203 & -0.99682 & & & & \\
\hline $\mathrm{C} 2$ & -1.6571 & -4.44651 & -1.00624 & \multicolumn{4}{|c|}{ C3_ES_Chelbot_PROD } \\
\hline $\mathrm{C} 3$ & -2.90812 & -4.2842 & -1.91053 & $\mathrm{C} 1$ & -3.73433 & -3.55763 & -1.69487 \\
\hline $\mathrm{C} 4$ & -3.08915 & -3.31582 & 0.298042 & $\mathrm{C} 2$ & -1.43563 & -4.35964 & -1.54081 \\
\hline H5 & -4.81524 & -4.08571 & -0.79452 & $\mathrm{C} 3$ & -2.57349 & -4.13509 & -2.57037 \\
\hline H6 & -2.67865 & -3.75283 & -2.83965 & $\mathrm{C} 4$ & -3.08564 & -3.49382 & -0.29419 \\
\hline $\mathrm{H} 7$ & -3.65869 & -3.01239 & 1.177278 & H5 & -4.5989 & -4.22958 & -1.67685 \\
\hline $\mathrm{H} 8$ & -4.2189 & -2.54299 & -1.41745 & H6 & -2.27101 & -3.45814 & -3.37569 \\
\hline H9 & -0.94828 & -5.20843 & -1.33104 & $\mathrm{H} 7$ & -3.78632 & -3.33831 & 0.526116 \\
\hline H10 & -3.30062 & -5.26744 & -2.1847 & $\mathrm{H} 8$ & -4.08259 & -2.57843 & -2.02384 \\
\hline $\mathrm{C} 11$ & -2.30239 & -4.63589 & 0.384183 & H9 & -0.64549 & -5.03535 & -1.86818 \\
\hline H12 & -1.58123 & -4.66096 & 1.207046 & $\mathrm{H} 10$ & -2.85425 & -5.08605 & -3.03158 \\
\hline H13 & -2.94635 & -5.51823 & 0.436763 & $\mathrm{C} 11$ & -2.22811 & -4.77192 & -0.27856 \\
\hline $\mathrm{C} 14$ & 0.377909 & -3.00157 & -0.27548 & H12 & -1.60789 & -4.87147 & 0.617247 \\
\hline N15 & 0.930582 & -1.9249 & 0.160199 & H13 & -2.80946 & -5.68654 & -0.42582 \\
\hline $\mathrm{C} 16$ & 2.3488 & -2.226 & 0.544272 & C14 & 0.390649 & -2.97744 & -0.35521 \\
\hline $\mathrm{C} 17$ & 2.389129 & -3.7848 & 0.344292 & N15 & 0.939 & -1.93696 & 0.185548 \\
\hline P18 & -1.91029 & -0.72241 & 0.40241 & $\mathrm{C} 16$ & 2.262718 & -2.33553 & 0.814969 \\
\hline Ir19 & 0.105397 & 0.081757 & -0.20228 & $\mathrm{C} 17$ & 2.174123 & -3.89096 & 0.658129 \\
\hline
\end{tabular}




\begin{tabular}{|c|c|c|c|c|c|c|c|}
\hline $\mathrm{C} 20$ & -2.16375 & -0.61926 & 2.209298 & P18 & -1.98692 & -0.92546 & 0.430301 \\
\hline $\mathrm{C} 21$ & -2.28074 & -0.39528 & 5.001425 & $\operatorname{Ir} 19$ & 0.230896 & -0.04724 & -0.0847 \\
\hline $\mathrm{C} 22$ & -1.92687 & -1.73676 & 3.019722 & $\mathrm{C} 20$ & -2.24544 & -1.14402 & 2.231037 \\
\hline $\mathrm{C} 23$ & -2.45175 & 0.616348 & 2.811423 & $\mathrm{C} 21$ & -2.52222 & -1.34378 & 5.01738 \\
\hline $\mathrm{C} 24$ & -2.51167 & 0.724981 & 4.198476 & $\mathrm{C} 22$ & -2.46055 & -2.38951 & 2.834967 \\
\hline $\mathrm{C} 25$ & -1.98902 & -1.62416 & 4.409958 & $\mathrm{C} 23$ & -2.13315 & -0.00302 & 3.043886 \\
\hline H26 & -2.62093 & 1.497405 & 2.201372 & $\mathrm{C} 24$ & -2.28226 & -0.10047 & 4.425595 \\
\hline $\mathrm{H} 27$ & -2.73448 & 1.685718 & 4.653539 & $\mathrm{C} 25$ & -2.597 & -2.48663 & 4.221317 \\
\hline $\mathrm{H} 28$ & -1.80183 & -2.49729 & 5.028376 & $\mathrm{H} 26$ & -1.91215 & 0.962815 & 2.597492 \\
\hline H29 & -2.32698 & -0.30802 & 6.083131 & $\mathrm{H} 27$ & -2.19833 & 0.790215 & 5.0417 \\
\hline $\mathrm{O} 30$ & 1.104072 & -4.12179 & -0.25827 & $\mathrm{H} 28$ & -2.75931 & -3.45873 & 4.677956 \\
\hline C31 & 2.62649 & -1.88796 & 2.004646 & $\mathrm{H} 29$ & -2.63444 & -1.42148 & 6.09497 \\
\hline C32 & 1.619179 & -1.4455 & 2.865453 & $\mathrm{O} 30$ & 1.034369 & -4.13384 & -0.21612 \\
\hline $\mathrm{C} 33$ & 3.911336 & -2.10609 & 2.524482 & $\mathrm{C} 31$ & 2.342458 & -2.04144 & 2.31035 \\
\hline C34 & 1.896373 & -1.18626 & 4.209149 & $\mathrm{C} 32$ & 1.197574 & -1.80554 & 3.075817 \\
\hline H35 & 0.615538 & -1.29965 & 2.493191 & $\mathrm{C} 33$ & 3.574476 & -2.19037 & 2.964853 \\
\hline C36 & 4.188486 & -1.84912 & 3.865446 & C34 & 1.287298 & -1.66414 & 4.461423 \\
\hline H37 & 4.704512 & -2.46476 & 1.873983 & H35 & 0.231104 & -1.72666 & 2.599794 \\
\hline C38 & 3.181969 & -1.37881 & 4.711978 & $\mathrm{C} 36$ & 3.662466 & -2.05701 & 4.349477 \\
\hline H39 & 1.095943 & -0.83796 & 4.856143 & H37 & 4.471219 & -2.40478 & 2.391472 \\
\hline $\mathrm{H} 40$ & 5.191433 & -2.01514 & 4.24805 & $\mathrm{C} 38$ & 2.51962 & -1.78261 & 5.102208 \\
\hline H41 & 3.399846 & -1.17427 & 5.756247 & H39 & 0.384658 & -1.46636 & 5.031168 \\
\hline $\mathrm{C} 42$ & -3.41111 & -0.01333 & -0.37431 & $\mathrm{H} 40$ & 4.626095 & -2.1675 & 4.838564 \\
\hline $\mathrm{C} 43$ & -4.5685 & 0.319822 & 0.340854 & H41 & 2.590025 & -1.67212 & 6.180605 \\
\hline $\mathrm{C} 44$ & -3.4101 & 0.100601 & -1.77453 & $\mathrm{C} 42$ & -3.54673 & -0.14124 & -0.15392 \\
\hline $\mathrm{C} 45$ & -5.69601 & 0.792948 & -0.33354 & $\mathrm{C} 43$ & -4.53415 & 0.366815 & 0.699986 \\
\hline H46 & -4.60122 & 0.211096 & 1.419419 & C44 & -3.72948 & -0.04193 & -1.54383 \\
\hline $\mathrm{C} 47$ & -4.53867 & 0.5666 & -2.44254 & $\mathrm{C} 45$ & -5.67703 & 0.973485 & 0.173825 \\
\hline $\mathrm{C} 48$ & -5.68176 & 0.924306 & -1.72127 & H46 & -4.41996 & 0.289834 & 1.776223 \\
\hline H49 & -6.58731 & 1.054037 & 0.229721 & C47 & -4.87707 & 0.551679 & -2.06492 \\
\hline $\mathrm{H} 50$ & -4.53133 & 0.645626 & -3.52623 & $\mathrm{C} 48$ & -5.85196 & 1.068253 & -1.20608 \\
\hline H51 & -6.56117 & 1.292364 & -2.24175 & H49 & -6.43606 & 1.361468 & 0.847098 \\
\hline H52 & 0.28258 & 0.492499 & 1.285983 & $\mathrm{H} 50$ & -5.02023 & 0.593205 & -3.14155 \\
\hline H53 & 1.856497 & 0.773653 & -0.62434 & H51 & -6.74763 & 1.529717 & -1.61172 \\
\hline H54 & -1.6708 & -2.68836 & 2.567142 & H52 & 0.630508 & 0.168779 & 1.395744 \\
\hline H55 & -2.53356 & -0.20047 & -2.33694 & H53 & 1.563305 & 2.443327 & -2.10695 \\
\hline H56 & 3.175331 & -4.11568 & -0.33556 & H54 & -2.49564 & -3.29131 & 2.235925 \\
\hline $\mathrm{C} 57$ & 4.690465 & -0.11278 & -2.44757 & H55 & -2.98453 & -0.45057 & -2.21861 \\
\hline C58 & 3.809906 & -1.1367 & -2.80935 & H56 & 3.05816 & -4.3361 & 0.202291 \\
\hline C59 & 3.096032 & -1.81886 & -1.82894 & C57 & 5.30449 & -0.535 & -1.70109 \\
\hline C60 & 3.247305 & -1.49875 & -0.46958 & C58 & 4.584077 & -1.64689 & -2.13996 \\
\hline C61 & 4.130568 & -0.47307 & -0.1195 & C59 & 3.629363 & -2.23129 & -1.30987 \\
\hline C62 & 4.846672 & 0.215223 & -1.10265 & C60 & 3.379142 & -1.7182 & -0.03067 \\
\hline H63 & 5.24829 & 0.422733 & -3.21059 & C61 & 4.089945 & -0.589 & 0.39049 \\
\hline H64 & 3.67583 & -1.40011 & -3.85461 & C62 & 5.051859 & -0.00753 & -0.43532 \\
\hline
\end{tabular}




\begin{tabular}{|c|c|c|c|c|c|c|c|}
\hline H65 & 2.397125 & -2.59413 & -2.13176 & H63 & 6.053466 & -0.08075 & -2.34334 \\
\hline H66 & 4.254757 & -0.18698 & 0.916668 & H64 & 4.765954 & -2.06096 & -3.12749 \\
\hline H67 & 5.515982 & 1.018031 & -0.80794 & H65 & 3.065622 & -3.08448 & -1.67971 \\
\hline C68 & -0.63392 & 3.263257 & -0.21128 & H66 & 3.882648 & -0.15165 & 1.360277 \\
\hline C69 & 0.017474 & 2.036464 & -0.88228 & H67 & 5.592166 & 0.870175 & -0.09334 \\
\hline $\mathrm{C} 70$ & 1.574521 & 1.873042 & -0.85507 & C68 & -0.91161 & 3.675132 & 0.098767 \\
\hline C71 & -0.33149 & 3.101839 & -3.25724 & C69 & -0.17357 & 2.762491 & -0.91317 \\
\hline $\mathrm{C} 72$ & -0.31878 & 0.82166 & -2.97616 & $\mathrm{C} 70$ & 1.171292 & 3.269402 & -1.50383 \\
\hline $\mathrm{C} 73$ & -0.77425 & 2.385102 & -4.54425 & C71 & -1.5966 & 3.402589 & -2.94597 \\
\hline H74 & 0.693534 & 3.494035 & -3.31968 & $\mathrm{C} 72$ & -0.99733 & 1.182798 & -2.67897 \\
\hline H75 & -1.00284 & 3.916639 & -2.98185 & C73 & -2.37106 & 2.506183 & -3.91871 \\
\hline H76 & -1.85384 & 2.466057 & -4.70556 & H74 & -0.80377 & 3.969588 & -3.44755 \\
\hline H77 & -0.24025 & 2.698467 & -5.44141 & $\mathrm{H} 75$ & -2.24378 & 4.099869 & -2.41104 \\
\hline N78 & -0.40244 & 1.988372 & -2.30547 & $\mathrm{H} 76$ & -3.40764 & 2.363013 & -3.6015 \\
\hline O79 & -0.46358 & 0.98327 & -4.29933 & H77 & -2.33329 & 2.83839 & -4.95617 \\
\hline O80 & -0.13061 & -0.2982 & -2.46338 & N78 & -1.03461 & 2.387237 & -2.04415 \\
\hline H81 & 2.459064 & -4.32137 & 1.291749 & O79 & -1.69796 & 1.224172 & -3.82733 \\
\hline N82 & -1.96971 & -2.36842 & 0.039461 & O80 & -0.45393 & 0.136854 & -2.31161 \\
\hline $\mathrm{C} 83$ & -1.01199 & -3.04607 & -0.86404 & H81 & 1.958406 & -4.37788 & 1.609781 \\
\hline H84 & -0.94098 & -2.54627 & -1.83945 & N82 & -2.0151 & -2.45713 & -0.2842 \\
\hline H85 & 1.96699 & 1.939305 & -1.87608 & $\mathrm{C} 83$ & -0.9046 & -2.96258 & -1.13197 \\
\hline C86 & 4.076681 & 4.215674 & 1.755303 & H84 & -0.74158 & -2.32223 & -2.00847 \\
\hline $\mathrm{C} 87$ & 3.727275 & 4.689898 & 0.489913 & H85 & 0.916737 & 4.079286 & -2.19702 \\
\hline $\mathrm{C} 88$ & 2.917367 & 3.919772 & -0.34499 & $\mathrm{C} 86$ & 4.232804 & 4.722049 & 1.173748 \\
\hline $\mathrm{C} 89$ & 2.440812 & 2.675027 & 0.079826 & $\mathrm{C} 87$ & 3.426933 & 5.621739 & 0.474416 \\
\hline C90 & 2.797315 & 2.203229 & 1.348203 & C88 & 2.440704 & 5.147983 & -0.39185 \\
\hline C91 & 3.610594 & 2.969734 & 2.181086 & $\mathrm{C} 89$ & 2.242928 & 3.772572 & -0.56617 \\
\hline H92 & 4.711037 & 4.812732 & 2.403844 & $\mathrm{C} 90$ & 3.065972 & 2.877833 & 0.12773 \\
\hline H93 & 4.088653 & 5.656452 & 0.15044 & C91 & 4.050319 & 3.349026 & 0.994673 \\
\hline H94 & 2.654171 & 4.28865 & -1.33432 & H92 & 5.001354 & 5.089317 & 1.847873 \\
\hline H95 & 2.424397 & 1.240989 & 1.686402 & H93 & 3.568295 & 6.691586 & 0.599562 \\
\hline H96 & 3.880096 & 2.591899 & 3.163317 & H94 & 1.817575 & 5.855286 & -0.93549 \\
\hline C97 & -0.35325 & 3.419922 & 1.292897 & H95 & 2.925945 & 1.812569 & -0.0083 \\
\hline H98 & -0.57019 & 2.491204 & 1.826491 & H96 & 4.677653 & 2.642074 & 1.531606 \\
\hline H99 & 0.67641 & 3.697727 & 1.508352 & C97 & -0.20241 & 3.694756 & 1.466703 \\
\hline H100 & -0.99846 & 4.206449 & 1.699437 & H98 & -0.14149 & 2.676294 & 1.870312 \\
\hline C101 & -2.15877 & 3.262884 & -0.42543 & H99 & 0.806861 & 4.098821 & 1.423916 \\
\hline H102 & -2.45913 & 3.098405 & -1.46323 & H100 & -0.78145 & 4.30264 & 2.168627 \\
\hline H103 & -2.62534 & 2.467864 & 0.159801 & C101 & -2.36666 & 3.232732 & 0.314484 \\
\hline H104 & -2.58328 & 4.216327 & -0.09539 & H102 & -2.97543 & 3.221295 & -0.59205 \\
\hline H105 & -0.2192 & 4.157279 & -0.70763 & H103 & -2.39219 & 2.219123 & 0.722088 \\
\hline \multirow[t]{2}{*}{$\mathrm{X}$} & 0.511818 & 2.053523 & -0.77184 & H104 & -2.8542 & 3.894846 & 1.036267 \\
\hline & & & & H105 & -0.89449 & 4.692923 & -0.31959 \\
\hline \multicolumn{4}{|c|}{ C3_ES_Chelbot_TS2 } & H106 & 1.728182 & 0.357927 & -0.43177 \\
\hline $\mathrm{C} 1$ & -3.68141 & -3.36122 & -1.85634 & H107 & 0.063298 & 1.836492 & -0.30471 \\
\hline
\end{tabular}




\begin{tabular}{|c|c|c|c|}
\hline $\mathrm{C} 2$ & -1.41707 & -4.24234 & -1.59908 \\
\hline $\mathrm{C} 3$ & -2.50391 & -3.98486 & -2.67772 \\
\hline $\mathrm{C} 4$ & -3.07329 & -3.26947 & -0.44108 \\
\hline H5 & -4.55861 & -4.01679 & -1.84497 \\
\hline H6 & -2.1444 & -3.32141 & -3.47057 \\
\hline H7 & -3.78195 & -3.05939 & 0.360619 \\
\hline H8 & -4.00132 & -2.38679 & -2.22501 \\
\hline H9 & -0.64903 & -4.96182 & -1.88241 \\
\hline H10 & -2.79846 & -4.92719 & -3.14771 \\
\hline $\mathrm{C} 11$ & -2.27231 & -4.58392 & -0.36166 \\
\hline H12 & -1.68488 & -4.69972 & 0.553866 \\
\hline H13 & -2.89671 & -5.47054 & -0.50296 \\
\hline $\mathrm{C} 14$ & 0.417925 & -2.91322 & -0.34543 \\
\hline N15 & 0.88584 & -1.91264 & 0.319402 \\
\hline $\mathrm{C} 16$ & 2.202731 & -2.31759 & 0.923178 \\
\hline $\mathrm{C} 17$ & 2.265812 & -3.84063 & 0.520313 \\
\hline P18 & -1.92274 & -0.78408 & 0.343949 \\
\hline Ir19 & 0.062728 & 0.156356 & -0.04692 \\
\hline $\mathrm{C} 20$ & -2.09303 & -1.09834 & 2.144596 \\
\hline $\mathrm{C} 21$ & -2.22985 & -1.55747 & 4.910269 \\
\hline $\mathrm{C} 22$ & -2.1031 & -2.4036 & 2.648373 \\
\hline $\mathrm{C} 23$ & -2.13109 & -0.02038 & 3.045761 \\
\hline $\mathrm{C} 24$ & -2.20773 & -0.24941 & 4.417352 \\
\hline $\mathrm{C} 25$ & -2.17082 & -2.63091 & 4.023895 \\
\hline $\mathrm{H} 26$ & -2.08073 & 0.999683 & 2.681054 \\
\hline $\mathrm{H} 27$ & -2.23929 & 0.593437 & 5.101976 \\
\hline $\mathrm{H} 28$ & -2.16779 & -3.64974 & 4.400145 \\
\hline H29 & -2.28068 & -1.7358 & 5.980529 \\
\hline $\mathrm{O} 30$ & 1.117307 & -4.04767 & -0.3441 \\
\hline C31 & 2.242126 & -2.18526 & 2.443986 \\
\hline $\mathrm{C} 32$ & 1.334746 & -1.41439 & 3.171472 \\
\hline $\mathrm{C} 33$ & 3.246284 & -2.87534 & 3.144246 \\
\hline $\mathrm{C} 34$ & 1.433469 & -1.32327 & 4.562303 \\
\hline H35 & 0.54057 & -0.89224 & 2.660654 \\
\hline C36 & 3.34523 & -2.78597 & 4.531214 \\
\hline H37 & 3.973017 & -3.47598 & 2.601628 \\
\hline C38 & 2.437504 & -2.00343 & 5.24913 \\
\hline H39 & 0.708485 & -0.72254 & 5.102736 \\
\hline $\mathrm{H} 40$ & 4.133416 & -3.32533 & 5.048895 \\
\hline H41 & 2.513279 & -1.93085 & 6.330108 \\
\hline $\mathrm{C} 42$ & -3.50423 & -0.0196 & -0.17477 \\
\hline $\mathrm{C} 43$ & -4.49135 & 0.388088 & 0.731878 \\
\hline $\mathrm{C} 44$ & -3.74422 & 0.107368 & -1.55295 \\
\hline $\mathrm{C} 45$ & -5.6883 & 0.93533 & 0.267541 \\
\hline H46 & -4.33865 & 0.277159 & 1.799394 \\
\hline
\end{tabular}




\begin{tabular}{|c|c|c|c|}
\hline $\mathrm{C} 47$ & -4.94123 & 0.650187 & -2.01233 \\
\hline $\mathrm{C} 48$ & -5.91373 & 1.073702 & -1.1015 \\
\hline H49 & -6.44729 & 1.245734 & 0.979713 \\
\hline H50 & -5.12692 & 0.723636 & -3.08054 \\
\hline H51 & -6.84898 & 1.494518 & -1.45941 \\
\hline H52 & 0.090123 & 0.535517 & 1.452153 \\
\hline H53 & 1.68063 & 1.536088 & -1.6961 \\
\hline H54 & -2.03461 & -3.24846 & 1.97655 \\
\hline H55 & -3.01304 & -0.2551 & -2.26649 \\
\hline H56 & 3.161687 & -4.09909 & -0.0458 \\
\hline $\mathrm{C} 57$ & 5.401829 & -0.27969 & -1.21734 \\
\hline C58 & 4.309498 & -0.81866 & -1.90057 \\
\hline C59 & 3.285492 & -1.44798 & -1.19439 \\
\hline C60 & 3.33006 & -1.54892 & 0.205333 \\
\hline C61 & 4.4192 & -0.98926 & 0.882717 \\
\hline C62 & 5.448519 & -0.36409 & 0.174061 \\
\hline H63 & 6.202386 & 0.209305 & -1.76485 \\
\hline H64 & 4.25387 & -0.75116 & -2.98344 \\
\hline H65 & 2.443864 & -1.85735 & -1.74516 \\
\hline H66 & 4.469037 & -1.02971 & 1.963985 \\
\hline H67 & 6.287324 & 0.062078 & 0.71722 \\
\hline C68 & -0.80057 & 3.225367 & 0.180761 \\
\hline C69 & -0.2314 & 2.124171 & -0.73929 \\
\hline $\mathrm{C} 70$ & 1.201209 & 2.479946 & -1.3985 \\
\hline C71 & -1.61469 & 3.077921 & -2.7763 \\
\hline $\mathrm{C} 72$ & -0.87481 & 0.902571 & -2.72411 \\
\hline $\mathrm{C} 73$ & -2.13317 & 2.292979 & -3.99522 \\
\hline H74 & -0.81385 & 3.782326 & -3.03905 \\
\hline $\mathrm{H} 75$ & -2.40718 & 3.630749 & -2.27011 \\
\hline H76 & -3.2028 & 2.081635 & -3.91684 \\
\hline H77 & -1.91529 & 2.76275 & -4.95474 \\
\hline N78 & -1.10288 & 1.981173 & -1.94733 \\
\hline O79 & -1.42365 & 1.022016 & -3.94354 \\
\hline $\mathrm{O} 80$ & -0.24517 & -0.11477 & -2.37816 \\
\hline H81 & 2.152202 & -4.50693 & 1.376643 \\
\hline N82 & -1.96406 & -2.26436 & -0.45809 \\
\hline $\mathrm{C} 83$ & -0.82963 & -2.86834 & -1.19528 \\
\hline H84 & -0.57715 & -2.27286 & -2.07913 \\
\hline H85 & 0.958414 & 2.95414 & -2.35696 \\
\hline $\mathrm{C} 86$ & 4.296773 & 5.040328 & 0.219116 \\
\hline C87 & 3.053652 & 5.574235 & -0.1285 \\
\hline C88 & 2.051378 & 4.746211 & -0.62778 \\
\hline $\mathrm{C} 89$ & 2.255968 & 3.364185 & -0.7693 \\
\hline C90 & 3.520911 & 2.852047 & -0.45424 \\
\hline C91 & 4.529693 & 3.677104 & 0.043164 \\
\hline
\end{tabular}




$\begin{array}{lrrr}\text { H92 } & 5.080128 & 5.685813 & 0.605966 \\ \text { H93 } & 2.868958 & 6.639488 & -0.02132 \\ \text { H94 } & 1.09917 & 5.18237 & -0.91658 \\ \text { H95 } & 3.735915 & 1.800817 & -0.61713 \\ \text { H96 } & 5.500404 & 3.250865 & 0.28218 \\ \text { C97 } & -0.04282 & 3.345864 & 1.516131 \\ \text { H98 } & -0.30181 & 2.511761 & 2.175149 \\ \text { H99 } & 1.039649 & 3.351101 & 1.394268 \\ \text { H100 } & -0.32492 & 4.274808 & 2.022359 \\ \text { C101 } & -2.29557 & 3.067988 & 0.491871 \\ \text { H102 } & -2.92452 & 2.922411 & -0.38873 \\ \text { H103 } & -2.46053 & 2.204492 & 1.135819 \\ \text { H104 } & -2.65844 & 3.95187 & 1.026783 \\ \text { H105 } & -0.68006 & 4.182286 & -0.3507 \\ \text { H106 } & 1.991109 & 1.051144 & 1.195641 \\ \text { H107 } & 2.467578 & 0.840205 & 0.659932\end{array}$

\section{C3_ER_Cheltop}

\section{C3_ER_Cheltop_SM}

$\begin{array}{lllll}\text { C1 } & -4.24858 & -3.40287 & 0.876158\end{array}$

$\begin{array}{llll}\mathrm{C} 2 & -2.19851 & -4.54636 & 0.222072\end{array}$

$\begin{array}{llll}\text { C3 } & -3.70397 & -4.46459 & -0.13559\end{array}$

$\begin{array}{llll}\text { C4 } & -2.98079 & -3.03809 & 1.685035\end{array}$

$\begin{array}{llll}\mathrm{H} 5 & -5.00531 & -3.82988 & 1.54224\end{array}$

H6 $\quad-3.86632 \quad-4.17636 \quad-1.17917$

H7 $\quad-3.16654 \quad-2.51518 \quad 2.623062$

$\begin{array}{llll}\text { H8 } & -4.69005 & -2.53279 & 0.388719\end{array}$

H9 $\quad-1.67552 \quad-5.41833 \quad-0.17013$

$\begin{array}{llll}\mathrm{H} 10 & -4.17723 & -5.4395 & 0.011934\end{array}$

$\begin{array}{llll}\mathrm{C} 11 & -2.23552 & -4.38247 & 1.758077\end{array}$

$\begin{array}{llll}\mathrm{H} 12 & -1.24827 & -4.30122 & 2.222813\end{array}$

H13 $\quad-2.81224 \quad-5.17038 \quad 2.250566$

$\begin{array}{llll}\text { C14 } & -0.04929 & -3.19441 & -0.3028\end{array}$

$\begin{array}{llll}\mathrm{N} 15 & 0.663073 & -2.12554 & -0.36302\end{array}$

$\begin{array}{llll}\mathrm{C} 16 & 2.108723 & -2.53303 & -0.27201\end{array}$

$\begin{array}{llll}\mathrm{C} 17 & 1.994708 & -4.06112 & -0.5708\end{array}$

$\begin{array}{llll}\mathrm{P} 18 & -1.92685 & -0.59942 & 0.626944\end{array}$

$\begin{array}{lllll}\text { Ir19 } & -0.12601 & -0.07979 & -0.60076\end{array}$

$\begin{array}{llll}\mathrm{C} 20 & -1.77477 & 0.092013 & 2.312401\end{array}$

$\begin{array}{llll}\mathrm{C} 21 & -1.26204 & 1.281777 & 4.802089\end{array}$

$\begin{array}{lllll}\mathrm{C} 22 & -1.28723 & -0.67666 & 3.377904\end{array}$

$\begin{array}{llll}\mathrm{C} 23 & -1.99657 & 1.465526 & 2.503436\end{array}$

$\begin{array}{llll}\mathrm{C} 24 & -1.74324 & 2.053451 & 3.741555\end{array}$

$\begin{array}{llll}\mathrm{C} 25 & -1.03854 & -0.08306 & 4.616192\end{array}$

$\begin{array}{llll}\mathrm{H} 26 & -2.35908 & 2.076643 & 1.684825\end{array}$

C3_ER_Cheltop_int2

\begin{tabular}{lrrr} 
C1 & -3.64301 & -3.342 & -1.96871 \\
C2 & -1.38485 & -4.21093 & -1.64029 \\
C3 & -2.43784 & -3.95995 & -2.75276 \\
C4 & -3.07706 & -3.23895 & -0.53632 \\
H5 & -4.51478 & -4.00482 & -1.97916 \\
H6 & -2.05735 & -3.29438 & -3.53389 \\
H7 & -3.81167 & -3.02735 & 0.241043 \\
H8 & -3.95996 & -2.37275 & -2.3521 \\
H9 & -0.60686 & -4.92936 & -1.89761 \\
H10 & -2.71222 & -4.90405 & -3.23129 \\
C11 & -2.27591 & -4.5511 & -0.42848 \\
H12 & -1.71426 & -4.66434 & 0.503192 \\
H13 & -2.89355 & -5.43976 & -0.58551 \\
C14 & 0.404818 & -2.89054 & -0.32633 \\
N15 & 0.87795 & -1.88895 & 0.332738 \\
C16 & 2.162049 & -2.31954 & 0.997783 \\
C17 & 2.190737 & -3.85033 & 0.634477 \\
P18 & -1.9584 & -0.74854 & 0.288742 \\
Ir19 & 0.164935 & 0.204216 & -0.13281 \\
C20 & -2.10105 & -1.0866 & 2.091348 \\
C21 & -2.24105 & -1.58468 & 4.852575 \\
C22 & -2.1699 & -2.39773 & 2.575872 \\
C23 & -2.08061 & -0.02465 & 3.011334 \\
C24 & -2.16112 & -0.27208 & 4.379964 \\
C25 & -2.23653 & -2.64456 & 3.948152 \\
H26 & -1.98644 & 0.998335 & 2.665624 \\
& & & \\
\hline
\end{tabular}




\begin{tabular}{|c|c|c|c|c|c|c|c|}
\hline $\mathrm{H} 27$ & -1.92598 & 3.115739 & 3.879585 & $\mathrm{H} 27$ & -2.14932 & 0.56071 & 5.077464 \\
\hline $\mathrm{H} 28$ & -0.66799 & -0.69078 & 5.436761 & $\mathrm{H} 28$ & -2.2776 & -3.66877 & 4.307221 \\
\hline H29 & -1.06391 & 1.741177 & 5.765789 & $\mathrm{H} 29$ & -2.29267 & -1.77798 & 5.92009 \\
\hline $\mathrm{O} 30$ & 0.59354 & -4.36538 & -0.31403 & $\mathrm{O} 30$ & 1.068448 & -4.04604 & -0.26674 \\
\hline C31 & 2.622259 & -2.35892 & 1.16244 & $\mathrm{C} 31$ & 2.173263 & -2.16289 & 2.516515 \\
\hline $\mathrm{C} 32$ & 1.812878 & -1.89252 & 2.195652 & $\mathrm{C} 32$ & 1.238365 & -1.41497 & 3.228407 \\
\hline $\mathrm{C} 33$ & 3.940743 & -2.74288 & 1.453906 & $\mathrm{C} 33$ & 3.20723 & -2.80017 & 3.223892 \\
\hline C34 & 2.312431 & -1.78603 & 3.495098 & $\mathrm{C} 34$ & 1.337898 & -1.29482 & 4.618064 \\
\hline H35 & 0.80116 & -1.58235 & 1.985305 & H35 & 0.430534 & -0.92738 & 2.707074 \\
\hline $\mathrm{C} 36$ & 4.438046 & -2.64467 & 2.750236 & $\mathrm{C} 36$ & 3.303741 & -2.68547 & 4.607849 \\
\hline H37 & 4.586342 & -3.10323 & 0.656334 & H37 & 3.960922 & -3.37007 & 2.684816 \\
\hline C38 & 3.623435 & -2.16002 & 3.777756 & $\mathrm{C} 38$ & 2.367035 & -1.92532 & 5.313566 \\
\hline H39 & 1.670324 & -1.39544 & 4.279544 & H39 & 0.59684 & -0.70666 & 5.149342 \\
\hline $\mathrm{H} 40$ & 5.462377 & -2.94024 & 2.95835 & $\mathrm{H} 40$ & 4.114522 & -3.18221 & 5.133048 \\
\hline H41 & 4.013202 & -2.07532 & 4.788103 & H41 & 2.44303 & -1.82865 & 6.392534 \\
\hline $\mathrm{C} 42$ & -3.58158 & -0.12243 & 0.003396 & $\mathrm{C} 42$ & -3.59498 & -0.03835 & -0.16662 \\
\hline $\mathrm{C} 43$ & -4.60661 & 0.330204 & 0.847351 & $\mathrm{C} 43$ & -4.55131 & 0.333242 & 0.787611 \\
\hline $\mathrm{C} 44$ & -3.87987 & -0.40833 & -1.33952 & $\mathrm{C} 44$ & -3.9035 & 0.107644 & -1.52884 \\
\hline $\mathrm{C} 45$ & -5.89822 & 0.513382 & 0.348975 & $\mathrm{C} 45$ & -5.78061 & 0.861629 & 0.388538 \\
\hline H46 & -4.40612 & 0.53439 & 1.893808 & H46 & -4.34809 & 0.212828 & 1.845716 \\
\hline $\mathrm{C} 47$ & -5.1718 & -0.23345 & -1.83025 & $\mathrm{C} 47$ & -5.13497 & 0.626976 & -1.92358 \\
\hline $\mathrm{C} 48$ & -6.18519 & 0.232727 & -0.98783 & $\mathrm{C} 48$ & -6.07584 & 1.015932 & -0.96532 \\
\hline H49 & -6.68165 & 0.870797 & 1.010891 & $\mathrm{H} 49$ & -6.5099 & 1.144413 & 1.142094 \\
\hline H50 & -5.38639 & -0.45527 & -2.87162 & $\mathrm{H} 50$ & -5.37182 & 0.709254 & -2.98109 \\
\hline H51 & -7.19122 & 0.373969 & -1.37124 & H51 & -7.03563 & 1.419723 & -1.2735 \\
\hline H52 & -0.8981 & -0.54383 & -1.88353 & H52 & 0.247393 & 0.567546 & 1.376663 \\
\hline H53 & 1.409335 & 0.461057 & -1.67983 & H53 & 1.342812 & 2.041705 & -2.28691 \\
\hline H54 & -1.08557 & -1.7332 & 3.239491 & $\mathrm{H} 54$ & -2.14988 & -3.23434 & 1.891416 \\
\hline H55 & -3.09717 & -0.76644 & -2.00083 & H55 & -3.19864 & -0.22024 & -2.28441 \\
\hline H56 & 2.204227 & -4.33156 & -1.60787 & H56 & 3.098247 & -4.15753 & 0.113064 \\
\hline C57 & 4.364205 & -0.28039 & -3.22523 & C57 & 5.450083 & -0.28326 & -0.98842 \\
\hline C58 & 3.541895 & -1.33511 & -3.62218 & C58 & 4.573699 & -1.10012 & -1.70844 \\
\hline C59 & 2.824133 & -2.05997 & -2.67248 & C59 & 3.523591 & -1.74052 & -1.05405 \\
\hline C60 & 2.929988 & -1.76078 & -1.30622 & $\mathrm{C} 60$ & 3.325286 & -1.57287 & 0.325557 \\
\hline C61 & 3.744627 & -0.68763 & -0.92082 & C61 & 4.185331 & -0.72515 & 1.030878 \\
\hline C62 & 4.455947 & 0.044947 & -1.87256 & C62 & 5.248825 & -0.09694 & 0.377753 \\
\hline H63 & 4.916825 & 0.289559 & -3.96607 & H63 & 6.277528 & 0.207292 & -1.49292 \\
\hline H64 & 3.444642 & -1.58297 & -4.67476 & H64 & 4.713525 & -1.24508 & -2.77584 \\
\hline H65 & 2.163517 & -2.85163 & -3.01334 & H65 & 2.854014 & -2.37803 & -1.62674 \\
\hline H66 & 3.819095 & -0.4187 & 0.126063 & H66 & 4.041203 & -0.56239 & 2.091621 \\
\hline H67 & 5.086296 & 0.869622 & -1.55136 & H67 & 5.918106 & 0.542018 & 0.945789 \\
\hline C68 & -1.24031 & 2.787857 & -1.78502 & C68 & -0.8588 & 3.214511 & 0.184472 \\
\hline C69 & -0.16018 & 1.953707 & -1.06278 & C69 & -0.25795 & 2.195762 & -0.81744 \\
\hline $\mathrm{C} 70$ & 1.14421 & 1.571587 & -1.84497 & $\mathrm{C} 70$ & 0.991321 & 2.808932 & -1.5869 \\
\hline C71 & 0.681335 & 4.089993 & 0.21131 & C71 & -1.87967 & 3.005975 & -2.76986 \\
\hline
\end{tabular}




\begin{tabular}{|c|c|c|c|c|c|c|c|}
\hline $\mathrm{C} 72$ & 0.933303 & 1.996982 & 1.109036 & C72 & -0.99679 & 0.882997 & -2.71721 \\
\hline $\mathrm{C} 73$ & 1.157755 & 4.170013 & 1.670497 & C73 & -2.39057 & 2.167577 & -3.95803 \\
\hline $\mathrm{H} 74$ & -0.13753 & 4.77855 & -0.00167 & $\mathrm{H} 74$ & -1.17039 & 3.779501 & -3.08386 \\
\hline H75 & 1.493071 & 4.27488 & -0.50732 & $\mathrm{H} 75$ & -2.69378 & 3.484488 & -2.22395 \\
\hline H76 & 2.027916 & 4.807761 & 1.826996 & H76 & -3.44511 & 1.904876 & -3.85014 \\
\hline $\mathrm{H} 77$ & 0.350068 & 4.465143 & 2.347887 & H77 & -2.21992 & 2.628417 & -4.9315 \\
\hline N78 & 0.255094 & 2.685692 & 0.162502 & N78 & -1.21933 & 1.971741 & -1.95805 \\
\hline O79 & 1.534553 & 2.802234 & 1.994194 & O79 & -1.61939 & 0.93375 & -3.9033 \\
\hline O80 & 1.03332 & 0.760221 & 1.157098 & O80 & -0.3115 & -0.10484 & -2.38448 \\
\hline H81 & 2.598643 & -4.67009 & 0.098754 & H81 & 2.024153 & -4.48548 & 1.505407 \\
\hline N82 & -2.01218 & -2.27816 & 0.841469 & N82 & -1.97523 & -2.22442 & -0.52942 \\
\hline $\mathrm{C} 83$ & -1.55239 & -3.20774 & -0.2213 & $\mathrm{C} 83$ & -0.81266 & -2.83566 & -1.22049 \\
\hline H84 & -1.91945 & -2.91651 & -1.21669 & H84 & -0.52645 & -2.2433 & -2.09527 \\
\hline $\mathrm{C} 85$ & 1.429962 & 2.168658 & -6.10242 & H85 & 0.593505 & 3.619824 & -2.20985 \\
\hline C86 & 1.67575 & 3.221285 & -5.21928 & C86 & 4.451846 & 4.573964 & 0.330182 \\
\hline $\mathrm{C} 87$ & 1.583128 & 3.014114 & -3.84295 & C87 & 3.261144 & 5.295305 & 0.214795 \\
\hline C88 & 1.236783 & 1.756973 & -3.33585 & C88 & 2.150006 & 4.714793 & -0.38973 \\
\hline $\mathrm{C} 89$ & 0.999274 & 0.705823 & -4.22574 & C89 & 2.189726 & 3.397545 & -0.87533 \\
\hline C90 & 1.094105 & 0.909324 & -5.60132 & C90 & 3.399925 & 2.698549 & -0.77949 \\
\hline H91 & 1.5033 & 2.328487 & -7.17422 & C91 & 4.51859 & 3.278304 & -0.1774 \\
\hline H92 & 1.942904 & 4.202407 & -5.60153 & H92 & 5.323446 & 5.027757 & 0.793108 \\
\hline H93 & 1.782758 & 3.835665 & -3.15807 & H93 & 3.203195 & 6.315 & 0.585274 \\
\hline H94 & 0.732758 & -0.26941 & -3.83108 & H94 & 1.234519 & 5.292507 & -0.48808 \\
\hline H95 & 0.90559 & 0.08489 & -6.28344 & H95 & 3.493864 & 1.705805 & -1.21118 \\
\hline H96 & 2.008898 & 2.037484 & -1.36006 & H96 & 5.447337 & 2.718932 & -0.12652 \\
\hline C97 & -2.37539 & 3.202775 & -0.8314 & C97 & -0.06893 & 3.303609 & 1.506533 \\
\hline H98 & -2.0229 & 3.684175 & 0.084488 & H98 & -0.32646 & 2.465495 & 2.160047 \\
\hline H99 & -3.04764 & 3.903648 & -1.33642 & H99 & 1.010091 & 3.286186 & 1.365043 \\
\hline H100 & -2.96752 & 2.33007 & -0.54796 & $\mathrm{H} 100$ & -0.32412 & 4.229422 & 2.033062 \\
\hline $\mathrm{C} 101$ & -1.87503 & 2.125358 & -3.01609 & C101 & -2.33603 & 2.996794 & 0.536178 \\
\hline H102 & -1.16726 & 1.954688 & -3.82284 & H102 & -2.99866 & 2.905767 & -0.32561 \\
\hline H103 & -2.32138 & 1.170245 & -2.73907 & H103 & -2.46209 & 2.087424 & 1.120759 \\
\hline H104 & -2.6719 & 2.769437 & -3.40286 & H104 & -2.6946 & 3.829948 & 1.149624 \\
\hline H105 & -0.74097 & 3.706022 & -2.13749 & H105 & -0.79168 & 4.200818 & -0.30435 \\
\hline \multirow[t]{2}{*}{$\mathrm{X}$} & 0.335144 & 1.892742 & -1.24452 & H106 & 1.739713 & 0.822751 & 0.183774 \\
\hline & & & & H107 & 1.806645 & 0.475789 & -0.59764 \\
\hline \multicolumn{8}{|c|}{ C3_ER_Cheltop_TS1 } \\
\hline $\mathrm{C} 1$ & -4.23697 & -3.40379 & 1.037604 & \multicolumn{4}{|c|}{ C3_ER_Cheltop_TS3 } \\
\hline $\mathrm{C} 2$ & -2.21525 & -4.53811 & 0.282813 & $\mathrm{C} 1$ & -4.17391 & -3.53776 & 1.247801 \\
\hline $\mathrm{C} 3$ & -3.7345 & -4.45052 & -0.01029 & $\mathrm{C} 2$ & -2.1636 & -4.5345 & 0.299761 \\
\hline $\mathrm{C} 4$ & -2.93366 & -3.03925 & 1.788053 & $\mathrm{C} 3$ & -3.70167 & -4.50714 & 0.116194 \\
\hline H5 & -4.95871 & -3.84224 & 1.734542 & $\mathrm{C} 4$ & -2.83619 & -3.12007 & 1.903142 \\
\hline H6 & -3.93999 & -4.14694 & -1.04179 & H5 & -4.79723 & -4.0525 & 1.986577 \\
\hline $\mathrm{H} 7$ & -3.07343 & -2.52002 & 2.736178 & H6 & -3.99192 & -4.16217 & -0.88136 \\
\hline $\mathrm{H} 8$ & -4.7069 & -2.53277 & 0.579937 & $\mathrm{H} 7$ & -2.93018 & -2.64016 & 2.876748 \\
\hline
\end{tabular}




\begin{tabular}{|c|c|c|c|c|c|c|c|}
\hline H9 & -1.71352 & -5.40991 & -0.13599 & $\mathrm{H} 8$ & -4.74112 & -2.68715 & 0.873269 \\
\hline H10 & -4.20183 & -5.42745 & 0.142464 & H9 & -1.65632 & -5.36863 & -0.18411 \\
\hline $\mathrm{C} 11$ & -2.18494 & -4.38295 & 1.82002 & H10 & -4.11283 & -5.51197 & 0.248048 \\
\hline H12 & -1.17844 & -4.30363 & 2.241419 & C11 & -2.02579 & -4.42616 & 1.835053 \\
\hline H13 & -2.73946 & -5.17339 & 2.333472 & H12 & -0.99492 & -4.31405 & 2.184016 \\
\hline $\mathrm{C} 14$ & -0.0814 & -3.18831 & -0.30531 & H13 & -2.504 & -5.25724 & 2.360976 \\
\hline N15 & 0.643368 & -2.13224 & -0.43258 & C14 & -0.12996 & -3.08624 & -0.34417 \\
\hline $\mathrm{C} 16$ & 2.090921 & -2.55746 & -0.38447 & N15 & 0.558306 & -2.00326 & -0.46557 \\
\hline $\mathrm{C} 17$ & 1.950272 & -4.10526 & -0.55063 & $\mathrm{C} 16$ & 2.024689 & -2.37885 & -0.45344 \\
\hline P18 & -1.9657 & -0.60077 & 0.636026 & $\mathrm{C} 17$ & 1.92601 & -3.9284 & -0.65016 \\
\hline Ir19 & -0.0877 & -0.05098 & -0.60624 & P18 & -2.08363 & -0.59104 & 0.763588 \\
\hline $\mathrm{C} 20$ & -1.87383 & 0.132562 & 2.307403 & Ir19 & -0.20852 & 0.007456 & -0.70942 \\
\hline $\mathrm{C} 21$ & -1.42639 & 1.390446 & 4.773565 & $\mathrm{C} 20$ & -1.93811 & 0.058438 & 2.469403 \\
\hline $\mathrm{C} 22$ & -1.25744 & -0.56303 & 3.357272 & $\mathrm{C} 21$ & -1.52665 & 1.140584 & 5.028787 \\
\hline $\mathrm{C} 23$ & -2.24852 & 1.47126 & 2.500243 & $\mathrm{C} 22$ & -1.2344 & -0.66373 & 3.445348 \\
\hline $\mathrm{C} 24$ & -2.02879 & 2.093043 & 3.728476 & $\mathrm{C} 23$ & -2.42027 & 1.338387 & 2.786728 \\
\hline $\mathrm{C} 25$ & -1.04127 & 0.06256 & 4.584289 & $\mathrm{C} 24$ & -2.22747 & 1.866176 & 4.063956 \\
\hline H26 & -2.71134 & 2.029504 & 1.695049 & $\mathrm{C} 25$ & -1.02797 & -0.12369 & 4.713677 \\
\hline $\mathrm{H} 27$ & -2.33575 & 3.125749 & 3.871008 & $\mathrm{H} 26$ & -2.95392 & 1.92142 & 2.043852 \\
\hline $\mathrm{H} 28$ & -0.57086 & -0.48897 & 5.393277 & $\mathrm{H} 27$ & -2.63462 & 2.843955 & 4.308701 \\
\hline H29 & -1.25641 & 1.875971 & 5.73006 & $\mathrm{H} 28$ & -0.48389 & -0.69688 & 5.458863 \\
\hline $\mathrm{O} 30$ & 0.546699 & -4.3669 & -0.27301 & $\mathrm{H} 29$ & -1.37429 & 1.556237 & 6.020518 \\
\hline $\mathrm{C} 31$ & 2.680056 & -2.28384 & 1.00556 & $\mathrm{O} 30$ & 0.535733 & -4.23937 & -0.35949 \\
\hline $\mathrm{C} 32$ & 1.884968 & -1.89087 & 2.080368 & $\mathrm{C} 31$ & 2.623819 & -2.12287 & 0.936338 \\
\hline $\mathrm{C} 33$ & 4.045358 & -2.53309 & 1.224749 & $\mathrm{C} 32$ & 1.853023 & -1.683 & 2.01034 \\
\hline $\mathrm{C} 34$ & 2.441624 & -1.71512 & 3.348114 & $\mathrm{C} 33$ & 3.970043 & -2.45173 & 1.167851 \\
\hline H35 & 0.835585 & -1.68915 & 1.925743 & C34 & 2.415396 & -1.52924 & 3.278408 \\
\hline C36 & 4.59977 & -2.36339 & 2.490416 & H35 & 0.814834 & -1.43705 & 1.859354 \\
\hline H37 & 4.676775 & -2.84811 & 0.398189 & $\mathrm{C} 36$ & 4.532551 & -2.30494 & 2.433098 \\
\hline C38 & 3.798395 & -1.9465 & 3.557258 & H37 & 4.586802 & -2.81657 & 0.350896 \\
\hline H39 & 1.808562 & -1.38029 & 4.165074 & C38 & 3.756133 & -1.83469 & 3.49549 \\
\hline $\mathrm{H} 40$ & 5.658517 & -2.55141 & 2.643984 & H39 & 1.796188 & -1.15936 & 4.090675 \\
\hline H41 & 4.233734 & -1.80462 & 4.542261 & $\mathrm{H} 40$ & 5.57764 & -2.55715 & 2.588886 \\
\hline $\mathrm{C} 42$ & -3.61124 & -0.15826 & -0.03348 & $\mathrm{H} 41$ & 4.195968 & -1.71371 & 4.481261 \\
\hline $\mathrm{C} 43$ & -4.69075 & 0.199727 & 0.789577 & $\mathrm{C} 42$ & -3.81763 & -0.28998 & 0.234054 \\
\hline $\mathrm{C} 44$ & -3.83181 & -0.34322 & -1.40733 & $\mathrm{C} 43$ & -4.86586 & -0.00383 & 1.121163 \\
\hline $\mathrm{C} 45$ & -5.95668 & 0.400633 & 0.239718 & C44 & -4.11651 & -0.55465 & -1.11309 \\
\hline H46 & -4.54959 & 0.319936 & 1.858643 & $\mathrm{C} 45$ & -6.18303 & 0.038101 & 0.661658 \\
\hline $\mathrm{C} 47$ & -5.10061 & -0.15226 & -1.95152 & $\mathrm{H} 46$ & -4.66377 & 0.176963 & 2.171543 \\
\hline $\mathrm{C} 48$ & -6.16365 & 0.228655 & -1.13025 & $\mathrm{C} 47$ & -5.4346 & -0.52498 & -1.56506 \\
\hline H49 & -6.7827 & 0.688323 & 0.883795 & $\mathrm{C} 48$ & -6.47248 & -0.22529 & -0.67875 \\
\hline H50 & -5.25682 & -0.29101 & -3.01734 & $\mathrm{H} 49$ & -6.98544 & 0.266988 & 1.357191 \\
\hline H51 & -7.15035 & 0.387963 & -1.55524 & $\mathrm{H} 50$ & -5.65058 & -0.72894 & -2.6098 \\
\hline H52 & -0.80705 & -0.47018 & -1.92904 & H51 & -7.49947 & -0.19702 & -1.0302 \\
\hline H53 & 1.251525 & 0.226844 & -1.45559 & H52 & -1.02025 & -0.54962 & -1.92828 \\
\hline
\end{tabular}




\begin{tabular}{|c|c|c|c|c|c|c|c|}
\hline H54 & -0.94196 & -1.591 & 3.215367 & H53 & 1.72159 & 2.527477 & -1.16878 \\
\hline H55 & -3.00516 & -0.62808 & -2.05025 & H54 & -0.8435 & -1.64797 & 3.21409 \\
\hline H56 & 2.159778 & -4.47268 & -1.55661 & H55 & -3.31237 & -0.77063 & -1.81049 \\
\hline $\mathrm{C} 57$ & 4.265378 & -0.62228 & -3.61509 & $\mathrm{H} 56$ & 2.134075 & -4.25923 & -1.66958 \\
\hline C58 & 3.4549 & -1.72636 & -3.87634 & C57 & 4.141677 & -0.59936 & -3.80512 \\
\hline C59 & 2.752238 & -2.34205 & -2.84157 & C58 & 3.091771 & -1.49604 & -4.01554 \\
\hline C60 & 2.868658 & -1.88696 & -1.52082 & C59 & 2.413107 & -2.04204 & -2.92887 \\
\hline C61 & 3.663171 & -0.75735 & -1.27609 & C60 & 2.772866 & -1.71436 & -1.6126 \\
\hline C62 & 4.35317 & -0.13035 & -2.31377 & C61 & 3.78943 & -0.77264 & -1.41492 \\
\hline H63 & 4.805531 & -0.13706 & -4.42229 & C62 & 4.474126 & -0.22692 & -2.50331 \\
\hline H64 & 3.356692 & -2.10313 & -4.89021 & H63 & 4.681405 & -0.17975 & -4.64905 \\
\hline H65 & 2.103388 & -3.1785 & -3.08312 & H64 & 2.801152 & -1.77137 & -5.02519 \\
\hline H66 & 3.744371 & -0.36799 & -0.26724 & $\mathrm{H} 65$ & 1.584362 & -2.72014 & -3.1136 \\
\hline H67 & 4.965157 & 0.741514 & -2.10039 & H66 & 4.057208 & -0.46601 & -0.41082 \\
\hline C68 & -1.21423 & 2.858865 & -1.70985 & H67 & 5.26941 & 0.491987 & -2.32837 \\
\hline C69 & -0.14824 & 2.030781 & -0.9646 & C68 & -1.74472 & 2.884943 & -1.57412 \\
\hline $\mathrm{C} 70$ & 1.110848 & 1.5549 & -1.65304 & C69 & -0.4359 & 2.338554 & -0.955 \\
\hline C71 & 0.58543 & 4.109454 & 0.445775 & $\mathrm{C} 70$ & 0.844195 & 2.987513 & -1.63784 \\
\hline $\mathrm{C} 72$ & 0.819649 & 1.980157 & 1.269154 & C71 & -0.27929 & 4.129116 & 0.97043 \\
\hline $\mathrm{C} 73$ & 0.942734 & 4.131703 & 1.940644 & $\mathrm{C} 72$ & 0.509064 & 1.992303 & 1.285203 \\
\hline H74 & -0.23169 & 4.789703 & 0.200734 & $\mathrm{C} 73$ & 0.183688 & 3.918564 & 2.421509 \\
\hline H75 & 1.445765 & 4.342036 & -0.19867 & $\mathrm{H} 74$ & -1.2642 & 4.59254 & 0.909279 \\
\hline H76 & 1.78012 & 4.781681 & 2.19478 & $\mathrm{H} 75$ & 0.429755 & 4.743695 & 0.406546 \\
\hline H77 & 0.075272 & 4.374053 & 2.562758 & H76 & 0.887585 & 4.673435 & 2.773308 \\
\hline N78 & 0.196579 & 2.700948 & 0.305887 & $\mathrm{H} 77$ & -0.65339 & 3.831909 & 3.117806 \\
\hline O79 & 1.329242 & 2.759828 & 2.232073 & N78 & -0.29775 & 2.733756 & 0.483452 \\
\hline $\mathrm{O} 80$ & 0.947665 & 0.747029 & 1.2716 & O79 & 0.867881 & 2.639222 & 2.398083 \\
\hline H81 & 2.544921 & -4.65831 & 0.174872 & O80 & 0.894917 & 0.838256 & 1.051822 \\
\hline N82 & -2.00716 & -2.27384 & 0.904676 & H81 & 2.546666 & -4.48105 & 0.053274 \\
\hline $\mathrm{C} 83$ & -1.58403 & -3.19817 & -0.17796 & N82 & -2.01352 & -2.27862 & 0.981213 \\
\hline H84 & -1.98428 & -2.90141 & -1.1587 & $\mathrm{C} 83$ & -1.62168 & -3.15344 & -0.15296 \\
\hline $\mathrm{C} 85$ & 1.726606 & 2.093438 & -5.87365 & H84 & -2.08381 & -2.84674 & -1.10204 \\
\hline $\mathrm{C} 86$ & 1.975347 & 3.128744 & -4.96989 & $\mathrm{C} 85$ & 1.207018 & 2.861139 & -5.95217 \\
\hline $\mathrm{C} 87$ & 1.782523 & 2.922795 & -3.60511 & C86 & 0.571408 & 3.93651 & -5.3266 \\
\hline $\mathrm{C} 88$ & 1.334152 & 1.682992 & -3.13131 & C87 & 0.469592 & 3.978625 & -3.93687 \\
\hline $\mathrm{C} 89$ & 1.094321 & 0.649345 & -4.03786 & C88 & 0.990167 & 2.945631 & -3.14357 \\
\hline $\mathrm{C} 90$ & 1.290546 & 0.85443 & -5.40304 & C89 & 1.654616 & 1.889966 & -3.78082 \\
\hline H91 & 1.87403 & 2.252987 & -6.93777 & C90 & 1.757587 & 1.84405 & -5.17149 \\
\hline H92 & 2.317142 & 4.095709 & -5.32771 & H91 & 1.286373 & 2.827794 & -7.03479 \\
\hline H93 & 1.975725 & 3.730972 & -2.90259 & H92 & 0.158827 & 4.746536 & -5.9215 \\
\hline H94 & 0.757907 & -0.31303 & -3.66846 & H93 & -0.02145 & 4.824981 & -3.46217 \\
\hline H95 & 1.101824 & 0.043332 & -6.10058 & H94 & 2.117976 & 1.108381 & -3.19184 \\
\hline H96 & 2.01225 & 1.837107 & -1.10037 & H95 & 2.280251 & 1.014164 & -5.6382 \\
\hline C97 & -2.41007 & 3.197392 & -0.8025 & H96 & 0.845648 & 4.043328 & -1.34881 \\
\hline H98 & -2.11585 & 3.624467 & 0.159458 & C97 & -2.951 & 2.928912 & -0.6296 \\
\hline
\end{tabular}




\begin{tabular}{|c|c|c|c|c|c|c|c|}
\hline H99 & -3.06351 & 3.91886 & -1.3027 & H98 & -2.79653 & 3.555426 & 0.251551 \\
\hline H100 & -3.00106 & 2.299935 & -0.60673 & H99 & -3.81787 & 3.329763 & -1.16489 \\
\hline C101 & -1.75216 & 2.244487 & -3.0099 & H100 & -3.21708 & 1.929716 & -0.2924 \\
\hline H102 & -0.99658 & 2.161507 & -3.78665 & C101 & -2.14769 & 2.146223 & -2.86327 \\
\hline H103 & -2.15892 & 1.250364 & -2.8201 & H102 & -1.30106 & 1.94138 & -3.51701 \\
\hline H104 & -2.5623 & 2.872087 & -3.39539 & H103 & -2.61038 & 1.190749 & -2.60853 \\
\hline H105 & -0.72062 & 3.806319 & -1.98683 & H104 & -2.87584 & 2.741054 & -3.42328 \\
\hline \multirow[t]{2}{*}{$\mathrm{X}$} & 0.335144 & 1.892742 & -1.24452 & H105 & -1.51825 & 3.926135 & -1.85453 \\
\hline & & & & H106 & 1.104133 & 0.10416 & -1.60944 \\
\hline \multicolumn{4}{|c|}{ C3_ER_Cheltop_int1 } & $\mathrm{H} 107$ & 0.114904 & 1.046027 & -1.83155 \\
\hline $\mathrm{C} 1$ & -4.24811 & -3.40733 & 0.803086 & & & & \\
\hline $\mathrm{C} 2$ & -2.18985 & -4.55236 & 0.175567 & \multicolumn{4}{|c|}{ C3_ER_Cheltop_PROD } \\
\hline $\mathrm{C} 3$ & -3.68894 & -4.4622 & -0.208 & $\mathrm{C} 1$ & -4.26675 & -3.67258 & 0.615979 \\
\hline $\mathrm{C} 4$ & -2.99282 & -3.05031 & 1.633905 & $\mathrm{C} 2$ & -2.1218 & -4.62818 & -0.05525 \\
\hline H5 & -5.01504 & -3.83888 & 1.454416 & $\mathrm{C} 3$ & -3.61261 & -4.57791 & -0.48109 \\
\hline H6 & -3.83165 & -4.16453 & -1.25186 & $\mathrm{C} 4$ & -3.05703 & -3.32986 & 1.516942 \\
\hline H7 & -3.19331 & -2.5321 & 2.571516 & H5 & -5.0251 & -4.21571 & 1.18934 \\
\hline H8 & -4.68157 & -2.53295 & 0.316267 & H6 & -3.74165 & -4.17818 & -1.49199 \\
\hline H9 & -1.6633 & -5.42391 & -0.21309 & $\mathrm{H} 7$ & -3.30099 & -2.91577 & 2.496103 \\
\hline H10 & -4.16831 & -5.43652 & -0.07767 & H8 & -4.73625 & -2.77643 & 0.207913 \\
\hline $\mathrm{C} 11$ & -2.25264 & -4.39728 & 1.711644 & H9 & -1.53944 & -5.42995 & -0.50896 \\
\hline H12 & -1.27282 & -4.32177 & 2.192787 & H10 & -4.03503 & -5.58662 & -0.47236 \\
\hline H13 & -2.83988 & -5.18627 & 2.189713 & $\mathrm{C} 11$ & -2.24177 & -4.63387 & 1.484689 \\
\hline $\mathrm{C} 14$ & -0.0273 & -3.2004 & -0.30039 & H12 & -1.28254 & -4.55527 & 2.005016 \\
\hline N15 & 0.679364 & -2.12709 & -0.33716 & H13 & -2.79388 & -5.49923 & 1.862213 \\
\hline C16 & 2.124962 & -2.5263 & -0.21621 & $\mathrm{C} 14$ & -0.02563 & -3.12019 & -0.26549 \\
\hline $\mathrm{C} 17$ & 2.026636 & -4.04805 & -0.54646 & N15 & 0.652759 & -2.01852 & -0.29784 \\
\hline P18 & -1.90389 & -0.60785 & 0.624448 & $\mathrm{C} 16$ & 2.133951 & -2.34172 & -0.23135 \\
\hline Ir19 & -0.11649 & -0.09114 & -0.62362 & $\mathrm{C} 17$ & 2.081967 & -3.90841 & -0.16193 \\
\hline $\mathrm{C} 20$ & -1.72672 & 0.063225 & 2.315852 & P18 & -2.09326 & -0.73994 & 0.729234 \\
\hline $\mathrm{C} 21$ & -1.21879 & 1.218265 & 4.822343 & Ir19 & -0.13178 & -0.14897 & -0.56584 \\
\hline $\mathrm{C} 22$ & -1.29919 & -0.73137 & 3.387349 & $\mathrm{C} 20$ & -1.94911 & -0.22305 & 2.478863 \\
\hline $\mathrm{C} 23$ & -1.89023 & 1.444918 & 2.508807 & $\mathrm{C} 21$ & -1.38944 & 0.705265 & 5.073425 \\
\hline $\mathrm{C} 24$ & -1.63879 & 2.015544 & 3.75474 & $\mathrm{C} 22$ & -1.35148 & -1.06792 & 3.424222 \\
\hline $\mathrm{C} 25$ & -1.05324 & -0.15435 & 4.634624 & $\mathrm{C} 23$ & -2.26196 & 1.096297 & 2.84868 \\
\hline H26 & -2.20453 & 2.074789 & 1.683938 & $\mathrm{C} 24$ & -1.98592 & 1.552634 & 4.136944 \\
\hline $\mathrm{H} 27$ & -1.77441 & 3.084683 & 3.893851 & $\mathrm{C} 25$ & -1.07609 & -0.60577 & 4.712407 \\
\hline $\mathrm{H} 28$ & -0.72933 & -0.78174 & 5.46021 & $\mathrm{H} 26$ & -2.72181 & 1.768936 & 2.129723 \\
\hline H29 & -1.02328 & 1.664189 & 5.792851 & $\mathrm{H} 27$ & -2.24339 & 2.571478 & 4.4144 \\
\hline $\mathrm{O} 30$ & 0.623376 & -4.367 & -0.31047 & $\mathrm{H} 28$ & -0.61547 & -1.27446 & 5.434251 \\
\hline C31 & 2.605647 & -2.37186 & 1.23196 & $\mathrm{H} 29$ & -1.1736 & 1.063146 & 6.075562 \\
\hline C32 & 1.788996 & -1.8707 & 2.242788 & $\mathrm{O} 30$ & 0.667528 & -4.25132 & -0.18437 \\
\hline $\mathrm{C} 33$ & 3.906854 & -2.78757 & 1.554141 & $\mathrm{C} 31$ & 2.76164 & -1.83765 & 1.06586 \\
\hline C34 & 2.263792 & -1.76778 & 3.551829 & $\mathrm{C} 32$ & 1.992218 & -1.36108 & 2.127628 \\
\hline H35 & 0.792442 & -1.53115 & 2.007884 & C33 & 4.145165 & -1.99225 & 1.2508 \\
\hline
\end{tabular}




\begin{tabular}{|c|c|c|c|c|c|c|c|}
\hline C36 & 4.379958 & -2.69146 & 2.859351 & C34 & 2.592126 & -1.00091 & 3.3353 \\
\hline H37 & 4.560612 & -3.16998 & 0.774192 & H35 & 0.923606 & -1.24532 & 2.019398 \\
\hline $\mathrm{C} 38$ & 3.557648 & -2.17623 & 3.865234 & $\mathrm{C} 36$ & 4.742825 & -1.64162 & 2.458466 \\
\hline H39 & 1.616536 & -1.35218 & 4.318873 & H37 & 4.758846 & -2.38191 & 0.443134 \\
\hline $\mathrm{H} 40$ & 5.391548 & -3.01213 & 3.090809 & $\mathrm{C} 38$ & 3.967723 & -1.13605 & 3.504888 \\
\hline H41 & 3.928305 & -2.09351 & 4.882891 & H39 & 1.971375 & -0.6093 & 4.136293 \\
\hline $\mathrm{C} 42$ & -3.56426 & -0.10201 & 0.03967 & $\mathrm{H} 40$ & 5.815419 & -1.76112 & 2.580891 \\
\hline $\mathrm{C} 43$ & -4.55538 & 0.380686 & 0.905932 & H41 & 4.435676 & -0.8554 & 4.443988 \\
\hline $\mathrm{C} 44$ & -3.89771 & -0.38602 & -1.29464 & $\mathrm{C} 42$ & -3.77867 & -0.23573 & 0.204778 \\
\hline $\mathrm{C} 45$ & -5.85131 & 0.598784 & 0.435092 & $\mathrm{C} 43$ & -4.83242 & 0.015874 & 1.095695 \\
\hline H46 & -4.32536 & 0.583035 & 1.946516 & C44 & -4.03445 & -0.23328 & -1.17659 \\
\hline $\mathrm{C} 47$ & -5.19309 & -0.1737 & -1.75821 & $\mathrm{C} 45$ & -6.11396 & 0.278878 & 0.61055 \\
\hline $\mathrm{C} 48$ & -6.17205 & 0.324665 & -0.89443 & $\mathrm{H} 46$ & -4.65618 & 0.004359 & 2.166855 \\
\hline H49 & -6.61094 & 0.97954 & 1.111632 & C47 & -5.31793 & 0.021617 & -1.65779 \\
\hline H50 & -5.43809 & -0.39142 & -2.79371 & $\mathrm{C} 48$ & -6.36071 & 0.281649 & -0.76438 \\
\hline H51 & -7.1814 & 0.495517 & -1.25717 & H49 & -6.92261 & 0.474954 & 1.308859 \\
\hline H52 & -0.90346 & -0.58102 & -1.88882 & $\mathrm{H} 50$ & -5.50311 & 0.022905 & -2.72808 \\
\hline H53 & 1.393004 & 0.43891 & -1.77407 & H51 & -7.36032 & 0.483987 & -1.13711 \\
\hline H54 & -1.13969 & -1.79503 & 3.247921 & $\mathrm{H} 52$ & -0.70661 & -0.74134 & -1.88963 \\
\hline H55 & -3.14004 & -0.77127 & -1.96974 & H53 & 1.664342 & 2.510185 & -1.33961 \\
\hline H56 & 2.248036 & -4.29336 & -1.58771 & H54 & -1.08698 & -2.08362 & 3.147017 \\
\hline $\mathrm{C} 57$ & 4.348209 & -0.15448 & -3.09485 & $\mathrm{H} 55$ & -3.22166 & -0.4323 & -1.87047 \\
\hline C58 & 3.518542 & -1.19073 & -3.52488 & H56 & 2.559862 & -4.40029 & -1.01002 \\
\hline C59 & 2.821912 & -1.96063 & -2.59579 & C57 & 3.806147 & -0.8761 & -3.96026 \\
\hline $\mathrm{C} 60$ & 2.953724 & -1.723 & -1.2193 & C58 & 2.943297 & -1.97246 & -3.94563 \\
\hline C61 & 3.776654 & -0.67029 & -0.79885 & C59 & 2.442104 & -2.44879 & -2.73539 \\
\hline C62 & 4.469797 & 0.105197 & -1.73047 & C60 & 2.793261 & -1.8431 & -1.52201 \\
\hline H63 & 4.883622 & 0.451519 & -3.81952 & C61 & 3.643716 & -0.73188 & -1.54973 \\
\hline H64 & 3.397339 & -1.38608 & -4.58587 & C62 & 4.151593 & -0.25747 & -2.75944 \\
\hline H65 & 2.151145 & -2.73462 & -2.9574 & H63 & 4.197514 & -0.50036 & -4.90097 \\
\hline H66 & 3.868504 & -0.44745 & 0.257741 & H64 & 2.657998 & -2.4563 & -4.87534 \\
\hline H67 & 5.107519 & 0.913973 & -1.38428 & H65 & 1.747777 & -3.28578 & -2.74901 \\
\hline C68 & -1.25734 & 2.752592 & -1.83533 & H66 & 3.898903 & -0.22157 & -0.62877 \\
\hline C69 & -0.15888 & 1.934141 & -1.12312 & H67 & 4.810748 & 0.605876 & -2.76218 \\
\hline $\mathrm{C} 70$ & 1.135685 & 1.550476 & -1.92553 & C68 & -1.77011 & 3.35101 & -1.52363 \\
\hline C71 & 0.701422 & 4.091289 & 0.102896 & C69 & -0.43466 & 2.744915 & -1.01147 \\
\hline $\mathrm{C} 72$ & 0.967784 & 2.013326 & 1.030969 & C70 & 0.859572 & 3.090628 & -1.80201 \\
\hline $\mathrm{C} 73$ & 1.201786 & 4.195465 & 1.55248 & C71 & -0.07164 & 4.481822 & 0.846769 \\
\hline H74 & -0.12109 & 4.7761 & -0.10829 & $\mathrm{C} 72$ & 0.429445 & 2.271054 & 1.29545 \\
\hline H75 & 1.501354 & 4.264344 & -0.6316 & $\mathrm{C} 73$ & 0.17306 & 4.264663 & 2.344684 \\
\hline H76 & 2.074722 & 4.835085 & 1.683988 & $\mathrm{H} 74$ & -0.97448 & 5.060878 & 0.646022 \\
\hline H77 & 0.405683 & 4.503034 & 2.238068 & $\mathrm{H} 75$ & 0.778801 & 4.973861 & 0.360319 \\
\hline N78 & 0.274806 & 2.686389 & 0.084182 & H76 & 0.873541 & 4.972524 & 2.788078 \\
\hline O79 & 1.583139 & 2.832843 & 1.893129 & $\mathrm{H} 77$ & -0.75983 & 4.251474 & 2.91802 \\
\hline $\mathrm{O} 80$ & 1.06656 & 0.777357 & 1.098932 & N78 & -0.20625 & 3.086009 & 0.40329 \\
\hline
\end{tabular}




\begin{tabular}{|c|c|c|c|c|c|c|c|}
\hline H81 & 2.626367 & -4.66822 & 0.116959 & O79 & 0.756296 & 2.941839 & 2.412915 \\
\hline N82 & -2.0094 & -2.2882 & 0.80949 & O80 & 0.683466 & 1.072865 & 1.180199 \\
\hline $\mathrm{C} 83$ & -1.53143 & -3.21418 & -0.24843 & H81 & 2.496237 & -4.28957 & 0.77115 \\
\hline H84 & -1.87774 & -2.91657 & -1.24924 & N82 & -2.11476 & -2.43393 & 0.794299 \\
\hline $\mathrm{C} 85$ & 1.348045 & 2.218715 & -6.1783 & $\mathrm{C} 83$ & -1.52831 & -3.22106 & -0.31676 \\
\hline C86 & 1.604241 & 3.257122 & -5.28135 & H84 & -1.83197 & -2.8464 & -1.30422 \\
\hline $\mathrm{C} 87$ & 1.536953 & 3.025198 & -3.90749 & $\mathrm{C} 85$ & 0.886794 & 2.58168 & -6.09768 \\
\hline C88 & 1.206226 & 1.757836 & -3.41625 & $\mathrm{C} 86$ & 0.67256 & 3.84214 & -5.53559 \\
\hline C89 & 0.956684 & 0.721235 & -4.31988 & $\mathrm{C} 87$ & 0.675388 & 3.998196 & -4.14913 \\
\hline C90 & 1.026147 & 0.949045 & -5.6934 & C88 & 0.880588 & 2.900135 & -3.30224 \\
\hline H91 & 1.400754 & 2.397733 & -7.24837 & C89 & 1.118418 & 1.644244 & -3.87493 \\
\hline H92 & 1.858882 & 4.246408 & -5.65104 & $\mathrm{C} 90$ & 1.118048 & 1.48763 & -5.26127 \\
\hline H93 & 1.743873 & 3.836097 & -3.21228 & H91 & 0.885641 & 2.457743 & -7.17661 \\
\hline H94 & 0.697361 & -0.26078 & -3.93705 & H92 & 0.506847 & 4.704498 & -6.17526 \\
\hline H95 & 0.826798 & 0.136676 & -6.38686 & H93 & 0.507897 & 4.984302 & -3.71995 \\
\hline H96 & 2.007019 & 2.011555 & -1.4491 & H94 & 1.300421 & 0.786941 & -3.23694 \\
\hline C97 & -2.37398 & 3.176806 & -0.86352 & H95 & 1.307383 & 0.504988 & -5.6846 \\
\hline H98 & -2.00478 & 3.684591 & 0.031543 & H96 & 1.064601 & 4.147302 & -1.59497 \\
\hline H99 & -3.06606 & 3.858927 & -1.36764 & C97 & -2.88915 & 3.263644 & -0.47365 \\
\hline H100 & -2.94945 & 2.305824 & -0.54335 & H98 & -2.65602 & 3.77273 & 0.464138 \\
\hline C101 & -1.91004 & 2.065256 & -3.04328 & H99 & -3.80506 & 3.707949 & -0.87328 \\
\hline H102 & -1.2186 & 1.905675 & -3.86645 & H100 & -3.11079 & 2.219078 & -0.2425 \\
\hline H103 & -2.32568 & 1.101075 & -2.75047 & C101 & -2.25545 & 2.666225 & -2.81468 \\
\hline H104 & -2.73147 & 2.687567 & -3.41416 & $\mathrm{H} 102$ & -1.56994 & 2.79278 & -3.64878 \\
\hline H105 & -0.77086 & 3.668125 & -2.21289 & $\mathrm{H} 103$ & -2.38958 & 1.593542 & -2.64225 \\
\hline \multirow[t]{2}{*}{$\mathrm{X}$} & 0.335144 & 1.892742 & -1.24452 & $\mathrm{H} 104$ & -3.22617 & 3.080232 & -3.10374 \\
\hline & & & & H105 & -1.5626 & 4.408594 & -1.74717 \\
\hline \multicolumn{4}{|c|}{ C3_ER_Cheltop_TS2 } & H106 & 1.192192 & 0.157971 & -1.38755 \\
\hline $\mathrm{C} 1$ & -4.17685 & -3.43818 & 1.005979 & H107 & -0.63743 & 1.627002 & -1.08584 \\
\hline $\mathrm{C} 2$ & -2.15991 & -4.48112 & 0.118916 & & & & \\
\hline C3 & -3.6871 & -4.39428 & -0.12836 & & & & \\
\hline $\mathrm{C} 4$ & -2.8513 & -3.05527 & 1.705867 & & & & \\
\hline H5 & -4.82221 & -3.95886 & 1.721568 & & & & \\
\hline H6 & -3.92205 & -4.01206 & -1.12693 & & & & \\
\hline $\mathrm{H} 7$ & -2.96074 & -2.57438 & 2.677412 & & & & \\
\hline $\mathrm{H} 8$ & -4.72994 & -2.57901 & 0.631905 & & & & \\
\hline H9 & -1.66751 & -5.33303 & -0.34795 & & & & \\
\hline H10 & -4.13797 & -5.38696 & -0.04308 & & & & \\
\hline $\mathrm{C} 11$ & -2.08036 & -4.38612 & 1.659261 & & & & \\
\hline H12 & -1.06114 & -4.31292 & 2.049305 & & & & \\
\hline H13 & -2.60798 & -5.2015 & 2.161882 & & & & \\
\hline $\mathrm{C} 14$ & -0.03018 & -3.112 & -0.34897 & & & & \\
\hline N15 & 0.715403 & -2.06246 & -0.38933 & & & & \\
\hline $\mathrm{C} 16$ & 2.157765 & -2.55361 & -0.25169 & & & & \\
\hline $\mathrm{C} 17$ & 1.976885 & -4.10576 & -0.38143 & & & & \\
\hline
\end{tabular}




\begin{tabular}{|c|c|c|c|}
\hline P18 & -1.99026 & -0.55544 & 0.60646 \\
\hline Ir19 & -0.24064 & 0.065871 & -0.61511 \\
\hline $\mathrm{C} 20$ & -1.84075 & 0.08007 & 2.310682 \\
\hline $\mathrm{C} 21$ & -1.35336 & 1.111739 & 4.873133 \\
\hline $\mathrm{C} 22$ & -1.14716 & -0.68124 & 3.265106 \\
\hline $\mathrm{C} 23$ & -2.27699 & 1.370396 & 2.650242 \\
\hline $\mathrm{C} 24$ & -2.04371 & 1.874605 & 3.929493 \\
\hline $\mathrm{C} 25$ & -0.90585 & -0.16673 & 4.537233 \\
\hline $\mathrm{H} 26$ & -2.79629 & 1.981349 & 1.922895 \\
\hline $\mathrm{H} 27$ & -2.40764 & 2.864528 & 4.19122 \\
\hline $\mathrm{H} 28$ & -0.37013 & -0.76799 & 5.2659 \\
\hline H29 & -1.16665 & 1.511102 & 5.865641 \\
\hline $\mathrm{O} 30$ & 0.550552 & -4.31232 & -0.28136 \\
\hline C31 & 2.722904 & -2.25972 & 1.140182 \\
\hline $\mathrm{C} 32$ & 2.009082 & -1.5838 & 2.124021 \\
\hline C33 & 3.995174 & -2.76635 & 1.456795 \\
\hline $\mathrm{C} 34$ & 2.555674 & -1.39353 & 3.395513 \\
\hline H35 & 1.037492 & -1.18172 & 1.895534 \\
\hline C36 & 4.538567 & -2.58591 & 2.724671 \\
\hline H37 & 4.570688 & -3.29353 & 0.699942 \\
\hline $\mathrm{C} 38$ & 3.817545 & -1.89464 & 3.702247 \\
\hline H39 & 1.986355 & -0.84284 & 4.138932 \\
\hline $\mathrm{H} 40$ & 5.52529 & -2.98114 & 2.948464 \\
\hline H41 & 4.243022 & -1.74731 & 4.690678 \\
\hline $\mathrm{C} 42$ & -3.70514 & -0.26474 & 0.03704 \\
\hline $\mathrm{C} 43$ & -4.74078 & 0.055213 & \\
\hline $\mathrm{C} 44$ & -4.02662 & -0.63668 & -1.27832 \\
\hline $\mathrm{C} 45$ & -6.06848 & 0.031734 & 0.500598 \\
\hline $\mathrm{H} 46$ & -4.52296 & 0.307521 & 1.960444 \\
\hline $\mathrm{C} 47$ & -5.35519 & -0.67096 & -1.69708 \\
\hline $\mathrm{C} 48$ & -6.3809 & -0.33388 & -0.80978 \\
\hline H49 & -6.8599 & 0.287311 & 1.199168 \\
\hline H50 & -5.58921 & -0.9589 & -2.71772 \\
\hline H51 & -7.41589 & -0.35881 & -1.13691 \\
\hline H52 & -1.0512 & -0.40472 & -1.87381 \\
\hline H53 & 1.604243 & 2.033348 & -1.52451 \\
\hline H54 & -0.78821 & -1.67173 & 3.009966 \\
\hline H55 & -3.23699 & -0.89639 & -1.97592 \\
\hline H56 & 2.309284 & -4.51035 & -1.33881 \\
\hline $\mathrm{C} 57$ & 4.48604 & -1.02211 & -3.57563 \\
\hline C58 & 3.458785 & -1.94488 & -3.78727 \\
\hline C59 & 2.731169 & -2.43399 & -2.70657 \\
\hline $\mathrm{C} 60$ & 3.013447 & -2.01625 & -1.39696 \\
\hline C61 & 4.037932 & -1.08542 & -1.19331 \\
\hline C62 & 4.769951 & -0.59389 & -2.27905 \\
\hline
\end{tabular}




\begin{tabular}{|c|c|c|c|}
\hline H63 & 5.059169 & -0.6403 & -4.41547 \\
\hline H64 & 3.221603 & -2.27858 & -4.79336 \\
\hline H65 & 1.912368 & -3.12271 & -2.89632 \\
\hline H66 & 4.261384 & -0.73194 & -0.19328 \\
\hline H67 & 5.563427 & 0.127526 & -2.10657 \\
\hline C68 & -1.82594 & 2.795402 & -1.51803 \\
\hline C69 & -0.53954 & 2.174965 & -0.91833 \\
\hline $\mathrm{C} 70$ & 0.751081 & 2.677219 & -1.75756 \\
\hline C71 & -0.26387 & 4.184056 & 0.813806 \\
\hline $\mathrm{C} 72$ & 0.603967 & 2.104505 & 1.225311 \\
\hline $\mathrm{C} 73$ & 0.337841 & 4.105342 & 2.22836 \\
\hline H74 & -1.26054 & 4.62477 & 0.810426 \\
\hline $\mathrm{H} 75$ & 0.369017 & 4.759736 & 0.126776 \\
\hline H76 & 1.049192 & 4.90012 & 2.454355 \\
\hline H77 & -0.43221 & 4.058668 & 3.003167 \\
\hline N78 & -0.28636 & 2.757303 & 0.449865 \\
\hline O79 & 1.053171 & 2.840366 & 2.245915 \\
\hline $\mathrm{O} 80$ & 0.99 & 0.938144 & 1.03372 \\
\hline H81 & 2.451591 & -4.6524 & 0.432234 \\
\hline N82 & -1.97116 & -2.23799 & 0.808409 \\
\hline $\mathrm{C} 83$ & -1.53666 & -3.12285 & -0.29666 \\
\hline H84 & & & -1.27613 \\
\hline $\mathrm{C} 85$ & 0.471733 & 3.547247 & -6.00807 \\
\hline C86 & -0.00924 & 4.447917 & -5.05685 \\
\hline $\mathrm{C} 87$ & 0.108751 & 4.159079 & -3.69936 \\
\hline $\mathrm{C} 88$ & 0.682333 & 2.95683 & -3.25118 \\
\hline $\mathrm{C} 89$ & 1.217507 & 2.095345 & -4.2194 \\
\hline C90 & 1.107863 & 2.382773 & -5.58083 \\
\hline H91 & 0.378717 & 3.766584 & -7.06782 \\
\hline H92 & -0.46774 & 5.381375 & -5.37106 \\
\hline H93 & -0.24445 & 4.888598 & -2.97533 \\
\hline H94 & 1.782586 & 1.229931 & -3.9066 \\
\hline H95 & 1.535918 & 1.697509 & -6.30753 \\
\hline H96 & 1.016175 & 3.636175 & -1.30036 \\
\hline C97 & -3.02773 & 2.95987 & -0.57784 \\
\hline H98 & -2.80676 & 3.540735 & 0.320343 \\
\hline H99 & -3.82688 & 3.486256 & -1.11082 \\
\hline H100 & -3.43455 & 2.001417 & -0.26957 \\
\hline $\mathrm{C} 101$ & -2.28892 & 2.070368 & -2.79435 \\
\hline H102 & -1.45954 & 1.758878 & -3.42805 \\
\hline H103 & -2.86086 & 1.181097 & -2.5311 \\
\hline H104 & -2.93719 & 2.726273 & -3.3846 \\
\hline H105 & -1.54777 & 3.815605 & -1.8139 \\
\hline H106 & 2.170064 & 0.082601 & -2.28846 \\
\hline H107 & 1.522537 & 0.013901 & -2.64664 \\
\hline
\end{tabular}


C3 ER Chelbot

C3_ER_Chelbot_SM

\begin{tabular}{|c|c|c|c|}
\hline $\mathrm{C} 1$ & -4.16247 & -2.95873 & -0.95958 \\
\hline $\mathrm{C} 2$ & -2.08426 & -4.23421 & -1.11606 \\
\hline $\mathrm{C} 3$ & -3.34145 & -3.85776 & -1.94525 \\
\hline $\mathrm{C} 4$ & -3.25484 & -2.93737 & 0.290598 \\
\hline H5 & -5.13432 & -3.40233 & -0.71983 \\
\hline H6 & -3.0825 & -3.34128 & -2.87486 \\
\hline H7 & -3.72516 & -2.57343 & 1.204659 \\
\hline $\mathrm{H} 8$ & -4.33844 & -1.95088 & -1.34004 \\
\hline H9 & -1.51971 & -5.08643 & -1.49506 \\
\hline H10 & -3.89434 & -4.76162 & -2.21589 \\
\hline $\mathrm{C} 11$ & -2.67699 & -4.36381 & 0.304223 \\
\hline H12 & -1.92464 & -4.5257 & 1.081746 \\
\hline H13 & -3.44503 & -5.13927 & 0.372556 \\
\hline $\mathrm{C} 14$ & 0.172051 & -3.1261 & -0.46 \\
\hline N15 & 0.971339 & -2.14999 & -0.17699 \\
\hline $\mathrm{C} 16$ & 2.297827 & -2.71908 & 0.275285 \\
\hline $\mathrm{C} 17$ & 1.936137 & -4.23983 & 0.36871 \\
\hline P18 & -1.63417 & -0.58196 & 0.429118 \\
\hline $\operatorname{Ir} 19$ & 0.558135 & -0.10417 & -0.60809 \\
\hline $\mathrm{C} 20$ & -1.64813 & -0.55929 & 2.259773 \\
\hline $\mathrm{C} 21$ & -1.48251 & -0.50155 & 5.062194 \\
\hline $\mathrm{C} 22$ & -1.68372 & -1.75012 & 2.999481 \\
\hline $\mathrm{C} 23$ & -1.50772 & 0.66191 & 2.940944 \\
\hline $\mathrm{C} 24$ & -1.42883 & 0.687585 & 4.331736 \\
\hline $\mathrm{C} 25$ & -1.6058 & -1.71889 & 4.392288 \\
\hline $\mathrm{H} 26$ & -1.45714 & 1.594778 & 2.394279 \\
\hline $\mathrm{H} 27$ & -1.31979 & 1.641329 & 4.839939 \\
\hline $\mathrm{H} 28$ & -1.63404 & -2.64903 & 4.952397 \\
\hline H29 & -1.42187 & -0.47965 & 6.146553 \\
\hline $\mathrm{O} 30$ & 0.624701 & -4.36086 & -0.25733 \\
\hline $\mathrm{C} 31$ & 2.715175 & -2.24987 & 1.666948 \\
\hline C32 & 1.824404 & -1.61469 & 2.534679 \\
\hline $\mathrm{C} 33$ & 3.990506 & -2.59299 & 2.14188 \\
\hline $\mathrm{C} 34$ & 2.21117 & -1.28088 & 3.833846 \\
\hline H35 & 0.824429 & -1.37546 & 2.207525 \\
\hline $\mathrm{C} 36$ & 4.37565 & -2.26491 & 3.440322 \\
\hline H37 & 4.691361 & -3.10652 & 1.489818 \\
\hline $\mathrm{C} 38$ & 3.489776 & -1.59735 & 4.288616 \\
\hline H39 & 1.499661 & -0.77923 & 4.483244 \\
\hline $\mathrm{H} 40$ & 5.370464 & -2.52895 & 3.78748 \\
\hline H41 & 3.794257 & -1.33591 & 5.298047 \\
\hline $\mathrm{C} 42$ & -3.07062 & 0.438329 & -0.08711 \\
\hline $\mathrm{C} 43$ & -3.95768 & 1.062592 & 0.79685 \\
\hline
\end{tabular}

jag_C3_ER_Chelbot_int2

\begin{tabular}{|c|c|c|c|}
\hline $\mathrm{C} 1$ & -3.98064 & -2.87851 & -1.56255 \\
\hline $\mathrm{C} 2$ & -1.97142 & -4.23452 & -1.30007 \\
\hline $\mathrm{C} 3$ & -3.05847 & -3.87029 & -2.3449 \\
\hline $\mathrm{C} 4$ & -3.26549 & -2.77463 & -0.19825 \\
\hline H5 & -4.98754 & -3.28614 & -1.42376 \\
\hline H6 & -2.62646 & -3.42486 & -3.24667 \\
\hline $\mathrm{H} 7$ & -3.85739 & -2.31033 & 0.590348 \\
\hline H8 & -4.08311 & -1.9052 & -2.04289 \\
\hline H9 & -1.4068 & -5.13951 & -1.52113 \\
\hline H10 & -3.60406 & -4.76731 & -2.65063 \\
\hline C11 & -2.77689 & -4.21882 & 0.017655 \\
\hline H12 & -2.15703 & -4.34498 & 0.910738 \\
\hline H13 & -3.5883 & -4.95192 & 0.028658 \\
\hline $\mathrm{C} 14$ & 0.257452 & -3.24788 & -0.43211 \\
\hline N15 & 1.022963 & -2.3415 & 0.066027 \\
\hline $\mathrm{C} 16$ & 2.257395 & -3.00326 & 0.613071 \\
\hline $\mathrm{C} 17$ & 2.066254 & -4.4705 & 0.096664 \\
\hline P18 & -1.50175 & -0.57464 & 0.241258 \\
\hline Ir19 & 0.680155 & -0.17332 & -0.31493 \\
\hline $\mathrm{C} 20$ & -1.71208 & -0.57676 & 2.065442 \\
\hline $\mathrm{C} 21$ & -1.95621 & -0.48352 & 4.85948 \\
\hline $\mathrm{C} 22$ & -2.23479 & -1.66955 & 2.767177 \\
\hline $\mathrm{C} 23$ & -1.27847 & 0.552572 & 2.78231 \\
\hline $\mathrm{C} 24$ & -1.40865 & 0.600596 & 4.168017 \\
\hline $\mathrm{C} 25$ & -2.35613 & -1.6202 & 4.157772 \\
\hline $\mathrm{H} 26$ & -0.81499 & 1.386821 & 2.264213 \\
\hline H27 & -1.06759 & 1.479826 & 4.70711 \\
\hline H28 & -2.75594 & -2.4779 & 4.690881 \\
\hline H29 & -2.05426 & -0.44758 & 5.94084 \\
\hline $\mathrm{O} 30$ & 0.701515 & -4.50792 & -0.41095 \\
\hline $\mathrm{C} 31$ & 2.282337 & -2.98186 & 2.141578 \\
\hline $\mathrm{C} 32$ & 1.357362 & -2.26918 & 2.904046 \\
\hline C33 & 3.274915 & -3.72335 & 2.802345 \\
\hline $\mathrm{C} 34$ & 1.422823 & -2.29068 & 4.300564 \\
\hline H35 & 0.585658 & -1.69267 & 2.416964 \\
\hline $\mathrm{C} 36$ & 3.34062 & -3.74553 & 4.193463 \\
\hline H37 & 4.014735 & -4.27235 & 2.223557 \\
\hline $\mathrm{C} 38$ & 2.412151 & -3.02551 & 4.950792 \\
\hline H39 & 0.689475 & -1.72865 & 4.870658 \\
\hline $\mathrm{H} 40$ & 4.118305 & -4.32238 & 4.68585 \\
\hline H41 & 2.462754 & -3.04148 & 6.035533 \\
\hline $\mathrm{C} 42$ & -2.77663 & 0.601839 & -0.36746 \\
\hline $\mathrm{C} 43$ & -3.37323 & 1.576111 & 0.445117 \\
\hline
\end{tabular}




\begin{tabular}{|c|c|c|c|c|c|c|c|}
\hline $\mathrm{C} 44$ & -3.27062 & 0.572715 & -1.47319 & $\mathrm{C} 44$ & -3.11386 & 0.55392 & -1.72933 \\
\hline $\mathrm{C} 45$ & -5.02089 & 1.822292 & 0.302518 & $\mathrm{C} 45$ & -4.28165 & 2.488439 & -0.09659 \\
\hline H46 & -3.82823 & 0.960116 & 1.868777 & $\mathrm{H} 46$ & -3.14435 & 1.624374 & 1.50374 \\
\hline $\mathrm{C} 47$ & -4.33383 & 1.328102 & -1.96066 & $\mathrm{C} 47$ & -4.03466 & 1.455684 & -2.2618 \\
\hline $\mathrm{C} 48$ & -5.20864 & 1.9607 & -1.07174 & $\mathrm{C} 48$ & -4.61615 & 2.433425 & -1.44947 \\
\hline H49 & -5.70428 & 2.302611 & 0.997054 & $\mathrm{H} 49$ & -4.7353 & 3.236489 & 0.547224 \\
\hline H50 & -4.48375 & 1.421335 & -3.03268 & $\mathrm{H} 50$ & -4.30573 & 1.392572 & -3.31253 \\
\hline H51 & -6.03671 & 2.552356 & -1.45113 & H51 & -5.33058 & 3.13748 & -1.86538 \\
\hline H52 & 1.232255 & 0.274951 & 0.732036 & $\mathrm{H} 52$ & 0.856304 & 0.196388 & 1.185598 \\
\hline H53 & 2.041136 & -0.16673 & -1.16949 & $\mathrm{H} 53$ & 1.930231 & 1.627962 & -2.38154 \\
\hline H54 & -1.74936 & -2.70472 & 2.490644 & H54 & -2.52309 & -2.57397 & 2.248344 \\
\hline H55 & -2.59798 & 0.071916 & -2.16408 & $\mathrm{H} 55$ & -2.66155 & -0.19391 & -2.37265 \\
\hline H56 & 2.630441 & -4.89079 & -0.16234 & H56 & 2.733184 & -4.73308 & -0.72775 \\
\hline $\mathrm{C} 57$ & 5.062536 & -1.846 & -2.95821 & C57 & 5.431445 & -0.75467 & -1.31211 \\
\hline C58 & 4.068543 & -2.81416 & -3.11075 & C58 & 4.54984 & -1.53859 & -2.06276 \\
\hline $\mathrm{C} 59$ & 3.205649 & -3.09994 & -2.05468 & C59 & 3.576191 & -2.30318 & -1.42243 \\
\hline C60 & 3.318497 & -2.42618 & -0.83091 & C60 & 3.463142 & -2.30316 & -0.02382 \\
\hline C61 & 4.299605 & -1.43691 & -0.69808 & C61 & 4.353574 & -1.5225 & 0.719839 \\
\hline C62 & 5.172486 & -1.1582 & -1.75044 & C62 & 5.332017 & -0.75605 & 0.079408 \\
\hline H63 & 5.742194 & -1.62677 & -3.77658 & H63 & 6.190154 & -0.15619 & -1.80788 \\
\hline H64 & 3.966549 & -3.34929 & -4.05037 & H64 & 4.614804 & -1.54867 & -3.14709 \\
\hline H65 & 2.427861 & -3.84595 & -2.19968 & H65 & 2.866354 & -2.86177 & -2.02652 \\
\hline H66 & 4.38079 & -0.87566 & 0.22538 & H66 & 4.27611 & -1.49198 & 1.800532 \\
\hline H67 & 5.933391 & -0.39309 & -1.62567 & $\mathrm{H} 67$ & 6.013393 & -0.15515 & 0.674918 \\
\hline C68 & 2.309309 & 2.455737 & -1.49248 & C68 & 1.267965 & 3.624811 & -2.61702 \\
\hline C69 & 0.934827 & 1.780133 & -1.62323 & C69 & 0.966953 & 2.140863 & -2.27297 \\
\hline $\mathrm{C} 70$ & -0.15309 & 1.9816 & -0.71704 & $\mathrm{C} 70$ & 0.39377 & 1.879605 & -0.8438 \\
\hline C71 & 0.175032 & 2.689067 & -3.91877 & C71 & -0.9903 & 2.103963 & -4.05254 \\
\hline $\mathrm{C} 72$ & -0.168 & 0.472023 & -3.38791 & $\mathrm{C} 72$ & -0.07348 & 0.143384 & -3.27566 \\
\hline $\mathrm{C} 73$ & -0.34023 & 1.903016 & -5.13678 & $\mathrm{C} 73$ & -1.43782 & 0.916168 & -4.91742 \\
\hline H74 & 1.050246 & 3.290774 & -4.16111 & $\mathrm{H} 74$ & -0.62476 & 2.928618 & -4.66089 \\
\hline H75 & -0.58792 & 3.336661 & -3.46649 & $\mathrm{H} 75$ & -1.78481 & 2.467628 & -3.39287 \\
\hline H76 & -1.20814 & 2.351157 & -5.62132 & $\mathrm{H} 76$ & -2.51871 & 0.847872 & -5.04619 \\
\hline H77 & 0.447452 & 1.725379 & -5.87479 & $\mathrm{H} 77$ & -0.94685 & 0.904423 & -5.89517 \\
\hline N78 & 0.50486 & 1.583079 & -3.01121 & N78 & 0.085507 & 1.479657 & -3.27198 \\
\hline O79 & -0.74132 & 0.616614 & -4.59082 & O79 & -0.99701 & -0.25243 & -4.17831 \\
\hline O80 & -0.29765 & -0.5654 & -2.72174 & O80 & 0.498928 & -0.7007 & -2.56511 \\
\hline H81 & 1.831765 & -4.56827 & 1.403657 & $\mathrm{H} 81$ & 2.144535 & -5.21322 & 0.888689 \\
\hline N82 & -2.02534 & -2.1575 & -0.01944 & N82 & -1.95835 & -2.07174 & -0.37376 \\
\hline $\mathrm{C} 83$ & -1.22942 & -2.95015 & -0.99106 & $\mathrm{C} 83$ & -1.06262 & -2.99079 & -1.12609 \\
\hline H84 & -1.13974 & -2.44407 & -1.95665 & H84 & -0.79183 & -2.56127 & -2.09773 \\
\hline H85 & -1.128 & 1.97256 & -1.19895 & H85 & -0.67982 & 2.081642 & -0.91194 \\
\hline $\mathrm{C} 86$ & -0.39296 & 4.457059 & 2.795261 & $\mathrm{C} 86$ & 1.91942 & 4.359174 & 2.367505 \\
\hline $\mathrm{C} 87$ & -1.42421 & 4.414711 & 1.856955 & C87 & 0.548644 & 4.250401 & 2.136297 \\
\hline C88 & -1.31236 & 3.597593 & 0.731763 & $\mathrm{C} 88$ & 0.074549 & 3.472479 & 1.077767 \\
\hline
\end{tabular}




\begin{tabular}{|c|c|c|c|c|c|c|c|}
\hline $\mathrm{C} 89$ & -0.16923 & 2.807728 & 0.518994 & C89 & 0.951682 & 2.769932 & 0.2371 \\
\hline C90 & 0.857271 & 2.854749 & 1.478022 & C90 & 2.330434 & 2.903611 & 0.477478 \\
\hline C91 & 0.747029 & 3.672669 & 2.598791 & C91 & 2.808725 & 3.687861 & 1.524393 \\
\hline H92 & -0.47652 & 5.091213 & 3.673177 & H92 & 2.2933 & 4.965252 & 3.187525 \\
\hline H93 & -2.31939 & 5.013105 & 2.000562 & H93 & -0.15486 & 4.780433 & 2.773226 \\
\hline H94 & -2.12809 & 3.558954 & 0.01425 & H94 & -0.99649 & 3.408295 & 0.893141 \\
\hline H95 & 1.732368 & 2.227258 & 1.365247 & H95 & 3.042701 & 2.385335 & -0.16082 \\
\hline H96 & 1.551994 & 3.690129 & 3.328051 & H96 & 3.880038 & 3.774795 & 1.685741 \\
\hline C97 & 3.320351 & 1.927417 & -2.51739 & C97 & 1.965817 & 3.776531 & -3.97815 \\
\hline H98 & 3.403517 & 0.838951 & -2.47912 & H98 & 2.836915 & 3.116051 & -4.05248 \\
\hline H99 & 3.044847 & 2.20957 & -3.53925 & H99 & 1.309334 & 3.549225 & -4.82434 \\
\hline H100 & 4.308763 & 2.35267 & -2.3169 & $\mathrm{H} 100$ & 2.316021 & 4.805328 & -4.10944 \\
\hline C101 & 2.210186 & 3.995759 & -1.5534 & C101 & 0.106503 & 4.618155 & -2.43587 \\
\hline H102 & 1.489046 & 4.385324 & -0.83206 & H102 & -0.38832 & 4.485015 & -1.47103 \\
\hline H103 & 3.185816 & 4.434558 & -1.32159 & H103 & 0.500016 & 5.639279 & -2.4627 \\
\hline H104 & 1.926762 & 4.355938 & -2.54702 & H104 & -0.6512 & 4.55254 & -3.22049 \\
\hline H105 & 2.680801 & 2.195098 & -0.49962 & H105 & 2.010597 & 3.901652 & -1.86166 \\
\hline $\mathrm{X}$ & 0.332262 & 1.867163 & -1.24276 & H107 & 2.413561 & 0.218992 & -0.11672 \\
\hline C3_ER & Chelbot_TS1 & & & H108 & 2.386856 & 0.00773 & -0.9041 \\
\hline $\mathrm{C} 1$ & -4.04644 & -3.46425 & -0.71608 & & & & \\
\hline $\mathrm{C} 2$ & -1.84423 & -4.39029 & -1.23333 & \multicolumn{4}{|c|}{ jag_C3_ER_Chelbot_TS3 } \\
\hline $\mathrm{C} 3$ & -3.22304 & -4.103 & -1.88623 & $\mathrm{C} 1$ & -4.012 & -2.73533 & -1.59376 \\
\hline $\mathrm{C} 4$ & -3.03158 & -3.4971 & 0.449095 & $\mathrm{C} 2$ & -2.00349 & -4.11457 & -1.44359 \\
\hline H5 & -4.93318 & -4.05787 & -0.47131 & $\mathrm{C} 3$ & -3.08622 & -3.65558 & -2.45668 \\
\hline H6 & -3.14036 & -3.44356 & -2.7556 & $\mathrm{C} 4$ & -3.29984 & -2.75221 & -0.22349 \\
\hline H7 & -3.45343 & -3.34172 & 1.442844 & H5 & -5.02015 & -3.15218 & -1.49745 \\
\hline H8 & -4.37348 & -2.44348 & -0.9267 & H6 & -2.6488 & -3.13192 & -3.31303 \\
\hline H9 & -1.21016 & -5.08718 & -1.78164 & $\mathrm{H} 7$ & -3.889 & -2.35529 & 0.603053 \\
\hline H10 & -3.67512 & -5.03915 & -2.22582 & H8 & -4.10901 & -1.72254 & -1.98651 \\
\hline $\mathrm{C} 11$ & -2.2647 & -4.8066 & 0.194982 & H9 & -1.4424 & -4.99995 & -1.7408 \\
\hline H12 & -1.42352 & -4.95549 & 0.877228 & $\mathrm{H} 10$ & -3.63093 & -4.52097 & -2.84431 \\
\hline H13 & -2.90782 & -5.69123 & 0.202801 & $\mathrm{C} 11$ & -2.81339 & -4.20937 & -0.13028 \\
\hline $\mathrm{C} 14$ & 0.283957 & -3.02344 & -0.59805 & H12 & -2.19671 & -4.40831 & 0.751455 \\
\hline N15 & 1.008106 & -1.9833 & -0.32666 & H13 & -3.62492 & -4.94066 & -0.18328 \\
\hline $\mathrm{C} 16$ & 2.367552 & -2.47742 & 0.128748 & $\mathrm{C} 14$ & 0.203929 & -3.19711 & -0.44986 \\
\hline $\mathrm{C} 17$ & 2.162501 & -4.04193 & 0.083344 & N15 & 1.02082 & -2.31578 & 0.022021 \\
\hline P18 & -1.75216 & -0.93888 & 0.700363 & $\mathrm{C} 16$ & 2.225764 & -3.02071 & 0.599144 \\
\hline Ir19 & 0.055377 & 0.062793 & -0.42751 & $\mathrm{C} 17$ & 1.877203 & -4.51748 & 0.271715 \\
\hline $\mathrm{C} 20$ & -1.59505 & -0.98887 & 2.515724 & P18 & -1.51434 & -0.55741 & 0.284461 \\
\hline $\mathrm{C} 21$ & -1.25069 & -0.94337 & 5.292134 & Ir19 & 0.829392 & -0.20234 & -0.28619 \\
\hline $\mathrm{C} 22$ & -1.47975 & -2.18672 & 3.229712 & $\mathrm{C} 20$ & -1.72257 & -0.57271 & 2.103038 \\
\hline $\mathrm{C} 23$ & -1.50547 & 0.236699 & 3.197202 & $\mathrm{C} 21$ & -1.92291 & -0.4747 & 4.897485 \\
\hline $\mathrm{C} 24$ & -1.34008 & 0.256823 & 4.579812 & $\mathrm{C} 22$ & -2.25907 & -1.65484 & 2.810322 \\
\hline $\mathrm{C} 25$ & -1.31213 & -2.16101 & 4.615524 & $\mathrm{C} 23$ & -1.25206 & 0.54711 & 2.811072 \\
\hline $\mathrm{H} 26$ & -1.55713 & 1.172607 & 2.645753 & $\mathrm{C} 24$ & -1.36218 & 0.598399 & 4.198833 \\
\hline
\end{tabular}




\begin{tabular}{|c|c|c|c|c|c|c|c|}
\hline $\mathrm{H} 27$ & -1.27669 & 1.207668 & 5.101256 & $\mathrm{C} 25$ & -2.35799 & -1.60341 & 4.202486 \\
\hline $\mathrm{H} 28$ & -1.22247 & -3.09378 & 5.164715 & $\mathrm{H} 26$ & -0.78612 & 1.373166 & 2.280057 \\
\hline H29 & -1.12206 & -0.92646 & 6.37057 & $\mathrm{H} 27$ & -0.99728 & 1.470149 & 4.734617 \\
\hline $\mathrm{O} 30$ & 0.83295 & -4.22812 & -0.45184 & $\mathrm{H} 28$ & -2.7695 & -2.45088 & 4.743136 \\
\hline $\mathrm{C} 31$ & 2.721528 & -2.11291 & 1.573256 & $\mathrm{H} 29$ & -2.00439 & -0.43686 & 5.980206 \\
\hline C32 & 1.889365 & -1.37885 & 2.416814 & $\mathrm{O} 30$ & 0.557571 & -4.4761 & -0.34142 \\
\hline $\mathrm{C} 33$ & 3.890286 & -2.67283 & 2.123425 & $\mathrm{C} 31$ & 2.329028 & -2.86367 & 2.116258 \\
\hline C34 & 2.231406 & -1.15705 & 3.753509 & $\mathrm{C} 32$ & 1.382276 & -2.18322 & 2.880503 \\
\hline H35 & 0.957843 & -0.98718 & 2.047808 & $\mathrm{C} 33$ & 3.383851 & -3.51564 & 2.776771 \\
\hline C36 & 4.235015 & -2.4537 & 3.454174 & C34 & 1.500326 & -2.12396 & 4.27244 \\
\hline H37 & 4.538278 & -3.28983 & 1.506754 & H35 & 0.547223 & -1.69623 & 2.402275 \\
\hline C38 & 3.408695 & -1.68371 & 4.275987 & $\mathrm{C} 36$ & 3.500066 & -3.46115 & 4.163565 \\
\hline H39 & 1.554254 & -0.58413 & 4.379656 & H37 & 4.127556 & -4.05868 & 2.19862 \\
\hline $\mathrm{H} 40$ & 5.147404 & -2.89056 & 3.849953 & $\mathrm{C} 38$ & 2.559122 & -2.75657 & 4.919818 \\
\hline H41 & 3.677117 & -1.51216 & 5.314395 & H39 & 0.750725 & -1.58211 & 4.840562 \\
\hline $\mathrm{C} 42$ & -3.33643 & -0.06356 & 0.411673 & $\mathrm{H} 40$ & 4.327353 & -3.96725 & 4.652911 \\
\hline $\mathrm{C} 43$ & -4.32139 & 0.056699 & 1.400843 & $\mathrm{H} 41$ & 2.651062 & -2.7099 & 6.001003 \\
\hline $\mathrm{C} 44$ & -3.58471 & 0.434204 & -0.87886 & $\mathrm{C} 42$ & -2.79119 & 0.638453 & -0.29319 \\
\hline $\mathrm{C} 45$ & -5.53502 & 0.679203 & 1.106524 & $\mathrm{C} 43$ & -3.47381 & 1.517243 & 0.558723 \\
\hline H46 & -4.14198 & -0.32458 & 2.401333 & $\mathrm{C} 44$ & -3.05118 & 0.689139 & -1.673 \\
\hline $\mathrm{C} 47$ & -4.80159 & 1.048281 & -1.1677 & $\mathrm{C} 45$ & -4.39512 & 2.430211 & 0.040389 \\
\hline $\mathrm{C} 48$ & -5.77534 & 1.177384 & -0.17379 & $\mathrm{H} 46$ & -3.29772 & 1.490354 & 1.628361 \\
\hline H49 & -6.29104 & 0.776213 & 1.880369 & $\mathrm{C} 47$ & -3.98149 & 1.592618 & -2.18467 \\
\hline H50 & -4.99094 & 1.428366 & -2.16763 & $\mathrm{C} 48$ & -4.65161 & 2.471601 & -1.32948 \\
\hline H51 & -6.72051 & 1.662925 & -0.3987 & $\mathrm{H} 49$ & -4.91974 & 3.102484 & 0.713386 \\
\hline H52 & 0.636068 & 0.434376 & 0.953909 & $\mathrm{H} 50$ & -4.19359 & 1.60617 & -3.25081 \\
\hline H53 & -0.93457 & 1.286653 & -0.21567 & H51 & -5.37493 & 3.176802 & -1.7285 \\
\hline H54 & -1.49954 & -3.1365 & 2.705756 & $\mathrm{H} 52$ & 1.090456 & 0.062014 & 1.219535 \\
\hline H55 & -2.82974 & 0.326939 & -1.6542 & H53 & 1.957465 & 1.769654 & -2.35906 \\
\hline H56 & 2.867565 & -4.5479 & -0.5771 & H54 & -2.57446 & -2.5512 & 2.290208 \\
\hline C57 & 5.406552 & -1.8087 & -2.90378 & H55 & -2.5413 & 0.005935 & -2.3454 \\
\hline C58 & 4.148684 & -2.31692 & -3.2376 & H56 & 2.561175 & -4.97728 & -0.44335 \\
\hline C59 & 3.174041 & -2.46572 & -2.25514 & C57 & 5.606568 & -1.5839 & -1.71472 \\
\hline C60 & 3.429426 & -2.10857 & -0.92062 & C58 & 4.586871 & -2.32435 & -2.31716 \\
\hline C61 & 4.677613 & -1.56359 & -0.6064 & C59 & 3.525699 & -2.79807 & -1.55079 \\
\hline C62 & 5.661625 & -1.42692 & -1.58939 & $\mathrm{C} 60$ & 3.462658 & -2.54538 & -0.17281 \\
\hline H63 & 6.174769 & -1.70448 & -3.66429 & C61 & 4.478439 & -1.78808 & 0.418355 \\
\hline H64 & 3.92989 & -2.60628 & -4.26159 & $\mathrm{C} 62$ & 5.546635 & -1.3167 & -0.34773 \\
\hline H65 & 2.205963 & -2.87581 & -2.52923 & H63 & 6.43932 & -1.21709 & -2.30816 \\
\hline H66 & 4.895028 & -1.24102 & 0.404317 & H64 & 4.616846 & -2.5298 & -3.38345 \\
\hline H67 & 6.628604 & -1.0113 & -1.32046 & H65 & 2.724047 & -3.34304 & -2.04325 \\
\hline C68 & 2.693942 & 1.977072 & -1.32243 & H66 & 4.436322 & -1.5527 & 1.475356 \\
\hline C69 & 1.23654 & 1.559297 & -1.58239 & H67 & 6.331087 & -0.73593 & 0.129284 \\
\hline $\mathrm{C} 70$ & 0.104461 & 2.231961 & -0.93567 & C68 & 1.273816 & 3.764729 & -2.5825 \\
\hline C71 & 1.106622 & 2.255408 & -4.09628 & C69 & 0.989798 & 2.274564 & -2.26464 \\
\hline
\end{tabular}




\begin{tabular}{|c|c|c|c|c|c|c|c|}
\hline $\mathrm{C} 72$ & -0.01579 & 0.400865 & -3.34079 & C70 & 0.419159 & 1.988379 & -0.82771 \\
\hline C73 & 0.556625 & 1.430962 & -5.26994 & C71 & -0.93907 & 2.217706 & -4.06158 \\
\hline H74 & 2.149033 & 2.534687 & -4.24074 & $\mathrm{C} 72$ & -0.01035 & 0.25541 & -3.27669 \\
\hline H75 & 0.520301 & 3.163592 & -3.89854 & C73 & -1.36959 & 1.026064 & -4.93044 \\
\hline H76 & -0.02043 & 2.00709 & -5.99355 & $\mathrm{H} 74$ & -0.56567 & 3.040042 & -4.66864 \\
\hline H77 & 1.344869 & 0.870208 & -5.78158 & H75 & -1.74474 & 2.584788 & -3.41666 \\
\hline N78 & 0.941108 & 1.29127 & -2.99689 & $\mathrm{H} 76$ & -2.4488 & 0.950207 & -5.07022 \\
\hline O79 & -0.33879 & 0.473228 & -4.63902 & H77 & -0.86999 & 1.02057 & -5.90393 \\
\hline O80 & -0.59216 & -0.39249 & -2.57561 & N78 & 0.127588 & 1.600881 & -3.26381 \\
\hline H81 & 2.182525 & -4.49436 & 1.075773 & O79 & -0.9275 & -0.1385 & -4.1894 \\
\hline N82 & -1.94802 & -2.51809 & 0.175222 & O80 & 0.561049 & -0.58487 & -2.57159 \\
\hline $\mathrm{C} 83$ & -1.17343 & -3.01343 & -0.99311 & H81 & 1.803675 & -5.13306 & 1.1677 \\
\hline H84 & -1.28225 & -2.36842 & -1.86839 & N82 & -1.99369 & -2.04192 & -0.33925 \\
\hline H85 & -0.69008 & 2.503883 & -1.63444 & $\mathrm{C} 83$ & -1.09558 & -2.89226 & -1.16295 \\
\hline $\mathrm{C} 86$ & 0.244302 & 5.197605 & 2.18891 & H84 & -0.80905 & -2.38757 & -2.0928 \\
\hline $\mathrm{C} 87$ & -0.55738 & 5.368053 & 1.061697 & H85 & -0.65963 & 1.897418 & -0.93612 \\
\hline C88 & -0.58648 & 4.385569 & 0.071624 & C86 & 1.109505 & 4.83202 & 2.358038 \\
\hline $\mathrm{C} 89$ & 0.187105 & 3.222924 & 0.18807 & C87 & -0.18355 & 4.56797 & 1.908414 \\
\hline $\mathrm{C} 90$ & 0.980556 & 3.055529 & 1.334278 & C88 & -0.39302 & 3.657404 & 0.870292 \\
\hline C91 & 1.011848 & 4.037161 & 2.320338 & C89 & 0.684591 & 2.993707 & 0.268484 \\
\hline H92 & 0.267521 & 5.958906 & 2.963118 & C90 & 1.979735 & 3.259025 & 0.740673 \\
\hline H93 & -1.16455 & 6.261773 & 0.951622 & C91 & 2.192437 & 4.174557 & 1.768149 \\
\hline H94 & -1.21725 & 4.527285 & -0.80332 & H92 & 1.274816 & 5.539511 & 3.16545 \\
\hline H95 & 1.570436 & 2.154562 & 1.457466 & H93 & -1.03254 & 5.070532 & 2.363713 \\
\hline H96 & 1.636014 & 3.893162 & 3.197695 & H94 & -1.40454 & 3.455354 & 0.525116 \\
\hline C97 & 3.668859 & 1.187585 & -2.20236 & H95 & 2.830257 & 2.730565 & 0.313457 \\
\hline H98 & 3.399218 & 0.136489 & -2.24905 & H96 & 3.202706 & 4.367472 & 2.117795 \\
\hline H99 & 3.695625 & 1.573259 & -3.22677 & C97 & 2.047816 & 3.925021 & -3.90062 \\
\hline $\mathrm{H} 100$ & 4.68378 & 1.267871 & -1.80703 & H98 & 2.957111 & 3.314592 & -3.90723 \\
\hline $\mathrm{C} 101$ & 2.951455 & 3.492084 & -1.4894 & H99 & 1.452758 & 3.639791 & -4.77452 \\
\hline H102 & 2.32968 & 4.101127 & -0.83208 & H100 & 2.344649 & 4.969682 & -4.03695 \\
\hline H103 & 3.999097 & 3.71216 & -1.25778 & C101 & 0.082584 & 4.73904 & -2.49807 \\
\hline H104 & 2.773769 & 3.822388 & -2.51849 & H102 & -0.56064 & 4.535265 & -1.63971 \\
\hline H105 & 2.90378 & 1.717739 & -0.28057 & H103 & 0.462826 & 5.758671 & -2.38302 \\
\hline \multirow[t]{2}{*}{$X$} & 0.332262 & 1.867163 & -1.24276 & H104 & -0.5366 & 4.737145 & -3.39835 \\
\hline & & & & H105 & 1.960288 & 4.055384 & -1.77973 \\
\hline \multicolumn{4}{|c|}{ C3_ER_Chelbot_INT1 } & H107 & 1.632158 & 1.140709 & -0.40673 \\
\hline $\mathrm{C} 1$ & -4.09098 & -2.80674 & -1.48151 & H108 & 2.416745 & -0.2278 & -0.52064 \\
\hline $\mathrm{C} 2$ & -2.12993 & -4.23952 & -1.26557 & & & & \\
\hline $\mathrm{C} 3$ & -3.22642 & -3.83432 & -2.28533 & \multicolumn{4}{|c|}{ jag_C3_ER_Chelbot_PROD } \\
\hline $\mathrm{C} 4$ & -3.3638 & -2.75963 & -0.11992 & $\mathrm{C} 1$ & -4.01655 & -2.80759 & -1.58036 \\
\hline H5 & -5.11855 & -3.1598 & -1.34587 & $\mathrm{C} 2$ & -1.97983 & -4.15199 & -1.50539 \\
\hline H6 & -2.80042 & -3.40824 & -3.19907 & $\mathrm{C} 3$ & -3.08118 & -3.67378 & -2.48845 \\
\hline $\mathrm{H} 7$ & -3.93375 & -2.29583 & 0.685279 & $\mathrm{C} 4$ & -3.3011 & -2.88207 & -0.21324 \\
\hline $\mathrm{H} 8$ & -4.13848 & -1.82023 & -1.94491 & H5 & -5.02029 & -3.23891 & -1.50526 \\
\hline
\end{tabular}




\begin{tabular}{|c|c|c|c|c|c|c|c|}
\hline H9 & -1.58913 & -5.15454 & -1.50817 & H6 & -2.66377 & -3.10957 & -3.32869 \\
\hline H10 & -3.81373 & -4.71033 & -2.57443 & $\mathrm{H} 7$ & -3.89626 & -2.54299 & 0.634902 \\
\hline $\mathrm{C} 11$ & -2.91759 & -4.22244 & 0.062781 & H8 & -4.12292 & -1.77723 & -1.92283 \\
\hline H12 & -2.29281 & -4.38237 & 0.94697 & H9 & -1.39944 & -5.00867 & -1.84734 \\
\hline H13 & -3.75078 & -4.93091 & 0.071166 & H10 & -3.61274 & -4.53445 & -2.90385 \\
\hline $\mathrm{C} 14$ & 0.148229 & -3.28522 & -0.45041 & $\mathrm{C} 11$ & -2.78144 & -4.33048 & -0.19538 \\
\hline N15 & 0.856372 & -2.36121 & 0.088198 & H12 & -2.15765 & -4.5573 & 0.67442 \\
\hline $\mathrm{C} 16$ & 2.188286 & -2.90165 & 0.458431 & $\mathrm{H} 13$ & -3.57539 & -5.07763 & -0.28302 \\
\hline $\mathrm{C} 17$ & 1.999161 & -4.41818 & 0.131104 & $\mathrm{C} 14$ & 0.204752 & -3.2235 & -0.47408 \\
\hline P18 & -1.56489 & -0.59349 & 0.311555 & N15 & 1.022059 & -2.34155 & 0.004248 \\
\hline Ir19 & 0.531074 & -0.24443 & -0.25293 & $\mathrm{C} 16$ & 2.253178 & -3.04554 & 0.54285 \\
\hline $\mathrm{C} 20$ & -1.80218 & -0.5906 & 2.129078 & $\mathrm{C} 17$ & 1.780557 & -4.53247 & 0.447995 \\
\hline $\mathrm{C} 21$ & -2.03512 & -0.50326 & 4.922381 & P18 & -1.56107 & -0.64539 & 0.335218 \\
\hline $\mathrm{C} 22$ & -2.26282 & -1.70839 & 2.83525 & Ir19 & 0.768721 & -0.31138 & -0.16947 \\
\hline $\mathrm{C} 23$ & -1.42929 & 0.564898 & 2.839112 & $\mathrm{C} 20$ & -1.80969 & -0.67278 & 2.147238 \\
\hline $\mathrm{C} 24$ & -1.55353 & 0.608315 & 4.224745 & $\mathrm{C} 21$ & -1.97619 & -0.62124 & 4.945992 \\
\hline $\mathrm{C} 25$ & -2.38051 & -1.66099 & 4.226677 & $\mathrm{C} 22$ & -2.27343 & -1.79223 & 2.847977 \\
\hline H26 & -1.01668 & 1.421771 & 2.315286 & $\mathrm{C} 23$ & -1.39751 & 0.464654 & 2.864902 \\
\hline $\mathrm{H} 27$ & -1.25964 & 1.506968 & 4.759502 & $\mathrm{C} 24$ & -1.49138 & 0.492725 & 4.253827 \\
\hline $\mathrm{H} 28$ & -2.73571 & -2.53517 & 4.76457 & $\mathrm{C} 25$ & -2.35595 & -1.76397 & 4.242504 \\
\hline H29 & -2.12908 & -0.469 & 6.004052 & $\mathrm{H} 26$ & -0.98525 & 1.3223 & 2.33779 \\
\hline $\mathrm{O} 30$ & 0.692243 & -4.50457 & -0.52298 & $\mathrm{H} 27$ & -1.17233 & 1.377509 & 4.797186 \\
\hline $\mathrm{C} 31$ & 2.48147 & -2.70372 & 1.940532 & $\mathrm{H} 28$ & -2.71368 & -2.63917 & 4.777156 \\
\hline $\mathrm{C} 32$ & 1.485854 & -2.298 & 2.832866 & $\mathrm{H} 29$ & -2.04366 & -0.60126 & 6.029945 \\
\hline $\mathrm{C} 33$ & 3.7669 & -2.97856 & 2.424167 & $\mathrm{O} 30$ & 0.565422 & -4.50135 & -0.35517 \\
\hline $\mathrm{C} 34$ & 1.77495 & -2.15452 & 4.190815 & C31 & 2.503357 & -2.74057 & 2.017661 \\
\hline H35 & 0.490595 & -2.07474 & 2.47052 & $\mathrm{C} 32$ & 1.459045 & -2.31605 & 2.847911 \\
\hline C36 & 4.052005 & -2.84364 & 3.781567 & $\mathrm{C} 33$ & 3.738379 & -3.05404 & 2.601702 \\
\hline H37 & 4.550717 & -3.28242 & 1.734228 & C34 & 1.659815 & -2.14632 & 4.217649 \\
\hline C38 & 3.056581 & -2.42613 & 4.668356 & H35 & 0.482347 & -2.10901 & 2.430973 \\
\hline H39 & 0.991949 & -1.82441 & 4.867687 & $\mathrm{C} 36$ & 3.937299 & -2.89117 & 3.973622 \\
\hline $\mathrm{H} 40$ & 5.052784 & -3.05692 & 4.145871 & H37 & 4.552453 & -3.42311 & 1.985632 \\
\hline H41 & 3.282378 & -2.31266 & 5.724755 & C38 & 2.903492 & -2.42406 & 4.786586 \\
\hline $\mathrm{C} 42$ & -2.79216 & 0.587897 & -0.35958 & H39 & 0.835388 & -1.797 & 4.83251 \\
\hline $\mathrm{C} 43$ & -3.49569 & 1.510278 & 0.424831 & $\mathrm{H} 40$ & 4.905362 & -3.13063 & 4.404523 \\
\hline $\mathrm{C} 44$ & -2.98341 & 0.581275 & -1.75178 & $\mathrm{H} 41$ & 3.063437 & -2.29017 & 5.852456 \\
\hline $\mathrm{C} 45$ & -4.37367 & 2.415717 & -0.1759 & $\mathrm{C} 42$ & -2.81568 & 0.557488 & -0.28286 \\
\hline H46 & -3.3726 & 1.522838 & 1.502177 & $\mathrm{C} 43$ & -3.5405 & 1.432196 & 0.537752 \\
\hline $\mathrm{C} 47$ & -3.86656 & 1.480007 & -2.34424 & $\mathrm{C} 44$ & -2.99022 & 0.631781 & -1.676 \\
\hline $\mathrm{C} 48$ & -4.55982 & 2.404905 & -1.55718 & $\mathrm{C} 45$ & -4.4163 & 2.365186 & -0.02349 \\
\hline H49 & -4.91762 & 3.124557 & 0.441674 & $\mathrm{H} 46$ & -3.43204 & 1.387163 & 1.616037 \\
\hline H50 & -4.0251 & 1.455748 & -3.41917 & $\mathrm{C} 47$ & -3.87502 & 1.554587 & -2.23119 \\
\hline H51 & -5.24833 & 3.106257 & -2.01939 & $\mathrm{C} 48$ & -4.5862 & 2.43048 & -1.40592 \\
\hline H52 & 0.721199 & 0.121231 & 1.243231 & H49 & -4.975 & 3.033297 & 0.625838 \\
\hline H53 & 2.103883 & 1.376409 & -2.05265 & $\mathrm{H} 50$ & -4.02312 & 1.582476 & -3.30757 \\
\hline
\end{tabular}




\begin{tabular}{|c|c|c|c|c|c|c|c|}
\hline H54 & -2.50772 & -2.62648 & 2.317034 & H51 & -5.27479 & 3.150635 & -1.83803 \\
\hline H55 & -2.44797 & -0.13379 & -2.36902 & H52 & 1.0547 & -0.16117 & 1.347757 \\
\hline H56 & 2.744611 & -4.83927 & -0.54387 & H53 & 2.010871 & 1.676168 & -2.08355 \\
\hline $\mathrm{C} 57$ & 4.567235 & -0.44255 & -2.20631 & $\mathrm{H} 54$ & -2.54582 & -2.6977 & 2.318115 \\
\hline C58 & 4.182349 & -1.72133 & -2.60795 & H55 & -2.45155 & -0.04981 & -2.32654 \\
\hline C59 & 3.470527 & -2.55073 & -1.73927 & H56 & 2.498866 & -5.19209 & -0.03787 \\
\hline C60 & 3.148923 & -2.12076 & -0.4505 & C57 & 5.371148 & -2.05573 & -2.30999 \\
\hline C61 & 3.536357 & -0.82716 & -0.04841 & $\mathrm{C} 58$ & 4.396291 & -3.00495 & -2.61979 \\
\hline C62 & 4.243195 & 0.002164 & -0.92413 & C59 & 3.421106 & -3.33457 & -1.68081 \\
\hline H63 & 5.124034 & 0.198646 & -2.88347 & C60 & 3.402687 & -2.72744 & -0.41785 \\
\hline H64 & 4.428535 & -2.07556 & -3.60465 & C61 & 4.369372 & -1.75807 & -0.12746 \\
\hline H65 & 3.14682 & -3.52358 & -2.09586 & C62 & 5.351851 & -1.43259 & -1.06262 \\
\hline H66 & 3.326659 & -0.4919 & 0.962639 & $\mathrm{H} 63$ & 6.13783 & -1.80272 & -3.03687 \\
\hline H67 & 4.559419 & 0.987672 & -0.59455 & H64 & 4.394166 & -3.48996 & -3.59167 \\
\hline C68 & 1.71383 & 3.425077 & -2.40923 & H65 & 2.657997 & -4.05899 & -1.95391 \\
\hline C69 & 1.199236 & 1.996283 & -2.09428 & H66 & 4.349612 & -1.24524 & 0.826149 \\
\hline $\mathrm{C} 70$ & 0.380633 & 1.81254 & -0.77585 & H67 & 6.100426 & -0.68541 & -0.81421 \\
\hline C71 & -0.58963 & 2.09958 & -4.02803 & C68 & 1.698653 & 3.739191 & -2.44813 \\
\hline $\mathrm{C} 72$ & 0.164015 & 0.082816 & -3.2251 & C69 & 1.140419 & 2.338497 & -2.09593 \\
\hline $\mathrm{C} 73$ & -1.07184 & 0.946048 & -4.9214 & $\mathrm{C} 70$ & 0.447678 & 2.189351 & -0.69207 \\
\hline H74 & -0.14683 & 2.903677 & -4.61395 & C71 & -0.74088 & 2.475152 & -3.92716 \\
\hline H75 & -1.38901 & 2.508786 & -3.39938 & $\mathrm{C} 72$ & 0.033522 & 0.424001 & -3.19935 \\
\hline H76 & -2.1477 & 0.945805 & -5.1006 & $\mathrm{C} 73$ & -1.19851 & 1.354391 & -4.87189 \\
\hline H77 & -0.53776 & 0.907919 & -5.87553 & $\mathrm{H} 74$ & -0.30708 & 3.307234 & -4.47719 \\
\hline N78 & 0.412098 & 1.406074 & -3.20895 & $\mathrm{H} 75$ & -1.54993 & 2.848212 & -3.28684 \\
\hline O79 & -0.73898 & -0.25133 & -4.17128 & H76 & -2.27132 & 1.351674 & -5.06746 \\
\hline $\mathrm{O} 80$ & 0.645034 & -0.79081 & -2.48201 & $\mathrm{H} 77$ & -0.64983 & 1.36396 & -5.81869 \\
\hline H81 & 1.949805 & -5.01838 & 1.040343 & N78 & 0.266298 & 1.760406 & -3.13554 \\
\hline N82 & -2.03512 & -2.09888 & -0.28789 & O79 & -0.86446 & 0.133398 & -4.16755 \\
\hline $\mathrm{C} 83$ & -1.19038 & -3.01988 & -1.09569 & O80 & 0.504733 & -0.47538 & -2.49857 \\
\hline H84 & -0.9444 & -2.57786 & -2.07002 & $\mathrm{H} 81$ & 1.509126 & -4.93085 & 1.426538 \\
\hline H85 & -0.6559 & 2.065792 & -1.02365 & N82 & -2.01174 & -2.14202 & -0.28888 \\
\hline $\mathrm{C} 86$ & 1.476662 & 4.231194 & 2.652851 & $\mathrm{C} 83$ & -1.10216 & -2.9203 & -1.17341 \\
\hline $\mathrm{C} 87$ & 0.16842 & 4.22948 & 2.167538 & H84 & -0.84028 & -2.36011 & -2.07808 \\
\hline $\mathrm{C} 88$ & -0.16548 & 3.471683 & 1.042057 & H85 & -0.59485 & 1.923004 & -0.84061 \\
\hline $\mathrm{C} 89$ & 0.791993 & 2.685588 & 0.38009 & $\mathrm{C} 86$ & 0.586994 & 5.297269 & 2.297997 \\
\hline C90 & 2.105478 & 2.705188 & 0.880277 & C87 & -0.62895 & 4.926494 & 1.723177 \\
\hline C91 & 2.445538 & 3.466707 & 1.99628 & C88 & -0.66714 & 3.92555 & 0.75099 \\
\hline H92 & 1.740926 & 4.823509 & 3.523761 & C89 & 0.513209 & 3.292641 & 0.340802 \\
\hline H93 & -0.5953 & 4.827294 & 2.6587 & $\mathrm{C} 90$ & 1.727017 & 3.652649 & 0.941362 \\
\hline H94 & -1.18818 & 3.483388 & 0.668918 & C91 & 1.764922 & 4.655474 & 1.90776 \\
\hline H95 & 2.86392 & 2.097948 & 0.394857 & H92 & 0.617492 & 6.076494 & 3.054031 \\
\hline H96 & 3.470063 & 3.462017 & 2.359131 & H93 & -1.54902 & 5.414802 & 2.031659 \\
\hline C97 & 2.570356 & 3.447136 & -3.68442 & H94 & -1.61675 & 3.633261 & 0.308345 \\
\hline H98 & 3.36074 & 2.689028 & -3.64489 & H95 & 2.641646 & 3.133371 & 0.663655 \\
\hline
\end{tabular}




\begin{tabular}{|c|c|c|c|c|c|c|c|}
\hline H99 & 1.980768 & 3.257212 & -4.58784 & H96 & 2.71147 & 4.929833 & 2.364379 \\
\hline H100 & 3.047819 & 4.424405 & -3.80649 & C97 & 2.541071 & 3.679928 & -3.73269 \\
\hline C101 & 0.662947 & 4.548641 & -2.39246 & H98 & 3.326913 & 2.919992 & -3.6655 \\
\hline H102 & 0.039299 & 4.502636 & -1.49687 & H99 & 1.930905 & 3.4468 & -4.61215 \\
\hline H103 & 1.171706 & 5.517842 & -2.38749 & H100 & 3.02155 & 4.646226 & -3.91418 \\
\hline H104 & 0.007682 & 4.542803 & -3.26724 & $\mathrm{C} 101$ & 0.703813 & 4.915921 & -2.48409 \\
\hline H105 & 2.387238 & 3.635049 & -1.57062 & H102 & -0.01253 & 4.886058 & -1.66145 \\
\hline \multirow[t]{2}{*}{$\mathrm{X}$} & 0.332262 & 1.867163 & -1.24276 & $\mathrm{H} 103$ & 1.259911 & 5.854216 & -2.39663 \\
\hline & & & & $\mathrm{H} 104$ & 0.14614 & 4.972166 & -3.42212 \\
\hline \multicolumn{4}{|c|}{ jag_C3_ER_Chelbot_TS2 } & $\mathrm{H} 105$ & 2.38753 & 3.946443 & -1.62141 \\
\hline $\mathrm{C} 1$ & -3.93835 & -2.71353 & -1.61154 & H107 & 1.095206 & 1.412215 & -0.10894 \\
\hline $\mathrm{C} 2$ & -1.94822 & -4.11256 & -1.40996 & H108 & 2.353954 & -0.16609 & -0.38121 \\
\hline $\mathrm{C} 3$ & -3.01803 & -3.66788 & -2.44291 & & & & \\
\hline $\mathrm{C} 4$ & -3.23306 & -2.69667 & -0.23871 & & & & \\
\hline H5 & -4.95068 & -3.11741 & -1.50608 & & & & \\
\hline H6 & -2.56753 & -3.17168 & -3.30865 & & & & \\
\hline H7 & -3.81893 & -2.26952 & 0.575367 & & & & \\
\hline H8 & -4.02504 & -1.71236 & -2.03494 & & & & \\
\hline H9 & -1.39594 & -5.01285 & -1.67749 & & & & \\
\hline H10 & -3.57076 & -4.53602 & -2.81215 & & & & \\
\hline $\mathrm{C} 11$ & -2.76658 & -4.1583 & -0.10134 & & & & \\
\hline H12 & -2.15694 & -4.34639 & 0.787458 & & & & \\
\hline H13 & -3.5901 & -4.8769 & -0.13766 & & & & \\
\hline $\mathrm{C} 14$ & 0.271274 & -3.1912 & -0.43425 & & & & \\
\hline N15 & 1.046664 & -2.29268 & 0.064292 & & & & \\
\hline $\mathrm{C} 16$ & 2.255605 & -2.9703 & 0.643295 & & & & \\
\hline $\mathrm{C} 17$ & 2.007553 & -4.4593 & 0.200706 & & & & \\
\hline P18 & -1.44647 & -0.53418 & 0.292733 & & & & \\
\hline Ir19 & 0.630642 & -0.13472 & -0.34062 & & & & \\
\hline $\mathrm{C} 20$ & -1.6352 & -0.61003 & 2.116049 & & & & \\
\hline $\mathrm{C} 21$ & -1.87272 & -0.63973 & 4.913751 & & & & \\
\hline $\mathrm{C} 22$ & -2.09244 & -1.75669 & 2.776128 & & & & \\
\hline $\mathrm{C} 23$ & -1.2641 & 0.514683 & 2.874361 & & & & \\
\hline $\mathrm{C} 24$ & -1.39146 & 0.499953 & 4.261742 & & & & \\
\hline $\mathrm{C} 25$ & -2.21082 & -1.76842 & 4.167921 & & & & \\
\hline $\mathrm{H} 26$ & -0.85565 & 1.394848 & 2.385931 & & & & \\
\hline $\mathrm{H} 27$ & -1.10118 & 1.376694 & 4.833601 & & & & \\
\hline $\mathrm{H} 28$ & -2.55878 & -2.6678 & 4.667472 & & & & \\
\hline H29 & -1.96804 & -0.65154 & 5.99545 & & & & \\
\hline $\mathrm{O} 30$ & 0.665949 & -4.46322 & -0.36379 & & & & \\
\hline C31 & 2.306781 & -2.87324 & 2.167851 & & & & \\
\hline $\mathrm{C} 32$ & 1.490874 & -2.01426 & 2.903449 & & & & \\
\hline $\mathrm{C} 33$ & 3.218629 & -3.68889 & 2.858258 & & & & \\
\hline $\mathrm{C} 34$ & 1.580194 & -1.96806 & 4.297551 & & & & \\
\hline H35 & 0.784096 & -1.37792 & 2.395775 & & & & \\
\hline
\end{tabular}




\begin{tabular}{|c|c|c|c|}
\hline $\mathrm{C} 36$ & 3.311017 & -3.64122 & 4.247275 \\
\hline H37 & 3.879437 & -4.352 & 2.304102 \\
\hline C38 & 2.488365 & -2.77803 & 4.975652 \\
\hline H39 & 0.928893 & -1.29321 & 4.843372 \\
\hline $\mathrm{H} 40$ & 4.02731 & -4.27707 & 4.759521 \\
\hline H41 & 2.558141 & -2.74074 & 6.058642 \\
\hline $\mathrm{C} 42$ & -2.74423 & 0.635362 & -0.26987 \\
\hline $\mathrm{C} 43$ & -3.44738 & 1.489025 & 0.589122 \\
\hline $\mathrm{C} 44$ & -3.01244 & 0.674544 & -1.64846 \\
\hline $\mathrm{C} 45$ & -4.40055 & 2.370801 & 0.074995 \\
\hline H46 & -3.26736 & 1.464508 & 1.657804 \\
\hline $\mathrm{C} 47$ & -3.97115 & 1.548411 & -2.15469 \\
\hline $\mathrm{C} 48$ & -4.66391 & 2.403703 & -1.29288 \\
\hline H49 & -4.943 & 3.026041 & 0.750013 \\
\hline H50 & -4.18637 & 1.560633 & -3.22004 \\
\hline H5 1 & -5.41117 & 3.085739 & -1.68726 \\
\hline H52 & 0.832294 & 0.290297 & 1.136683 \\
\hline H53 & 1.991924 & 1.703458 & -2.30892 \\
\hline H54 & -2.33147 & -2.65384 & 2.219396 \\
\hline H55 & -2.47454 & 0.011494 & -2.31992 \\
\hline H56 & 2.693958 & -4.79274 & -0.58062 \\
\hline C57 & 5.69995 & -1.33801 & -1.44137 \\
\hline C58 & 4.629551 & -1.89549 & -2.14518 \\
\hline C59 & 3.538269 & -2.4169 & -1.4548 \\
\hline C60 & 3.496933 & -2.39373 & -0.05219 \\
\hline C61 & 4.566038 & -1.822 & 0.643636 \\
\hline C62 & 5.661984 & -1.30081 & -0.04912 \\
\hline H63 & 6.55383 & -0.93292 & -1.97651 \\
\hline H64 & 4.642854 & -1.92181 & -3.23109 \\
\hline H65 & 2.698177 & -2.81598 & -2.01678 \\
\hline H66 & 4.549257 & -1.77651 & 1.7263 \\
\hline H67 & 6.485744 & -0.86281 & 0.50701 \\
\hline C68 & 1.248223 & 3.664067 & -2.62831 \\
\hline C69 & 1.0011 & 2.171349 & -2.2773 \\
\hline $\mathrm{C} 70$ & 0.333892 & 1.903567 & -0.89098 \\
\hline C71 & -0.88843 & 1.991412 & -4.11181 \\
\hline C72 & 0.146961 & 0.108107 & -3.28964 \\
\hline $\mathrm{C} 73$ & -1.29296 & 0.749917 & -4.92372 \\
\hline H74 & -0.56405 & 2.806314 & -4.75729 \\
\hline $\mathrm{H} 75$ & -1.69095 & 2.34937 & -3.45922 \\
\hline H76 & -2.3715 & 0.619157 & -5.02249 \\
\hline H77 & -0.82587 & 0.727932 & -5.91262 \\
\hline N78 & 0.221717 & 1.450154 & -3.31815 \\
\hline O79 & -0.77211 & -0.36784 & -4.1565 \\
\hline O80 & 0.792387 & -0.67613 & -2.56799 \\
\hline
\end{tabular}




$\begin{array}{lrrr}\text { H81 } & 2.01526 & -5.16092 & 1.033633 \\ \text { N82 } & -1.91532 & -2.00493 & -0.37435 \\ \text { C83 } & -1.02277 & -2.89758 & -1.15789 \\ \text { H84 } & -0.72636 & -2.42906 & -2.10386 \\ \text { H85 } & -0.73382 & 2.091403 & -1.0369 \\ \text { C86 } & 1.583404 & 4.379187 & 2.436092 \\ \text { C87 } & 0.231317 & 4.214188 & 2.133408 \\ \text { C88 } & -0.15291 & 3.441708 & 1.036759 \\ \text { C89 } & 0.798423 & 2.802731 & 0.224756 \\ \text { C90 } & 2.155806 & 2.997413 & 0.531949 \\ \text { C91 } & 2.543951 & 3.774395 & 1.622061 \\ \text { H92 } & 1.886444 & 4.981967 & 3.287193 \\ \text { H93 } & -0.52658 & 4.696749 & 2.745363 \\ \text { H94 } & -1.2093 & 3.328009 & 0.800711 \\ \text { H95 } & 2.91436 & 2.53783 & -0.09359 \\ \text { H96 } & 3.600763 & 3.90925 & 1.836453 \\ \text { C97 } & 1.991623 & 3.828135 & -3.96315 \\ \text { H98 } & 2.892933 & 3.206213 & -3.99487 \\ \text { H99 } & 1.376276 & 3.559339 & -4.82801 \\ \text { H100 } & 2.30001 & 4.869712 & -4.0981 \\ \text { C101 } & 0.032529 & 4.601154 & -2.51609 \\ \text { H102 } & -0.49233 & 4.468025 & -1.56709 \\ \text { H103 } & 0.375315 & 5.639957 & -2.55593 \\ \text { H104 } & -0.6892 & 4.477899 & -3.32748 \\ \text { H105 } & 1.942313 & 3.987905 & -1.84608 \\ \text { H107 } & 3.310868 & 0.471611 & -0.37389 \\ \text { H108 } & 3.287537 & 0.320133 & -1.10657 \\ & & & \end{array}$

C3_ZS_Cheltop

C3_ZS_Cheltop_SM

$\begin{array}{lrrrlrrr}\text { C1 } & -3.9825 & -3.39713 & -0.74319 & \mathrm{C} 1 & -3.7376 & -4.15979 & -0.76753 \\ \mathrm{C} 2 & -1.7632 & -4.41248 & -0.77251 & \mathrm{C} 2 & -1.432 & -4.92519 & -0.55026 \\ \mathrm{C} 3 & -3.0528 & -4.29503 & -1.62635 & \mathrm{C} 3 & -2.6547 & -5.02208 & -1.49666 \\ \mathrm{C} 4 & -3.0978 & -3.13606 & 0.497881 & \mathrm{C} 4 & -2.9794 & -3.68937 & 0.496529 \\ \mathrm{H} 5 & -4.8983 & -3.92313 & -0.45436 & \mathrm{H} 5 & -4.6097 & -4.75812 & -0.48455 \\ \text { H6 } & -2.8584 & -3.86286 & -2.61329 & \mathrm{H} 6 & -2.4282 & -4.65487 & -2.50254 \\ \text { H7 } & -3.6204 & -2.74861 & 1.372277 & \mathrm{H} 7 & -3.5991 & -3.28239 & 1.295316 \\ \text { H8 } & -4.273 & -2.466 & -1.23281 & \mathrm{H} 8 & -4.0903 & -3.32031 & -1.36709 \\ \text { H9 } & -1.0871 & -5.21641 & -1.06389 & \text { H9 } & -0.6593 & -5.67511 & -0.71646 \\ \text { H10 } & -3.486 & -5.28678 & -1.78401 & \text { H10 } & -2.9689 & -6.06521 & -1.59248 \\ \text { C11 } & -2.3435 & -4.46683 & 0.658105 & \text { C11 } & -2.1124 & -4.91296 & 0.8363 \\ \text { H12 } & -1.5851 & -4.44182 & 1.44604 & \text { H12 } & -1.4262 & -4.73742 & 1.67059 \\ \text { H13 } & -3.0059 & -5.32316 & 0.813004 & \text { H13 } & -2.6975 & -5.81664 & 1.028415 \\ \text { C14 } & 0.32964 & -2.96266 & -0.27405 & \text { C14 } & 0.47108 & -3.26417 & -0.02371 \\ \text { N15 } & 0.98401 & -1.87155 & -0.05726 & \text { N15 } & 0.93988 & -2.12147 & 0.334915\end{array}$




\begin{tabular}{|c|c|c|c|c|c|c|c|}
\hline $\mathrm{C} 16$ & 2.32103 & -2.22668 & 0.558691 & C16 & 2.30778 & -2.35072 & 0.940872 \\
\hline $\mathrm{C} 17$ & 2.32208 & -3.77854 & 0.351499 & $\mathrm{C} 17$ & 2.52132 & -3.87585 & 0.654105 \\
\hline P18 & -1.8892 & -0.56048 & 0.26754 & P18 & -2.0564 & -1.03234 & 0.131689 \\
\hline Ir19 & 0.31609 & 0.07191 & -0.64967 & Ir19 & 0.03653 & -0.19328 & -0.42745 \\
\hline $\mathrm{C} 20$ & -2.2825 & -0.27233 & 2.032207 & $\mathrm{C} 20$ & -2.5999 & -0.70382 & 1.859106 \\
\hline $\mathrm{C} 21$ & -2.7077 & 0.214137 & 4.76517 & $\mathrm{C} 21$ & -3.2091 & -0.1671 & 4.556167 \\
\hline $\mathrm{C} 22$ & -2.0092 & -1.26689 & 2.984809 & $\mathrm{C} 22$ & -2.1183 & -1.53418 & 2.882844 \\
\hline $\mathrm{C} 23$ & -2.765 & 0.972762 & 2.468162 & $\mathrm{C} 23$ & -3.4236 & 0.379044 & 2.206667 \\
\hline $\mathrm{C} 24$ & -2.973 & 1.211003 & 3.825363 & $\mathrm{C} 24$ & -3.7209 & 0.644946 & 3.543337 \\
\hline $\mathrm{C} 25$ & -2.2257 & -1.02519 & 4.340898 & $\mathrm{C} 25$ & -2.4164 & -1.26529 & 4.218784 \\
\hline H26 & -2.9872 & 1.757406 & 1.755898 & $\mathrm{H} 26$ & -3.8595 & 1.000343 & 1.435235 \\
\hline $\mathrm{H} 27$ & -3.3438 & 2.180879 & 4.143893 & $\mathrm{H} 27$ & -4.3655 & 1.483966 & 3.790869 \\
\hline $\mathrm{H} 28$ & -2.0117 & -1.80622 & 5.064843 & $\mathrm{H} 28$ & -2.0284 & -1.91936 & 4.99442 \\
\hline $\mathrm{H} 29$ & -2.8743 & 0.401608 & 5.822092 & $\mathrm{H} 29$ & -3.4402 & 0.042843 & 5.59625 \\
\hline $\mathrm{O} 30$ & 0.9477 & -4.10796 & -0.00103 & $\mathrm{O} 30$ & 1.24134 & -4.34086 & 0.147558 \\
\hline C31 & 2.35088 & -1.93246 & 2.061578 & C 31 & 2.32196 & -2.11945 & 2.453381 \\
\hline C32 & 1.31717 & -1.28778 & 2.735512 & $\mathrm{C} 32$ & 1.23682 & -1.59399 & 3.147793 \\
\hline C33 & 3.46863 & -2.36598 & 2.792426 & $\mathrm{C} 33$ & 3.48614 & -2.45099 & 3.16584 \\
\hline C34 & 1.39442 & -1.07034 & 4.112693 & $\mathrm{C} 34$ & 1.30573 & -1.39205 & 4.528236 \\
\hline H35 & 0.45896 & -0.92485 & 2.191456 & H35 & 0.352 & -1.30103 & 2.610501 \\
\hline C36 & 3.54931 & -2.14905 & 4.164828 & $\mathrm{C} 36$ & 3.55548 & -2.25661 & 4.542703 \\
\hline H37 & 4.29237 & -2.85675 & 2.278972 & H37 & 4.35258 & -2.8435 & 2.638747 \\
\hline C38 & 2.50845 & -1.4971 & 4.831146 & $\mathrm{C} 38$ & 2.46192 & -1.72291 & 5.230427 \\
\hline H39 & 0.57602 & -0.56196 & 4.613712 & H39 & 0.44988 & -0.96321 & 5.042171 \\
\hline $\mathrm{H} 40$ & 4.4252 & -2.48433 & 4.71274 & $\mathrm{H} 40$ & 4.4648 & -2.51418 & 5.078011 \\
\hline H41 & 2.57059 & -1.3238 & 5.901709 & $\mathrm{H} 41$ & 2.5182 & -1.5645 & 6.303616 \\
\hline $\mathrm{C} 42$ & -3.3552 & 0.064711 & -0.66294 & $\mathrm{C} 42$ & -3.5059 & -0.69772 & -0.93287 \\
\hline $\mathrm{C} 43$ & -4.6009 & 0.297382 & -0.06158 & $\mathrm{C} 43$ & -4.8176 & -0.83564 & -0.44913 \\
\hline $\mathrm{C} 44$ & -3.2508 & 0.177469 & -2.06033 & $\mathrm{C} 44$ & -3.3001 & -0.54858 & -2.31151 \\
\hline $\mathrm{C} 45$ & -5.7027 & 0.670074 & -0.83362 & $\mathrm{C} 45$ & -5.8984 & -0.81198 & -1.32918 \\
\hline H46 & -4.7213 & 0.189345 & 1.010628 & $\mathrm{H} 46$ & -5.0013 & -0.97959 & 0.610312 \\
\hline $\mathrm{C} 47$ & -4.3536 & 0.545513 & -2.83013 & $\mathrm{C} 47$ & -4.384 & -0.53364 & -3.18825 \\
\hline $\mathrm{C} 48$ & -5.5832 & 0.802022 & -2.2176 & $\mathrm{C} 48$ & -5.6855 & -0.66581 & -2.7015 \\
\hline H49 & -6.6575 & 0.852884 & -0.34896 & H49 & -6.9075 & -0.91888 & -0.94148 \\
\hline H50 & -4.2526 & 0.631355 & -3.90841 & $\mathrm{H} 50$ & -4.2102 & -0.40952 & -4.253 \\
\hline H51 & -6.4418 & 1.093309 & -2.81522 & $\mathrm{H} 51$ & -6.5283 & -0.65375 & -3.38593 \\
\hline H52 & -0.278 & -0.51192 & -1.96569 & H52 & -0.2656 & -0.82386 & -1.8367 \\
\hline H53 & 1.72312 & 0.018467 & -1.38447 & $\mathrm{H} 53$ & 1.4913 & 2.42071 & -0.29219 \\
\hline H54 & -1.6123 & -2.22501 & 2.667749 & H54 & -1.5102 & -2.39589 & 2.631909 \\
\hline H55 & -2.304 & -0.02859 & -2.54969 & H55 & -2.2917 & -0.43277 & -2.6936 \\
\hline H56 & 2.96013 & -4.11436 & -0.46819 & H56 & 3.2779 & -4.08512 & -0.10448 \\
\hline C57 & 5.38909 & -0.18815 & -1.74243 & C57 & 4.83632 & 0.391301 & -1.26731 \\
\hline C58 & 4.64442 & -1.2223 & -2.31284 & C58 & 4.39103 & -0.79103 & -1.86378 \\
\hline C59 & 3.67557 & -1.88073 & -1.55887 & C59 & 3.62799 & -1.69943 & -1.13127 \\
\hline C60 & 3.44125 & -1.53001 & -0.22158 & C60 & 3.30143 & -1.44583 & 0.207408 \\
\hline
\end{tabular}




\begin{tabular}{|c|c|c|c|c|c|c|c|}
\hline C61 & 4.18192 & -0.48283 & 0.336297 & C61 & 3.75252 & -0.25864 & 0.797945 \\
\hline C62 & 5.14882 & 0.182018 & -0.4195 & C62 & 4.51625 & 0.652745 & 0.064992 \\
\hline H63 & 6.14786 & 0.324595 & -2.32684 & H63 & 5.43075 & 1.099959 & -1.83643 \\
\hline H64 & 4.81325 & -1.51227 & -3.34587 & H64 & 4.63402 & -1.00395 & -2.90052 \\
\hline H65 & 3.08002 & -2.65579 & -2.03404 & H65 & 3.25573 & -2.59104 & -1.62821 \\
\hline H66 & 3.99335 & -0.17132 & 1.356091 & H66 & 3.48854 & -0.0352 & 1.825336 \\
\hline H67 & 5.71955 & 0.98767 & 0.034945 & H67 & 4.85908 & 1.56821 & 0.539122 \\
\hline C68 & 1.09976 & 2.184765 & -2.9974 & C68 & 0.61954 & 4.232777 & -0.98429 \\
\hline C69 & 0.72311 & 2.029038 & -1.51524 & C69 & 0.47036 & 2.815358 & -0.36949 \\
\hline $\mathrm{C} 70$ & -0.6365 & 2.027849 & -1.07288 & $\mathrm{C} 70$ & -0.3684 & 1.781021 & -1.19394 \\
\hline H71 & -1.3414 & 1.839404 & -1.8711 & H71 & 0.12005 & 1.751331 & -2.17207 \\
\hline $\mathrm{C} 72$ & -1.2567 & 2.891685 & -0.02846 & $\mathrm{C} 72$ & -1.7801 & 2.237385 & -1.43712 \\
\hline $\mathrm{C} 73$ & -2.494 & 3.467449 & -0.37839 & C73 & -2.2329 & 2.490674 & -2.74035 \\
\hline $\mathrm{C} 74$ & -0.7233 & 3.206348 & 1.233934 & C74 & -2.6927 & 2.435501 & -0.39206 \\
\hline $\mathrm{C} 75$ & -3.1499 & 4.352326 & 0.474519 & C75 & -3.5329 & 2.932917 & -2.98667 \\
\hline H76 & -2.9489 & 3.20921 & -1.33088 & H76 & -1.5608 & 2.32184 & -3.57856 \\
\hline C77 & -1.3723 & 4.106141 & 2.07915 & C77 & -3.9919 & 2.880278 & -0.62724 \\
\hline $\mathrm{H} 78$ & 0.15165 & 2.699014 & 1.609064 & H78 & -2.3883 & 2.202501 & 0.618987 \\
\hline C79 & -2.5826 & 4.68962 & 1.704875 & C79 & -4.4205 & 3.134903 & -1.93038 \\
\hline H80 & -4.1035 & 4.778263 & 0.176008 & H80 & -3.8544 & 3.114044 & -4.00859 \\
\hline H81 & -0.9381 & 4.327624 & 3.050099 & H81 & -4.6761 & 3.017064 & 0.20715 \\
\hline H82 & -3.0865 & 5.385187 & 2.369746 & H82 & -5.4339 & 3.475646 & -2.12008 \\
\hline $\mathrm{C} 83$ & 2.16468 & 4.04194 & -0.72817 & $\mathrm{C} 83$ & -0.7229 & 4.060095 & 1.656579 \\
\hline $\mathrm{C} 84$ & 2.03844 & 2.114143 & 0.546573 & $\mathrm{C} 84$ & -0.0161 & 1.865865 & 1.84657 \\
\hline $\mathrm{C} 85$ & 3.2258 & 4.039402 & 0.390344 & $\mathrm{C} 85$ & -0.8247 & 3.565468 & 3.114851 \\
\hline H86 & 1.33201 & 4.726794 & -0.5272 & H86 & -1.7007 & 4.203193 & 1.188538 \\
\hline H87 & 2.59721 & 4.272823 & -1.70367 & H87 & -0.1655 & 4.994315 & 1.586522 \\
\hline H88 & 4.23325 & 3.865243 & 0.001162 & H88 & -0.0809 & 4.031958 & 3.767636 \\
\hline H89 & 3.21596 & 4.936214 & 1.010168 & H89 & -1.8188 & 3.68607 & 3.547654 \\
\hline N90 & 1.71021 & 2.651726 & -0.64702 & N90 & -0.0034 & 2.938811 & 1.035855 \\
\hline O91 & 2.87277 & 2.906447 & 1.232989 & O91 & -0.5383 & 2.144989 & 3.051984 \\
\hline O92 & 1.60677 & 1.047171 & 1.004686 & O92 & 0.38397 & 0.719762 & 1.585328 \\
\hline H93 & 2.56858 & -4.32406 & 1.260484 & H93 & 2.74333 & -4.44132 & 1.557728 \\
\hline N94 & -1.9616 & -2.24698 & 0.126793 & N94 & -1.9113 & -2.72187 & 0.117564 \\
\hline C95 & -1.0863 & -3.01881 & -0.78487 & C95 & -0.8895 & -3.47394 & -0.6391 \\
\hline H96 & -1.0701 & -2.60576 & -1.80287 & H96 & -0.8168 & -3.14834 & -1.68664 \\
\hline C97 & 2.51758 & 1.68591 & -3.30861 & C97 & 1.7652 & 4.996274 & -0.28963 \\
\hline H98 & 2.82564 & 2.021413 & -4.30402 & H98 & 1.7662 & 6.048927 & -0.58843 \\
\hline H99 & 3.25446 & 2.04314 & -2.58374 & H99 & 1.72078 & 4.95623 & 0.802357 \\
\hline H100 & 2.55352 & 0.593142 & -3.28845 & H100 & 2.73041 & 4.567942 & -0.58464 \\
\hline C101 & 0.09302 & 1.580093 & -3.98267 & C101 & 0.90929 & 4.189731 & -2.49148 \\
\hline H102 & 0.03463 & 0.495229 & -3.85661 & H102 & 1.76381 & 3.537814 & -2.71031 \\
\hline H103 & -0.9119 & 1.998579 & -3.87343 & H103 & 0.05217 & 3.843484 & -3.06905 \\
\hline H104 & 0.4195 & 1.785086 & -5.00638 & H104 & 1.16325 & 5.193263 & -2.84652 \\
\hline H105 & 1.08242 & 3.275146 & -3.16346 & H105 & -0.3265 & 4.771469 & -0.84293 \\
\hline
\end{tabular}


C3_ZS_Cheltop_TS1$$
\mathrm{C} 1
$$

$\mathrm{C} 2$

C3

C4

H5

H6

$\mathrm{H} 7$

H8

H9

H10

C11

H12

H13

C14

N15

C16

C17

P18

Ir19

C20

C21

$\mathrm{C} 22$

$\mathrm{C} 23$

C24

C25

H 26

H27

H28

H29

O30

C31

C32

C33

C34

H35

C36

H37

C38

H39

H40

H4 1

C42

C43
$-3.91$

$-1.6608$

$-2.9491$

$-3.0448$

$-4.8186$

$-2.7627$

$-3.5858$

$-4.212$

$-0.9576$

$-3.3528$

$-2.2494$

$-1.497$

$-2.8844$

0.39179

1.00515

2.36602

2.41361

$-1.8945$

0.14087

$-2.2189$

$-2.5651$

$-1.9696$

$-2.6428$

$-2.8099$

$-2.1467$

$-2.8472$

$-3.1364$

$-1.9523$

$-3.63089$

$-0.75081$

$-4.58155$

$-0.77398$

$-4.49655$

$-1.63398$

$-3.3646$

$-4.1791$

0.502611

$-4.05438$

$-3.00428$

$-2.69729$

$-5.36003$

$-5.49962$

$-4.67313$

$-0.48216$

$-2.61798$

1.377872

$-1.23025$

$-1.06979$

$-1.79807$

$-4.63363$

$-5.55259$

0.651305

1.444612

$-3.06336$

0.790649

$-1.95275$

$-0.2698$

$-0.04248$

$-2.26487$

0.520077

$-3.82331$

0.335154

$-0.75864$

0.333207

$\begin{array}{ll}-0.0026 & -0.53731\end{array}$

$-0.49817$

2.114683

$-0.02649$

4.858484

$-1.51598$

3.046768

0.760085

2.572792

$0.992894 \quad 3.936373$

$-1.28027$

4.410581

$\begin{array}{ll}1.557901 & 1.868008\end{array}$

$1.971798 \quad 4.275951$

$-2.07779$

5.121962

$-2.6998$

$0.155421 \quad 5.920827$

1.05944

$-4.19522$

$-0.04456$

2.45415

$-1.95778$

1.38484

$-1.44976$

2.018061

2.750322

3.6483

$-2.26713$

2.688585

1.50483

$-1.22995$

4.123914

0.46419

$-1.19136$

2.25049

3.77094

$-2.0491$

4.057707

4.49383

$-2.66225$

2.130435

2.69696

$-1.52368$

4.781013

0.65927

$-0.82146$

4.669488

4.7055

$-2.28414$

4.55893

2.79342

$-3.3921$

$-1.34691$

5.848429

$-4.6165$

$-0.08984$

$-0.50653$

$0.062927 \quad 0.162903$
H106

H107

1.73351

0.347158

$-0.61627$

1.51709

0.051438

$-1.35887$

C3_ZS_Cheltop_TS3

C1
C2
C3
C4
H5
H6
H7
H8
H9

H9

H10

C11

H12

H13

C14

N15

$\mathrm{C} 16$

C17

P18

Ir19

C20

$\mathrm{C} 21$

$\mathrm{C} 22$

$\mathrm{C} 23$

$\mathrm{C} 24$

$\mathrm{C} 25$

$\mathrm{H} 26$

$\mathrm{H} 27$

$\mathrm{H} 28$

H29

O30

C31

$\mathrm{C} 32$

$\mathrm{C} 33$

C34

H35

C36

H37

C38

H39

$\mathrm{H} 40$

H41 $\begin{array}{rrr}-3.7035 & -4.30885 & -0.77303 \\ -1.3634 & -4.96963 & -0.53593 \\ -2.5698 & -5.10392 & -1.50255 \\ -2.9828 & -3.82418 & 0.505637 \\ -4.5503 & -4.95318 & -0.51435 \\ -2.348 & -4.70777 & -2.49872 \\ -3.6313 & -3.45583 & 1.300976 \\ -4.088 & -3.47261 & -1.35855 \\ -0.5572 & -5.68285 & -0.7048\end{array}$

$\begin{array}{lll}-2.8339 & -6.15837 & -1.62245\end{array}$

$\begin{array}{lll}-2.0631 & -5.01059 & 0.840208\end{array}$

$\begin{array}{lll}-1.3946 & -4.8156 & 1.684245\end{array}$

$\begin{array}{lll}-2.6064 & -5.94408 & 1.011308\end{array}$

$\begin{array}{llll}0.45449 & -3.21383 & 0.034232\end{array}$

$\begin{array}{llll}0.92178 & -2.04434 & 0.323096\end{array}$

$\begin{array}{llll}2.28042 & -2.22378 & 0.978105\end{array}$

$\begin{array}{llll}2.52015 & -3.7501 & 0.736559\end{array}$

$\begin{array}{lll}-2.1402 & -1.11693 & 0.162751\end{array}$

$\begin{array}{lll}0.07188 & -0.24591 & -0.52912\end{array}$

$\begin{array}{lll}-2.6946 & -0.81578 & 1.892165\end{array}$

$\begin{array}{lll}-3.3157 & -0.28866 & 4.588139\end{array}$

$\begin{array}{lll}-2.2649 & -1.67949 & 2.910715\end{array}$

$\begin{array}{lll}-3.4647 & 0.30364 & 2.246915\end{array}$

$\begin{array}{lll}-3.7645 & 0.568593 & 3.582774\end{array}$

$\begin{array}{lll}-2.5749 & -1.42012 & 4.245445\end{array}$

$\begin{array}{lll}-3.865 & 0.950268 & 1.477098\end{array}$

$\begin{array}{lll}-4.366 & 1.437612 & 3.836093\end{array}$

$\begin{array}{lll}-2.2374 & -2.10738 & 5.016287\end{array}$

$\begin{array}{lll}-3.5545 & -0.08546 & 5.628127\end{array}$

$\begin{array}{lll}1.23277 & -4.26168 & 0.29257\end{array}$

$\begin{array}{lll}2.23057 & -1.97755 & 2.489511\end{array}$

$\begin{array}{lll}1.08399 & -1.55828 & 3.155965\end{array}$

$\begin{array}{lll}3.39625 & -2.22032 & 3.233982\end{array}$

$\begin{array}{llll}1.0966 & -1.36228 & 4.538742\end{array}$

$\begin{array}{lll}0.18925 & -1.34272 & 2.597543\end{array}$

$\begin{array}{lll}3.41016 & -2.03416 & 4.613355\end{array}$

$\begin{array}{lll}4.30485 & -2.53728 & 2.727935\end{array}$

$\begin{array}{llll}2.25672 & -1.59818 & 5.272162\end{array}$

$\begin{array}{llll}0.19289 & -1.01307 & 5.030254\end{array}$

$\begin{array}{lll}4.32185 & -2.22224 & 5.173285\end{array}$

$\begin{array}{llll}2.26884 & -1.44323 & 6.347447\end{array}$ 


\begin{tabular}{|c|c|c|c|c|c|c|c|}
\hline $\mathrm{C} 44$ & -3.3451 & 0.156321 & -1.88887 & $\mathrm{C} 42$ & -3.6231 & -0.77465 & -0.86957 \\
\hline $\mathrm{C} 45$ & -5.7548 & 0.481733 & -0.52705 & $\mathrm{C} 43$ & -4.9328 & -0.91727 & -0.3837 \\
\hline $\mathrm{H} 46$ & -4.6896 & -0.14178 & 1.225516 & $\mathrm{C} 44$ & -3.4315 & -0.51578 & -2.23415 \\
\hline $\mathrm{C} 47$ & -4.4852 & 0.56886 & -2.57693 & $\mathrm{C} 45$ & -6.0228 & -0.79361 & -1.2447 \\
\hline $\mathrm{C} 48$ & -5.6932 & 0.740943 & -1.89669 & $\mathrm{H} 46$ & -5.1058 & -1.12888 & 0.666886 \\
\hline $\mathrm{H} 49$ & -6.6916 & 0.602089 & 0.009444 & $\mathrm{C} 47$ & -4.5223 & -0.39665 & -3.09451 \\
\hline H50 & -4.43 & 0.757688 & -3.64538 & $\mathrm{C} 48$ & -5.8215 & -0.53534 & -2.60279 \\
\hline H5 1 & -6.5798 & 1.068543 & -2.43125 & H49 & -7.0306 & -0.90637 & -0.85479 \\
\hline H52 & -0.4406 & -0.58929 & -1.8569 & $\mathrm{H} 50$ & -4.3569 & -0.19028 & -4.14788 \\
\hline H53 & 1.50773 & 0.4522 & -1.39359 & H51 & -6.6716 & -0.44394 & -3.27239 \\
\hline H54 & -1.6195 & -2.48555 & 2.709389 & H52 & 0.05352 & -1.07572 & -1.85972 \\
\hline H55 & -2.4149 & 0.017838 & -2.42951 & $\mathrm{H} 53$ & 1.5718 & 2.334211 & -0.39753 \\
\hline H56 & 3.08423 & -4.15662 & -0.4589 & H54 & -1.6863 & -2.5606 & 2.658095 \\
\hline C57 & 5.4102 & -0.25761 & -1.8489 & $\mathrm{H} 55$ & -2.4253 & -0.40569 & -2.62573 \\
\hline C58 & 4.68722 & -1.32584 & -2.38282 & H56 & 3.2502 & -3.97119 & -0.04415 \\
\hline C59 & 3.70013 & -1.95102 & -1.62236 & C57 & 4.92059 & 0.446754 & -1.18575 \\
\hline C60 & 3.43373 & -1.54002 & -0.30877 & C58 & 4.49951 & -0.75059 & -1.76711 \\
\hline C61 & 4.13543 & -0.4406 & 0.201231 & C59 & 3.68733 & -1.62597 & -1.04982 \\
\hline C62 & 5.11759 & 0.192437 & -0.56215 & $\mathrm{C} 60$ & 3.28881 & -1.32665 & 0.258959 \\
\hline H63 & 6.18631 & 0.224565 & -2.43626 & C61 & 3.70875 & -0.121 & 0.831094 \\
\hline H64 & 4.89042 & -1.67322 & -3.39174 & C62 & 4.5211 & 0.758276 & 0.114417 \\
\hline H65 & 3.13399 & -2.76498 & -2.06745 & H63 & 5.55488 & 1.130635 & -1.74286 \\
\hline H66 & 3.92273 & -0.08528 & 1.202473 & H64 & 4.79645 & -1 & -2.78167 \\
\hline H67 & 5.6645 & 1.031587 & -0.14036 & $\mathrm{H} 65$ & 3.33399 & -2.52828 & -1.54088 \\
\hline C68 & 1.37409 & 2.091291 & -2.95459 & H66 & 3.38424 & 0.140654 & 1.831515 \\
\hline C69 & 0.90983 & 1.88153 & -1.49978 & H67 & 4.84277 & 1.688568 & 0.575181 \\
\hline $\mathrm{C} 70$ & -0.5246 & 1.916966 & -1.16603 & C68 & 0.79136 & 4.242575 & -1.01953 \\
\hline H71 & -1.1501 & 1.804603 & -2.04546 & C69 & 0.58921 & 2.812254 & -0.43721 \\
\hline $\mathrm{C} 72$ & -1.1164 & 2.97213 & -0.27627 & $\mathrm{C} 70$ & -0.3231 & 1.908398 & -1.32692 \\
\hline $\mathrm{C} 73$ & -1.943 & 3.926776 & -0.89593 & H71 & 0.14077 & 1.970092 & -2.31207 \\
\hline C74 & -0.9013 & 3.091127 & 1.105744 & $\mathrm{C} 72$ & -1.7577 & 2.366717 & -1.44575 \\
\hline $\mathrm{C} 75$ & -2.5104 & 4.973689 & -0.173 & $\mathrm{C} 73$ & -2.2796 & 2.718212 & -2.69791 \\
\hline H76 & -2.1423 & 3.843322 & -1.96179 & C74 & -2.6197 & 2.400693 & -0.34261 \\
\hline C77 & -1.4602 & 4.14685 & 1.829348 & $\mathrm{C} 75$ & -3.6116 & 3.109815 & -2.83893 \\
\hline H78 & -0.3438 & 2.331837 & 1.637202 & H76 & -1.6446 & 2.665475 & -3.57881 \\
\hline C79 & -2.2616 & 5.095292 & 1.195486 & C77 & -3.9469 & 2.802609 & -0.47586 \\
\hline H80 & -3.1475 & 5.694178 & -0.67819 & $\mathrm{H} 78$ & -2.2617 & 2.067475 & 0.62235 \\
\hline H81 & -1.2784 & 4.211956 & 2.898904 & C79 & -4.4506 & 3.15924 & -1.72683 \\
\hline H82 & -2.6998 & 5.912006 & 1.761771 & H80 & -3.9961 & 3.366094 & -3.82179 \\
\hline $\mathrm{C} 83$ & 2.14994 & 3.949141 & -0.57458 & H81 & -4.598 & 2.817878 & 0.394356 \\
\hline $\mathrm{C} 84$ & 1.88702 & 2.056346 & 0.731206 & H82 & -5.4896 & 3.455396 & -1.83458 \\
\hline $\mathrm{C} 85$ & 3.00501 & 4.031088 & 0.704255 & $\mathrm{C} 83$ & -0.5756 & 4.053318 & 1.594783 \\
\hline H86 & 1.24854 & 4.571071 & -0.52483 & $\mathrm{C} 84$ & 0.08366 & 1.84138 & 1.761543 \\
\hline H87 & 2.7194 & 4.201694 & -1.47095 & $\mathrm{C} 85$ & -0.6308 & 3.562215 & 3.055282 \\
\hline H88 & 4.0711 & 3.899721 & 0.497033 & H86 & -1.5688 & 4.197218 & 1.158361 \\
\hline
\end{tabular}




\begin{tabular}{|c|c|c|c|c|c|c|c|}
\hline H89 & 2.8458 & 4.941639 & 1.281978 & H87 & -0.0169 & 4.984954 & 1.505753 \\
\hline N90 & 1.78969 & 2.528226 & -0.53809 & H88 & 0.16253 & 3.996259 & 3.671123 \\
\hline O91 & 2.565 & 2.904304 & 1.513238 & H89 & -1.5977 & 3.722531 & 3.533734 \\
\hline O92 & 1.41138 & 0.995151 & 1.152714 & N90 & 0.1204 & 2.924472 & 0.959146 \\
\hline H93 & 2.65266 & -4.34421 & 1.261074 & O91 & -0.4073 & 2.131447 & 2.976296 \\
\hline N94 & -1.9365 & -2.43773 & 0.149965 & O92 & 0.42694 & 0.688309 & 1.476885 \\
\hline C95 & -1.0294 & -3.16641 & -0.76805 & H93 & 2.78602 & -4.27954 & 1.649801 \\
\hline H96 & -1.0343 & -2.74172 & -1.7813 & N94 & -1.9627 & -2.80318 & 0.145774 \\
\hline C97 & 2.85395 & 1.750256 & -3.15634 & C95 & -0.8905 & -3.49354 & -0.59803 \\
\hline H98 & 3.17849 & 2.053686 & -4.15606 & H96 & -0.8179 & -3.15922 & -1.64275 \\
\hline H99 & 3.50629 & 2.232909 & -2.42473 & C97 & 1.97797 & 4.926278 & -0.31328 \\
\hline H100 & 3.01056 & 0.671739 & -3.06306 & H98 & 2.02045 & 5.987636 & -0.57608 \\
\hline $\mathrm{C} 101$ & 0.51847 & 1.334646 & -3.97813 & H99 & 1.93787 & 4.849801 & 0.776792 \\
\hline H102 & 0.57625 & 0.256293 & -3.79968 & H100 & 2.91896 & 4.465253 & -0.63377 \\
\hline H103 & -0.5332 & 1.631349 & -3.95598 & C101 & 1.04546 & 4.257512 & -2.5347 \\
\hline H104 & 0.89425 & 1.534529 & -4.98554 & H102 & 1.85864 & 3.5762 & -2.81122 \\
\hline H105 & 1.22566 & 3.169104 & -3.12834 & H103 & 0.15662 & 3.993632 & -3.11055 \\
\hline $\mathrm{X}$ & 0 & 0 & 0 & H104 & 1.34124 & 5.264822 & -2.84392 \\
\hline & & & & H105 & -0.1275 & 4.815356 & -0.83933 \\
\hline \multicolumn{4}{|c|}{ C3_ZS_Cheltop_INT1 } & H106 & 1.53035 & 0.14181 & -1.03154 \\
\hline $\mathrm{C} 1$ & -3.775 & -3.59274 & -1.4966 & H107 & -0.5987 & 0.498669 & -1.74381 \\
\hline $\mathrm{C} 2$ & -1.6 & -4.576 & -0.94604 & & & & \\
\hline $\mathrm{C} 3$ & -2.641 & -4.49469 & -2.09664 & \multicolumn{4}{|c|}{ C3_ZS_Cheltop_PROD } \\
\hline $\mathrm{C} 4$ & -3.2128 & -3.26743 & -0.09513 & $\mathrm{C} 1$ & -3.6213 & -4.5574 & -0.57437 \\
\hline H5 & -4.7247 & -4.13207 & -1.42037 & $\mathrm{C} 2$ & -1.2541 & -5.14108 & -0.46282 \\
\hline H6 & -2.211 & -4.08094 & -3.01426 & $\mathrm{C} 3$ & -2.5077 & -5.33251 & -1.35532 \\
\hline H7 & -3.9209 & -2.84378 & 0.618627 & $\mathrm{C} 4$ & -2.853 & -4.05082 & 0.668342 \\
\hline H8 & -3.9495 & -2.68055 & -2.07191 & H5 & -4.4425 & -5.21751 & -0.2763 \\
\hline H9 & -0.8793 & -5.39021 & -1.03086 & H6 & -2.3564 & -4.95179 & -2.3703 \\
\hline H10 & -3.012 & -5.49581 & -2.33324 & $\mathrm{H} 7$ & -3.4746 & -3.70869 & 1.495984 \\
\hline C11 & -2.5173 & -4.58368 & 0.296334 & $\mathrm{H} 8$ & -4.0484 & -3.729 & -1.14314 \\
\hline H12 & -1.9747 & -4.53444 & 1.243917 & H9 & -0.4299 & -5.82252 & -0.67284 \\
\hline H13 & -3.1988 & -5.43891 & 0.312738 & H10 & -2.7451 & -6.397 & -1.43767 \\
\hline $\mathrm{C} 14$ & 0.27475 & -3.07158 & 0.064446 & $\mathrm{C} 11$ & -1.8718 & -5.20119 & 0.952056 \\
\hline N15 & 0.8996 & -1.96467 & 0.258982 & $\mathrm{H} 12$ & -1.1652 & -4.97539 & 1.756313 \\
\hline $\mathrm{C} 16$ & 2.07136 & -2.2253 & 1.156048 & H13 & -2.3679 & -6.15464 & 1.15455 \\
\hline $\mathrm{C} 17$ & 1.72484 & -3.6687 & 1.659285 & C14 & 0.52596 & -3.30366 & -0.04938 \\
\hline P18 & -1.9343 & -0.71875 & 0.014156 & N15 & 0.98376 & -2.10547 & 0.120783 \\
\hline Ir19 & 0.15034 & -0.01561 & -0.45145 & $\mathrm{C} 16$ & 2.35118 & -2.20387 & 0.777918 \\
\hline $\mathrm{C} 20$ & -2.2917 & -0.51682 & 1.80394 & $\mathrm{C} 17$ & 2.65831 & -3.70554 & 0.512692 \\
\hline $\mathrm{C} 21$ & -2.5102 & -0.16175 & 4.582705 & P18 & -2.0959 & -1.31184 & 0.254542 \\
\hline $\mathrm{C} 22$ & -2.1097 & -1.59938 & 2.677713 & Ir19 & 0.03309 & -0.39534 & -0.46867 \\
\hline $\mathrm{C} 23$ & -2.5765 & 0.749392 & 2.339839 & $\mathrm{C} 20$ & -2.6864 & -0.96114 & 1.957182 \\
\hline $\mathrm{C} 24$ & -2.6882 & 0.92145 & 3.719272 & $\mathrm{C} 21$ & -3.3987 & -0.27784 & 4.590768 \\
\hline $\mathrm{C} 25$ & -2.2219 & -1.42242 & 4.056431 & $\mathrm{C} 22$ & -2.3166 & -1.78124 & 3.032876 \\
\hline
\end{tabular}




\begin{tabular}{|c|c|c|c|c|c|c|c|}
\hline $\mathrm{H} 26$ & -2.7006 & 1.605836 & 1.688882 & $\mathrm{C} 23$ & -3.4293 & 0.201321 & 2.220871 \\
\hline $\mathrm{H} 27$ & -2.9045 & 1.909789 & 4.114076 & $\mathrm{C} 24$ & -3.7756 & 0.543068 & 3.526847 \\
\hline $\mathrm{H} 28$ & -2.0788 & -2.27013 & 4.720446 & $\mathrm{C} 25$ & -2.6769 & -1.44454 & 4.338001 \\
\hline H29 & -2.5918 & -0.02442 & 5.656941 & $\mathrm{H} 26$ & -3.7712 & 0.823301 & 1.400316 \\
\hline $\mathrm{O} 30$ & 0.70572 & -4.14987 & 0.73142 & $\mathrm{H} 27$ & -4.3557 & 1.443168 & 3.712431 \\
\hline C31 & 2.12061 & -1.27102 & 2.341874 & $\mathrm{H} 28$ & -2.3931 & -2.09873 & 5.157753 \\
\hline $\mathrm{C} 32$ & 1.16527 & -0.27809 & 2.565047 & $\mathrm{H} 29$ & -3.6751 & -0.01601 & 5.607998 \\
\hline $\mathrm{C} 33$ & 3.13745 & -1.43815 & 3.294206 & $\mathrm{O} 30$ & 1.35264 & -4.30169 & 0.257148 \\
\hline C34 & 1.2251 & 0.547175 & 3.688947 & $\mathrm{C} 31$ & 2.25292 & -1.98992 & 2.29345 \\
\hline H35 & 0.31467 & -0.16436 & 1.897435 & $\mathrm{C} 32$ & 1.06243 & -1.68253 & 2.94527 \\
\hline $\mathrm{C} 36$ & 3.20038 & -0.62263 & 4.421713 & $\mathrm{C} 33$ & 3.42015 & -2.152 & 3.057466 \\
\hline H37 & 3.89711 & -2.19951 & 3.138383 & $\mathrm{C} 34$ & 1.03307 & -1.51975 & 4.332137 \\
\hline $\mathrm{C} 38$ & 2.24575 & 0.377936 & 4.621669 & H35 & 0.15917 & -1.53189 & 2.376346 \\
\hline H39 & 0.45631 & 1.29976 & 3.8319 & $\mathrm{C} 36$ & 3.39236 & -1.99753 & 4.440049 \\
\hline $\mathrm{H} 40$ & 3.99918 & -0.76617 & 5.143647 & H37 & 4.3615 & -2.37824 & 2.56322 \\
\hline H41 & 2.29524 & 1.011754 & 5.502392 & C38 & 2.19407 & -1.67621 & 5.083893 \\
\hline $\mathrm{C} 42$ & -3.3393 & 0.034811 & -0.8805 & H39 & 0.09528 & -1.25791 & 4.812808 \\
\hline $\mathrm{C} 43$ & -4.556 & 0.352476 & -0.26127 & $\mathrm{H} 40$ & 4.30622 & -2.12084 & 5.014128 \\
\hline $\mathrm{C} 44$ & -3.2016 & 0.24118 & -2.26412 & $\mathrm{H} 41$ & 2.17192 & -1.54749 & 6.162318 \\
\hline $\mathrm{C} 45$ & -5.6077 & 0.885682 & -1.00978 & $\mathrm{C} 42$ & -3.5577 & -0.89683 & -0.78122 \\
\hline $\mathrm{H} 46$ & -4.687 & 0.191005 & 0.803678 & $\mathrm{C} 43$ & -4.8734 & -1.12257 & -0.33984 \\
\hline C47 & -4.2536 & 0.771408 & -3.00772 & $\mathrm{C} 44$ & -3.3566 & -0.36561 & -2.06368 \\
\hline $\mathrm{C} 48$ & -5.459 & 1.100967 & -2.38035 & $\mathrm{C} 45$ & -5.9573 & -0.80904 & -1.15753 \\
\hline $\mathrm{H} 49$ & -6.5441 & 1.132345 & -0.51785 & $\mathrm{H} 46$ & -5.0523 & -1.53001 & 0.651097 \\
\hline H50 & -4.1318 & 0.932844 & -4.0749 & $\mathrm{C} 47$ & -4.4431 & -0.04813 & -2.87993 \\
\hline H5 1 & -6.2772 & 1.519921 & -2.95851 & $\mathrm{C} 48$ & -5.7451 & -0.2659 & -2.42841 \\
\hline H52 & -0.0822 & -0.42782 & -1.91848 & $\mathrm{H} 49$ & -6.9686 & -0.98557 & -0.80179 \\
\hline H53 & 0.91132 & 1.784722 & -2.73638 & $\mathrm{H} 50$ & -4.2701 & 0.382785 & -3.86154 \\
\hline H54 & -1.8654 & -2.57814 & 2.279956 & H51 & -6.5917 & -0.01386 & -3.06032 \\
\hline H55 & -2.2654 & -0.00863 & -2.75545 & H52 & -0.1584 & -1.05495 & -1.86805 \\
\hline H56 & 2.5682 & -4.35782 & 1.627458 & $\mathrm{H} 53$ & 1.34781 & 2.234774 & -0.39873 \\
\hline C57 & 5.61452 & -2.25936 & -1.36428 & H54 & -1.7434 & -2.68413 & 2.852234 \\
\hline C58 & 4.64914 & -3.2619 & -1.47285 & H55 & -2.3465 & -0.18672 & -2.41827 \\
\hline C59 & 3.52544 & -3.23837 & -0.65018 & H56 & 3.27379 & -3.88478 & -0.37115 \\
\hline C60 & 3.34572 & -2.21711 & 0.294636 & C57 & 4.93522 & 0.651001 & -1.21606 \\
\hline C61 & 4.31147 & -1.21164 & 0.38589 & C58 & 4.60069 & -0.54924 & -1.84499 \\
\hline C62 & 5.439 & -1.23556 & -0.43504 & C59 & 3.79966 & -1.48257 & -1.1896 \\
\hline H63 & 6.49461 & -2.27763 & -2.00082 & C60 & 3.32752 & -1.23865 & 0.105945 \\
\hline H64 & 4.77224 & -4.06417 & -2.19482 & C61 & 3.65757 & -0.02607 & 0.723652 \\
\hline H65 & 2.78407 & -4.0268 & -0.75387 & C62 & 4.45732 & 0.909963 & 0.068982 \\
\hline H66 & 4.18081 & -0.39236 & 1.080457 & H63 & 5.56281 & 1.377733 & -1.7244 \\
\hline H67 & 6.17878 & -0.44483 & -0.34753 & H64 & 4.95785 & -0.75778 & -2.8493 \\
\hline C68 & 0.24467 & 3.811325 & -2.79744 & H65 & 3.51775 & -2.38689 & -1.72037 \\
\hline C69 & 0.64965 & 2.549386 & -1.99459 & H66 & 3.26816 & 0.198287 & 1.709534 \\
\hline $\mathrm{C} 70$ & -0.4359 & 1.924864 & -1.06901 & $\mathrm{H} 67$ & 4.70763 & 1.843067 & 0.566961 \\
\hline
\end{tabular}




\begin{tabular}{|c|c|c|c|c|c|c|c|}
\hline H71 & -1.3187 & 1.829074 & -1.69939 & C68 & 0.87419 & 4.214919 & -1.05562 \\
\hline $\mathrm{C} 72$ & -0.8148 & 2.793762 & 0.11024 & C69 & 0.44656 & 2.849626 & -0.47657 \\
\hline $\mathrm{C} 73$ & -2.0258 & 3.50798 & 0.11357 & $\mathrm{C} 70$ & -0.5474 & 2.055606 & -1.36904 \\
\hline $\mathrm{C} 74$ & -0.0114 & 2.888425 & 1.263902 & H71 & -0.0648 & 1.780351 & -2.30429 \\
\hline $\mathrm{C} 75$ & -2.4148 & 4.282545 & 1.209213 & $\mathrm{C} 72$ & -1.9007 & 2.688943 & -1.6101 \\
\hline H76 & -2.6869 & 3.435027 & -0.74554 & C73 & -2.225 & 3.273979 & -2.83797 \\
\hline $\mathrm{C} 77$ & -0.3935 & 3.663187 & 2.359049 & $\mathrm{C} 74$ & -2.8715 & 2.662637 & -0.59931 \\
\hline $\mathrm{H} 78$ & 0.91795 & 2.330142 & 1.329558 & $\mathrm{C} 75$ & -3.4831 & 3.847623 & -3.03709 \\
\hline C79 & -1.6007 & 4.365574 & 2.34082 & H76 & -1.5019 & 3.272745 & -3.64835 \\
\hline H80 & -3.3595 & 4.818627 & 1.178883 & C77 & -4.1279 & 3.22718 & -0.7953 \\
\hline H81 & 0.25245 & 3.711555 & 3.231972 & $\mathrm{H} 78$ & -2.6421 & 2.16818 & 0.338948 \\
\hline H82 & -1.9 & 4.969362 & 3.192456 & C79 & -4.4356 & 3.82767 & -2.01835 \\
\hline $\mathrm{C} 83$ & 2.38703 & 4.097468 & -0.71774 & H80 & -3.7209 & 4.301139 & -3.99495 \\
\hline $\mathrm{C} 84$ & 2.53266 & 1.807914 & -0.61935 & H81 & -4.8714 & 3.184382 & -0.00421 \\
\hline C85 & 3.74365 & 3.656882 & -0.1421 & H82 & -5.4163 & 4.265217 & -2.17993 \\
\hline H86 & 1.70413 & 4.476919 & 0.049998 & C83 & -0.7744 & 4.114747 & 1.496899 \\
\hline H87 & 2.49937 & 4.848359 & -1.49948 & $\mathrm{C} 84$ & -0.0349 & 1.923297 & 1.754138 \\
\hline H88 & 4.56114 & 3.785301 & -0.8585 & $\mathrm{C} 85$ & -0.7938 & 3.685413 & 2.978016 \\
\hline H89 & 3.99967 & 4.132581 & 0.804692 & H86 & -1.7763 & 4.220352 & 1.071959 \\
\hline N90 & 1.90339 & 2.813137 & -1.24497 & H87 & -0.2386 & 5.05419 & 1.353575 \\
\hline O91 & 3.58266 & 2.230963 & 0.10227 & H88 & -0.003 & 4.167413 & 3.56121 \\
\hline O92 & 2.22367 & 0.598981 & -0.63056 & H89 & -1.7566 & 3.844177 & 3.465583 \\
\hline H93 & 1.27842 & -3.65946 & 2.656875 & N90 & -0.0616 & 2.978957 & 0.900925 \\
\hline N94 & -2.0344 & -2.37767 & -0.26651 & O91 & -0.5332 & 2.259895 & 2.958356 \\
\hline C95 & -0.9318 & -3.18544 & -0.83842 & O92 & 0.36848 & 0.780295 & 1.524938 \\
\hline H96 & -0.6232 & -2.81404 & -1.82383 & H93 & 3.0907 & -4.20746 & 1.375865 \\
\hline C97 & 1.30797 & 4.137455 & -3.86368 & N94 & -1.8889 & -2.99336 & 0.264407 \\
\hline H98 & 1.09238 & 5.09622 & -4.34492 & C95 & -0.8489 & -3.64742 & -0.56643 \\
\hline H99 & 2.32526 & 4.183004 & -3.46521 & H96 & -0.8774 & -3.30971 & -1.61198 \\
\hline H100 & 1.30525 & 3.366674 & -4.64332 & C97 & 2.01826 & 4.811874 & -0.21628 \\
\hline C101 & -1.1118 & 3.628601 & -3.49809 & H98 & 2.26559 & 5.817725 & -0.56815 \\
\hline H102 & -1.1178 & 2.713879 & -4.10384 & H99 & 1.78557 & 4.878083 & 0.849715 \\
\hline H103 & -1.9466 & 3.574113 & -2.79782 & H100 & 2.91691 & 4.19181 & -0.31401 \\
\hline H104 & -1.2995 & 4.470655 & -4.17154 & C101 & 1.34846 & 4.074443 & -2.51218 \\
\hline \multirow[t]{2}{*}{ H105 } & 0.14957 & 4.653262 & -2.09788 & H102 & 2.12025 & 3.300162 & -2.59753 \\
\hline & & & & H103 & 0.53734 & 3.8253 & -3.19878 \\
\hline \multicolumn{2}{|c|}{ C3_ZS_Cheltop_TS2 } & & & $\mathrm{H} 104$ & 1.78436 & 5.017481 & -2.85527 \\
\hline $\mathrm{C} 1$ & -3.7143 & -4.14033 & -0.8828 & $\mathrm{H} 105$ & 0.00858 & 4.891409 & -1.03911 \\
\hline $\mathrm{C} 2$ & -1.4083 & -4.90543 & -0.6499 & H106 & 1.4367 & 0.080955 & -1.06961 \\
\hline $\mathrm{C} 3$ & -2.6176 & -4.98212 & -1.61608 & H107 & -0.9092 & 1.117059 & -0.8077 \\
\hline $\mathrm{C} 4$ & -2.9741 & -3.69225 & 0.399187 & & & & \\
\hline H5 & -4.5862 & -4.7492 & -0.62197 & & & & \\
\hline H6 & -2.3775 & -4.58913 & -2.60899 & & & & \\
\hline $\mathrm{H} 7$ & -3.6053 & -3.30213 & 1.197983 & & & & \\
\hline H8 & -4.0649 & -3.29063 & -1.46945 & & & & \\
\hline
\end{tabular}




\begin{tabular}{|c|c|c|c|}
\hline H9 & -0.6332 & -5.65227 & -0.81928 \\
\hline H10 & -2.9281 & -6.02304 & -1.74268 \\
\hline C11 & -2.1078 & -4.92027 & 0.726849 \\
\hline H12 & -1.4328 & -4.7599 & 1.573234 \\
\hline H13 & -2.6939 & -5.82809 & 0.894533 \\
\hline $\mathrm{C} 14$ & 0.47555 & -3.24371 & -0.04475 \\
\hline N15 & 0.93222 & -2.0996 & 0.326698 \\
\hline $\mathrm{C} 16$ & 2.28836 & -2.32164 & 0.953298 \\
\hline $\mathrm{C} 17$ & 2.48668 & -3.86376 & 0.73203 \\
\hline P18 & -2.036 & -1.02985 & 0.090818 \\
\hline Ir19 & -0.0368 & -0.18229 & -0.38279 \\
\hline $\mathrm{C} 20$ & -2.5787 & -0.729 & 1.819034 \\
\hline $\mathrm{C} 21$ & -3.1834 & -0.25152 & 4.521759 \\
\hline $\mathrm{C} 22$ & -2.1051 & -1.58941 & 2.821706 \\
\hline $\mathrm{C} 23$ & -3.3872 & 0.358137 & 2.189081 \\
\hline $\mathrm{C} 24$ & -3.6831 & 0.594244 & 3.530743 \\
\hline $\mathrm{C} 25$ & -2.4021 & -1.34987 & 4.162232 \\
\hline $\mathrm{H} 26$ & -3.8101 & 1.003853 & 1.430901 \\
\hline $\mathrm{H} 27$ & -4.3162 & 1.434914 & 3.800715 \\
\hline $\mathrm{H} 28$ & -2.0214 & -2.02446 & 4.923506 \\
\hline H29 & -3.413 & -0.06428 & 5.56669 \\
\hline $\mathrm{O} 30$ & 1.23696 & -4.32155 & 0.152152 \\
\hline $\mathrm{C} 31$ & 2.30125 & -2.04126 & 2.456965 \\
\hline C32 & 1.20836 & -1.52504 & 3.144915 \\
\hline $\mathrm{C} 33$ & 3.47157 & -2.34201 & 3.173528 \\
\hline C34 & 1.27524 & -1.30307 & 4.522412 \\
\hline H35 & 0.31551 & -1.2609 & 2.605322 \\
\hline C36 & 3.53868 & -2.12984 & 4.5474 \\
\hline H37 & 4.34295 & -2.72754 & 2.64948 \\
\hline C38 & 2.43541 & -1.60842 & 5.22911 \\
\hline H39 & 0.41213 & -0.88345 & 5.031633 \\
\hline $\mathrm{H} 40$ & 4.4525 & -2.36569 & 5.085033 \\
\hline H41 & 2.48798 & -1.43655 & 6.300413 \\
\hline $\mathrm{C} 42$ & -3.4697 & -0.67781 & -0.98697 \\
\hline $\mathrm{C} 43$ & -4.7832 & -0.7922 & -0.4999 \\
\hline $\mathrm{C} 44$ & -3.2634 & -0.57599 & -2.37032 \\
\hline $\mathrm{C} 45$ & -5.8636 & -0.79377 & -1.38131 \\
\hline H46 & -4.9704 & -0.90249 & 0.562553 \\
\hline $\mathrm{C} 47$ & -4.3468 & -0.5885 & -3.24704 \\
\hline $\mathrm{C} 48$ & -5.6495 & -0.69661 & -2.7572 \\
\hline H49 & -6.8729 & -0.88165 & -0.98963 \\
\hline H50 & -4.172 & -0.50401 & -4.31545 \\
\hline H51 & -6.4918 & -0.70494 & -3.44229 \\
\hline H52 & -0.2787 & -0.78902 & -1.81011 \\
\hline H53 & 1.43881 & 2.429561 & -0.32464 \\
\hline
\end{tabular}




\begin{tabular}{|c|c|c|c|}
\hline H54 & -1.5043 & -2.44942 & 2.549775 \\
\hline H55 & -2.2541 & -0.48025 & -2.75561 \\
\hline H56 & 3.2919 & -4.11465 & 0.039888 \\
\hline C57 & 5.1019 & 0.120271 & -1.27686 \\
\hline $\mathrm{C} 58$ & 4.59336 & -1.05562 & -1.83196 \\
\hline C59 & 3.69896 & -1.84001 & -1.10602 \\
\hline $\mathrm{C} 60$ & 3.31089 & -1.47431 & 0.190012 \\
\hline C61 & 3.81533 & -0.28716 & 0.733946 \\
\hline C62 & 4.70408 & 0.505296 & 0.003513 \\
\hline H63 & 5.79996 & 0.732486 & -1.84023 \\
\hline H64 & 4.88896 & -1.35984 & -2.83171 \\
\hline H65 & 3.28978 & -2.73349 & -1.57 \\
\hline H66 & 3.50683 & 0.02292 & 1.725967 \\
\hline H67 & 5.08999 & 1.421237 & 0.442275 \\
\hline C68 & 0.54595 & 4.22739 & -1.02625 \\
\hline C69 & 0.41615 & 2.821715 & -0.3813 \\
\hline $\mathrm{C} 70$ & -0.4489 & 1.773788 & -1.16305 \\
\hline H71 & 0.03277 & 1.7035 & -2.14425 \\
\hline $\mathrm{C} 72$ & -1.8553 & 2.249838 & -1.40081 \\
\hline $\mathrm{C} 73$ & -2.3329 & 2.471906 & -2.70036 \\
\hline $\mathrm{C} 74$ & -2.7402 & 2.497692 & -0.34239 \\
\hline $\mathrm{C} 75$ & -3.6312 & 2.928917 & -2.92976 \\
\hline H76 & -1.6837 & 2.265369 & -3.54805 \\
\hline $\mathrm{C} 77$ & -4.0373 & 2.956311 & -0.56005 \\
\hline H78 & -2.4124 & 2.288285 & 0.66623 \\
\hline C79 & -4.4913 & 3.177453 & -1.86091 \\
\hline H80 & -3.9738 & 3.083971 & -3.94904 \\
\hline H81 & -4.6989 & 3.129918 & 0.28563 \\
\hline H82 & -5.5029 & 3.529795 & -2.03864 \\
\hline $\mathrm{C} 83$ & -0.7053 & 4.072239 & 1.694843 \\
\hline $\mathrm{C} 84$ & 0.07568 & 1.899275 & 1.852638 \\
\hline $\mathrm{C} 85$ & -0.7141 & 3.576553 & 3.158937 \\
\hline H86 & -1.7099 & 4.204066 & 1.283988 \\
\hline H87 & -0.165 & 5.013656 & 1.591363 \\
\hline H88 & 0.04773 & 4.06749 & 3.771345 \\
\hline H89 & -1.6877 & 3.666554 & 3.642613 \\
\hline N90 & -0.0067 & 2.960858 & 1.036051 \\
\hline O91 & -0.3848 & 2.164354 & 3.085363 \\
\hline O92 & 0.51692 & 0.773671 & 1.563023 \\
\hline H93 & 2.6297 & -4.40009 & 1.669535 \\
\hline N94 & -1.9021 & -2.71692 & 0.047217 \\
\hline C95 & -0.8653 & -3.45327 & -0.70251 \\
\hline H96 & -0.7697 & -3.10747 & -1.74133 \\
\hline C97 & 1.70015 & 5.011922 & -0.37121 \\
\hline H98 & 1.6917 & 6.056384 & -0.69739 \\
\hline
\end{tabular}




$\begin{array}{llll}\text { H99 } & 1.67375 & 5.001517 & 0.721877 \\ \text { H100 } & 2.66208 & 4.579516 & -0.67072 \\ \text { C101 } & 0.80414 & 4.155383 & -2.53824 \\ \text { H102 } & 1.65702 & 3.502998 & -2.76106 \\ \text { H103 } & -0.0633 & 3.793635 & -3.09019 \\ \text { H104 } & 1.04567 & 5.152635 & -2.91877 \\ \text { H105 } & -0.4016 & 4.761621 & -0.87492 \\ \text { H106 } & 2.24919 & 0.591353 & -1.18911 \\ \text { H107 } & 1.9958 & 0.311567 & -1.83756\end{array}$

\section{C3_ZR_Cheltop}

C3_ZR_Cheltop_SM

\begin{tabular}{|c|c|c|c|c|c|c|}
\hline-3.42767 & -4.99928 & 0.479053 & $\mathrm{C} 1$ & -4.04825 & -3.70792 & 1.012992 \\
\hline-1.07632 & -5.06677 & -0.16005 & $\mathrm{C} 2$ & -1.9792 & -4.72145 & 0.235016 \\
\hline-2.47179 & -5.55021 & -0.62902 & $\mathrm{C} 3$ & -3.50205 & -4.72514 & -0.04046 \\
\hline-2.44773 & -4.25965 & 1.419601 & $\mathrm{C} 4$ & -2.76 & -3.26768 & 1.751572 \\
\hline-3.92578 & -5.80825 & 1.023599 & H5 & -4.73054 & -4.18775 & 1.722404 \\
\hline-2.72446 & -5.17828 & -1.6271 & H6 & -3.73764 & -4.43444 & -1.06916 \\
\hline-2.84639 & -4.00546 & 2.401367 & $\mathrm{H} 7$ & -2.92599 & -2.76692 & 2.70453 \\
\hline-4.2017 & -4.34184 & 0.084042 & $\mathrm{H} 8$ & -4.57931 & -2.87114 & 0.561246 \\
\hline-0.23432 & -5.58061 & -0.62223 & H9 & -1.42924 & -5.55957 & -0.19259 \\
\hline-2.49288 & -6.64278 & -0.67149 & $\mathrm{H} 10$ & -3.90919 & -5.72776 & 0.118339 \\
\hline-1.20833 & -5.16983 & 1.375254 & $\mathrm{C} 11$ & -1.93794 & -4.56798 & 1.771924 \\
\hline-0.35672 & -4.76126 & 1.92764 & H12 & -0.93228 & -4.43476 & 2.181759 \\
\hline-1.41156 & -6.18957 & 1.714036 & H13 & -2.44246 & -5.38834 & 2.290096 \\
\hline 0.343637 & -2.90881 & -0.32145 & $\mathrm{C} 14$ & 0.068039 & -3.27072 & -0.36372 \\
\hline 0.617797 & -1.65944 & -0.16291 & N15 & 0.725981 & -2.17015 & -0.4416 \\
\hline 2.110915 & -1.46655 & -0.38425 & $\mathrm{C} 16$ & 2.191876 & -2.51519 & -0.31192 \\
\hline 2.587364 & -2.95173 & -0.44308 & $\mathrm{C} 17$ & 2.14716 & -4.02739 & -0.67101 \\
\hline-2.54607 & -1.49083 & 0.626891 & P18 & -1.95202 & -0.75675 & 0.642316 \\
\hline-0.74214 & 0.008598 & -0.05545 & $\operatorname{Ir} 19$ & -0.13697 & -0.13893 & -0.6763 \\
\hline-3.15637 & -1.11929 & 2.307915 & $\mathrm{C} 20$ & -1.93831 & -0.0852 & 2.3461 \\
\hline-3.81411 & -0.25983 & 4.904451 & $\mathrm{C} 21$ & -1.7045 & 1.084397 & 4.891056 \\
\hline-2.39589 & -1.53501 & 3.413806 & $\mathrm{C} 22$ & -1.29752 & -0.77028 & 3.389358 \\
\hline-4.24099 & -0.25313 & 2.520374 & $\mathrm{C} 23$ & -2.46 & 1.194817 & 2.593705 \\
\hline-4.56959 & 0.166839 & 3.81054 & $\mathrm{C} 24$ & -2.34871 & 1.77067 & 3.858899 \\
\hline-2.7274 & -1.1138 & 4.700168 & $\mathrm{C} 25$ & -1.1837 & -0.18811 & 4.651593 \\
\hline-4.83341 & 0.102073 & 1.683925 & $\mathrm{H} 26$ & -2.95836 & 1.740253 & 1.800765 \\
\hline-5.42115 & 0.825411 & 3.95831 & $\mathrm{H} 27$ & -2.77457 & 2.754057 & 4.039657 \\
\hline-2.12771 & -1.44353 & 5.543398 & $\mathrm{H} 28$ & -0.68912 & -0.73362 & 5.450106 \\
\hline-4.07096 & 0.067071 & 5.907833 & H29 & -1.61662 & 1.534331 & 5.875428 \\
\hline 1.367497 & -3.73463 & -0.55405 & $\mathrm{O} 30$ & 0.770934 & -4.407 & -0.37184 \\
\hline 2.786102 & -0.80053 & 0.808679 & C31 & 2.638369 & -2.38495 & 1.151956 \\
\hline 2.180973 & -0.81334 & 2.068814 & $\mathrm{C} 32$ & 1.760272 & -2.02653 & 2.171628 \\
\hline 4.088303 & -0.29117 & 0.687809 & C33 & 3.968007 & -2.69164 & 1.478231 \\
\hline
\end{tabular}




\begin{tabular}{|c|c|c|c|c|c|c|c|}
\hline C34 & 2.831787 & -0.26469 & 3.174009 & C34 & 2.199799 & -1.95382 & 3.494336 \\
\hline H35 & 1.186292 & -1.22162 & 2.187385 & H35 & 0.741626 & -1.77114 & 1.927233 \\
\hline C36 & 4.743273 & 0.244827 & 1.795902 & $\mathrm{C} 36$ & 4.406276 & -2.62908 & 2.798097 \\
\hline H37 & 4.590628 & -0.3026 & -0.27381 & H37 & 4.668203 & -2.96374 & 0.692386 \\
\hline $\mathrm{C} 38$ & 4.112107 & 0.272108 & 3.041183 & $\mathrm{C} 38$ & 3.52112 & -2.25554 & 3.813298 \\
\hline H39 & 2.330197 & -0.25528 & 4.13737 & H39 & 1.505488 & -1.6454 & 4.270714 \\
\hline $\mathrm{H} 40$ & 5.747219 & 0.644208 & 1.68376 & $\mathrm{H} 40$ & 5.43973 & -2.86558 & 3.034388 \\
\hline H41 & 4.619502 & 0.699724 & 3.901403 & H41 & 3.864698 & -2.1986 & 4.842115 \\
\hline $\mathrm{C} 42$ & -4.02203 & -1.64208 & -0.45836 & $\mathrm{C} 42$ & -3.64214 & -0.4778 & -0.01453 \\
\hline $\mathrm{C} 43$ & -5.29444 & -2.01298 & 0.008349 & $\mathrm{C} 43$ & -4.74183 & -0.23665 & 0.822926 \\
\hline $\mathrm{C} 44$ & -3.81647 & -1.51141 & -1.841 & C44 & -3.86232 & -0.69748 & -1.38419 \\
\hline $\mathrm{C} 45$ & -6.33915 & -2.22659 & -0.89005 & $\mathrm{C} 45$ & -6.03139 & -0.18926 & 0.293165 \\
\hline $\mathrm{H} 46$ & -5.47045 & -2.14381 & 1.071482 & $\mathrm{H} 46$ & -4.60221 & -0.09367 & 1.888864 \\
\hline C47 & -4.86112 & -1.73847 & -2.73778 & C47 & -5.1529 & -0.66211 & -1.90685 \\
\hline $\mathrm{C} 48$ & -6.12624 & -2.09063 & -2.26467 & $\mathrm{C} 48$ & -6.24053 & -0.40201 & -1.07004 \\
\hline H49 & -7.32008 & -2.50596 & -0.5161 & H49 & -6.87389 & 0.007151 & 0.949963 \\
\hline H50 & -4.68747 & -1.63125 & -3.80473 & $\mathrm{H} 50$ & -5.30811 & -0.82795 & -2.96891 \\
\hline H51 & -6.94214 & -2.25963 & -2.96111 & H51 & -7.24611 & -0.36635 & -1.47853 \\
\hline H52 & -0.9641 & -0.20249 & -1.58421 & $\mathrm{H} 52$ & -1.02533 & -0.56929 & -1.89993 \\
\hline H53 & 0.603721 & 0.70044 & -0.51552 & $\mathrm{H} 53$ & 1.82407 & 1.934043 & -1.0084 \\
\hline H54 & -1.52663 & -2.16616 & 3.26072 & H54 & -0.88531 & -1.75781 & 3.218586 \\
\hline H55 & -2.83929 & -1.21677 & -2.21048 & $\mathrm{H} 55$ & -3.02059 & -0.87606 & -2.0449 \\
\hline H56 & 3.22176 & -3.17837 & -1.29987 & H56 & 2.320471 & -4.24483 & -1.72748 \\
\hline $\mathrm{C} 57$ & 2.321582 & 0.587778 & -4.21352 & C57 & 4.2689 & 0.170129 & -3.02006 \\
\hline C58 & 2.014686 & -0.77314 & -4.14429 & C58 & 3.673177 & -0.99382 & -3.51075 \\
\hline $\mathrm{C} 59$ & 1.991402 & -1.42516 & -2.91217 & C59 & 3.050436 & -1.8898 & -2.63963 \\
\hline $\mathrm{C} 60$ & 2.276879 & -0.73721 & -1.72451 & C60 & 3.017315 & -1.6454 & -1.26051 \\
\hline C61 & 2.575563 & 0.630247 & -1.80541 & C61 & 3.620658 & -0.47315 & -0.77729 \\
\hline C62 & 2.601756 & 1.284365 & -3.03829 & C62 & 4.241509 & 0.424243 & -1.64805 \\
\hline H63 & 2.343903 & 1.096566 & -5.17272 & H63 & 4.749803 & 0.86802 & -3.6988 \\
\hline H64 & 1.793317 & -1.32933 & -5.0507 & H64 & 3.687318 & -1.20558 & -4.57593 \\
\hline H65 & 1.727386 & -2.47966 & -2.88384 & H65 & 2.564479 & -2.76438 & -3.06046 \\
\hline H66 & 2.779374 & 1.200601 & -0.90674 & H66 & 3.573091 & -0.24459 & 0.280608 \\
\hline H67 & 2.838969 & 2.344021 & -3.07225 & H67 & 4.693874 & 1.328521 & -1.25091 \\
\hline C68 & -3.37037 & 1.810127 & -0.49846 & C68 & -1.53135 & 2.731533 & -1.54233 \\
\hline C69 & -1.99558 & 1.794022 & 0.208331 & C69 & -0.35681 & 2.037547 & -0.81149 \\
\hline $\mathrm{C} 70$ & -0.78518 & 2.165951 & -0.47248 & C70 & 1.018176 & 2.528895 & -1.44575 \\
\hline H71 & -0.86157 & 2.263781 & -1.55008 & H71 & 1.004688 & 2.293321 & -2.5135 \\
\hline $\mathrm{C} 72$ & 0.237111 & 3.104047 & 0.106612 & $\mathrm{C} 72$ & 1.400541 & 3.97954 & -1.24622 \\
\hline $\mathrm{C} 73$ & 1.243911 & 2.754967 & 1.014981 & $\mathrm{C} 73$ & 2.261755 & 4.32726 & -0.1933 \\
\hline $\mathrm{C} 74$ & 0.141255 & 4.441415 & -0.31288 & C74 & 0.934449 & 5.003921 & -2.08309 \\
\hline $\mathrm{C} 75$ & 2.120615 & 3.724479 & 1.50613 & $\mathrm{C} 75$ & 2.607977 & 5.657297 & 0.048605 \\
\hline H76 & 1.355637 & 1.725691 & 1.333058 & H76 & 2.665509 & 3.542453 & 0.443958 \\
\hline C77 & 1.016515 & 5.408282 & 0.179155 & C77 & 1.277677 & 6.336352 & -1.845 \\
\hline H78 & -0.62742 & 4.723938 & -1.02925 & $\mathrm{H} 78$ & 0.306494 & 4.75673 & -2.9353 \\
\hline
\end{tabular}




\begin{tabular}{|c|c|c|c|c|c|c|c|}
\hline C79 & 2.00888 & 5.052267 & 1.095543 & C79 & 2.10584 & 6.670413 & -0.77131 \\
\hline H80 & 2.896732 & 3.427687 & 2.205952 & H80 & 3.277641 & 5.901939 & 0.868949 \\
\hline H81 & 0.926633 & 6.437406 & -0.15734 & H81 & 0.902927 & 7.114462 & -2.50418 \\
\hline H82 & 2.693689 & 5.804302 & 1.476796 & H82 & 2.372863 & 7.706937 & -0.58776 \\
\hline $\mathrm{C} 83$ & -2.26456 & 3.417386 & 2.256212 & $\mathrm{C} 83$ & -0.58832 & 3.745983 & 1.265506 \\
\hline $\mathrm{C} 84$ & -1.16508 & 1.414925 & 2.476444 & $\mathrm{C} 84$ & 0.53149 & 1.732297 & 1.411706 \\
\hline $\mathrm{C} 85$ & -2.01 & 3.089677 & 3.732503 & C85 & 0.326214 & 3.700887 & 2.512399 \\
\hline H86 & -3.28111 & 3.76281 & 2.094297 & H86 & -1.64203 & 3.80595 & 1.54916 \\
\hline H87 & -1.56032 & 4.155563 & 1.857353 & H87 & -0.34771 & 4.578185 & 0.604267 \\
\hline H88 & -1.53896 & 3.897483 & 4.293031 & H88 & 1.166075 & 4.395125 & 2.439018 \\
\hline H89 & -2.91812 & 2.750595 & 4.240893 & H89 & -0.21444 & 3.858501 & 3.44654 \\
\hline N90 & -2.01116 & 2.090075 & 1.649971 & N90 & -0.29666 & 2.44947 & 0.634061 \\
\hline O91 & -1.08719 & 1.971033 & 3.688848 & O91 & 0.867303 & 2.349787 & 2.546083 \\
\hline O92 & -0.50664 & 0.407783 & 2.185037 & O92 & 0.966781 & 0.59557 & 1.128987 \\
\hline H93 & 3.084848 & -3.25259 & 0.480306 & H93 & 2.805421 & -4.63385 & -0.05179 \\
\hline N94 & -1.87492 & -3.04733 & 0.762315 & N94 & -1.87523 & -2.44395 & 0.869288 \\
\hline C95 & -1.03227 & -3.52829 & -0.359 & C95 & -1.42839 & -3.34704 & -0.22671 \\
\hline H96 & -1.45464 & -3.26274 & -1.34117 & H96 & -1.85503 & -3.06363 & -1.20001 \\
\hline C97 & -3.25087 & 1.834973 & -2.02974 & C97 & -2.8909 & 2.719906 & -0.8342 \\
\hline H98 & -2.56416 & 1.075944 & -2.40638 & H98 & -2.87489 & 3.229021 & 0.131039 \\
\hline H99 & -4.23217 & 1.644289 & -2.47331 & H99 & -3.6279 & 3.24399 & -1.4519 \\
\hline H100 & -2.91182 & 2.814856 & -2.38537 & H100 & -3.25767 & 1.708994 & -0.68442 \\
\hline $\mathrm{C} 101$ & -4.30004 & 2.959375 & -0.06328 & $\mathrm{C} 101$ & -1.70994 & 2.189399 & -2.97052 \\
\hline H102 & -4.6357 & 2.846688 & 0.970915 & $\mathrm{H} 102$ & -0.77746 & 2.178565 & -3.54159 \\
\hline H103 & -3.82318 & 3.939212 & -0.18037 & H103 & -2.09496 & 1.166793 & -2.94353 \\
\hline H104 & -5.19623 & 2.954035 & -0.69179 & H104 & -2.42572 & 2.808119 & -3.52152 \\
\hline H105 & -3.88348 & 0.884143 & -0.22924 & H105 & -1.25057 & 3.790536 & -1.63366 \\
\hline \multirow[t]{2}{*}{$\mathrm{X}$} & -1.52878 & 1.835509 & -0.4773 & H106 & 1.39454 & 0.10461 & -1.54425 \\
\hline & & & & H107 & 0.813622 & 0.006655 & -2.13567 \\
\hline \multicolumn{8}{|c|}{ C3_ZR_Cheltop_TS1 } \\
\hline $\mathrm{C} 1$ & -3.33943 & -5.09715 & 0.414086 & \multicolumn{4}{|c|}{ C3_ZR_Cheltop_TS3 } \\
\hline $\mathrm{C} 2$ & -0.97801 & -5.11036 & -0.19421 & $\mathrm{C} 1$ & -4.09624 & -3.79857 & 0.772442 \\
\hline $\mathrm{C} 3$ & -2.35639 & -5.62371 & -0.6825 & $\mathrm{C} 2$ & -1.96725 & -4.7499 & 0.07422 \\
\hline $\mathrm{C} 4$ & -2.38775 & -4.34241 & 1.371528 & $\mathrm{C} 3$ & -3.46996 & -4.74996 & -0.29848 \\
\hline H5 & -3.83085 & -5.91819 & 0.946416 & $\mathrm{C} 4$ & -2.86148 & -3.38455 & 1.610061 \\
\hline H6 & -2.60298 & -5.25479 & -1.68321 & H5 & -4.81353 & -4.32526 & 1.410508 \\
\hline H7 & -2.80471 & -4.10025 & 2.348941 & H6 & -3.6418 & -4.40382 & -1.32286 \\
\hline H8 & -4.11906 & -4.44978 & 0.011861 & $\mathrm{H} 7$ & -3.08931 & -2.93089 & 2.574517 \\
\hline H9 & -0.11802 & -5.60225 & -0.64709 & $\mathrm{H} 8$ & -4.6113 & -2.94377 & 0.335428 \\
\hline H10 & -2.35363 & -6.71632 & -0.72803 & H9 & -1.38322 & -5.56176 & -0.35878 \\
\hline $\mathrm{C} 11$ & -1.12682 & -5.2235 & 1.338969 & $\mathrm{H} 10$ & -3.8749 & -5.7632 & -0.22387 \\
\hline H12 & -0.29169 & -4.79687 & 1.902811 & $\mathrm{C} 11$ & -2.02593 & -4.67589 & 1.616452 \\
\hline H13 & -1.30882 & -6.24949 & 1.670621 & H12 & -1.04964 & -4.55571 & 2.095247 \\
\hline $\mathrm{C} 14$ & 0.392069 & -2.91542 & -0.32938 & H13 & -2.55171 & -5.52689 & 2.058451 \\
\hline N15 & 0.620199 & -1.65852 & -0.17185 & $\mathrm{C} 14$ & 0.100802 & -3.2539 & -0.32013 \\
\hline
\end{tabular}




\begin{tabular}{|c|c|c|c|c|c|c|c|}
\hline $\mathrm{C} 16$ & 2.106337 & -1.41687 & -0.33658 & N15 & 0.753395 & -2.15026 & -0.39112 \\
\hline $\mathrm{C} 17$ & 2.638021 & -2.88274 & -0.48067 & $\mathrm{C} 16$ & 2.222503 & -2.46804 & -0.2396 \\
\hline P18 & -2.51562 & -1.5642 & 0.630303 & $\mathrm{C} 17$ & 2.203716 & -4.01353 & -0.46457 \\
\hline Ir19 & -0.84634 & -0.04572 & -0.08317 & P18 & -2.03999 & -0.81503 & 0.677837 \\
\hline $\mathrm{C} 20$ & -3.04679 & -1.20148 & 2.336987 & Ir19 & -0.07992 & -0.10995 & -0.65665 \\
\hline $\mathrm{C} 21$ & -3.60158 & -0.35085 & 4.95183 & $\mathrm{C} 20$ & -2.00301 & -0.20454 & 2.39839 \\
\hline $\mathrm{C} 22$ & -2.27038 & -1.65748 & 3.414224 & $\mathrm{C} 21$ & -1.74116 & 0.878128 & 4.971821 \\
\hline $\mathrm{C} 23$ & -4.08611 & -0.29066 & 2.58215 & $\mathrm{C} 22$ & -1.42157 & -0.95701 & 3.427685 \\
\hline $\mathrm{C} 24$ & -4.36473 & 0.125249 & 3.884773 & $\mathrm{C} 23$ & -2.4485 & 1.099873 & 2.66954 \\
\hline $\mathrm{C} 25$ & -2.55362 & -1.24221 & 4.712948 & $\mathrm{C} 24$ & -2.32114 & 1.633641 & 3.950836 \\
\hline $\mathrm{H} 26$ & -4.68164 & 0.094648 & 1.760244 & $\mathrm{C} 25$ & -1.2949 & -0.41688 & 4.707381 \\
\hline H27 & -5.18248 & 0.817671 & 4.064607 & $\mathrm{H} 26$ & -2.90121 & 1.694396 & 1.882905 \\
\hline $\mathrm{H} 28$ & -1.94784 & -1.60474 & 5.538057 & $\mathrm{H} 27$ & -2.68393 & 2.637625 & 4.154425 \\
\hline H29 & -3.81954 & -0.02665 & 5.96526 & $\mathrm{H} 28$ & -0.84917 & -1.01204 & 5.499112 \\
\hline $\mathrm{O} 30$ & 1.44439 & -3.71097 & -0.5451 & $\mathrm{H} 29$ & -1.64175 & 1.295621 & 5.969482 \\
\hline C31 & 2.727112 & -0.79952 & 0.912217 & $\mathrm{O} 30$ & 0.808812 & -4.38499 & -0.26402 \\
\hline $\mathrm{C} 32$ & 2.070743 & -0.8553 & 2.144522 & C31 & 2.677047 & -2.21286 & 1.204081 \\
\hline $\mathrm{C} 33$ & 4.030926 & -0.28323 & 0.861857 & $\mathrm{C} 32$ & 1.764975 & -1.96687 & 2.22875 \\
\hline C34 & 2.675384 & -0.34096 & 3.292568 & $\mathrm{C} 33$ & 4.038554 & -2.33413 & 1.526464 \\
\hline H35 & 1.072257 & -1.26702 & 2.206602 & C34 & 2.197608 & -1.80383 & 3.545229 \\
\hline $\mathrm{C} 36$ & 4.638924 & 0.219522 & 2.010642 & H35 & 0.715997 & -1.86567 & 1.995816 \\
\hline H37 & 4.570297 & -0.26218 & -0.08042 & $\mathrm{C} 36$ & 4.471342 & -2.17987 & 2.841513 \\
\hline C38 & 3.957027 & 0.20395 & 3.229476 & H37 & 4.764671 & -2.5359 & 0.744174 \\
\hline H39 & 2.135542 & -0.36345 & 4.234653 & C38 & 3.550915 & -1.90602 & 3.857046 \\
\hline $\mathrm{H} 40$ & 5.64438 & 0.626389 & 1.951287 & H39 & 1.470341 & -1.57954 & 4.320438 \\
\hline H41 & 4.427151 & 0.605615 & 4.122642 & $\mathrm{H} 40$ & 5.529069 & -2.26697 & 3.073453 \\
\hline $\mathrm{C} 42$ & -4.05985 & -1.67954 & -0.35768 & $\mathrm{H} 41$ & 3.89043 & -1.77369 & 4.880508 \\
\hline $\mathrm{C} 43$ & -5.27456 & -2.11585 & 0.200088 & $\mathrm{C} 42$ & -3.74492 & -0.49473 & 0.072961 \\
\hline $\mathrm{C} 44$ & -3.99523 & -1.42923 & -1.73666 & $\mathrm{C} 43$ & -4.82354 & -0.25152 & 0.935842 \\
\hline $\mathrm{C} 45$ & -6.3991 & -2.28454 & -0.60591 & C44 & -3.99094 & -0.64374 & -1.30171 \\
\hline H46 & -5.34384 & -2.32261 & 1.263816 & $\mathrm{C} 45$ & -6.11778 & -0.13451 & 0.42735 \\
\hline $\mathrm{C} 47$ & -5.12146 & -1.60705 & -2.5414 & $\mathrm{H} 46$ & -4.66272 & -0.15479 & 2.004265 \\
\hline $\mathrm{C} 48$ & -6.32521 & -2.03358 & -1.97873 & $\mathrm{C} 47$ & -5.28506 & -0.53818 & -1.80477 \\
\hline H49 & -7.33316 & -2.6165 & -0.16186 & $\mathrm{C} 48$ & -6.3516 & -0.27665 & -0.94084 \\
\hline $\mathrm{H} 50$ & -5.05835 & -1.40615 & -3.60696 & H49 & -6.94456 & 0.063959 & 1.103522 \\
\hline H51 & -7.20326 & -2.16649 & -2.60351 & $\mathrm{H} 50$ & -5.46076 & -0.64755 & -2.87088 \\
\hline H52 & -1.10952 & -0.32876 & -1.59764 & H51 & -7.36006 & -0.1832 & -1.33324 \\
\hline H53 & 0.335167 & 0.868692 & -0.62722 & H52 & -1.08873 & -0.56604 & -1.78024 \\
\hline H54 & -1.43134 & -2.32036 & 3.228584 & $\mathrm{H} 53$ & 1.891189 & 1.968537 & -1.04992 \\
\hline H55 & -3.06421 & -1.08337 & -2.17291 & $\mathrm{H} 54$ & -1.06356 & -1.9611 & 3.230689 \\
\hline H56 & 3.221172 & -3.05305 & -1.38629 & H55 & -3.1656 & -0.82112 & -1.98465 \\
\hline $\mathrm{C} 57$ & 2.269449 & 0.878293 & -4.02202 & H56 & 2.484442 & -4.32665 & -1.47214 \\
\hline C58 & 1.967556 & -0.48551 & -4.03701 & C57 & 4.260118 & -0.1143 & -3.27425 \\
\hline C59 & 1.967269 & -1.21442 & -2.85008 & C58 & 3.575202 & -1.27978 & -3.62474 \\
\hline C60 & 2.27396 & -0.6028 & -1.62601 & C59 & 2.956635 & -2.05534 & -2.64309 \\
\hline
\end{tabular}




\begin{tabular}{|c|c|c|c|c|c|c|c|}
\hline C61 & 2.571226 & 0.765302 & -1.62362 & C60 & 3.017476 & -1.69142 & -1.29193 \\
\hline C62 & 2.570839 & 1.499309 & -2.81154 & C61 & 3.697581 & -0.51202 & -0.95278 \\
\hline H63 & 2.268936 & 1.448597 & -4.9465 & C62 & 4.315758 & 0.266239 & -1.93279 \\
\hline H64 & 1.727816 & -0.98071 & -4.97356 & H63 & 4.743556 & 0.488675 & -4.03726 \\
\hline H65 & 1.698283 & -2.26755 & -2.88231 & H64 & 3.519535 & -1.58789 & -4.6649 \\
\hline H66 & 2.78857 & 1.276851 & -0.69506 & H65 & 2.403319 & -2.93669 & -2.95337 \\
\hline H67 & 2.80055 & 2.560747 & -2.78018 & H66 & 3.728581 & -0.18839 & 0.08063 \\
\hline C68 & -3.28177 & 1.908127 & -0.69198 & H67 & 4.835855 & 1.175244 & -1.6435 \\
\hline C69 & -1.93526 & 1.80131 & 0.064831 & C68 & -1.47732 & 2.720463 & -1.55914 \\
\hline $\mathrm{C} 70$ & -0.65601 & 2.111446 & -0.59181 & C69 & -0.28132 & 2.062959 & -0.8288 \\
\hline H71 & -0.71565 & 2.212906 & -1.67131 & $\mathrm{C} 70$ & 1.078998 & 2.56529 & -1.47042 \\
\hline $\mathrm{C} 72$ & 0.341703 & 3.069449 & 0.001052 & H71 & 1.05324 & 2.326705 & -2.53699 \\
\hline $\mathrm{C} 73$ & 1.28685 & 2.730358 & 0.975421 & $\mathrm{C} 72$ & 1.454195 & 4.016416 & -1.26366 \\
\hline C74 & 0.287484 & 4.392216 & -0.4644 & C73 & 2.341045 & 4.345526 & -0.22618 \\
\hline $\mathrm{C} 75$ & 2.153678 & 3.699991 & 1.483453 & $\mathrm{C} 74$ & 0.951858 & 5.057448 & -2.05817 \\
\hline H76 & 1.358014 & 1.708212 & 1.329438 & C75 & 2.677745 & 5.671707 & 0.045382 \\
\hline C77 & 1.149419 & 5.360203 & 0.048422 & $\mathrm{H} 76$ & 2.771569 & 3.546708 & 0.375214 \\
\hline H78 & -0.43687 & 4.662928 & -1.22956 & C77 & 1.282564 & 6.387146 & -1.7884 \\
\hline C79 & 2.085218 & 5.016228 & 1.027137 & $\mathrm{H} 78$ & 0.303506 & 4.827783 & -2.89978 \\
\hline H80 & 2.886499 & 3.414159 & 2.23254 & C79 & 2.136663 & 6.699753 & -0.72912 \\
\hline H81 & 1.094581 & 6.380506 & -0.3206 & H80 & 3.367145 & 5.90235 & 0.853432 \\
\hline H82 & 2.760796 & 5.769357 & 1.422225 & H81 & 0.877345 & 7.180761 & -2.41004 \\
\hline $\mathrm{C} 83$ & -2.2004 & 3.519579 & 2.058818 & H82 & 2.391449 & 7.734615 & -0.51921 \\
\hline $\mathrm{C} 84$ & -1.18666 & 1.485621 & 2.36388 & $\mathrm{C} 83$ & -0.53115 & 3.793354 & 1.238435 \\
\hline $\mathrm{C} 85$ & -1.98614 & 3.228868 & 3.549703 & $\mathrm{C} 84$ & 0.456759 & 1.726906 & 1.458604 \\
\hline H86 & -3.20001 & 3.897031 & 1.867719 & $\mathrm{C} 85$ & 0.357897 & 3.727406 & 2.499569 \\
\hline H87 & -1.46327 & 4.221609 & 1.655202 & H86 & -1.59067 & 3.865638 & 1.499945 \\
\hline H88 & -1.49412 & 4.035386 & 4.093909 & H87 & -0.26562 & 4.623769 & 0.584586 \\
\hline H89 & -2.91608 & 2.943834 & 4.05209 & H88 & 1.266215 & 4.325873 & 2.398713 \\
\hline N90 & -1.98398 & 2.165674 & 1.495123 & H89 & -0.16837 & 3.989395 & 3.418094 \\
\hline O91 & -1.10817 & 2.074036 & 3.560048 & N90 & -0.24742 & 2.489803 & 0.614725 \\
\hline O92 & -0.55897 & 0.446862 & 2.115617 & O91 & 0.757395 & 2.329573 & 2.607475 \\
\hline H93 & 3.208414 & -3.19737 & 0.394493 & O92 & 0.814764 & 0.546596 & 1.230735 \\
\hline N94 & -1.83826 & -3.11703 & 0.723071 & H93 & 2.801491 & -4.55052 & 0.27019 \\
\hline C95 & -0.96755 & -3.57092 & -0.38788 & N94 & -1.93989 & -2.50692 & 0.822906 \\
\hline H96 & -1.38236 & -3.30885 & -1.37408 & C95 & -1.40234 & -3.35031 & -0.28064 \\
\hline C97 & -3.10726 & 1.888518 & -2.21924 & H96 & -1.76522 & -3.02458 & -1.26597 \\
\hline H98 & -2.44655 & 1.087631 & -2.55661 & C97 & -2.82108 & 2.670843 & -0.8252 \\
\hline H99 & -4.08111 & 1.739719 & -2.69468 & H98 & -2.81168 & 3.213121 & 0.122037 \\
\hline H100 & -2.70713 & 2.84206 & -2.58372 & H99 & -3.59913 & 3.130954 & -1.44322 \\
\hline C101 & -4.15499 & 3.123543 & -0.32364 & H100 & -3.12812 & 1.648139 & -0.63032 \\
\hline H102 & -4.54282 & 3.057081 & 0.696048 & C101 & -1.66111 & 2.16241 & -2.9805 \\
\hline H103 & -3.61416 & 4.069987 & -0.43916 & H102 & -0.73353 & 2.156427 & -3.55945 \\
\hline H104 & -5.02275 & 3.159889 & -0.99003 & H103 & -2.03567 & 1.135729 & -2.94205 \\
\hline H105 & -3.85779 & 1.018983 & -0.41679 & H104 & -2.39001 & 2.766065 & -3.53089 \\
\hline
\end{tabular}




\begin{tabular}{|c|c|c|c|c|c|c|c|}
\hline \multirow[t]{2}{*}{$\mathrm{X}$} & \multirow[t]{2}{*}{-1.52878} & \multirow[t]{2}{*}{1.835509} & \multirow[t]{2}{*}{-0.4773} & H105 & -1.22423 & 3.785339 & -1.66326 \\
\hline & & & & H106 & 1.373267 & 0.154683 & -1.24307 \\
\hline \multicolumn{4}{|c|}{ C3_ZR_Cheltop_INT1 } & H107 & 0.360374 & -0.09257 & -2.17009 \\
\hline $\mathrm{C} 1$ & -4.16881 & -3.50899 & 0.506112 & & & & \\
\hline $\mathrm{C} 2$ & -2.03965 & -4.58626 & 0.001888 & \multicolumn{4}{|c|}{ C3_ZR_Cheltop_PROD } \\
\hline $\mathrm{C} 3$ & -3.51107 & -4.52878 & -0.48183 & $\mathrm{C} 1$ & -4.09679 & -4.04262 & 0.439749 \\
\hline $\mathrm{C} 4$ & -2.9837 & -3.1361 & 1.427926 & $\mathrm{C} 2$ & -1.88516 & -4.92213 & -0.08026 \\
\hline H5 & -4.96996 & -3.97141 & 1.091729 & $\mathrm{C} 3$ & -3.34824 & -4.951 & -0.59184 \\
\hline H6 & -3.59065 & -4.21593 & -1.52784 & $\mathrm{C} 4$ & -2.96634 & -3.66201 & 1.426152 \\
\hline H7 & -3.25526 & -2.6401 & 2.359979 & H5 & -4.88842 & -4.58965 & 0.961883 \\
\hline H8 & -4.58635 & -2.63467 & 0.004602 & H6 & -3.43607 & -4.5877 & -1.62081 \\
\hline H9 & -1.46428 & -5.43527 & -0.3664 & $\mathrm{H} 7$ & -3.307 & -3.26592 & 2.382567 \\
\hline H10 & -3.96976 & -5.51827 & -0.40083 & H8 & -4.54804 & -3.15841 & -0.01427 \\
\hline $\mathrm{C} 11$ & -2.21201 & -4.46334 & 1.532112 & H9 & -1.23401 & -5.69158 & -0.49552 \\
\hline H12 & -1.27078 & -4.37058 & 2.082655 & $\mathrm{H} 10$ & -3.72954 & -5.97602 & -0.57337 \\
\hline H13 & -2.80734 & -5.27735 & 1.954972 & $\mathrm{C} 11$ & -2.09654 & -4.93151 & 1.450527 \\
\hline $\mathrm{C} 14$ & 0.114089 & -3.1731 & -0.29997 & H12 & -1.17529 & -4.80856 & 2.027391 \\
\hline N15 & 0.804969 & -2.09257 & -0.21828 & H13 & -2.63319 & -5.82057 & 1.793913 \\
\hline $\mathrm{C} 16$ & 2.259598 & -2.46234 & -0.18792 & $\mathrm{C} 14$ & 0.13068 & -3.28926 & -0.27381 \\
\hline $\mathrm{C} 17$ & 2.188668 & -3.99546 & -0.51573 & N15 & 0.723453 & -2.1401 & -0.32536 \\
\hline P18 & -1.90602 & -0.64746 & 0.535682 & $\mathrm{C} 16$ & 2.205187 & -2.35396 & -0.05016 \\
\hline $\operatorname{Ir} 19$ & 0.002896 & -0.04179 & -0.45029 & $\mathrm{C} 17$ & 2.289334 & -3.87585 & -0.35086 \\
\hline $\mathrm{C} 20$ & -1.90991 & -0.03114 & 2.248604 & P18 & -2.03108 & -1.0282 & 0.755381 \\
\hline $\mathrm{C} 21$ & -1.52752 & 1.058013 & 4.799838 & $\operatorname{Ir} 19$ & -0.18 & -0.35528 & -0.65146 \\
\hline $\mathrm{C} 22$ & -1.39165 & -0.82106 & 3.283906 & $\mathrm{C} 20$ & -1.9677 & -0.52344 & 2.517177 \\
\hline $\mathrm{C} 23$ & -2.22585 & 1.314009 & 2.498288 & $\mathrm{C} 21$ & -1.71841 & 0.420869 & 5.153291 \\
\hline $\mathrm{C} 24$ & -2.03754 & 1.85158 & 3.770072 & $\mathrm{C} 22$ & -1.65892 & -1.40505 & 3.560874 \\
\hline $\mathrm{C} 25$ & -1.2085 & -0.27796 & 4.555018 & $\mathrm{C} 23$ & -2.13341 & 0.84145 & 2.807367 \\
\hline $\mathrm{H} 26$ & -2.60555 & 1.942061 & 1.69866 & $\mathrm{C} 24$ & -2.00812 & 1.308713 & 4.113448 \\
\hline $\mathrm{H} 27$ & -2.28875 & 2.891798 & 3.958427 & $\mathrm{C} 25$ & -1.54512 & -0.93422 & 4.871653 \\
\hline $\mathrm{H} 28$ & -0.80947 & -0.89826 & 5.352441 & $\mathrm{H} 26$ & -2.36517 & 1.539226 & 2.006855 \\
\hline $\mathrm{H} 29$ & -1.37646 & 1.481025 & 5.788596 & $\mathrm{H} 27$ & -2.14244 & 2.366324 & 4.323716 \\
\hline $\mathrm{O} 30$ & 0.776185 & -4.32882 & -0.39754 & $\mathrm{H} 28$ & -1.31461 & -1.63188 & 5.67206 \\
\hline C31 & 2.838274 & -2.26456 & 1.212629 & $\mathrm{H} 29$ & -1.62629 & 0.783657 & 6.172535 \\
\hline C32 & 2.074336 & -1.77405 & 2.271891 & $\mathrm{O} 30$ & 0.922281 & -4.35298 & -0.15835 \\
\hline $\mathrm{C} 33$ & 4.164632 & -2.6538 & 1.459255 & C31 & 2.51758 & -2.1429 & 1.43766 \\
\hline C34 & 2.633082 & -1.63801 & 3.5445 & $\mathrm{C} 32$ & 1.560097 & -1.77272 & 2.378275 \\
\hline H35 & 1.049782 & -1.47556 & 2.10786 & C33 & 3.831013 & -2.38841 & 1.869086 \\
\hline C36 & 4.72069 & -2.52427 & 2.728843 & $\mathrm{C} 34$ & 1.906213 & -1.63164 & 3.724124 \\
\hline H37 & 4.771542 & -3.05021 & 0.649063 & H35 & 0.546193 & -1.56853 & 2.070045 \\
\hline C38 & 3.955718 & -2.00739 & 3.777933 & C36 & 4.176078 & -2.25611 & 3.210286 \\
\hline H39 & 2.02495 & -1.2334 & 4.348859 & H37 & 4.592584 & -2.66627 & 1.145068 \\
\hline $\mathrm{H} 40$ & 5.750873 & -2.82431 & 2.898472 & C38 & 3.210949 & -1.87235 & 4.145106 \\
\hline H41 & 4.390405 & -1.89956 & 4.767593 & H39 & 1.146275 & -1.32352 & 4.435216 \\
\hline $\mathrm{C} 42$ & -3.50326 & -0.16208 & -0.21818 & $\mathrm{H} 40$ & 5.198119 & -2.447 & 3.524688 \\
\hline
\end{tabular}




\begin{tabular}{|c|c|c|c|c|c|c|c|}
\hline $\mathrm{C} 43$ & -4.6144 & 0.201497 & 0.557499 & $\mathrm{H} 41$ & 3.478973 & -1.76225 & 5.191988 \\
\hline $\mathrm{C} 44$ & -3.65872 & -0.32131 & -1.60454 & $\mathrm{C} 42$ & -3.71906 & -0.54524 & 0.21562 \\
\hline $\mathrm{C} 45$ & -5.85035 & 0.428996 & -0.05031 & $\mathrm{C} 43$ & -4.77097 & -0.28861 & 1.106341 \\
\hline $\mathrm{H} 46$ & -4.52313 & 0.305212 & 1.63355 & $\mathrm{C} 44$ & -3.96823 & -0.5138 & -1.16766 \\
\hline $\mathrm{C} 47$ & -4.89591 & -0.10075 & -2.20576 & $\mathrm{C} 45$ & -6.04644 & 0.003278 & 0.621363 \\
\hline $\mathrm{C} 48$ & -5.99448 & 0.282053 & -1.43087 & $\mathrm{H} 46$ & -4.59633 & -0.31121 & 2.177546 \\
\hline $\mathrm{H} 49$ & -6.70158 & 0.718329 & 0.559175 & $\mathrm{C} 47$ & -5.24536 & -0.23031 & -1.64818 \\
\hline H50 & -5.0018 & -0.2207 & -3.27995 & $\mathrm{C} 48$ & -6.28587 & 0.032158 & -0.75356 \\
\hline H51 & -6.95711 & 0.461151 & -1.90019 & H49 & -6.85447 & 0.204884 & 1.318821 \\
\hline H52 & -0.58754 & -0.49503 & -1.83036 & $\mathrm{H} 50$ & -5.42729 & -0.2059 & -2.71886 \\
\hline H53 & 1.675255 & 0.601349 & -1.35197 & H51 & -7.28015 & 0.258969 & -1.12724 \\
\hline $\mathrm{H} 54$ & -1.11229 & -1.85157 & 3.090307 & $\mathrm{H} 52$ & -0.86383 & -1.01244 & -1.89314 \\
\hline $\mathrm{H} 55$ & -2.80604 & -0.60918 & -2.21116 & $\mathrm{H} 53$ & 1.478939 & 1.962218 & -1.61077 \\
\hline H56 & 2.501406 & -4.23505 & -1.53463 & H54 & -1.47995 & -2.4542 & 3.352447 \\
\hline C57 & 4.143622 & -0.2551 & -3.42499 & H55 & -3.15469 & -0.70044 & -1.86338 \\
\hline C58 & 3.070523 & -1.118 & -3.66719 & H56 & 2.568873 & -4.11303 & -1.37944 \\
\hline C59 & 2.495079 & -1.82451 & -2.61526 & C57 & 4.424899 & 0.287389 & -2.67331 \\
\hline C60 & 2.97756 & -1.6885 & -1.30213 & C58 & 3.884776 & -0.89819 & -3.17246 \\
\hline C61 & 4.041405 & -0.81267 & -1.07016 & C59 & 3.200133 & -1.76794 & -2.32446 \\
\hline C62 & 4.621998 & -0.10402 & -2.12598 & $\mathrm{C} 60$ & 3.053602 & -1.47419 & -0.96366 \\
\hline $\mathrm{H} 63$ & 4.594497 & 0.300216 & -4.24235 & C61 & 3.591133 & -0.27628 & -0.4736 \\
\hline H64 & 2.681303 & -1.2375 & -4.67444 & C62 & 4.272543 & 0.595594 & -1.32093 \\
\hline H65 & 1.642499 & -2.46835 & -2.81413 & H63 & 4.958236 & 0.96553 & -3.33336 \\
\hline H66 & 4.414964 & -0.66412 & -0.06431 & H64 & 3.989563 & -1.1454 & -4.22489 \\
\hline H67 & 5.436121 & 0.584429 & -1.92117 & H65 & 2.753445 & -2.66026 & -2.75184 \\
\hline C68 & -1.01604 & 2.82103 & -1.72386 & H66 & 3.450251 & -0.01176 & 0.567471 \\
\hline C69 & -0.02291 & 2.00203 & -0.87771 & H67 & 4.68554 & 1.518601 & -0.92273 \\
\hline $\mathrm{C} 70$ & 1.318599 & 1.65783 & -1.6137 & C68 & -1.80027 & 3.235484 & -1.45325 \\
\hline H71 & 1.136887 & 1.583456 & -2.68374 & C69 & -0.50647 & 2.514691 & -1.00054 \\
\hline $\mathrm{C} 72$ & 2.512067 & 2.537494 & -1.33726 & C70 & 0.738614 & 2.70347 & -1.91536 \\
\hline $\mathrm{C} 73$ & 3.324749 & 2.357653 & -0.21103 & H71 & 0.461773 & 2.451004 & -2.94153 \\
\hline $\mathrm{C} 74$ & 2.790397 & 3.592198 & -2.21555 & $\mathrm{C} 72$ & 1.350897 & 4.082673 & -1.82073 \\
\hline $\mathrm{C} 75$ & 4.384307 & 3.229528 & 0.041538 & $\mathrm{C} 73$ & 2.456756 & 4.284062 & -0.981 \\
\hline $\mathrm{H} 76$ & 3.137375 & 1.524494 & 0.459169 & C74 & 0.829999 & 5.184268 & -2.51402 \\
\hline C77 & 3.853701 & 4.461202 & -1.96623 & C75 & 3.009661 & 5.55478 & -0.81321 \\
\hline H78 & 2.172065 & 3.732702 & -3.09915 & H76 & 2.889179 & 3.431682 & -0.46099 \\
\hline $\mathrm{C} 79$ & 4.650993 & 4.284722 & -0.83369 & C77 & 1.379298 & 6.457215 & -2.34733 \\
\hline H80 & 5.006951 & 3.078553 & 0.918941 & $\mathrm{H} 78$ & -0.00819 & 5.046799 & -3.19259 \\
\hline H81 & 4.060031 & 5.273383 & -2.65765 & C79 & 2.464709 & 6.649083 & -1.48897 \\
\hline H82 & 5.479195 & 4.960122 & -0.63971 & H80 & 3.869309 & 5.689592 & -0.16219 \\
\hline $\mathrm{C} 83$ & 0.480585 & 4.164482 & 0.573524 & H81 & 0.961828 & 7.299659 & -2.89164 \\
\hline C84 & 0.758787 & 2.039441 & 1.422353 & H82 & 2.891054 & 7.639549 & -1.36026 \\
\hline $\mathrm{C} 85$ & 0.794052 & 4.199421 & 2.083073 & $\mathrm{C} 83$ & -0.26741 & 4.174904 & 0.982672 \\
\hline H86 & -0.39661 & 4.76341 & 0.322686 & $\mathrm{C} 84$ & 0.605711 & 2.021948 & 1.168595 \\
\hline H87 & 1.325616 & 4.49353 & -0.03774 & $\mathrm{C} 85$ & 0.79025 & 4.090237 & 2.099288 \\
\hline
\end{tabular}




\begin{tabular}{|c|c|c|c|c|c|c|c|}
\hline H88 & 1.633957 & 4.845304 & 2.340257 & H86 & -1.27227 & 4.357446 & 1.375741 \\
\hline H89 & -0.07985 & 4.466603 & 2.685019 & H87 & -0.02351 & 4.947676 & 0.253114 \\
\hline N90 & 0.231728 & 2.725134 & 0.389567 & H88 & 1.719449 & 4.59329 & 1.819108 \\
\hline O91 & 1.159077 & 2.832748 & 2.420673 & H89 & 0.438881 & 4.461446 & 3.062747 \\
\hline O92 & 0.8975 & 0.8037 & 1.461012 & N90 & -0.17477 & 2.827715 & 0.395331 \\
\hline H93 & 2.738228 & -4.60739 & 0.197615 & O91 & 1.070829 & 2.674793 & 2.242359 \\
\hline N94 & -1.9751 & -2.33218 & 0.679515 & O92 & 0.872883 & 0.8321 & 0.983386 \\
\hline C95 & -1.39066 & -3.22158 & -0.34925 & H93 & 2.930423 & -4.41182 & 0.345737 \\
\hline H96 & -1.66917 & -2.9188 & -1.37052 & N94 & -2.0068 & -2.72545 & 0.77891 \\
\hline C97 & -2.323 & 3.110926 & -0.96829 & C95 & -1.36021 & -3.4839 & -0.32501 \\
\hline H98 & -2.15067 & 3.540773 & 0.022244 & H96 & -1.67734 & -3.1263 & -1.31475 \\
\hline H99 & -2.93944 & 3.816733 & -1.53388 & C97 & -2.97824 & 2.982361 & -0.50003 \\
\hline H100 & -2.90118 & 2.195754 & -0.83766 & H98 & -2.77829 & 3.300267 & 0.525364 \\
\hline C101 & -1.34254 & 2.196347 & -3.08706 & H99 & -3.86118 & 3.529027 & -0.84466 \\
\hline H102 & -0.47022 & 2.11717 & -3.74207 & H100 & -3.23716 & 1.921439 & -0.47961 \\
\hline H103 & -1.76226 & 1.196931 & -2.96091 & C101 & -2.19623 & 2.812016 & -2.87673 \\
\hline H104 & -2.08101 & 2.81295 & -3.60907 & H102 & -1.45467 & 3.09252 & -3.62815 \\
\hline \multirow[t]{2}{*}{ H105 } & -0.52391 & 3.788502 & -1.92576 & H103 & -2.34028 & 1.726081 & -2.92971 \\
\hline & & & & H104 & -3.14167 & 3.286554 & -3.15381 \\
\hline \multicolumn{4}{|c|}{ C3_ZR_Cheltop_TS2 } & H105 & -1.57166 & 4.309596 & -1.46331 \\
\hline $\mathrm{C} 1$ & -3.98524 & -3.88293 & 0.681175 & H106 & 0.992971 & -0.06361 & -1.69198 \\
\hline $\mathrm{C} 2$ & -1.82005 & -4.74932 & -0.02122 & H107 & -0.83815 & 1.420418 & -1.05735 \\
\hline $\mathrm{C} 3$ & -3.3232 & -4.8089 & -0.39123 & & & & \\
\hline $\mathrm{C} 4$ & -2.7683 & -3.42863 & 1.522743 & & & & \\
\hline H5 & -4.6862 & -4.43561 & 1.315278 & & & & \\
\hline H6 & -3.51052 & -4.4698 & -1.41511 & & & & \\
\hline H7 & -3.01474 & -2.98963 & 2.489112 & & & & \\
\hline H8 & -4.52662 & -3.04274 & 0.246129 & & & & \\
\hline H9 & -1.20364 & -5.53391 & -0.45951 & & & & \\
\hline H10 & -3.68798 & -5.83713 & -0.31507 & & & & \\
\hline $\mathrm{C} 11$ & -1.88052 & -4.68561 & 1.521458 & & & & \\
\hline H12 & -0.90958 & -4.52668 & 1.999985 & & & & \\
\hline H13 & -2.37033 & -5.55995 & 1.95921 & & & & \\
\hline $\mathrm{C} 14$ & 0.175 & -3.14618 & -0.42073 & & & & \\
\hline N15 & 0.765578 & -2.00379 & -0.43106 & & & & \\
\hline $\mathrm{C} 16$ & 2.246123 & -2.26439 & -0.28724 & & & & \\
\hline $\mathrm{C} 17$ & 2.302232 & -3.77706 & -0.65444 & & & & \\
\hline P18 & -1.99793 & -0.82781 & 0.61814 & & & & \\
\hline $\operatorname{Ir} 19$ & -0.2979 & -0.05879 & -0.57307 & & & & \\
\hline $\mathrm{C} 20$ & -1.95251 & -0.23698 & 2.348416 & & & & \\
\hline $\mathrm{C} 21$ & -1.69324 & 0.804446 & 4.942641 & & & & \\
\hline $\mathrm{C} 22$ & -1.4005 & -1.01699 & 3.374571 & & & & \\
\hline $\mathrm{C} 23$ & -2.37294 & 1.072138 & 2.635272 & & & & \\
\hline $\mathrm{C} 24$ & -2.24414 & 1.586359 & 3.924692 & & & & \\
\hline $\mathrm{C} 25$ & -1.27835 & -0.4987 & 4.66369 & & & & \\
\hline
\end{tabular}




\begin{tabular}{|c|c|c|c|}
\hline H26 & -2.80139 & 1.687366 & 1.853224 \\
\hline $\mathrm{H} 27$ & -2.58187 & 2.597354 & 4.136319 \\
\hline $\mathrm{H} 28$ & -0.85857 & -1.11707 & 5.451935 \\
\hline H29 & -1.59414 & 1.205529 & 5.94688 \\
\hline $\mathrm{O} 30$ & 0.941475 & -4.2396 & -0.42589 \\
\hline C31 & 2.671494 & -2.11982 & 1.183345 \\
\hline C32 & 1.763252 & -1.84751 & 2.202437 \\
\hline C33 & 4.019419 & -2.3261 & 1.513892 \\
\hline C34 & 2.188264 & -1.76501 & 3.52969 \\
\hline H35 & 0.729173 & -1.66417 & 1.957088 \\
\hline $\mathrm{C} 36$ & 4.444329 & -2.25442 & 2.837342 \\
\hline H37 & 4.743527 & -2.52666 & 0.728473 \\
\hline C38 & 3.526832 & -1.97033 & 3.853138 \\
\hline H39 & 1.466881 & -1.52427 & 4.305264 \\
\hline $\mathrm{H} 40$ & 5.492016 & -2.41361 & 3.076061 \\
\hline H41 & 3.858807 & -1.90612 & 4.885489 \\
\hline $\mathrm{C} 42$ & -3.70002 & -0.53486 & 0.010257 \\
\hline $\mathrm{C} 43$ & -4.77059 & -0.32988 & 0.893557 \\
\hline $\mathrm{C} 44$ & -3.96232 & -0.71209 & -1.35778 \\
\hline $\mathrm{C} 45$ & -6.07831 & -0.28245 & 0.409657 \\
\hline H46 & -4.5925 & -0.21141 & 1.956965 \\
\hline $\mathrm{C} 47$ & -5.26985 & -0.66989 & -1.83469 \\
\hline $\mathrm{C} 48$ & -6.33075 & -0.45134 & -0.952 \\
\hline H49 & -6.90031 & -0.11705 & 1.100133 \\
\hline H50 & -5.46091 & -0.80292 & -2.89552 \\
\hline H51 & -7.3501 & -0.41443 & -1.32497 \\
\hline H52 & -1.10979 & -0.50009 & -1.8372 \\
\hline H53 & 1.415467 & 1.300055 & -1.44288 \\
\hline H54 & -1.05717 & -2.02433 & 3.16864 \\
\hline H55 & -3.14081 & -0.87414 & -2.04749 \\
\hline H56 & 2.535195 & -3.98084 & -1.70101 \\
\hline C57 & 4.543432 & 0.405966 & -2.84612 \\
\hline C58 & 4.103117 & -0.82021 & -3.34333 \\
\hline $\mathrm{C} 59$ & 3.368501 & -1.68763 & -2.53262 \\
\hline C60 & 3.065855 & -1.35363 & -1.20625 \\
\hline C61 & 3.505494 & -0.11196 & -0.71989 \\
\hline C62 & 4.239351 & 0.755673 & -1.52985 \\
\hline H63 & 5.1188 & 1.079638 & -3.47411 \\
\hline H64 & 4.327171 & -1.10655 & -4.36673 \\
\hline H65 & 3.022674 & -2.61895 & -2.96748 \\
\hline H66 & 3.271265 & 0.16915 & 0.301459 \\
\hline H67 & 4.57267 & 1.708972 & -1.13297 \\
\hline C68 & -1.78142 & 2.761061 & -1.44996 \\
\hline C69 & -0.60596 & 2.015559 & -0.77901 \\
\hline $\mathrm{C} 70$ & 0.724671 & 2.149384 & -1.62794 \\
\hline
\end{tabular}




\begin{tabular}{lrrr} 
H71 & 0.473431 & 2.0746 & -2.68425 \\
C72 & 1.521492 & 3.412556 & -1.39065 \\
C73 & 2.506844 & 3.479488 & -0.39616 \\
C74 & 1.252689 & 4.560191 & -2.14852 \\
C75 & 3.191692 & 4.67018 & -0.14842 \\
H76 & 2.737284 & 2.595234 & 0.189754 \\
C77 & 1.931075 & 5.754048 & -1.8984 \\
H78 & 0.507909 & 4.518517 & -2.93948 \\
C79 & 2.898816 & 5.814408 & -0.8934 \\
H80 & 3.954184 & 4.703267 & 0.625474 \\
H81 & 1.707035 & 6.636 & -2.49188 \\
H82 & 3.428477 & 6.742956 & -0.70081 \\
C83 & -0.48701 & 3.973941 & 1.050756 \\
C84 & 0.474633 & 1.9 & 1.384183 \\
C85 & 0.457112 & 3.979658 & 2.278704 \\
H86 & -1.51942 & 4.19798 & 1.330276 \\
H87 & -0.16425 & 4.682079 & 0.285696 \\
H88 & 1.347587 & 4.589296 & 2.114738 \\
H89 & -0.03979 & 4.278499 & 3.203258 \\
N90 & -0.35932 & 2.584515 & 0.582028 \\
O91 & 0.89237 & 2.60116 & 2.43921 \\
O92 & 0.856548 & 0.731041 & 1.173597 \\
H93 & 2.967122 & -4.34739 & -0.00836 \\
N94 & -1.88249 & -2.51579 & 0.737858 \\
C95 & -1.31906 & -3.32493 & -0.37369 \\
H96 & -1.70418 & -3.00863 & -1.35402 \\
C97 & -3.07624 & 2.807662 & -0.62511 \\
H98 & -2.94988 & 3.266606 & 0.357284 \\
H99 & -3.8269 & 3.402774 & -1.15538 \\
H100 & -3.48933 & 1.810269 & -0.48733 \\
C101 & -2.12221 & 2.211609 & -2.84551 \\
H102 & -1.26434 & 2.178554 & -3.52152 \\
H103 & -2.52067 & 1.198036 & -2.76657 \\
H104 & -2.88443 & 2.840375 & -3.31654 \\
\hline & -1.4441 & 3.80245 & -1.58497 \\
\hline 1.195932 & -0.30113 & -2.95842 \\
H106 & 0.503769 & -0.27478 & -3.22418
\end{tabular}

C3_ZS_Chelbot

C3_ZS_Chelbot_SM

$\begin{array}{llll}\text { C1 } & -3.79966 & -3.44435 & -1.29761\end{array}$

C2 $\quad-1.52645 \quad-4.33553 \quad-1.22873$

$\begin{array}{llll}\text { C3 } & -2.73555 & -4.17059 & -2.18718\end{array}$

$\begin{array}{llll}\text { C4 } & -3.03668 & -3.26023 & 0.032425\end{array}$

H5 $\quad-4.68885 \quad-4.06414 \quad-1.14223$

C3_ZS_Chelbot_INT2

$\begin{array}{llll}\text { C1 } & -3.75023 & -3.44428 & -1.46494 \\ \text { C2 } & -1.54938 & -4.41945 & -1.07503 \\ \text { C3 } & -2.63794 & -4.28781 & -2.17153 \\ \text { C4 } & -3.12083 & -3.16724 & -0.08256 \\ \text { H5 } & -4.67708 & -4.01518 & -1.34578\end{array}$




\begin{tabular}{|c|c|c|c|c|c|c|c|}
\hline H6 & -2.47343 & -3.60082 & -3.08425 & H6 & -2.25319 & -3.80744 & -3.07646 \\
\hline H7 & -3.65228 & -2.98187 & 0.888381 & $\mathrm{H} 7$ & -3.81307 & -2.78556 & 0.667985 \\
\hline H8 & -4.12598 & -2.48768 & -1.70743 & H8 & -3.99517 & -2.52087 & -1.98952 \\
\hline H9 & -0.79217 & -5.08004 & -1.5367 & H9 & -0.82705 & -5.21896 & -1.23754 \\
\hline H10 & -3.08999 & -5.15188 & -2.51454 & $\mathrm{H} 10$ & -3.00602 & -5.2772 & -2.45676 \\
\hline $\mathrm{C} 11$ & -2.22916 & -4.56731 & 0.12707 & $\mathrm{C} 11$ & -2.4007 & -4.49747 & 0.209719 \\
\hline H12 & -1.54074 & -4.60091 & 0.976913 & H12 & -1.80518 & -4.48976 & 1.127558 \\
\hline H13 & -2.85777 & -5.46209 & 0.13285 & H13 & -3.07607 & -5.35746 & 0.222241 \\
\hline $\mathrm{C} 14$ & 0.429156 & -2.90221 & -0.32813 & $\mathrm{C} 14$ & 0.40533 & -3.02 & -0.12743 \\
\hline N15 & 1.006666 & -1.82654 & 0.087315 & N15 & 0.976495 & -1.95737 & 0.323075 \\
\hline $\mathrm{C} 16$ & 2.382772 & -2.19424 & 0.605014 & $\mathrm{C} 16$ & 2.326165 & -2.34275 & 0.877168 \\
\hline $\mathrm{C} 17$ & 2.252397 & -3.74862 & 0.660058 & $\mathrm{C} 17$ & 2.245159 & -3.90668 & 0.801315 \\
\hline P18 & -1.85716 & -0.67337 & 0.334943 & P18 & -1.80935 & -0.66996 & 0.317875 \\
\hline Ir19 & 0.318295 & 0.191319 & -0.35401 & $\operatorname{Ir} 19$ & 0.314328 & 0.092851 & -0.31879 \\
\hline $\mathrm{C} 20$ & -1.9784 & -0.686 & 2.165398 & $\mathrm{C} 20$ & -1.90806 & -0.69876 & 2.158825 \\
\hline $\mathrm{C} 21$ & -1.94525 & -0.63074 & 4.973078 & $\mathrm{C} 21$ & -2.01122 & -0.70538 & 4.969021 \\
\hline $\mathrm{C} 22$ & -1.93619 & -1.87835 & 2.898717 & $\mathrm{C} 22$ & -2.17422 & -1.88145 & 2.860367 \\
\hline $\mathrm{C} 23$ & -1.98453 & 0.53702 & 2.856947 & $\mathrm{C} 23$ & -1.6704 & 0.478994 & 2.888631 \\
\hline $\mathrm{C} 24$ & -1.97527 & 0.564489 & 4.249356 & $\mathrm{C} 24$ & -1.73211 & 0.477651 & 4.280151 \\
\hline $\mathrm{C} 25$ & -1.92072 & -1.84861 & 4.294778 & $\mathrm{C} 25$ & -2.22212 & -1.8839 & 4.255745 \\
\hline $\mathrm{H} 26$ & -1.98471 & 1.472943 & 2.310057 & $\mathrm{H} 26$ & -1.41112 & 1.397334 & 2.376082 \\
\hline $\mathrm{H} 27$ & -1.98145 & 1.518352 & 4.769131 & $\mathrm{H} 27$ & -1.54895 & 1.399083 & 4.825708 \\
\hline $\mathrm{H} 28$ & -1.88041 & -2.78044 & 4.851275 & $\mathrm{H} 28$ & -2.41967 & -2.81246 & 4.783222 \\
\hline H29 & -1.93194 & -0.61044 & 6.059004 & $\mathrm{H} 29$ & -2.0512 & -0.70879 & 6.054379 \\
\hline $\mathrm{O} 30$ & 1.074409 & -4.0559 & -0.14127 & $\mathrm{O} 30$ & 1.038862 & -4.18381 & 0.036874 \\
\hline C31 & 2.655773 & -1.69109 & 2.017398 & C31 & 2.528845 & -1.92101 & 2.325928 \\
\hline C32 & 1.6288 & -1.24947 & 2.855021 & $\mathrm{C} 32$ & 1.548041 & -1.26219 & 3.066561 \\
\hline $\mathrm{C} 33$ & 3.954181 & -1.80284 & 2.536884 & $\mathrm{C} 33$ & 3.73355 & -2.27556 & 2.954 \\
\hline $\mathrm{C} 34$ & 1.901031 & -0.8774 & 4.172624 & C34 & 1.777551 & -0.9341 & 4.405302 \\
\hline H35 & 0.614761 & -1.19208 & 2.485765 & H35 & 0.605436 & -1.0052 & 2.6071 \\
\hline C36 & 4.224577 & -1.43668 & 3.853796 & C36 & 3.96001 & -1.95231 & 4.289045 \\
\hline H37 & 4.761611 & -2.1623 & 1.905247 & H37 & 4.505735 & -2.79076 & 2.38764 \\
\hline C38 & 3.199328 & -0.96198 & 4.673913 & C38 & 2.98186 & -1.27209 & 5.018489 \\
\hline H39 & 1.088104 & -0.52599 & 4.801205 & H39 & 1.002038 & -0.41729 & 4.962063 \\
\hline $\mathrm{H} 40$ & 5.238032 & -1.51866 & 4.236188 & $\mathrm{H} 40$ & 4.900877 & -2.22628 & 4.757479 \\
\hline $\mathrm{H} 41$ & 3.412523 & -0.66801 & 5.69766 & H41 & 3.159503 & -1.0134 & 6.058333 \\
\hline $\mathrm{C} 42$ & -3.44751 & 0.039784 & -0.2504 & $\mathrm{C} 42$ & -3.40543 & 0.058362 & -0.21252 \\
\hline $\mathrm{C} 43$ & -4.46565 & 0.475696 & 0.606205 & $\mathrm{C} 43$ & -4.33169 & 0.597357 & 0.688167 \\
\hline $\mathrm{C} 44$ & -3.64125 & 0.120185 & -1.64015 & $\mathrm{C} 44$ & -3.70044 & 0.057905 & -1.58535 \\
\hline $\mathrm{C} 45$ & -5.64458 & 1.011407 & 0.08193 & $\mathrm{C} 45$ & -5.52688 & 1.146238 & 0.219557 \\
\hline $\mathrm{H} 46$ & -4.34485 & 0.405498 & 1.68225 & H46 & -4.12565 & 0.597948 & 1.753294 \\
\hline $\mathrm{C} 47$ & -4.81904 & 0.65364 & -2.15844 & $\mathrm{C} 47$ & -4.89102 & 0.611 & -2.0492 \\
\hline $\mathrm{C} 48$ & -5.82201 & 1.111316 & -1.29771 & $\mathrm{C} 48$ & -5.80573 & 1.161888 & -1.14651 \\
\hline H49 & -6.42561 & 1.347793 & 0.757728 & H49 & -6.23999 & 1.56058 & 0.926271 \\
\hline $\mathrm{H} 50$ & -4.95977 & 0.702468 & -3.23495 & H50 & -5.1137 & 0.59828 & -3.11266 \\
\hline
\end{tabular}




\begin{tabular}{|c|c|c|c|c|c|c|c|}
\hline H51 & -6.7394 & 1.529389 & -1.70103 & H51 & -6.73607 & 1.590768 & -1.50727 \\
\hline H52 & 0.681668 & 0.604731 & 1.095665 & H52 & 0.478218 & 0.637759 & 1.132868 \\
\hline H53 & 1.888502 & 0.314765 & -0.58516 & H53 & 1.740688 & 1.53362 & -2.432 \\
\hline H54 & -1.88573 & -2.83159 & 2.386372 & $\mathrm{H} 54$ & -2.32342 & -2.8123 & 2.33072 \\
\hline H55 & -2.88234 & -0.26333 & -2.31376 & H55 & -3.015 & -0.39849 & -2.29029 \\
\hline H56 & 3.105699 & -4.27993 & 0.239506 & H56 & 3.088112 & -4.36283 & 0.280835 \\
\hline C57 & 5.176147 & -0.84002 & -2.43866 & C57 & 5.103771 & -0.63532 & -1.99461 \\
\hline C58 & 4.417824 & -1.99494 & -2.6342 & $\mathrm{C} 58$ & 4.229922 & -1.66129 & -2.36313 \\
\hline C59 & 3.535797 & -2.42883 & -1.64562 & C59 & 3.377613 & -2.2242 & -1.41579 \\
\hline C60 & 3.40223 & -1.72374 & -0.441 & C60 & 3.381477 & -1.77737 & -0.086 \\
\hline C61 & 4.150038 & -0.55054 & -0.26708 & C61 & 4.261064 & -0.75014 & 0.27381 \\
\hline C62 & 5.033246 & -0.11605 & -1.25624 & C62 & 5.117552 & -0.18517 & -0.67488 \\
\hline H63 & 5.86251 & -0.49929 & -3.2081 & $\mathrm{H} 63$ & 5.767466 & -0.19218 & -2.73129 \\
\hline H64 & 4.512195 & -2.56065 & -3.55678 & H64 & 4.208969 & -2.01977 & -3.38817 \\
\hline H65 & 2.944355 & -3.3225 & -1.8274 & H65 & 2.688606 & -3.00576 & -1.72633 \\
\hline H66 & 4.047576 & 0.023866 & 0.6469 & H66 & 4.271966 & -0.37579 & 1.29113 \\
\hline H67 & 5.603498 & 0.795479 & -1.10267 & H67 & 5.793021 & 0.612209 & -0.37823 \\
\hline C68 & -0.80095 & 3.322061 & -0.21356 & C68 & -0.60698 & 3.202018 & -0.44636 \\
\hline C69 & -0.16946 & 2.180599 & -1.02677 & C69 & -0.03252 & 2.012649 & -1.25399 \\
\hline $\mathrm{C} 70$ & 1.275227 & 2.022899 & -1.04517 & $\mathrm{C} 70$ & 1.342016 & 2.422099 & -1.93156 \\
\hline H71 & 1.793935 & 2.471395 & -0.20294 & $\mathrm{H} 71$ & 2.053138 & 2.679734 & -1.14228 \\
\hline $\mathrm{C} 72$ & 2.061803 & 2.11181 & -2.32132 & $\mathrm{C} 72$ & 1.296647 & 3.527084 & -2.96424 \\
\hline $\mathrm{C} 73$ & 2.505902 & 3.397129 & -2.67696 & $\mathrm{C} 73$ & 1.321223 & 4.884089 & -2.61118 \\
\hline $\mathrm{C} 74$ & 2.34916 & 1.040213 & -3.16987 & $\mathrm{C} 74$ & 1.226392 & 3.198004 & -4.32664 \\
\hline $\mathrm{C} 75$ & 3.197021 & 3.607598 & -3.8687 & $\mathrm{C} 75$ & 1.240151 & 5.879053 & -3.58657 \\
\hline H76 & 2.306077 & 4.235423 & -2.01247 & H76 & 1.412897 & 5.166858 & -1.56605 \\
\hline $\mathrm{C} 77$ & 3.037744 & 1.252991 & -4.36567 & C77 & 1.144042 & 4.188878 & -5.30541 \\
\hline H78 & 2.044007 & 0.038347 & -2.89284 & $\mathrm{H} 78$ & 1.243198 & 2.150261 & -4.62124 \\
\hline C79 & 3.457856 & 2.53292 & -4.72286 & C79 & 1.141979 & 5.535113 & -4.93594 \\
\hline H80 & 3.533437 & 4.607526 & -4.1285 & H80 & 1.259703 & 6.924781 & -3.29243 \\
\hline H81 & 3.252494 & 0.408028 & -5.0134 & $\mathrm{H} 81$ & 1.093804 & 3.911677 & -6.35502 \\
\hline H82 & 3.993504 & 2.694653 & -5.65396 & H82 & 1.079644 & 6.310373 & -5.69403 \\
\hline $\mathrm{C} 83$ & -1.05436 & 3.145787 & -3.27797 & $\mathrm{C} 83$ & -1.79824 & 2.566101 & -3.20811 \\
\hline $\mathrm{C} 84$ & -0.75856 & 0.872557 & -3.01119 & $\mathrm{C} 84$ & -0.81093 & 0.489663 & -2.97785 \\
\hline $\mathrm{C} 85$ & -1.67341 & 2.363948 & -4.45001 & $\mathrm{C} 85$ & -1.92035 & 1.74246 & -4.50668 \\
\hline H86 & -0.11481 & 3.643055 & -3.54466 & H86 & -1.328 & 3.533783 & -3.38013 \\
\hline H87 & -1.7477 & 3.878216 & -2.86046 & H87 & -2.76776 & 2.711045 & -2.72285 \\
\hline H88 & -2.76653 & 2.347837 & -4.40039 & H88 & -2.94451 & 1.646894 & -4.86941 \\
\hline H89 & -1.34867 & 2.705584 & -5.43283 & H89 & -1.27524 & 2.125835 & -5.30091 \\
\hline N90 & -0.80047 & 2.043621 & -2.34093 & N90 & -0.94748 & 1.685285 & -2.39385 \\
\hline O91 & -1.20215 & 0.99882 & -4.26874 & O91 & -1.44774 & 0.406953 & -4.15569 \\
\hline O92 & -0.38622 & -0.22136 & -2.55253 & O92 & -0.1978 & -0.48891 & -2.49687 \\
\hline H93 & 2.055625 & -4.09861 & 1.674906 & H93 & 2.121578 & -4.35855 & 1.786948 \\
\hline N94 & -1.93371 & -2.27836 & -0.17242 & N94 & -1.95159 & -2.25 & -0.25528 \\
\hline C95 & -0.9095 & -2.93138 & -1.02311 & C95 & -0.87997 & -3.03157 & -0.91791 \\
\hline
\end{tabular}




\begin{tabular}{|c|c|c|c|c|c|c|c|}
\hline H96 & -0.78175 & -2.40568 & -1.97583 & H96 & -0.63211 & -2.59868 & -1.89385 \\
\hline C97 & -2.32249 & 3.207897 & -0.06731 & C97 & -2.04015 & 3.026089 & 0.065809 \\
\hline H98 & -2.73955 & 4.16126 & 0.272268 & H98 & -2.4106 & 3.976503 & 0.464536 \\
\hline H99 & -2.81861 & 2.928824 & -0.99974 & H99 & -2.74107 & 2.693379 & -0.69944 \\
\hline H100 & -2.59257 & 2.449973 & 0.667636 & H100 & -2.08602 & 2.298072 & 0.873769 \\
\hline $\mathrm{C} 101$ & -0.14497 & 3.542552 & 1.157206 & C101 & 0.297069 & 3.608859 & 0.729321 \\
\hline H102 & -0.1797 & 2.63138 & 1.760199 & H102 & 0.372138 & 2.797583 & 1.459782 \\
\hline H103 & 0.901073 & 3.851691 & 1.078523 & H103 & 1.3133 & 3.867137 & 0.420768 \\
\hline H104 & -0.67448 & 4.33462 & 1.69576 & H104 & -0.12079 & 4.484773 & 1.2363 \\
\hline \multirow[t]{2}{*}{ H105 } & -0.59912 & 4.231462 & -0.80532 & H105 & -0.63061 & 4.057682 & -1.13745 \\
\hline & & & & H106 & 1.951417 & 0.617822 & -0.12919 \\
\hline \multicolumn{4}{|c|}{ C3_ZS_Chelbot_TS1 } & H107 & 1.959393 & 0.244907 & -0.88899 \\
\hline $\mathrm{C} 1$ & -3.77593 & -3.44484 & -1.26031 & & & & \\
\hline $\mathrm{C} 2$ & -1.5019 & -4.33488 & -1.20938 & \multicolumn{4}{|c|}{ C3_ZS_Chelbot_TS3 } \\
\hline $\mathrm{C} 3$ & -2.71883 & -4.17131 & -2.15817 & $\mathrm{C} 1$ & -3.77689 & -3.40923 & -1.57868 \\
\hline $\mathrm{C} 4$ & -3.00516 & -3.26653 & 0.066385 & $\mathrm{C} 2$ & -1.54655 & -4.33801 & -1.24718 \\
\hline H5 & -4.66613 & -4.06245 & -1.10207 & $\mathrm{C} 3$ & -2.64318 & -4.17961 & -2.3324 \\
\hline H6 & -2.46469 & -3.60233 & -3.05806 & $\mathrm{C} 4$ & -3.15265 & -3.19663 & -0.18206 \\
\hline $\mathrm{H} 7$ & -3.61664 & -2.99287 & 0.926887 & H5 & -4.68829 & -4.01069 & -1.49532 \\
\hline H8 & -4.10198 & -2.48611 & -1.66566 & H6 & -2.27607 & -3.64159 & -3.212 \\
\hline H9 & -0.76757 & -5.07637 & -1.52462 & $\mathrm{H} 7$ & -3.857 & -2.87853 & 0.586339 \\
\hline H10 & -3.07566 & -5.15308 & -2.48159 & H8 & -4.04644 & -2.46216 & -2.04688 \\
\hline $\mathrm{C} 11$ & -2.19464 & -4.57252 & 0.150817 & H9 & -0.80095 & -5.10392 & -1.45772 \\
\hline H12 & -1.50074 & -4.60588 & 0.996243 & H10 & -2.9829 & -5.16312 & -2.66896 \\
\hline H13 & -2.82113 & -5.46882 & 0.157723 & $\mathrm{C} 11$ & -2.39106 & -4.51746 & 0.033093 \\
\hline C14 & 0.460238 & -2.89096 & -0.33058 & H12 & -1.79479 & -4.53897 & 0.950432 \\
\hline N15 & 1.032018 & -1.81046 & 0.078378 & H13 & -3.03571 & -5.4002 & -0.00406 \\
\hline $\mathrm{C} 16$ & 2.412682 & -2.16388 & 0.584943 & $\mathrm{C} 14$ & 0.371836 & -2.93321 & -0.23554 \\
\hline $\mathrm{C} 17$ & 2.304591 & -3.72148 & 0.630678 & N15 & 0.964548 & -1.88976 & 0.244537 \\
\hline P18 & -1.82859 & -0.67719 & 0.359109 & $\mathrm{C} 16$ & 2.316257 & -2.30677 & 0.78952 \\
\hline Ir19 & 0.295375 & 0.204808 & -0.33683 & $\mathrm{C} 17$ & 2.181037 & -3.86222 & 0.717324 \\
\hline $\mathrm{C} 20$ & -1.95857 & -0.67384 & 2.187404 & P18 & -1.88087 & -0.68643 & 0.362552 \\
\hline $\mathrm{C} 21$ & -1.9475 & -0.58476 & 4.992918 & Ir19 & 0.395203 & 0.137373 & -0.14334 \\
\hline $\mathrm{C} 22$ & -1.91885 & -1.85762 & 2.933964 & $\mathrm{C} 20$ & -2.03336 & -0.81458 & 2.195006 \\
\hline $\mathrm{C} 23$ & -1.97444 & 0.557584 & 2.863304 & $\mathrm{C} 21$ & -2.17754 & -0.90494 & 5.002916 \\
\hline $\mathrm{C} 24$ & -1.97574 & 0.601872 & 4.254811 & $\mathrm{C} 22$ & -2.35749 & -2.00737 & 2.854848 \\
\hline $\mathrm{C} 25$ & -1.91439 & -1.8108 & 4.329935 & $\mathrm{C} 23$ & -1.75809 & 0.327954 & 2.967044 \\
\hline $\mathrm{H} 26$ & -1.97381 & 1.486046 & 2.303818 & $\mathrm{C} 24$ & -1.84026 & 0.286663 & 4.357014 \\
\hline $\mathrm{H} 27$ & -1.98916 & 1.561986 & 4.762684 & $\mathrm{C} 25$ & -2.42462 & -2.05148 & 4.249183 \\
\hline H28 & -1.87625 & -2.73582 & 4.897817 & H26 & -1.4513 & 1.249175 & 2.483649 \\
\hline H29 & -1.94314 & -0.55119 & 6.078527 & $\mathrm{H} 27$ & -1.62682 & 1.180954 & 4.935196 \\
\hline $\mathrm{O} 30$ & 1.118829 & -4.03966 & -0.15521 & $\mathrm{H} 28$ & -2.66867 & -2.98665 & 4.744448 \\
\hline $\mathrm{C} 31$ & 2.685456 & -1.66526 & 1.999358 & $\mathrm{H} 29$ & -2.23406 & -0.94122 & 6.086888 \\
\hline C32 & 1.654782 & -1.24309 & 2.842306 & $\mathrm{O} 30$ & 0.997021 & -4.10239 & -0.09336 \\
\hline C33 & 3.987049 & -1.7573 & 2.514198 & C31 & 2.524558 & -1.92089 & 2.248036 \\
\hline
\end{tabular}




\begin{tabular}{|c|c|c|c|c|c|c|c|}
\hline C34 & 1.924951 & -0.86891 & 4.159549 & $\mathrm{C} 32$ & 1.466731 & -1.5155 & 3.065612 \\
\hline H35 & 0.63883 & -1.20224 & 2.477049 & $\mathrm{C} 33$ & 3.788612 & -2.12129 & 2.822963 \\
\hline C36 & 4.255941 & -1.38918 & 3.831148 & $\mathrm{C} 34$ & 1.678897 & -1.26471 & 4.422554 \\
\hline H37 & 4.798068 & -2.10235 & 1.879161 & H35 & 0.474389 & -1.39793 & 2.652879 \\
\hline C38 & 3.226255 & -0.93255 & 4.6558 & $\mathrm{C} 36$ & 3.997223 & -1.87866 & 4.178948 \\
\hline H39 & 1.108374 & -0.53182 & 4.791164 & H37 & 4.616444 & -2.45785 & 2.20537 \\
\hline $\mathrm{H} 40$ & 5.271858 & -1.45527 & 4.209905 & C38 & 2.943621 & -1.43916 & 4.981867 \\
\hline H41 & 3.438614 & -0.63632 & 5.679054 & H39 & 0.844543 & -0.93895 & 5.035806 \\
\hline $\mathrm{C} 42$ & -3.41032 & 0.038384 & -0.23961 & $\mathrm{H} 40$ & 4.984328 & -2.03053 & 4.605884 \\
\hline $\mathrm{C} 43$ & -4.43564 & 0.473967 & 0.608031 & $\mathrm{H} 41$ & 3.108472 & -1.24264 & 6.037221 \\
\hline $\mathrm{C} 44$ & -3.59048 & 0.113428 & -1.6311 & $\mathrm{C} 42$ & -3.46927 & 0.0808 & -0.15047 \\
\hline $\mathrm{C} 45$ & -5.61177 & 1.002787 & 0.071908 & $\mathrm{C} 43$ & -4.3863 & 0.631768 & 0.752266 \\
\hline H46 & -4.32327 & 0.407783 & 1.685137 & C44 & -3.74779 & 0.134363 & -1.52675 \\
\hline $\mathrm{C} 47$ & -4.76522 & 0.639915 & -2.16119 & $\mathrm{C} 45$ & -5.55694 & 1.234423 & 0.28612 \\
\hline $\mathrm{C} 48$ & -5.77627 & 1.094626 & -1.3093 & $\mathrm{H} 46$ & -4.19301 & 0.59574 & 1.819355 \\
\hline H49 & -6.40047 & 1.339805 & 0.738397 & C47 & -4.91729 & 0.733314 & -1.98831 \\
\hline H50 & -4.89781 & 0.685605 & -3.23884 & $\mathrm{C} 48$ & -5.82415 & 1.290076 & -1.08105 \\
\hline H51 & -6.6923 & 1.50704 & -1.72212 & H49 & -6.26242 & 1.655924 & 0.996329 \\
\hline H52 & 0.642254 & 0.609789 & 1.120284 & $\mathrm{H} 50$ & -5.13007 & 0.755736 & -3.05387 \\
\hline H53 & 1.868241 & 0.456635 & -0.59671 & H51 & -6.73715 & 1.756229 & -1.43946 \\
\hline H54 & -1.8615 & -2.81661 & 2.432401 & H52 & 0.580581 & 0.432393 & 1.372975 \\
\hline H55 & -2.82335 & -0.26894 & -2.29558 & H53 & 1.849203 & 1.619074 & -2.21304 \\
\hline H56 & 3.158594 & -4.2386 & 0.193976 & H54 & -2.53914 & -2.91435 & 2.293976 \\
\hline $\mathrm{C} 57$ & 5.180103 & -0.7539 & -2.4572 & $\mathrm{H} 55$ & -3.05992 & -0.31141 & -2.23841 \\
\hline C58 & 4.434668 & -1.91629 & -2.65882 & H56 & 3.028658 & -4.35404 & 0.240233 \\
\hline C59 & 3.5577 & -2.36532 & -1.67225 & C57 & 5.115569 & -0.7084 & -2.12005 \\
\hline C60 & 3.417479 & -1.66875 & -0.46345 & C58 & 4.299215 & -1.79601 & -2.43515 \\
\hline C61 & 4.148255 & -0.48595 & -0.28559 & C59 & 3.436059 & -2.32024 & -1.47598 \\
\hline C62 & 5.026589 & -0.03638 & -1.27236 & C60 & 3.370422 & -1.77035 & -0.18772 \\
\hline H63 & 5.863049 & -0.40174 & -3.22454 & C61 & 4.183094 & -0.67166 & 0.113809 \\
\hline H64 & 4.535412 & -2.47617 & -3.58431 & C62 & 5.054868 & -0.15031 & -0.84355 \\
\hline H65 & 2.976911 & -3.26522 & -1.85772 & H63 & 5.792312 & -0.29973 & -2.86501 \\
\hline H66 & 4.040197 & 0.082083 & 0.631589 & H64 & 4.333439 & -2.236 & -3.42774 \\
\hline H67 & 5.584847 & 0.881854 & -1.11539 & H65 & 2.793969 & -3.15347 & -1.7506 \\
\hline C68 & -0.7413 & 3.3491 & -0.2103 & H66 & 4.127431 & -0.20959 & 1.092653 \\
\hline C69 & -0.12148 & 2.190382 & -1.01012 & H67 & 5.682385 & 0.699322 & -0.58963 \\
\hline $\mathrm{C} 70$ & 1.336222 & 2.019712 & -1.01909 & C68 & -0.63834 & 3.387669 & -0.48266 \\
\hline H71 & 1.846072 & 2.47554 & -0.17493 & C69 & 0.019565 & 2.201782 & -1.22763 \\
\hline $\mathrm{C} 72$ & 2.120305 & 2.126307 & -2.29581 & $\mathrm{C} 70$ & 1.40046 & 2.560668 & -1.88662 \\
\hline $\mathrm{C} 73$ & 2.57817 & 3.410662 & -2.63444 & $\mathrm{H} 71$ & 2.067763 & 2.954448 & -1.11581 \\
\hline $\mathrm{C} 74$ & 2.372827 & 1.065707 & -3.16872 & $\mathrm{C} 72$ & 1.318417 & 3.502348 & -3.06558 \\
\hline C75 & 3.248028 & 3.632044 & -3.83629 & $\mathrm{C} 73$ & 1.227805 & 4.891823 & -2.90059 \\
\hline H76 & 2.403324 & 4.239851 & -1.95191 & $\mathrm{C} 74$ & 1.306817 & 2.982579 & -4.36829 \\
\hline $\mathrm{C} 77$ & 3.039658 & 1.289851 & -4.37465 & $\mathrm{C} 75$ & 1.0963 & 5.734987 & -4.0046 \\
\hline H78 & 2.058361 & 0.063705 & -2.90148 & $\mathrm{H} 76$ & 1.267027 & 5.319982 & -1.90261 \\
\hline
\end{tabular}




\begin{tabular}{|c|c|c|c|c|c|c|c|}
\hline C79 & 3.472481 & 2.570098 & -4.71626 & C77 & 1.176178 & 3.822383 & -5.47513 \\
\hline H80 & 3.595347 & 4.631051 & -4.08477 & $\mathrm{H} 78$ & 1.409564 & 1.909314 & -4.51478 \\
\hline H81 & 3.227448 & 0.454255 & -5.04269 & C79 & 1.060894 & 5.202096 & -5.29467 \\
\hline H82 & 3.990281 & 2.741291 & -5.65573 & H80 & 1.026451 & 6.809065 & -3.85796 \\
\hline $\mathrm{C} 83$ & -0.96489 & 3.181887 & -3.27539 & H81 & 1.175055 & 3.401876 & -6.4771 \\
\hline $\mathrm{C} 84$ & -0.71761 & 0.903716 & -3.00525 & H82 & 0.958866 & 5.858981 & -6.15344 \\
\hline $\mathrm{C} 85$ & -1.59256 & 2.411345 & -4.45059 & $\mathrm{C} 83$ & -1.76305 & 2.530114 & -3.14681 \\
\hline H86 & -0.01581 & 3.662545 & -3.538 & $\mathrm{C} 84$ & -0.63927 & 0.515022 & -2.86962 \\
\hline H87 & -1.64728 & 3.926838 & -2.862 & $\mathrm{C} 85$ & -1.86743 & 1.647426 & -4.40919 \\
\hline H88 & -2.68614 & 2.414964 & -4.40658 & H86 & -1.32274 & 3.501764 & -3.37263 \\
\hline H89 & -1.25675 & 2.746474 & -5.43196 & H87 & -2.73137 & 2.666554 & -2.65871 \\
\hline N90 & -0.73599 & 2.075341 & -2.33643 & H88 & -2.89482 & 1.465449 & -4.72718 \\
\hline O91 & -1.14743 & 1.037674 & -4.26664 & H89 & -1.28052 & 2.045974 & -5.23946 \\
\hline O92 & -0.37166 & -0.19787 & -2.54223 & N90 & -0.8741 & 1.713204 & -2.3013 \\
\hline H93 & 2.126582 & -4.08144 & 1.645976 & O91 & -1.28752 & 0.365022 & -4.03144 \\
\hline N94 & -1.90335 & -2.28457 & -0.13769 & O92 & 0.055702 & -0.38931 & -2.37921 \\
\hline C95 & -0.8881 & -2.92884 & -1.00624 & H93 & 1.997117 & -4.2991 & 1.699956 \\
\hline H96 & -0.77787 & -2.39927 & -1.95928 & N94 & -2.01528 & -2.23595 & -0.29798 \\
\hline C97 & -2.26693 & 3.247506 & -0.08707 & C95 & -0.92349 & -2.93723 & -1.01507 \\
\hline H98 & -2.68165 & 4.197051 & 0.266401 & H96 & -0.69478 & -2.44519 & -1.96666 \\
\hline H99 & -2.7539 & 2.991144 & -1.03161 & C97 & -2.01468 & 3.06898 & 0.108839 \\
\hline H100 & -2.55132 & 2.475331 & 0.627562 & H98 & -2.46609 & 3.981009 & 0.512012 \\
\hline $\mathrm{C} 101$ & -0.10304 & 3.564337 & 1.169217 & H99 & -2.71183 & 2.640358 & -0.6099 \\
\hline H102 & -0.16186 & 2.657314 & 1.776455 & H100 & -1.91963 & 2.354753 & 0.927808 \\
\hline H103 & 0.94902 & 3.856939 & 1.105457 & C101 & 0.260634 & 3.965516 & 0.622817 \\
\hline H104 & -0.62716 & 4.367748 & 1.69631 & $\mathrm{H} 102$ & 0.455637 & 3.214637 & 1.398234 \\
\hline H105 & -0.52106 & 4.255593 & -0.79979 & $\mathrm{H} 103$ & 1.223812 & 4.326736 & 0.254673 \\
\hline $\mathrm{X}$ & 0 & 0 & 0 & H104 & -0.24304 & 4.810252 & 1.101654 \\
\hline & & & & H105 & -0.76909 & 4.173234 & -1.24021 \\
\hline C3_ZS & nelbot_INT1 & & & H106 & 0.780395 & 1.650332 & 0.028914 \\
\hline $\mathrm{C} 1$ & -3.86714 & -3.49565 & -1.04459 & H107 & 1.946964 & 0.42717 & -0.30181 \\
\hline $\mathrm{C} 2$ & -1.61234 & -4.43598 & -1.02658 & & & & \\
\hline $\mathrm{C} 3$ & -2.85772 & -4.28916 & -1.94076 & \multicolumn{4}{|c|}{ C3_ZS_Chelbot_PROD } \\
\hline $\mathrm{C} 4$ & -3.05305 & -3.28624 & 0.250898 & $\mathrm{C} 1$ & -3.86701 & -3.58765 & -1.17036 \\
\hline H5 & -4.77059 & -4.0783 & -0.83687 & $\mathrm{C} 2$ & -1.5816 & -4.4505 & -1.13453 \\
\hline H6 & -2.62283 & -3.77081 & -2.87571 & $\mathrm{C} 3$ & -2.79723 & -4.27619 & -2.08318 \\
\hline H7 & -3.63099 & -2.96776 & 1.119241 & $\mathrm{C} 4$ & -3.10609 & -3.44363 & 0.166522 \\
\hline H8 & -4.17533 & -2.54332 & -1.4796 & H5 & -4.75298 & -4.21764 & -1.03894 \\
\hline H9 & -0.90169 & -5.20335 & -1.334 & H6 & -2.54725 & -3.68191 & -2.96789 \\
\hline H10 & -3.24739 & -5.27674 & -2.20297 & $\mathrm{H} 7$ & -3.72708 & -3.20586 & 1.030753 \\
\hline $\mathrm{C} 11$ & -2.26477 & -4.6039 & 0.363186 & $\mathrm{H} 8$ & -4.19831 & -2.61889 & -1.54778 \\
\hline H12 & -1.54562 & -4.61598 & 1.188101 & H9 & -0.83626 & -5.17194 & -1.46941 \\
\hline H13 & -2.90762 & -5.48628 & 0.427405 & $\mathrm{H} 10$ & -3.13998 & -5.25406 & -2.43292 \\
\hline $\mathrm{C} 14$ & 0.415709 & -2.98534 & -0.2993 & $\mathrm{C} 11$ & -2.2784 & -4.7406 & 0.214365 \\
\hline N15 & 0.980825 & -1.90046 & 0.100692 & H12 & -1.58987 & -4.78885 & 1.06337 \\
\hline
\end{tabular}




\begin{tabular}{|c|c|c|c|c|c|c|c|}
\hline $\mathrm{C} 16$ & 2.377932 & -2.21969 & 0.548556 & H13 & -2.89048 & -5.64665 & 0.188429 \\
\hline $\mathrm{C} 17$ & 2.35054 & -3.78656 & 0.494668 & C14 & 0.363243 & -3.0087 & -0.21871 \\
\hline P18 & -1.83208 & -0.71421 & 0.405865 & N15 & 0.966965 & -1.93913 & 0.192842 \\
\hline Ir19 & 0.139123 & 0.090396 & -0.3147 & $\mathrm{C} 16$ & 2.360533 & -2.31106 & 0.673789 \\
\hline $\mathrm{C} 20$ & -1.97328 & -0.63626 & 2.22913 & $\mathrm{C} 17$ & 2.212608 & -3.86311 & 0.720786 \\
\hline $\mathrm{C} 21$ & -1.97906 & -0.44791 & 5.027153 & P18 & -1.92986 & -0.83339 & 0.502179 \\
\hline $\mathrm{C} 22$ & -1.88074 & -1.79024 & 3.016284 & Ir19 & 0.278946 & -0.04765 & -0.10712 \\
\hline $\mathrm{C} 23$ & -2.0522 & 0.617186 & 2.858553 & $\mathrm{C} 20$ & -2.13695 & -0.8426 & 2.322886 \\
\hline $\mathrm{C} 24$ & -2.05986 & 0.709558 & 4.24747 & $\mathrm{C} 21$ & -2.20493 & -0.76308 & 5.130964 \\
\hline $\mathrm{C} 25$ & -1.88657 & -1.69446 & 4.409505 & $\mathrm{C} 22$ & -2.12806 & -2.02872 & 3.068356 \\
\hline $\mathrm{H} 26$ & -2.09381 & 1.523687 & 2.264679 & $\mathrm{C} 23$ & -2.15204 & 0.385691 & 3.004961 \\
\hline H27 & -2.12058 & 1.684662 & 4.722174 & $\mathrm{C} 24$ & -2.19434 & 0.425532 & 4.396168 \\
\hline $\mathrm{H} 28$ & -1.8081 & -2.59598 & 5.010153 & $\mathrm{C} 25$ & -2.16403 & -1.98706 & 4.464212 \\
\hline H29 & -1.98127 & -0.37518 & 6.110938 & $\mathrm{H} 26$ & -2.11449 & 1.317931 & 2.449578 \\
\hline $\mathrm{O} 30$ & 1.112609 & -4.12084 & -0.1991 & $\mathrm{H} 27$ & -2.20877 & 1.383538 & 4.908106 \\
\hline C31 & 2.66203 & -1.79038 & 1.984137 & $\mathrm{H} 28$ & -2.15492 & -2.91473 & 5.029326 \\
\hline $\mathrm{C} 32$ & 1.653657 & -1.34966 & 2.842342 & $\mathrm{H} 29$ & -2.2345 & -0.73282 & 6.216288 \\
\hline $\mathrm{C} 33$ & 3.961568 & -1.95047 & 2.48882 & $\mathrm{O} 30$ & 1.013468 & -4.1601 & -0.05447 \\
\hline C34 & 1.942751 & -1.0351 & 4.171837 & $\mathrm{C} 31$ & 2.640181 & -1.83203 & 2.093611 \\
\hline H35 & 0.641896 & -1.24608 & 2.47918 & C32 & 1.585716 & -1.58563 & 2.981542 \\
\hline $\mathrm{C} 36$ & 4.249259 & -1.64165 & 3.816396 & $\mathrm{C} 33$ & 3.953766 & -1.80069 & 2.578841 \\
\hline H37 & 4.756401 & -2.30056 & 1.835396 & $\mathrm{C} 34$ & 1.840589 & -1.255 & 4.31119 \\
\hline $\mathrm{C} 38$ & 3.240354 & -1.17377 & 4.661463 & H35 & 0.5601 & -1.64196 & 2.639156 \\
\hline H39 & 1.142969 & -0.6849 & 4.818129 & $\mathrm{C} 36$ & 4.207909 & -1.47559 & 3.911659 \\
\hline $\mathrm{H} 40$ & 5.262631 & -1.76211 & 4.188751 & H37 & 4.783882 & -2.02526 & 1.916519 \\
\hline H41 & 3.467139 & -0.92536 & 5.694334 & $\mathrm{C} 38$ & 3.153717 & -1.19014 & 4.779153 \\
\hline $\mathrm{C} 42$ & -3.3743 & 0.030086 & -0.24789 & H39 & 1.005595 & -1.05061 & 4.975417 \\
\hline $\mathrm{C} 43$ & -4.41622 & 0.494077 & 0.564297 & $\mathrm{H} 40$ & 5.233289 & -1.4452 & 4.268874 \\
\hline $\mathrm{C} 44$ & -3.51483 & 0.080107 & -1.64491 & $\mathrm{H} 41$ & 3.354825 & -0.92865 & 5.814113 \\
\hline $\mathrm{C} 45$ & -5.56853 & 1.030857 & -0.0136 & $\mathrm{C} 42$ & -3.45071 & -0.04473 & -0.1665 \\
\hline H46 & -4.3382 & 0.440625 & 1.64477 & $\mathrm{C} 43$ & -4.46546 & 0.526575 & 0.60919 \\
\hline $\mathrm{C} 47$ & -4.66598 & 0.61467 & -2.21616 & C44 & -3.5543 & -0.00019 & -1.56804 \\
\hline $\mathrm{C} 48$ & -5.69203 & 1.101374 & -1.40024 & $\mathrm{C} 45$ & -5.55571 & 1.147785 & -0.00622 \\
\hline H49 & -6.37013 & 1.391474 & 0.624526 & $\mathrm{H} 46$ & -4.41415 & 0.48849 & 1.692413 \\
\hline H50 & -4.76878 & 0.643885 & -3.29755 & C47 & -4.64395 & 0.614729 & -2.17917 \\
\hline H51 & -6.5892 & 1.521431 & -1.84562 & $\mathrm{C} 48$ & -5.6462 & 1.198214 & -1.39677 \\
\hline H52 & 0.435022 & 0.538428 & 1.146957 & $\mathrm{H} 49$ & -6.33868 & 1.586177 & 0.605956 \\
\hline H53 & 1.885237 & 0.859331 & -0.83792 & $\mathrm{H} 50$ & -4.71823 & 0.62748 & -3.26343 \\
\hline H54 & -1.77295 & -2.7615 & 2.548031 & H51 & -6.49757 & 1.678605 & -1.87015 \\
\hline H55 & -2.7336 & -0.32413 & -2.27922 & $\mathrm{H} 52$ & 0.61502 & 0.261026 & 1.3761 \\
\hline H56 & 3.182676 & -4.22662 & -0.05501 & $\mathrm{H} 53$ & 1.825206 & 1.824707 & -1.88888 \\
\hline $\mathrm{C} 57$ & 5.035773 & -0.50388 & -2.43298 & H54 & -2.06599 & -2.98757 & 2.566751 \\
\hline C58 & 4.301522 & -1.65702 & -2.71372 & $\mathrm{H} 55$ & -2.79178 & -0.47098 & -2.18052 \\
\hline C59 & 3.455256 & -2.1993 & -1.7482 & H56 & 3.051804 & -4.39836 & 0.278028 \\
\hline $\mathrm{C} 60$ & 3.338706 & -1.60964 & -0.48118 & C57 & 4.944933 & -0.91295 & -2.52291 \\
\hline
\end{tabular}




\begin{tabular}{|c|c|c|c|c|c|c|c|}
\hline C61 & 4.057912 & -0.43642 & -0.22029 & C58 & 4.215272 & -2.09404 & -2.66263 \\
\hline C62 & 4.902289 & 0.109842 & -1.18875 & C59 & 3.420192 & -2.55165 & -1.61329 \\
\hline H63 & 5.692529 & -0.07716 & -3.18504 & C60 & 3.340271 & -1.84195 & -0.40713 \\
\hline H64 & 4.385148 & -2.13472 & -3.68582 & C61 & 4.062559 & -0.64861 & -0.28532 \\
\hline H65 & 2.878189 & -3.08742 & -1.9935 & C62 & 4.864911 & -0.19274 & -1.33118 \\
\hline H66 & 3.968688 & 0.047935 & 0.745925 & H63 & 5.569651 & -0.557 & -3.33728 \\
\hline H67 & 5.453635 & 1.019337 & -0.96855 & H64 & 4.263794 & -2.66103 & -3.58787 \\
\hline C68 & -0.60136 & 3.274113 & -0.35147 & H65 & 2.843711 & -3.46175 & -1.75697 \\
\hline C69 & -0.03452 & 2.021722 & -1.04687 & H66 & 3.988995 & -0.06486 & 0.62388 \\
\hline $\mathrm{C} 70$ & 1.529001 & 1.932435 & -1.05798 & H67 & 5.423434 & 0.731641 & -1.2137 \\
\hline H71 & 1.933054 & 2.481155 & -0.20942 & C68 & -0.81401 & 3.701828 & -0.505 \\
\hline $\mathrm{C} 72$ & 2.232673 & 2.306457 & -2.3369 & C69 & -0.08695 & 2.539966 & -1.22669 \\
\hline $\mathrm{C} 73$ & 2.672248 & 3.625836 & -2.50938 & $\mathrm{C} 70$ & 1.389494 & 2.792707 & -1.63657 \\
\hline C74 & 2.405643 & 1.388517 & -3.37741 & H71 & 1.940335 & 3.157269 & -0.76743 \\
\hline $\mathrm{C} 75$ & 3.255218 & 4.025057 & -3.71285 & $\mathrm{C} 72$ & 1.518563 & 3.722145 & -2.82183 \\
\hline H76 & 2.554476 & 4.342686 & -1.69975 & $\mathrm{C} 73$ & 1.457674 & 5.115937 & -2.68981 \\
\hline $\mathrm{C} 77$ & 2.982809 & 1.788823 & -4.58285 & C74 & 1.662478 & 3.17602 & -4.10601 \\
\hline H78 & 2.105192 & 0.355274 & -3.23533 & $\mathrm{C} 75$ & 1.517981 & 5.941272 & -3.81406 \\
\hline $\mathrm{C} 79$ & 3.404328 & 3.108323 & -4.75628 & H76 & 1.368116 & 5.561673 & -1.70277 \\
\hline H80 & 3.593483 & 5.050254 & -3.83501 & $\mathrm{C} 77$ & 1.723561 & 3.997791 & -5.23203 \\
\hline H81 & 3.110853 & 1.06578 & -5.38348 & $\mathrm{H} 78$ & 1.736649 & 2.096384 & -4.22031 \\
\hline H82 & 3.855612 & 3.419447 & -5.6941 & C79 & 1.644021 & 5.384453 & -5.08842 \\
\hline $\mathrm{C} 83$ & -0.76816 & 3.018391 & -3.38751 & H80 & 1.470842 & 7.020032 & -3.69505 \\
\hline $\mathrm{C} 84$ & -0.58674 & 0.744452 & -3.04942 & H81 & 1.842849 & 3.557459 & -6.21834 \\
\hline $\mathrm{C} 85$ & -1.3351 & 2.229259 & -4.58362 & H82 & 1.692033 & 6.027634 & -5.96232 \\
\hline H86 & 0.188196 & 3.499327 & -3.6132 & $\mathrm{C} 83$ & -1.61103 & 2.930912 & -3.28985 \\
\hline H87 & -1.47133 & 3.76869 & -3.02159 & $\mathrm{C} 84$ & -0.72394 & 0.811556 & -2.9017 \\
\hline H88 & -2.42782 & 2.268696 & -4.62675 & $\mathrm{C} 85$ & -1.65682 & 2.062898 & -4.55989 \\
\hline H89 & -0.91383 & 2.524147 & -5.5448 & H86 & -1.08735 & 3.871379 & -3.4657 \\
\hline N90 & -0.58457 & 1.92995 & -2.41557 & H87 & -2.60821 & 3.134676 & -2.8878 \\
\hline O91 & -0.9543 & 0.845944 & -4.33161 & H88 & -2.64513 & 2.010224 & -5.01773 \\
\hline O92 & -0.295 & -0.35483 & -2.53377 & H89 & -0.91528 & 2.379946 & -5.29769 \\
\hline H93 & 2.292542 & -4.22237 & 1.493268 & N90 & -0.85687 & 2.057529 & -2.37682 \\
\hline N94 & -1.93674 & -2.33879 & -0.02316 & O91 & -1.30065 & 0.728737 & -4.11167 \\
\hline C95 & -0.96692 & -3.03385 & -0.90477 & O92 & -0.20406 & -0.18001 & -2.37881 \\
\hline H96 & -0.89162 & -2.54792 & -1.88494 & H93 & 2.034033 & -4.21746 & 1.737162 \\
\hline C97 & -2.13619 & 3.249847 & -0.28306 & N94 & -2.01572 & -2.44415 & 0.006624 \\
\hline H98 & -2.51976 & 4.229475 & 0.020031 & C95 & -0.99204 & -3.03968 & -0.88623 \\
\hline H99 & -2.6053 & 2.976742 & -1.23168 & H96 & -0.88857 & -2.47969 & -1.8243 \\
\hline H100 & -2.47531 & 2.51655 & 0.450319 & C97 & -2.21275 & 3.276718 & -0.03532 \\
\hline C101 & -0.03006 & 3.523597 & 1.052677 & H98 & -2.75881 & 4.137632 & 0.3601 \\
\hline H102 & -0.17111 & 2.649043 & 1.693684 & H99 & -2.81929 & 2.817391 & -0.81702 \\
\hline H103 & 1.036951 & 3.764362 & 1.045764 & H100 & -2.1243 & 2.539558 & 0.767939 \\
\hline H104 & -0.54287 & 4.3741 & 1.513299 & $\mathrm{C} 101$ & -0.00053 & 4.203792 & 0.698554 \\
\hline H105 & -0.30504 & 4.138053 & -0.97136 & H102 & 0.234439 & 3.375776 & 1.378808 \\
\hline
\end{tabular}


C3_ZS_Chelbot_TS2

\begin{tabular}{|c|c|c|c|}
\hline $\mathrm{C} 1$ & -3.76072 & -3.46184 & -1.38819 \\
\hline $\mathrm{C} 2$ & -1.5185 & -4.39179 & -1.11872 \\
\hline C3 & -2.65125 & -4.24252 & -2.16953 \\
\hline $\mathrm{C} 4$ & -3.08706 & -3.22574 & -0.01968 \\
\hline H5 & -4.66694 & -4.06347 & -1.26187 \\
\hline H6 & -2.31234 & -3.71298 & -3.06551 \\
\hline H7 & -3.75462 & -2.89488 & 0.776458 \\
\hline $\mathrm{H} 8$ & -4.04801 & -2.52295 & -1.86263 \\
\hline H9 & -0.78202 & -5.16381 & -1.34114 \\
\hline H10 & -3.00297 & -5.22849 & -2.48555 \\
\hline $\mathrm{C} 11$ & -2.32138 & -4.54678 & 0.189609 \\
\hline H12 & -1.69383 & -4.56253 & 1.085699 \\
\hline H13 & -2.97442 & -5.42398 & 0.189927 \\
\hline $\mathrm{C} 14$ & 0.429822 & -2.95281 & -0.18358 \\
\hline N15 & 0.964612 & -1.87664 & 0.279781 \\
\hline $\mathrm{C} 16$ & 2.375102 & -2.1909 & 0.6919 \\
\hline $\mathrm{C} 17$ & 2.372391 & -3.75723 & 0.591887 \\
\hline P18 & -1.84293 & -0.69913 & 0.389213 \\
\hline Ir19 & 0.136313 & 0.151505 & -0.16543 \\
\hline $\mathrm{C} 20$ & -1.97499 & -0.76774 & 2.220683 \\
\hline $\mathrm{C} 21$ & -2.02304 & -0.84252 & 5.026898 \\
\hline $\mathrm{C} 22$ & -2.01928 & -1.99166 & 2.898022 \\
\hline $\mathrm{C} 23$ & -1.93737 & 0.42253 & 2.967949 \\
\hline $\mathrm{C} 24$ & -1.96981 & 0.38485 & 4.359226 \\
\hline $\mathrm{C} 25$ & -2.04291 & -2.02736 & 4.293841 \\
\hline H26 & -1.85774 & 1.37998 & 2.465257 \\
\hline $\mathrm{H} 27$ & -1.94223 & 1.313178 & 4.922533 \\
\hline $\mathrm{H} 28$ & -2.06809 & -2.98524 & 4.805106 \\
\hline H29 & -2.04018 & -0.87191 & 6.112462 \\
\hline $\mathrm{O} 30$ & 1.144115 & -4.07949 & -0.12057 \\
\hline C31 & 2.667406 & -1.77214 & 2.127318 \\
\hline C32 & 1.698162 & -1.17666 & 2.939993 \\
\hline C33 & 3.917862 & -2.07684 & 2.687451 \\
\hline $\mathrm{C} 34$ & 1.98836 & -0.8442 & 4.264757 \\
\hline H35 & 0.712323 & -0.9745 & 2.546968 \\
\hline C36 & 4.206059 & -1.74915 & 4.011496 \\
\hline H37 & 4.677225 & -2.56727 & 2.08387 \\
\hline C38 & 3.244496 & -1.11889 & 4.804785 \\
\hline H39 & 1.216731 & -0.379 & 4.871711 \\
\hline $\mathrm{H} 40$ & 5.183724 & -1.98598 & 4.421331 \\
\hline H41 & 3.47107 & -0.85819 & 5.834324 \\
\hline $\mathrm{C} 42$ & -3.40844 & 0.030915 & -0.20636 \\
\hline $\mathrm{C} 43$ & -4.39476 & 0.537434 & 0.647701 \\
\hline
\end{tabular}

$\begin{array}{lrrr}\text { H103 } & 0.939601 & 4.679982 & 0.411199 \\ \text { H104 } & -0.58485 & 4.940577 & 1.257282 \\ \text { H105 } & -0.90428 & 4.521882 & -1.22915 \\ \text { H106 } & -0.1176 & 1.763073 & -0.38246 \\ \text { H107 } & 1.792077 & 0.372584 & -0.36784\end{array}$




\begin{tabular}{|c|c|c|c|}
\hline $\mathrm{C} 44$ & -3.61933 & 0.049476 & -1.59529 \\
\hline $\mathrm{C} 45$ & -5.56814 & 1.07663 & 0.117483 \\
\hline H46 & -4.2545 & 0.515212 & 1.723094 \\
\hline $\mathrm{C} 47$ & -4.7881 & 0.593928 & -2.11983 \\
\hline $\mathrm{C} 48$ & -5.76294 & 1.114958 & -1.26269 \\
\hline H49 & -6.33029 & 1.4652 & 0.786554 \\
\hline H50 & -4.94767 & 0.598479 & -3.19457 \\
\hline H51 & -6.67652 & 1.537166 & -1.67106 \\
\hline H52 & 0.188189 & 0.675502 & 1.296074 \\
\hline H53 & 1.883042 & 1.376416 & -1.68324 \\
\hline H54 & -2.00914 & -2.92233 & 2.346688 \\
\hline H55 & -2.88353 & -0.38639 & -2.26243 \\
\hline H56 & 3.208629 & -4.15562 & 0.016549 \\
\hline C57 & 4.842256 & -0.46862 & -2.47046 \\
\hline C58 & 3.774796 & -1.31875 & -2.7651 \\
\hline C59 & 3.01536 & -1.86861 & -1.7355 \\
\hline $\mathrm{C} 60$ & 3.297827 & -1.58153 & -0.38924 \\
\hline C61 & 4.378388 & -0.73796 & -0.10706 \\
\hline C62 & 5.141977 & -0.18608 & -1.13908 \\
\hline H63 & 5.43215 & -0.03149 & -3.27082 \\
\hline H64 & 3.527486 & -1.55193 & -3.79695 \\
\hline H65 & 2.184129 & -2.51512 & -1.99702 \\
\hline H66 & 4.615931 & -0.48111 & 0.918181 \\
\hline H67 & 5.969269 & 0.474137 & -0.8952 \\
\hline C68 & -0.76158 & 3.271041 & -0.33962 \\
\hline C69 & -0.14525 & 2.04535 & -1.05148 \\
\hline $\mathrm{C} 70$ & 1.364898 & 2.323674 & -1.47201 \\
\hline H71 & 1.888592 & 2.765671 & -0.62555 \\
\hline $\mathrm{C} 72$ & 1.539736 & 3.195363 & -2.69524 \\
\hline $\mathrm{C} 73$ & 1.501474 & 4.593953 & -2.60338 \\
\hline $\mathrm{C} 74$ & 1.715453 & 2.611246 & -3.9576 \\
\hline $\mathrm{C} 75$ & 1.602621 & 5.386473 & -3.74769 \\
\hline H76 & 1.397225 & 5.066006 & -1.62989 \\
\hline $\mathrm{C} 77$ & 1.820095 & 3.401316 & -5.10366 \\
\hline H78 & 1.779374 & 1.528026 & -4.03912 \\
\hline C79 & 1.753466 & 4.792303 & -5.0025 \\
\hline H80 & 1.56912 & 6.468813 & -3.65919 \\
\hline H81 & 1.961614 & 2.931824 & -6.07359 \\
\hline H82 & 1.83318 & 5.409682 & -5.89259 \\
\hline $\mathrm{C} 83$ & -1.59266 & 2.695411 & -3.21356 \\
\hline $\mathrm{C} 84$ & -0.66544 & 0.595891 & -2.92555 \\
\hline $\mathrm{C} 85$ & -1.60795 & 1.888447 & -4.53217 \\
\hline H86 & -1.05034 & 3.635653 & -3.32305 \\
\hline H87 & -2.60008 & 2.89961 & -2.83892 \\
\hline H88 & -2.59893 & 1.804737 & -4.98097 \\
\hline
\end{tabular}




$\begin{array}{lrrr}\text { H89 } & -0.89645 & 2.280298 & -5.26194 \\ \text { N90 } & -0.89047 & 1.767844 & -2.31625 \\ \text { O91 } & -1.17067 & 0.546725 & -4.16633 \\ \text { O92 } & -0.07694 & -0.38136 & -2.41539 \\ \text { H93 } & 2.321379 & -4.23419 & 1.572748 \\ \text { N94 } & -1.94764 & -2.27404 & -0.19804 \\ \text { C95 } & -0.8815 & -2.9939 & -0.92706 \\ \text { H96 } & -0.67773 & -2.51887 & -1.89464 \\ \text { C97 } & -2.23853 & 3.097608 & 0.031933 \\ \text { H98 } & -2.66606 & 4.057841 & 0.338182 \\ \text { H99 } & -2.84834 & 2.700195 & -0.77951 \\ \text { H100 } & -2.34359 & 2.409499 & 0.87104 \\ \text { C101 } & 0.015307 & 3.693025 & 0.91923 \\ \text { H102 } & 0.073087 & 2.864703 & 1.632952 \\ \text { H103 } & 1.035089 & 4.025827 & 0.708418 \\ \text { H104 } & -0.49866 & 4.526103 & 1.409487 \\ \text { H105 } & -0.70235 & 4.11117 & -1.04903 \\ \text { H106 } & 2.116397 & 1.057975 & 0.892576 \\ \text { H107 } & 2.528315 & 0.737021 & 0.356272\end{array}$

\section{C3_ZR_Chelbot}

C3_ZR_Chelbot_SM

$\begin{array}{lrrr}\text { C1 } & -4.21652 & -2.94392 & 1.121838 \\ \text { C2 } & -2.20859 & -4.24784 & 0.653239 \\ \text { C3 } & -3.71566 & -4.17308 & 0.294898 \\ \text { C4 } & -2.92291 & -2.47574 & 1.826573 \\ \text { H5 } & -4.965 & -3.23304 & 1.867033 \\ \text { H6 } & -3.88076 & -4.0532 & -0.78058 \\ \text { H7 } & -3.06798 & -1.78553 & 2.656694 \\ \text { H8 } & -4.65393 & -2.16417 & 0.49822 \\ \text { H9 } & -1.7287 & -5.19659 & 0.416817 \\ \text { H10 } & -4.21713 & -5.09487 & 0.603061 \\ \text { C11 } & -2.21911 & -3.80897 & 2.133135 \\ \text { H12 } & -1.22462 & -3.67605 & 2.569894 \\ \text { H13 } & -2.81392 & -4.47316 & 2.766494 \\ \text { C14 } & -0.0145 & -3.11205 & -0.13523 \\ \text { N15 } & 0.818947 & -2.13676 & -0.27555 \\ \text { C16 } & 2.19706 & -2.7214 & -0.52892 \\ \text { C17 } & 1.931055 & -4.23502 & -0.25357 \\ \text { P18 } & -1.86464 & -0.34886 & 0.181523 \\ \text { Ir19 } & 0.44357 & -0.02243 & -0.46204 \\ \text { C20 } & -2.27295 & 0.737486 & 1.586155 \\ \text { C21 } & -2.59362 & 2.490097 & 3.752764 \\ \text { C22 } & -1.65536 & 0.466598 & 2.819937 \\ \text { C23 } & -3.03022 & 1.908058 & 1.443134\end{array}$

C3_ZR_Chelbot_INT2

$\begin{array}{lrrr}\text { C1 } & -4.25596 & -3.0884 & -0.67751 \\ \text { C2 } & -2.13637 & -4.28408 & -0.8835 \\ \text { C3 } & -3.45882 & -4.01753 & -1.65133 \\ \text { C4 } & -3.25246 & -2.90055 & 0.484326 \\ \text { H5 } & -5.16212 & -3.57841 & -0.30566 \\ \text { H6 } & -3.28095 & -3.55056 & -2.62581 \\ \text { H7 } & -3.66835 & -2.47324 & 1.396806 \\ \text { H8 } & -4.55504 & -2.14431 & -1.13124 \\ \text { H9 } & -1.57071 & -5.14953 & -1.22844 \\ \text { H10 } & -3.98581 & -4.95868 & -1.83195 \\ \text { C11 } & -2.61962 & -4.2996 & 0.583735 \\ \text { H12 } & -1.81021 & -4.35776 & 1.317605 \\ \text { H13 } & -3.35145 & -5.08779 & 0.781659 \\ \text { C14 } & 0.121669 & -3.09627 & -0.46512 \\ \text { N15 } & 0.863027 & -2.10455 & -0.12261 \\ \text { C16 } & 2.247223 & -2.61782 & 0.155324 \\ \text { C17 } & 2.017945 & -4.16898 & 0.068319 \\ \text { P18 } & -1.86832 & -0.43784 & 0.074375 \\ \text { Ir19 } & 0.34326 & 0.040969 & -0.47435 \\ \text { C20 } & -2.1679 & 0.019014 & 1.814285 \\ \text { C21 } & -2.2944 & 0.781642 & 4.509212 \\ \text { C22 } & -1.88101 & -0.90905 & 2.826608 \\ \text { C23 } & -2.50089 & 1.337005 & 2.160346\end{array}$




\begin{tabular}{|c|c|c|c|c|c|c|c|}
\hline $\mathrm{C} 24$ & -3.18929 & 2.776458 & 2.525611 & $\mathrm{C} 24$ & -2.5662 & 1.710614 & 3.50258 \\
\hline $\mathrm{C} 25$ & -1.82268 & 1.333004 & 3.896662 & $\mathrm{C} 25$ & -1.9502 & -0.5285 & 4.166677 \\
\hline H26 & -3.47646 & 2.164658 & 0.489931 & $\mathrm{H} 26$ & -2.69178 & 2.07892 & 1.394317 \\
\hline $\mathrm{H} 27$ & -3.77836 & 3.680672 & 2.40186 & $\mathrm{H} 27$ & -2.82312 & 2.734972 & 3.756681 \\
\hline $\mathrm{H} 28$ & -1.34774 & 1.109049 & 4.847558 & $\mathrm{H} 28$ & -1.73344 & -1.25679 & 4.943246 \\
\hline H29 & -2.72138 & 3.167164 & 4.592386 & $\mathrm{H} 29$ & -2.34502 & 1.076862 & 5.553151 \\
\hline $\mathrm{O} 30$ & 0.485638 & -4.35054 & -0.17942 & O30 & 0.669656 & -4.31628 & -0.45745 \\
\hline C31 & 3.234277 & -2.24123 & 0.481078 & C31 & 2.710993 & -2.25974 & 1.563376 \\
\hline C32 & 2.854172 & -1.676 & 1.703655 & $\mathrm{C} 32$ & 1.863741 & -1.6419 & 2.488062 \\
\hline $\mathrm{C} 33$ & 4.591622 & -2.50687 & 0.253476 & $\mathrm{C} 33$ & 3.988474 & -2.6619 & 1.983926 \\
\hline C34 & 3.815251 & -1.33001 & 2.653322 & C34 & 2.298258 & -1.39241 & 3.79185 \\
\hline H35 & 1.806321 & -1.49302 & 1.907831 & H35 & 0.863304 & -1.35212 & 2.19556 \\
\hline C36 & 5.551677 & -2.1659 & 1.205929 & $\mathrm{C} 36$ & 4.420004 & -2.41658 & 3.286376 \\
\hline H37 & 4.903563 & -2.97576 & -0.67493 & H37 & 4.65333 & -3.16365 & 1.285236 \\
\hline $\mathrm{C} 38$ & 5.167863 & -1.56497 & 2.404798 & C38 & 3.578297 & -1.77067 & 4.195523 \\
\hline H39 & 3.503743 & -0.87827 & 3.591077 & H39 & 1.624244 & -0.90329 & 4.489972 \\
\hline $\mathrm{H} 40$ & 6.599813 & -2.3707 & 1.007933 & $\mathrm{H} 40$ & 5.414825 & -2.73006 & 3.589815 \\
\hline H41 & 5.915892 & -1.29208 & 3.143433 & $\mathrm{H} 41$ & 3.915965 & -1.57506 & 5.208938 \\
\hline $\mathrm{C} 42$ & -3.20959 & -0.25349 & -1.05862 & $\mathrm{C} 42$ & -3.30086 & 0.161913 & -0.8979 \\
\hline $\mathrm{C} 43$ & -4.54327 & 0.067682 & -0.76053 & $\mathrm{C} 43$ & -4.47754 & 0.663323 & -0.33006 \\
\hline $\mathrm{C} 44$ & -2.87983 & -0.67835 & -2.3567 & C44 & -3.25706 & -0.07164 & -2.28197 \\
\hline $\mathrm{C} 45$ & -5.52074 & -0.0068 & -1.75127 & $\mathrm{C} 45$ & -5.57299 & 0.969147 & -1.13973 \\
\hline H46 & -4.82557 & 0.367744 & 0.243336 & H46 & -4.55187 & 0.817346 & 0.739841 \\
\hline $\mathrm{C} 47$ & -3.86568 & -0.76988 & -3.33934 & $\mathrm{C} 47$ & -4.34966 & 0.23566 & -3.0881 \\
\hline $\mathrm{C} 48$ & -5.18449 & -0.4262 & -3.04034 & $\mathrm{C} 48$ & -5.51062 & 0.767915 & -2.51856 \\
\hline H49 & -6.54772 & 0.256163 & -1.5142 & H49 & -6.47849 & 1.363797 & -0.68791 \\
\hline H50 & -3.59978 & -1.09725 & -4.34043 & $\mathrm{H} 50$ & -4.30176 & 0.042447 & -4.15664 \\
\hline H51 & -5.95071 & -0.48618 & -3.80787 & H51 & -6.36559 & 1.008494 & -3.144 \\
\hline H52 & 0.645051 & 0.162578 & 1.066484 & H52 & 0.40047 & 0.499997 & 1.018 \\
\hline H53 & 2.029701 & -0.07706 & -0.56749 & $\mathrm{H} 53$ & 1.741568 & 1.732302 & -2.53119 \\
\hline H54 & -1.0371 & -0.41977 & 2.928618 & H54 & -1.59116 & -1.92159 & 2.564402 \\
\hline H55 & -1.84619 & -0.90565 & -2.60519 & H55 & -2.38266 & -0.52378 & -2.73059 \\
\hline H56 & 2.291223 & -4.89514 & -1.04279 & H56 & 2.704944 & -4.67379 & -0.61167 \\
\hline $\mathrm{C} 57$ & 2.925968 & -1.86614 & -4.72741 & C57 & 4.652468 & -1.18439 & -3.17684 \\
\hline C58 & 2.016611 & -2.85214 & -4.34397 & $\mathrm{C} 58$ & 3.587322 & -2.06522 & -3.3792 \\
\hline C59 & 1.821273 & -3.13479 & -2.99424 & C59 & 2.850208 & -2.52866 & -2.29296 \\
\hline $\mathrm{C} 60$ & 2.529178 & -2.44322 & -2.00169 & C60 & 3.158877 & -2.12842 & -0.9837 \\
\hline C61 & 3.432877 & -1.45123 & -2.39764 & C61 & 4.226327 & -1.24425 & -0.79198 \\
\hline C62 & 3.632812 & -1.16865 & -3.74923 & C62 & 4.968436 & -0.7775 & -1.88071 \\
\hline H63 & 3.079416 & -1.64383 & -5.77936 & H63 & 5.231688 & -0.82208 & -4.02158 \\
\hline H64 & 1.455038 & -3.39893 & -5.09574 & H64 & 3.327569 & -2.38864 & -4.38331 \\
\hline H65 & 1.089616 & -3.89076 & -2.71921 & H65 & 2.010625 & -3.19252 & -2.47653 \\
\hline H66 & 3.98487 & -0.89125 & -1.6546 & H66 & 4.480217 & -0.90707 & 0.206828 \\
\hline H67 & 4.349505 & -0.4027 & -4.03442 & H67 & 5.796261 & -0.09496 & -1.70967 \\
\hline C68 & 2.465488 & 2.507405 & -0.24266 & C68 & 1.096119 & 3.716227 & -2.94917 \\
\hline
\end{tabular}




\begin{tabular}{|c|c|c|c|c|c|c|c|}
\hline C69 & 1.24798 & 1.918139 & -0.97523 & C69 & 0.784658 & 2.269143 & -2.47878 \\
\hline $\mathrm{C} 70$ & -0.09223 & 2.12739 & -0.51047 & $\mathrm{C} 70$ & 0.282515 & 2.115589 & -1.01348 \\
\hline H71 & -0.15605 & 2.423168 & 0.531472 & H71 & 1.120146 & 2.50071 & -0.42457 \\
\hline $\mathrm{C} 72$ & -1.18225 & 2.76164 & -1.31438 & C72 & -0.89232 & 2.972601 & -0.63121 \\
\hline $\mathrm{C} 73$ & -1.67947 & 2.307169 & -2.54349 & C73 & -2.09278 & 3.023884 & -1.35195 \\
\hline $\mathrm{C} 74$ & -1.71754 & 3.953567 & -0.79016 & $\mathrm{C} 74$ & -0.7834 & 3.803626 & 0.499431 \\
\hline $\mathrm{C} 75$ & -2.66696 & 3.018931 & -3.22605 & C75 & -3.13428 & 3.871763 & -0.97255 \\
\hline H76 & -1.34206 & 1.366479 & -2.95007 & H76 & -2.23762 & 2.3676 & -2.19763 \\
\hline C77 & -2.69767 & 4.669481 & -1.47441 & C77 & -1.81709 & 4.655448 & 0.882858 \\
\hline $\mathrm{H} 78$ & -1.359 & 4.319016 & 0.168784 & $\mathrm{H} 78$ & 0.132241 & 3.776108 & 1.085686 \\
\hline C79 & -3.17889 & 4.205291 & -2.70081 & C79 & -3.0015 & 4.696713 & 0.143579 \\
\hline $\mathrm{H} 80$ & -3.0477 & 2.627984 & -4.16532 & H80 & -4.05472 & 3.876092 & -1.54967 \\
\hline H81 & -3.08882 & 5.588119 & -1.04591 & H81 & -1.69577 & 5.290324 & 1.756429 \\
\hline H82 & -3.94928 & 4.755927 & -3.2323 & H82 & -3.80896 & 5.361862 & 0.435384 \\
\hline $\mathrm{C} 83$ & 1.720448 & 3.081158 & -3.23612 & C83 & -0.90954 & 2.188204 & -4.54136 \\
\hline $\mathrm{C} 84$ & 0.956708 & 0.892903 & -3.18789 & C84 & -0.22711 & 0.251538 & -3.49323 \\
\hline $\mathrm{C} 85$ & 1.785861 & 2.433048 & -4.63325 & $\mathrm{C} 85$ & -1.16733 & 0.964369 & -5.43454 \\
\hline H86 & 2.665167 & 3.551617 & -2.9552 & H86 & -0.39264 & 2.981787 & -5.07809 \\
\hline H87 & 0.907032 & 3.80994 & -3.14045 & H87 & -1.83183 & 2.599505 & -4.12041 \\
\hline H88 & 1.293496 & 3.015814 & -5.41215 & H88 & -2.1927 & 0.897763 & -5.79991 \\
\hline H89 & 2.812113 & 2.200903 & -4.93325 & H89 & -0.46981 & 0.904613 & -6.27589 \\
\hline N90 & 1.439524 & 1.897542 & -2.41911 & N90 & -0.07643 & 1.587629 & -3.48821 \\
\hline O91 & 1.070252 & 1.176013 & -4.49251 & O91 & -0.92393 & -0.17411 & -4.56839 \\
\hline O92 & 0.433237 & -0.15187 & -2.77618 & O92 & 0.160346 & -0.57302 & -2.64768 \\
\hline H93 & 2.334705 & -4.55032 & 0.709856 & H93 & 2.043936 & -4.6438 & 1.05041 \\
\hline N94 & -1.94463 & -1.91462 & 0.849378 & N94 & -2.05932 & -2.1208 & 0.037119 \\
\hline C95 & -1.51783 & -3.02952 & -0.02075 & C95 & -1.31193 & -2.9743 & -0.91562 \\
\hline H96 & -1.88903 & -2.92365 & -1.05365 & H96 & -1.27868 & -2.55232 & -1.92929 \\
\hline C97 & 2.345648 & 2.526671 & 1.286042 & C97 & 1.8095 & 4.539395 & -1.86541 \\
\hline H98 & 3.217197 & 3.034777 & 1.709588 & H98 & 2.129699 & 5.498464 & -2.28432 \\
\hline H99 & 1.456433 & 3.056842 & 1.637901 & H99 & 1.165163 & 4.749512 & -1.011 \\
\hline H100 & 2.321386 & 1.507047 & 1.680008 & H100 & 2.708296 & 4.022617 & -1.5068 \\
\hline $\mathrm{C} 101$ & 3.795623 & 1.857814 & -0.65106 & C101 & 1.987428 & 3.694447 & -4.20726 \\
\hline H102 & 3.876284 & 1.718789 & -1.73315 & H102 & 1.635111 & 3.0129 & -4.987 \\
\hline H103 & 4.634022 & 2.480297 & -0.32331 & H103 & 2.066199 & 4.694221 & -4.6451 \\
\hline H104 & 3.900941 & 0.878071 & -0.17477 & H104 & 3.000023 & 3.37071 & -3.93912 \\
\hline \multirow[t]{2}{*}{ H105 } & 2.487743 & 3.558994 & -0.57544 & H105 & 0.144199 & 4.217668 & -3.16782 \\
\hline & & & & H106 & 2.015286 & 0.395229 & -0.12616 \\
\hline \multicolumn{4}{|c|}{ C3_ZR_Chelbot_TS1 } & H107 & 2.053062 & 0.219239 & -0.94206 \\
\hline $\mathrm{C} 1$ & -4.25737 & -3.20512 & 0.941546 & & & & \\
\hline $\mathrm{C} 2$ & -2.16515 & -4.42446 & 0.664067 & \multicolumn{4}{|c|}{ C3_ZR_Chelbot_TS3 } \\
\hline $\mathrm{C} 3$ & -3.63754 & -4.42084 & 0.177519 & $\mathrm{C} 1$ & -4.21821 & -3.17512 & -0.93054 \\
\hline $\mathrm{C} 4$ & -3.0567 & -2.6892 & 1.769711 & $\mathrm{C} 2$ & -2.04941 & -4.28667 & -1.09864 \\
\hline H5 & -5.06679 & -3.51558 & 1.610456 & $\mathrm{C} 3$ & -3.35532 & -4.04691 & -1.90187 \\
\hline H6 & -3.71546 & -4.31998 & -0.90975 & $\mathrm{C} 4$ & -3.25904 & -2.98074 & 0.265129 \\
\hline
\end{tabular}




\begin{tabular}{|c|c|c|c|c|c|c|c|}
\hline H7 & -3.3129 & -2.01096 & 2.582884 & H5 & -5.11768 & -3.70714 & -0.60266 \\
\hline $\mathrm{H} 8$ & -4.65643 & -2.44209 & 0.272758 & H6 & -3.1615 & -3.54818 & -2.85721 \\
\hline H9 & -1.62062 & -5.34793 & 0.470834 & $\mathrm{H} 7$ & -3.71957 & -2.58887 & 1.172405 \\
\hline H10 & -4.12492 & -5.35967 & 0.455664 & H8 & -4.53367 & -2.22718 & -1.366 \\
\hline $\mathrm{C} 11$ & -2.3281 & -3.9935 & 2.137783 & H9 & -1.44348 & -5.12371 & -1.44501 \\
\hline H12 & -1.38441 & -3.81974 & 2.663165 & H10 & -3.8405 & -5.00135 & -2.12458 \\
\hline H13 & -2.94906 & -4.68675 & 2.712257 & $\mathrm{C} 11$ & -2.57671 & -4.35688 & 0.352688 \\
\hline $\mathrm{C} 14$ & 0.026798 & -3.1879 & -0.01965 & H12 & -1.78688 & -4.40336 & 1.108745 \\
\hline N15 & 0.801081 & -2.1729 & -0.19728 & H13 & -3.28185 & -5.17798 & 0.509525 \\
\hline $\mathrm{C} 16$ & 2.197439 & -2.68214 & -0.46835 & C14 & 0.144111 & -3.04461 & -0.55803 \\
\hline $\mathrm{C} 17$ & 2.033372 & -4.19587 & -0.12132 & N15 & 0.871926 & -2.04637 & -0.19501 \\
\hline P18 & -1.93497 & -0.51394 & 0.240788 & $\mathrm{C} 16$ & 2.226116 & -2.56166 & 0.22966 \\
\hline Ir19 & 0.258475 & -0.07233 & -0.37705 & $\mathrm{C} 17$ & 1.996385 & -4.11063 & 0.137179 \\
\hline $\mathrm{C} 20$ & -2.40698 & 0.607713 & 1.594656 & P18 & -1.90974 & -0.46773 & 0.011619 \\
\hline $\mathrm{C} 21$ & -2.79473 & 2.475528 & 3.646149 & Ir19 & 0.454693 & 0.04955 & -0.54822 \\
\hline $\mathrm{C} 22$ & -1.98906 & 0.319317 & 2.903142 & $\mathrm{C} 20$ & -2.11103 & -0.11136 & 1.794885 \\
\hline $\mathrm{C} 23$ & -2.99303 & 1.851853 & 1.316486 & $\mathrm{C} 21$ & -2.10907 & 0.533643 & 4.521472 \\
\hline $\mathrm{C} 24$ & -3.18639 & 2.778056 & 2.341953 & $\mathrm{C} 22$ & -1.95877 & -1.11663 & 2.757623 \\
\hline $\mathrm{C} 25$ & -2.19296 & 1.245478 & 3.924055 & $\mathrm{C} 23$ & -2.24926 & 1.223978 & 2.206708 \\
\hline $\mathrm{H} 26$ & -3.27943 & 2.112503 & 0.30332 & $\mathrm{C} 24$ & -2.25015 & 1.540685 & 3.563277 \\
\hline $\mathrm{H} 27$ & -3.63868 & 3.739113 & 2.115024 & $\mathrm{C} 25$ & -1.96312 & -0.79354 & 4.116114 \\
\hline $\mathrm{H} 28$ & -1.87581 & 1.010421 & 4.936043 & $\mathrm{H} 26$ & -2.34572 & 2.018284 & 1.474655 \\
\hline H29 & -2.94888 & 3.197717 & 4.442678 & $\mathrm{H} 27$ & -2.35568 & 2.577593 & 3.870036 \\
\hline $\mathrm{O} 30$ & 0.596865 & -4.4001 & -0.02778 & $\mathrm{H} 28$ & -1.84738 & -1.58076 & 4.855955 \\
\hline C31 & 3.239056 & -2.08122 & 0.470459 & $\mathrm{H} 29$ & -2.10879 & 0.783551 & 5.57871 \\
\hline C32 & 2.868406 & -1.49583 & 1.686712 & $\mathrm{O} 30$ & 0.685769 & -4.26144 & -0.47481 \\
\hline $\mathrm{C} 33$ & 4.604169 & -2.23127 & 0.187076 & $\mathrm{C} 31$ & 2.535408 & -2.21277 & 1.68208 \\
\hline C34 & 3.83706 & -1.01513 & 2.569233 & $\mathrm{C} 32$ & 1.558459 & -1.70073 & 2.53986 \\
\hline H35 & 1.819095 & -1.40269 & 1.938961 & $\mathrm{C} 33$ & 3.79377 & -2.53756 & 2.211125 \\
\hline $\mathrm{C} 36$ & 5.572462 & -1.75368 & 1.0708 & $\mathrm{C} 34$ & 1.842999 & -1.47187 & 3.886172 \\
\hline H37 & 4.915924 & -2.7155 & -0.73346 & H35 & 0.570031 & -1.47861 & 2.163541 \\
\hline C38 & 5.193181 & -1.1294 & 2.260267 & $\mathrm{C} 36$ & 4.076495 & -2.31586 & 3.558023 \\
\hline H39 & 3.526928 & -0.54918 & 3.500409 & H37 & 4.560371 & -2.95831 & 1.565734 \\
\hline $\mathrm{H} 40$ & 6.624166 & -1.87045 & 0.825668 & $\mathrm{C} 38$ & 3.103518 & -1.77299 & 4.39974 \\
\hline H41 & 5.946393 & -0.74999 & 2.944195 & H39 & 1.068529 & -1.06001 & 4.527265 \\
\hline $\mathrm{C} 42$ & -3.24447 & -0.40871 & -1.03571 & $\mathrm{H} 40$ & 5.058968 & -2.56626 & 3.948349 \\
\hline $\mathrm{C} 43$ & -4.57276 & -0.06002 & -0.74178 & $\mathrm{H} 41$ & 3.327463 & -1.59434 & 5.447508 \\
\hline $\mathrm{C} 44$ & -2.91247 & -0.8109 & -2.3398 & $\mathrm{C} 42$ & -3.43373 & 0.20279 & -0.78308 \\
\hline $\mathrm{C} 45$ & -5.54441 & -0.09161 & -1.74061 & $\mathrm{C} 43$ & -4.52733 & 0.717987 & -0.07919 \\
\hline $\mathrm{H} 46$ & -4.85128 & 0.237681 & 0.264261 & $\mathrm{C} 44$ & -3.52165 & 0.081659 & -2.1802 \\
\hline $\mathrm{C} 47$ & -3.89233 & -0.85292 & -3.33245 & $\mathrm{C} 45$ & -5.672 & 1.137746 & -0.7622 \\
\hline $\mathrm{C} 48$ & -5.20644 & -0.48812 & -3.03645 & $\mathrm{H} 46$ & -4.49372 & 0.803494 & 1.001341 \\
\hline H49 & -6.56637 & 0.192606 & -1.50606 & $\mathrm{C} 47$ & -4.66253 & 0.501895 & -2.85983 \\
\hline H50 & -3.62472 & -1.15842 & -4.33994 & $\mathrm{C} 48$ & -5.74228 & 1.041792 & -2.15217 \\
\hline H51 & -5.96648 & -0.5106 & -3.81216 & $\mathrm{H} 49$ & -6.51116 & 1.540105 & -0.20152 \\
\hline
\end{tabular}




\begin{tabular}{|c|c|c|c|c|c|c|c|}
\hline H52 & 0.440278 & 0.084173 & 1.15842 & $\mathrm{H} 50$ & -4.71835 & 0.395272 & -3.94027 \\
\hline H53 & 1.86831 & 0.317359 & -0.59038 & $\mathrm{H} 51$ & -6.63332 & 1.370401 & -2.67919 \\
\hline H54 & -1.48816 & -0.62062 & 3.11586 & $\mathrm{H} 52$ & 0.552303 & 0.305987 & 0.983879 \\
\hline H55 & -1.88336 & -1.0529 & -2.58854 & $\mathrm{H} 53$ & 1.679679 & 1.687643 & -2.7629 \\
\hline H56 & 2.427902 & -4.86776 & -0.88403 & H54 & -1.8147 & -2.14695 & 2.449804 \\
\hline $\mathrm{C} 57$ & 2.646714 & -1.93252 & -4.72961 & $\mathrm{H} 55$ & -2.70771 & -0.36344 & -2.7414 \\
\hline C58 & 1.828242 & -2.96208 & -4.26297 & H56 & 2.726918 & -4.62508 & -0.48802 \\
\hline C59 & 1.729582 & -3.21262 & -2.89671 & C57 & 4.977717 & -1.12396 & -2.80688 \\
\hline C60 & 2.44214 & -2.44207 & -1.96579 & C58 & 4.004234 & -2.07769 & -3.11107 \\
\hline C61 & 3.250958 & -1.40684 & -2.44542 & C59 & 3.152354 & -2.5462 & -2.11466 \\
\hline C62 & 3.359358 & -1.15984 & -3.81522 & C60 & 3.255556 & -2.07561 & -0.79839 \\
\hline H63 & 2.7241 & -1.73536 & -5.79478 & C61 & 4.22422 & -1.10845 & -0.50632 \\
\hline H64 & 1.262636 & -3.56919 & -4.9638 & C62 & 5.083358 & -0.64131 & -1.50318 \\
\hline H65 & 1.068933 & -4.00697 & -2.55841 & H63 & 5.648752 & -0.76185 & -3.5807 \\
\hline H66 & 3.801898 & -0.78563 & -1.7538 & H64 & 3.907707 & -2.45608 & -4.1247 \\
\hline H67 & 4.005807 & -0.35811 & -4.1637 & H65 & 2.383864 & -3.26901 & -2.37668 \\
\hline C68 & 2.636455 & 2.404166 & -0.24224 & H66 & 4.303286 & -0.70481 & 0.496472 \\
\hline C69 & 1.403406 & 1.777333 & -0.92357 & H67 & 5.834712 & 0.103466 & -1.25572 \\
\hline $\mathrm{C} 70$ & 0.04975 & 2.0483 & -0.41573 & C68 & 1.039768 & 3.673031 & -3.2634 \\
\hline H71 & 0.051917 & 2.388221 & 0.616187 & C69 & 0.747901 & 2.260826 & -2.67778 \\
\hline $\mathrm{C} 72$ & -0.94932 & 2.844561 & -1.20218 & $\mathrm{C} 70$ & 0.395602 & 2.242903 & -1.16787 \\
\hline $\mathrm{C} 73$ & -1.67241 & 2.371555 & -2.30493 & $\mathrm{H} 71$ & 1.260483 & 2.778225 & -0.75432 \\
\hline $\mathrm{C} 74$ & -1.18439 & 4.165213 & -0.77771 & $\mathrm{C} 72$ & -0.7762 & 3.03963 & -0.66912 \\
\hline $\mathrm{C} 75$ & -2.59381 & 3.188057 & -2.96344 & $\mathrm{C} 73$ & -2.04293 & 3.010194 & -1.26079 \\
\hline H76 & -1.55306 & 1.348178 & -2.62764 & $\mathrm{C} 74$ & -0.58329 & 3.895835 & 0.429095 \\
\hline $\mathrm{C} 77$ & -2.10011 & 4.983943 & -1.43506 & $\mathrm{C} 75$ & -3.08025 & 3.81288 & -0.78578 \\
\hline H78 & -0.6468 & 4.547701 & 0.086935 & $\mathrm{H} 76$ & -2.23755 & 2.328797 & -2.07415 \\
\hline C79 & -2.8108 & 4.497206 & -2.53482 & C77 & -1.61664 & 4.69502 & 0.913426 \\
\hline H80 & -3.15444 & 2.786827 & -3.80329 & $\mathrm{H} 78$ & 0.391565 & 3.933043 & 0.910699 \\
\hline H81 & -2.26417 & 5.999429 & -1.08482 & C79 & -2.87337 & 4.659738 & 0.303576 \\
\hline H82 & -3.53256 & 5.129998 & -3.04338 & H80 & -4.05549 & 3.757743 & -1.26027 \\
\hline $\mathrm{C} 83$ & 1.814573 & 2.950593 & -3.17802 & H81 & -1.439 & 5.349374 & 1.762585 \\
\hline $\mathrm{C} 84$ & 0.852302 & 0.841652 & -3.12521 & $\mathrm{H} 82$ & -3.68052 & 5.284499 & 0.675284 \\
\hline $\mathrm{C} 85$ & 1.688353 & 2.357505 & -4.59428 & $\mathrm{C} 83$ & -1.17082 & 2.129644 & -4.51806 \\
\hline H86 & 2.815866 & 3.339299 & -2.98163 & $\mathrm{C} 84$ & -0.3312 & 0.214372 & -3.52983 \\
\hline H87 & 1.072394 & 3.731576 & -2.97612 & $\mathrm{C} 85$ & -1.52262 & 0.882243 & -5.34711 \\
\hline H88 & 1.178002 & 3.013168 & -5.30016 & H86 & -0.71943 & 2.907113 & -5.13298 \\
\hline H89 & 2.655878 & 2.049462 & -5.00165 & H87 & -2.04173 & 2.554141 & -4.00933 \\
\hline N90 & 1.526683 & 1.756236 & -2.37603 & $\mathrm{H} 88$ & -2.58539 & 0.796242 & -5.57621 \\
\hline O91 & 0.878655 & 1.164276 & -4.42359 & H89 & -0.93868 & 0.815159 & -6.27021 \\
\hline O92 & 0.265918 & -0.16019 & -2.70061 & N90 & -0.21973 & 1.556713 & -3.55384 \\
\hline H93 & 2.464844 & -4.43919 & 0.851179 & O91 & -1.15234 & -0.23728 & -4.50137 \\
\hline N94 & -2.01016 & -2.0818 & 0.896864 & O92 & 0.197393 & -0.58005 & -2.74061 \\
\hline C95 & -1.48334 & -3.17327 & 0.044746 & $\mathrm{H} 93$ & 1.952306 & -4.57746 & 1.122408 \\
\hline H96 & -1.8062 & -3.06981 & -1.00516 & N94 & -2.09074 & -2.14402 & -0.13494 \\
\hline
\end{tabular}




\begin{tabular}{|c|c|c|c|c|c|c|c|}
\hline C97 & 2.543235 & 2.408693 & 1.28839 & C95 & -1.27158 & -2.94799 & -1.07346 \\
\hline H98 & 3.458512 & 2.838192 & 1.705893 & H96 & -1.21088 & -2.5 & -2.07307 \\
\hline H99 & 1.703017 & 2.999955 & 1.65984 & C97 & 1.851326 & 4.563928 & -2.30989 \\
\hline H100 & 2.44498 & 1.386924 & 1.665381 & H98 & 2.131374 & 5.488303 & -2.82456 \\
\hline C101 & 3.959994 & 1.762522 & -0.68229 & H99 & 1.285708 & 4.845583 & -1.41888 \\
\hline H102 & 4.022308 & 1.634758 & -1.76704 & H100 & 2.780693 & 4.07165 & -1.99783 \\
\hline H103 & 4.803351 & 2.382578 & -0.36436 & C101 & 1.807454 & 3.544827 & -4.59325 \\
\hline H104 & 4.080044 & 0.77977 & -0.21293 & H102 & 1.360359 & 2.828352 & -5.28879 \\
\hline H105 & 2.621023 & 3.452434 & -0.58052 & H103 & 1.869291 & 4.512615 & -5.1001 \\
\hline \multirow[t]{2}{*}{$X$} & \multirow[t]{2}{*}{0} & \multirow{2}{*}{\multicolumn{2}{|c|}{0}} & H104 & 2.831701 & 3.205036 & -4.40073 \\
\hline & & & & H105 & 0.078188 & 4.175526 & -3.43089 \\
\hline \multicolumn{4}{|c|}{ C3_ZR_Chelbot_INT1 } & H106 & 2.032027 & 0.121197 & -0.7808 \\
\hline $\mathrm{C} 1$ & -4.23933 & -3.19229 & 0.91985 & H107 & 1.298462 & 1.321552 & -0.23247 \\
\hline $\mathrm{C} 2$ & -2.16468 & -4.43755 & 0.641566 & & & & \\
\hline $\mathrm{C} 3$ & -3.63943 & -4.42341 & 0.164499 & \multicolumn{4}{|c|}{ C3_ZR_Chelbot_PROD } \\
\hline $\mathrm{C} 4$ & -3.03039 & -2.68661 & 1.742571 & $\mathrm{C} 1$ & -3.99134 & -3.54621 & -1.33026 \\
\hline H5 & -5.05267 & -3.48444 & 1.592062 & $\mathrm{C} 2$ & -1.73604 & -4.48176 & -1.32272 \\
\hline H6 & -3.72271 & -4.33334 & -0.92322 & $\mathrm{C} 3$ & -2.97647 & -4.31617 & -2.23979 \\
\hline H7 & -3.27931 & -2.00419 & 2.553573 & $\mathrm{C} 4$ & -3.18068 & -3.35633 & -0.02853 \\
\hline H8 & -4.62666 & -2.42831 & 0.244808 & H5 & -4.88974 & -4.14067 & -1.13331 \\
\hline H9 & -1.62824 & -5.36659 & 0.450766 & H6 & -2.73711 & -3.7761 & -3.16145 \\
\hline H10 & -4.13595 & -5.35324 & 0.456259 & $\mathrm{H} 7$ & -3.7664 & -3.05314 & 0.839581 \\
\hline $\mathrm{C} 11$ & -2.31317 & -3.9957 & 2.11338 & H8 & -4.30949 & -2.59064 & -1.74923 \\
\hline H12 & -1.36462 & -3.82758 & 2.631808 & H9 & -1.02682 & -5.24493 & -1.64293 \\
\hline H13 & -2.93778 & -4.67874 & 2.696137 & $\mathrm{H} 10$ & -3.36239 & -5.2982 & -2.52774 \\
\hline $\mathrm{C} 14$ & 0.035695 & -3.23848 & -0.07425 & $\mathrm{C} 11$ & -2.39438 & -4.67637 & 0.062637 \\
\hline N15 & 0.804372 & -2.2197 & -0.22512 & H12 & -1.67961 & -4.69983 & 0.89091 \\
\hline $\mathrm{C} 16$ & 2.199635 & -2.70115 & -0.50777 & H13 & -3.03558 & -5.56141 & 0.106135 \\
\hline $\mathrm{C} 17$ & 2.038988 & -4.23977 & -0.26109 & $\mathrm{C} 14$ & 0.291581 & -3.06843 & -0.56376 \\
\hline P18 & -1.88672 & -0.51556 & 0.223606 & N15 & 0.945595 & -2.01167 & -0.20298 \\
\hline Ir19 & 0.208571 & -0.1153 & -0.33082 & $\mathrm{C} 16$ & 2.332989 & -2.42151 & 0.260229 \\
\hline $\mathrm{C} 20$ & -2.44956 & 0.61371 & 1.52999 & $\mathrm{C} 17$ & 2.161978 & -3.97397 & 0.291795 \\
\hline $\mathrm{C} 21$ & -2.95049 & 2.529793 & 3.507231 & P18 & -1.92075 & -0.77208 & 0.118369 \\
\hline $\mathrm{C} 22$ & -2.10456 & 0.357168 & 2.865851 & Ir19 & 0.333539 & -0.08013 & -0.47957 \\
\hline $\mathrm{C} 23$ & -3.01412 & 1.851881 & 1.186159 & $\mathrm{C} 20$ & -2.1105 & -0.62058 & 1.930281 \\
\hline $\mathrm{C} 24$ & -3.26503 & 2.802092 & 2.175773 & $\mathrm{C} 21$ & -2.14445 & -0.26058 & 4.711994 \\
\hline $\mathrm{C} 25$ & -2.36685 & 1.307219 & 3.850134 & $\mathrm{C} 22$ & -2.18217 & -1.72664 & 2.784985 \\
\hline H26 & -3.23346 & 2.090952 & 0.151195 & $\mathrm{C} 23$ & -2.0294 & 0.669285 & 2.483886 \\
\hline $\mathrm{H} 27$ & -3.69703 & 3.759378 & 1.899225 & $\mathrm{C} 24$ & -2.05476 & 0.847568 & 3.864429 \\
\hline $\mathrm{H} 28$ & -2.10588 & 1.098896 & 4.88366 & $\mathrm{C} 25$ & -2.20118 & -1.54457 & 4.170565 \\
\hline H29 & -3.14694 & 3.272217 & 4.275355 & $\mathrm{H} 26$ & -1.92804 & 1.534962 & 1.833762 \\
\hline $\mathrm{O} 30$ & 0.603607 & -4.45178 & -0.14037 & $\mathrm{H} 27$ & -1.99215 & 1.849252 & 4.28011 \\
\hline C31 & 3.219542 & -2.14032 & 0.481007 & $\mathrm{H} 28$ & -2.25492 & -2.40984 & 4.825172 \\
\hline C32 & 2.826242 & -1.53009 & 1.677327 & H29 & -2.15889 & -0.12172 & 5.7892 \\
\hline $\mathrm{C} 33$ & 4.588608 & -2.33483 & 0.243158 & $\mathrm{O} 30$ & 0.910885 & -4.2378 & -0.40569 \\
\hline
\end{tabular}




\begin{tabular}{|c|c|c|c|c|c|c|c|}
\hline $\mathrm{C} 34$ & 3.781917 & -1.07018 & 2.587292 & C31 & 2.623803 & -1.96193 & 1.685595 \\
\hline H35 & 1.77328 & -1.40601 & 1.897237 & C32 & 1.57535 & -1.6764 & 2.568752 \\
\hline C36 & 5.541296 & -1.88296 & 1.155359 & $\mathrm{C} 33$ & 3.933915 & -1.9893 & 2.180517 \\
\hline H37 & 4.913466 & -2.82945 & -0.66799 & $\mathrm{C} 34$ & 1.83522 & -1.36173 & 3.901231 \\
\hline C38 & 5.142222 & -1.2349 & 2.326809 & H35 & 0.550121 & -1.68813 & 2.220784 \\
\hline H39 & 3.45615 & -0.58707 & 3.504339 & $\mathrm{C} 36$ & 4.193266 & -1.68187 & 3.516459 \\
\hline $\mathrm{H} 40$ & 6.596569 & -2.03553 & 0.947828 & H37 & 4.758276 & -2.2468 & 1.523363 \\
\hline H41 & 5.884451 & -0.87507 & 3.033118 & C38 & 3.146483 & -1.35471 & 4.378354 \\
\hline $\mathrm{C} 42$ & -3.1109 & -0.43864 & -1.14043 & H39 & 1.005239 & -1.12321 & 4.560472 \\
\hline $\mathrm{C} 43$ & -4.45739 & -0.11283 & -0.90371 & $\mathrm{H} 40$ & 5.216766 & -1.69785 & 3.880155 \\
\hline $\mathrm{C} 44$ & -2.71855 & -0.83709 & -2.42916 & $\mathrm{H} 41$ & 3.351584 & -1.1062 & 5.415706 \\
\hline $\mathrm{C} 45$ & -5.3854 & -0.1633 & -1.94251 & $\mathrm{C} 42$ & -3.42831 & 0.021433 & -0.58895 \\
\hline $\mathrm{H} 46$ & -4.78507 & 0.183131 & 0.087578 & $\mathrm{C} 43$ & -4.38016 & 0.734165 & 0.150913 \\
\hline $\mathrm{C} 47$ & -3.65541 & -0.8937 & -3.46174 & C44 & -3.57191 & -0.05625 & -1.98544 \\
\hline $\mathrm{C} 48$ & -4.98712 & -0.55296 & -3.22287 & $\mathrm{C} 45$ & -5.43996 & 1.37692 & -0.49661 \\
\hline H49 & -6.42086 & 0.103158 & -1.75056 & $\mathrm{H} 46$ & -4.30014 & 0.79741 & 1.230978 \\
\hline $\mathrm{H} 50$ & -3.33885 & -1.19472 & -4.45633 & C47 & -4.62928 & 0.584017 & -2.62758 \\
\hline H51 & -5.713 & -0.58912 & -4.0301 & $\mathrm{C} 48$ & -5.56364 & 1.31437 & -1.8851 \\
\hline H52 & 0.33306 & 0.011689 & 1.218594 & $\mathrm{H} 49$ & -6.17158 & 1.924796 & 0.090945 \\
\hline H53 & 2.07818 & 0.822325 & -0.78257 & $\mathrm{H} 50$ & -4.73189 & 0.502498 & -3.70643 \\
\hline H54 & -1.60763 & -0.57193 & 3.129201 & H51 & -6.38805 & 1.815833 & -2.38363 \\
\hline H55 & -1.67867 & -1.0629 & -2.63848 & H52 & 0.639647 & 0.251027 & 1.002766 \\
\hline H56 & 2.409456 & -4.85871 & -1.07898 & $\mathrm{H} 53$ & 1.512485 & 1.472658 & -2.55182 \\
\hline $\mathrm{C} 57$ & 2.776642 & -1.65677 & -4.69703 & H54 & -2.19433 & -2.73193 & 2.378809 \\
\hline C58 & 1.9412 & -2.71292 & -4.32605 & H55 & -2.86002 & -0.62562 & -2.57253 \\
\hline $\mathrm{C} 59$ & 1.797564 & -3.05569 & -2.98395 & H56 & 2.960265 & -4.51435 & -0.21635 \\
\hline $\mathrm{C} 60$ & 2.483593 & -2.35656 & -1.97995 & C57 & 4.937644 & -1.06502 & -2.93959 \\
\hline C61 & 3.310043 & -1.29458 & -2.36193 & C58 & 4.129558 & -2.19106 & -3.10355 \\
\hline C62 & 3.461828 & -0.95275 & -3.70812 & C59 & 3.328035 & -2.63284 & -2.0526 \\
\hline H63 & 2.890478 & -1.39009 & -5.74357 & C60 & 3.31831 & -1.96333 & -0.82127 \\
\hline H64 & 1.396407 & -3.26815 & -5.08405 & C61 & 4.116745 & -0.82268 & -0.67512 \\
\hline H65 & 1.12255 & -3.86598 & -2.71945 & C62 & 4.926774 & -0.3832 & -1.72279 \\
\hline H66 & 3.843897 & -0.72484 & -1.61186 & H63 & 5.57 & -0.72299 & -3.75422 \\
\hline H67 & 4.119865 & -0.13051 & -3.97717 & H64 & 4.121972 & -2.72656 & -4.04868 \\
\hline C68 & 2.806322 & 2.784248 & -0.59527 & H65 & 2.688167 & -3.49688 & -2.21352 \\
\hline C69 & 1.663286 & 1.847357 & -1.06117 & H66 & 4.09771 & -0.26551 & 0.253682 \\
\hline $\mathrm{C} 70$ & 0.266099 & 1.975282 & -0.40227 & H67 & 5.54809 & 0.497628 & -1.58558 \\
\hline H71 & 0.383889 & 2.329021 & 0.622428 & C68 & 1.467396 & 3.498842 & -3.20628 \\
\hline $\mathrm{C} 72$ & -0.70713 & 2.894172 & -1.09493 & C69 & 0.770785 & 2.275493 & -2.57005 \\
\hline $\mathrm{C} 73$ & -1.54045 & 2.49056 & -2.14944 & $\mathrm{C} 70$ & 0.300309 & 2.475579 & -1.10183 \\
\hline $\mathrm{C} 74$ & -0.78622 & 4.235682 & -0.68102 & $\mathrm{H} 71$ & 1.163351 & 2.611197 & -0.45348 \\
\hline $\mathrm{C} 75$ & -2.40844 & 3.389112 & -2.77283 & $\mathrm{C} 72$ & -0.723 & 3.570935 & -0.87106 \\
\hline H76 & -1.53549 & 1.457843 & -2.46948 & $\mathrm{C} 73$ & -2.09269 & 3.3045 & -0.98899 \\
\hline $\mathrm{C} 77$ & -1.65106 & 5.138394 & -1.30095 & $\mathrm{C} 74$ & -0.31137 & 4.871754 & -0.55349 \\
\hline H78 & -0.16249 & 4.57261 & 0.144264 & $\mathrm{C} 75$ & -3.03334 & 4.319689 & -0.81939 \\
\hline
\end{tabular}




\begin{tabular}{|c|c|c|c|c|c|c|c|}
\hline C79 & -2.4666 & 4.719773 & -2.35539 & H76 & -2.42789 & 2.296241 & -1.20717 \\
\hline H80 & -3.0479 & 3.040087 & -3.57938 & C77 & -1.25112 & 5.890238 & -0.39174 \\
\hline H81 & -1.69028 & 6.169093 & -0.95853 & $\mathrm{H} 78$ & 0.743735 & 5.093433 & -0.42716 \\
\hline H82 & -3.14322 & 5.419645 & -2.83716 & C79 & -2.61348 & 5.618255 & -0.52743 \\
\hline $\mathrm{C} 83$ & 1.665454 & 2.975128 & -3.41518 & H80 & -4.0902 & 4.086523 & -0.90826 \\
\hline $\mathrm{C} 84$ & 0.838254 & 0.839741 & -3.1359 & H81 & -0.91682 & 6.895188 & -0.15029 \\
\hline $\mathrm{C} 85$ & 1.395239 & 2.302421 & -4.77273 & H82 & -3.34281 & 6.411961 & -0.39433 \\
\hline H86 & 2.653252 & 3.43726 & -3.37564 & $\mathrm{C} 83$ & -1.30862 & 2.517154 & -4.18624 \\
\hline H87 & 0.905237 & 3.718551 & -3.15387 & $\mathrm{C} 84$ & -0.5824 & 0.434411 & -3.47316 \\
\hline H88 & 0.772574 & 2.896591 & -5.44178 & $\mathrm{C} 85$ & -1.82441 & 1.407239 & -5.11703 \\
\hline H89 & 2.318584 & 2.008474 & -5.28137 & H86 & -0.85292 & 3.341977 & -4.73533 \\
\hline N90 & 1.568928 & 1.80769 & -2.53055 & H87 & -2.09209 & 2.917636 & -3.53665 \\
\hline O91 & 0.666246 & 1.090574 & -4.43992 & H88 & -2.90171 & 1.440895 & -5.28079 \\
\hline O92 & 0.351514 & -0.16335 & -2.5946 & H89 & -1.30136 & 1.387806 & -6.07833 \\
\hline H93 & 2.498858 & -4.54959 & 0.6788 & N90 & -0.32008 & 1.76041 & -3.40947 \\
\hline N94 & -1.98268 & -2.09191 & 0.859294 & O91 & -1.51684 & 0.173423 & -4.41296 \\
\hline C95 & -1.47365 & -3.19896 & 0.009547 & O92 & -0.09676 & -0.46804 & -2.78078 \\
\hline H96 & -1.80595 & -3.09347 & -1.03729 & H93 & 2.047658 & -4.3456 & 1.311009 \\
\hline C97 & 2.910491 & 2.818103 & 0.936414 & N94 & -2.06095 & -2.40592 & -0.27029 \\
\hline H98 & 3.799162 & 3.382356 & 1.23436 & C95 & -1.09204 & -3.08081 & -1.17018 \\
\hline H99 & 2.044626 & 3.291226 & 1.405032 & H96 & -1.00652 & -2.57196 & -2.13756 \\
\hline H100 & 3.006251 & 1.803011 & 1.341203 & C97 & 2.691787 & 3.911714 & -2.37023 \\
\hline C101 & 4.156678 & 2.349424 & -1.19196 & H98 & 3.219997 & 4.734543 & -2.86075 \\
\hline H102 & 4.117726 & 2.199149 & -2.27456 & H99 & 2.435086 & 4.244929 & -1.36277 \\
\hline H103 & 4.927471 & 3.096919 & -0.98149 & $\mathrm{H} 100$ & 3.393293 & 3.073802 & -2.27682 \\
\hline H104 & 4.483128 & 1.405018 & -0.7393 & C101 & 1.941228 & 3.169391 & -4.6343 \\
\hline \multirow[t]{2}{*}{ H105 } & 2.554848 & 3.794708 & -0.94768 & H102 & 1.132958 & 2.863775 & -5.30351 \\
\hline & & & & $\mathrm{H} 103$ & 2.427895 & 4.041086 & -5.08155 \\
\hline \multicolumn{4}{|c|}{ C3_ZR_Chelbot_TS2 } & H104 & 2.672459 & 2.352965 & -4.61289 \\
\hline $\mathrm{C} 1$ & -4.20889 & -3.12099 & -0.45781 & H105 & 0.761002 & 4.339507 & -3.23558 \\
\hline $\mathrm{C} 2$ & -2.10398 & -4.32199 & -0.75642 & H106 & 1.870265 & 0.27823 & -0.71043 \\
\hline $\mathrm{C} 3$ & -3.46948 & -4.0765 & -1.45166 & H107 & -0.3393 & 1.577574 & -0.74075 \\
\hline $\mathrm{C} 4$ & -3.14849 & -2.91973 & 0.648492 & & & & \\
\hline H5 & -5.10037 & -3.59331 & -0.03187 & & & & \\
\hline H6 & -3.35074 & -3.63722 & -2.44748 & & & & \\
\hline H7 & -3.52047 & -2.48004 & 1.573576 & & & & \\
\hline H8 & -4.51946 & -2.1808 & -0.91349 & & & & \\
\hline H9 & -1.5519 & -5.18988 & -1.11685 & & & & \\
\hline H10 & -4.00487 & -5.02223 & -1.57324 & & & & \\
\hline $\mathrm{C} 11$ & -2.50487 & -4.31455 & 0.735745 & & & & \\
\hline H12 & -1.65604 & -4.35664 & 1.425054 & & & & \\
\hline H13 & -3.22103 & -5.10206 & 0.986596 & & & & \\
\hline $\mathrm{C} 14$ & 0.167897 & -3.1041 & -0.50403 & & & & \\
\hline N15 & 0.897243 & -2.08954 & -0.20541 & & & & \\
\hline $\mathrm{C} 16$ & 2.282591 & -2.56733 & 0.097778 & & & & \\
\hline
\end{tabular}




\begin{tabular}{|c|c|c|c|}
\hline $\mathrm{C} 17$ & 2.141688 & -4.11496 & -0.16697 \\
\hline P18 & -1.76925 & -0.46762 & 0.162986 \\
\hline Ir19 & 0.270487 & 0.016136 & -0.56511 \\
\hline $\mathrm{C} 20$ & -1.99038 & -0.00409 & 1.910211 \\
\hline $\mathrm{C} 21$ & -2.03481 & 0.78258 & 4.594724 \\
\hline $\mathrm{C} 22$ & -1.73025 & -0.93887 & 2.922789 \\
\hline $\mathrm{C} 23$ & -2.25248 & 1.332272 & 2.249153 \\
\hline $\mathrm{C} 24$ & -2.27872 & 1.718582 & 3.587382 \\
\hline $\mathrm{C} 25$ & -1.75874 & -0.54451 & 4.260562 \\
\hline H26 & -2.41511 & 2.075462 & 1.477666 \\
\hline $\mathrm{H} 27$ & -2.48309 & 2.754758 & 3.840775 \\
\hline $\mathrm{H} 28$ & -1.56162 & -1.27467 & 5.040322 \\
\hline H29 & -2.05384 & 1.087934 & 5.636978 \\
\hline $\mathrm{O} 30$ & 0.742211 & -4.3078 & -0.51606 \\
\hline C31 & 2.637079 & -2.32664 & 1.565644 \\
\hline C32 & 1.772667 & -1.65921 & 2.437713 \\
\hline $\mathrm{C} 33$ & 3.825306 & -2.86739 & 2.081412 \\
\hline C34 & 2.103501 & -1.50024 & 3.784645 \\
\hline H35 & 0.838606 & -1.26041 & 2.069817 \\
\hline $\mathrm{C} 36$ & 4.157187 & -2.70748 & 3.425119 \\
\hline H37 & 4.50058 & -3.41457 & 1.428297 \\
\hline C38 & 3.299087 & -2.01502 & 4.281963 \\
\hline H39 & 1.414338 & -0.97378 & 4.439588 \\
\hline $\mathrm{H} 40$ & 5.085284 & -3.12664 & 3.802666 \\
\hline H41 & 3.558475 & -1.88942 & 5.329078 \\
\hline $\mathrm{C} 42$ & -3.21604 & 0.149191 & -0.76931 \\
\hline $\mathrm{C} 43$ & -4.35307 & 0.698841 & -0.16601 \\
\hline $\mathrm{C} 44$ & -3.23966 & -0.13806 & -2.14483 \\
\hline $\mathrm{C} 45$ & -5.4817 & 0.989378 & -0.93542 \\
\hline $\mathrm{H} 46$ & -4.3728 & 0.898673 & 0.898684 \\
\hline C47 & -4.36484 & 0.155491 & -2.90872 \\
\hline $\mathrm{C} 48$ & -5.48944 & 0.727952 & -2.30524 \\
\hline H49 & -6.35702 & 1.420363 & -0.45828 \\
\hline $\mathrm{H} 50$ & -4.36881 & -0.0755 & -3.9705 \\
\hline H51 & -6.37072 & 0.955871 & -2.89787 \\
\hline H52 & 0.506534 & 0.478395 & 0.902394 \\
\hline H53 & 1.710037 & 1.589955 & -2.53459 \\
\hline H54 & -1.48562 & -1.96425 & 2.666476 \\
\hline H55 & -2.38691 & -0.60952 & -2.61639 \\
\hline H56 & 2.746324 & -4.46048 & -1.00777 \\
\hline $\mathrm{C} 57$ & 4.857778 & -0.75288 & -2.90473 \\
\hline C58 & 3.661022 & -1.37934 & -3.25823 \\
\hline C59 & 2.865116 & -1.96667 & -2.27748 \\
\hline C60 & 3.241758 & -1.93305 & -0.92523 \\
\hline C61 & 4.43959 & -1.30007 & -0.5809 \\
\hline
\end{tabular}




\begin{tabular}{|c|c|c|c|}
\hline C62 & 5.243615 & -0.71936 & -1.56488 \\
\hline H63 & 5.483401 & -0.29608 & -3.66625 \\
\hline H64 & 3.344274 & -1.41114 & -4.29693 \\
\hline H65 & 1.92336 & -2.42025 & -2.56808 \\
\hline H66 & 4.742775 & & 0.45688 \\
\hline H67 & 6.169677 & -0.23022 & -1.27658 \\
\hline C68 & 1.252289 & 3.624233 & -2.96111 \\
\hline C69 & 0.798328 & 2.205177 & -2.52408 \\
\hline $\mathrm{C} 70$ & 0.19877 & 2.078314 & -1.09327 \\
\hline H71 & 1.01039 & 2.436468 & -0.45151 \\
\hline $\mathrm{C} 72$ & -0.96827 & 2.978197 & -0.78647 \\
\hline $\mathrm{C} 73$ & -2.1548 & 3.005133 & -1.53346 \\
\hline C74 & -0.87646 & 3.860325 & 0.306133 \\
\hline $\mathrm{C} 75$ & -3.19501 & 3.879935 & -1.21824 \\
\hline H76 & -2.29389 & 2.301844 & -2.34227 \\
\hline $\mathrm{C} 77$ & -1.91347 & 4.733877 & 0.63054 \\
\hline H78 & 0.024989 & 3.853163 & 0.91467 \\
\hline C79 & -3.08057 & 4.752087 & -0.13601 \\
\hline H80 & -4.10413 & 3.863717 & -1.81298 \\
\hline H81 & -1.80674 & & 1.478548 \\
\hline H82 & -3.88932 & 5.434266 & 0.109593 \\
\hline $\mathrm{C} 83$ & -0.84306 & 2.29292 & -4.61829 \\
\hline $\mathrm{C} 84$ & -0.3063 & 0.287301 & -3.59884 \\
\hline $\mathrm{C} 85$ & -1.27968 & 1.097398 & -5.48646 \\
\hline H86 & -0.23974 & 3.002965 & -5.18356 \\
\hline H87 & -1.68844 & 2.832203 & -4.18187 \\
\hline H88 & -2.33044 & 1.131039 & -5.77701 \\
\hline H89 & -0.65535 & 0.976095 & -6.37662 \\
\hline N90 & -0.06213 & 1.607128 & -3.57949 \\
\hline O91 & -1.084 & -0.06732 & -4.64158 \\
\hline O92 & 0.078425 & -0.57131 & -2.78415 \\
\hline H93 & 2.347715 & -4.71606 & 0.71895 \\
\hline N94 & -1.9804 & -2.14594 & 0.126764 \\
\hline C95 & -1.2918 & -3.00803 & -0.86222 \\
\hline H96 & -1.33228 & -2.59979 & -1.88146 \\
\hline C97 & 1.977854 & 4.37744 & -1.83597 \\
\hline H98 & 2.395184 & 5.310049 & -2.2282 \\
\hline H99 & 1.310213 & 4.634868 & -1.01275 \\
\hline H100 & 2.811512 & 3.78615 & -1.43867 \\
\hline C101 & 2.188216 & 3.536146 & -4.18202 \\
\hline H102 & 1.799453 & 2.905834 & -4.98741 \\
\hline H103 & 2.380186 & 4.530503 & -4.59647 \\
\hline H104 & 3.152754 & 3.110031 & -3.88171 \\
\hline H105 & 0.355333 & 4.203061 & -3.21728 \\
\hline H106 & 2.609021 & 0.994144 & -0.01636 \\
\hline
\end{tabular}




\section{References}

[1] Massaro, L.; Yang, J.; Krajangsri, S.; Silvi, E.; Singh, T.; Andersson, P. G. J. Org. Chem. 2019, 84, 21, 13540-13548

[2] Adam, W.; Bosio, S. G.; Turro, N. J. J. Am. Chem. Soc, 2002, 124(47),14004-14005.

[3] Gourdet, B; Lam, H. W. J. Am. Chem. Soc, 2009, 131(11), 3802-3803.

[4] Song, P.; Yu, P.; Lin, J. S.; Li, Y.; Yang, N. Y.; Liu, X. Y. Org. lett., 2017,19(6), 13301333.

[5] Tischler, A. N.; Tischler, M. H. Tetrahedron Lett., 1978, 19(37), 3407-3410.

[6] Terada, M.; Soga, K.; Momiyama, N. Angew. Chem. Int. Ed, 2008, 47(22), 4122-4125.

[7] Chang, L.; Kuang, Y.; Qin, B.; Zhou, X.; Liu, X.; Lin, L.; Feng, X. Org. lett., 2010, 12(10), 2214-2217.

[8] Kim, M. J.; Kim, W. H.; Han, K.; Choi, Y. K.; Park, J. Org. lett., 2007, 9(6), 1157-1159.

[9] Kotani, E.; Kobayashi, S.; Ishii, Y.; Tobinaga, S., Chem. Pharm. Bull., 1984, 32(11), 4281-4291.

[10] Roe, C.; Hobbs, H.; Stockman, R. A. J. Org. Chem. 2011. 76(22), 9452-9459.

[11] Dai, X.; Nakai, T.; Romero, J. A.; Fu, G. C. Angew. Chem., Int. Ed, 2007, $119(23)$, 44454447.

[12] Kameyama, M.; Kamigata, N. Bull. Chem. Soc. Jpn, 1987, 60(10), 3687-3691.

[13]Jaguar, version 7.9, Schrodinger, LLC, New York, NY, 2011.

[14] (a) Becke, A. D.; J. Chem. Phys. 1993, 98, 5648-5652. (b) Lee, C.; Yang, W.; Parr, R. G. Phys. Rev. B. 1988, 37, 785-789.

[15] Grimme, S.; Antony, J.; Ehrlich, S.; Krieg, H. J. Chem. Phys. 2010, 132, 154104.

[16] Hay, P. J.; Wadt, W. R. J. Chem. Phys. 1985, 82, 270-283.

[17] (a) Goodman, J. M.; Silva, M. A. Tetrahedron Lett. 2003, 44, 8233-8236. (b) Goodman, J. M.; Silva, M. A. Tetrahedron Lett. 2005, 46, 2067-2069. 